\title{
Foodstuff Concentrations and Relocation Considerations Following a Tritium Oxide Release from SRS Tritium Facilities
}

by

A. Blanchard

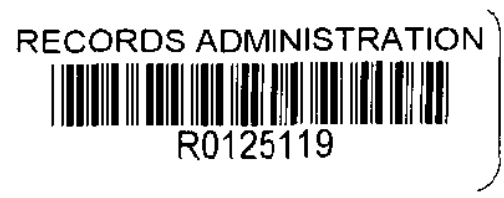

Westinghouse Savannah River Company

Savannah River Site

Aiken, South Carolina 29808

D. Marx

Westinghouse Safety Management Solutions

DOE Contract No. DE-AC09-96SR18500

This paper was prepared in connection with work done under the above contract number with the U. S.

Department of Energy. By acceptance of this paper, the publisher and/or recipient acknowledges the U. S.

Government's right to retain a nonexclusive, royalty-free license in and to any copyright covering this paper, along with the right to reproduce and to authorize others to reproduce all or part of the copyrighted paper. 
Keywords:

Ingestion Pathways

Tritium Oxide Release

Foodstuff Concentrations

Consequence Analysis

\section{Foodstuff Concentrations and Relocation Considerations Following a Tritium Oxide Release from SRS Tritium Facilities (U)}

D.R. Marx

April 1999

Westinghouse Savannah River Company Aiken, SC 29808

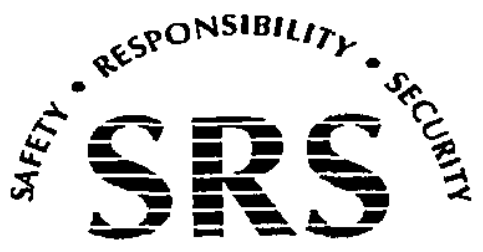

SAVANNAH RIVER SITE

PREPARED FOR THE U.S. DEPARTMENT OF ENERGY UNDER CONTRACT NO. DE-AC09-96SR18500 


\section{DISCLAIMER}

This report was prepared as an account of work sponsored by an agency of the United States Government. Neither the United States Government nor any agency thereof, nor any of their employees, makes any warranty, express or implied, or assumes any legal liability or responsibility for the accuracy, completeness, or usefulness of any information, apparatus, product, or process disclosed, or represents that its use would not infringe privately owned rights. Reference herein to any specific commercial product, process, or service by trade name, trademark, manufacturer, or otherwise does not necessarily constitute or imply its endorsement, recommendation, or favoring by the United States Government or any agency thereof. The views and opinions of authors expressed herein do not necessarily state or reflect those of the United States Government or any agency thereof.

This report has been reproduced directly from the best available copy.

Available to DOE and DOE contractors from the Office of Scientific and Technical Information, P.O. Box 62, Oak Ridge, TN 37831; prices available from (615) 576-8401.

Available to the public from the National Technical Information Service, U.S. Department of Commerce; 5285 Port Royal Road, Springfield, VA 22161. 
Foodstuff Concentrations and Relocation Considerations Following

WSRC-TR-99-00118

a Tritium Oxide Release from SRS Tritium Facilities (U)

This Page Intentionally Left Blank

iv 


\section{EXECUTIVE SUMMARY}

The ingestion pathway consequences following an accidental tritium release from the Savannah River Site (SRS) Tritium Facilities are evaluated. Two releases, the Design Basis Fire (scenario 4-RD-1.2) and the Beyond Design Basis Fire (scenario 4- RD-1.3), are examined for both average and adverse meteorological conditions.

Tritium concentrations in vegetables, wheat grain, meat, and milk are calculated using the tritium dispersion and dose assessment model UFOTRI. For the design basis fire with average and adverse meteorological conditions, the peak HTO and one week concentrations for all foodstuffs exceed the DIL at $100 \mathrm{~km}$. At one year for both meteorological conditions, wheat grain continues to exceed the DIL at $100 \mathrm{~km}$.

For the beyond design basis fire with average and adverse meteorological conditions, the peak HTO and one week concentrations for all foodstuffs exceed the DIL at $100 \mathrm{~km}$. At one year and average conditions, wheat grain exceeds the DIL at $100 \mathrm{~km}$ and the milk concentration at the site boundary is equal to the DIL. At one year and under adverse meteorological conditions, meat and milk exceed the DIL at the site boundary $(11.54 \mathrm{~km})$ and wheat grain exceeds the DIL at $100 \mathrm{~km}$.

Additionally, the possible need for relocation of individuals in the area surrounding SRS following the accidental releases is examined. For the beyond design basis fire with adverse meteorological conditions, relocation would be recommended for individuals living downwind between the site boundary and approximately $15 \mathrm{~km}$ from the release. 


\section{Table of Contents}

1.0 INTRODUCTION............................................................................................ 1

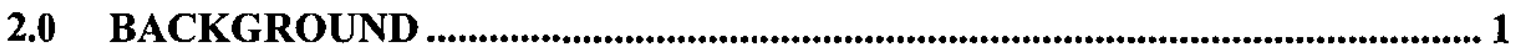

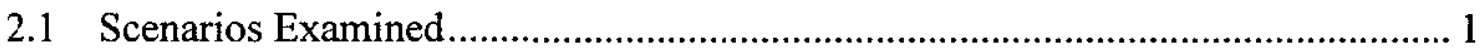

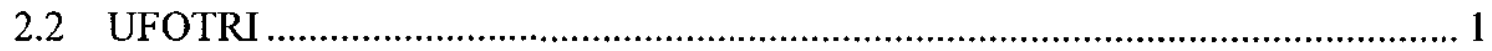

3.0 FDA GUIDANCE AND EVALUATION CRITERIA ..................................... 2

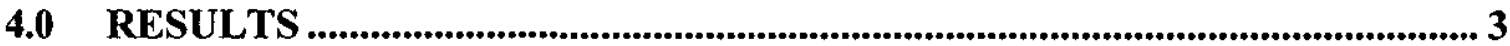

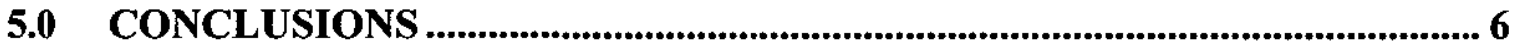

6.0 REFERENCES........................................................................................................ 7

APPENDIX A ANNUAL CONCENTRATIONS RESULTING IN EPA

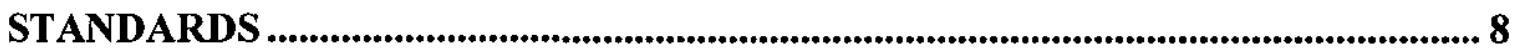

APPENDIX B RADIONUCLIDE CONCENTRATIONS IN FOODSTUFFS ......... 10 


\section{List of Tables}

Table 1. Input Parameters for Tritium Facilities Release Scenarios................... 1

Table 2. Meteorological Input Parameters for Tritium Facilities for Average and Adverse Conditions. 1

Table 3. Additional Input parameters for UFOTRI................................. 2

Table 4. Calculated DILs for HTO and OBT ..................................... 3

Table 5. Tritium Concentrations for DB Fire, Average Met Conditions (Max HTO)......4

Table 6. Tritium Concentrations for DB Fire, Average Met Conditions (1 week)........ 4

Table 7. Tritium Concentrations for DB Fire, Average Met Conditions (1 year)........ 4

Table 8. Tritium Concentrations for DB Fire, Adverse Met Conditions (Max HTO).....4

Table 9. Tritium Concentrations for DB Fire, Adverse Met Conditions (1 week)....... 4

Table 10. Tritium Concentrations for DB Fire, Adverse Met Conditions (1 year)........ 4

Table 11. Tritium Concentrations for BDB Fire, Average Met Conditions (Max HTO)...5

Table 12. Tritium Concentrations for BDB Fire, Average Met Conditions (1 week).....5

Table 13. Tritium Concentrations for BDB Fire, Average Met Conditions (1 year),..... 5

Table 14. Tritium Concentrations for BDB Fire, Adverse Met Conditions (Max HTO)... 5

Table 15. Tritium Concentrations for BDB Fire, Adverse Met Conditions (1 week)..... 5

Table 16. Tritium Concentrations for BDB Fire, Adverse Met Conditions (1 year).......5

Table 17. Inhalation and Skin Absorption Dose due to 1 year Re-emission..............6 


\section{$1.0 \quad$ INTRODUCTION}

Hypothetical accidental releases of radionuclides from nuclear facilities may cause contamination of food and forage. Numerous pathways exist for deposited radionuclides to reach man. The major pathways considered by recent Food and Drug Administration (FDA) guidance (FDA 1998) are analyzed in this report for two accidental tritium releases from the Savannah River Site (SRS) Tritium Facilities. Additionally, the potential need for relocation of individuals living in the area surrounding SRS is examined.

\subsection{BACKGROUND}

The scenarios examined and the tritium dispersion and dose assessment model UFOTRI are described below.

\subsection{Scenarios Examined}

Two accidental release scenarios, both with average and adverse meteorological conditions, are examined. The release scenarios, 4-RD-1.2 and 4-RD-1.3, are design basis (DB) and beyond design basis (BDB) fires, respectively, and are described in the Tritium Facilities EPHA, SEHA-H-00006 (Davis 1998). Parameters describing the two scenarios are listed in Table 1.

Table 1. Input Parameters for Tritium Facilities

Release Scenarios 4-RD-1.2 and 4-RD-1.3

\begin{tabular}{|l|c|c|}
\hline Parameter & DB Fire (4-RD-1.2) & BDB Fire* (4-RD-1.3) \\
\hline Release Amount & $1.9 \mathrm{E}+7 \mathrm{Ci}$ & $1.0 \mathrm{E}+8 \mathrm{Ci}$ \\
\hline Chemical Form & Tritium Oxide & Tritium Oxide \\
\hline Release Height & $200 \mathrm{ft}$ & $200 \mathrm{ft}$ \\
\hline Release Duration & $30 \mathrm{~min}$ & $30 \mathrm{~min}$ \\
\hline \multicolumn{2}{|l}{${ }^{*}$ BDB Fire has an estimated frequency of less than $10^{-6}$ per year } \\
\hline
\end{tabular}

Parameters for the average and adverse meteorological conditions are listed in Table 2 (Davis 1998).

Table 2. Meteorological Input Parameters for Tritium Facilities for Average and Adverse Conditions

\begin{tabular}{|l|c|c|}
\hline Parameter & Average Met. & Adverse Met. \\
\hline Release Height & $200 \mathrm{ft}$ & $200 \mathrm{ft}$ \\
\hline Wind Speed Height & $10 \mathrm{~m}$ & $10 \mathrm{~m}$ \\
\hline Stability Class & $\mathrm{C}$ & $\mathrm{E}$ \\
\hline Wind Speed & $2.5 \mathrm{~m} / \mathrm{s}$ & $1.7 \mathrm{~m} / \mathrm{s}$ \\
\hline Inversion Layer & $500 \mathrm{~m}$ & $200 \mathrm{~m}$ \\
\hline Temperature & $25^{\circ} \mathrm{C}$ & $29^{\circ} \mathrm{C}$ \\
\hline
\end{tabular}

\section{$2.2 \quad$ UFOTRI}

The tritium dispersion and dose assessment model UFOTRI (Unfallfolgenmodell für Tritiumfreisetzungen) has been chosen to model the SRS Tritium Facilities release. UFOTRI was developed at the German Karlsruhe laboratory to assess the radiological consequences of accidental atmospheric tritium releases (Raskob 1990, 1993). UFOTRI explicitly addresses all 
applicable biophysics of tritium dispersion, its interaction in the environment, and the ultimate uptake by downwind receptors. UFOTRI realistically models the atmospheric dispersion, resuspension, and downwind deposition of tritium. Additionally, UFOTRI couples the shortterm atmospheric dispersion model with a first-order compartment module that dynamically describes the long-term behavior of tritium in the environment (Raskob 1990, 1993).

Table 3 provides a listing of some of the additional parameters required in UFOTRI (the input file for the beyond design basis fire with average meteorological parameters is located in Appendix A).

Table 3. Additional Input parameters for UFOTRI

\begin{tabular}{|c|c|c|c|}
\hline \multicolumn{2}{|c|}{ Additional Information } & \multicolumn{2}{|l|}{ Meteorology } \\
\hline Chemical Forms & $0 \% \mathrm{HT} / 100 \%$ HTO & No Rainfall & $0 \mathrm{~mm} / \mathrm{h}$ \\
\hline \multicolumn{2}{|c|}{ No building wake effect model assumed } & Thermal energy of release & $0 \mathrm{cal} / \mathrm{sec}$ \\
\hline Initial soil water conten & $35 \%$ & Wind direction (in degrees) & 315 \\
\hline Relative humidity & 0.8 & Dispersion parameters & Briggs \\
\hline \multicolumn{2}{|l|}{ Soil and vegetation } & \multicolumn{2}{|c|}{ Code coefficients } \\
\hline Soil type & Sandy loam & HTO dry deposition velocity* & $0.5 \mathrm{~cm} / \mathrm{s}$ \\
\hline Pore volume (default) & $50 \%$ & HT dry deposition velocity* & $0.05 \mathrm{~cm} / \mathrm{s}$ \\
\hline
\end{tabular}

\subsection{FDa Guidance and Evaluation Criteria}

The Food and Drug Administration (FDA) Derived Intervention Levels (DILs) are limits on the concentrations permitted in human food distributed in commerce to prevent consumption of undesirable amounts of radionuclides and apply in the first year after an accident (FDA 1998). The DIL corresponds to the concentration in food present throughout the relevant period of time that, in the absence of any intervention, could lead to an individual receiving a radiation dose equal to the Protective Action Guide (PAG). The PAGs for the ingestion pathway are $0.5 \mathrm{rem}$ committed effective dose equivalent or 5 rem committed dose equivalent to an individual tissue or organ, whichever is most limiting.

FDA (1998) places selected radionuclides into five primary groups, each with common characteristics. These groups are:
- $\mathrm{Sr}-90$
- I-131
- $\mathrm{Cs}-134+\mathrm{Cs}-137$

- $\mathrm{Pu}-238+\mathrm{Pu}-239+\mathrm{Am}-241$

- Ru-103 + Ru-106

The five radionuclide groups are not sufficient to cover accidents at all types of facilities or locations. Appendix E of FDA (1998) presents DILs for 15 additional radionuclides. DILs for tritiated water (HTO) and organically bound tritium (OBT) were derived using methodology consistent with FDA (1998) and are listed in Table 4 (Tharakan 1999). 
Table 4 Calculated DILs for HTO and OBT

\begin{tabular}{|c|c|c|c|}
\hline $\begin{array}{c}\text { Radionuclide } \\
\text { Group }\end{array}$ & DIL (Ci/g) & $\begin{array}{c}\text { Radionuclide } / \\
\text { Group }\end{array}$ & DIL (Ci $/ \mathrm{g})$ \\
\hline HTO & $5.9 \mathrm{E}-09$ & OBT & $2.4 \mathrm{E}-09$ \\
\hline
\end{tabular}

To evaluate the release, calculated concentrations in vegetables, wheat grain, meat, and milk for multiple distances and times following the release will be compared with the HTO and OBT DILs. Tritium concentrations in grass, although calculated in UFOTRI, were not examined since grass is not directly consumed by humans. However, tritium concentrations in grass are indirectly incorporated via the meat and milk pathways. In all instances, the HTO and OBT concentrations will be summed prior to comparison with the appropriate DIL.

For short-term concentrations, the time at which the peak HTO concentration occurs in each food type will be examined. The summed HTO and OBT concentrations will be compared with the HTO DIL. In the time immediately following the release, the HTO concentration quickly reaches its peak and is the dominant form of tritium present in foodstuffs.

For long-term concentrations, two evaluation times - one week (168 hours) and one year ( 8760 hours) - will be examined. The OBT DIL will be used for these comparisons because the tritium will be incorporated into carbon compounds that have a greater biological half-life than HTO. For plants, the HTO half-life is approximately one hour while the OBT half-life ranges from 8 hours to over 10 days. Examination of long-term concentrations is useful to determine if intervention is still required.

Concentrations for both the short and long-term will be examined at the facility boundary $(0.15$ $\mathrm{km}$ ), site boundary $(11.54 \mathrm{~km}), 1 \mathrm{~km}, 10 \mathrm{~km}, 50 \mathrm{~km}$, and $100 \mathrm{~km}$ from the release. The facility and site boundary concentrations may be used to determine if any intervention will be necessary. The $100 \mathrm{~km}$ value is used for comparison with the particulate concentrations. (The code used to calculate concentrations for particulates, HOTSPOT, calculates up to a maximum distance of 100 $\mathrm{km}$.) The remaining distances are chosen for additional examination.

In addition to examining vegetation concentration, the potential need for relocation of individuals living in the area surround SRS will be examined. Relocation is necessary if the 1 year inhalation and skin absorption dose due to resuspension of tritium exceeds 2 rem. In UFOTRI, this is calculated as the difference between the inhalation dose with re-emission and dose due to the HTO plume.

\subsection{Results}

Calculated tritium concentrations for average and adverse meteorological conditions for the design basis fire are presented in Tables 5 to 7 and 8 to 10, respectively, and in Tables 11 to 13 and 14 to 16 for the beyond design basis fire (summarized from Appendix B). Concentrations listed are the sum of the HTO and OBT concentrations. Values in bold surpass the appropriate DIL. 
Table 5. Tritium Concentrations for Design Basis Fire, Average Met Conditions (Max HTO)

\begin{tabular}{lccccccc}
\hline Pathway & $\mathrm{DIL}$ & $0.15 \mathrm{~km}$ & $1 \mathrm{~km}$ & $10 \mathrm{~km}$ & $11.54 \mathrm{~km}$ & $50 \mathrm{~km}$ & $100 \mathrm{~km}$ \\
& $\mathrm{Ci} / \mathrm{g}$ & $\mathrm{Ci} / \mathrm{g}$ & $\mathrm{Ci} / \mathrm{g}$ & $\mathrm{Ci} / \mathrm{g}$ & $\mathrm{Ci} / \mathrm{g}$ & $\mathrm{Ci} / \mathrm{g}$ & $\mathrm{C} / \mathrm{g}$ \\
\hline Vegetable & $5.9 \mathrm{E}-09$ & $\mathbf{1 . 2 E - 0 3}$ & $\mathbf{2 . 6 E - 0 4}$ & $\mathbf{1 . 3 E - 0 5}$ & $\mathbf{1 . 3 E - 0 5}$ & $\mathbf{3 . 9 2 E - 0 6}$ & $\mathbf{1 . 5 E - 0 6}$ \\
Wheat grain & $5.9 \mathrm{E}-09$ & $\mathbf{5 . 5 E - 0 4}$ & $\mathbf{1 . 2 E}-04$ & $\mathbf{6 . 6 E}-06$ & $\mathbf{6 . 4 E}-06$ & $\mathbf{1 . 9 7 E - 0 6}$ & $\mathbf{9 . 9 E - 0 7}$ \\
Meat & $5.9 \mathrm{E}-09$ & $\mathbf{5 . 3 E - 0 5}$ & $\mathbf{1 . 1 E}-05$ & $\mathbf{6 . 7 E - 0 7}$ & $\mathbf{6 . 4 E - 0 7}$ & $\mathbf{1 . 2 0 E - 0 7}$ & $\mathbf{4 . 0 E - 0 8}$ \\
Milk & $5.9 \mathrm{E}-09$ & $\mathbf{6 . 7 E - 0 5}$ & $\mathbf{1 . 4 E - 0 5}$ & $\mathbf{8 . 5 E - 0 7}$ & $\mathbf{8 . 1 E - 0 7}$ & $\mathbf{1 . 5 1 E - 0 7}$ & $\mathbf{5 . 0 E - 0 8}$ \\
\hline
\end{tabular}

Table 6. Tritium Concentrations for Design Basis Fire, Average Met Conditions (1 week)

\begin{tabular}{lccccccc}
\hline Pathway & DIL & $0.15 \mathrm{~km}$ & $1 \mathrm{~km}$ & $10 \mathrm{~km}$ & $11.54 \mathrm{~km}$ & $50 \mathrm{~km}$ & $100 \mathrm{~km}$ \\
& $\mathrm{Ci} / \mathrm{g}$ & $\mathrm{Ci} / \mathrm{g}$ & $\mathrm{Ci} / \mathrm{g}$ & $\mathrm{Ci} / \mathrm{g}$ & $\mathrm{Ci} / \mathrm{g}$ & $\mathrm{Ci} / \mathrm{g}$ & $\mathrm{Ci} / \mathrm{g}$ \\
\hline Vegetable & $2.4 \mathrm{E}-09$ & $\mathbf{1 . 4 E - 0 5}$ & $\mathbf{3 . 4 E}-06$ & $\mathbf{2 . 4 E - 0 7}$ & $\mathbf{2 . 5 E}-07$ & $\mathbf{7 . 8 E}-08$ & $\mathbf{4 . 5 E - 0 8}$ \\
Wheat grain & $2.4 \mathrm{E}-09$ & $\mathbf{9 . 6 E - 0 6}$ & $\mathbf{2 . 2 E - 0 6}$ & $\mathbf{1 . 6 E - 0 7}$ & $\mathbf{1 . 7 E - 0 7}$ & $\mathbf{5 . 2 E}-08$ & $\mathbf{3 . 1 E}-08$ \\
Meat & $2.4 \mathrm{E}-09$ & $\mathbf{1 . 9 E - 0 5}$ & $\mathbf{4 . 3 E}-06$ & $\mathbf{2 . 9 E - 0 7}$ & $\mathbf{2 . 9 E}-07$ & $\mathbf{7 . 4 E - 0 8}$ & $\mathbf{3 . 6 E - 0 8}$ \\
Milk & $2.4 \mathrm{E}-09$ & $\mathbf{3 . 3 E - 0 5}$ & $\mathbf{7 . 6 E - 0 6}$ & $\mathbf{5 . 1 E - 0 7}$ & $\mathbf{5 . 2 E - 0 7}$ & $\mathbf{1 . 4 E - 0 7}$ & $\mathbf{6 . 9 E - 0 8}$ \\
\hline
\end{tabular}

Table 7. Tritium Concentrations for Design Basis Fire, Average Met Conditions (1 year)

\begin{tabular}{lccccccc}
\hline Pathway & DIL & $\begin{array}{c}0.15 \mathrm{~km} \\
\mathrm{Ci} / \mathrm{g}\end{array}$ & $\begin{array}{c}1 \mathrm{~km} \\
\mathrm{Ci} / \mathrm{g}\end{array}$ & $\begin{array}{c}10 \mathrm{~km} \\
\mathrm{Ci} / \mathrm{g}\end{array}$ & $\begin{array}{c}11.54 \mathrm{~km} \\
\mathrm{Ci} / \mathrm{g}\end{array}$ & $\begin{array}{c}50 \mathrm{~km} \\
\mathrm{Ci} / \mathrm{g}\end{array}$ & $\begin{array}{c}100 \mathrm{~km} \\
\mathrm{Ci} / \mathrm{g}\end{array}$ \\
\hline Vegetable & $2.4 \mathrm{E}-09$ & $9.8 \mathrm{E}-10$ & $2.3 \mathrm{E}-10$ & $1.5 \mathrm{E}-11$ & $1.6 \mathrm{E}-11$ & $4.7 \mathrm{E}-12$ & $2.6 \mathrm{E}-12$ \\
Wheat grain & $2.4 \mathrm{E}-09$ & $\mathbf{6 . 3 E - 0 6}$ & $\mathbf{1 . 5 E}-06$ & $\mathbf{1 . 0 E}-07$ & $\mathbf{1 . 1 E - 0 7}$ & $\mathbf{3 . 4 E}-\mathbf{0 8}$ & $\mathbf{2 . 0 E}-08$ \\
Meat & $2.4 \mathrm{E}-09$ & $\mathbf{2 . 1 E - 0 8}$ & $\mathbf{4 . 8 E - 0 9}$ & $3.3 \mathrm{E}-10$ & $3.4 \mathrm{E}-10$ & $1.0 \mathrm{E}-10$ & $5.8 \mathrm{E}-11$ \\
Milk & $2.4 \mathrm{E}-09$ & $\mathbf{3 . 3 E - 0 8}$ & $\mathbf{7 . 7 E - 0 9}$ & $5.2 \mathrm{E}-10$ & $5.4 \mathrm{E}-10$ & $1.6 \mathrm{E}-10$ & $9.2 \mathrm{E}-11$ \\
\hline
\end{tabular}

Table 8. Tritium Concentrations for Design Basis Fire, Adverse Met Conditions (Max HTO)

\begin{tabular}{lccccccc}
\hline Pathway & DIL & $0.15 \mathrm{~km}$ & $1 \mathrm{~km}$ & $10 \mathrm{~km}$ & $11.54 \mathrm{~km}$ & $50 \mathrm{~km}$ & $100 \mathrm{~km}$ \\
& $\mathrm{Ci} / \mathrm{g}$ & $\mathrm{Ci} / \mathrm{g}$ & $\mathrm{Ci} / \mathrm{g}$ & $\mathrm{Ci} / \mathrm{g}$ & $\mathrm{Ci} / \mathrm{g}$ & $\mathrm{Ci} / \mathrm{g}$ & $\mathrm{Ci} / \mathrm{g}$ \\
\hline Vegetable & $5.9 \mathrm{E}-09$ & $\mathbf{1 . 8 E - 0 5}$ & $\mathbf{5 . 6 E - 0 4}$ & $\mathbf{4 . 8 E - 0 5}$ & $\mathbf{5 . 0 E}-05$ & $\mathbf{1 . 2 E - 0 5}$ & $\mathbf{5 . 1 E}-06$ \\
Wheat grain & $5.9 \mathrm{E}-09$ & $\mathbf{5 . 6 E - 0 6}$ & $\mathbf{1 . 7 E - 0 4}$ & $\mathbf{1 . 5 E - 0 5}$ & $\mathbf{1 . 4 E}-05$ & $\mathbf{3 . 9 E}-06$ & $1.7 \mathrm{E}-06$ \\
Meat & $5.9 \mathrm{E}-09$ & $\mathbf{6 . 8 E - 0 7}$ & $\mathbf{2 . 1 E - 0 5}$ & $\mathbf{1 . 9 E - 0 6}$ & $\mathbf{1 . 7 E - 0 6}$ & $\mathbf{2 . 8 E}-07$ & $\mathbf{8 . 9 E}-08$ \\
Milk & $5.9 \mathrm{E}-09$ & $\mathbf{8 . 7 E - 0 7}$ & $\mathbf{2 . 7 E - 0 5}$ & $\mathbf{2 . 4 E - 0 6}$ & $\mathbf{2 . 2 E - 0 6}$ & $\mathbf{3 . 7 E - 0 7}$ & $\mathbf{1 . 1 E - 0 7}$ \\
\hline
\end{tabular}

Table 9. Tritium Concentrations for Design Basis Fire, Adverse Met Conditions (1 week)

\begin{tabular}{lccccccc}
\hline Pathway & DIL & $0.15 \mathrm{~km}$ & $1 \mathrm{~km}$ & $10 \mathrm{~km}$ & $11.54 \mathrm{~km}$ & $50 \mathrm{~km}$ & $100 \mathrm{~km}$ \\
& $\mathrm{Ci} / \mathrm{g}$ & $\mathrm{Ci} / \mathrm{g}$ & $\mathrm{Ci} / \mathrm{g}$ & $\mathrm{Ci} / \mathrm{g}$ & $\mathrm{Ci} / \mathrm{g}$ & $\mathrm{Ci} / \mathrm{g}$ & $\mathrm{Ci} / \mathrm{g}$ \\
\hline Vegetable & $2.4 \mathrm{E}-09$ & $\mathbf{2 . 2 E - 0 7}$ & $\mathbf{7 . 0 E}-06$ & $\mathbf{8 . 2 E - 0 7}$ & $\mathbf{9 . 0 E - 0 7}$ & $\mathbf{2 . 5 E - 0 7}$ & $\mathbf{1 . 2 E - 0 7}$ \\
Wheat grain & $2.4 \mathrm{E}-09$ & $\mathbf{1 . 1 E - 0 7}$ & $\mathbf{3 . 6 E - 0 6}$ & $\mathbf{4 . 5 E - 0 7}$ & $\mathbf{5 . 1 E - 0 7}$ & $\mathbf{1 . 5 E - 0 7}$ & $\mathbf{8 . 6 E - 0 8}$ \\
Meat & $2.4 \mathrm{E}-09$ & $\mathbf{2 . 6 E - 0 7}$ & $\mathbf{8 . 1 E - 0 6}$ & $\mathbf{9 . 1 E - 0 7}$ & $\mathbf{9 . 6 E - 0 7}$ & $\mathbf{2 . 2 E - 0 7}$ & $1.0 \mathrm{E}-07$ \\
Milk & $2.4 \mathrm{E}-09$ & $\mathbf{4 . 3 E - 0 7}$ & $\mathbf{1 . 4 E - 0 5}$ & $\mathbf{1 . 5 E - 0 6}$ & $\mathbf{1 . 6 E - 0 6}$ & $\mathbf{3 . 9 E - 0 7}$ & $\mathbf{1 . 9 E - 0 7}$ \\
\hline
\end{tabular}

Table 10. Tritium Concentrations for Design Basis Fire, Adverse Met Conditions (1 year)

\begin{tabular}{|c|c|c|c|c|c|c|c|}
\hline Pathway & $\begin{array}{l}\mathrm{DIL} \\
\mathrm{Ci} / \mathrm{g}\end{array}$ & $\begin{array}{c}0.15 \mathrm{~km} \\
\mathrm{Ci} / \mathrm{g}\end{array}$ & $\begin{array}{l}1 \mathrm{~km} \\
\mathrm{Ci} / \mathrm{g}\end{array}$ & $\begin{array}{c}10 \mathrm{~km} \\
\mathrm{Ci} / \mathrm{g}\end{array}$ & $\begin{array}{c}11.54 \mathrm{~km} \\
\mathrm{Ci} / \mathrm{g}\end{array}$ & $\begin{array}{c}50 \mathrm{~km} \\
\mathrm{Ci} / \mathrm{g}\end{array}$ & $\begin{array}{c}100 \mathrm{~km} \\
\mathrm{Ci} / \mathrm{g}\end{array}$ \\
\hline Vegetable & $2.4 \mathrm{E}-09$ & $1.2 \mathrm{E}-11$ & $3.8 \mathrm{E}-10$ & $4.2 \mathrm{E}-11$ & $4.6 \mathrm{E}-11$ & $1.2 \mathrm{E}-11$ & $5.79 \mathrm{E}-12$ \\
\hline Wheat grain & 2.4 E-09 & $7.4 \mathrm{E}-08$ & $2.4 E-06$ & 2.8E-07 & $3.1 \mathrm{E}-07$ & 8.6E-08 & $4.42 \mathrm{E}-08$ \\
\hline Meat & 2.4 E-09 & $2.4 \mathrm{E}-10$ & $7.8 E-09$ & $9.0 \mathrm{E}-10$ & $9.8 \mathrm{E}-10$ & $2.6 \mathrm{E}-10$ & $1.33 \mathrm{E}-10$ \\
\hline Milk & 2.4 E-09 & $3.8 \mathrm{E}-10$ & 1.2E-08 & $1.4 \mathrm{E}-09$ & $1.5 \mathrm{E}-09$ & 4.1E-10 & $2.04 \mathrm{E}-10$ \\
\hline
\end{tabular}


Table 11. Tritium Concentrations for Beyond Design Basis Fire, Average Met Conditions (Max HTO)

\begin{tabular}{lccccccc}
\hline Pathway & DIL & $\begin{array}{c}0.15 \mathrm{~km} \\
\mathrm{Ci} / \mathrm{g}\end{array}$ & $\begin{array}{c}1 \mathrm{~km} \\
\mathrm{Ci} / \mathrm{g}\end{array}$ & $\begin{array}{c}10 \mathrm{~km} \\
\mathrm{Ci} / \mathrm{g}\end{array}$ & $\begin{array}{c}11.54 \mathrm{~km} \\
\mathrm{Ci} / \mathrm{g}\end{array}$ & $\begin{array}{c}50 \mathrm{~km} \\
\mathrm{Ci} / \mathrm{g}\end{array}$ & $\begin{array}{c}100 \mathrm{~km} \\
\mathrm{Ci} / \mathrm{g}\end{array}$ \\
\hline Vegetable & $5.9 \mathrm{E}-09$ & $\mathbf{4 . 4 E - 0 3}$ & $\mathbf{1 . 0 E}-03$ & $\mathbf{5 . 3 E}-05$ & $\mathbf{5 . 2 E - 0 5}$ & $\mathbf{1 . 5 E - 0 5}$ & $\mathbf{5 . 9 E - 0 6}$ \\
Wheat grain & $5.9 \mathrm{E}-09$ & $\mathbf{2 . 5 E}-03$ & $\mathbf{5 . 3 E}-04$ & $\mathbf{3 . 0 E}-05$ & $\mathbf{3 . 0 E}-05$ & $\mathbf{9 . 3 E}-06$ & $\mathbf{4 . 5 E - 0 6}$ \\
Meat & $5.9 \mathrm{E}-09$ & $\mathbf{2 . 2 E - 0 4}$ & $\mathbf{4 . 8 E}-05$ & $\mathbf{2 . 8 E - 0 6}$ & $\mathbf{2 . 7 E - 0 6}$ & $\mathbf{5 . 5 E}-07$ & $\mathbf{1 . 8 E - 0 7}$ \\
Milk & $5.9 \mathrm{E}-09$ & $\mathbf{2 . 8 E - 0 4}$ & $\mathbf{6 . 0 E}-05$ & $\mathbf{3 . 6 E}-06$ & $\mathbf{3 . 4 E - 0 6}$ & $\mathbf{6 . 9 E - 0 7}$ & $\mathbf{2 . 2 E - 0 7}$ \\
\hline
\end{tabular}

Table 12. Tritium Concentrations for Beyond Design Basis Fire, Average Met Conditions (1 week)

\begin{tabular}{lccccccc}
\hline Pathway & DIL & $0.15 \mathrm{~km}$ & $1 \mathrm{~km}$ & $10 \mathrm{~km}$ & $11.54 \mathrm{~km}$ & $50 \mathrm{~km}$ & $100 \mathrm{~km}$ \\
& $\mathrm{Ci} / \mathrm{g}$ & $\mathrm{Ci} / \mathrm{g}$ & $\mathrm{Ci} / \mathrm{g}$ & $\mathrm{Ci} / \mathrm{g}$ & $\mathrm{Ci} / \mathrm{g}$ & $\mathrm{Ci} / \mathrm{g}$ & $\mathrm{Ci} / \mathrm{g}$ \\
\hline Vegetable & $2.4 \mathrm{E}-09$ & $\mathbf{6 . 6 E - 0 5}$ & $\mathbf{1 . 5 E - 0 5}$ & $\mathbf{1 . 1 E - 0 6}$ & $\mathbf{1 . 1 E - 0 6}$ & $\mathbf{3 . 5 E - 0 7}$ & $\mathbf{2 . 1 E - 0 7}$ \\
Wheat grain & $2.4 \mathrm{E}-09$ & $\mathbf{4 . 5 E - 0 5}$ & $\mathbf{1 . 0 E - 0 5}$ & $\mathbf{7 . 3 E}-07$ & $\mathbf{7 . 7 E - 0 7}$ & $\mathbf{2 . 5 E - 0 7}$ & $\mathbf{1 . 5 E - 0 7}$ \\
Meat & $2.4 \mathrm{E}-09$ & $\mathbf{8 . 7 E - 0 5}$ & $\mathbf{2 . 0 E - 0 5}$ & $\mathbf{1 . 3 E - 0 6}$ & $\mathbf{1 . 3 E - 0 6}$ & $\mathbf{3 . 5 E - 0 7}$ & $\mathbf{1 . 7 E - 0 7}$ \\
Milk & $2.4 \mathrm{E}-09$ & $\mathbf{1 . 5 E - 0 4}$ & $\mathbf{3 . 4 E - 0 5}$ & $\mathbf{2 . 3 E - 0 6}$ & $\mathbf{2 . 3 E - 0 6}$ & $\mathbf{6 . 3 E - 0 7}$ & $\mathbf{3 . 1 E - 0 7}$ \\
\hline
\end{tabular}

Table 13. Tritium Concentrations for Beyond Design Basis Fire, Average Met Conditions (1 year)

\begin{tabular}{lccccccc}
\hline Pathway & $\mathrm{DIL}$ & $\mathbf{0 . 1 5} \mathrm{km}$ & $1 \mathrm{~km}$ & $10 \mathrm{~km}$ & $11.54 \mathrm{~km}$ & $50 \mathrm{~km}$ & $100 \mathrm{~km}$ \\
& $\mathrm{Ci} / \mathrm{g}$ & $\mathrm{Ci} / \mathrm{g}$ & $\mathrm{Ci} / \mathrm{g}$ & $\mathrm{Ci} / \mathrm{g}$ & $\mathrm{Ci} / \mathrm{g}$ & $\mathrm{Ci} / \mathrm{g}$ & $\mathrm{Ci} / \mathrm{g}$ \\
\hline Vegetable & $2.4 \mathrm{E}-09$ & $\mathbf{4 . 8 E - 0 9}$ & $1.1 \mathrm{E}-09$ & $7.5 \mathrm{E}-11$ & $7.8 \mathrm{E}-11$ & $2.3 \mathrm{E}-11$ & $1.3 \mathrm{E}-11$ \\
Wheat grain & $2.4 \mathrm{E}-09$ & $\mathbf{2 . 8 E - 0 5}$ & $\mathbf{6 . 5 E}-06$ & $\mathbf{4 . 5 E}-07$ & $\mathbf{4 . 7 E - 0 7}$ & $\mathbf{1 . 5 E}-07$ & $\mathbf{8 . 8 E}-08$ \\
Meat & $2.4 \mathrm{E}-09$ & $\mathbf{9 . 1 E - 0 8}$ & $\mathbf{2 . 1 E}-08$ & $1.4 \mathrm{E}-09$ & $1.5 \mathrm{E}-09$ & $4.6 \mathrm{E}-10$ & $2.6 \mathrm{E}-10$ \\
Milk & $2.4 \mathrm{E}-09$ & $\mathbf{1 . 5 E - 0 7}$ & $\mathbf{3 . 4 E - 0 8}$ & $2.3 \mathrm{E}-09$ & $\mathbf{2 . 4 E - 0 9}$ & $7.3 \mathrm{E}-10$ & $4.2 \mathrm{E}-10$ \\
\hline
\end{tabular}

Table 14. Tritium Concentrations for Beyond Design Basis Fire, Adverse Met Conditions (Max HTO)

\begin{tabular}{lccccccc}
\hline Pathway & $\mathrm{DIL}$ & $0.15 \mathrm{~km}$ & $1 \mathrm{~km}$ & $10 \mathrm{~km}$ & $11.54 \mathrm{~km}$ & $50 \mathrm{~km}$ & $100 \mathrm{~km}$ \\
& $\mathrm{Ci} / \mathrm{g}$ & $\mathrm{Ci} / \mathrm{g}$ & $\mathrm{Ci} / \mathrm{g}$ & $\mathrm{Ci} / \mathrm{g}$ & $\mathrm{Ci} / \mathrm{g}$ & $\mathrm{Ci} / \mathrm{g}$ & $\mathrm{Ci} / \mathrm{g}$ \\
\hline Vegetable & $5.9 \mathrm{E}-09$ & $9.5 \mathrm{E}-05$ & $\mathbf{3 . 0 E}-03$ & $\mathbf{2 . 5 E}-04$ & $\mathbf{2 . 6 E - 0 4}$ & $\mathbf{6 . 5 E}-05$ & $\mathbf{2 . 7 E - 0 5}$ \\
Wheat grain & $5.9 \mathrm{E}-09$ & $\mathbf{2 . 9 E - 0 5}$ & $\mathbf{9 . 0 E - 0 4}$ & $\mathbf{7 . 9 E}-05$ & $\mathbf{7 . 6 E - 0 5}$ & $\mathbf{2 . 1 E - 0 5}$ & $\mathbf{8 . 7 E - 0 6}$ \\
Meat & $5.9 \mathrm{E}-09$ & $\mathbf{3 . 6 E - 0 6}$ & $\mathbf{1 . 1 E - 0 4}$ & $\mathbf{9 . 9 E}-06$ & $\mathbf{9 . 0 E - 0 6}$ & $\mathbf{1 . 5 E}-06$ & $\mathbf{4 . 7 E - 0 7}$ \\
Milk & $5.9 \mathrm{E}-09$ & $\mathbf{4 . 6 E - 0 6}$ & $\mathbf{1 . 4 E - 0 4}$ & $\mathbf{1 . 2 E - 0 5}$ & $\mathbf{1 . 1 E - 0 5}$ & $\mathbf{1 . 9 E - 0 6}$ & $\mathbf{5 . 9 E - 0 7}$ \\
\hline
\end{tabular}

Table 15. Tritium Concentrations for Beyond Design Basis Fire, Adverse Met Conditions (1 week)

\begin{tabular}{lccccccc}
\hline Pathway & $\mathrm{DIL}$ & $0.15 \mathrm{~km}$ & $1 \mathrm{~km}$ & $10 \mathrm{~km}$ & $11.54 \mathrm{~km}$ & $50 \mathrm{~km}$ & $100 \mathrm{~km}$ \\
& $\mathrm{Ci} / \mathrm{g}$ & $\mathrm{Ci} / \mathrm{g}$ & $\mathrm{Ci} / \mathrm{g}$ & $\mathrm{Ci} / \mathrm{g}$ & $\mathrm{Ci} / \mathrm{g}$ & $\mathrm{Ci} / \mathrm{g}$ & $\mathrm{C} / \mathrm{g}$ \\
\hline Vegetable & $2.4 \mathrm{E}-09$ & $\mathbf{1 . 1 E - 0 6}$ & $\mathbf{3 . 7 E - 0 5}$ & $\mathbf{4 . 3 E - 0 6}$ & $\mathbf{4 . 7 E - 0 6}$ & $\mathbf{1 . 3 E}-06$ & $\mathbf{6 . 5 E - 0 7}$ \\
Wheat grain & $2.4 \mathrm{E}-09$ & $\mathbf{5 . 8 E - 0 7}$ & $\mathbf{1 . 9 E - 0 5}$ & $\mathbf{2 . 4 E - 0 6}$ & $\mathbf{2 . 7 E - 0 6}$ & $\mathbf{8 . 0 E - 0 7}$ & $\mathbf{4 . 5 E - 0 7}$ \\
Meat & $2.4 \mathrm{E}-09$ & $\mathbf{1 . 3 E - 0 6}$ & $\mathbf{4 . 3 E - 0 5}$ & $\mathbf{4 . 8 E - 0 6}$ & $\mathbf{5 . 1 E - 0 6}$ & $\mathbf{1 . 2 E - 0 6}$ & $\mathbf{5 . 5 E - 0 7}$ \\
Milk & $2.4 \mathrm{E}-09$ & $\mathbf{2 . 2 E - 0 6}$ & $\mathbf{7 . 2 E - 0 5}$ & $\mathbf{8 . 1 E - 0 6}$ & $\mathbf{8 . 6 E - 0 6}$ & $\mathbf{2 . 1 E - 0 6}$ & $\mathbf{1 . 0 E - 0 6}$ \\
\hline
\end{tabular}

Table 16. Tritium Concentrations for Beyond Design Basis Fire, Adverse Met Conditions (1 year)

\begin{tabular}{lccccccc}
\hline Pathway & DIL & $0.15 \mathrm{~km}$ & $1 \mathrm{~km}$ & $10 \mathrm{~km}$ & $11.54 \mathrm{~km}$ & $50 \mathrm{~km}$ & $100 \mathrm{~km}$ \\
& $\mathrm{Ci} / \mathrm{g}$ & $\mathrm{Ci} / \mathrm{g}$ & $\mathrm{Ci} / \mathrm{g}$ & $\mathrm{Ci} / \mathrm{g}$ & $\mathrm{Ci} / \mathrm{g}$ & $\mathrm{Ci} / \mathrm{g}$ & $\mathrm{Ci} / \mathrm{g}$ \\
\hline Vegetable & $2.4 \mathrm{E}-09$ & $6.2 \mathrm{E}-11$ & $2.0 \mathrm{E}-09$ & $2.2 \mathrm{E}-10$ & $2.4 \mathrm{E}-10$ & $6.3 \mathrm{E}-11$ & $3.0 \mathrm{E}-11$ \\
Wheat grain & $2.4 \mathrm{E}-09$ & $\mathbf{3 . 9 E - 0 7}$ & $\mathbf{1 . 3 E - 0 5}$ & $\mathbf{1 . 5 E}-06$ & $\mathbf{1 . 6 E - 0 6}$ & $\mathbf{4 . 5 E - 0 7}$ & $\mathbf{2 . 3 E}-07$ \\
Meat & $2.4 \mathrm{E}-09$ & $1.3 \mathrm{E}-09$ & $\mathbf{4 . 1 E - 0 8}$ & $\mathbf{4 . 7 E - 0 9}$ & $\mathbf{5 . 2 E - 0 9}$ & $1.4 \mathrm{E}-09$ & $7.0 \mathrm{E}-10$ \\
Milk & $2.4 \mathrm{E}-09$ & $2.0 \mathrm{E}-09$ & $\mathbf{6 . 4 E - 0 8}$ & $\mathbf{7 . 3 E - 0 9}$ & $\mathbf{8 . 0 E}-09$ & $2.1 \mathrm{E}-09$ & $1.1 \mathrm{E}-09$ \\
\hline
\end{tabular}

The doses due to re-suspension at multiple distances are shown in Table 17 (taken from Appendix B). Values in bold exceed the relocation dose criteria of 2 rem. It is evident that relocation is not necessary for the design basis fire or the beyond design basis fire with average 
met conditions since the site boundary is $11.54 \mathrm{~km}$ from the release. For the beyond design basis fire with adverse meteorological conditions, relocation would be recommended for individuals living downwind between the site boundary and approximately $15.00 \mathrm{~km}$ from the release.

Table 17. Inhalation and Skin Absorption Dose due to 1 year Re-emission (rem)

\begin{tabular}{|c|c|c|c|c|}
\hline $\begin{array}{c}\text { Distance } \\
(\mathrm{m})\end{array}$ & $\begin{array}{c}\text { DB Fire - } \\
\text { Average Met }\end{array}$ & $\begin{array}{c}\text { DB Fire - } \\
\text { Adverse Met }\end{array}$ & $\begin{array}{c}\text { BDB Fire - } \\
\text { Average Met }\end{array}$ & $\begin{array}{c}\text { BDB Fire - } \\
\text { Adverse Met }\end{array}$ \\
\hline 150 & 0.05 & 0.00 & 0.26 & 0.00 \\
\hline 500 & 0.43 & 0.14 & 2.24 & 0.74 \\
\hline 1000 & 0.41 & 0.52 & 2.14 & 2.74 \\
\hline 3500 & 0.15 & 0.39 & 0.79 & 2.05 \\
\hline 5000 & 0.11 & 0.41 & 0.57 & $\mathbf{2 . 1 6}$ \\
\hline 7500 & 0.07 & 0.35 & 0.37 & 1.83 \\
\hline 10000 & 0.07 & 0.33 & 0.36 & 1.71 \\
\hline 10800 & 0.09 & 0.42 & 0.46 & 2.20 \\
\hline 11540 & 0.09 & 0.47 & 0.48 & 2.49 \\
\hline 15000 & 0.06 & 0.30 & 0.32 & 1.49 \\
\hline 18000 & 0.06 & 0.29 & 0.32 & 1.42 \\
\hline 21000 & 0.06 & 0.28 & 0.32 & 1.38 \\
\hline 30000 & 0.04 & 0.17 & 0.19 & 0.91 \\
\hline 50000 & 0.03 & 0.12 & 0.16 & 0.66 \\
\hline 75000 & 0.03 & 0.09 & 0.14 & 0.48 \\
\hline 100000 & 0.02 & 0.07 & 0.12 & 0.39 \\
\hline
\end{tabular}

\subsection{ConClusions}

Tritium concentrations in vegetables, wheat grain, meat, and milk have been calculated for the Tritium Facilities design and beyond design basis fires with average and adverse meteorological conditions. Peak HTO, one week, and one year concentrations were evaluated and compared with the DILs. Bold values in Tables 5 to 16 indicate the concentrations exceeding the DIL.

For the design basis fire with average (defined as $\mathrm{C}$ stability and $2.5 \mathrm{~m} / \mathrm{s}$ windspeed) and adverse (defined as E stability and $1.7 \mathrm{~m} / \mathrm{s}$ windspeed) meteorological conditions, the peak HTO and one week concentrations for all foodstuffs exceed the DIL at $100 \mathrm{~km}$. At one year for both meteorological conditions, wheat grain continues to exceed the DIL at $100 \mathrm{~km}$.

For the beyond design basis fire with average and adverse meteorological conditions, the peak HTO and one week concentrations for all foodstuffs exceed the DIL at $100 \mathrm{~km}$. At one year and average conditions, wheat grain exceeds the DIL at $100 \mathrm{~km}$ and the milk concentration at the site boundary is equal to the DIL. At one year and under adverse meteorological conditions, meat and milk exceed the DIL at the site boundary $(11.54 \mathrm{~km})$ and wheat grain exceeds the DIL at 100 $\mathrm{km}$.

Additionally, for the beyond design basis fire with adverse meteorological conditions, relocation would be recommended for individuals living downwind between the site boundary and approximately $15 \mathrm{~km}$ from the release. It should be noted, however, that the likelihood of this scenario is in the beyond extremely unlikely category (less than once in one million years). 


\subsection{REFERENCES}

FDA (1998). Accidental Contamination of Human Foods and Animal Feeds: Recommendations for States and Local Agencies. Food and Drug Administration, Rockville, MD.

M.W. Davis (1998). Emergency Preparedness Hazards Assessment for Tritium Facilities, Westinghouse Savannah River Company, Aiken, SC, S-EHA-A-00006.

W. Raskob (1990). UFOTRI: Program for Assessing the Off-Site Consequences from Accidental Tritium Releases. Kernforschungszentrum Karlsruhe, KfK 4605.

W. Raskob (1993). Description of the New Version 4.0 of the Tritium Model UFOTRI Including User Guide. Kernforschungszentrum Karlsruhe, KfK 5194.

B.K. Tharakan (1999). Derived Intervention levels for Tritium Based on Food and Drug Administration Methodology Using ICRP 56 Dose Coefficients (U). Westinghouse Savannah River Company, Aiken, SC, WSRC-TR-99-00064. 


\section{APpendix A Annual Concentrations Resulting in EPA Standards}

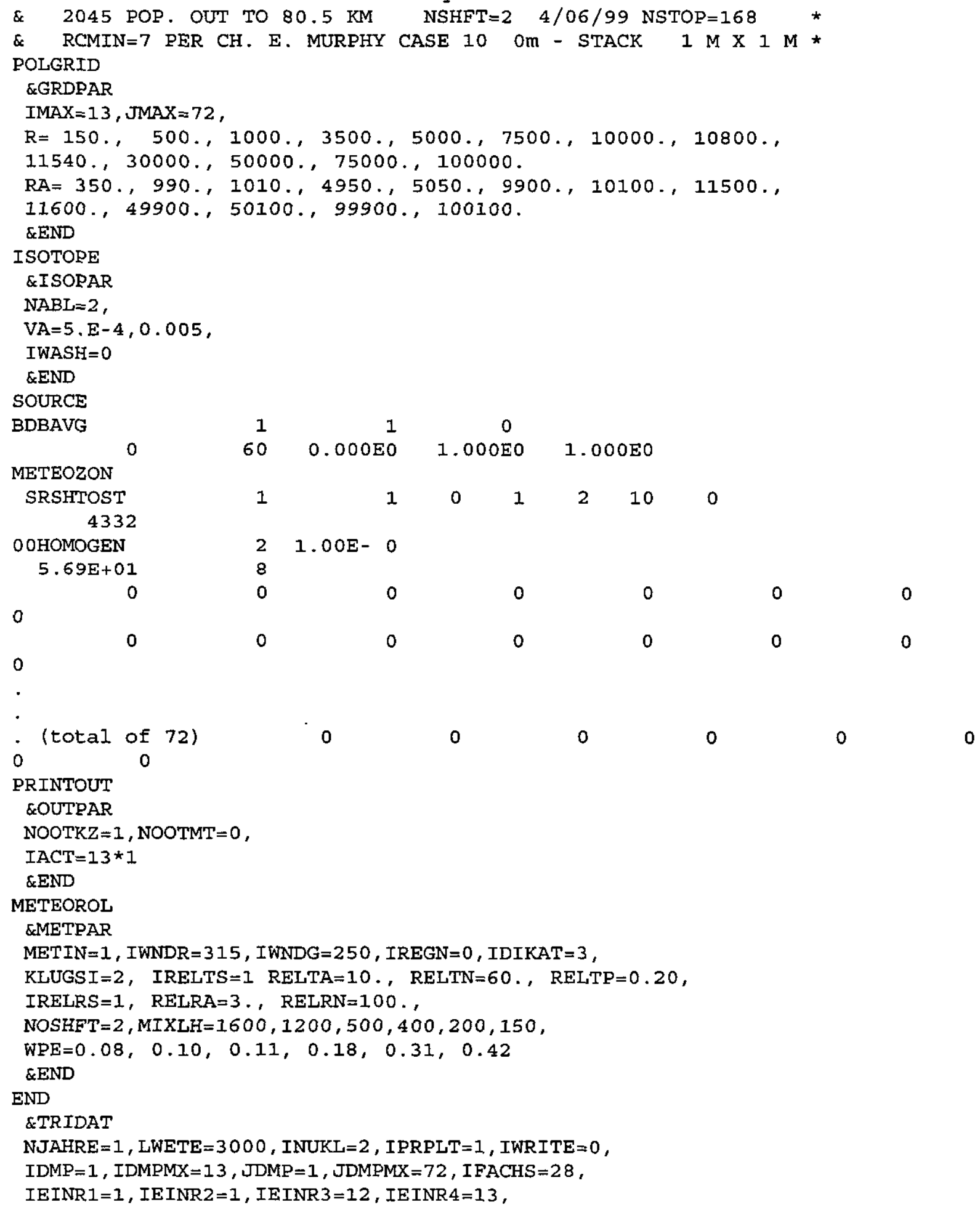


$\mathrm{LT} 1=1, \mathrm{LT} 2=365, \mathrm{LT} 3=366, \mathrm{LT} 4=730$,

NSTOP $=70$, BODFAK=1. E+2, REMRE =0.3, IZFREI=1800, IFLENG =0, BODEIN=0.35, $\operatorname{IRFIX}=0, \operatorname{REFEST}=5.0, \mathrm{ITEMPE}=2500, \mathrm{ISTRBE}=200, \mathrm{ISTRGR}=200$,

RELFA $=0.8, \mathrm{ARAU}=0.1$, IZUSD $=0$, IZUAUS $=0, \operatorname{KAUSDR}=0,1,0,0,0,0,0,0,0,0,0,0$,

$Q(2,1)=1.0, Q(1,1)=0.0, Q 1=3.7 \mathrm{E} 18$,

NBOX $=10, \mathrm{NINI}=10, \mathrm{NINT}=10, \mathrm{NOUT}=10, \mathrm{NTIM}=3, \mathrm{ITIMD}=9, \mathrm{NCOW}=250$, WWCOW $=350$, WOCOW $=150$.

TIMEDO=0.,3 .,7 , 14.,21.,30.,90.,180.,200.,365.,370.,3650., $F A K C 1=0.5, F A K C 2=0.35, F A K C P=0.05, F A K C W=0.1$,

$\mathrm{RCMIN}=7$. , $\mathrm{LEAF}=5, \mathrm{BEWG}=269 ., \mathrm{BEWGZ}=156 \ldots$,

RCMIN2 $=2,, \operatorname{LEAF2}=3,, \mathrm{BEWG} 2=680,, \mathrm{BEWGZ2}=170 \ldots$,

RCMINP $=2$, , LEAFP $=4.7, \mathrm{BEWGP}=2700 ., \mathrm{BEWGZP}=300 \ldots$

RCMINW=2 . LEAFW=7 , BEWGW=2000 , BEWGZW=500 . ,

IPOT=233, IWHEAT $=261$, IMILK=315, IVEGE=124, IMEAT=206, DOSF=1 . 7E-11,

$\mathrm{DOSHT}=1.7 \mathrm{E}-15, \mathrm{DOSFOB}=4 . \mathrm{OE}-11, \mathrm{BRRATE}=3.33 \mathrm{E}-4, \mathrm{SKRATE}=1.665 \mathrm{E}-04$,

IMODEL $=1$, IARTBO $=2$, WILTP $=0.1$, SOILMX $=0.5, \operatorname{IBODEX}=1$,

IMONT=1, IRRIGA $=0$, IRRIGG $=0$, IFLAGS $=1$, IFLAGR=1, IDEPHT=1 ,

IBODFX $=0, I C O V E R=1, I R E L I N=181, I P H O T O=1, I A R I V=0$

\&END 


\section{APPENDIX B RADIONUCLIDE CONCENTRATIONS IN FOODSTUFFS}

\begin{tabular}{|c|c|c|c|c|c|c|c|c|}
\hline & \multicolumn{7}{|c|}{ Tritium Facilities, Beyond Design Basis Fire, Adverse Met Conditions } & \\
\hline & \multicolumn{4}{|c|}{ Concentration Values for $150 \mathrm{~m}$ from release } & & & & \\
\hline \multicolumn{9}{|c|}{ Only Wheat Seed is examined since this is the edible portion of the Wheat. The "WHEAT" category is } \\
\hline \multicolumn{9}{|c|}{ actually the unedible plant body and is not considered in the dose estimate. } \\
\hline & & & & & & & & \\
\hline & \multicolumn{2}{|c|}{ VEGETABLES } & \multicolumn{2}{|c|}{ WHEAT SEED } & \multicolumn{2}{|l|}{$\overline{\text { MEAT }}$} & \multicolumn{2}{|l|}{ MILK } \\
\hline \multirow[t]{3}{*}{ HOUR } & $\mathrm{HTO}$ & OBT & HTO & OBT & HTO & OBT & HTO & $\mathrm{T}$ \\
\hline & $(\mathrm{Bq} / \mathrm{g})$ & $(\mathrm{Bq} / \mathrm{g})$ & $(\mathrm{Bq} / \mathrm{g})$ & $(\mathrm{Bq} / \mathrm{g})$ & $q / g)$ & (Bo & (B & ) \\
\hline & & & & & & & & \\
\hline 1 & $3.49 E+06$ & $1.69 \mathrm{E}+04$ & $6.32 \mathrm{E}+05$ & $2.09 E+03$ & $6.51 E+04$ & $1.30 \mathrm{E}+01$ & $7.89 E+03$ & $3.37 \mathrm{E}+03$ \\
\hline 2 & $1.38 \mathrm{E}+06$ & $2.33 \mathrm{E}+04$ & $9.49 \mathrm{E}+05$ & $3.75 \mathrm{E}+03$ & $9.76 \mathrm{E}+04$ & $3.31 \mathrm{E}+01$ & $1.75 \mathrm{E}+04$ & $7.56 \mathrm{E}+03$ \\
\hline 3 & $5.67 \mathrm{E}+05$ & $2.59 \mathrm{E}+04$ & $1.07 \mathrm{E}+06$ & $5.06 \mathrm{E}+03$ & $1.17 \mathrm{E}+05$ & $5.73 \mathrm{E}+01$ & $2.71 \mathrm{E}+04$ & $1.1^{\prime}$ \\
\hline 4 & $2.54 \mathrm{E}+05$ & $2.69 \mathrm{E}+04$ & $1.08 \mathrm{E}+06$ & $6.12 \mathrm{E}+03$ & $1.28 \mathrm{E}+05$ & $8.39 \mathrm{E}+01$ & $3.59 \mathrm{E}+04$ & $1.56 \mathrm{E}+04$ \\
\hline 5 & $1.33 \mathrm{E}+05$ & $2.73 E+04$ & $1.02 \mathrm{E}+06$ & $6.95 \mathrm{E}+03$ & $1.34 \overline{\mathrm{E}}+05$ & $1.12 \mathrm{E}+02$ & $4.37 \mathrm{E}+04$ & $1.91 \mathrm{E}+04$ \\
\hline 6 & $8.64 \mathrm{E}+04$ & $2.75 \mathrm{E}+04$ & $9.20 \mathrm{E}+05$ & $7.62 \mathrm{E}+03$ & $1.33 \mathrm{E}+05$ & $1.26 \mathrm{E}+02$ & $1.29 \mathrm{E}+05$ & 4.0 \\
\hline 7 & $6.79 \mathrm{E}+04$ & $2.77 \mathrm{E}+04$ & $8.13 \mathrm{E}+05$ & $8.16 \mathrm{E}+03$ & $1.32 \overline{\mathrm{E}+05}$ & $1.40 \mathrm{E}+02$ & $1.28 \mathrm{E}+05$ & 3.9 \\
\hline 8 & $6.03 E+04$ & $2.77 \mathrm{E}+04$ & $7.05 \mathrm{E}+05$ & $8.58 \mathrm{E}+03$ & $1.31 \bar{E}+05$ & $1.54 \mathrm{E}+02$ & $1.28 \mathrm{E}+05$ & $3.98 \mathrm{E}+04$ \\
\hline 9 & $5.69 \mathrm{E}+04$ & $2.78 \mathrm{E}+04$ & $6.03 \mathrm{E}+05$ & $8.93 \mathrm{E}+03$ & $1.29 \mathrm{E}+05$ & $1.68 \mathrm{E}+02$ & $1.27 \mathrm{E}+05$ & $3.96 \mathrm{E}+0$ \\
\hline 10 & $5.51 \mathrm{E}+04$ & $2.79 \mathrm{E}+04$ & $5.10 \mathrm{E}+05$ & $9.20 \mathrm{E}+03$ & $1.28 \mathrm{E}+05$ & $1.82 \mathrm{E}+02$ & $1.27 \mathrm{E}+05$ & 3.9 \\
\hline 11 & $5.39 \mathrm{E}+04$ & $2.80 \mathrm{E}+04$ & $4.29 \mathrm{E}+05$ & $9.43 \mathrm{E}+03$ & $1.27 \overline{\mathrm{E}}+05$ & $1.95 \mathrm{E}+02$ & $1.26 \mathrm{E}+05$ & $E+02$ \\
\hline 12 & $5.30 \mathrm{E}+04$ & $2.80 \mathrm{E}+04$ & $3.58 \mathrm{E}+05$ & $9.61 \mathrm{E}+03$ & $1.26 \mathrm{E}+05$ & $2.09 \mathrm{E}+02$ & $1.26 \mathrm{E}+05$ & $3.91 \mathrm{E}+0$ \\
\hline 13 & $5.22 \mathrm{E}+04$ & $2.81 \mathrm{E}+04$ & $2.98 \mathrm{E}+05$ & $9.75 \mathrm{E}+03$ & $1.25 \mathrm{E}+05$ & $2.22 \mathrm{E}+02$ & $1.25 \mathrm{E}+05$ & $3.89 \mathrm{E}+0$ \\
\hline 14 & $5.14 \mathrm{E}+04$ & $2.81 \mathrm{E}+04$ & $2.47 \mathrm{E}+05$ & $9.87 \bar{E}+03$ & $1.24 \mathrm{E}+05$ & $2.35 \mathrm{E}+02$ & $1.24 \mathrm{E}+05$ & $3.88 \mathrm{E}+0$ \\
\hline 15 & $5.06 \mathrm{E}+04$ & $2.82 \mathrm{E}+04$ & $2.05 \mathrm{E}+05$ & $9.97 \mathrm{E}+03$ & $1.23 \mathrm{E}+05$ & $2.48 \mathrm{E}+02$ & $1.24 \mathrm{E}+05$ & $3.86 \mathrm{E}+0$ \\
\hline 16 & $4.99 \mathrm{E}+04$ & $2.83 E+04$ & $1 . \overline{70 \mathrm{E}+05}$ & $1.01 \mathrm{E}+04$ & $1.22 \mathrm{E}+05$ & $2.61 \mathrm{E}+02$ & $1.23 \mathrm{E}+05$ & $3.85 \mathrm{E}+0$ \\
\hline 17 & $4.91 \mathrm{E}+04$ & $2.83 \mathrm{E}+04$ & $1.41 \mathrm{E}+05$ & $1.01 \mathrm{E}+04$ & $1.21 \mathrm{E}+05$ & $2.74 \mathrm{E}+02$ & $1.23 \mathrm{E}+05$ & $3.83 \mathrm{E}+0$ \\
\hline 18 & $4.84 E+04$ & $2.84 E+04$ & $1.17 \mathrm{E}+05$ & $1.02 \bar{E}+04$ & $1.21 \bar{E}+05$ & $3.02 \mathrm{E}+02$ & $1.17 E+05$ & $5.49 \mathrm{E}+04$ \\
\hline 19 & $4.78 \mathrm{E}+04$ & $2.84 \mathrm{E}+04$ & $9 . \overline{81 \mathrm{E}+04}$ & $1.02 \mathrm{E}+04$ & $1.20 \overline{\mathrm{E}+05}$ & $3.30 \mathrm{E}+02$ & $1.17 \mathrm{E}+05$ & $5.49 \mathrm{E}+0$ \\
\hline 20 & $4.71 \mathrm{E}+04$ & $2.85 \mathrm{E}+04$ & $8.23 \mathrm{E}+04$ & $1.03 \mathrm{E}+04$ & $1.20 \overline{\mathrm{E}+05}$ & $3.58 \mathrm{E}+02$ & $1.16 \mathrm{E}+05$ & $5.48 \mathrm{E}+0$ \\
\hline 21 & $4.64 \mathrm{E}+04$ & $2.85 \mathrm{E}+04$ & $6 . \overline{96 \mathrm{E}+04}$ & $1.03 \mathrm{E}+04$ & $1.19 \overline{\mathrm{E}+05}$ & $3.86 \mathrm{E}+02$ & $1.16 \mathrm{E}+05$ & $5.47 \bar{E}+04$ \\
\hline 22 & $4.58 \mathrm{E}+04$ & $2.86 \mathrm{E}+04$ & $5.92 \mathrm{E}+04$ & $1.03 E+04$ & $1.19 \overline{\mathrm{E}+05}$ & $4.14 \mathrm{E}+02$ & $1.16 \mathrm{E}+05$ & $5.47 \mathrm{E}+0$ \\
\hline 23 & $4.52 \mathrm{E}+04$ & $2.86 \mathrm{E}+04$ & $5 . \overline{08 \mathrm{E}+04}$ & $1.0 \overline{\mathrm{E}+04}$ & $1.18 \overline{\mathrm{E}+05}$ & $4.41 \mathrm{E}+02$ & $1.16 \mathrm{E}+05$ & $5.46 \mathrm{E}+0$ \\
\hline 24 & $4.46 \mathrm{E}+04$ & $2.87 \mathrm{E}+04$ & $4 . \overline{40 E+04}$ & $1.04 \mathrm{E}+04$ & $1.18 \overline{\mathrm{E}+05}$ & $4.69 \mathrm{E}+02$ & $1.15 \mathrm{E}+05$ & $5.45 \mathrm{E}+04$ \\
\hline 25 & $4.40 \mathrm{E}+04$ & $2.87 \mathrm{E}+04$ & $3.85 \mathrm{E}+04$ & $1.04 \mathrm{E}+04$ & $1.17 \overline{\mathrm{E}+05}$ & $4.96 \mathrm{E}+02$ & $1.15 \mathrm{E}+05$ & $5.45 \mathrm{E}+04$ \\
\hline 26 & $4.34 \mathrm{E}+04$ & $2.87 \mathrm{E}+04$ & $3.40 \mathrm{E}+04$ & $1.05 \bar{E}+04$ & $1.17 \mathrm{E}+05$ & $5.24 \mathrm{E}+02$ & $1.15 \mathrm{E}+05$ & $5.44 \mathrm{E}+04$ \\
\hline 27 & $4.29 \mathrm{E}+04$ & $2.88 \mathrm{E}+04$ & $3 . \overline{04 \mathrm{E}+04}$ & $1.05 \mathrm{E}+04$ & $1.16 \mathrm{E}+05$ & $5.51 \mathrm{E}+02$ & $1.15 \mathrm{E}+05$ & $5.43 \mathrm{E}+04$ \\
\hline 28 & $4.23 E+04$ & $2.88 \mathrm{E}+04$ & $2.75 \mathrm{E}+04$ & $1.05 \mathrm{E}+04$ & $1.16 \mathrm{E}+05$ & $5.79 \mathrm{E}+02$ & $1.14 \mathrm{E}+05$ & $5.42 \mathrm{E}+04$ \\
\hline 29 & $4.18 \mathrm{E}+04$ & $2.89 \mathrm{E}+04$ & $2.52 \mathrm{E}+04$ & $1.05 \mathrm{E}+04$ & $1.15 \mathrm{E}+05$ & $6.06 \mathrm{E}+02$ & $1.14 \mathrm{E}+05$ & $5.42 \mathrm{E}+04$ \\
\hline 30 & $4.13 \mathrm{E}+04$ & $2.89 \mathrm{E}+04$ & $2.32 \mathrm{E}+04$ & $1.05 \mathrm{E}+04$ & $1.14 \mathrm{E}+05$ & $6.18 \mathrm{E}+02$ & $1.11 \mathrm{E}+05$ & $3.45 \mathrm{E}+04$ \\
\hline 31 & $4.08 \mathrm{E}+04$ & $2.89 \mathrm{E}+04$ & $2.17 \mathrm{E}+04$ & $1.05 \mathrm{E}+04$ & $1.13 \mathrm{E}+05$ & $6.29 \mathrm{E}+02$ & $1.10 \mathrm{E}+05$ & $3.44 \mathrm{E}+04$ \\
\hline 32 & $4.03 \mathrm{E}+04$ & $2.90 \mathrm{E}+04$ & $2.04 \mathrm{E}+04$ & $1.06 \mathrm{E}+04$ & $1.12 \mathrm{E}+05$ & $6.41 \mathrm{E}+02$ & $1.10 \mathrm{E}+05$ & $3.43 \mathrm{E}+04$ \\
\hline 33 & $3.99 \mathrm{E}+04$ & $2.90 \mathrm{E}+04$ & $1.93 E+04$ & $1.06 \mathrm{E}+04$ & $1.11 \mathrm{E}+05$ & $6.52 \mathrm{E}+02$ & $1.09 \mathrm{E}+05$ & $3.41 \mathrm{E}+04$ \\
\hline 34 & $3.94 \mathrm{E}+04$ & $2.91 \mathrm{E}+04$ & $1.85 \mathrm{E}+04$ & $1.06 \mathrm{E}+04$ & $1.10 \mathrm{E}+05$ & $6.64 \mathrm{E}+02$ & $1.09 \mathrm{E}+05$ & $3.40 \mathrm{E}+04$ \\
\hline 35 & $3.90 \mathrm{E}+04$ & $2.91 \mathrm{E}+04$ & $1.77 \mathrm{E}+04$ & $1.06 \mathrm{E}+04$ & $1.09 \mathrm{E}+05$ & $6.75 \mathrm{E}+02$ & $1.08 \mathrm{E}+05$ & $3.38 \mathrm{E}+04$ \\
\hline 36 & $3.85 \mathrm{E}+04$ & $2.91 \mathrm{E}+04$ & $1.71 \mathrm{E}+04$ & $1.06 \mathrm{E}+04$ & $1.08 \mathrm{E}+05$ & $6.86 \mathrm{E}+02$ & $1.08 \mathrm{E}+05$ & $3.37 \mathrm{E}+04$ \\
\hline 37 & $3.81 \mathrm{E}+04$ & $2.92 \mathrm{E}+04$ & $1.66 \mathrm{E}+04$ & $1.06 \mathrm{E}+04$ & $1.08 \mathrm{E}+05$ & $6.97 \mathrm{E}+02$ & $1.07 \mathrm{E}+05$ & $3.36 \mathrm{E}+04$ \\
\hline
\end{tabular}




\begin{tabular}{|c|c|c|c|c|c|c|c|c|}
\hline 38 & $3.77 \mathrm{E}+04$ & $2.92 \mathrm{E}+04$ & $1.62 \mathrm{E}+04$ & $1.07 \mathrm{E}+04$ & $1.07 \overline{\mathrm{E}+05}$ & $7.08 \mathrm{E}+02$ & $1.07 \mathrm{E}+05$ & \\
\hline 39 & $3.73 \mathrm{E}+04$ & $2.92 \mathrm{E}+04$ & $1.58 \mathrm{E}+04$ & $1.07 \mathrm{E}+04$ & $1.06 \mathrm{E}+05$ & $7.19 \mathrm{E}+02$ & $1.07 \mathrm{E}+05$ & $3.33 \mathrm{E}+$ \\
\hline 40 & $3.69 \mathrm{E}+04$ & $2.93 \mathrm{E}+04$ & $1.55 \mathrm{E}+04$ & $1.07 \mathrm{E}+04$ & $1.05 \mathrm{E}+05$ & $7.30 \mathrm{E}+02$ & $.06 \mathrm{E}+05$ & $31 \mathrm{E}+$ \\
\hline 41 & $3.66 \mathrm{E}+04$ & $2.93 \mathrm{E}+04$ & $1.52 \mathrm{E}+04$ & $1.07 \mathrm{E}+04$ & $1.04 \mathrm{E}+05$ & $7.41 \mathrm{E}+02$ & $.06 \mathrm{E}+05$ & $.30 \mathrm{E}+$ \\
\hline 42 & $3.62 \mathrm{E}+04$ & $93 \bar{E}+04$ & $1.49 \mathrm{E}+04$ & $1.07 \mathrm{E}+04$ & $1.04 \mathrm{E}+05$ & $7.67 \mathrm{E}+02$ & $.00 \mathrm{E}+05$ & $.02 \mathrm{E}+$ \\
\hline 43 & $3.59 \mathrm{E}+04$ & $2.93 \mathrm{E}+04$ & $1.4 \overline{7}+04$ & $1.07 \mathrm{E}+04$ & $1.03 \mathrm{E}+05$ & $7.93 \mathrm{E}+02$ & $1.00 \mathrm{E}+05$ & $5.01 \mathrm{E}+$ \\
\hline 44 & $3.55 \mathrm{E}+04$ & $2.94 \mathrm{E}+04$ & $1.45 \mathrm{E}+04$ & $1.07 \mathrm{E}+04$ & $1.03 E+05$ & $8.19 \mathrm{E}+02$ & $9.98 \mathrm{E}+04$ & $5.00 \mathrm{E}+0$ \\
\hline 45 & $3.52 \mathrm{E}+04$ & $2.94 \mathrm{E}+04$ & $1.43 \mathrm{E}+04$ & $1.08 \mathrm{E}+04$ & $1.02 \mathrm{E}+05$ & $8.45 \mathrm{E}+02$ & $9.96 \mathrm{E}+04$ & $4.99 \mathrm{E}+\mathrm{C}$ \\
\hline 46 & $3.49 \mathrm{E}+04$ & $2.94 \mathrm{E}+04$ & $1.41 \mathrm{E}+04$ & $1.08 \mathrm{E}+04$ & $1.02 \mathrm{E}+05$ & $8.70 \mathrm{E}+02$ & $9.94 \mathrm{E}+04$ & $4.98 \mathrm{E}+\mathrm{C}$ \\
\hline 47 & $3.46 \mathrm{E}+04$ & $2.94 \mathrm{E}+04$ & $1.40 \mathrm{E}+04$ & $1.08 \mathrm{E}+04$ & $1.01 \mathrm{E}+05$ & $8.96 \mathrm{E}+02$ & $9.91 \mathrm{E}+04$ & $4.98 \mathrm{E}+0$ \\
\hline 48 & $3 . \overline{43 E}+04$ & $2 . \overline{95 E+04}$ & $1.38 \mathrm{E}+04$ & $1.08 \mathrm{E}+04$ & $1.01 \mathrm{E}+05$ & $9.22 \mathrm{E}+02$ & $9.89 \mathrm{E}+04$ & $4.97 \mathrm{E}+0$ \\
\hline 49 & $3.40 \mathrm{E}+04$ & $2.95 \mathrm{E}+04$ & $1.37 \mathrm{E}+04$ & $1.08 \mathrm{E}+04$ & $1.00 \mathrm{E}+05$ & $9.47 \mathrm{E}+02$ & $9.86 \mathrm{E}+04$ & $4.96 \mathrm{E}+0$ \\
\hline 50 & $3.37 \mathrm{E}+04$ & $2.95 \mathrm{E}+04$ & $1.35 \mathrm{E}+04$ & $1.08 \mathrm{E}+04$ & $9.96 \mathrm{E}+04$ & $9.73 \mathrm{E}+02$ & $9.84 \mathrm{E}+04$ & $4.95 \mathrm{E}+0$ \\
\hline 51 & $3.34 \mathrm{E}+04$ & $2.95 \mathrm{E}+04$ & $1.34 \mathrm{E}+04$ & $1.08 \mathrm{E}+04$ & $9.91 \mathrm{E}+04$ & $9.98 \mathrm{E}+02$ & $9.82 E+04$ & $4.95 \mathrm{E}+0$ \\
\hline 52 & $3.32 \mathrm{E}+04$ & $2.96 \mathrm{E}+04$ & $1.33 \mathrm{E}+04$ & $1.08 \bar{E}+04$ & $9.86 \mathrm{E}+04$ & $1.02 \mathrm{E}+03$ & $9.79 \mathrm{E}+04$ & $4.94 E+0$ \\
\hline 53 & $3.29 \mathrm{E}+04$ & $2.96 \mathrm{E}+04$ & $1.31 \mathrm{E}+04$ & $1.09 \mathrm{E}+04$ & $9.81 \mathrm{E}+04$ & $1.05 \mathrm{E}+03$ & $9.77 \mathrm{E}+04$ & $4.93 \mathrm{E}+0$ \\
\hline 54 & $3.27 \mathrm{E}+04$ & $2.96 \mathrm{E}+04$ & $1.30 \mathrm{E}+04$ & $1.09 \mathrm{E}+04$ & $9.73 \mathrm{E}+04$ & $1.06 \mathrm{E}+03$ & $9.43 \mathrm{E}+04$ & $2.96 \mathrm{E}+0$ \\
\hline 55 & $3.24 \mathrm{E}+04$ & $2.96 \mathrm{E}+04$ & $1.29 \mathrm{E}+04$ & $1.09 \mathrm{E}+04$ & $9.65 \mathrm{E}+04$ & $1.07 \mathrm{E}+03$ & $9.39 \mathrm{E}+04$ & $2.94 \mathrm{E}+0$ \\
\hline 56 & $3.22 \mathrm{E}+04$ & $2.96 \mathrm{E}+04$ & $1.28 \mathrm{E}+04$ & $1.09 \mathrm{E}+04$ & $9.57 E+04$ & $1.08 \mathrm{E}+03$ & $9.35 E+04$ & $2.93 \mathrm{E}+0$ \\
\hline 57 & $3.20 \mathrm{E}+04$ & $2.97 \mathrm{E}+04$ & $1.27 \mathrm{E}+04$ & $1.09 \mathrm{E}+04$ & $9.49 \mathrm{E}+04$ & $1.09 \mathrm{E}+03$ & $9.31 \mathrm{E}+04$ & $2.92 \mathrm{E}+0$ \\
\hline 58 & $3.17 \mathrm{E}+04$ & $2.97 \mathrm{E}+04$ & $1.26 \mathrm{E}+04$ & $1.09 \mathrm{E}+04$ & $9.41 \mathrm{E}+04$ & $1.10 \mathrm{E}+03$ & $9.27 \mathrm{E}+04$ & $2.91 \mathrm{E}+0$ \\
\hline 59 & $3.15 \mathrm{E}+04$ & $2.97 \mathrm{E}+04$ & $1.25 \mathrm{E}+04$ & $1.09 \mathrm{E}+04$ & $9.33 \mathrm{E}+04$ & $1.11 \mathrm{E}+03$ & $9.23 \mathrm{E}+04$ & $2 . \overline{90 \mathrm{E}+0}$ \\
\hline 60 & $3.13 E+04$ & $2.97 \mathrm{E}+04$ & $1.24 \mathrm{E}+04$ & $1.09 \mathrm{E}+04$ & $9.25 \mathrm{E}+04$ & $1.11 \mathrm{E}+03$ & $9.20 \mathrm{E}+04$ & $2.88 \mathrm{E}+0$ \\
\hline 61 & $11 \mathrm{E}+04$ & $2.97 \mathrm{E}+04$ & $1.23 \mathrm{E}+04$ & $1.10 \mathrm{E}+04$ & $9.17 \mathrm{E}+04$ & $1.12 \mathrm{E}+03$ & $9.16 \mathrm{E}+04$ & $2.87 \mathrm{E}+0$ \\
\hline 62 & $3.09 \mathrm{E}+04$ & $2.98 \mathrm{E}+04$ & $1.22 \mathrm{E}+04$ & $1.10 \mathrm{E}+04$ & $9.10 \mathrm{E}+04$ & $1.13 \mathrm{E}+03$ & $9.12 \mathrm{E}+04$ & $2.86 \mathrm{E}+0$ \\
\hline 63 & $3.08 \mathrm{E}+04$ & $2.98 \mathrm{E}+04$ & $1.22 \mathrm{E}+04$ & $1.10 \mathrm{E}+04$ & $9.02 \mathrm{E}+04$ & $1.14 \mathrm{E}+03$ & $9.08 \mathrm{E}+04$ & $2.85 \mathrm{E}+0$ \\
\hline 64 & $3.06 \mathrm{E}+04$ & $2.98 \mathrm{E}+04$ & $1.21 \mathrm{E}+04$ & $1.10 \mathrm{E}+04$ & $8.94 \mathrm{E}+04$ & $1.15 \mathrm{E}+03$ & $9.04 \mathrm{E}+04$ & $2 . \overline{84 \mathrm{E}+0}$ \\
\hline 65 & $3.04 \mathrm{E}+04$ & $2.98 \mathrm{E}+04$ & $1.20 \mathrm{E}+04$ & $1.10 \mathrm{E}+04$ & $8.87 \mathrm{E}+04$ & $1.16 \mathrm{E}+03$ & $9.01 \mathrm{E}+04$ & $2.83 \mathrm{E}+0$ \\
\hline 66 & $3.02 \mathrm{E}+04$ & $2.98 \mathrm{E}+04$ & $1.19 E+04$ & $1.10 \mathrm{E}+04$ & $8.83 \mathrm{E}+04$ & $1.18 \mathrm{E}+03$ & $8.56 \mathrm{E}+04$ & $4.56 \mathrm{E}+0$ \\
\hline 67 & $3.01 \mathrm{E}+04$ & $2.99 \mathrm{E}+04$ & $1.18 \mathrm{E}+04$ & $1.10 \mathrm{E}+04$ & $8.78 \mathrm{E}+04$ & $1.21 \mathrm{E}+03$ & $8.53 E+04$ & $4.55 \mathrm{E}+0$ \\
\hline 68 & $2.99 \mathrm{E}+04$ & $2.99 \mathrm{E}+04$ & $1.18 \mathrm{E}+04$ & $1.10 \mathrm{E}+04$ & $8.74 \mathrm{E}+04$ & $1.23 \mathrm{E}+03$ & $8.51 \mathrm{E}+04$ & $4.55 \mathrm{E}+0$ \\
\hline 69 & $2.98 \mathrm{E}+04$ & $2.99 \overline{\mathrm{E}}+04$ & $1.17 \mathrm{E}+04$ & $1.10 \mathrm{E}+04$ & $8.70 \mathrm{E}+04$ & $1.26 \mathrm{E}+03$ & $8.49 \mathrm{E}+04$ & $4.54 \overline{\mathrm{E}+0}$ \\
\hline 168 & $1.25 \mathrm{E}+04$ & $2.99 \mathrm{E}+04$ & $9.58 \mathrm{E}+03$ & $1.19 \mathrm{E}+04$ & $4.71 \mathrm{E}+04$ & $2.72 \mathrm{E}+03$ & $4.93 \mathrm{E}+04$ & $3.35 \mathrm{E}+0$ \\
\hline 336 & $5.95 \mathrm{E}+03$ & $2.50 \mathrm{E}+04$ & $4.86 \mathrm{E}+03$ & $1.26 \mathrm{E}+04$ & $1.82 \mathrm{E}+04$ & $4.91 \mathrm{E}+03$ & $2.93 \mathrm{E}+04$ & $2.52 \mathrm{E}+0$ \\
\hline 504 & $4.37 \mathrm{E}+03$ & $2.07 \mathrm{E}+04$ & $3.33 \mathrm{E}+03$ & $1.31 \mathrm{E}+04$ & $8.49 \mathrm{E}+03$ & $6.07 \mathrm{E}+03$ & $1.22 \mathrm{E}+04$ & $1.65 \mathrm{E}+04$ \\
\hline 720 & $3.19 \mathrm{E}+03$ & $1.61 \mathrm{E}+04$ & $2.23 \mathrm{E}+03$ & $1.34 \mathrm{E}+04$ & $4.19 \mathrm{E}+03$ & $6.68 \mathrm{E}+03$ & $5.81 \mathrm{E}+03$ & $1.14 \mathrm{E}+04$ \\
\hline 2160 & $5.98 \mathrm{E}+02$ & $3.02 \mathrm{E}+03$ & $2.51 \mathrm{E}+02$ & $1.41 \mathrm{E}+04$ & $3.72 \mathrm{E}+02$ & $3.88 \mathrm{E}+03$ & $1.31 \mathrm{E}+03$ & $3.89 \mathrm{E}+0$ \\
\hline 4320 & $5.75 \mathrm{E}+01$ & $2.55 \mathrm{E}+02$ & $1.26 \mathrm{E}+03$ & $1.35 \mathrm{E}+04$ & $6.00 \mathrm{E}+01$ & $9.22 \mathrm{E}+02$ & $1.56 \mathrm{E}+02$ & $6.06 \mathrm{E}+02$ \\
\hline 4800 & $3.42 \mathrm{E}+01$ & $1.48 \mathrm{E}+02$ & $1.26 \mathrm{E}+03$ & $1.35 \mathrm{E}+04$ & $4.34 \mathrm{E}+01$ & $6.63 \mathrm{E}+02$ & $5.12 \mathrm{E}+01$ & $1.97 \mathrm{E}+02$ \\
\hline 8760 & $4.70 \mathrm{E}-01$ & $1.81 \mathrm{E}+00$ & $1.23 \mathrm{E}+03$ & $1.31 \mathrm{E}+04$ & $3.47 \mathrm{E}+00$ & $4.35 \mathrm{E}+01$ & $1.58 \mathrm{E}+01$ & $5.75 \mathrm{E}+01$ \\
\hline $\mathbf{T}$ & $\begin{array}{l}2.4 \mathrm{E} 6 \\
\mathrm{pCi} / \mathrm{L}\end{array}$ & $\begin{array}{l}8.88 \mathrm{E}+4 \\
\mathrm{~Bq} / \mathrm{kg}\end{array}$ & $8 \mathrm{~Bq} / \mathrm{g}$ & & & & & \\
\hline $\mathbf{0}$ & $\begin{array}{l}5.9 \mathrm{E} 6 \\
\mathrm{pCi} / \mathrm{L} \\
\end{array}$ & $\begin{array}{l}2.18 \mathrm{E}+5 \\
\mathrm{~Bq} / \mathrm{kg}\end{array}$ & $\mathrm{Bq} / \mathrm{g}$ & & & & & \\
\hline & \multicolumn{2}{|c|}{ VEGETABLES } & \multicolumn{2}{|c|}{ WHEAT SEED } & \multicolumn{2}{|l|}{ MEAT } & \multicolumn{2}{|l|}{ MILK } \\
\hline \multirow[t]{2}{*}{ HOUR } & U & $3 T$ & & $\mathrm{BT}$ & $\mathrm{rO}$ & & TO & B1 \\
\hline & $\mathrm{q} / \mathrm{g})$ & $q / g)$ & (g) & $q / g)$ & $q / g)$ & $\mathrm{g} / \mathrm{g}$ & $q / g)$ & $\mathrm{q} / \mathrm{s}$ \\
\hline
\end{tabular}


Foodstuff Concentrations and Relocation Considerations Following

a Tritium Oxide Release from SRS Tritium Facilities (U)

\begin{tabular}{|c|c|c|c|c|c|c|c|c|}
\hline Peak HTO & & & & & & & & \\
\hline $1 / 4 / 5 / 6$ & $3.49 \mathrm{E}+06$ & $1.69 \mathrm{E}+04$ & $1.08 \mathrm{E}+06$ & $6.12 \mathrm{E}+03$ & $1.34 \mathrm{E}+05$ & $1.12 \mathrm{E}+02$ & $1.29 \mathrm{E}+05$ & $4.01 \mathrm{E}+04$ \\
\hline SUM & $3.51 \mathrm{E}+06$ & & $1.08 \mathrm{E}+06$ & & $1.34 \mathrm{E}+05$ & & $1.69 \mathrm{E}+05$ & \\
\hline$\overline{\mathrm{Ci} / \mathrm{g}}$ & $9.48 \mathrm{E}-05$ & & $2.92 \mathrm{E}-05$ & & $3.62 \mathrm{E}-06$ & & $4.56 \mathrm{E}-06$ & \\
\hline & & & & & & & & \\
\hline DIL HTO & $5.9 \mathrm{E}-09$ & & & & & & & \\
\hline$(\mathrm{Ci} / \mathrm{g})$ & & & & & & & & \\
\hline & & & & & & & & \\
\hline 168 & $1.25 \mathrm{E}+04$ & $2.99 \mathrm{E}+04$ & $9.58 \mathrm{E}+03$ & $1.19 \mathrm{E}+04$ & $4.71 \mathrm{E}+04$ & $2.72 \mathrm{E}+03$ & $4.93 \mathrm{E}+04$ & $3.35 \overline{\mathrm{E}}+04$ \\
\hline SUM & $4.24 E+04$ & & $2.15 \mathrm{E}+04$ & & $4.98 \mathrm{E}+04$ & & $8.28 \mathrm{E}+04$ & \\
\hline $\mathrm{Ci} / \mathrm{g}$ & $1.15 \mathrm{E}-06$ & & $5.80 \mathrm{E}-07$ & & $1.35 E-06$ & & $2.24 \mathrm{E}-06$ & \\
\hline & & & & & & & & \\
\hline DIL OBT & $2.4 \mathrm{E}-09$ & & & & & & & \\
\hline $\mathrm{Ci} / \mathrm{g}$ & & & & & & & & \\
\hline & & & & & & & & \\
\hline 8760 & $4.70 \mathrm{E}-01$ & $1.81 \mathrm{E}+00$ & $1.23 \mathrm{E}+03$ & $1.31 \mathrm{E}+04$ & $3.47 \mathrm{E}+00$ & $4.35 \mathrm{E}+01$ & $1.58 \mathrm{E}+01$ & $5.75 \mathrm{E}+01$ \\
\hline SUM & $2.28 \mathrm{E}+00$ & & $1.43 \mathrm{E}+04$ & & $4.69 \mathrm{E}+01$ & & $7.33 \mathrm{E}+01$ & \\
\hline $\mathrm{Ci} / \mathrm{g}$ & $6.17 \mathrm{E}-11$ & & $3.87 \mathrm{E}-07$ & & $1.27 \mathrm{E}-09$ & & $1.98 \mathrm{E}-09$ & \\
\hline$\overline{\text { DIL OBT }}$ & $24 \mathrm{~F}-09$ & & & & & & & \\
\hline $\mathrm{Ci} / \mathrm{g}$ & & & & & & & & \\
\hline & & & & & & & & \\
\hline & & & & & & & & \\
\hline
\end{tabular}




\begin{tabular}{|c|c|c|c|c|c|c|c|c|}
\hline & \multicolumn{7}{|c|}{ Tritium Facilities, Beyond Design Basis Fire, Adverse Met Conditions } & \\
\hline & \multicolumn{4}{|c|}{ Concentration Values for $1 \mathrm{~km}$ from release } & & & & \\
\hline \multicolumn{9}{|c|}{ Only Wheat Seed is examined since this is the edible portion of the Wheat. The "WHEAT" category is } \\
\hline \multicolumn{9}{|c|}{ actually the unedible plant body and is not considered in the dose estimate. } \\
\hline & & & & & & & & \\
\hline & \multicolumn{2}{|c|}{ VEGETABLES } & \multicolumn{2}{|c|}{ WHEAT SEED } & \multicolumn{2}{|l|}{ MEAT } & \multicolumn{2}{|l|}{ MILK } \\
\hline \multirow[t]{3}{*}{ HOUR } & HTO & $\widehat{\mathrm{OBT}}$ & HTO & OBT & HTO & $\overline{\mathrm{OBT}}$ & HTO & OBT \\
\hline & $(\mathrm{Bq} / \mathrm{g})$ & $(\mathrm{Bq} / \mathrm{g})$ & $(\mathrm{Bq} / \mathrm{g})$ & $(\mathrm{Bq} / \mathrm{g})$ & $(\mathrm{Bq} / \mathrm{g})$ & $(\mathrm{Bq} / \mathrm{g})$ & $(\mathrm{Bq} / \mathrm{g})$ & $\overline{\mathrm{BO}}$ \\
\hline & & & & & & & & \\
\hline 1 & $1.10 \mathrm{E}+08$ & $5.06 \mathrm{E}+05$ & $1.90 \mathrm{E}+07$ & $6.27 \mathrm{E}+04$ & $1.96 \mathrm{E}+06$ & $3.92 \mathrm{E}+02$ & $2.37 \mathrm{E}+05$ & $1.01 \mathrm{E}+$ \\
\hline$\overline{2}$ & $4.58 \mathrm{E}+07$ & $7.09 \mathrm{E}+05$ & $2.88 \mathrm{E}+07$ & $1.14 \mathrm{E}+05$ & $2.97 \mathrm{E}+06$ & $1.00 \mathrm{E}+03$ & $5.31 \mathrm{E}+05$ & $2.29 \mathrm{E}+05$ \\
\hline 3 & $2.03 \mathrm{E}+07$ & $7.93 \mathrm{E}+05$ & $3.28 \mathrm{E}+07$ & $1.55 \mathrm{E}+05$ & $3.57 \mathrm{E}+06$ & $1.74 \mathrm{E}+03$ & $8.23 E+05$ & $3.57 \mathrm{E}+05$ \\
\hline 4 & $1.00 \mathrm{E}+07$ & $8.30 \mathrm{E}+05$ & $3.32 \mathrm{E}+07$ & $1.87 \mathrm{E}+05$ & $3.93 \mathrm{E}+06$ & $2.56 \mathrm{E}+03$ & $1.10 \mathrm{E}+06$ & $4.76 \mathrm{E}+05$ \\
\hline 5 & $5.80 \mathrm{E}+06$ & $8.48 \mathrm{E}+05$ & $3.15 \mathrm{E}+07$ & $2.14 \mathrm{E}+05$ & $4.14 \mathrm{E}+06$ & $3.43 \mathrm{E}+03$ & $1.34 \mathrm{E}+06$ & $5.84 \mathrm{E}+05$ \\
\hline 6 & $4.00 \mathrm{E}+06$ & $8.57 \mathrm{E}+05$ & $2.88 \mathrm{E}+07$ & $2.35 \mathrm{E}+05$ & $4.11 \mathrm{E}+06$ & $3.87 \mathrm{E}+03$ & $3.98 \mathrm{E}+06$ & $1.24 \mathrm{E}+0$ \\
\hline 7 & $3.20 \mathrm{E}+06$ & $8.64 \mathrm{E}+05$ & $2.56 \mathrm{E}+07$ & $2.53 \mathrm{E}+05$ & $4.08 \mathrm{E}+06$ & $4.30 \mathrm{E}+03$ & $3.97 \mathrm{E}+06$ & $1.24 \mathrm{E}+0$ \\
\hline 8 & $2.81 E+06$ & $8.68 \mathrm{E}+05$ & $2.23 \mathrm{E}+07$ & $2.67 \mathrm{E}+05$ & $4.04 \mathrm{E}+06$ & $4.74 \mathrm{E}+03$ & $3.95 \mathrm{E}+06$ & $1.23 \mathrm{E}+06$ \\
\hline 9 & $2.59 \mathrm{E}+06$ & $8.72 \mathrm{E}+05$ & $1.92 \mathrm{E}+07$ & $2.78 \mathrm{E}+05$ & $4.01 E+06$ & $5.16 \mathrm{E}+03$ & $3.93 E+06$ & $1.23 \mathrm{E}+0$ \\
\hline 10 & $2.46 \mathrm{E}+06$ & $8.76 \mathrm{E}+05$ & $1.64 \mathrm{E}+07$ & $2.87 \mathrm{E}+05$ & $3.98 \mathrm{E}+06$ & $5.59 \mathrm{E}+03$ & $3.92 \mathrm{E}+06$ & $1.22 \mathrm{E}+0$ \\
\hline 11 & $2.36 \mathrm{E}+06$ & $8.79 \mathrm{E}+05$ & $1.38 \mathrm{E}+07$ & $2.94 \mathrm{E}+05$ & $3.94 \mathrm{E}+06$ & $6.01 E+03$ & $3.90 \mathrm{E}+06$ & $1.22 \mathrm{E}+0$ \\
\hline 12 & $2.29 \mathrm{E}+06$ & $8.83 \mathrm{E}+05$ & $1.17 \mathrm{E}+07$ & $3.01 \mathrm{E}+05$ & $3.91 \mathrm{E}+06$ & $6.43 \mathrm{E}+03$ & $3.89 \mathrm{E}+06$ & $1.21 \mathrm{E}+0$ \\
\hline 13 & $2.23 \mathrm{E}+06$ & $8.86 \mathrm{E}+05$ & $9.78 \mathrm{E}+06$ & $3.06 \mathrm{E}+05$ & $3.88 \mathrm{E}+06$ & $6.84 \mathrm{E}+03$ & $3.87 \mathrm{E}+06$ & $1.21 \mathrm{E}+0$ \\
\hline 14 & $2.17 \mathrm{E}+06$ & $8.89 \mathrm{E}+05$ & $8.20 \mathrm{E}+06$ & $3.10 \mathrm{E}+05$ & $3.85 \mathrm{E}+06$ & $7.25 \mathrm{E}+03$ & $3.85 \mathrm{E}+06$ & $1.20 \mathrm{E}+0$ \\
\hline 15 & $2.12 \mathrm{E}+06$ & $8.92 \mathrm{E}+05$ & $6.87 \mathrm{E}+06$ & $3.13 \mathrm{E}+05$ & $3.81 \mathrm{E}+06$ & $7.65 \mathrm{E}+03$ & $3.84 \mathrm{E}+06$ & $1.20 \mathrm{E}+06$ \\
\hline 16 & $2.08 \mathrm{E}+06$ & $8.94 \mathrm{E}+05$ & $5.76 \mathrm{E}+06$ & $3.16 \mathrm{E}+05$ & $3.78 \mathrm{E}+06$ & $8.05 E+03$ & $3.82 \mathrm{E}+06$ & $1.19 \mathrm{E}+0$ \\
\hline 17 & $2.03 \mathrm{E}+06$ & $8.97 \mathrm{E}+05$ & $4.85 \mathrm{E}+06$ & $3.19 \mathrm{E}+05$ & $3.75 \mathrm{E}+06$ & $8.45 E+03$ & $3.81 \mathrm{E}+06$ & $1.19 \mathrm{E}+0$ \\
\hline 18 & $1.99 \mathrm{E}+06$ & $9.00 \mathrm{E}+05$ & $4.09 \mathrm{E}+06$ & $3.21 \mathrm{E}+05$ & $3.74 \mathrm{E}+06$ & $9.33 \mathrm{E}+03$ & $3.63 \mathrm{E}+06$ & $1.72 \mathrm{E}+0$ \\
\hline 19 & $1.95 \mathrm{E}+06$ & $9.02 \mathrm{E}+05$ & $3.47 \mathrm{E}+06$ & $3.23 \mathrm{E}+05$ & $3.73 E+06$ & $1.02 \bar{E}+04$ & $3.62 \mathrm{E}+06$ & $1.72 \mathrm{E}+0$ \\
\hline 20 & $1.91 \mathrm{E}+06$ & $9.04 \mathrm{E}+05$ & $2.97 \mathrm{E}+06$ & $3.25 \mathrm{E}+05$ & $3.72 \mathrm{E}+06$ & $\mathrm{E}+04$ & $3.62 \mathrm{E}+06$ & $1.72 \mathrm{E}+0 \mathrm{C}$ \\
\hline 21 & $1.87 \mathrm{E}+06$ & $9.07 \mathrm{E}+05$ & $2.55 \mathrm{E}+06$ & $3.26 \mathrm{E}+05$ & $3.71 \mathrm{E}+06$ & $1.20 \mathrm{E}+04$ & $3.61 \mathrm{E}+06$ & $1.71 \mathrm{E}+06$ \\
\hline 22 & $1.84 \mathrm{E}+06$ & $9.09 \vec{E}+05$ & $2.21 \mathrm{E}+06$ & $3.28 \mathrm{E}+05$ & $3.70 \mathrm{E}+06$ & $1.28 \mathrm{E}+04$ & $3.61 \mathrm{E}+06$ & $1.71 \mathrm{E}+06$ \\
\hline 23 & $1.80 \mathrm{E}+06$ & $9.11 E+05$ & $1.94 \mathrm{E}+06$ & $3.29 \mathrm{E}+05$ & $3.69 \mathrm{E}+06$ & $\mathrm{E}+04$ & $3.60 \mathrm{E}+06$ & $1.71 \mathrm{E}+06$ \\
\hline 24 & $1.76 \mathrm{E}+06$ & $9.13 E+05$ & $1.71 \mathrm{E}+06$ & $3.30 \mathrm{E}+05$ & $3.68 \mathrm{E}+06$ & $1.46 \mathrm{E}+04$ & $3.60 \mathrm{E}+06$ & $1.71 \mathrm{E}+06$ \\
\hline 25 & $1.72 \mathrm{E}+06$ & $9.15 \mathrm{E}+05$ & $1.52 \mathrm{E}+06$ & $3.31 \mathrm{E}+05$ & $3.67 \mathrm{E}+06$ & $1.55 \mathrm{E}+04$ & $3.59 \mathrm{E}+06$ & $1.71 \mathrm{E}+06$ \\
\hline 26 & $1.69 \mathrm{E}+06$ & $9.17 \mathrm{E}+05$ & $1.37 \mathrm{E}+06$ & $3.32 \mathrm{E}+05$ & $3.65 \mathrm{E}+06$ & $1.63 \mathrm{E}+04$ & $3.59 \mathrm{E}+06$ & $1.71 \mathrm{E}+06$ \\
\hline 27 & $1.66 \mathrm{E}+06$ & $9.19 \mathrm{E}+05$ & $1.25 \mathrm{E}+06$ & $3.33 \mathrm{E}+05$ & $3.64 \mathrm{E}+06$ & $1.72 \mathrm{E}+04$ & $3.58 \mathrm{E}+06$ & $1.71 \mathrm{E}+06$ \\
\hline 28 & $1.63 \mathrm{E}+06$ & $9.21 \mathrm{E}+05$ & $1.14 \mathrm{E}+06$ & $3.34 \mathrm{E}+05$ & $3.63 \mathrm{E}+06$ & $1.81 \mathrm{E}+04$ & $3.58 \mathrm{E}+06$ & $1.71 \mathrm{E}+06$ \\
\hline 29 & $1.61 \mathrm{E}+06$ & $9.23 \mathrm{E}+05$ & $1.06 \mathrm{E}+06$ & $3.34 \mathrm{E}+05$ & $3.62 \mathrm{E}+06$ & $1.89 \mathrm{E}+04$ & $3.57 \mathrm{E}+06$ & $1.70 \mathrm{E}+06$ \\
\hline 30 & $1.58 \mathrm{E}+06$ & $9.25 \mathrm{E}+05$ & $9.86 \mathrm{E}+05$ & $3.35 \mathrm{E}+05$ & $3.59 \mathrm{E}+06$ & $1.93 \mathrm{E}+04$ & $3.48 \mathrm{E}+06$ & $1.09 \overline{\mathrm{E}+06}$ \\
\hline 31 & $1.56 \mathrm{E}+06$ & $9.27 \mathrm{E}+05$ & $9.26 \mathrm{E}+05$ & $3.36 \mathrm{E}+05$ & $3.56 \mathrm{E}+06$ & $1.97 \mathrm{E}+04$ & $3.46 \mathrm{E}+06$ & $1.08 \mathrm{E}+06$ \\
\hline 32 & $1.54 \mathrm{E}+06$ & $9.28 \mathrm{E}+05$ & $8.77 \mathrm{E}+05$ & $3.37 \mathrm{E}+05$ & $3.53 \mathrm{E}+06$ & $2.00 \mathrm{E}+04$ & $3.45 \mathrm{E}+06$ & $1.08 \mathrm{E}+06$ \\
\hline 33 & $1.51 \mathrm{E}+06$ & $9.30 \mathrm{E}+05$ & $8.35 \mathrm{E}+05$ & $3.37 \mathrm{E}+05$ & $3.50 \mathrm{E}+06$ & $2.04 \mathrm{E}+04$ & $3.43 \mathrm{E}+06$ & $1.07 \overline{\mathrm{E}}+06$ \\
\hline 34 & $1.49 \mathrm{E}+06$ & $9.31 \mathrm{E}+05$ & $8.00 \mathrm{E}+05$ & $3.38 \mathrm{E}+05$ & $3.47 \mathrm{E}+06$ & $2.08 \mathrm{E}+04$ & $3.42 \mathrm{E}+06$ & $1.07 \mathrm{E}+06$ \\
\hline 35 & $1.47 \mathrm{E}+06$ & $9.33 \mathrm{E}+05$ & $7.70 \mathrm{E}+05$ & $3.39 \mathrm{E}+05$ & $3.44 \mathrm{E}+06$ & $2.11 \mathrm{E}+04$ & $3.40 \mathrm{E}+06$ & $1.06 \mathrm{E}+06$ \\
\hline 36 & $1.45 \mathrm{E}+06$ & $9.34 \mathrm{E}+05$ & $7.44 \mathrm{E}+05$ & $3.39 \mathrm{E}+05$ & $3.41 \mathrm{E}+06$ & $2.15 \mathrm{E}+04$ & $3.39 \mathrm{E}+06$ & $1.06 \mathrm{E}+06$ \\
\hline 37 & $1.40 \mathrm{E}+06$ & $9.36 \mathrm{E}+05$ & $7.22 \mathrm{E}+05$ & $3.40 \mathrm{E}+05$ & $3.38 \mathrm{E}+06$ & $2.18 \mathrm{E}+04$ & $3.38 \mathrm{E}+06$ & $1.06 \mathrm{E}+06$ \\
\hline 38 & $1.37 \mathrm{E}+06$ & $9.37 \mathrm{E}+05$ & $7.00 \mathrm{E}+05$ & $3.41 \mathrm{E}+05$ & $3.35 \mathrm{E}+06$ & $2.22 \mathrm{E}+04$ & $3.36 \mathrm{E}+06$ & $1.05 \mathrm{E}+06$ \\
\hline
\end{tabular}




\begin{tabular}{|c|c|c|c|c|c|c|c|c|}
\hline 39 & $1.35 \mathrm{E}+06$ & $9.39 \mathrm{E}+05$ & $6.80 \mathrm{E}+05$ & $3.41 \mathrm{E}+05$ & $3.33 \mathrm{E}+06$ & $2.25 \mathrm{E}+04$ & $3.35 \mathrm{E}+06$ & $1.05 \mathrm{E}$ \\
\hline 40 & $1.33 \mathrm{E}+06$ & $9.40 \mathrm{E}+05$ & $6.61 \mathrm{E}+05$ & $3.42 \mathrm{E}+05$ & $3.30 \mathrm{E}+06$ & $2.28 \mathrm{E}+04$ & $3.34 \mathrm{E}+06$ & $1.04 \mathrm{E}+\mathrm{C}$ \\
\hline 41 & $1.31 \mathrm{E}+06$ & $9.41 \mathrm{E}+05$ & $6.43 \mathrm{E}+05$ & $3.43 \mathrm{E}+05$ & $3.27 \mathrm{E}+06$ & $2.32 \mathrm{E}+04$ & $3.32 \mathrm{E}+06$ & $1.04 \mathrm{E}+$ \\
\hline 42 & $1.30 \mathrm{E}+06$ & $9.42 \mathrm{E}+05$ & $6.28 \mathrm{E}+05$ & $3.43 \mathrm{E}+05$ & $3.26 \mathrm{E}+06$ & $2.40 \mathrm{E}+04$ & $3.16 \mathrm{E}+06$ & $1.59 \mathrm{E}+0 \mathrm{c}$ \\
\hline 43 & $1.28 \mathrm{E}+06$ & $9.43 \mathrm{E}+05$ & $6.13 \mathrm{E}+05$ & $3.44 \mathrm{E}+05$ & $3.24 \mathrm{E}+06$ & $2.48 \mathrm{E}+04$ & $3.15 \mathrm{E}+06$ & $1.59 \bar{E}+0$ \\
\hline 44 & $1.27 \mathrm{E}+06$ & $9.44 \mathrm{E}+05$ & $6.00 \mathrm{E}+05$ & $3.44 \mathrm{E}+05$ & $3.23 \mathrm{E}+06$ & $2.57 \mathrm{E}+04$ & $3.14 \mathrm{E}+06$ & $1.59 \mathrm{E}+$ \\
\hline 45 & $1.25 \mathrm{E}+06$ & $9.46 \mathrm{E}+05$ & $5.88 \mathrm{E}+05$ & $3.45 \mathrm{E}+05$ & $3.22 \mathrm{E}+06$ & $2.65 \mathrm{E}+04$ & $3.14 \mathrm{E}+06$ & $1.59 \mathrm{E}+\mathrm{C}$ \\
\hline 46 & $1.24 \mathrm{E}+06$ & $9.47 \mathrm{E}+05$ & $5.77 \mathrm{E}+05$ & $3.45 \mathrm{E}+05$ & $3.20 \mathrm{E}+06$ & $2.73 \mathrm{E}+04$ & $3.13 E+06$ & $1.58 \mathrm{E}+\mathrm{C}$ \\
\hline 47 & $1.22 \mathrm{E}+06$ & $9.48 \mathrm{E}+05$ & $5.66 \mathrm{E}+05$ & $3.46 \mathrm{E}+05$ & $3.19 \mathrm{E}+06$ & $2.81 \mathrm{E}+04$ & $3.12 \mathrm{E}+06$ & $1.58 \mathrm{E}+$ \\
\hline 48 & $1.21 \mathrm{E}+06$ & $9.49 \mathrm{E}+05$ & $5.57 \mathrm{E}+05$ & $3.46 \mathrm{E}+05$ & $3.17 \mathrm{E}+06$ & $2.90 \mathrm{E}+04$ & $3.12 \mathrm{E}+06$ & $1.58 \mathrm{E}+\mathrm{C}$ \\
\hline 49 & $1.19 \mathrm{E}+06$ & $9.50 \mathrm{E}+05$ & $5.48 \mathrm{E}+05$ & $3.47 \mathrm{E}+05$ & $3.16 \mathrm{E}+06$ & $2.98 \mathrm{E}+04$ & $3.11 \mathrm{E}+06$ & $1.58 \mathrm{E}+$ \\
\hline 50 & $1.18 E+06$ & $9.51 \mathrm{E}+05$ & $5.40 \mathrm{E}+05$ & $3.47 \mathrm{E}+05$ & $3.15 \mathrm{E}+06$ & $3.06 \mathrm{E}+04$ & $3.10 \mathrm{E}+06$ & $1.58 \mathrm{E}^{+}$ \\
\hline 51 & $1.17 \mathrm{E}+06$ & $9.52 \mathrm{E}+05$ & $5.32 \mathrm{E}+05$ & $3.48 \mathrm{E}+05$ & $3.13 \mathrm{E}+06$ & $3.14 \mathrm{E}+04$ & $3.10 \mathrm{E}+06$ & $1.57 \mathrm{E}+\mathrm{C}$ \\
\hline 52 & $1.16 \mathrm{E}+06$ & $9.52 \mathrm{E}+05$ & $5.24 \mathrm{E}+05$ & $3.48 \mathrm{E}+05$ & $3.12 \mathrm{E}+06$ & $3.22 \mathrm{E}+04$ & $3.09 \mathrm{E}+06$ & $1.57 \mathrm{E}+\mathrm{C}$ \\
\hline 53 & $1.15 \mathrm{E}+06$ & $9.53 \mathrm{E}+05$ & $5.17 \mathrm{E}+05$ & $3.49 \mathrm{E}+05$ & $3.10 \mathrm{E}+06$ & $3.30 \mathrm{E}+04$ & $3.08 \mathrm{E}+06$ & $1.57 \mathrm{E}+$ \\
\hline 54 & $1.14 \mathrm{E}+06$ & $9.54 \mathrm{E}+05$ & $5.10 \mathrm{E}+05$ & $3.49 \mathrm{E}+05$ & $3.08 \mathrm{E}+06$ & $3.33 E+04$ & $2.98 \mathrm{E}+06$ & $9.36 \mathrm{E}+$ \\
\hline 55 & $1.13 \mathrm{E}+06$ & $9.55 \mathrm{E}+05$ & $5.04 \mathrm{E}+05$ & $3.50 \mathrm{E}+05$ & $3.05 E+06$ & $3.36 \mathrm{E}+04$ & $2.97 \mathrm{E}+06$ & $9.32 \mathrm{E}+0$ \\
\hline 56 & $1.12 \mathrm{E}+06$ & $9.56 \mathrm{E}+05$ & $4.97 \mathrm{E}+05$ & $3.50 \mathrm{E}+05$ & $3.03 \mathrm{E}+06$ & $3.39 \mathrm{E}+04$ & $2.96 \mathrm{E}+06$ & $9.28 \mathrm{E}+0$ \\
\hline 57 & $1.11 \mathrm{E}+06$ & $9.57 \mathrm{E}+05$ & $4.91 \mathrm{E}+05$ & $3.51 \mathrm{E}+05$ & $3.00 \mathrm{E}+06$ & $3.42 \mathrm{E}+04$ & $2.95 \mathrm{E}+06$ & $9.24 \mathrm{E}+0$ \\
\hline 58 & $1.10 \mathrm{E}+06$ & $9.57 \mathrm{E}+05$ & $4.85 \mathrm{E}+05$ & $3.51 \mathrm{E}+05$ & $2.98 \mathrm{E}+06$ & $3.45 \mathrm{E}+04$ & $2.93 \mathrm{E}+06$ & $9.20 \mathrm{E}+0$ \\
\hline 59 & $1.09 \mathrm{E}+06$ & $9.58 \mathrm{E}+05$ & $4.79 \mathrm{E}+05$ & $3.52 \mathrm{E}+05$ & $2.95 \mathrm{E}+06$ & $3.48 \mathrm{E}+04$ & $2.92 \mathrm{E}+06$ & $9.17 \mathrm{E}+0$ \\
\hline 60 & $1.08 \mathrm{E}+06$ & $9.59 \mathrm{E}+05$ & $4.74 \mathrm{E}+05$ & $3.52 \mathrm{E}+05$ & $2.93 \mathrm{E}+06$ & $3.51 \mathrm{E}+04$ & $2.91 \mathrm{E}+06$ & $9.13 \mathrm{E}+0$ \\
\hline 61 & $1.07 \mathrm{E}+06$ & $9.60 \mathrm{E}+05$ & $4.68 \mathrm{E}+05$ & $3.52 \mathrm{E}+05$ & $2.90 \mathrm{E}+06$ & $3.54 \mathrm{E}+04$ & $2.90 \mathrm{E}+06$ & $9.09 E+c$ \\
\hline 62 & $1.06 \mathrm{E}+06$ & $9.60 \mathrm{E}+05$ & $4.63 \mathrm{E}+05$ & $3.53 \mathrm{E}+05$ & $2.88 \mathrm{E}+06$ & $3.57 \mathrm{E}+04$ & $2.89 \mathrm{E}+06$ & $9.05 E+c$ \\
\hline 63 & $1.05 E+06$ & $9.61 E+05$ & $4.58 \mathrm{E}+05$ & $3.53 E+05$ & $2.85 E+06$ & $3.60 \mathrm{E}+04$ & $2.87 \mathrm{E}+06$ & $9.02 E+$ \\
\hline 64 & $1.05 \mathrm{E}+06$ & $9.62 \mathrm{E}+05$ & $4.53 \mathrm{E}+05$ & $3.54 \mathrm{E}+05$ & $2.83 E+06$ & $3.62 \mathrm{E}+04$ & $2.86 \mathrm{E}+06$ & $8.98 \mathrm{E}+0$ \\
\hline 65 & $1.04 \mathrm{E}+06$ & $9.62 \mathrm{E}+05$ & $4.48 \mathrm{E}+05$ & $3.54 \mathrm{E}+05$ & $2.81 \mathrm{E}+06$ & $3.65 \mathrm{E}+04$ & $2.85 \mathrm{E}+06$ & $8.94 \mathrm{E}+0$ \\
\hline 66 & $1.00 \mathrm{E}+06$ & $9.63 \mathrm{E}+05$ & $4.43 \mathrm{E}+05$ & $3.55 \mathrm{E}+05$ & $2.79 \mathrm{E}+06$ & $3.73 \mathrm{E}+04$ & $2.71 \mathrm{E}+06$ & $1.46 \mathrm{E}+0$ \\
\hline 67 & $9.87 \mathrm{E}+05$ & $9.64 \mathrm{E}+05$ & $4.36 \mathrm{E}+05$ & $3.55 \mathrm{E}+05$ & $2.78 \mathrm{E}+06$ & $3.81 \mathrm{E}+04$ & $2.70 \mathrm{E}+06$ & $1.46 \mathrm{E}+0$ \\
\hline 68 & $9.77 \mathrm{E}+05$ & $9.64 \mathrm{E}+05$ & $4.28 \mathrm{E}+05$ & $3.55 \mathrm{E}+05$ & $2.77 \mathrm{E}+06$ & $3.88 \mathrm{E}+04$ & $2.70 \mathrm{E}+06$ & $1.45 \mathrm{E}+0$ \\
\hline 69 & $9.70 \mathrm{E}+05$ & $9.65 \mathrm{E}+05$ & $4.20 \mathrm{E}+05$ & $3.56 \mathrm{E}+05$ & $2.76 \mathrm{E}+06$ & $3.96 \mathrm{E}+04$ & $2.69 \mathrm{E}+06$ & $1.45 \mathrm{E}+0$ \\
\hline 168 & $4.06 \mathrm{E}+05$ & $9.66 \mathrm{E}+05$ & $3.12 \mathrm{E}+05$ & $3.84 \mathrm{E}+05$ & $1.50 \mathrm{E}+06$ & $8.67 \mathrm{E}+04$ & $1.57 \mathrm{E}+06$ & $1.08 \mathrm{E}+0$ \\
\hline 336 & $1.92 \mathrm{E}+05$ & $8.08 \mathrm{E}+05$ & $1.58 \mathrm{E}+05$ & $4.08 \mathrm{E}+05$ & $5.84 \mathrm{E}+05$ & $1.58 \mathrm{E}+05$ & $9.34 \mathrm{E}+05$ & $8.12 \mathrm{E}+0$ \\
\hline 504 & $1.41 \mathrm{E}+05$ & $6.67 \mathrm{E}+05$ & $1.08 \mathrm{E}+05$ & $4.22 \mathrm{E}+05$ & $2.74 \mathrm{E}+05$ & $1.95 \mathrm{E}+05$ & $3.91 \mathrm{E}+05$ & $5.33 \mathrm{E}+05$ \\
\hline 720 & $1.03 \mathrm{E}+05$ & $5.20 \mathrm{E}+05$ & $7.22 \mathrm{E}+04$ & $4.34 \mathrm{E}+05$ & $1.35 \mathrm{E}+05$ & $2.15 \mathrm{E}+05$ & $1.87 \mathrm{E}+05$ & $3.67 \mathrm{E}+05$ \\
\hline 2160 & $1.93 \mathrm{E}+04$ & $9.76 \mathrm{E}+04$ & $8.12 E+03$ & $4.56 \mathrm{E}+05$ & $1.20 \mathrm{E}+04$ & $1.25 \mathrm{E}+05$ & $4.24 \mathrm{E}+04$ & $1.26 \mathrm{E}+0$ \\
\hline 4320 & $1.85 E+03$ & $8.24 \mathrm{E}+03$ & $4.09 \mathrm{E}+04$ & $4.36 \mathrm{E}+05$ & $1.93 \mathrm{E}+03$ & $2.98 \mathrm{E}+04$ & $5.03 \mathrm{E}+03$ & $1.96 \mathrm{E}+04$ \\
\hline 4800 & $1.10 \mathrm{E}+03$ & $4.79 \mathrm{E}+03$ & $4.08 \mathrm{E}+04$ & $4.34 \mathrm{E}+05$ & $1.40 \mathrm{E}+03$ & $2.14 \mathrm{E}+04$ & $1.65 \mathrm{E}+03$ & $6.36 \mathrm{E}+03$ \\
\hline 8760 & $1.51 \mathrm{E}+01$ & $5.85 \mathrm{E}+01$ & $3.97 \mathrm{E}+04$ & $4.23 \mathrm{E}+05$ & $1.12 \mathrm{E}+02$ & $1.40 \mathrm{E}+03$ & $5.08 \mathrm{E}+02$ & $1.86 \mathrm{E}+0$ \\
\hline$\overline{\mathbf{B T}}$ & $\begin{array}{l}2.4 \mathrm{E} 6 \\
\mathrm{pCi} / \mathrm{L}\end{array}$ & $\begin{array}{l}8.88 \mathrm{E}+4 \\
\mathrm{~Bq} / \mathrm{kg}\end{array}$ & $8 \mathrm{~Bq} / \mathrm{g}$ & & & & & \\
\hline TO & $\begin{array}{l}5.9 \mathrm{E} 6 \\
\mathrm{pCi} / \mathrm{L}\end{array}$ & $\begin{array}{l}2.18 \mathrm{E}+5 \\
\mathrm{~Bq} / \mathrm{kg} \\
\end{array}$ & $218.3 \mathrm{~Bq} / \mathrm{g}$ & & & & & \\
\hline & & & & & & & & \\
\hline & \multicolumn{2}{|c|}{ VEGETABLES } & \multicolumn{2}{|c|}{ WHEAT SEED } & \multicolumn{2}{|l|}{ MEAT } & \multicolumn{2}{|l|}{ MILK } \\
\hline \multirow[t]{2}{*}{ HOUR } & $\mathrm{O}$ & OBT & HTO & OBT & HTO & OBT & HTO & OBT \\
\hline & $(\mathrm{Bq} / \mathrm{g})$ & $(\mathrm{Bq} / \mathrm{g})$ & $(\mathrm{Bq} / \mathrm{g})$ & $(\mathrm{Bq} / \mathrm{g})$ & $(\mathrm{Bq} / \mathrm{g})$ & $(\mathrm{Bq} / \mathrm{g})$ & $(\mathrm{Bq} / \mathrm{g})$ & $(\mathrm{Bq} / \mathrm{g})$ \\
\hline ak HTO & & & & & & & & \\
\hline
\end{tabular}


Foodstuff Concentrations and Relocation Considerations Following

WSRC-TR-99-00118

a Tritium Oxide Release from SRS Tritium Facilities (U)

\begin{tabular}{|c|c|c|c|c|c|c|c|c|}
\hline $1 / 4 / 5 / 6$ & $1.10 \mathrm{E}+08$ & $5.06 \mathrm{E}+05$ & $3.32 \mathrm{E}+07$ & $1.87 \mathrm{E}+05$ & $4.14 \mathrm{E}+06$ & $3.43 \mathrm{E}+03$ & $3.98 \mathrm{E}+06$ & $1.24 \mathrm{E}+06$ \\
\hline$\overline{\mathrm{SUM}}$ & $1.10 \mathrm{E}+08$ & & $3.34 \mathrm{E}+07$ & & $4.15 \mathrm{E}+06$ & & $5.22 \mathrm{E}+06$ & \\
\hline $\mathrm{Ci} / \mathrm{g}$ & $2.97 \mathrm{E}-03$ & & 9.02E-04 & & 1.12E-04 & & $1.41 \mathrm{E}-04$ & \\
\hline & & & & & & & & \\
\hline DIL HTO & $5.9 \overline{\mathrm{E}}-09$ & & & . & & & & \\
\hline \multicolumn{9}{|l|}{$(\mathrm{Ci} / \mathrm{g})$} \\
\hline & & & & & & & & \\
\hline 168 & $4.06 \mathrm{E}+05$ & $9.66 \mathrm{E}+05$ & $3.12 \mathrm{E}+05$ & $3.84 \mathrm{E}+05$ & $1.50 \mathrm{E}+06$ & $8.67 \mathrm{E}+04$ & $1.57 \mathrm{E}+06$ & $1.08 \mathrm{E}+06$ \\
\hline SUM & $1.37 \mathrm{E}+06$ & & $6.96 \mathrm{E}+05$ & & $1.59 \mathrm{E}+06$ & & $2.65 \mathrm{E}+06$ & \\
\hline $\mathrm{Ci} / \mathrm{g}$ & $3.71 \mathrm{E}-05$ & & 1.88E-05 & & $4.29 \mathrm{E}-05$ & & $7.15 E-05$ & \\
\hline & & & & & & & & \\
\hline DIL OBT & $2.4 \mathrm{E}-09$ & & & & & & & \\
\hline \multicolumn{9}{|l|}{$\mathrm{Ci} / \mathrm{g}$} \\
\hline & & & & & & & & \\
\hline 8760 & $1.51 \mathrm{E}+01$ & $5.85 \bar{E}+01$ & $3.97 \mathrm{E}+04$ & $4.23 \mathrm{E}+05$ & $1.12 \mathrm{E}+02$ & $1.40 \mathrm{E}+03$ & $5.08 \mathrm{E}+02$ & $1.86 \mathrm{E}+03$ \\
\hline SUM & $7.36 \mathrm{E}+01$ & & $4.63 \mathrm{E}+05$ & & $1.51 \mathrm{E}+03$ & & $2.37 \mathrm{E}+03$ & \\
\hline $\mathrm{Ci} / \mathrm{g}$ & $1.99 \mathrm{E}-09$ & & $1.25 \mathrm{E}-05$ & & $4.09 \mathrm{E}-08$ & & $6.39 \mathrm{E}-08$ & \\
\hline & & & & & & & & \\
\hline DIL OBT & 2.4E-09 & & & & & & & \\
\hline $\mathrm{Ci} / \mathrm{g}$ & & & & & & & & \\
\hline & & & & & & & & \\
\hline
\end{tabular}




\begin{tabular}{|c|c|c|c|c|c|c|c|c|}
\hline & \multicolumn{7}{|c|}{ Tritium Facilities, Beyond Design Basis Fire, Adverse Met Conditions } & \\
\hline & \multicolumn{4}{|c|}{ Concentration Values for $10 \mathrm{~km}$ from release } & & & & \\
\hline \multicolumn{9}{|c|}{ Only Wheat Seed is examined since this is the edible portion of the Wheat. The "WHEAT" category is } \\
\hline \multicolumn{9}{|c|}{ actually the unedible plant body and is not considered in the dose estimate. } \\
\hline & & & & & & & & \\
\hline & \multicolumn{2}{|c|}{ VEGETABLES } & \multicolumn{2}{|c|}{ WHEAT SEED } & \multicolumn{2}{|l|}{ MEAT } & \multicolumn{2}{|l|}{ MILK } \\
\hline \multirow[t]{3}{*}{ HOUR } & HTO & OBT & HTO & $\widehat{\mathrm{OBT}}$ & HTO & OBT & HTO & $\overline{\mathrm{BT}}$ \\
\hline & $(\mathrm{Bq} / \mathrm{g})$ & $(\mathrm{Bq} / \mathrm{g})$ & $(\mathrm{Bq} / \mathrm{g})$ & $(\mathrm{Bq} / \mathrm{g})$ & $q / g)$ & $\mathrm{Ba}$ & $(\mathrm{Bq} / \mathrm{g})$ & $\overline{3 a /}$ \\
\hline & & & & & & & & \\
\hline $\mathrm{I}$ & $9.39 \mathrm{E}+06$ & $3.59 \mathrm{E}+04$ & $1.35 \mathrm{E}+06$ & $4.45 \mathrm{E}+03$ & $1.41 \mathrm{E}+05$ & $2.80 \mathrm{E}+01$ & $1.71 \mathrm{E}+04$ & $7.26 \mathrm{E}+03$ \\
\hline 2 & $5.58 \mathrm{E}+06$ & $5.67 \mathrm{E}+04$ & $2.28 \mathrm{E}+06$ & $8.82 \mathrm{E}+03$ & $2.36 \mathrm{E}+05$ & $7.62 \mathrm{E}+01$ & $4.06 \mathrm{E}+04$ & $1.74 \mathrm{E}+04$ \\
\hline 3 & $3.03 \mathrm{E}+06$ & $6.70 \mathrm{E}+04$ & $2.74 \mathrm{E}+06$ & $1.26 \mathrm{E}+04$ & $2.98 \mathrm{E}+05$ & $1.38 \mathrm{E}+02$ & $6.54 \mathrm{E}+04$ & $2.83 \mathrm{E}+04$ \\
\hline 4 & $1.83 \mathrm{E}+06$ & $7.25 \mathrm{E}+04$ & $2.90 \mathrm{E}+06$ & $1.57 \mathrm{E}+04$ & $3.39 \mathrm{E}+05$ & $2.08 \mathrm{E}+02$ & $8.93 \mathrm{E}+04$ & $3.88 \mathrm{E}+04$ \\
\hline 5 & $1.24 \mathrm{E}+06$ & $7.58 \mathrm{E}+04$ & $2.85 \mathrm{E}+06$ & $1.84 \mathrm{E}+04$ & $3.66 \mathrm{E}+05$ & $2.85 \mathrm{E}+02$ & $1.12 \mathrm{E}+05$ & $4.85 \mathrm{E}+04$ \\
\hline 6 & $9.41 \mathrm{E}+05$ & $7.80 \mathrm{E}+04$ & $2.68 \mathrm{E}+06$ & $2.06 \mathrm{E}+04$ & $3.64 \mathrm{E}+05$ & $3.24 \mathrm{E}+02$ & $3.52 \mathrm{E}+05$ & $1.10 \mathrm{E}+05$ \\
\hline 7 & $7.75 \mathrm{E}+05$ & $7.97 \mathrm{E}+04$ & $2.45 \mathrm{E}+06$ & $2.24 \mathrm{E}+04$ & $3.61 \mathrm{E}+05$ & $3.62 \mathrm{E}+02$ & $3.51 \mathrm{E}+05$ & $1.09 \mathrm{E}+05$ \\
\hline 8 & $6.76 \mathrm{E}+05$ & $8.10 \mathrm{E}+04$ & $2.20 \mathrm{E}+06$ & $2.39 \mathrm{E}+04$ & $3.58 \mathrm{E}+05$ & $4.01 \mathrm{E}+02$ & $3.50 \mathrm{E}+05$ & $1.09 \mathrm{E}+05$ \\
\hline 9 & $6.11 \mathrm{E}+05$ & $8.21 \mathrm{E}+04$ & $1.95 \mathrm{E}+06$ & $2.52 \mathrm{E}+04$ & $3.55 \mathrm{E}+05$ & $2+02$ & $3.48 \mathrm{E}+05$ & $1.09 \mathrm{E}+05$ \\
\hline 10 & $5.67 \mathrm{E}+05$ & $8.32 \mathrm{E}+04$ & $1.71 \mathrm{E}+06$ & $2.63 \mathrm{E}+04$ & $3.53 \mathrm{E}+05$ & $4.76 \mathrm{E}+02$ & $3.47 \mathrm{E}+05$ & $1.08 \mathrm{E}+05$ \\
\hline 11 & $5.34 \mathrm{E}+05$ & $8.41 E+04$ & $1.49 \mathrm{E}+06$ & $2.72 \mathrm{E}+04$ & $3.50 \mathrm{E}+05$ & $5.14 \mathrm{E}+02$ & $3.46 \mathrm{E}+05$ & $1.08 \mathrm{E}+05$ \\
\hline 12 & $5.09 \mathrm{E}+05$ & $8.50 \mathrm{E}+04$ & $1.30 \mathrm{E}+06$ & $2.80 \mathrm{E}+04$ & $3.47 \mathrm{E}+05$ & $5.51 \mathrm{E}+02$ & $3.44 \mathrm{E}+05$ & $1.07 \mathrm{E}+05$ \\
\hline 13 & $4.87 \mathrm{E}+05$ & $8.58 \mathrm{E}+04$ & $1.13 \mathrm{E}+06$ & $2.87 \mathrm{E}+04$ & $3.44 \mathrm{E}+05$ & $5.87 \mathrm{E}+02$ & $3.43 \mathrm{E}+05$ & $1.07 \mathrm{E}+05$ \\
\hline 14 & $4.69 \mathrm{E}+05$ & $8.66 \mathrm{E}+04$ & $9 . \overline{77 \mathrm{E}+05}$ & $2.93 \mathrm{E}+04$ & $3.42 \mathrm{E}+05$ & $6 . \overline{24 \mathrm{E}+02}$ & $3.42 \mathrm{E}+05$ & $1.07 \mathrm{E}+05$ \\
\hline 15 & $4.53 \mathrm{E}+05$ & $8.74 \mathrm{E}+04$ & $8.51 \mathrm{E}+05$ & $2.99 \mathrm{E}+04$ & $3.39 \mathrm{E}+05$ & $6.60 \mathrm{E}+02$ & $3.40 \mathrm{E}+05$ & $1.06 \mathrm{E}+05$ \\
\hline 16 & $4.38 \mathrm{E}+05$ & $8.81 \mathrm{E}+04$ & $7.44 \mathrm{E}+05$ & $3.03 \mathrm{E}+04$ & $3.36 \mathrm{E}+05$ & +02 & $3.39 \mathrm{E}+05$ & $1.06 \mathrm{E}+05$ \\
\hline 17 & $4.25 \mathrm{E}+05$ & $8.88 \mathrm{E}+04$ & $6.53 \mathrm{E}+05$ & $3.07 \mathrm{E}+04$ & $3.34 \mathrm{E}+05$ & $7.31 \mathrm{E}+02$ & $3.38 \mathrm{E}+05$ & $1.05 \mathrm{E}+05$ \\
\hline 18 & $4.12 \mathrm{E}+05$ & $8.95 E+04$ & $5.77 \mathrm{E}+05$ & $3.11 \mathrm{E}+04$ & $3.36 \mathrm{E}+05$ & $8.14 E+02$ & $3.25 \mathrm{E}+05$ & $1.60 \mathrm{E}+05$ \\
\hline 19 & $4.01 \mathrm{E}+05$ & $9.01 \mathrm{E}+04$ & $5.13 \mathrm{E}+05$ & $3.15 \mathrm{E}+04$ & $3.37 \mathrm{E}+05$ & $8.98 \mathrm{E}+02$ & $3.26 \mathrm{E}+05$ & $1.60 \mathrm{E}+05$ \\
\hline 20 & $3.90 \mathrm{E}+05$ & $9.07 \mathrm{E}+04$ & $4.59 \mathrm{E}+05$ & $3.18 \mathrm{E}+04$ & $3.39 \mathrm{E}+05$ & $9.83 E+02$ & $3.27 \mathrm{E}+05$ & $1.60 \mathrm{E}+05$ \\
\hline 21 & $3.80 \mathrm{E}+05$ & $9.13 \mathrm{E}+04$ & $4.14 \mathrm{E}+05$ & $3.21 \mathrm{E}+04$ & $3.40 \mathrm{E}+05$ & $1.07 \mathrm{E}+03$ & $3.27 \mathrm{E}+05$ & $1.61 \mathrm{E}+05$ \\
\hline 22 & $3.70 \mathrm{E}+05$ & $9.19 E+04$ & $3.77 \bar{E}+05$ & $3.24 \mathrm{E}+04$ & $3.41 \mathrm{E}+05$ & $1.15 \mathrm{E}+03$ & $3.28 \mathrm{E}+05$ & $1.61 \mathrm{E}+05$ \\
\hline 23 & $3.61 \mathrm{E}+05$ & $9.25 \mathrm{E}+04$ & $3.45 \mathrm{E}+05$ & $3.26 \mathrm{E}+04$ & $3.43 \mathrm{E}+05$ & $1.24 \mathrm{E}+03$ & $3.29 \mathrm{E}+05$ & $1.62 \mathrm{E}+05$ \\
\hline 24 & $3.52 \mathrm{E}+05$ & $9.30 \mathrm{E}+04$ & $3.18 \mathrm{E}+05$ & $3.29 \mathrm{E}+04$ & $3.44 \mathrm{E}+05$ & $1.32 \mathrm{E}+03$ & $3.29 \mathrm{E}+05$ & $1.62 \mathrm{E}+05$ \\
\hline 25 & $3.43 \mathrm{E}+05$ & $9.36 \mathrm{E}+04$ & $2.96 \mathrm{E}+05$ & $3.31 \mathrm{E}+04$ & $3.45 \mathrm{E}+05$ & $1.41 \mathrm{E}+03$ & $3.30 \mathrm{E}+05$ & $1.62 \mathrm{E}+05$ \\
\hline 26 & $3.35 \mathrm{E}+05$ & $9.41 \mathrm{E}+04$ & $2.77 \mathrm{E}+05$ & $3.33 \mathrm{E}+04$ & $3.45 \mathrm{E}+05$ & $1.50 \mathrm{E}+03$ & $3.31 \mathrm{E}+05$ & $1.63 \mathrm{E}+05$ \\
\hline 27 & $3.27 \mathrm{E}+05$ & $9.46 \mathrm{E}+04$ & $2.60 \mathrm{E}+05$ & $3.35 \mathrm{E}+04$ & $3.46 \mathrm{E}+05$ & $1.58 \mathrm{E}+03$ & $3.31 \mathrm{E}+05$ & $1.63 \mathrm{E}+05$ \\
\hline 28 & $3.21 \mathrm{E}+05$ & $9.51 E+04$ & $2.46 \mathrm{E}+05$ & $3.37 \mathrm{E}+04$ & $3.47 \mathrm{E}+05$ & $1.67 \mathrm{E}+03$ & $3.32 \mathrm{E}+05$ & $1.63 \mathrm{E}+05$ \\
\hline 29 & $3.14 \mathrm{E}+05$ & $9.55 \mathrm{E}+04$ & $2.34 \mathrm{E}+05$ & $3.39 \mathrm{E}+04$ & $3.48 \mathrm{E}+05$ & $1.76 \mathrm{E}+03$ & $3.32 \mathrm{E}+05$ & $1.64 \mathrm{E}+05$ \\
\hline 30 & $3.08 \mathrm{E}+05$ & $9.60 \mathrm{E}+04$ & $2.24 \mathrm{E}+05$ & $3.41 \mathrm{E}+04$ & $3.45 \mathrm{E}+05$ & $1.79 \mathrm{E}+03$ & $3.34 \mathrm{E}+05$ & $1.04 \mathrm{E}+05$ \\
\hline 31 & $3.02 \mathrm{E}+05$ & $9.64 \mathrm{E}+04$ & $2.15 \mathrm{E}+05$ & $3.43 \mathrm{E}+04$ & $3.42 \mathrm{E}+05$ & $1.83 \mathrm{E}+03$ & $3.33 \mathrm{E}+05$ & $1.04 \mathrm{E}+05$ \\
\hline 32 & $2.96 \mathrm{E}+05$ & $9.69 \mathrm{E}+04$ & $2.07 \mathrm{E}+05$ & $3.45 \mathrm{E}+04$ & $3.39 \mathrm{E}+05$ & $1.86 \mathrm{E}+03$ & $3.32 \mathrm{E}+05$ & $1.04 \mathrm{E}+05$ \\
\hline 33 & $2.90 \mathrm{E}+05$ & $9.73 \mathrm{E}+04$ & $2.00 \mathrm{E}+05$ & $3.47 \mathrm{E}+04$ & $3.37 \mathrm{E}+05$ & $1.90 \mathrm{E}+03$ & $3.30 \mathrm{E}+05$ & $1.03 \mathrm{E}+05$ \\
\hline 34 & $2.85 \mathrm{E}+05$ & $9.77 \mathrm{E}+04$ & $1.93 E+05$ & $3.48 \mathrm{E}+04$ & $3.34 \mathrm{E}+05$ & $1.93 \mathrm{E}+03$ & $3.29 \mathrm{E}+05$ & $1.03 \mathrm{E}+05$ \\
\hline 35 & $2.80 \mathrm{E}+05$ & $9.81 \mathrm{E}+04$ & $1.88 \mathrm{E}+05$ & $3.50 \mathrm{E}+04$ & $3.31 \mathrm{E}+05$ & $1.97 \mathrm{E}+03$ & $3.28 \mathrm{E}+05$ & $1.02 \mathrm{E}+05$ \\
\hline 36 & $2.74 \mathrm{E}+05$ & $9.85 \mathrm{E}+04$ & $1.83 \mathrm{E}+05$ & $3.52 \mathrm{E}+04$ & $3.29 \mathrm{E}+05$ & $2.00 \mathrm{E}+03$ & $3.26 \mathrm{E}+05$ & $1.02 \mathrm{E}+05$ \\
\hline 37 & $2.69 \mathrm{E}+05$ & $9.89 \mathrm{E}+04$ & $1.78 \mathrm{E}+05$ & $3.53 \mathrm{E}+04$ & $3.26 \mathrm{E}+05$ & $2.03 \mathrm{E}+03$ & $3.25 \mathrm{E}+05$ & $1.02 \mathrm{E}+05$ \\
\hline 38 & $2.63 \mathrm{E}+05$ & $9.92 \mathrm{E}+04$ & $1.74 \mathrm{E}+05$ & $3.55 \mathrm{E}+04$ & $3.23 \mathrm{E}+05$ & $2.07 \mathrm{E}+03$ & $3.24 \mathrm{E}+05$ & $1.01 \mathrm{E}+05$ \\
\hline
\end{tabular}


Foodstuff Concentrations and Relocation Considerations Following a Tritium Oxide Release from SRS Tritium Facilities (U)

\begin{tabular}{|c|c|c|c|c|c|c|c|c|}
\hline 39 & $2.59 \mathrm{E}+05$ & $9.96 \mathrm{E}+04$ & $1.70 \mathrm{E}+05$ & $3.56 \mathrm{E}+04$ & $3.21 \mathrm{E}+05$ & $2.10 \mathrm{E}+03$ & $3.22 \mathrm{E}+05$ & $1.01 \mathrm{E}+05$ \\
\hline 40 & $2.54 \mathrm{E}+05$ & $9.99 \mathrm{E}+04$ & $1.67 \mathrm{E}+05$ & $3.58 \mathrm{E}+04$ & $3.18 \mathrm{E}+05$ & $2.13 \mathrm{E}+03$ & $3.21 \mathrm{E}+05$ & $1.00 \mathrm{E}+0$ \\
\hline 41 & $2.49 \mathrm{E}+05$ & $1.00 \mathrm{E}+05$ & $1.63 \mathrm{E}+05$ & $3.59 \mathrm{E}+04$ & $3.15 \mathrm{E}+05$ & $2.17 \mathrm{E}+03$ & $3.20 \mathrm{E}+05$ & $1.00 \mathrm{E}+05$ \\
\hline 42 & $2.45 \mathrm{E}+05$ & $1.01 \mathrm{E}+05$ & $1.60 \mathrm{E}+05$ & $3.61 \mathrm{E}+04$ & $3.16 \mathrm{E}+05$ & $2.25 \mathrm{E}+03$ & $3.06 \mathrm{E}+05$ & $1.61 \mathrm{E}+05$ \\
\hline 43 & $2.40 \mathrm{E}+05$ & $1.01 \mathrm{E}+05$ & $1.57 \mathrm{E}+05$ & $3.62 \mathrm{E}+04$ & $3.16 \mathrm{E}+05$ & $2.34 \mathrm{E}+03$ & $3.06 \mathrm{E}+05$ & $1.61 \mathrm{E}+0$ \\
\hline 44 & $2.36 \mathrm{E}+05$ & $1.01 \mathrm{E}+05$ & $1.55 \mathrm{E}+05$ & $3.64 \mathrm{E}+04$ & $3.16 \mathrm{E}+05$ & $2.42 \mathrm{E}+03$ & $3.06 \mathrm{E}+05$ & $1.61 \mathrm{E}+0$ \\
\hline 45 & $2.32 \mathrm{E}+05$ & $1.02 \mathrm{E}+05$ & $1.52 \mathrm{E}+05$ & $3.65 \mathrm{E}+04$ & $3.16 \mathrm{E}+05$ & $2.51 \mathrm{E}+03$ & $3.06 \mathrm{E}+05$ & $1.61 \mathrm{E}+0$ \\
\hline 46 & $2.28 \mathrm{E}+05$ & $1.02 \mathrm{E}+05$ & $1.50 \mathrm{E}+05$ & $3.67 \mathrm{E}+04$ & $3.16 \mathrm{E}+05$ & $2.60 \mathrm{E}+03$ & $3.06 \mathrm{E}+05$ & $1.61 \mathrm{E}+0$ \\
\hline 47 & $2.24 \mathrm{E}+05$ & $1.02 \mathrm{E}+05$ & $1.47 \mathrm{E}+05$ & $3.68 \mathrm{E}+04$ & $3.15 \mathrm{E}+05$ & $2.68 \mathrm{E}+03$ & $3.06 \mathrm{E}+05$ & $1.61 \mathrm{E}+0$ \\
\hline 48 & $2.20 \mathrm{E}+05$ & $1.02 \mathrm{E}+05$ & $1.45 \mathrm{E}+05$ & $3.69 \mathrm{E}+04$ & $3.15 \mathrm{E}+05$ & $2.77 \mathrm{E}+03$ & $3.06 \mathrm{E}+05$ & $1.61 \mathrm{E}+05$ \\
\hline 49 & $2.17 \mathrm{E}+05$ & $1.03 \mathrm{E}+05$ & $1.43 \mathrm{E}+05$ & $3.71 \mathrm{E}+04$ & $3.15 \mathrm{E}+05$ & $2.86 \mathrm{E}+03$ & $3.06 \mathrm{E}+05$ & $1.61 \mathrm{E}+05$ \\
\hline 50 & $2.14 \mathrm{E}+05$ & $1.03 \mathrm{E}+05$ & $1.41 \mathrm{E}+05$ & $3.72 \bar{E}+04$ & $3.15 \mathrm{E}+05$ & $2.94 \mathrm{E}+03$ & $3.06 \mathrm{E}+05$ & $1.61 \mathrm{E}+0$ \\
\hline 51 & $2.10 \mathrm{E}+05$ & $1.03 \mathrm{E}+05$ & $1.39 \mathrm{E}+05$ & $3.73 E+04$ & $3.14 \mathrm{E}+05$ & $3.03 \mathrm{E}+03$ & $3.06 \mathrm{E}+05$ & $1.62 \mathrm{E}+05$ \\
\hline 52 & $2.07 \mathrm{E}+05$ & $1.03 \mathrm{E}+05$ & $1.37 \mathrm{E}+05$ & $3.75 \mathrm{E}+04$ & $3.14 \mathrm{E}+05$ & $3.12 \mathrm{E}+03$ & $3.05 \mathrm{E}+05$ & $1.62 \mathrm{E}+0$ \\
\hline 53 & $2.05 \mathrm{E}+05$ & $1.04 \mathrm{E}+05$ & $1.35 \mathrm{E}+05$ & $3.76 \mathrm{E}+04$ & $3.14 \mathrm{E}+05$ & $3.20 \mathrm{E}+03$ & $3.05 \mathrm{E}+05$ & $1.62 \mathrm{E}+0$ \\
\hline 54 & $2.02 \mathrm{E}+05$ & $1.04 \mathrm{E}+05$ & $1.33 \mathrm{E}+05$ & $3.77 \mathrm{E}+04$ & $3.11 \mathrm{E}+05$ & $3.23 \mathrm{E}+03$ & $3.02 \mathrm{E}+05$ & $9.45 \mathrm{E}+0$ \\
\hline 55 & $1.99 \mathrm{E}+05$ & $1.04 \mathrm{E}+05$ & $1.31 \mathrm{E}+05$ & $3.78 \mathrm{E}+04$ & $3.09 \mathrm{E}+05$ & $3.26 \mathrm{E}+03$ & $3.00 \mathrm{E}+05$ & $9.41 \mathrm{E}+0$ \\
\hline 56 & $1.96 \mathrm{E}+05$ & $1.04 \mathrm{E}+05$ & $1.29 \mathrm{E}+05$ & $3.79 \mathrm{E}+04$ & $3.06 \mathrm{E}+05$ & $3.29 \mathrm{E}+03$ & $2.99 \mathrm{E}+05$ & $9.38 \mathrm{E}+0$ \\
\hline 57 & $1.94 \mathrm{E}+05$ & $1.05 \mathrm{E}+05$ & $1.27 \mathrm{E}+05$ & $3.81 \mathrm{E}+04$ & $3.04 \mathrm{E}+05$ & $3.33 \mathrm{E}+03$ & $2.98 \mathrm{E}+05$ & $9.34 \mathrm{E}+04$ \\
\hline$\overline{58}$ & $1.91 \mathrm{E}+05$ & $1.05 \mathrm{E}+05$ & $1.25 \mathrm{E}+05$ & $3.82 \mathrm{E}+04$ & $3.01 \mathrm{E}+05$ & $3.36 \mathrm{E}+03$ & $2.97 \mathrm{E}+05$ & $9.30 \mathrm{E}+0$ \\
\hline 59 & $1.89 \mathrm{E}+05$ & $1.05 \mathrm{E}+05$ & $1.23 \mathrm{E}+05$ & $3.83 E+04$ & $2.99 \mathrm{E}+05$ & $3.39 \mathrm{E}+03$ & $2.95 \mathrm{E}+05$ & $9.26 \mathrm{E}+0$ \\
\hline 60 & $1.86 \mathrm{E}+05$ & $1.05 \mathrm{E}+05$ & $1.21 \mathrm{E}+05$ & $3.84 \mathrm{E}+04$ & $2.96 \mathrm{E}+05$ & $3.41 \mathrm{E}+03$ & $2.94 \mathrm{E}+05$ & $9.23 \mathrm{E}+0$ \\
\hline 61 & $1.84 \mathrm{E}+05$ & $1.06 \mathrm{E}+05$ & $1.19 \mathrm{E}+05$ & $3.85 \mathrm{E}+04$ & $2.94 \mathrm{E}+05$ & $3.44 \mathrm{E}+03$ & $2.93 \mathrm{E}+05$ & $9.19 \mathrm{E}+0$ \\
\hline 62 & $1.82 \mathrm{E}+05$ & $1.06 \mathrm{E}+05$ & $1.17 \mathrm{E}+05$ & $3.86 \mathrm{E}+04$ & $2.91 \mathrm{E}+05$ & $3.47 \mathrm{E}+03$ & $2.92 \mathrm{E}+05$ & $9.15 \mathrm{E}+04$ \\
\hline 63 & $1.80 \mathrm{E}+05$ & $1.06 \mathrm{E}+05$ & $1.15 \mathrm{E}+05$ & $3.87 \mathrm{E}+04$ & $2.89 \mathrm{E}+05$ & $3.50 \mathrm{E}+03$ & $2.91 \mathrm{E}+05$ & $9.12 \mathrm{E}+04$ \\
\hline 64 & $1.77 \mathrm{E}+05$ & $1.06 \mathrm{E}+05$ & $1.13 \mathrm{E}+05$ & $3.88 \mathrm{E}+04$ & $2.87 \mathrm{E}+05$ & $3.53 \mathrm{E}+03$ & $2.90 \mathrm{E}+05$ & $9.08 \mathrm{E}+04$ \\
\hline 65 & $1.75 \mathrm{E}+05$ & $1.06 \mathrm{E}+05$ & $1.11 \mathrm{E}+05$ & $3.89 \mathrm{E}+04$ & $2.84 \mathrm{E}+05$ & $3.56 \mathrm{E}+03$ & $2.88 \mathrm{E}+05$ & $9.04 \mathrm{E}+04$ \\
\hline 66 & $1.72 \mathrm{E}+05$ & $1.06 \mathrm{E}+05$ & $1.09 \mathrm{E}+05$ & $3.90 \mathrm{E}+04$ & $2.84 \mathrm{E}+05$ & $3.64 \mathrm{E}+03$ & $2.75 \mathrm{E}+05$ & $1.55 \mathrm{E}+05$ \\
\hline 67 & $1.70 \mathrm{E}+05$ & $1.07 \mathrm{E}+05$ & $1.07 \mathrm{E}+05$ & $3.91 \mathrm{E}+04$ & $2.83 \mathrm{E}+05$ & $3.73 E+03$ & $2.75 \mathrm{E}+05$ & $1.55 \mathrm{E}+05$ \\
\hline 68 & $1.68 \mathrm{E}+05$ & $1.07 \mathrm{E}+05$ & $1.06 \mathrm{E}+05$ & $3.92 \mathrm{E}+04$ & $2.83 \mathrm{E}+05$ & $3.81 \mathrm{E}+03$ & $2.74 \mathrm{E}+05$ & $1.55 \mathrm{E}+05$ \\
\hline 69 & $1.66 \mathrm{E}+05$ & $1.07 \mathrm{E}+05$ & $1.04 \mathrm{E}+05$ & $3.93 \mathrm{E}+04$ & $2.82 \mathrm{E}+05$ & $3.89 \mathrm{E}+03$ & $2.74 \mathrm{E}+05$ & $1.54 \mathrm{E}+05$ \\
\hline 168 & $4.76 \mathrm{E}+04$ & $1.12 \mathrm{E}+05$ & $4.26 \mathrm{E}+04$ & $4.51 \mathrm{E}+04$ & $1.68 \mathrm{E}+05$ & $9.28 \mathrm{E}+03$ & $1.75 \mathrm{E}+05$ & $1.24 \mathrm{E}+05$ \\
\hline 336 & $2.17 \mathrm{E}+04$ & $9.35 \mathrm{E}+04$ & $1.87 \mathrm{E}+04$ & $4.79 \mathrm{E}+04$ & $6.67 \mathrm{E}+04$ & $1.78 \mathrm{E}+04$ & $1.06 \mathrm{E}+05$ & $9.48 \mathrm{E}+04$ \\
\hline 504 & $1.59 \mathrm{E}+04$ & $7.72 \mathrm{E}+04$ & $1.27 \mathrm{E}+04$ & $4.96 \mathrm{E}+04$ & $3.16 \mathrm{E}+04$ & $2.23 E+04$ & $4.49 \mathrm{E}+04$ & $6.27 \mathrm{E}+04$ \\
\hline 720 & $1.15 \mathrm{E}+04$ & $6.02 \mathrm{E}+04$ & $8.47 \mathrm{E}+03$ & $5.10 \mathrm{E}+04$ & $1.58 \mathrm{E}+04$ & $2.48 \mathrm{E}+04$ & $2.18 \mathrm{E}+04$ & $4.34 \mathrm{E}+04$ \\
\hline 2160 & $2.16 \mathrm{E}+03$ & $1.12 \mathrm{E}+04$ & $9.49 \mathrm{E}+02$ & $5.37 \mathrm{E}+04$ & $1.39 \mathrm{E}+03$ & $1.46 \mathrm{E}+04$ & $4.96 \mathrm{E}+03$ & $1.48 \mathrm{E}+04$ \\
\hline 4320 & $2.07 \mathrm{E}+02$ & $9.37 \mathrm{E}+02$ & $4.78 \mathrm{E}+03$ & $5.12 \mathrm{E}+04$ & $2.20 \mathrm{E}+02$ & $3.47 \mathrm{E}+03$ & $5.78 \mathrm{E}+02$ & $2.28 \mathrm{E}+03$ \\
\hline 4800 & $1.23 \mathrm{E}+02$ & $5.44 \mathrm{E}+02$ & $4.77 \mathrm{E}+03$ & $5.11 \mathrm{E}+04$ & $1.59 \mathrm{E}+02$ & $2.49 \mathrm{E}+03$ & $1.87 \mathrm{E}+02$ & $7.36 \mathrm{E}+02$ \\
\hline 8760 & $1.69 \mathrm{E}+00$ & $6.58 \mathrm{E}+00$ & $4.65 \mathrm{E}+03$ & $4.98 \mathrm{E}+04$ & $1.26 \mathrm{E}+01$ & $1.63 \mathrm{E}+02$ & $5.75 \mathrm{E}+01$ & $2.14 \mathrm{E}+02$ \\
\hline BT & $\begin{array}{l}2.4 \mathrm{E} 6 \\
\mathrm{pCi} / \mathrm{L}\end{array}$ & $\begin{array}{l}8.88 \mathrm{E}+4 \\
\mathrm{~Bq} / \mathrm{kg}\end{array}$ & $8 \mathrm{~Bq} / \mathrm{g}$ & & & & & \\
\hline TO & $\begin{array}{l}5.9 \mathrm{E} 6 \\
\mathrm{pCi} / \mathrm{L}\end{array}$ & $\begin{array}{l}2.18 \mathrm{E}+5 \\
\mathrm{~Bq} / \mathrm{kg}\end{array}$ & $218.3 \mathrm{~Bq} / \mathrm{g}$ & & & & & \\
\hline & & & & & & & & \\
\hline & \multicolumn{2}{|c|}{ VEGETABLES } & \multicolumn{2}{|c|}{ WHEAT SEED } & \multicolumn{2}{|l|}{ MEAT } & \multicolumn{2}{|l|}{ MILK } \\
\hline \multirow[t]{2}{*}{ HŌUR } & TO & OBT & $\Gamma \mathrm{O}$ & OBT & HTO & OBT & HTO & $\overline{\mathrm{OBT}}$ \\
\hline & $(\mathrm{Bq} / \mathrm{g})$ & $(\mathrm{Bq} / \mathrm{g})$ & $(\mathrm{Bq} / \mathrm{g})$ & $(\mathrm{Bq} / \mathrm{g})$ & $(\mathrm{Bq} / \mathrm{g})$ & $(\mathrm{Bq} / \mathrm{g})$ & $(\mathrm{Bq} / \mathrm{g})$ & $(\mathrm{Bq} / \mathrm{g})$ \\
\hline eak $\mathrm{HTO}$ & & & & & & & & \\
\hline
\end{tabular}


a Tritium Oxide Release from SRS Tritium Facilities (U)

\begin{tabular}{|c|c|c|c|c|c|c|c|c|}
\hline $1 / 4 / 5 / 6$ & $9.39 \mathrm{E}+06$ & $3.59 \mathrm{E}+04$ & $2.90 \mathrm{E}+06$ & $1.57 \mathrm{E}+04$ & $3.66 \overline{\mathrm{E}}+05$ & $2.85 \mathrm{E}+02$ & $3.52 \mathrm{E}+05$ & $1.10 \mathrm{E}+05$ \\
\hline SUM & $9.43 \mathrm{E}+06$ & & $2.91 \mathrm{E}+06$ & & $3.66 \mathrm{E}+05$ & & $4.62 \mathrm{E}+05$ & \\
\hline $\mathrm{Ci} / \mathrm{g}$ & $2.55 \mathrm{E}-04$ & & $7.87 \mathrm{E}-05$ & & $9.90 \mathrm{E}-06$ & & $1.25 \mathrm{E}-05$ & \\
\hline & & & & & & & & \\
\hline DIL HTO & $5.9 \mathrm{E}-09$ & & & & & & & \\
\hline \multicolumn{9}{|l|}{$(\mathrm{Ci} / \mathrm{g})$} \\
\hline & & & & & & & & \\
\hline 168 & $4.76 \mathrm{E}+04$ & $1.12 \mathrm{E}+05$ & $4.26 \mathrm{E}+04$ & $4.51 \mathrm{E}+04$ & $1.68 \mathrm{E}+05$ & $9.28 \mathrm{E}+03$ & $1 . \overline{75 E+05}$ & $1.24 \mathrm{E}+05$ \\
\hline$\overline{\text { SUM }}$ & $1.60 \mathrm{E}+05$ & & $8.77 \mathrm{E}+04$ & & $1.77 \mathrm{E}+05$ & & $2.99 \mathrm{E}+05$ & \\
\hline$\overline{\mathrm{Ci} / \mathrm{g}}$ & $4.31 \mathrm{E}-06$ & & $2.37 \mathrm{E}-06$ & & $4.79 \mathrm{E}-06$ & & $8.09 E-06$ & \\
\hline & & & & & & & & \\
\hline DIL OBT & $2.4 \mathrm{E}-09$ & & & & & & & \\
\hline \multicolumn{9}{|l|}{$\mathrm{Ci} / \mathrm{g}$} \\
\hline & & & & & & & & \\
\hline 8760 & $1.69 \mathrm{E}+00$ & $6.58 \mathrm{E}+00$ & $4.65 \mathrm{E}+03$ & $4.98 \mathrm{E}+04$ & $1.26 \overline{\mathrm{E}}+01$ & $1.63 \mathrm{E}+02$ & $5.75 \mathrm{E}+01$ & $2.14 \mathrm{E}+02$ \\
\hline SUM & $8.27 \mathrm{E}+00$ & & $5.44 \mathrm{E}+04$ & & $1.75 \mathrm{E}+02$ & & $2.72 \mathrm{E}+02$ & \\
\hline$\overline{\mathrm{Ci} / \mathrm{g}}$ & $2.23 \mathrm{E}-10$ & & 1.47E-06 & & $4.73 E-09$ & & $7.35 \mathrm{E}-09$ & \\
\hline & & & & & & & & \\
\hline DIL OBT & $2.4 \mathrm{E}-09$ & & & & & & & \\
\hline$\overline{\mathrm{Ci}} / \mathrm{g}$ & & & & & & & & \\
\hline
\end{tabular}




\begin{tabular}{|c|c|c|c|c|c|c|c|c|}
\hline & \multicolumn{7}{|c|}{ Tritium Facilities, Beyond Design Basis Fire, Adverse Met Conditions } & \\
\hline & \multicolumn{4}{|c|}{ Concentration Values for $11.54 \mathrm{~km}$ from release } & & & & \\
\hline \multicolumn{9}{|c|}{ Only Wheat Seed is examined since this is the edible portion of the Wheat. The "WHEAT" category is } \\
\hline \multicolumn{9}{|c|}{ actually the unedible plant body and is not considered in the dose estimate. } \\
\hline & & & & & & & & \\
\hline & \multicolumn{2}{|c|}{ VEGETABLES } & \multicolumn{2}{|c|}{ WHEAT SEED } & \multicolumn{2}{|l|}{ MEAT } & \multicolumn{2}{|l|}{ MILK } \\
\hline \multirow[t]{3}{*}{ HOUR } & HTO & TOBT & HTO & OBT & HTO & $\overline{\mathrm{OBT}}$ & HTO & BT \\
\hline & $(\mathrm{Bq} / \mathrm{g})$ & $(\mathrm{Bq} / \mathrm{g})$ & $(\mathrm{Bq} / \mathrm{g})$ & $(\mathrm{Bq} / \mathrm{g})$ & $(\mathrm{Bq} / \mathrm{g})$ & 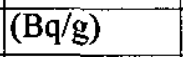 & $(\mathrm{Bq} / \mathrm{g})$ & $3 \mathrm{a} / \mathrm{g})$ \\
\hline & & & & & & & & \\
\hline 1 & $2.21 \mathrm{E}+06$ & $1.44 \mathrm{E}+03$ & $5.39 \mathrm{E}+04$ & $1.78 \mathrm{E}+02$ & $7.51 \mathrm{E}+03$ & $1.32 \mathrm{E}+00$ & $9.10 \mathrm{E}+02$ & $3.61 \mathrm{E}+02$ \\
\hline 2 & $9.66 \mathrm{E}+06$ & $3.84 \mathrm{E}+04$ & $1.44 \mathrm{E}+06$ & $4.81 E+03$ & $1.51 \mathrm{E}+05$ & $3.14 \mathrm{E}+01$ & $1.71 \mathrm{E}+04$ & $7.24 \mathrm{E}+03$ \\
\hline 3 & $5.05 E+06$ & $5.63 \mathrm{E}+04$ & $2.26 \mathrm{E}+06$ & $8.92 \mathrm{E}+03$ & $2.36 \mathrm{E}+05$ & $7.98 \mathrm{E}+01$ & $3.82 \mathrm{E}+04$ & $1.64 \mathrm{E}+0$ \\
\hline 4 & $2.92 \mathrm{E}+06$ & $6.56 \mathrm{E}+04$ & $2 . \overline{67 \mathrm{E}+06}$ & $1.25 \mathrm{E}+04$ & $2.94 \mathrm{E}+05$ & $1.41 \mathrm{E}+02$ & $6.07 \mathrm{E}+04$ & $2.62 \mathrm{E}+04$ \\
\hline 5 & $1.90 \mathrm{E}+06$ & $7.10 \mathrm{E}+04$ & $2.81 \mathrm{E}+06$ & $1.55 \mathrm{E}+04$ & $3.33 \mathrm{E}+05$ & $2.10 \mathrm{E}+02$ & $8.25 \mathrm{E}+04$ & $3.58 \mathrm{E}+04$ \\
\hline 6 & $1.39 \mathrm{E}+06$ & $7.44 \mathrm{E}+04$ & $2.76 \mathrm{E}+06$ & $1.80 \mathrm{E}+04$ & $3.31 \mathrm{E}+05$ & $2.45 \mathrm{E}+02$ & $3.21 \mathrm{E}+05$ & $9.99 \mathrm{E}+0$ \\
\hline$\overline{7}$ & $1.11 \mathrm{E}+06$ & $7.69 \mathrm{E}+04$ & $2.61 \mathrm{E}+06$ & $2.02 \mathrm{E}+04$ & $3.29 \mathrm{E}+05$ & $2.81 E+02$ & $3.20 \mathrm{E}+05$ & $9.95 \mathrm{E}+04$ \\
\hline 8 & $9.46 \mathrm{E}+05$ & $7.88 \mathrm{E}+04$ & $2.40 \mathrm{E}+06$ & $2.20 \mathrm{E}+04$ & $3.26 \mathrm{E}+05$ & $3.16 \mathrm{E}+02$ & $3.19 \mathrm{E}+05$ & $9.92 \mathrm{E}+04$ \\
\hline 9 & $8.44 \mathrm{E}+05$ & $8.05 \mathrm{E}+04$ & $2.17 \mathrm{E}+06$ & $2.36 \mathrm{E}+04$ & $3.24 \mathrm{E}+05$ & $3.50 \mathrm{E}+02$ & $3.17 \mathrm{E}+05$ & $9.89 \mathrm{E}+04$ \\
\hline 10 & $7.76 \mathrm{E}+05$ & $8.19 \mathrm{E}+04$ & $1.94 \mathrm{E}+06$ & $2.49 \mathrm{E}+04$ & $3.22 \mathrm{E}+05$ & $3.84 \mathrm{E}+02$ & $3.16 \mathrm{E}+05$ & $9.85 \mathrm{E}+04$ \\
\hline 11 & $7.27 \mathrm{E}+05$ & $8.33 \mathrm{E}+04$ & $1.72 \mathrm{E}+06$ & $2.61 \mathrm{E}+04$ & $3.19 \mathrm{E}+05$ & $\mathrm{E}+02$ & $3.15 \mathrm{E}+05$ & $9.82 \mathrm{E}+0$ \\
\hline 12 & $6.89 \mathrm{E}+05$ & $8.45 \mathrm{E}+04$ & $1.51 \mathrm{E}+06$ & $2.70 \mathrm{E}+04$ & $3.17 \mathrm{E}+05$ & $4.52 \mathrm{E}+02$ & $3.14 \mathrm{E}+05$ & $9.78 \mathrm{E}+0$ \\
\hline 13 & $6.58 \mathrm{E}+05$ & $8.57 \mathrm{E}+04$ & $1.33 \mathrm{E}+06$ & $2.79 \mathrm{E}+04$ & $3.15 \mathrm{E}+05$ & $4.86 \mathrm{E}+02$ & $3.13 \mathrm{E}+05$ & $9.75 \mathrm{E}+0$ \\
\hline 14 & $6.32 \mathrm{E}+05$ & $8.68 \mathrm{E}+04$ & $1.17 \mathrm{E}+06$ & $2.87 \mathrm{E}+04$ & $3.12 \mathrm{E}+05$ & $5.19 \mathrm{E}+02$ & $3.12 \mathrm{E}+05$ & $9.71 \mathrm{E}+04$ \\
\hline 15 & $6.09 \mathrm{E}+05$ & $8.78 \mathrm{E}+04$ & $1.04 \mathrm{E}+06$ & $2.93 \mathrm{E}+04$ & $3.10 \mathrm{E}+05$ & $5.52 \mathrm{E}+02$ & $3.11 \mathrm{E}+05$ & $9.67 \mathrm{E}+0$ \\
\hline 16 & $5.89 \mathrm{E}+05$ & $8.89 \mathrm{E}+04$ & $9.16 \mathrm{E}+05$ & $3.00 \mathrm{E}+04$ & $3.07 \mathrm{E}+05$ & $5.85 \mathrm{E}+02$ & $3.09 \mathrm{E}+05$ & $9.64 \mathrm{E}+0$ \\
\hline 17 & $5.70 \mathrm{E}+05$ & $8.98 \mathrm{E}+04$ & $8.14 \mathrm{E}+05$ & $3.05 \mathrm{E}+04$ & $3.05 \mathrm{E}+05$ & $6.17 \mathrm{E}+02$ & $3.08 \mathrm{E}+05$ & $9.60 \mathrm{E}+04$ \\
\hline 18 & $5.52 \mathrm{E}+05$ & $9.08 \mathrm{E}+04$ & $7.28 \mathrm{E}+05$ & $3.10 \mathrm{E}+04$ & $3.09 \mathrm{E}+05$ & $6.99 \mathrm{E}+02$ & $2.99 \mathrm{E}+05$ & $1.52 \mathrm{E}+05$ \\
\hline 19 & $5.36 \mathrm{E}+05$ & $9.17 \mathrm{E}+04$ & $6.54 \mathrm{E}+05$ & $3.15 \mathrm{E}+04$ & $3.12 \mathrm{E}+05$ & $7.81 \mathrm{E}+02$ & $3.01 \mathrm{E}+05$ & $1.53 \mathrm{E}+0$ \\
\hline 20 & $5.21 \mathrm{E}+05$ & $9.25 \mathrm{E}+04$ & $5.92 \mathrm{E}+05$ & $3.19 \mathrm{E}+04$ & $3.15 \mathrm{E}+05$ & $E+02$ & $3.02 \mathrm{E}+05$ & $1.54 \mathrm{E}+0$ \\
\hline 21 & $5.07 \mathrm{E}+05$ & $9.34 \mathrm{E}+04$ & $5.40 \mathrm{E}+05$ & $3.23 \mathrm{E}+04$ & $3.18 \mathrm{E}+05$ & $9.47 \mathrm{E}+02$ & $3.04 \mathrm{E}+05$ & $1.55 \mathrm{E}+0$ \\
\hline 22 & $4.94 \mathrm{E}+05$ & $9.42 \mathrm{E}+04$ & $4.95 \mathrm{E}+05$ & $3.27 \mathrm{E}+04$ & $3.21 \mathrm{E}+05$ & $1.03 E+03$ & $3.05 \mathrm{E}+05$ & $1.55 \mathrm{E}+0$ \\
\hline 23 & $4.82 \mathrm{E}+05$ & $9.50 \mathrm{E}+04$ & $4.58 \mathrm{E}+05$ & $3.30 \mathrm{E}+04$ & $3.24 \mathrm{E}+05$ & $1.12 \mathrm{E}+03$ & $3.07 \mathrm{E}+05$ & $1.56 \mathrm{E}+0$ \\
\hline 24 & $4.69 \mathrm{E}+05$ & $9.58 \mathrm{E}+04$ & $4.26 \mathrm{E}+05$ & $3.34 \mathrm{E}+04$ & $3.26 \mathrm{E}+05$ & $1.20 \mathrm{E}+03$ & $3.08 \mathrm{E}+05$ & $1.57 \mathrm{E}+0$ \\
\hline 25 & $4.58 \mathrm{E}+05$ & $9.65 \mathrm{E}+04$ & $3.98 \mathrm{E}+05$ & $3.37 \mathrm{E}+04$ & $3.29 \mathrm{E}+05$ & $1.29 \mathrm{E}+03$ & $3.09 \mathrm{E}+05$ & $1.57 \mathrm{E}+0$ \\
\hline 26 & $4.47 \mathrm{E}+05$ & $9.72 \mathrm{E}+04$ & $3.75 \mathrm{E}+05$ & $3.40 \mathrm{E}+04$ & $3.31 \mathrm{E}+05$ & $1.37 \mathrm{E}+03$ & $3.11 \mathrm{E}+05$ & $1.58 \mathrm{E}+0$ \\
\hline 27 & $4.36 \mathrm{E}+05$ & $9.79 \mathrm{E}+04$ & $3.55 \mathrm{E}+05$ & $3.43 \mathrm{E}+04$ & $3.33 \mathrm{E}+05$ & $1.46 \mathrm{E}+03$ & $3.12 \mathrm{E}+05$ & $1.59 \mathrm{E}+05$ \\
\hline 28 & $4.27 \mathrm{E}+05$ & $9.86 \mathrm{E}+04$ & $3 . \overline{37 \mathrm{E}+05}$ & $3.46 \mathrm{E}+04$ & $3.36 \mathrm{E}+05$ & $1.55 \mathrm{E}+03$ & $3.13 \mathrm{E}+05$ & $1.59 \mathrm{E}+05$ \\
\hline 29 & $4.18 \mathrm{E}+05$ & $9.93 \mathrm{E}+04$ & $3.22 \mathrm{E}+05$ & $3.48 \mathrm{E}+04$ & $3.38 \mathrm{E}+05$ & $1.64 \mathrm{E}+03$ & $3.14 \mathrm{E}+05$ & $1.60 \mathrm{E}+0$ \\
\hline 30 & $4.09 \mathrm{E}+05$ & $9.99 \mathrm{E}+04$ & $3.09 \mathrm{E}+05$ & $3.5 \mathrm{iE}+04$ & $3.35 \mathrm{E}+05$ & $1.67 \mathrm{E}+03$ & $3.25 \mathrm{E}+05$ & $1.01 \mathrm{E}+0$ \\
\hline 31 & $4.01 \mathrm{E}+05$ & $1.01 \mathrm{E}+05$ & $2.97 \mathrm{E}+05$ & $3.54 \mathrm{E}+04$ & $3.32 \mathrm{E}+05$ & $1.71 \mathrm{E}+03$ & $3.23 \mathrm{E}+05$ & $1.01 \mathrm{E}+05$ \\
\hline 32 & $3.93 \mathrm{E}+05$ & $1.01 \mathrm{E}+05$ & $2.87 \mathrm{E}+05$ & $3.56 \mathrm{E}+04$ & $3.30 \mathrm{E}+05$ & $1.74 \mathrm{E}+03$ & $3.22 \mathrm{E}+05$ & $1.01 \mathrm{E}+0$ \\
\hline 33 & $3.85 \mathrm{E}+05$ & $1.02 \mathrm{E}+05$ & $2.78 \mathrm{E}+05$ & $3.59 \mathrm{E}+04$ & $3.27 \mathrm{E}+05$ & $1.78 \mathrm{E}+03$ & $3.21 \mathrm{E}+05$ & $1.00 \mathrm{E}+05$ \\
\hline 34 & $3.77 \mathrm{E}+05$ & $1.02 \mathrm{E}+05$ & $2.70 \mathrm{E}+05$ & $3.61 \mathrm{E}+04$ & $3.24 \mathrm{E}+05$ & $1.81 \mathrm{E}+03$ & $3.20 \mathrm{E}+05$ & $9.98 \mathrm{E}+04$ \\
\hline 35 & $3.70 \mathrm{E}+05$ & $1.03 \mathrm{E}+05$ & $2.62 \mathrm{E}+05$ & $3.63 \mathrm{E}+04$ & $3.22 \mathrm{E}+05$ & $1.84 \mathrm{E}+03$ & $3.18 \mathrm{E}+05$ & $9.94 \mathrm{E}+04$ \\
\hline 36 & $3.62 \mathrm{E}+05$ & $1.03 \mathrm{E}+05$ & $2.56 \mathrm{E}+05$ & $3.66 \mathrm{E}+04$ & $3.19 \mathrm{E}+05$ & $1.88 \mathrm{E}+03$ & $3.17 \mathrm{E}+05$ & $9.90 \mathrm{E}+0$ \\
\hline 37 & $3.55 \mathrm{E}+05$ & $1.04 \mathrm{E}+05$ & $2.50 \mathrm{E}+05$ & $3.68 \mathrm{E}+04$ & $3.17 \mathrm{E}+05$ & $1.91 \mathrm{E}+03$ & $3.16 \mathrm{E}+05$ & $9.86 \mathrm{E}+04$ \\
\hline 38 & $3.47 \mathrm{E}+05$ & $1.05 \mathrm{E}+05$ & $2.44 \mathrm{E}+05$ & $3.70 \mathrm{E}+04$ & $3.14 E+05$ & $1.94 \mathrm{E}+03$ & $3.15 \mathrm{E}+05$ & $9.83 \mathrm{E}+04$ \\
\hline
\end{tabular}




\begin{tabular}{|c|c|c|c|c|c|c|c|c|}
\hline 39 & $3.40 \mathrm{E}+05$ & $1.05 \mathrm{E}+05$ & $2.39 \mathrm{E}+05$ & $3.72 \mathrm{E}+04$ & $3.12 \mathrm{E}+05$ & $1.97 \mathrm{E}+03$ & $3.13 \mathrm{E}+05$ & $9.79 \mathrm{E}+0$ \\
\hline 40 & $3.34 \mathrm{E}+05$ & $1.06 \mathrm{E}+05$ & $2.34 \mathrm{E}+05$ & $3.75 E+04$ & $3.09 \mathrm{E}+05$ & $2.00 \mathrm{E}+03$ & $3.12 \mathrm{E}+05$ & $.75 \mathrm{E}+0$ \\
\hline 41 & $3.27 \mathrm{E}+05$ & $1.06 \mathrm{E}+05$ & $2.29 \mathrm{E}+05$ & $3.77 \mathrm{E}+04$ & $3.07 \mathrm{E}+05$ & $2.04 \mathrm{E}+03$ & $3.11 \mathrm{E}+05$ & $9.7 \overline{1 E+0}$ \\
\hline 42 & $3.21 \mathrm{E}+05$ & $1.06 \mathrm{E}+05$ & $2.25 \mathrm{E}+05$ & $3.79 \mathrm{E}+04$ & $3.08 \mathrm{E}+05$ & $2.13 E+03$ & $2.98 \overline{\mathrm{E}}+05$ & $.63 \mathrm{E}+0$ \\
\hline 43 & $3.15 \bar{E}+05$ & $1.07 \mathrm{E}+05$ & $2.21 \mathrm{E}+05$ & $3.81 \mathrm{E}+04$ & $3.09 \mathrm{E}+05$ & $2.21 \mathrm{E}+03$ & $2.99 \overline{\mathrm{E}}+05$ & $1.63 \mathrm{E}+0$ \\
\hline 44 & $3.09 \mathrm{E}+05$ & $1.07 \mathrm{E}+05$ & $2.17 \mathrm{E}+05$ & $3.83 \mathrm{E}+04$ & $3.10 \mathrm{E}+05$ & $2.30 \mathrm{E}+03$ & $2.99 \mathrm{E}+05$ & $1.64 \mathrm{E}+0$ \\
\hline 45 & $3.03 \mathrm{E}+05$ & $1.08 \mathrm{E}+05$ & $2.13 \mathrm{E}+05$ & $3.85 \mathrm{E}+04$ & $3.11 \mathrm{E}+05$ & $2.39 \mathrm{E}+03$ & $3.00 \mathrm{E}+05$ & $1.6 \overline{\mathrm{E}+0}$ \\
\hline 46 & $2.97 \mathrm{E}+05$ & $1.08 \mathrm{E}+05$ & $2.10 \mathrm{E}+05$ & $3.87 \mathrm{E}+04$ & $3.12 \mathrm{E}+05$ & $2.48 \mathrm{E}+03$ & $3.00 \overline{\mathrm{E}}+05$ & $1.64 \mathrm{E}+0$ \\
\hline 47 & $2.92 \mathrm{E}+05$ & $1.09 \mathrm{E}+05$ & $2.06 \mathrm{E}+05$ & $3.89 \mathrm{E}+04$ & $3.12 \mathrm{E}+05$ & $2.57 \mathrm{E}+03$ & $3.01 \mathrm{E}+05$ & $1.64 \mathrm{E}+0$ \\
\hline 48 & $2.86 \mathrm{E}+05$ & $1.09 \mathrm{E}+05$ & $2.03 E+05$ & $3.90 \mathrm{E}+04$ & $3.13 \mathrm{E}+05$ & $2.66 \mathrm{E}+03$ & $3.01 \mathrm{E}+05$ & $1.64 \mathrm{E}+0$ \\
\hline$\overline{49}$ & $2.81 \mathrm{E}+05$ & $1.09 \mathrm{E}+05$ & $2.00 \mathrm{E}+05$ & $3.92 \mathrm{E}+04$ & $3.13 \mathrm{E}+05$ & $2.75 \mathrm{E}+03$ & $3.01 \mathrm{E}+05$ & $1.65 \mathrm{E}+0$ \\
\hline 50 & $2.76 \mathrm{E}+05$ & $1.10 \mathrm{E}+05$ & $1.97 \mathrm{E}+05$ & $3.94 \mathrm{E}+04$ & $3.14 \mathrm{E}+05$ & $2.84 \mathrm{E}+03$ & $3.02 \mathrm{E}+05$ & $1.65 \mathrm{E}+0$ \\
\hline 51 & $2.72 \mathrm{E}+05$ & $1.10 \mathrm{E}+05$ & $1.95 \mathrm{E} \div 05$ & $3.96 \mathrm{E}+04$ & $3.14 \mathrm{E}+05$ & $2.94 \mathrm{E}+03$ & $3.02 \mathrm{E}+05$ & $1.65 \mathrm{E}+0$ \\
\hline 52 & $2.68 \mathrm{E}+05$ & $1.11 \mathrm{E}+05$ & $1.92 \mathrm{E}+05$ & $3.98 \mathrm{E}+04$ & $3.15 \mathrm{E}+05$ & $3.03 \mathrm{E}+03$ & $3.02 \mathrm{E}+05$ & $1.65 \mathrm{E}+0$ \\
\hline 53 & $2.64 \mathrm{E}+05$ & $1.11 \mathrm{E}+05$ & $1.89 \mathrm{E}+05$ & $4.00 \mathrm{E}+04$ & $3.15 \mathrm{E}+05$ & $3.12 \mathrm{E}+03$ & $3.03 \bar{E}+05$ & $1.65 \mathrm{E}+0$ \\
\hline 54 & $2.60 \mathrm{E}+05$ & $1.11 \mathrm{E}+05$ & $1.86 \mathrm{E}+05$ & $4.01 \mathrm{E}+04$ & $3.13 \mathrm{E}+05$ & $3.15 \mathrm{E}+03$ & $3.03 \mathrm{E}+05$ & $9.49 \mathrm{E}+04$ \\
\hline 55 & $2.56 \mathrm{E}+05$ & $1.12 \mathrm{E}+05$ & $1.83 E+05$ & $4.03 \mathrm{E}+04$ & $3.10 \mathrm{E}+05$ & $3.18 \mathrm{E}+03$ & $3.02 \mathrm{E}+05$ & $9.45 \mathrm{E}+0$ \\
\hline 56 & $2.52 \mathrm{E}+05$ & $1.12 \mathrm{E}+05$ & $1.80 \mathrm{E}+05$ & $4.05 \mathrm{E}+04$ & $3.07 \mathrm{E}+05$ & $3.21 \mathrm{E}+03$ & $3.00 \mathrm{E}+05$ & $\overline{9.42 \mathrm{E}+0}$ \\
\hline 57 & $2.49 \mathrm{E}+05$ & $1.12 \mathrm{E}+05$ & $1.77 \mathrm{E}+05$ & $4.06 \mathrm{E}+04$ & $3.05 \mathrm{E}+05$ & $3.24 \mathrm{E}+03$ & $2.99 \overline{\mathrm{E}+05}$ & $9 . \overline{8 \mathrm{E}+02}$ \\
\hline 58 & $2.45 \mathrm{E}+05$ & $1.13 \mathrm{E}+05$ & $1.74 \mathrm{E}+05$ & $4.08 \mathrm{E}+04$ & $3.02 \mathrm{E}+05$ & $3.27 \mathrm{E}+03$ & $2.98 \mathrm{E}+05$ & $9.3 \overline{4 E+02}$ \\
\hline 59 & $2.41 \mathrm{E}+05$ & $1.13 \mathrm{E}+05$ & $1.71 \mathrm{E}+05$ & $4.09 \mathrm{E}+04$ & $3.00 \mathrm{E}+05$ & $3.30 \mathrm{E}+03$ & $2.97 \mathrm{E}+05$ & $\overline{9.30 \mathrm{E}+0}$ \\
\hline 60 & $2.38 \mathrm{E}+05$ & $1.13 E+05$ & $1.68 \mathrm{E}+05$ & $4.11 E+04$ & $2.98 \mathrm{E}+05$ & $3.33 E+03$ & $2.96 \mathrm{E}+05$ & $9.27 \mathrm{E}+04$ \\
\hline 61 & $2.35 \mathrm{E}+05$ & $1.13 \mathrm{E}+05$ & $1.65 \mathrm{E}+05$ & $4.12 \mathrm{E}+04$ & $2.95 \mathrm{E}+05$ & $3.36 \mathrm{E}+03$ & $2.94 \mathrm{E}+05$ & $9.23 \mathrm{E}+02$ \\
\hline 62 & $2.31 \mathrm{E}+05$ & $1.14 \mathrm{E}+05$ & $1.62 \mathrm{E}+05$ & $4.14 \mathrm{E}+04$ & $2.93 \mathrm{E}+05$ & $3.39 \mathrm{E}+03$ & $2.93 \mathrm{E}+05$ & $9.19 \mathrm{E}+0$ \\
\hline 63 & $2.28 \mathrm{E}+05$ & $1.14 \mathrm{E}+05$ & $1.59 \mathrm{E}+05$ & $4.15 \mathrm{E}+04$ & $2.90 \mathrm{E}+05$ & $3.42 \mathrm{E}+03$ & $2.92 \mathrm{E}+05$ & $9.16 \mathrm{E}+04$ \\
\hline 64 & $2.25 \mathrm{E}+05$ & $1.14 \mathrm{E}+05$ & $1.56 \mathrm{E}+05$ & $4.17 \mathrm{E}+04$ & $2.88 \mathrm{E}+05$ & $3.45 \mathrm{E}+03$ & $2.91 \mathrm{E}+05$ & $9.12 \mathrm{E}+04$ \\
\hline 65 & $2.22 \mathrm{E}+05$ & $1.15 \mathrm{E}+05$ & $1.53 \mathrm{E}+05$ & $4.18 \mathrm{E}+04$ & $2.86 \mathrm{E}+05$ & $3.47 \mathrm{E}+03$ & $2.90 \mathrm{E}+05$ & $9.09 \mathrm{E}+0$ \\
\hline 66 & $2.18 \mathrm{E}+05$ & $1.15 \mathrm{E}+05$ & $1.51 \mathrm{E}+05$ & $4.20 \mathrm{E}+04$ & $2.86 \mathrm{E}+05$ & $3.56 \mathrm{E}+03$ & $2.77 \mathrm{E}+05$ & $1.61 \mathrm{E}+05$ \\
\hline 67 & $2.15 \mathrm{E}+05$ & $1.15 \mathrm{E}+05$ & $1.48 \mathrm{E}+05$ & $4.21 \mathrm{E}+04$ & $2.86 \mathrm{E}+05$ & $3.65 \mathrm{E}+03$ & $2.77 \mathrm{E}+05$ & $1.61 \mathrm{E}+05$ \\
\hline 68 & $2.12 \mathrm{E}+05$ & $1.15 \mathrm{E}+05$ & $1.45 \mathrm{E}+05$ & $4.22 \mathrm{E}+04$ & $2.86 \mathrm{E}+05$ & $3.74 \mathrm{E}+03$ & $2.77 \mathrm{E}+05$ & $1.61 \mathrm{E}+05$ \\
\hline 69 & $2.09 \mathrm{E}+05$ & $1.16 \mathrm{E}+05$ & $1.43 \mathrm{E}+05$ & $4.24 \mathrm{E}+04$ & $2.86 \mathrm{E}+05$ & $3.83 \mathrm{E}+03$ & $2.77 \mathrm{E}+05$ & $1.61 \mathrm{E}+05$ \\
\hline 168 & $5.25 \mathrm{E}+04$ & $1.23 \mathrm{E}+05$ & $4.97 \mathrm{E}+04$ & $4.96 \mathrm{E}+04$ & $1.78 \mathrm{E}+05$ & $9.66 \mathrm{E}+03$ & $1.85 \mathrm{E}+05$ & $1.35 \mathrm{E}+05$ \\
\hline 336 & $2.34 \mathrm{E}+04$ & $1.03 \mathrm{E}+05$ & $2.06 \mathrm{E}+04$ & $5.28 \mathrm{E}+04$ & $7.17 \mathrm{E}+04$ & $1.91 \mathrm{E}+04$ & $1.13 \mathrm{E}+05$ & $1.04 \mathrm{E}+05$ \\
\hline 504 & $1.70 \mathrm{E}+04$ & $8.46 \mathrm{E}+04$ & $1.40 \mathrm{E}+04$ & $5.46 \mathrm{E}+04$ & $3.44 \mathrm{E}+04$ & $2.42 \mathrm{E}+04$ & $4.86 \mathrm{E}+04$ & $6.90 \mathrm{E}+04$ \\
\hline 720 & $1.24 \mathrm{E}+04$ & $6.58 \mathrm{E}+04$ & $9.31 \mathrm{E}+03$ & $5.62 \mathrm{E}+04$ & $1.73 \mathrm{E}+04$ & $2.70 \mathrm{E}+04$ & $2.38 \mathrm{E}+04$ & $4.78 \mathrm{E}+04$ \\
\hline 2160 & $2.30 \mathrm{E}+03$ & $1.22 \mathrm{E}+04$ & $1.04 \mathrm{E}+03$ & $5.91 \mathrm{E}+04$ & $1.51 \mathrm{E}+03$ & $1.60 \mathrm{E}+04$ & $5.44 \mathrm{E}+03$ & $1.63 \mathrm{E}+04$ \\
\hline 4320 & $2.21 \mathrm{E}+02$ & $1.01 \mathrm{E}+03$ & $5.25 \mathrm{E}+03$ & $5.64 \mathrm{E}+04$ & $2.37 \mathrm{E}+02$ & $3.80 \mathrm{E}+03$ & $6.26 \mathrm{E}+02$ & $2.49 \mathrm{E}+0$ \\
\hline 4800 & $1.32 \mathrm{E}+02$ & $5.88 \mathrm{E}+02$ & $5.23 \mathrm{E}+03$ & $5.62 \mathrm{E}+04$ & $1.71 \mathrm{E}+02$ & $2.72 \mathrm{E}+03$ & $2.02 \mathrm{E}+02$ & $8.02 \mathrm{E}+0$ \\
\hline 8760 & $1.81 \mathrm{E}+00$ & $7.06 \mathrm{E}+00$ & $5.10 \mathrm{E}+03$ & $5.48 \mathrm{E}+04$ & $1.34 \mathrm{E}+01$ & $1.77 \mathrm{E}+02$ & $6.17 \mathrm{E}+01$ & $2.33 \mathrm{E}+02$ \\
\hline $\mathbf{T}$ & $\begin{array}{l}86 \\
\mathrm{~L}\end{array}$ & $\begin{array}{l}\mathrm{E}+4 \\
\mathrm{~g}\end{array}$ & $8 \mathrm{~Bq} / \mathrm{g}$ & & & & & \\
\hline 0 & $\begin{array}{l}5.9 \mathrm{E} 6 \\
\mathrm{pCi} / \mathrm{L}\end{array}$ & $\begin{array}{l}2.18 \mathrm{E}+5 \\
\mathrm{~Bq} / \mathrm{kg}\end{array}$ & $8.3 \mathrm{~Bq} / \mathrm{g}$ & & & & & \\
\hline & \multicolumn{2}{|c|}{ VEGETABLES } & \multicolumn{2}{|c|}{ WHEAT SEED } & \multicolumn{2}{|l|}{ MEAT } & \multicolumn{2}{|l|}{ MILK } \\
\hline \multirow[t]{2}{*}{ HOUR } & $\mathrm{O}$ & $\widehat{\mathrm{OBT}}$ & HTO & OBT & HTO & $\overline{\mathrm{OBT}}$ & HTO & $\overline{\mathrm{DBT}}$ \\
\hline & $(\mathrm{Bq} / \mathrm{g})$ & $(\mathrm{Bq} / \mathrm{g})$ & $(\overline{\mathrm{Bq} / \mathrm{g}})$ & $(\mathrm{Bq} / \mathrm{g})$ & $(\mathrm{Bq} / \mathrm{g})$ & $(\mathrm{Bq} / \mathrm{g})$ & $(\mathrm{Bq} / \mathrm{g})$ & $\mathrm{Bq} / \mathrm{g})$ \\
\hline 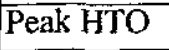 & & & & & & & & \\
\hline
\end{tabular}




\begin{tabular}{|c|c|c|c|c|c|c|c|c|}
\hline$2 \longdiv { 2 / 5 / 6 }$ & $9.66 \mathrm{E}+06$ & $3.84 \mathrm{E}+04$ & $2.81 E+06$ & $1.55 \mathrm{E}+04$ & $3.33 \mathrm{E}+05$ & $2.10 E+02$ & $3.21 \mathrm{E}+05$ & $9.99 \mathrm{E}+04$ \\
\hline SUM & $9.69 \mathrm{E}+06$ & & $2.82 \mathrm{E}+06$ & & $3.33 \mathrm{E}+05$ & & $4.21 \mathrm{E}+05$ & \\
\hline$\overline{\mathrm{C}} / \mathrm{g}$ & $2.62 \mathrm{E}-04$ & & $7.63 \mathrm{E}-05$ & & $9.01 \mathrm{E}-06$ & & $1.14 \mathrm{E}-0 \mathrm{~S}$ & \\
\hline & & & & & & & & \\
\hline DIL HTO & $5.9 \mathrm{E}-09$ & & & & & & & \\
\hline \multicolumn{9}{|l|}{$(\mathrm{Ci} / \mathrm{g})$} \\
\hline & & & & & & & & \\
\hline 168 & $5.25 \mathrm{E}+04$ & $1.23 \mathrm{E}+05$ & $4.97 \overline{\mathrm{E}}+04$ & $4.96 \overline{\mathrm{E}}+04$ & $1.78 \mathrm{E}+05$ & $9.66 \mathrm{E}+03$ & $1.85 \mathrm{E}+05$ & $1.35 \mathrm{E}+05$ \\
\hline SUM & $1.75 \mathrm{E}+05$ & & $9.93 \mathrm{E}+04$ & & $1.87 \mathrm{E}+05$ & & $3.19 \bar{E}+05$ & \\
\hline $\mathrm{Ci} / \mathrm{g}$ & $4.74 \mathrm{E}-06$ & & $2.68 \mathrm{E}-06$ & & $5.06 \mathrm{E}-06$ & & $8.63 \mathrm{E}-06$ & \\
\hline & & & & & & & & \\
\hline DIL OBT & $2.4 \mathrm{E}-09$ & & & & & & & \\
\hline \multicolumn{9}{|l|}{$\mathrm{Ci} / \mathrm{g}$} \\
\hline & & & & & & & & \\
\hline 8760 & $1.81 \mathrm{E}+00$ & $7.06 \bar{E}+00$ & $5.10 \mathrm{E}+03$ & $5.48 \mathrm{E}+04$ & $1.34 \mathrm{E}+01$ & $1.77 \mathrm{E}+02$ & $6.17 \mathrm{E}+01$ & $2.33 \mathrm{E}+02$ \\
\hline SUM & $8.87 \mathrm{E}+00$ & & $5.99 \mathrm{E}+04$ & & $1.91 \overline{\mathrm{E}}+02$ & & $2.95 \mathrm{E}+02$ & \\
\hline$\overline{\mathrm{Ci}} / \mathrm{g}$ & $2.40 \mathrm{E}-10$ & & $1.62 \mathrm{E}-06$ & & $5.15 \mathrm{E}-09$ & & $7.97 \mathrm{E}-09$ & \\
\hline & & & & & & & & \\
\hline DIL OBT & $2.4 \mathrm{E}-09$ & & & & & & & \\
\hline $\mathrm{Ci} / \mathrm{g}$ & & & & & & & & \\
\hline
\end{tabular}




\begin{tabular}{|c|c|c|c|c|c|c|c|c|}
\hline & \multicolumn{7}{|c|}{ Tritium Facilities, Beyond Design Basis Fire, Adverse Met Conditions } & \\
\hline & \multicolumn{4}{|c|}{ Concentration Values for $50 \mathrm{~km}$ from release } & & & & \\
\hline \multicolumn{9}{|c|}{ Only Wheat Seed is examined since this is the edible portion of the Wheat. The "WHEAT" category is } \\
\hline \multicolumn{9}{|c|}{ actually the unedible plant body and is not considered in the dose estimate. } \\
\hline & & & & & & & & \\
\hline & \multicolumn{2}{|c|}{ VEGETABLES } & \multicolumn{2}{|c|}{ WHEAT SEED } & \multicolumn{2}{|l|}{ MEAT } & \multicolumn{2}{|l|}{ MILK } \\
\hline \multirow{3}{*}{ HOUR } & HTO & $\mathrm{OBT}$ & HTO & OBT & HTO & OBT & HTO & OBT \\
\hline & $(\mathrm{Bq} / \mathrm{g})$ & $/ \mathrm{g})$ & $(\overline{\mathrm{Bq} / \mathrm{g})}$ & $(\mathrm{Bq} / \mathrm{g})$ & g) & D & $(\mathrm{Bq} / \mathrm{g})$ & $\overline{\mathrm{Bq} / \mathrm{g})}$ \\
\hline & & & & & & & & \\
\hline 1 & $7.87 \mathrm{E}+04$ & $0.00 \mathrm{E}+00$ & $0.00 \mathrm{E}+00$ & $0.00 \overline{\mathrm{E}+00}$ & $8.08 \mathrm{E}+01$ & $8.69 \mathrm{E}-03$ & $9.78 \mathrm{E}+00$ & $3.05 \mathrm{E}+0$ \\
\hline 2 & $1.87 \mathrm{E}+05$ & $1.46 \mathrm{E}+02$ & $5.26 \mathrm{E}+03$ & $1.74 \mathrm{E}+01$ & $7.24 \mathrm{E}+02$ & $1.39 \mathrm{E}-01$ & $8.67 \mathrm{E}+01$ & $3.40 \mathrm{E}+0$ \\
\hline 3 & $3.67 \mathrm{E}+05$ & $4.93 \mathrm{E}+02$ & $1.83 \mathrm{E}+04$ & $6 . \overline{56 E+01}$ & $2.27 \mathrm{E}+03$ & $5.69 \mathrm{E}-01$ & $2.98 \mathrm{E}+02$ & $1.22 \mathrm{E}+0$ \\
\hline 4 & $3.60 \mathrm{E}+05$ & $1.17 \mathrm{E}+03$ & $4.42 \overline{\mathrm{E}}+04$ & $1.69 \overline{\mathrm{E}+02}$ & $5.02 \mathrm{E}+03$ & $1.57 \mathrm{E}+00$ & $7.13 \mathrm{E}+02$ & $2.9 \overline{9 \mathrm{E}+02}$ \\
\hline 5 & $2.39 \mathrm{E}+06$ & $1.14 \mathrm{E}+04$ & $4.29 \mathrm{E}+05$ & $1 . \overline{48} \overline{E+03}$ & $4.51 \mathrm{E}+04$ & $1.06 \mathrm{E}+01$ & $4.30 \mathrm{E}+03$ & $1.83 \mathrm{E}+03$ \\
\hline 6 & $1.18 \mathrm{E}+06$ & $1.61 \mathrm{E}+04$ & $6.47 \mathrm{E}+05$ & $2 . \overline{62 \overline{\mathrm{E}}+03}$ & $4.50 \mathrm{E}+04$ & $1.55 \mathrm{E}+01$ & $4,37 \mathrm{E}+04$ & $1.3 \overline{6 \mathrm{E}+0}$ \\
\hline 7 & $6.18 \mathrm{E}+05$ & $1.83 \mathrm{E}+04$ & $7.43 \mathrm{E}+05$ & $3.56 \mathrm{E}+03$ & $4.48 \mathrm{E}+04$ & $2.03 \mathrm{E}+01$ & $4.35 \mathrm{E}+04$ & $1.36 \mathrm{E}+0$ \\
\hline 8 & $3.79 \mathrm{E}+05$ & $1 . \overline{94 \mathrm{E}+04}$ & $7.62 \mathrm{E}+05$ & $4.34 \mathrm{E}+03$ & $4.45 \mathrm{E}+04$ & $2.50 \mathrm{E}+01$ & $4.34 \mathrm{E}+04$ & $1.35 \mathrm{E}+04$ \\
\hline 9 & $2.73 \mathrm{E}+05$ & $2.01 \mathrm{E}+04$ & $7.35 \mathrm{E}+05$ & $4.99 \mathrm{E}+03$ & $4.43 \mathrm{E}+04$ & $2.98 \mathrm{E}+01$ & $4.33 \mathrm{E}+04$ & $1.35 \mathrm{E}+0$ \\
\hline 10 & $2.22 \mathrm{E}+05$ & $2.06 \mathrm{E}+04$ & $6.84 \bar{E}+05$ & $5.53 \mathrm{E}+03$ & $4.40 \mathrm{E}+04$ & $3.45 \mathrm{E}+01$ & $4.32 \mathrm{E}+04$ & $1.34 \mathrm{E}+04$ \\
\hline 11 & $1.95 \mathrm{E}+05$ & $2.10 \mathrm{E}+04$ & $6.20 \mathrm{E}+05$ & $5.98 \mathrm{E}+03$ & $4.37 \mathrm{E}+04$ & $3.92 \mathrm{E}+01$ & $4.30 \mathrm{E}+04$ & $1.34 \mathrm{E}+04$ \\
\hline 12 & $1.79 \mathrm{E}+05$ & $2.13 \mathrm{E}+04$ & $5.53 \mathrm{E}+05$ & $6.36 \mathrm{E}+03$ & $4.34 \mathrm{E}+04$ & $4.38 \mathrm{E}+01$ & $4.29 \mathrm{E}+04$ & $1.34 \mathrm{E}+0$ \\
\hline 13 & $1.69 \mathrm{E}+05$ & $2.16 \mathrm{E}+04$ & $4.88 \overrightarrow{\mathrm{E}}+05$ & $6.6 \overline{8 E}+03$ & $4.31 \mathrm{E}+04$ & $4.84 \mathrm{E}+01$ & $4.28 \mathrm{E}+04$ & $1.3 \overline{3 \mathrm{E}+0}$ \\
\hline 14 & $1.61 \mathrm{E}+05$ & $2.19 \mathrm{E}+04$ & $4.28 \mathrm{E}+05$ & $6.95 \mathrm{E}+03$ & $4.28 \mathrm{E}+04$ & $5.30 \mathrm{E}+0 \mathrm{l}$ & $4.26 \mathrm{E}+04$ & $1.33 \mathrm{E}+04$ \\
\hline 15 & $1.55 \mathrm{E}+05$ & $2.22 \mathrm{E}+04$ & $3.74 \mathrm{E}+05$ & $7.18 \mathrm{E}+03$ & $4.25 \mathrm{E}+04$ & $5.75 \mathrm{E}+01$ & $4.25 \mathrm{E}+04$ & $1.32 \mathrm{E}+0$ \\
\hline 16 & $1.50 \mathrm{E}+05$ & $2.24 \mathrm{E}+04$ & $3.27 \mathrm{E}+05$ & $7.39 \mathrm{E}+03$ & $4.23 \mathrm{E}+04$ & $6.21 \mathrm{E}+01$ & $4.23 \mathrm{E}+04$ & $1.32 \mathrm{E}+0$ \\
\hline 17 & $1.46 \mathrm{E}+05$ & $2.27 \mathrm{E}+04$ & $2.85 \mathrm{E}+05$ & $7.57 \mathrm{E}+03$ & $4.20 \mathrm{E}+04$ & $6.65 \mathrm{E}+01$ & $4.22 \mathrm{E}+04$ & $1.32 \mathrm{E}+04$ \\
\hline 18 & $1.42 \mathrm{E}+05$ & $2.29 \mathrm{E}+04$ & $2.50 \mathrm{E}+05$ & $7.73 \mathrm{E}+03$ & $4.32 \mathrm{E}+04$ & $8.34 \mathrm{E}+01$ & $4.19 \mathrm{E}+04$ & $2.80 \mathrm{E}+0$ \\
\hline 19 & $1.38 \mathrm{E}+05$ & $2.31 \mathrm{E}+04$ & $2.20 \mathrm{E}+05$ & $7.87 \mathrm{E}+03$ & $4.44 \mathrm{E}+04$ & $1.01 \mathrm{E}+02$ & $4.25 \mathrm{E}+04$ & $2.83 \mathrm{E}+0$ \\
\hline 20 & $1.35 \mathrm{E}+05$ & $2.34 \mathrm{E}+04$ & $1.95 \mathrm{E}+05$ & $8.00 \mathrm{E}+03$ & $4.55 \mathrm{E}+04$ & $1.18 \mathrm{E}+02$ & $4.30 \mathrm{E}+04$ & $2.85 \mathrm{E}+04$ \\
\hline 21 & $1 . \overline{32 \mathrm{E}+05}$ & $2.36 \mathrm{E}+04$ & $1.73 \mathrm{E}+05$ & $8.11 \mathrm{E}+03$ & $4.66 \mathrm{E}+04$ & $1.36 \mathrm{E}+02$ & $4.36 \mathrm{E}+04$ & $2.87 \mathrm{E}+04$ \\
\hline 22 & $1.29 \mathrm{E}+05$ & $2.38 \mathrm{E}+04$ & $1.55 \mathrm{E}+05$ & $8.22 \mathrm{E}+03$ & $4.77 \overline{\mathrm{E}}+04$ & $1.53 \mathrm{E}+02$ & $4.4 \overline{1 E+04}$ & $2.90 \mathrm{E}+04$ \\
\hline 23 & $1.26 \mathrm{E}+05$ & $2.40 \mathrm{E}+04$ & $1.40 \mathrm{E}+05$ & $8.32 \mathrm{E}+03$ & $4.87 \mathrm{E}+04$ & $1.71 \mathrm{E}+02$ & $4.46 \mathrm{E}+04$ & $2.92 \mathrm{E}+0$ \\
\hline 24 & $1.24 \mathrm{E}+05$ & $2.42 \mathrm{E}+04$ & $1.28 \mathrm{E}+05$ & $8.42 E+03$ & $4.97 \mathrm{E}+04$ & $1.90 \mathrm{E}+02$ & $.51 \mathrm{E}+04$ & $2.94 \mathrm{E}+04$ \\
\hline 25 & $1.21 \mathrm{E}+05$ & $2.44 \mathrm{E}+04$ & $1.18 \mathrm{E}+05$ & $8.51 \mathrm{E}+03$ & $5.06 \mathrm{E}+04$ & $2.08 \mathrm{E}+02$ & $4.56 \mathrm{E}+04$ & $2.97 \mathrm{E}+04$ \\
\hline 26 & $1.19 \mathrm{E}+05$ & $2.46 \mathrm{E}+04$ & $1.09 \mathrm{E}+05$ & $8.59 \mathrm{E}+03$ & $5.16 \mathrm{E}+04$ & $2.27 \mathrm{E}+02$ & $4.61 \mathrm{E}+04$ & $2.99 \mathrm{E}+04$ \\
\hline 27 & $1.17 \mathrm{E}+05$ & $2.48 \mathrm{E}+04$ & $1.02 \mathrm{E}+05$ & $8.67 \mathrm{E}+03$ & $5.25 \mathrm{E}+04$ & $2.45 \mathrm{E}+02$ & $4.66 \mathrm{E}+04$ & $3.01 \mathrm{E}+04$ \\
\hline 28 & $1.15 \mathrm{E}+05$ & $2.50 E+04$ & $9.55 \mathrm{E}+04$ & $8.75 \mathrm{E}+03$ & $5.33 \mathrm{E}+04$ & $2.64 \mathrm{E}+02$ & $4.70 \mathrm{E}+04$ & $3.03 \mathrm{E}+04$ \\
\hline 29 & $1.13 \mathrm{E}+05$ & $2.52 \mathrm{E}+04$ & $9.04 \mathrm{E}+04$ & $8.83 \mathrm{E}+03$ & $5.42 \mathrm{E}+04$ & $2.83 \mathrm{E}+02$ & $4.75 \mathrm{E}+04$ & $3 . \overline{05 \mathrm{E}+04}$ \\
\hline 30 & $1.12 \mathrm{E}+05$ & $2.53 \mathrm{E}+04$ & $8.61 \bar{E}+04$ & $8.90 \mathrm{E}+03$ & $5.38 \mathrm{E}+04$ & $2.89 \mathrm{E}+02$ & $5.21 \mathrm{E}+04$ & $1.63 \mathrm{E}+04$ \\
\hline 31 & $1.11 \mathrm{E}+05$ & $2.55 \mathrm{E}+04$ & $8.25 \mathrm{E}+04$ & $8.97 \mathrm{E}+03$ & $5.34 \mathrm{E}+04$ & $2.95 \mathrm{E}+02$ & $5.19 \mathrm{E}+04$ & $1.62 \bar{E}+04$ \\
\hline 32 & $1.09 \mathrm{E}+05$ & $2.57 \mathrm{E}+04$ & $7.95 \mathrm{E}+04$ & $9.04 E+03$ & $5.30 \mathrm{E}+04$ & $3.00 \mathrm{E}+02$ & $5.17 \mathrm{E}+04$ & $1.62 \mathrm{E}+04$ \\
\hline 33 & $1.08 \mathrm{E}+05$ & $2.59 \mathrm{E}+04$ & $7.69 \mathrm{E}+04$ & $9.11 \mathrm{E}+03$ & $5.26 \mathrm{E}+04$ & $3.06 \mathrm{E}+02$ & $5.15 \mathrm{E}+04$ & $1 . \overline{61 E}+04$ \\
\hline 34 & $1.07 \mathrm{E}+05$ & $2.60 \mathrm{E}+04$ & $7.48 \mathrm{E}+04$ & $9.18 \mathrm{E}+03$ & $5.22 \mathrm{E}+04$ & $3.11 \mathrm{E}+02$ & $5.14 \mathrm{E}+04$ & $1.61 \mathrm{E}+04$ \\
\hline 35 & $1.05 \mathrm{E}+05$ & $2.62 \mathrm{E}+04$ & $7.30 \mathrm{E}+04$ & $9.24 \mathrm{E}+03$ & $5.18 \mathrm{E}+04$ & $3.16 \mathrm{E}+02$ & $5.12 \mathrm{E}+04$ & $1.60 \mathrm{E}+04$ \\
\hline 36 & $1.04 \mathrm{E}+05$ & $2.63 \mathrm{E}+04$ & $7.14 \mathrm{E}+04$ & $9.31 \mathrm{E}+03$ & $5.14 \mathrm{E}+04$ & $3.22 \mathrm{E}+02$ & $5.10 \mathrm{E}+04$ & $1.59 \mathrm{E}+04$ \\
\hline 37 & $1.03 \mathrm{E}+05$ & $2.65 \mathrm{E}+04$ & $7.01 \mathrm{E}+04$ & $9.37 \mathrm{E}+03$ & $5.10 \mathrm{E}+04$ & $3.27 \mathrm{E}+02$ & $5.08 \mathrm{E}+04$ & $1.59 E+04$ \\
\hline 38 & $1.02 \mathrm{E}+05$ & $2.67 \mathrm{E}+04$ & $6.90 \mathrm{E}+04$ & $9.44 \mathrm{E}+03$ & $5.06 \mathrm{E}+04$ & $3.32 \mathrm{E}+02$ & $5.06 \mathrm{E}+04$ & $1.58 \mathrm{E}+04$ \\
\hline
\end{tabular}




\begin{tabular}{|c|c|c|c|c|c|c|c|c|}
\hline 39 & $1.00 \mathrm{E}+05$ & $2.68 \mathrm{E}+04$ & $6.80 \mathrm{E}+04$ & $9.50 \mathrm{E}+03$ & $5.02 \mathrm{E}+04$ & $3.37 \mathrm{E}+02$ & $5.04 \mathrm{E}+04$ & $1.58 \mathrm{E}+0$ \\
\hline 40 & $9.91 E+04$ & $2.70 \mathrm{E}+04$ & $6.71 \mathrm{E}+04$ & $9.56 \mathrm{E}+03$ & $4.98 \mathrm{E}+04$ & $3.42 \mathrm{E}+02$ & $5.02 \mathrm{E}+04$ & $1.57 \mathrm{E}+04$ \\
\hline 41 & $9.79 E+04$ & $2.71 \mathrm{E}+04$ & $6.64 \overline{\mathrm{E}+04}$ & $9.62 \mathrm{E}+03$ & $4.95 \overline{\mathrm{E}+04}$ & $3.47 \bar{E}+02$ & $5.00 \mathrm{E}+04$ & $1.56 \mathrm{E}+0$ \\
\hline 42 & $9.67 \mathrm{E}+04$ & $2.73 E+04$ & $6.57 \mathrm{E}+04$ & $9.69 E+03$ & $5.02 \mathrm{E}+04$ & $3.67 \mathrm{E}+02$ & $4.86 \mathrm{E}+04$ & $3.31 \mathrm{E}+$ \\
\hline 43 & $9.55 \mathrm{E}+04$ & $2.74 \mathrm{E}+04$ & $6.51 \mathrm{E}+04$ & $9.75 \bar{E}+03$ & $5.08 \mathrm{E}+04$ & $3.87 \mathrm{E}+02$ & $4.89 \mathrm{E}+04$ & $3.33 \mathrm{E}+0$ \\
\hline$\overline{44}$ & $9.43 \mathrm{E}+04$ & $2.75 \mathrm{E}+04$ & $6.46 \mathrm{E}+04$ & $9.81 \mathrm{E}+03$ & $5.15 \mathrm{E}+04$ & $4.08 \mathrm{E}+02$ & $4 . \overline{93} \mathrm{E}+04$ & $3.34 \mathrm{E}+0$ \\
\hline 45 & $9.31 \mathrm{E}+04$ & $2.77 \mathrm{E}+04$ & $6.42 \mathrm{E}+04$ & $9.87 \mathrm{E}+03$ & $5.21 \mathrm{E}+04$ & $4.28 \mathrm{E}+02$ & $4.96 \mathrm{E}+04$ & $3.35 \mathrm{E}+0$ \\
\hline$\overline{46}$ & $9.19 \bar{E}+04$ & $2.78 \mathrm{E}+04$ & $6.37 \mathrm{E}+04$ & $9.93 \mathrm{E}+03$ & $5.27 \mathrm{E}+04$ & $4.48 \mathrm{E}+02$ & $4.99 E+04$ & $3.37 \mathrm{E}+04$ \\
\hline 47 & $9.08 \mathrm{E}+04$ & $2.79 \mathrm{E}+04$ & $6.33 \mathrm{E}+04$ & $9.99 E+03$ & $5.33 E+04$ & $4.69 \mathrm{E}+02$ & $5.02 \mathrm{E}+04$ & $3.38 \mathrm{E}+\mathrm{c}$ \\
\hline 48 & $8.96 \mathrm{E}+04$ & $2.81 \mathrm{E}+04$ & $6.30 \mathrm{E}+04$ & $1.01 E+04$ & $5.39 \mathrm{E}+04$ & $4.89 E+02$ & $5.05 \bar{E}+04$ & $3.39 \mathrm{E}+\mathrm{C}$ \\
\hline 49 & $8.85 \mathrm{E}+04$ & $2.82 \mathrm{E}+04$ & $6.27 \mathrm{E}+04$ & $1.01 \mathrm{E}+04$ & $5.44 \mathrm{E}+04$ & $5.10 \mathrm{E}+02$ & $5.08 \mathrm{E}+04$ & $3.41 \mathrm{E}+04$ \\
\hline 50 & $8.75 E+04$ & $2.83 \mathrm{E}+04$ & $6.24 \mathrm{E}+04$ & $1.02 \mathrm{E}+04$ & $5.50 \mathrm{E}+04$ & $5.31 \mathrm{E}+02$ & $5.10 \mathrm{E}+04$ & $3.42 \mathrm{E}+0$ \\
\hline 51 & $8.64 \mathrm{E}+04$ & $2.85 E+04$ & $6.20 \mathrm{E}+04$ & $1.02 \mathrm{E}+04$ & $5.55 E+04$ & $5.52 \mathrm{E}+02$ & $5.13 \mathrm{E}+04$ & $3.43 \mathrm{E}+0$ \\
\hline 52 & $8.54 \mathrm{E}+04$ & $2.86 \mathrm{E}+04$ & $6.16 \mathrm{E}+04$ & $1.03 \mathrm{E}+04$ & $5.60 \mathrm{E}+04$ & $5.73 \mathrm{E}+02$ & $5.16 \mathrm{E}+04$ & $3.44 \mathrm{E}+0$ \\
\hline 53 & $8.45 \mathrm{E}+04$ & $2.87 \mathrm{E}+04$ & $6.12 \mathrm{E}+04$ & $1.04 \mathrm{E}+04$ & $5.65 \mathrm{E}+04$ & $5.94 \mathrm{E}+02$ & $5.18 \mathrm{E}+04$ & $3.46 \mathrm{E}+04$ \\
\hline 54 & $8.35 \mathrm{E}+04$ & $2.88 \mathrm{E}+04$ & $6.07 \mathrm{E}+04$ & $1.04 \mathrm{E}+04$ & $5.60 \mathrm{E}+04$ & $5.99 E+02$ & $5.43 \mathrm{E}+04$ & $1.70 \mathrm{E}^{+}$ \\
\hline 55 & $8.26 \mathrm{E}+04$ & $2.89 \mathrm{E}+\overline{04}$ & $6.02 \mathrm{E}+04$ & $1.05 \mathrm{E}+04$ & $5.56 \mathrm{E}+04$ & $6.05 \mathrm{E}+02$ & $5.41 E+04$ & $1.70 \mathrm{E}+0$ \\
\hline 56 & $8.16 \mathrm{E}+04$ & $2.91 \mathrm{E}+04$ & $5.96 \mathrm{E}+04$ & $1.05 \mathrm{E}+04$ & $5.52 \mathrm{E}+04$ & $6.10 \mathrm{E}+0 \overline{2}$ & $5.39 E+04$ & $1.69 \mathrm{E}+0$ \\
\hline 57 & $8.07 \mathrm{E}+04$ & $2.92 \mathrm{E}+04$ & $5.91 \mathrm{E}+04$ & $1.06 \mathrm{E}+04$ & $5.47 \mathrm{E}+04$ & $6.16 \mathrm{E}+02$ & $5.37 \mathrm{E}+04$ & $1.68 \mathrm{E}+\mathrm{C}$ \\
\hline 58 & $7.98 \bar{E}+04$ & $2.93 E+04$ & $5.84 \mathrm{E}+04$ & $1.06 \mathrm{E}+04$ & $5.43 \mathrm{E}+04$ & $6.21 \mathrm{E}+02$ & $5.35 \mathrm{E}+04$ & $1.68 \mathrm{E}+0$ \\
\hline$\overline{59}$ & $7.89 \mathrm{E}+04$ & $2.94 \mathrm{E}+04$ & $5.78 \mathrm{E}+04$ & $1.07 \mathrm{E}+04$ & $5.39 \mathrm{E}+04$ & $6.27 \mathrm{E}+02$ & $5.33 \mathrm{E}+04$ & $1.67 \mathrm{E}+02$ \\
\hline 60 & $7.80 \mathrm{E}+04$ & $2.95 \mathrm{E}+04$ & $5.72 \mathrm{E}+04$ & $1.07 \mathrm{E}+04$ & $5.34 \mathrm{E}+04$ & $6.32 \mathrm{E}+02$ & $5.30 \mathrm{E}+04$ & $1.66 \mathrm{E}+0$ \\
\hline 61 & $7.71 \mathrm{E}+04$ & $2.96 \mathrm{E}+04$ & $5.65 \mathrm{E}+04$ & $1.08 \mathrm{E}+04$ & $5.30 \mathrm{E}+04$ & $6.37 \mathrm{E}+02$ & $5.28 \mathrm{E}+04$ & $1.66 \mathrm{E}+0$ \\
\hline 62 & $7.62 \mathrm{E}+04$ & $2.97 \mathrm{E}+04$ & $5.58 \mathrm{E}+04$ & $1.08 \mathrm{E}+04$ & $5.26 \mathrm{E}+04$ & $6.42 \mathrm{E}+02$ & $5.26 \mathrm{E}+04$ & $1.65 \mathrm{E}+0$ \\
\hline 63 & $7.53 \mathrm{E}+04$ & $2.98 \mathrm{E}+04$ & $5.51 \mathrm{E}+04$ & $1.09 \mathrm{E}+04$ & $5.22 \mathrm{E}+04$ & $6.48 \mathrm{E}+02$ & $5.24 \mathrm{E}+04$ & $1.65 \mathrm{E}+02$ \\
\hline 64 & $7.44 \mathrm{E}+04$ & $2.99 \mathrm{E}+04$ & $5.45 \mathrm{E}+04$ & $1.09 \mathrm{E}+04$ & $5.18 \mathrm{E}+04$ & $6.53 \mathrm{E}+02$ & $5 . \overline{22 \mathrm{E}+04}$ & $1.64 \mathrm{E}+0$ \\
\hline 65 & $7.35 \mathrm{E}+04$ & $3.00 \mathrm{E}+04$ & $5.38 \mathrm{E}+04$ & $1.10 \mathrm{E}+04$ & $5.14 \mathrm{E}+04$ & $6.58 \mathrm{E}+02$ & $5.20 \mathrm{E}+04$ & $1.63 \mathrm{E}+02$ \\
\hline 66 & $7.25 \mathrm{E}+04$ & $3.01 \mathrm{E}+04$ & $5.31 \mathrm{E}+04$ & $1.10 \mathrm{E}+04$ & $5.18 \mathrm{E}+04$ & $6.79 \mathrm{E}+02$ & $5.02 \mathrm{E}+04$ & $3.55 \mathrm{E}+04$ \\
\hline 67 & $7.16 \mathrm{E}+04$ & $3.02 \mathrm{E}+04$ & $5.24 \mathrm{E}+04$ & $1.11 \mathrm{E}+04$ & $5.21 \mathrm{E}+04$ & $7.00 \mathrm{E}+02$ & $5.03 \mathrm{E}+04$ & $3.55 \mathrm{E}+02$ \\
\hline 68 & $7.07 \mathrm{E}+04$ & $3.03 \mathrm{E}+04$ & $5.17 \mathrm{E}+04$ & $1.11 \mathrm{E}+04$ & $5.25 \mathrm{E}+04$ & $7.22 \mathrm{E}+02$ & $5.05 \mathrm{E}+04$ & $3.5 \overline{6 \mathrm{E}+04}$ \\
\hline 69 & $6.99 \mathrm{E}+04$ & $3.04 \mathrm{E}+04$ & $5.09 \mathrm{E}+04$ & $1.12 \mathrm{E}+04$ & $5.28 \mathrm{E}+04$ & $7.43 \mathrm{E}+02$ & $5.07 \mathrm{E}+04$ & $3.57 \mathrm{E}+0$ \\
\hline 168 & $1.44 \mathrm{E}+04$ & $3.38 \mathrm{E}+04$ & $1.56 \mathrm{E}+04$ & $1.39 \mathrm{E}+04$ & $4.08 \mathrm{E}+04$ & $2.22 \mathrm{E}+03$ & $4.19 \mathrm{E}+04$ & $3.47 \mathrm{E}+0$ \\
\hline$\overline{336}$ & $6.21 \mathrm{E}+03$ & $2.82 \mathrm{E}+04$ & $5.68 \mathrm{E}+03$ & $1.48 \mathrm{E}+04$ & $1.78 \mathrm{E}+04$ & $4.83 \mathrm{E}+03$ & $2.68 \mathrm{E}+04$ & $2.75 \mathrm{E}+0$ \\
\hline 504 & $4.48 \mathrm{E}+03$ & $2.32 \mathrm{E}+04$ & $3.83 \mathrm{E}+03$ & $1.53 \mathrm{E}+04$ & $9.01 \mathrm{E}+03$ & $6.30 \mathrm{E}+03$ & $1.24 \mathrm{E}+04$ & $1.89 \mathrm{E}+04$ \\
\hline 720 & $3.24 \mathrm{E}+03$ & $1.81 \mathrm{E}+04$ & $2.54 \mathrm{E}+03$ & $1.57 \mathrm{E}+04$ & $4.70 \mathrm{E}+03$ & $7.15 \mathrm{E}+03$ & $6.34 E+03$ & $1.32 \mathrm{E}+04$ \\
\hline 2160 & $6.02 \mathrm{E}+02$ & $3.31 \mathrm{E}+03$ & $2.83 \mathrm{E}+02$ & $1.65 \mathrm{E}+04$ & $4.11 \mathrm{E}+02$ & $4.33 \mathrm{E}+03$ & $1.49 \mathrm{E}+03$ & $4.50 \mathrm{E}+03$ \\
\hline 4320 & $5.79 \mathrm{E}+01$ & $2.72 \mathrm{E}+02$ & $1.43 \mathrm{E}+03$ & $1.58 \mathrm{E}+04$ & $6.29 \mathrm{E}+01$ & $1.03 \mathrm{E}+03$ & $1.68 \mathrm{E}+02$ & $6.76 \mathrm{E}+02$ \\
\hline 4800 & $3.44 \mathrm{E}+01$ & $1.57 \mathrm{E}+02$ & $1.43 \mathrm{E}+03$ & $1.57 \mathrm{E}+04$ & $4.53 \mathrm{E}+01$ & $7.37 \mathrm{E}+02$ & $5.35 \mathrm{E}+01$ & $2.16 \mathrm{E}+02$ \\
\hline $8 \overline{860}$ & $4.73 \mathrm{E}-01$ & $1.86 \mathrm{E}+00$ & $1.39 \mathrm{E}+03$ & $1.53 \mathrm{E}+04$ & $3.53 \mathrm{E}+00$ & $4.78 \mathrm{E}+01$ & $1.63 \mathrm{E}+01$ & $6.27 \overline{\mathrm{E}+0}$ \\
\hline $\mathbf{3 T}$ & $\begin{array}{l}2.4 \mathrm{E} 6 \\
\mathrm{pCi} / \mathrm{L}\end{array}$ & $\begin{array}{l}8.88 \mathrm{E}+4 \\
\mathrm{~Bq} / \mathrm{kg}\end{array}$ & $8 \mathrm{~Bq} / \mathrm{g}$ & & & & & \\
\hline $\mathbf{0}$ & $\begin{array}{l}5.9 \mathrm{E} 6 \\
\mathrm{pCi} / \mathrm{L}\end{array}$ & $\begin{array}{l}2.18 \mathrm{E}+5 \\
\mathrm{~Bq} / \mathrm{kg}\end{array}$ & $218.3 \mathrm{~Bq} / \mathrm{g}$ & & & & & \\
\hline & \multicolumn{2}{|c|}{ VEGETABLES } & \multicolumn{2}{|c|}{ WHEAT SEED } & \multicolumn{2}{|l|}{ MEAT } & \multicolumn{2}{|l|}{$\overline{\text { MILK }}$} \\
\hline \multirow[t]{2}{*}{$\overline{\mathrm{HOUR}}$} & 0 & OBT & IHTO & OBT & HTO & OBT & HTO & OBT \\
\hline & $(\mathrm{Bq} / \mathrm{g})$ & $(\mathrm{Bq} / \mathrm{g})$ & $(\mathrm{Bq} / \mathrm{g})$ & $(\mathrm{Bq} / \mathrm{g})$ & $(\mathrm{Bq} / \mathrm{g})$ & $(\mathrm{Bq} / \mathrm{g})$ & $(\mathrm{Bq} / \mathrm{g})$ & $(\overline{B q} / \mathrm{g})$ \\
\hline eak HTO & & & & & & & & \\
\hline
\end{tabular}




\begin{tabular}{|c|c|c|c|c|c|c|c|c|}
\hline $5 / 8 / 29 / 54$ & $2.39 \mathrm{E}+06$ & $1.14 \mathrm{E}+04$ & $7.62 \mathrm{E}+05$ & $4.34 \mathrm{E}+03$ & $5.42 \mathrm{E}+04$ & $2.83 \mathrm{E}+02$ & $5.43 \mathrm{E}+04$ & $1.70 \mathrm{E}+04$ \\
\hline SUM & $2.40 \mathrm{E}+06$ & & $7.67 \mathrm{E}+05$ & & $5.45 \mathrm{E}+04$ & & $7.13 \mathrm{E}+04$ & \\
\hline$\overline{\mathrm{Ci} / \mathrm{g}}$ & $6.48 E-05$ & & $2.07 \mathrm{E}-05$ & & $1.47 \mathrm{E}-06$ & & $1.93 \mathrm{E}-06$ & \\
\hline & & & & & & & & \\
\hline DIL HTO & $5.9 \mathrm{E}-09$ & & & & & & & \\
\hline \multicolumn{9}{|l|}{$(\mathrm{Ci} / \mathrm{g})$} \\
\hline & & & & & & & & \\
\hline 168 & $1.44 \mathrm{E}+04$ & $3.38 \mathrm{E}+04$ & $1.56 \mathrm{E}+04$ & $1.39 \mathrm{E}+04$ & $4.08 \mathrm{E}+04$ & $2.22 \mathrm{E}+03$ & $4.19 \mathrm{E}+04$ & $3.47 \mathrm{E}+04$ \\
\hline SUM & $4.82 \mathrm{E}+04$ & & $2.95 \mathrm{E}+04$ & & $4.30 \mathrm{E}+04$ & & $7.65 \mathrm{E}+04$ & \\
\hline $\mathrm{Ci} / \mathrm{g}$ & $1.30 \mathrm{E}-06$ & & $7.97 \mathrm{E}-07$ & & $1.16 \mathrm{E}-06$ & & $2.07 E-06$ & \\
\hline & & & & & & & & \\
\hline DIL OBT & $2.4 \mathrm{E}-09$ & & & & & & & \\
\hline \multicolumn{9}{|l|}{$\overline{\mathrm{Ci} / \mathrm{g}}$} \\
\hline & & & & & & & & \\
\hline 8760 & $4.73 \mathrm{E}-01$ & $1.86 \mathrm{E}+00$ & $1.39 \mathrm{E}+03$ & $1.53 \mathrm{E}+04$ & $3.53 \mathrm{E}+00$ & $4.78 \mathrm{E}+01$ & $1.63 \mathrm{E}+01$ & $6.27 \mathrm{E}+01$ \\
\hline SUM & $2.34 \mathrm{E}+00$ & & $1.67 \mathrm{E}+04$ & & $5.13 \mathrm{E}+01$ & & $7.90 \mathrm{E}+01$ & \\
\hline $\mathrm{Ci} / \mathrm{g}$ & $6.31 \mathrm{E}-11$ & & 4.52E-07 & & $1.39 \mathrm{E}-09$ & & $2.14 \mathrm{E}-09$ & \\
\hline DIL OBT & $2.4 \mathrm{E}-09$ & & & & & & & \\
\hline $\mathrm{Ci} / \mathrm{g}$ & & & & & & & & \\
\hline
\end{tabular}




\begin{tabular}{|c|c|c|c|c|c|c|c|c|}
\hline & \multicolumn{7}{|c|}{ Tritium Facilities, Beyond Design Basis Fire, Adverse Met Conditions } & \\
\hline & \multicolumn{4}{|c|}{ Concentration Values for $100 \mathrm{~km}$ from release } & & & & \\
\hline \multicolumn{9}{|c|}{ Only Wheat Seed is examined since this is the edible portion of the Wheat. The "WHEAT" category is } \\
\hline \multicolumn{9}{|c|}{ actually the unedible plant body and is not considered in the dose estimate. } \\
\hline & & & & & & & & \\
\hline & \multicolumn{2}{|c|}{ VEGETABLES } & \multicolumn{2}{|c|}{ WHEAT SEED } & \multicolumn{2}{|l|}{ MEAT } & \multicolumn{2}{|l|}{ MILK } \\
\hline \multirow[t]{3}{*}{ HOUR } & HTO & OBT & HTO & $\overline{\mathrm{OBT}}$ & HTO & OBT & HTO & OBT \\
\hline & $(\mathrm{Bq} / \mathrm{g})$ & $(\mathrm{Bq} / \mathrm{g})$ & $(\mathrm{Bq} / \mathrm{g})$ & $(\mathrm{Bq} / \mathrm{g})$ & $(\mathrm{Bq} / \mathrm{g})$ & $(\mathrm{Bq} / \mathrm{g})$ & $(\mathrm{Bq} / \mathrm{g})$ & $\mathrm{Ba} / \mathrm{g})$ \\
\hline & & & & & & & & \\
\hline 1 & $2.67 \mathrm{E}+03$ & $0.00 \mathrm{E}+00$ & $0.00 \mathrm{E}+00$ & $0.00 \mathrm{E}+00$ & $2.74 \mathrm{E}+00$ & $2.95 \mathrm{E}-04$ & $3.32 \mathrm{E}-01$ & $1.04 \mathrm{E}-01$ \\
\hline 2 & $8.12 \mathrm{E}+03$ & $4.97 \mathrm{E}+00$ & $1.79 \mathrm{E}+02$ & $5.90 \mathrm{E}-01$ & $2.64 \mathrm{E}+01$ & $4.91 \mathrm{E}-03$ & $3.14 \mathrm{E}+00$ & $1.22 \mathrm{E}+00$ \\
\hline 3 & $1.91 \mathrm{E}+04$ & $2.00 \mathrm{E}+01$ & $7.41 \mathrm{E}+02$ & $2.62 \mathrm{E}+00$ & $9.58 \mathrm{E}+01$ & $2.27 \mathrm{E}-02$ & $1.21 \mathrm{E}+01$ & $4.90 \mathrm{E}+0$ \\
\hline 4 & $2.50 \mathrm{E}+04$ & $5.54 \mathrm{E}+01$ & $2.08 \mathrm{E}+03$ & $7.74 \mathrm{E}+00$ & $2.43 \mathrm{E}+02$ & $7.03 \mathrm{E}-02$ & $3.24 \mathrm{E}+01$ & $1.35 \mathrm{E}+01$ \\
\hline 5 & $5.15 \mathrm{E}+04$ & $1.02 \mathrm{E}+02$ & $3.87 \mathrm{E}+03$ & $1.57 \mathrm{E}+01$ & $4.68 \mathrm{E}+02$ & $1.62 \mathrm{E}-01$ & $6.75 \mathrm{E}+01$ & $2.83 \mathrm{E}+01$ \\
\hline 6 & $5.94 \mathrm{E}+04$ & $1.97 \mathrm{E}+02$ & $7.44 \mathrm{E}+03$ & $3.12 \mathrm{E}+01$ & $5.04 \mathrm{E}+02$ & $\mathrm{E}-01$ & $4.89 \mathrm{E}+02$ & $1.52 \mathrm{E}+02$ \\
\hline 7 & $9.42 \mathrm{E}+04$ & $3.08 \mathrm{E}+02$ & $1.16 \mathrm{E}+04$ & $5.22 \mathrm{E}+01$ & $5.72 \mathrm{E}+02$ & $2.78 \mathrm{E}-01$ & $5.22 \mathrm{E}+02$ & $1.62 \mathrm{E}+02$ \\
\hline 8 & $1.36 \mathrm{E}+05$ & $4.82 \mathrm{E}+02$ & $1.80 \mathrm{E}+04$ & $8.45 \mathrm{E}+01$ & $6.68 \mathrm{E}+02$ & $3.49 \mathrm{E}-01$ & $5.63 \mathrm{E}+02$ & $1.75 \mathrm{E}+02$ \\
\hline 9 & $1.83 \mathrm{E}+05$ & $9.92 \mathrm{E}+02$ & $3.67 \mathrm{E}+04$ & $1.64 \mathrm{E}+02$ & $8.77 \mathrm{E}+02$ & $4.43 \mathrm{E}-01$ & $6.35 \mathrm{E}+02$ & $\mathrm{E}+02$ \\
\hline 10 & $9.88 \mathrm{E}+05$ & $5.22 \mathrm{E}+03$ & $1.95 \mathrm{E}+05$ & $7.22 \mathrm{E}+02$ & $3.08 \mathrm{E}+03$ & $7.75 \mathrm{E}-01$ & $1.11 \mathrm{E}+03$ & $3.44 \mathrm{E}+02$ \\
\hline 11 & $4.65 \mathrm{E}+05$ & $7.05 \mathrm{E}+03$ & $2.79 \mathrm{E}+05$ & $1.19 \mathrm{E}+03$ & $3.13 \mathrm{E}+03$ & $1.11 \mathrm{E}+00$ & $1.43 \mathrm{E}+03$ & $4.44 \mathrm{E}+02$ \\
\hline 12 & $2.49 \mathrm{E}+05$ & $7.90 \mathrm{E}+03$ & $3.15 \mathrm{E}+05$ & $1.58 \mathrm{E}+03$ & $3.16 \mathrm{E}+03$ & $1.45 \mathrm{E}+00$ & $1.66 \mathrm{E}+03$ & $5.17 \mathrm{E}+02$ \\
\hline 13 & $1.57 \mathrm{E}+05$ & $8.36 \mathrm{E}+03$ & $3.20 \mathrm{E}+05$ & $1.90 \mathrm{E}+03$ & $3.18 \mathrm{E}+03$ & $i+00$ & $1.84 \mathrm{E}+03$ & $5.72 \mathrm{E}+02$ \\
\hline 14 & $1.16 \mathrm{E}+05$ & $8.64 \mathrm{E}+03$ & $3.07 \mathrm{E}+05$ & $2.16 \mathrm{E}+03$ & $3.20 \mathrm{E}+03$ & $2.13 \mathrm{E}+00$ & $1.98 \mathrm{E}+03$ & $6.16 \mathrm{E}+02$ \\
\hline 15 & $9.59 \mathrm{E}+04$ & $8.84 \mathrm{E}+03$ & $2.85 \mathrm{E}+05$ & $2.39 \mathrm{E}+03$ & $3.21 \mathrm{E}+03$ & $2.48 \mathrm{E}+00$ & $2.09 \mathrm{E}+03$ & $6.51 \mathrm{E}+02$ \\
\hline 16 & $8.56 \mathrm{E}+04$ & $9.00 \mathrm{E}+03$ & $2.58 \mathrm{E}+05$ & $2.57 \mathrm{E}+03$ & $3.22 \mathrm{E}+03$ & $E+00$ & $2.19 \mathrm{E}+03$ & $6.81 \mathrm{E}+02$ \\
\hline 17 & $7.96 \mathrm{E}+04$ & $9.15 \mathrm{E}+03$ & $2.30 \mathrm{E}+05$ & $2.73 \mathrm{E}+03$ & $3.23 \mathrm{E}+03$ & $\mathrm{E}+00$ & $2.26 \mathrm{E}+03$ & $7.05 \mathrm{E}+02$ \\
\hline 18 & $7.56 \mathrm{E}+04$ & $9.29 \mathrm{E}+03$ & $2.04 \mathrm{E}+05$ & $2.87 \mathrm{E}+03$ & $4.28 \mathrm{E}+03$ & $8.60 \mathrm{E}+00$ & $4.15 \mathrm{E}+03$ & $7.32 \mathrm{E}+03$ \\
\hline 19 & $7.28 \mathrm{E}+04$ & $9.42 \mathrm{E}+03$ & $1.79 \mathrm{E}+05$ & $2.98 \mathrm{E}+03$ & $5.19 \mathrm{E}+03$ & $1.42 \mathrm{E}+01$ & $4.59 \mathrm{E}+03$ & $7.51 \mathrm{E}+03$ \\
\hline 20 & $7.05 \mathrm{E}+04$ & $9.54 \mathrm{E}+03$ & $1.57 \mathrm{E}+05$ & $3.08 \mathrm{E}+03$ & $6.02 \mathrm{E}+03$ & $2.00 \mathrm{E}+01$ & $5.01 \mathrm{E}+03$ & $7.69 \mathrm{E}+0$ \\
\hline 21 & $6.86 \mathrm{E}+04$ & $9.66 \mathrm{E}+03$ & $1.37 \mathrm{E}+05$ & $3.17 \mathrm{E}+03$ & $6.79 \mathrm{E}+03$ & $2.59 \mathrm{E}+01$ & $5.40 \mathrm{E}+03$ & $7.85 \mathrm{E}+03$ \\
\hline 22 & $6.70 \mathrm{E}+04$ & $9.77 \mathrm{E}+03$ & $1.21 \mathrm{E}+05$ & $3.24 \mathrm{E}+03$ & $7.51 \mathrm{E}+03$ & $3.19 \mathrm{E}+01$ & $5.77 \mathrm{E}+03$ & $8.01 \mathrm{E}+03$ \\
\hline 23 & $6.56 \mathrm{E}+04$ & $9.88 \mathrm{E}+03$ & $1.06 \mathrm{E}+05$ & $3.31 \mathrm{E}+03$ & $8.20 \mathrm{E}+03$ & $3.81 \mathrm{E}+01$ & $6.14 \mathrm{E}+03$ & $8.17 \mathrm{E}+0$ \\
\hline 24 & $6.43 \mathrm{E}+04$ & $9.99 \mathrm{E}+03$ & $9.42 \mathrm{E}+04$ & $3.38 \mathrm{E}+03$ & $8.87 \mathrm{E}+03$ & $4.44 \mathrm{E}+01$ & $6.49 \mathrm{E}+03$ & $8.31 \mathrm{E}+03$ \\
\hline 25 & $6.32 E+04$ & $1.01 \mathrm{E}+04$ & $8.41 E+04$ & $3.43 \mathrm{E}+03$ & $9.52 \mathrm{E}+03$ & $5.08 \mathrm{E}+01$ & $6.83 \mathrm{E}+03$ & $8.46 \mathrm{E}+03$ \\
\hline 26 & $6.21 \mathrm{E}+04$ & $1.02 \mathrm{E}+04$ & $7.55 \mathrm{E}+04$ & $3.49 \mathrm{E}+03$ & $1.02 \mathrm{E}+04$ & $5.74 \mathrm{E}+01$ & $7.16 \mathrm{E}+03$ & $8.60 \mathrm{E}+03$ \\
\hline 27 & $6.11 \mathrm{E}+04$ & $1.03 \mathrm{E}+04$ & $6.84 \mathrm{E}+04$ & $3.54 \mathrm{E}+03$ & $1.08 \mathrm{E}+04$ & $6.41 \mathrm{E}+01$ & $7.49 \mathrm{E}+03$ & $8.74 \mathrm{E}+03$ \\
\hline 28 & $6.04 \mathrm{E}+04$ & $1.04 \mathrm{E}+04$ & $6.25 \mathrm{E}+04$ & $3.58 \mathrm{E}+03$ & $1.14 \mathrm{E}+04$ & $7.08 \mathrm{E}+01$ & $7.81 \mathrm{E}+03$ & $8.88 \mathrm{E}+03$ \\
\hline 29 & $5.98 \mathrm{E}+04$ & $1.05 \mathrm{E}+04$ & $5.76 \mathrm{E}+04$ & $3.63 \mathrm{E}+03$ & $1.20 \mathrm{E}+04$ & $7.77 \mathrm{E}+01$ & $8.13 E+03$ & $9.01 \mathrm{E}+03$ \\
\hline 30 & $5.94 \mathrm{E}+04$ & $1.06 \mathrm{E}+04$ & $5.36 \mathrm{E}+04$ & $3.67 \mathrm{E}+03$ & $1.19 \mathrm{E}+04$ & $7.89 \mathrm{E}+01$ & $1.15 \mathrm{E}+04$ & $3.61 \mathrm{E}+03$ \\
\hline 31 & $5.89 \mathrm{E}+04$ & $1.07 \mathrm{E}+04$ & $5.03 \mathrm{E}+04$ & $3.71 \mathrm{E}+03$ & $1.18 \mathrm{E}+04$ & $8.02 \mathrm{E}+01$ & $1.15 \mathrm{E}+04$ & $3.60 \mathrm{E}+03$ \\
\hline 32 & $5.86 \mathrm{E}+04$ & $1.08 \mathrm{E}+04$ & $4.76 \mathrm{E}+04$ & $3.75 \mathrm{E}+03$ & $1.18 \mathrm{E}+04$ & $8.14 \mathrm{E}+01$ & $1.15 \mathrm{E}+04$ & $3.58 \mathrm{E}+03$ \\
\hline 33 & $5.82 \mathrm{E}+04$ & $1.09 \mathrm{E}+04$ & $4.54 \mathrm{E}+04$ & $3.79 \mathrm{E}+03$ & $1.17 \mathrm{E}+04$ & $8.26 \mathrm{E}+01$ & $1.14 \mathrm{E}+04$ & $3.57 \mathrm{E}+03$ \\
\hline 34 & $5.79 \mathrm{E}+04$ & $1.10 \mathrm{E}+04$ & $4.37 \mathrm{E}+04$ & $3.83 \mathrm{E}+03$ & $1.16 \mathrm{E}+04$ & $8.38 \mathrm{E}+01$ & $1.14 \mathrm{E}+04$ & $3.56 \mathrm{E}+03$ \\
\hline 35 & $5.76 \mathrm{E}+04$ & $1.11 \mathrm{E}+04$ & $4.23 \mathrm{E}+04$ & $3.86 \mathrm{E}+03$ & $1.15 \mathrm{E}+04$ & $8.49 \mathrm{E}+01$ & $1.14 \mathrm{E}+04$ & $3.55 \mathrm{E}+03$ \\
\hline 36 & $5.74 \mathrm{E}+04$ & $1.12 \mathrm{E}+04$ & $4.11 \mathrm{E}+04$ & $3.90 \mathrm{E}+03$ & $1.15 \mathrm{E}+04$ & $8.61 \mathrm{E}+01$ & $1.13 \mathrm{E}+04$ & $3.54 \mathrm{E}+03$ \\
\hline 37 & $5.71 \mathrm{E}+04$ & $1.13 \mathrm{E}+04$ & $4.02 \bar{E}+04$ & $3.94 \mathrm{E}+03$ & $1.14 \mathrm{E}+04$ & $8.73 \mathrm{E}+01$ & $1.13 \mathrm{E}+04$ & $3.53 \mathrm{E}+03$ \\
\hline 38 & $5.69 \mathrm{E}+04$ & $1.14 \mathrm{E}+04$ & $3.95 \mathrm{E}+04$ & $3.98 \mathrm{E}+03$ & $1.13 \mathrm{E}+04$ & $8.84 \mathrm{E}+01$ & $1.13 \mathrm{E}+04$ & $3.52 \mathrm{E}+03$ \\
\hline
\end{tabular}




\begin{tabular}{|c|c|c|c|c|c|c|c|c|}
\hline 39 & $5.66 \mathrm{E}+04$ & $1.14 \mathrm{E}+04$ & $3.90 \mathrm{E}+04$ & $4.01 \mathrm{E}+03$ & $1.12 \mathrm{E}+04$ & $8.96 \mathrm{E}+01$ & $1.12 \mathrm{E}+04$ & $3.51 \mathrm{E}+0$ \\
\hline 40 & $5.64 \mathrm{E}+04$ & $1.15 \mathrm{E}+04$ & $3.86 \mathrm{E}+04$ & $4.05 \mathrm{E}+03$ & $1.12 \mathrm{E}+04$ & $9.07 \mathrm{E}+01$ & $1.12 \mathrm{E}+04$ & $350 \overline{\mathrm{E}}+0$ \\
\hline 41 & $5.62 \mathrm{E}+04$ & $1.16 \bar{E}+04$ & $3.83 \mathrm{E}+04$ & $4.08 \mathrm{E}+03$ & $1.11 \mathrm{E}+04$ & $9.18 \mathrm{E}+01$ & $1.11 \mathrm{E}+04$ & $3.49 \mathrm{E}+03$ \\
\hline 42 & $5.59 \mathrm{E}+04$ & $1.17 \mathrm{E}+04$ & $3.81 \mathrm{E}+04$ & $4.12 \mathrm{E}+03$ & $1.17 \mathrm{E}+04$ & $9.94 \mathrm{E}+01$ & $1.13 E+04$ & $1.13 \mathrm{E}+0$ \\
\hline 43 & $5.57 \mathrm{E}+04$ & $1.18 \mathrm{E}+04$ & $3.80 \mathrm{E}+04$ & $4.16 \mathrm{E}+03$ & $1.22 \mathrm{E}+04$ & $1.07 \mathrm{E}+02$ & $1.16 \mathrm{E}+04$ & $1.14 \mathrm{E}+0$ \\
\hline 44 & $5.54 \mathrm{E}+04$ & $1.19 \mathrm{E}+04$ & $3.80 \mathrm{E}+04$ & $4.19 \mathrm{E}+03$ & $1.27 \mathrm{E}+04$ & $1.15 \mathrm{E}+02$ & $1.18 \mathrm{E}+04$ & $1.15 \mathrm{E}+$ \\
\hline 45 & $5.51 \mathrm{E}+04$ & $1.20 \mathrm{E}+04$ & $3.80 \mathrm{E}+04$ & $4.23 \mathrm{E}+03$ & $1.32 \mathrm{E}+04$ & $1.23 \mathrm{E}+02$ & $1.21 \mathrm{E}+04$ & $1.16 \mathrm{E}+0$ \\
\hline 46 & $5.49 \mathrm{E}+04$ & $1.21 \mathrm{E}+04$ & $3.81 \mathrm{E}+04$ & $4.27 \mathrm{E}+03$ & $1.38 \mathrm{E}+04$ & $1.31 \mathrm{E}+02$ & $1.23 \mathrm{E}+04$ & $1.17 \mathrm{E}+0$ \\
\hline 47 & $5.46 \mathrm{E}+04$ & $1.21 \mathrm{E}+04$ & $3.82 \mathrm{E}+04$ & $4.30 \mathrm{E}+03$ & $1.43 \mathrm{E}+04$ & $1.39 \mathrm{E}+02$ & $1.26 \mathrm{E}+04$ & $1.19 \mathrm{E}+\mathrm{C}$ \\
\hline 48 & $5.43 \mathrm{E}+04$ & $1.22 \mathrm{E}+04$ & $3.83 \mathrm{E}+04$ & $4.34 \mathrm{E}+03$ & $1.48 \mathrm{E}+04$ & $1.47 \mathrm{E}+02$ & $1.28 \mathrm{E}+04$ & $1.20 \mathrm{E}+04$ \\
\hline 49 & $5.40 \mathrm{E}+04$ & $1.23 \mathrm{E}+04$ & $3.85 \mathrm{E}+04$ & $4.38 \mathrm{E}+03$ & $1.52 \mathrm{E}+04$ & $1.55 \mathrm{E}+02$ & $1.31 \mathrm{E}+04$ & $1.21 \mathrm{E}-$ \\
\hline 50 & $5.38 \mathrm{E}+04$ & $1.24 \mathrm{E}+04$ & $3.86 \mathrm{E}+04$ & $4.42 \mathrm{E}+03$ & $1.57 \mathrm{E}+04$ & $1.64 \mathrm{E}+02$ & $1.33 \mathrm{E}+04$ & $1.22 \mathrm{E}+04$ \\
\hline 51 & $5.35 \mathrm{E}+04$ & $1.25 \mathrm{E}+04$ & $3.8 \overline{8 E}+04$ & $4.45 \mathrm{E}+03$ & $1.62 \mathrm{E}+04$ & $1.72 \mathrm{E}+02$ & $1.35 \mathrm{E}+04$ & $1.23 \mathrm{E}+04$ \\
\hline 52 & $5.32 \mathrm{E}+04$ & $1.26 \mathrm{E}+04$ & $3.89 \mathrm{E}+04$ & $4.49 \mathrm{E}+03$ & $1.67 \mathrm{E}+04$ & $1.81 \mathrm{E}+02$ & $1.38 \mathrm{E}+04$ & $1.24 \mathrm{E}+04$ \\
\hline 53 & $5.29 \mathrm{E}+04$ & $1.26 \mathrm{E}+04$ & $3.90 \mathrm{E}+04$ & $4.53 \mathrm{E}+03$ & $1.71 \mathrm{E}+04$ & $1.89 \mathrm{E}+02$ & $1.40 \mathrm{E}+04$ & $1.25 \mathrm{E}+0$ \\
\hline 54 & $5.26 \mathrm{E}+04$ & $1.27 \mathrm{E}+04$ & $3.90 \mathrm{E}+04$ & $4.57 \mathrm{E}+03$ & $1.70 \mathrm{E}+04$ & $1.91 \mathrm{E}+02$ & $1.65 \mathrm{E}+04$ & $5.17 \mathrm{E}+0$ \\
\hline 55 & $5.23 \mathrm{E}+04$ & $1.28 \mathrm{E}+04$ & $3.91 \mathrm{E}+04$ & $4.61 E+03$ & $1.69 \mathrm{E}+04$ & $1.93 \mathrm{E}+02$ & $1.64 \mathrm{E}+04$ & $5.15 \mathrm{E}+0$. \\
\hline 56 & $5.20 \mathrm{E}+04$ & $1.29 \mathrm{E}+04$ & $3.90 \mathrm{E}+04$ & $4.64 \mathrm{E}+03$ & $1.68 \mathrm{E}+04$ & $1.94 \mathrm{E}+02$ & $1.64 \mathrm{E}+04$ & $5.13 \mathrm{E}+0$ \\
\hline 57 & $5.17 \mathrm{E}+04$ & $1.30 \mathrm{E}+04$ & $3.90 \mathrm{E}+04$ & $4.68 \mathrm{E}+03$ & $1.67 \mathrm{E}+04$ & $1.96 \mathrm{E}+02$ & $1.63 \mathrm{E}+04$ & $5.12 \mathrm{E}+03$ \\
\hline 58 & $5.14 \mathrm{E}+04$ & $1.30 \mathrm{E}+04$ & $3.89 \mathrm{E}+04$ & $4.72 \mathrm{E}+03$ & $1.65 \mathrm{E}+04$ & $1.98 \mathrm{E}+02$ & $1.63 \mathrm{E}+04$ & $\overline{5.10 \mathrm{E}+03}$ \\
\hline 59 & $5.11 \mathrm{E}+04$ & $1.31 \mathrm{E}+04$ & $3.88 \mathrm{E}+04$ & $4.76 \mathrm{E}+03$ & $1.64 \mathrm{E}+04$ & $1.99 \mathrm{E}+02$ & $1.62 \mathrm{E}+04$ & $5.08 \mathrm{E}+03$ \\
\hline 60 & $5.08 \mathrm{E}+04$ & $1.32 \mathrm{E}+04$ & $3.86 \mathrm{E}+04$ & $4.79 \mathrm{E}+03$ & $1.63 \mathrm{E}+04$ & $2.01 \mathrm{E}+02$ & $1.61 \mathrm{E}+04$ & $5.06 \mathrm{E}+03$ \\
\hline 61 & $5.04 \mathrm{E}+04$ & $1.33 \mathrm{E}+04$ & $3.85 \mathrm{E}+04$ & $4.83 \mathrm{E}+03$ & $1.62 \mathrm{E}+04$ & $2.02 \mathrm{E}+02$ & $1.61 \mathrm{E}+04$ & $5.05 \mathrm{E}+03$ \\
\hline 62 & $5.01 \mathrm{E}+04$ & $1.34 \mathrm{E}+04$ & $3.83 \mathrm{E}+04$ & $4.87 \mathrm{E}+03$ & $1.61 \mathrm{E}+04$ & $2.04 \mathrm{E}+02$ & $1.60 \mathrm{E}+04$ & $5.03 \mathrm{E}+03$ \\
\hline 63 & $4.97 \mathrm{E}+04$ & $1.34 \mathrm{E}+04$ & $3.81 \mathrm{E}+04$ & $4.90 \mathrm{E}+03$ & $1.60 \mathrm{E}+04$ & $2.06 \mathrm{E}+02$ & $1.60 \mathrm{E}+04$ & $5.01 \mathrm{lE}+03$ \\
\hline 64 & $4.93 \mathrm{E}+04$ & $1.35 \mathrm{E}+04$ & $3.79 \mathrm{E}+04$ & $4.94 \mathrm{E}+03$ & $1.58 \mathrm{E}+04$ & $2.07 \mathrm{E}+02$ & $1.59 \mathrm{E}+04$ & $4.99 \mathrm{E}+03$ \\
\hline 65 & $4.89 \mathrm{E}+04$ & $1.36 \mathrm{E}+04$ & $3.77 \mathrm{E}+04$ & $4.97 \mathrm{E}+03$ & $1.57 \mathrm{E}+04$ & $2.09 \mathrm{E}+02$ & $1.59 \mathrm{E}+04$ & $4.98 \mathrm{E}+03$ \\
\hline 66 & $4.85 \mathrm{E}+04$ & $1.37 \mathrm{E}+04$ & $3.75 \mathrm{E}+04$ & $5.01 \mathrm{E}+03$ & 1.61 & $2.18 \mathrm{E}+02$ & $1.57 \mathrm{E}+04$ & $1.40 \mathrm{E}+04$ \\
\hline 67 & $4.80 \mathrm{E}+04$ & $1.37 \mathrm{E}+04$ & $3.72 \mathrm{E}+04$ & $5.05 \mathrm{E}+03$ & $1.66 \mathrm{E}+04$ & $2.27 \mathrm{E}+02$ & $1.59 \mathrm{E}+04$ & $1.41 \mathrm{E}+0$ \\
\hline 68 & $4.76 \mathrm{E}+04$ & $1.38 \mathrm{E}+04$ & $3.69 \mathrm{E}+04$ & $5.08 \mathrm{E}+03$ & $1.70 \mathrm{E}+04$ & $2.36 \mathrm{E}+02$ & $1.60 \mathrm{E}+04$ & $1.42 \mathrm{E}+04$ \\
\hline 69 & $4.71 \mathrm{E}+04$ & $1.39 \mathrm{E}+04$ & $3.67 \mathrm{E}+04$ & $5.12 \mathrm{E}+03$ & $1.74 \mathrm{E}+04$ & $2.45 \mathrm{E}+02$ & $1.62 \mathrm{E}+04$ & $1.43 \mathrm{E}+04$ \\
\hline 168 & $7.27 \mathrm{E}+03$ & $1.69 \mathrm{E}+04$ & $9.66 \mathrm{E}+03$ & $7.16 \mathrm{E}+03$ & $1.95 \mathrm{E}+04$ & $9.57 \mathrm{E}+02$ & $1.98 \mathrm{E}+04$ & $1.73 \mathrm{E}+0$ \\
\hline 336 & $3.01 \mathrm{E}+03$ & $1.41 \mathrm{E}+04$ & $2.92 \mathrm{E}+03$ & $7.63 \mathrm{E}+03$ & $8.83 E+03$ & $2.32 \mathrm{E}+03$ & $1.30 \mathrm{E}+04$ & $1.39 \mathrm{E}+0$ \\
\hline 504 & $2.16 \mathrm{E}+03$ & $1.16 \mathrm{E}+04$ & $1.96 \mathrm{E}+03$ & $7.89 \mathrm{E}+03$ & $4.56 \mathrm{E}+03$ & $3.09 \mathrm{E}+03$ & $6.19 E+03$ & $9.66 \mathrm{E}+03$ \\
\hline 720 & $1.56 \mathrm{E}+03$ & $9.01 \mathrm{E}+03$ & $1.30 \mathrm{E}+03$ & $8.10 \mathrm{E}+03$ & $2.40 \mathrm{E}+03$ & $3.56 \mathrm{E}+03$ & $3.23 \mathrm{E}+03$ & $6.79 \mathrm{E}+03$ \\
\hline 2160 & $2.89 \mathrm{E}+02$ & $1.64 \mathrm{E}+03$ & $1.44 \mathrm{E}+02$ & $8.51 \mathrm{E}+03$ & $2.09 \mathrm{E}+02$ & $2.19 \mathrm{E}+03$ & $7.63 \mathrm{E}+02$ & $2.31 \mathrm{E}+03$ \\
\hline 4320 & $2.78 \mathrm{E}+01$ & $1.33 \mathrm{E}+02$ & $7.27 \mathrm{E}+02$ & $8.13 \mathrm{E}+03$ & $3.15 \mathrm{E}+01$ & $5.20 \mathrm{E}+02$ & $8.49 \mathrm{E}+01$ & $3.43 \mathrm{E}+02$ \\
\hline 4800 & $1.65 \mathrm{E}+01$ & $7.69 \mathrm{E}+01$ & $7.25 \mathrm{E}+02$ & $8.11 \mathrm{E}+03$ & $2.27 \mathrm{E}+01$ & $3.73 \mathrm{E}+02$ & $2.68 \mathrm{E}+01$ & $1.09 \mathrm{E}+02$ \\
\hline 8760 & $2.27 \mathrm{E}-01$ & $9.01 \mathrm{E}-01$ & $7.07 \mathrm{E}+02$ & $7.90 \mathrm{E}+03$ & $1.76 \mathrm{E}+00$ & $2.41 \mathrm{E}+01$ & $8.15 \mathrm{E}+00$ & $3.16 \mathrm{E}+0$ \\
\hline$\overline{\mathbf{B T}}$ & $\begin{array}{l}\mathrm{E6} 6 \\
\mathrm{~L}\end{array}$ & $\begin{array}{l}8.88 \mathrm{E}+4 \\
\mathrm{~Bq} / \mathrm{kg}\end{array}$ & $88.8 \mathrm{~Bq} / \mathrm{g}$ & & & & & \\
\hline$\overline{\mathrm{O}}$ & $\begin{array}{l}5.9 \mathrm{E} 6 \\
\mathrm{pCi} / \mathrm{L}\end{array}$ & $\begin{array}{l}2.18 \mathrm{E}+5 \\
\mathrm{~Bq} / \mathrm{kg}\end{array}$ & $218.3 \mathrm{~Bq} / \mathrm{g}$ & & & & & \\
\hline & \multicolumn{2}{|c|}{ VEGETABLES } & \multicolumn{2}{|c|}{ WHEAT SEED } & \multicolumn{2}{|l|}{ MEAT } & \multicolumn{2}{|l|}{ MILK } \\
\hline \multirow[t]{2}{*}{ HOUR } & $\overline{\mathrm{CO}}$ & $\widehat{\mathrm{OBT}}$ & HTO & OBT & HTO & $\overline{3 T}$ & HTO & $\overline{\mathrm{OBT}}$ \\
\hline & $q / g)$ & $(\overline{B q} / \mathrm{g})$ & $(\mathrm{Bq} / \mathrm{g})$ & $(\mathrm{Bq} / \mathrm{g})$ & $(\mathrm{Bq} / \mathrm{g})$ & $(\mathrm{Bq} / \mathrm{g})$ & $(\mathrm{Bq} / \mathrm{g})$ & $(\overline{B q} / \mathrm{g})$ \\
\hline & & & & & & & & \\
\hline
\end{tabular}




\begin{tabular}{|c|c|c|c|c|c|c|c|c|}
\hline $\begin{array}{l}10 / 13 / 53 / 5 \\
4\end{array}$ & $9 . \overline{88 \mathrm{E}+05}$ & $5.22 \mathrm{E}+03$ & $3.20 \mathrm{E}+05$ & $1.90 \mathrm{E}+03$ & $1.71 \mathrm{E}+04$ & $1.89 \mathrm{E}+02$ & $1.65 \mathrm{E}+04$ & $5.17 \mathrm{E}+03$ \\
\hline SUM & $9.93 \mathrm{E}+05$ & & $3.22 \mathrm{E}+05$ & & $1.73 E+04$ & & $2.16 E+04$ & \\
\hline $\mathrm{Ci} / \mathrm{g}$ & $2.68 E-05$ & & $8.70 \mathrm{E}-06$ & & $4.68 \mathrm{E}-07$ & & $5.85 \mathrm{E}-07$ & \\
\hline & & & & & & & & \\
\hline DIL $\overline{\text { HTO }}$ & $5.9 \mathrm{E}-09$ & & & & & & & \\
\hline \multicolumn{9}{|l|}{$(\mathrm{Ci} / \mathrm{g})$} \\
\hline & & & & & & & & \\
\hline 168 & $7.27 \mathrm{E}+03$ & $1.69 E+04$ & $9.66 \mathrm{E}+03$ & $7.16 \mathrm{E}+03$ & $1.95 \mathrm{E}+04$ & $9.57 \mathrm{E}+02$ & $1.98 \mathrm{E}+04$ & $1.73 E+04$ \\
\hline SŪM & $2.42 \mathrm{E}+04$ & & $1.68 \mathrm{E}+04$ & & $2.04 \mathrm{E}+04$ & & $3.71 \mathrm{E}+04$ & \\
\hline $\mathrm{Ci} / \mathrm{g}$ & 6.53E-07 & & 4.55E-07 & & 5.52E-07 & & $1.00 \mathrm{E}-06$ & \\
\hline DIL OBT & $2.4 \mathrm{E}-09$ & & & & & & & \\
\hline \multicolumn{9}{|l|}{$\mathrm{Ci} / \mathrm{g}$} \\
\hline & & & & & & & & \\
\hline 8760 & $2.27 \mathrm{E}-01$ & $9.01 \mathrm{E}-01$ & $7.07 \mathrm{E}+02$ & $7.90 \mathrm{E}+03$ & $1.76 \mathrm{E}+00$ & $2.41 \mathrm{E}+01$ & $8.15 \mathrm{E}+00$ & $3.16 \mathrm{E}+01$ \\
\hline SUM & $1.13 \mathrm{E}+00$ & & $8.61 E+03$ & & $2.59 \mathrm{E}+01$ & & $3.98 \mathrm{E}+01$ & \\
\hline $\mathrm{Ci} / \mathrm{g}$ & $3.05 \mathrm{E}-11$ & & $2.33 \mathrm{E}-07$ & & $6.99 \mathrm{E}-10$ & & $1.07 E-09$ & \\
\hline DIL OBT & $2.4 \mathrm{E}-09$ & & & & & & & \\
\hline $\mathrm{Ci} / \mathrm{g}$ & & & & & & & & \\
\hline
\end{tabular}




\begin{tabular}{|c|c|c|c|c|c|c|c|c|}
\hline & \multicolumn{7}{|c|}{ Tritium Facilities, Beyond Design Basis Fire, Average Met Conditions } & \\
\hline & \multicolumn{4}{|c|}{ Concentration Values for $150 \mathrm{~m}$ from release } & & & & \\
\hline \multicolumn{9}{|c|}{ Only Wheat Seed is examined since this is the edible portion of the Wheat. The "WHEAT" category is } \\
\hline \multicolumn{9}{|c|}{ ctually the unedible plant body and is not considered in the dose estimate. } \\
\hline & & & & & & & & \\
\hline & \multicolumn{2}{|c|}{ VEGETABLES } & \multicolumn{2}{|c|}{ WHEAT SEED } & \multicolumn{2}{|l|}{ MEÁT } & \multicolumn{2}{|l|}{ MILK } \\
\hline \multirow[t]{3}{*}{ HOUR } & HTO & OBT & HTO & $\overline{\mathrm{OBT}}$ & HTO & OBT & HTO & 1 \\
\hline & (g) & $(\mathrm{Bq} / \mathrm{g})$ & $(\mathrm{Bq} / \mathrm{g})$ & $(\mathrm{Bq} / \mathrm{g})$ & $(\mathrm{Bq} / \mathrm{g})$ & (E & $(\mathrm{Bq} / \mathrm{g})$ & ) \\
\hline & & & & & & & & \\
\hline 1 & $1.60 \mathrm{E}+08$ & $9.80 \mathrm{E}+05$ & $6.57 \mathrm{E}+07$ & $2.17 \mathrm{E}+05$ & $4.91 \mathrm{E}+06$ & $1.0 \overline{1 E}+03$ & $5.94 \mathrm{E}+05$ & $2.58 \mathrm{E}+05$ \\
\hline 2 & $4.81 \mathrm{E}+07$ & $1.25 \mathrm{E}+06$ & $9.04 \mathrm{E}+07$ & $3.62 \mathrm{E}+05$ & $6.84 \mathrm{E}+06$ & $2.43 E+03$ & $1.27 \bar{E}+06$ & $5.51 E+05$ \\
\hline 3 & $1.67 \mathrm{E}+07$ & $1.33 \mathrm{E}+06$ & $9.35 \mathrm{E}+07$ & $4.60 \mathrm{E}+05$ & $7.70 \mathrm{E}+06$ & $4.04 \mathrm{E}+03$ & $1.89 \mathrm{E}+06$ & $8.24 \mathrm{E}+05$ \\
\hline 4 & $7.65 \mathrm{E}+06$ & $1.36 \mathrm{E}+06$ & $8.60 \mathrm{E}+07$ & $5.25 \mathrm{E}+05$ & $8.07 \mathrm{E}+06$ & $5.74 \mathrm{E}+03$ & $2.42 \mathrm{E}+06$ & $1.06 \mathrm{E}+06$ \\
\hline 5 & $5.10 \mathrm{E}+06$ & $1.37 \mathrm{E}+06$ & $7.42 \mathrm{E}+07$ & $5.69 \mathrm{E}+05$ & $8.22 \mathrm{E}+06$ & $7.47 \mathrm{E}+03$ & $2.89 \mathrm{E}+06$ & $1.27 \mathrm{E}+06$ \\
\hline 6 & $4.34 \mathrm{E}+06$ & $1.38 \mathrm{E}+06$ & $6.16 \mathrm{E}+07$ & $5.99 \mathrm{E}+05$ & $8.15 \mathrm{E}+06$ & $8.34 \mathrm{E}+03$ & $7.90 \mathrm{E}+06$ & $2.46 \mathrm{E}+06$ \\
\hline 7 & $4.07 \mathrm{E}+06$ & $1.38 \mathrm{E}+06$ & $4.97 \mathrm{E}+07$ & $6.20 \mathrm{E}+05$ & $8.08 \mathrm{E}+06$ & $9.20 \mathrm{E}+03$ & $7.87 \mathrm{E}+06$ & $2.45 \mathrm{E}+06$ \\
\hline 8 & $3.94 \mathrm{E}+06$ & $1.39 \mathrm{E}+06$ & $3.94 \mathrm{E}+07$ & $6.34 \mathrm{E}+05$ & $8.01 \mathrm{E}+06$ & $1.01 \mathrm{E}+04$ & $7.83 \mathrm{E}+06$ & $2.44 \mathrm{E}+06$ \\
\hline 9 & $3.85 \mathrm{E}+06$ & $1.39 \mathrm{E}+06$ & $3.09 \mathrm{E}+07$ & $6.43 \mathrm{E}+05$ & $7.95 \mathrm{E}+06$ & $1.09 \mathrm{E}+04$ & $7.80 \mathrm{E}+06$ & $2.43 \mathrm{E}+06$ \\
\hline 10 & $3.77 \mathrm{E}+06$ & $1.40 \mathrm{E}+06$ & $2.40 \mathrm{E}+07$ & $6.50 \mathrm{E}+0 \mathrm{5}$ & $7.88 \mathrm{E}+06$ & $1.18 \mathrm{E}+04$ & $7.77 \mathrm{E}+06$ & $2.42 \mathrm{E}+06$ \\
\hline 11 & $3.69 \mathrm{E}+06$ & $1.40 \mathrm{E}+06$ & $1.85 \mathrm{E}+07$ & $6.55 \mathrm{E}+05$ & $7.82 \mathrm{E}+06$ & $1.26 \mathrm{E}+04$ & $7.74 \mathrm{E}+06$ & $2.41 \mathrm{E}+06$ \\
\hline 12 & $3.62 \mathrm{E}+06$ & $1.41 \mathrm{E}+06$ & $1.42 \mathrm{E}+07$ & $6.59 \mathrm{E}+05$ & $7.75 \mathrm{E}+06$ & $1.34 \mathrm{E}+04$ & $7.70 \mathrm{E}+06$ & $2.40 \mathrm{E}+0$ \\
\hline 13 & $3.54 \mathrm{E}+06$ & $1.41 \mathrm{E}+06$ & $1.09 \mathrm{E}+07$ & $6.62 \mathrm{E}+05$ & $7.68 \mathrm{E}+06$ & $1.42 \mathrm{E}+04$ & $7.67 \mathrm{E}+06$ & $2.39 \bar{E}+06$ \\
\hline 14 & $3.47 \mathrm{E}+06$ & $1.42 \mathrm{E}+06$ & $8.45 \mathrm{E}+06$ & $6.64 \mathrm{E}+05$ & $7.62 \mathrm{E}+06$ & $1.50 \mathrm{E}+04$ & $7.64 \overline{\mathrm{E}+06}$ & $2.38 \mathrm{E}+06$ \\
\hline 15 & $3 . \overline{41 E+06}$ & $1.42 \mathrm{E}+06$ & $6.57 \mathrm{E}+06$ & $6.66 \mathrm{E}+05$ & $7.56 \mathrm{E}+06$ & $1.58 \mathrm{E}+04$ & $7.61 \mathrm{E}+06$ & $2.37 \mathrm{E}+06$ \\
\hline 16 & $3.34 \mathrm{E}+06$ & $1.42 \mathrm{E}+06$ & $5.16 \mathrm{E}+06$ & $6.68 \mathrm{E}+05$ & $7.49 \mathrm{E}+06$ & $1.66 \mathrm{E}+04$ & $7.58 \mathrm{E}+06$ & $2.36 \mathrm{E}+06$ \\
\hline 17 & $3.28 \mathrm{E}+06$ & $1.43 \mathrm{E}+06$ & $4.11 \mathrm{E}+06$ & $6.70 \mathrm{E}+05$ & $7.43 \mathrm{E}+06$ & $1.74 \mathrm{E}+04$ & $7.55 \mathrm{E}+06$ & $2.35 \mathrm{E}+06$ \\
\hline 18 & $3.21 \mathrm{E}+06$ & $1.43 \mathrm{E}+06$ & $3 . \overline{32 \mathrm{E}+06}$ & $6.71 \mathrm{E}+05$ & $7.41 \mathrm{E}+06$ & $1.91 \mathrm{E}+04$ & $7.18 \mathrm{E}+06$ & $3.34 \mathrm{E}+06$ \\
\hline 19 & $3.15 \mathrm{E}+06$ & $1.44 \mathrm{E}+06$ & $2.73 \mathrm{E}+06$ & $6.72 E+05$ & $7.38 \mathrm{E}+06$ & $2.08 \mathrm{E}+04$ & $7.17 \mathrm{E}+06$ & $3.33 \mathrm{E}+0$ \\
\hline 20 & $3.10 \mathrm{E}+06$ & $1.44 \mathrm{E}+06$ & $2.30 \mathrm{E}+06$ & $6.73 \mathrm{E}+05$ & $7 . \overline{35 \mathrm{E}+06}$ & $2.25 \mathrm{E}+04$ & $7.15 \mathrm{E}+06$ & $3.33 \mathrm{E}+06$ \\
\hline 21 & $3.04 \mathrm{E}+06$ & $1 . \overline{44 \mathrm{E}+06}$ & $1.98 \mathrm{E}+06$ & $6.75 \mathrm{E}+05$ & $7.33 \mathrm{E}+06$ & $2.42 \mathrm{E}+04$ & $7.14 \mathrm{E}+06$ & $3.33 \mathrm{E}+06$ \\
\hline 22 & $2.99 \mathrm{E}+06$ & $1.45 \mathrm{E}+06$ & $1.74 \mathrm{E}+06$ & $6.76 \mathrm{E}+05$ & $7.30 \mathrm{E}+06$ & $2.58 \mathrm{E}+04$ & $7.13 E+06$ & $3.32 \mathrm{E}+06$ \\
\hline 23 & $2.94 \mathrm{E}+06$ & $1.45 \mathrm{E}+06$ & $1.56 \mathrm{E}+06$ & $6.77 \mathrm{E}+05$ & $7.27 \mathrm{E}+06$ & $2.75 \mathrm{E}+04$ & $7.11 \mathrm{E}+06$ & $3.32 \mathrm{E}+06$ \\
\hline 24 & $2.89 \mathrm{E}+06$ & $1.45 \mathrm{E}+06$ & $1.42 \mathrm{E}+06$ & $6.78 \mathrm{E}+05$ & $7.24 \mathrm{E}+06$ & $2.92 \mathrm{E}+04$ & $7.10 \mathrm{E}+06$ & $3.32 \mathrm{E}+06$ \\
\hline 25 & $2.84 \mathrm{E}+06$ & $1.46 \mathrm{E}+06$ & $1.32 \mathrm{E}+06$ & $6.79 \mathrm{E}+05$ & $7.21 \mathrm{E}+06$ & $3.09 \mathrm{E}+04$ & $7.09 \mathrm{E}+06$ & $3.31 \mathrm{E}+06$ \\
\hline 26 & $2.80 \mathrm{E}+06$ & $1.46 \mathrm{E}+06$ & $1.24 \mathrm{E}+06$ & $6.80 \mathrm{E}+05$ & $7.19 \mathrm{E}+06$ & $3.25 \mathrm{E}+04$ & $7.07 \mathrm{E}+06$ & $3.31 \mathrm{E}+06$ \\
\hline 27 & $2.76 \mathrm{E}+06$ & $1.46 \mathrm{E}+06$ & $1.18 \mathrm{E}+06$ & $6.81 \mathrm{E}+05$ & $7.16 \mathrm{E}+06$ & $3 . \overline{42 \mathrm{E}+04}$ & $7.06 \mathrm{E}+06$ & $3.31 \mathrm{E}+06$ \\
\hline 28 & $2.72 \mathrm{E}+06$ & $1.46 \mathrm{E}+06$ & $1.13 \mathrm{E}+06$ & $6.82 \mathrm{E}+05$ & $7.13 \bar{E}+06$ & $3.58 \mathrm{E}+04$ & $7.05 \mathrm{E}+06$ & $3.30 \mathrm{E}+06$ \\
\hline 29 & $2.68 \mathrm{E}+06$ & $1.47 E+06$ & $1.09 \mathrm{E}+06$ & $6.83 \mathrm{E}+05$ & $7.10 \mathrm{E}+06$ & $3.75 \mathrm{E}+04$ & $7.03 E+06$ & $3.30 \mathrm{E}+06$ \\
\hline 30 & $2.64 \mathrm{E}+06$ & $1.47 \mathrm{E}+06$ & $1.06 \mathrm{E}+06$ & $6.84 \mathrm{E}+05$ & $7.04 \mathrm{E}+06$ & $3.82 \mathrm{E}+04$ & $6.82 \mathrm{E}+06$ & $2.13 \mathrm{E}+06$ \\
\hline 31 & $2.60 \mathrm{E}+06$ & $1.47 \mathrm{E}+06$ & $1.03 E+06$ & $6.85 \mathrm{E}+05$ & $6.98 \mathrm{E}+06$ & $3.89 \mathrm{E}+04$ & $6.79 \mathrm{E}+06$ & $2.12 \mathrm{E}+06$ \\
\hline 32 & $2.57 \mathrm{E}+06$ & $1.47 \mathrm{E}+06$ & $1.01 \mathrm{E}+06$ & $6.86 \mathrm{E}+05$ & $6.92 \mathrm{E}+06$ & $3.96 \mathrm{E}+04$ & $6.76 \mathrm{E}+06$ & $2.11 \mathrm{E}+06$ \\
\hline 33 & $2.53 \mathrm{E}+06$ & $1.48 \mathrm{E}+06$ & $9.91 \mathrm{E}+05$ & $6.87 \mathrm{E}+05$ & $6.86 \mathrm{E}+06$ & $4.04 \mathrm{E}+04$ & $6.74 \mathrm{E}+06$ & $2.11 \mathrm{E}+06$ \\
\hline 34 & $2.50 \mathrm{E}+06$ & $1.48 \mathrm{E}+06$ & $9.74 \mathrm{E}+05$ & $6.87 \bar{E}+05$ & $6.81 \mathrm{E}+06$ & $4.11 \mathrm{E}+04$ & $6.71 \mathrm{E}+06$ & $2.10 \mathrm{E}+06$ \\
\hline 35 & $2.47 \mathrm{E}+06$ & $1.48 \mathrm{E}+06$ & $9.57 \mathrm{E}+05$ & $6.88 \mathrm{E}+05$ & $6.75 \mathrm{E}+06$ & $4.18 \mathrm{E}+04$ & $6.68 \mathrm{E}+06$ & $2.09 \mathrm{E}+0 \mathrm{C}$ \\
\hline 36 & $2 . \overline{44 \mathrm{E}+06}$ & $1.48 \mathrm{E}+06$ & $9 . \overline{43 E+05}$ & $6.89 \mathrm{E}+05$ & $6.69 \mathrm{E}+06$ & $4.24 \mathrm{E}+04$ & $6.65 \mathrm{E}+06$ & $2.08 \mathrm{E}+06$ \\
\hline 37 & $2.41 \mathrm{E}+06$ & $1.49 \mathrm{E}+06$ & $9.29 \mathrm{E}+05$ & $6.90 \mathrm{E}+05$ & $6.64 \mathrm{E}+06$ & $4.31 \mathrm{E}+04$ & $6.62 \mathrm{E}+06$ & $2.07 \mathrm{E}+06$ \\
\hline 38 & $2.39 \mathrm{E}+06$ & $1.49 \mathrm{E}+06$ & $9.16 \mathrm{E}+05$ & $6.91 \mathrm{E}+05$ & $6.58 \mathrm{E}+06$ & $4.38 \mathrm{E}+04$ & $6.60 \mathrm{E}+06$ & $2.06 \mathrm{E}+06$ \\
\hline
\end{tabular}


Foodstuff Concentrations and Relocation Considerations Following

\begin{tabular}{|c|c|c|c|c|c|c|c|c|}
\hline 39 & $2.36 \mathrm{E}+06$ & $1.49 \mathrm{E}+06$ & $9.04 \mathrm{E}+05$ & $6.92 \mathrm{E}+05$ & $6.53 \mathrm{E}+06$ & $4.45 \mathrm{E}+04$ & $6.57 \mathrm{E}+06$ & $2.05 \mathrm{E}+0$ \\
\hline 40 & $2.34 \bar{E}+06$ & $1.49 \mathrm{E}+06$ & $8.9 \bar{E}+05$ & $6.93 \mathrm{E}+05$ & $6 . \overline{47 \mathrm{E}+06}$ & $4.51 \mathrm{E}+04$ & $6.54 \mathrm{E}+06$ & $2.05 \mathrm{E}+0$ \\
\hline 41 & $2 . \overline{32} \overline{\mathrm{E}+06}$ & $1.49 \mathrm{E}+06$ & $8.82 \mathrm{E}+05$ & $6.93 \mathrm{E}+05$ & $6.42 \mathrm{E}+06$ & $4.58 \mathrm{E}+04$ & $6.52 \mathrm{E}+06$ & $2.04 \mathrm{E}+$ \\
\hline 42 & $2.29 \bar{E}+06$ & $1.50 \mathrm{E}+06$ & $8.7 \overline{1 E}+05$ & $6.94 \mathrm{E}+05$ & $6.39 \mathrm{E}+06$ & $4.74 \mathrm{E}+04$ & $6.19 \mathrm{E}+06$ & $3.06 \mathrm{E}+$ \\
\hline 43 & $2.27 \mathrm{E}+06$ & $1.50 \mathrm{E}+06$ & $8.61 \mathrm{E}+05$ & $6.95 \mathrm{E}+05$ & $6.36 \mathrm{E}+06$ & $4.90 \overline{\mathrm{E}}+04$ & $6.18 \mathrm{E}+06$ & $3.06 \mathrm{E}+$ \\
\hline 44 & $2.25 \mathrm{E}+06$ & $1.50 \mathrm{E}+06$ & $8.52 \mathrm{E}+05$ & $6.96 \mathrm{E}+05$ & $6.33 \mathrm{E}+06$ & $5.05 \mathrm{E}+04$ & $6.16 \mathrm{E}+06$ & $3.06 \mathrm{E}+$ \\
\hline 45 & $2.23 \overline{\mathrm{E}+06}$ & $1.50 \mathrm{E}+06$ & $8.43 \mathrm{E}+05$ & $6.97 \mathrm{E}+05$ & $6 . \overline{30 \mathrm{E}+06}$ & $5.21 \mathrm{E}+04$ & $6.15 \mathrm{E}+06$ & $3.05 \mathrm{E}+$ \\
\hline 46 & $2.21 \mathrm{E}+06$ & $1.50 \mathrm{E}+06$ & $8.3 \overline{4 E}+05$ & $6.97 \mathrm{E}+05$ & $6.28 \mathrm{E}+06$ & $5.37 \mathrm{E}+04$ & $6.14 \mathrm{E}+06$ & $3.05 \mathrm{E}+$ \\
\hline 47 & $2.20 \overline{\mathrm{E}}+06$ & $1.51 \mathrm{E}+06$ & $8.26 \mathrm{E}+05$ & $6.98 \mathrm{E}+05$ & $6.25 \mathrm{E}+06$ & $5.52 \mathrm{E}+04$ & $6.12 \mathrm{E}+06$ & $3.04 \mathrm{E}+\mathrm{C}$ \\
\hline 48 & $2.18 \mathrm{E}+06$ & $1.5 \overline{\mathrm{IE}+06}$ & $8.18 \mathrm{E}+05$ & $6.99 \mathrm{E}+05$ & $6.22 \mathrm{E}+06$ & $5.68 \mathrm{E}+04$ & $6.11 \mathrm{E}+06$ & $3.04 \mathrm{E}+06$ \\
\hline 49 & $2.16 \mathrm{E}+06$ & $1.51 \mathrm{E}+06$ & $8.10 \mathrm{E}+05$ & $7.00 \mathrm{E}+05$ & $6.19 \mathrm{E}+06$ & $5.84 \mathrm{E}+04$ & $6.10 \mathrm{E}+06$ & $3.03 \mathrm{E}-$ \\
\hline 50 & $2.15 \mathrm{E}+06$ & $1.51 \mathrm{E}+06$ & $8.02 \mathrm{E}+05$ & $7.01 \mathrm{E}+05$ & $6.16 \mathrm{E}+06$ & $5.99 \mathrm{E}+04$ & $6.08 \mathrm{E}+06$ & $3.03 E+c$ \\
\hline 51 & $2.13 \mathrm{E}+06$ & $1.51 \mathrm{E}+06$ & $7.95 \mathrm{E}+05$ & $7.01 \mathrm{E}+05$ & $6.14 \mathrm{E}+06$ & $6.14 \mathrm{E}+04$ & $6.07 \mathrm{E}+06$ & $3.03 \mathrm{E}+$ \\
\hline 52 & $2.12 \mathrm{E}+06$ & $1.51 \mathrm{E}+06$ & $7.88 \mathrm{E}+05$ & $7.02 \mathrm{E}+05$ & $6.11 \mathrm{E}+06$ & $6.30 \mathrm{E}+04$ & $6.06 \mathrm{E}+06$ & $3.02 \mathrm{E}+0$ \\
\hline 53 & $2.11 \overline{\mathrm{E}+06}$ & $1.52 \mathrm{E}+06$ & $7.82 \mathrm{E}+05$ & $7.03 \mathrm{E}+05$ & $6.08 \mathrm{E}+06$ & $6.45 \mathrm{E}+04$ & $6.04 \mathrm{E}+06$ & $3.02 \mathrm{E}+0$ \\
\hline 54 & $2.09 \mathrm{E}+06$ & $1.52 \mathrm{E}+06$ & $7.75 \mathrm{E}+05$ & $7 . \overline{04 E} \div 05$ & $6,03 \mathrm{E}+06$ & $6.51 \mathrm{E}+04$ & $5.85 \mathrm{E}+06$ & $1.83 \mathrm{E}+0$ \\
\hline 55 & $2.08 \mathrm{E}+06$ & $1.52 \mathrm{E}+06$ & $7.69 \mathrm{E}+05$ & $7 . \overline{04 E}+05$ & $5.98 \mathrm{E}+06$ & $6.57 \mathrm{E}+04$ & $5.82 E+06$ & $1.83 \mathrm{E}+0$ \\
\hline 56 & $2.07 \mathrm{E}+06$ & $1.52 \mathrm{E}+06$ & $7.63 \mathrm{E}+05$ & $7.05 \mathrm{E}+05$ & $5.93 \mathrm{E}+06$ & $6.63 \mathrm{E}+04$ & $5.80 \mathrm{E}+06$ & $1.82 \mathrm{E}+\mathrm{C}$ \\
\hline 57 & $2.05 \mathrm{E}+06$ & $1.52 \mathrm{E}+06$ & $7.58 \mathrm{E}+05$ & $7.06 \mathrm{E}+05$ & $5.88 \mathrm{E}+06$ & $6.69 \mathrm{E}+04$ & $5.77 \mathrm{E}+06$ & $1.81 \mathrm{E}+0$ \\
\hline 58 & $2.04 \mathrm{E}+06$ & $1.52 \mathrm{E}+06$ & $7.52 \mathrm{E}+05$ & $7 . \overline{06 \mathrm{E}}+05$ & $5.83 \mathrm{E}+06$ & $6.75 \mathrm{E}+04$ & $5.75 \mathrm{E}+06$ & $1.80 \mathrm{E}+0$ \\
\hline 59 & $2.03 \mathrm{E}+06$ & $1.52 \mathrm{E}+06$ & $7.47 \mathrm{E}+05$ & $7.07 \mathrm{E}+05$ & $5.78 \mathrm{E}+06$ & $6.80 \mathrm{E}+04$ & $5.72 \mathrm{E}+06$ & $1.80 \mathrm{E}+0$ \\
\hline 60 & $2.02 \mathrm{E}+06$ & $1.53 \mathrm{E}+06$ & $7.42 \mathrm{E}+05$ & $7.08 \mathrm{E}+05$ & $5.73 \mathrm{E}+06$ & $6.86 \mathrm{E}+04$ & $5.70 \mathrm{E}+06$ & $1.79 \mathrm{E}+0$ \\
\hline 61 & $2.01 \overline{\mathrm{E}}+06$ & $1.5 \overline{3 \mathrm{E}}+06$ & $7.37 \mathrm{E}+05$ & $7.09 \mathrm{E}+05$ & $5.69 \mathrm{E}+06$ & $6.92 \mathrm{E}+04$ & $5.68 \mathrm{E}+06$ & $1.78 \mathrm{E}+0$ \\
\hline 62 & $2.00 \mathrm{E}+06$ & $1.53 \mathrm{E}+06$ & $7.33 \mathrm{E}+05$ & $7.09 \mathrm{E}+05$ & $5.64 \mathrm{E}+06$ & $6.97 \mathrm{E}+04$ & $5.65 \mathrm{E}+06$ & $1.77 \mathrm{E}+0$ \\
\hline 63 & $1.99 \mathrm{E}+06$ & $1.53 \mathrm{E}+06$ & $7.28 \mathrm{E}+05$ & $7.10 \mathrm{E}+05$ & $5.59 \mathrm{E}+06$ & $7.03 \mathrm{E}+04$ & $5.63 \mathrm{E}+06$ & $1.77 \mathrm{E}+0$ \\
\hline 64 & $1.98 \mathrm{E}+06$ & $1.53 \mathrm{E}+06$ & $7.24 \mathrm{E}+05$ & $7.11 \mathrm{E}+05$ & $5.55 \mathrm{E}+06$ & $7.08 \mathrm{E}+04$ & $5.61 \mathrm{E}+06$ & $1.76 \mathrm{E}+0$ \\
\hline 65 & $1.97 \mathrm{E}+06$ & $1.53 \mathrm{E}+06$ & $7.20 \mathrm{E}+05$ & $7.11 \mathrm{E}+05$ & $5.50 \mathrm{E}+06$ & $7.14 \mathrm{E}+04$ & $5.58 \mathrm{E}+06$ & $1.75 \mathrm{E}+0$ \\
\hline 66 & $1.96 \bar{E}+06$ & $1.53 \bar{E}+06$ & $7.16 \mathrm{E}+05$ & $7.12 \mathrm{E}+05$ & $5.47 \mathrm{E}+06$ & $7.29 \mathrm{E}+04$ & $5.31 \mathrm{E}+06$ & $2.80 \mathrm{E}+0$ \\
\hline 67 & $1.95 \mathrm{E}+06$ & $1.54 \mathrm{E}+06$ & $7.12 \mathrm{E}+05$ & $7.13 E+05$ & $5.45 \mathrm{E}+06$ & $7.43 \mathrm{E}+04$ & $5.29 \mathrm{E}+06$ & $2.80 \mathrm{E}+0$ \\
\hline 68 & $1.95 \mathrm{E}+06$ & $1.54 \mathrm{E}+06$ & $7.08 \mathrm{E}+05$ & $7.13 \mathrm{E}+05$ & $5.43 \mathrm{E}+06$ & $7.58 \mathrm{E}+04$ & $5.28 \mathrm{E}+06$ & $2.79 \mathrm{E}+0$ \\
\hline 69 & $1.94 \mathrm{E}+06$ & $1.5 \overline{4 E+06}$ & $7.04 \mathrm{E}+05$ & $7.14 \mathrm{E}+05$ & $5.40 \mathrm{E}+06$ & $7.72 \mathrm{E}+04$ & $5.27 \mathrm{E}+06$ & $\overline{2.79 \mathrm{E}+0}$ \\
\hline$\overline{168}$ & $9.01 \mathrm{E}+05$ & $1.54 \mathrm{E}+06$ & $8.80 \mathrm{E}+05$ & $7.75 \mathrm{E}+05$ & $3.05 \mathrm{E}+06$ & $1.82 \mathrm{E}+05$ & $3.36 \mathrm{E}+06$ & $2.17 \mathrm{E}+0$ \\
\hline 336 & $4.77 \bar{E}+05$ & $1.31 \mathrm{E}+06$ & $4.68 \mathrm{E}+05$ & $8.43 \mathrm{E}+05$ & $1.28 \mathrm{E}+06$ & $3.20 \mathrm{E}+05$ & $1.96 \mathrm{E}+06$ & $1.62 \mathrm{E}+0$ \\
\hline 504 & $3.55 \mathrm{E}+05$ & $1.11 \mathrm{E}+06$ & $3.24 \mathrm{E}+05$ & $8.85 \mathrm{E}+05$ & $6.27 \mathrm{E}+05$ & $3.95 \mathrm{E}+05$ & $8.76 \mathrm{E}+05$ & $1.08 \mathrm{E}+0$ \\
\hline 720 & $2.59 \mathrm{E}+05$ & $8.80 \mathrm{E}+05$ & $2.18 \mathrm{E}+05$ & $9.22 \mathrm{E}+05$ & $3.14 \mathrm{E}+05$ & $4.36 \mathrm{E}+05$ & $4.33 \mathrm{E}+05$ & $\overline{7.56 \mathrm{E}+0}$ \\
\hline 2160 & $4.89 \mathrm{E}+04$ & $1.83 \mathrm{E}+05$ & $2.47 \mathrm{E}+04$ & $9.95 \overline{\mathrm{E}+05}$ & $2.80 \mathrm{E}+04$ & $2.57 \mathrm{E}+05$ & $9.73 E+04$ & $2.63 \mathrm{E}+0$ \\
\hline 4320 & $4.70 \mathrm{E}+03$ & $1.74 \mathrm{E}+04$ & $1.24 \mathrm{E}+05$ & $9.37 \mathrm{E}+05$ & $4.92 \mathrm{E}+03$ & $6.22 \mathrm{E}+04$ & $1.22 \mathrm{E}+04$ & $4.21 \mathrm{E}+0$ \\
\hline 4800 & $2.80 \mathrm{E}+03$ & $1.03 \mathrm{E}+04$ & $1.24 \mathrm{E}+05$ & $9.34 \mathrm{E}+05$ & $3.60 \mathrm{E}+03$ & $4.49 \mathrm{E}+04$ & $4.22 \mathrm{E}+03$ & $1.40 \mathrm{E}$ \\
\hline 8760 & $3.84 \mathrm{E}+01$ & $1.39 \mathrm{E}+02$ & $1.20 \mathrm{E}+05$ & $9.10 \mathrm{E}+05$ & $3.05 \mathrm{E}+02$ & $3.07 \mathrm{E}+03$ & $1.33 \mathrm{E}+03$ & $4.18 \mathrm{E}+$ \\
\hline BT & & $\begin{array}{l}8.88 \mathrm{E}+4 \\
\mathrm{~Bq} / \mathrm{kg}\end{array}$ & $8 \mathrm{~Bq} / \mathrm{g}$ & & & & & \\
\hline ro & $\begin{array}{l}5.9 \mathrm{E} 6 \\
\mathrm{pCi} / \mathrm{L}\end{array}$ & $\begin{array}{l}2.18 \mathrm{E}+5 \\
\mathrm{~Bq} / \mathrm{kg}\end{array}$ & $8.3 \mathrm{~Bq} / \mathrm{g}$ & & & & & \\
\hline & \multirow{2}{*}{\multicolumn{2}{|c|}{ VEGETABLES }} & & & & & & \\
\hline & & & \multicolumn{2}{|c|}{ WHEAT SEED } & \multicolumn{2}{|l|}{ MEAT } & \multicolumn{2}{|l|}{ MILK } \\
\hline \multirow[t]{2}{*}{ HOUR } & HTO & OBT & TO & $\mathrm{OBT}$ & HTO & $\mathrm{OBT}$ & HTO & OBT \\
\hline & $(\mathrm{Bq} / \mathrm{g})$ & $(\mathrm{Bq} / \mathrm{g})$ & $(\mathrm{Bq} / \mathrm{g})$ & $(\mathrm{Bq} / \mathrm{g})$ & $(\mathrm{Bq} / \mathrm{g})$ & $(\mathrm{Bq} / \mathrm{g})$ & $(\mathrm{Bq} / \mathrm{g})$ & $(\mathrm{Bq} / \mathrm{g})$ \\
\hline [O & & & & & & & & \\
\hline
\end{tabular}


Foodstuff Concentrations and Relocation Considerations Following

a Tritium Oxide Release from SRS Tritium Facilities (U)

\begin{tabular}{|c|c|c|c|c|c|c|c|c|}
\hline$[1 / 3 / 5 / 6$ & $1.60 \mathrm{E}+08$ & $9.80 \mathrm{E}+05$ & $9.35 \mathrm{E}+07$ & $\overline{4.60 \mathrm{E}+05}$ & $8.22 \mathrm{E}+06$ & $7.47 \mathrm{E}+03$ & $7.90 \mathrm{E}+06$ & $2.46 \mathrm{E}+06$ \\
\hline$\overline{\text { SUM }}$ & $1.61 \mathrm{E}+08$ & & $9.40 \mathrm{E}+07$ & & $8.23 \mathrm{E}+06$ & & $1.04 \mathrm{E}+07$ & \\
\hline $\mathrm{Ci} / \mathrm{g}$ & $4.35 E-03$ & & $2.54 \mathrm{E}-03$ & & $2.22 \mathrm{E}-04$ & & $2.80 \mathrm{E}-04$ & \\
\hline & & & & & & & & \\
\hline DIL HTO & $5.9 \mathrm{E}-09$ & & & & & & & \\
\hline \multicolumn{9}{|l|}{$(\mathrm{Ci} / \mathrm{g})$} \\
\hline & & & & & & & & \\
\hline 168 & $9.01 \mathrm{E}+05$ & $1.54 \mathrm{E}+06$ & $8.80 \mathrm{E}+05$ & $7.75 \mathrm{E}+05$ & $3.05 \mathrm{E}+06$ & $1.82 \mathrm{E}+05$ & $3.36 \mathrm{E}+06$ & $2,17 \mathrm{E}+06$ \\
\hline SUM & $2.4 \overline{4 E}+06$ & & $1.66 \mathrm{E}+06$ & & $3.23 \mathrm{E}+06$ & & $5.53 \mathrm{E}+06$ & \\
\hline $\mathrm{Ci} / \mathrm{g}$ & $6.59 \mathrm{E}-05$ & & $4.47 \mathrm{E}-05$ & & $8.72 \mathrm{E}-05$ & & $1.49 \mathrm{E}-04$ & \\
\hline & & & & & & & & \\
\hline DIL OBT & $2.4 \mathrm{E}-09$ & & & & & & & \\
\hline \multicolumn{9}{|l|}{$\mathrm{Ci} / \mathrm{g}$} \\
\hline & & & & & & & & \\
\hline 8760 & $3.84 \mathrm{E}+01$ & $1.39 \mathrm{E}+02$ & $1.20 \mathrm{E}+05$ & $9.10 \mathrm{E}+05$ & $3.05 \mathrm{E}+02$ & $3.07 \mathrm{E}+03$ & $1.33 \mathrm{E}+03$ & $4.18 \mathrm{E}+03$ \\
\hline SUM & $1.78 \mathrm{E}+02$ & & $1.0 \bar{E}+06$ & & $3.37 \mathrm{E}+03$ & & $5.51 \mathrm{E}+03$ & \\
\hline $\mathrm{Ci} / \mathrm{g}$ & $4.80 \mathrm{E}-09$ & & $2.79 \mathrm{E}-05$ & & $9.11 \mathrm{E}-08$ & & $1.49 \mathrm{E}-07$ & \\
\hline & & & & & & & & \\
\hline DIL OBT & $2.4 \mathrm{E}-09$ & & & & & & & \\
\hline $\mathrm{Ci} / \mathrm{g}$ & & & & & & & & \\
\hline
\end{tabular}




\begin{tabular}{|c|c|c|c|c|c|c|c|c|}
\hline & \multicolumn{7}{|c|}{ Tritium Facilities, Beyond Design Basis Fire, Average Met Conditions } & \\
\hline & \multicolumn{4}{|c|}{ Concentration Values for $1 \mathrm{~km}$ from release } & & & & \\
\hline \multicolumn{9}{|c|}{ Only Wheat Seed is examined since this is the edible portion of the Wheat. The "WHEAT" category is } \\
\hline \multicolumn{9}{|c|}{ ctually the unedible plant body and is not considered in the dose estimate. } \\
\hline & & & & & & & & \\
\hline & \multicolumn{2}{|c|}{ VEGETABLES } & \multicolumn{2}{|c|}{ WHEAT SEED } & \multicolumn{2}{|l|}{ MEAT } & \multicolumn{2}{|l|}{ MILK } \\
\hline \multirow[t]{3}{*}{ HOUR } & HTO & OBT & HTO & OBT & HTO & OBT & HTO & $\overline{\mathrm{OBT}}$ \\
\hline & $(\mathrm{Bq} / \mathrm{g})$ & $(\mathrm{Bq} / \mathrm{g})$ & $(\mathrm{Bq} / \mathrm{g})$ & $(\mathrm{Bq} / \mathrm{g})$ & $(B q / g)$ & 18 & $(\mathrm{~Bq} / \mathrm{g})$ & $3 a$ \\
\hline & & & & & & & & \\
\hline 1 & $3.66 \mathrm{E}+07$ & $1.97 \mathrm{E}+05$ & $1.32 \mathrm{E}+07$ & $4.36 \mathrm{E}+04$ & $9.90 \mathrm{E}+05$ & $2.03 E+02$ & $1.20 \mathrm{E}+05$ & $5.19 \mathrm{E}+04$ \\
\hline 2 & $1.31 \mathrm{E}+07$ & $2.59 \mathrm{E}+05$ & $1.87 \mathrm{E}+07$ & $7.44 \mathrm{E}+04$ & $1.42 \mathrm{E}+06$ & $4.97 \mathrm{E}+02$ & $2.59 \mathrm{E}+05$ & $1.13 \mathrm{E}+05$ \\
\hline 3 & $5.70 \mathrm{E}+06$ & $2.82 \mathrm{E}+05$ & $1.97 \mathrm{E}+07$ & $9.58 \mathrm{E}+04$ & $1.62 \mathrm{E}+06$ & $8.36 \mathrm{E}+02$ & $3.90 \mathrm{E}+05$ & $1.71 E+05$ \\
\hline 4 & $3.20 \mathrm{E}+06$ & $2.91 E+05$ & $1.84 \mathrm{E}+07$ & $1.11 \mathrm{E}+05$ & $1.72 \mathrm{E}+06$ & $1.20 \mathrm{E}+03$ & $5.06 \mathrm{E}+05$ & $2.22 \mathrm{E}+05$ \\
\hline 5 & $2.29 \mathrm{E}+06$ & $2.96 \mathrm{E}+05$ & $1.62 \mathrm{E}+07$ & $1.21 \mathrm{E}+05$ & $1.77 \mathrm{E}+06$ & $8+03$ & $6.07 \mathrm{E}+05$ & $2.66 \mathrm{E}+0$ \\
\hline 6 & $1.90 \mathrm{E}+06$ & $3.00 \mathrm{E}+05$ & $1.37 \mathrm{E}+07$ & $1.28 \mathrm{E}+05$ & $1.76 \mathrm{E}+06$ & $1.76 \mathrm{E}+03$ & $1.70 \bar{E}+06$ & $5.30 \mathrm{E}+0$ \\
\hline 7 & $1.71 \mathrm{E}+06$ & $3.03 E+05$ & $1.12 \mathrm{E}+07$ & $1.34 \mathrm{E}+05$ & $1.74 \mathrm{E}+06$ & $1.94 \mathrm{E}+03$ & $1.70 \mathrm{E}+06$ & $5.28 \mathrm{E}+05$ \\
\hline 8 & $1.60 \mathrm{E}+06$ & $3.05 \mathrm{E}+05$ & $9.09 \mathrm{E}+06$ & $1.37 \mathrm{E}+05$ & $1.73 \mathrm{E}+06$ & +03 & $1.69 \mathrm{E}+06$ & $5.26 \mathrm{E}+0$ \\
\hline 9 & $1.52 \mathrm{E}+06$ & $3.07 \mathrm{E}+05$ & $7.27 \mathrm{E}+06$ & $1.40 \mathrm{E}+05$ & $1.71 \mathrm{E}+06$ & $2.31 \mathrm{E}+03$ & $1.68 \mathrm{E}+06$ & $5.24 \mathrm{E}+05$ \\
\hline 10 & $1.45 \mathrm{E}+06$ & $3.10 \mathrm{E}+05$ & $5.79 \mathrm{E}+06$ & $1.42 \mathrm{E}+05$ & $1.70 \mathrm{E}+06$ & $2.49 \mathrm{E}+03$ & $1.67 \mathrm{E}+06$ & $5.22 \mathrm{E}+05$ \\
\hline 11 & $1.40 \mathrm{E}+06$ & $3.12 \mathrm{E}+05$ & $4.60 \mathrm{E}+06$ & $1.44 \mathrm{E}+05$ & $1.69 \mathrm{E}+06$ & $2.67 \mathrm{E}+03$ & $1.67 \mathrm{E}+06$ & $5.20 \mathrm{E}+05$ \\
\hline 12 & $1.35 \mathrm{E}+06$ & $3.14 \mathrm{E}+05$ & $3.66 \mathrm{E}+06$ & $1.45 \mathrm{E}+05$ & $1.67 \mathrm{E}+06$ & $2.85 \mathrm{E}+03$ & $1.66 \mathrm{E}+06$ & $5.18 \mathrm{E}+0$ \\
\hline 13 & $1.30 \mathrm{E}+06$ & $3.16 \mathrm{E}+05$ & $2.93 E+06$ & $1.46 \mathrm{E}+05$ & $1.66 \mathrm{E}+06$ & $2+03$ & $1.65 \mathrm{E}+06$ & $5.15 \mathrm{E}+05$ \\
\hline 14 & $1.26 \mathrm{E}+06$ & $3.17 \mathrm{E}+05$ & $2.36 \mathrm{E}+06$ & $1.47 \mathrm{E}+05$ & $1.64 \mathrm{E}+06$ & $3.20 \mathrm{E}+03$ & $1.65 \mathrm{E}+06$ & $5.13 \mathrm{E}+05$ \\
\hline 15 & $1.23 \mathrm{E}+06$ & $3.19 \mathrm{E}+05$ & $1.93 \mathrm{E}+06$ & $1.48 \mathrm{E}+05$ & $1.63 \mathrm{E}+06$ & +03 & $1.64 \mathrm{E}+06$ & $5.11 \mathrm{E}+05$ \\
\hline 16 & $1.18 \mathrm{E}+06$ & $3.21 \mathrm{E}+05$ & $1.60 \mathrm{E}+06$ & $1.49 \mathrm{E}+05$ & $1.62 \mathrm{E}+06$ & $3.55 \mathrm{E}+03$ & $1.63 \mathrm{E}+06$ & $5.09 \mathrm{E}+05$ \\
\hline 17 & $1.14 \mathrm{E}+06$ & $3.22 \mathrm{E}+05$ & $1.35 \mathrm{E}+06$ & $1.50 \mathrm{E}+05$ & $1.60 \mathrm{E}+06$ & $3.72 \mathrm{E}+03$ & $1.63 E+06$ & $5.07 \mathrm{E}+05$ \\
\hline 18 & $1.11 \mathrm{E}+06$ & $3.24 \mathrm{E}+05$ & $1.15 \mathrm{E}+06$ & $1.50 \mathrm{E}+05$ & $1.60 \mathrm{E}+06$ & $4.09 \mathrm{E}+03$ & $1.55 \mathrm{E}+06$ & $7.33 \mathrm{E}+05$ \\
\hline 19 & $1.08 \mathrm{E}+06$ & $3.25 \mathrm{E}+05$ & $1.00 \mathrm{E}+06$ & $1.51 \mathrm{E}+05$ & $1.60 \mathrm{E}+06$ & $4.47 \mathrm{E}+03$ & $1.55 \mathrm{E}+06$ & $7.33 \mathrm{E}+05$ \\
\hline 20 & $1.05 \mathrm{E}+06$ & $3.27 \mathrm{E}+05$ & $8.85 \mathrm{E}+05$ & $1.52 \mathrm{E}+05$ & $1.60 \mathrm{E}+06$ & $4.84 \mathrm{E}+03$ & $1.55 \mathrm{E}+06$ & $7.34 \mathrm{E}+05$ \\
\hline 21 & $1.03 \mathrm{E}+06$ & $3.28 \mathrm{E}+05$ & $7.95 \mathrm{E}+05$ & $1.52 \mathrm{E}+05$ & $1.60 \mathrm{E}+06$ & $5.22 \mathrm{E}+03$ & $1.55 \mathrm{E}+06$ & $7.34 \mathrm{E}+05$ \\
\hline 22 & $9.98 \mathrm{E}+05$ & $3.29 \mathrm{E}+05$ & $7.24 \mathrm{E}+05$ & $1.53 \mathrm{E}+05$ & $1.60 \mathrm{E}+06$ & $5.59 \mathrm{E}+03$ & $1.55 \mathrm{E}+06$ & $7.34 \mathrm{E}+0$ \\
\hline 23 & $9.73 \mathrm{E}+05$ & $3.31 \mathrm{E}+05$ & $6.67 \mathrm{E}+05$ & $1.53 \mathrm{E}+05$ & $1.60 \mathrm{E}+06$ & $5.97 \mathrm{E}+03$ & $1.55 \mathrm{E}+06$ & $7.34 \mathrm{E}+05$ \\
\hline 24 & $9.48 \mathrm{E}+05$ & $3.32 \mathrm{E}+05$ & $6.22 \mathrm{E}+05$ & $1.54 \mathrm{E}+05$ & $1.59 \mathrm{E}+06$ & $6.34 \mathrm{E}+03$ & $1.55 \mathrm{E}+06$ & $7.34 \mathrm{E}+05$ \\
\hline 25 & $9.25 \mathrm{E}+05$ & $3.33 \mathrm{E}+05$ & $5.84 \mathrm{E}+05$ & $1.54 \mathrm{E}+05$ & $1.59 \mathrm{E}+06$ & $6.72 \mathrm{E}+03$ & $1.55 \mathrm{E}+06$ & $7 . \overline{34 \mathrm{E}+0}$ \\
\hline 26 & $9.03 \mathrm{E}+05$ & $3.34 \mathrm{E}+05$ & $5.53 \mathrm{E}+05$ & $1.55 \mathrm{E}+05$ & $1.59 \mathrm{E}+06$ & $7.09 \mathrm{E}+03$ & $1.55 \mathrm{E}+06$ & $7.34 \mathrm{E}+05$ \\
\hline 27 & $8.81 E+05$ & $3.35 \mathrm{E}+05$ & $5.26 \mathrm{E}+05$ & $1.55 \mathrm{E}+05$ & $1.58 \mathrm{E}+06$ & $7.47 \mathrm{E}+03$ & $1.55 \mathrm{E}+06$ & $7.34 \mathrm{E}+05$ \\
\hline 28 & $8.60 \mathrm{E}+05$ & $3.36 \mathrm{E}+05$ & $5.03 \mathrm{E}+05$ & $1.55 \mathrm{E}+05$ & $1.58 \mathrm{E}+06$ & $7.84 \mathrm{E}+03$ & $1.55 \mathrm{E}+06$ & $7.34 \mathrm{E}+05$ \\
\hline 29 & $8.40 \mathrm{E}+05$ & $3.37 \mathrm{E}+05$ & $4.83 \mathrm{E}+05$ & $1.56 \mathrm{E}+05$ & $1.58 \mathrm{E}+06$ & $8.21 \mathrm{E}+03$ & $1.54 \mathrm{E}+06$ & $7.34 \mathrm{E}+05$ \\
\hline 30 & $8.21 \mathrm{E}+05$ & $3.38 \mathrm{E}+05$ & $4.64 \mathrm{E}+05$ & $1.56 \mathrm{E}+05$ & $1.56 \mathrm{E}+06$ & $8.38 \mathrm{E}+03$ & $1.51 \mathrm{E}+06$ & $4.73 \mathrm{E}+05$ \\
\hline 31 & $8.03 E+05$ & $3.39 \mathrm{E}+05$ & $4.48 \mathrm{E}+05$ & $1.57 \mathrm{E}+05$ & $1.55 \mathrm{E}+06$ & $8.54 \mathrm{E}+03$ & $1.51 \mathrm{E}+06$ & $4.71 \mathrm{E}+05$ \\
\hline 32 & $7.86 \mathrm{E}+05$ & $3.40 \mathrm{E}+05$ & $4.33 \mathrm{E}+05$ & $1.57 \mathrm{E}+05$ & $1.54 \mathrm{E}+06$ & $8.70 \mathrm{E}+03$ & $1.50 \mathrm{E}+06$ & $4.69 \mathrm{E}+05$ \\
\hline 33 & $7.69 \mathrm{E}+05$ & $3.41 \mathrm{E}+05$ & $4.18 \mathrm{E}+05$ & $1.57 \mathrm{E}+05$ & $1.52 \mathrm{E}+06$ & $8.85 \mathrm{E}+03$ & $1.50 \mathrm{E}+06$ & $4.67 \mathrm{E}+05$ \\
\hline 34 & $7.32 \mathrm{E}+05$ & $3.42 \mathrm{E}+05$ & $4.05 \mathrm{E}+05$ & $1.58 \mathrm{E}+05$ & $1.51 \mathrm{E}+06$ & $9.01 \mathrm{E}+03$ & $1.49 \mathrm{E}+06$ & $4.66 \mathrm{E}+05$ \\
\hline 35 & $7.11 \mathrm{E}+05$ & $3.43 \mathrm{E}+05$ & $3.90 \mathrm{E}+05$ & $1.58 \mathrm{E}+05$ & $1.50 \mathrm{E}+06$ & $9.17 \mathrm{E}+03$ & $1.48 \mathrm{E}+06$ & $4.64 \mathrm{E}+05$ \\
\hline 36 & $6.95 \mathrm{E}+05$ & $3.44 \mathrm{E}+05$ & $3.76 \mathrm{E}+05$ & $1.58 \mathrm{E}+05$ & $1.49 \mathrm{E}+06$ & $9.32 \mathrm{E}+03$ & $1.48 \mathrm{E}+06$ & $4.62 \mathrm{E}+05$ \\
\hline 37 & $6.81 \mathrm{E}+05$ & $3.44 \mathrm{E}+05$ & $3.61 \mathrm{E}+05$ & $1.59 \mathrm{E}+05$ & $1.47 \mathrm{E}+06$ & $9.47 \mathrm{E}+03$ & $1.47 \mathrm{E}+06$ & $4.60 \mathrm{E}+05$ \\
\hline 38 & $6.69 \mathrm{E}+05$ & $3.45 \mathrm{E}+05$ & $3.48 \mathrm{E}+05$ & $1.59 \mathrm{E}+05$ & $1.46 \mathrm{E}+06$ & $9.62 \mathrm{E}+03$ & $1.47 \mathrm{E}+06$ & $4.58 \mathrm{E}+\overline{05}$ \\
\hline
\end{tabular}




\begin{tabular}{|c|c|c|c|c|c|c|c|c|}
\hline 39 & $6.57 \mathrm{E}+05$ & $3.46 \mathrm{E}+05$ & $3.35 \mathrm{E}+05$ & $1.59 \mathrm{E}+05$ & $1.45 \mathrm{E}+06$ & $9.77 \mathrm{E}+03$ & $1.46 \mathrm{E}+06$ & $4.56 \mathrm{E}+0$ \\
\hline 40 & $6.45 \mathrm{E}+05$ & $3.46 \mathrm{E}+05$ & $3.24 \mathrm{E}+05$ & $1.60 \mathrm{E}+05$ & $1.44 \mathrm{E}+06$ & $9.92 \mathrm{E}+03$ & $1.45 \mathrm{E}+06$ & $4.54 \mathrm{E}+0$ \\
\hline 41 & $6.34 \mathrm{E}+05$ & $3.47 \mathrm{E}+05$ & $3.14 \mathrm{E}+05$ & $1.60 \mathrm{E}+05$ & $1.43 \mathrm{E}+06$ & $1.01 \mathrm{E}+04$ & $1.45 \mathrm{E}+06$ & $4.52 \mathrm{E}+05$ \\
\hline 42 & $6.24 \mathrm{E}+05$ & $3.48 \mathrm{E}+05$ & $3.04 \mathrm{E}+05$ & $1.60 \mathrm{E}+05$ & $1.42 \mathrm{E}+06$ & $1.04 \mathrm{E}+04$ & $1.38 \mathrm{E}+06$ & $6.93 \mathrm{E}+05$ \\
\hline 43 & $.14 \mathrm{E}+05$ & $3.48 \mathrm{E}+05$ & $2.95 \mathrm{E}+05$ & $1.60 \mathrm{E}+05$ & $1.42 \mathrm{E}+06$ & $1.08 \mathrm{E}+04$ & $1.37 \mathrm{E}+06$ & $6.92 \mathrm{E}+0$ \\
\hline 44 & $6.05 \mathrm{E}+05$ & $3.49 \mathrm{E}+05$ & $2.87 \mathrm{E}+05$ & $1.61 \mathrm{E}+05$ & $1.41 \mathrm{E}+06$ & $1.12 \mathrm{E}+04$ & $1.37 \mathrm{E}+06$ & $6.92 \mathrm{E}+05$ \\
\hline 45 & $5.96 \mathrm{E}+05$ & $3.50 \mathrm{E}+05$ & $2.80 \mathrm{E}+05$ & $1.61 \mathrm{E}+05$ & $1.41 \mathrm{E}+06$ & $1.15 \mathrm{E}+04$ & $1.37 \mathrm{E}+06$ & $6.91 \mathrm{E}+0$ \\
\hline 46 & $5.89 \mathrm{E}+05$ & $3.50 \mathrm{E}+05$ & $2.73 \mathrm{E}+05$ & $1.61 \mathrm{E}+05$ & $1.40 \mathrm{E}+06$ & $1.19 \mathrm{E}+04$ & $1.37 \mathrm{E}+06$ & $6.90 \mathrm{E}+0$ \\
\hline 47 & $5.72 \mathrm{E}+05$ & $3.51 \mathrm{E}+05$ & $2.66 \mathrm{E}+05$ & $1.61 \mathrm{E}+05$ & $1.40 \mathrm{E}+06$ & $1.22 \mathrm{E}+04$ & $1.37 \mathrm{E}+06$ & $6.89 \mathrm{E}+0$ \\
\hline 48 & $5.62 \mathrm{E}+05$ & $3.51 \mathrm{E}+05$ & $2.59 \mathrm{E}+05$ & $1.62 \mathrm{E}+05$ & $1.39 \mathrm{E}+06$ & $1.26 \mathrm{E}+04$ & $1.36 \mathrm{E}+06$ & $6.89 \mathrm{E}+0$ \\
\hline 49 & $5.55 \mathrm{E}+05$ & $3.52 \mathrm{E}+05$ & $2.52 \mathrm{E}+05$ & $1.62 \mathrm{E}+05$ & $1.38 \mathrm{E}+06$ & $1.29 \mathrm{E}+04$ & $1.36 \mathrm{E}+06$ & $6.88 \mathrm{E}+0$ \\
\hline 50 & $5.49 \mathrm{E}+05$ & $3.52 \mathrm{E}+05$ & $2.45 \mathrm{E}+05$ & $1.62 \mathrm{E}+05$ & $1.38 \mathrm{E}+06$ & $1.33 \mathrm{E}+04$ & $1.36 \mathrm{E}+06$ & $6.87 \mathrm{E}+$ \\
\hline 51 & $5.44 \mathrm{E}+05$ & $3.53 \mathrm{E}+05$ & $2.39 \mathrm{E}+05$ & $1.62 \mathrm{E}+05$ & $1.37 \mathrm{E}+06$ & $1.37 \mathrm{E}+04$ & $1.35 \mathrm{E}+06$ & $6.86 \mathrm{E}+0$ \\
\hline 52 & $5.39 \mathrm{E}+05$ & $3.53 \mathrm{E}+05$ & $2.34 \mathrm{E}+05$ & $1.63 \mathrm{E}+05$ & $1.37 \mathrm{E}+06$ & $1.40 \mathrm{E}+04$ & $1.35 \mathrm{E}+06$ & $6.85 \mathrm{E}+0$ \\
\hline 53 & $5.34 \mathrm{E}+05$ & $3.53 \mathrm{E}+05$ & $2.29 \mathrm{E}+05$ & $1.63 \mathrm{E}+05$ & $1.36 \mathrm{E}+06$ & $1.44 \mathrm{E}+04$ & $1.35 \mathrm{E}+06$ & $6.85 \mathrm{E}+$ \\
\hline 54 & $5.29 \mathrm{E}+05$ & $3.54 \mathrm{E}+05$ & $2.24 \mathrm{E}+05$ & $1.63 \mathrm{E}+05$ & $1.35 \mathrm{E}+06$ & $1.45 \mathrm{E}+04$ & $1.31 \mathrm{E}+06$ & $4.11 \mathrm{E}+0$ \\
\hline 55 & $5.24 \mathrm{E}+05$ & $3.54 \mathrm{E}+05$ & $2.20 \mathrm{E}+05$ & $1.63 \mathrm{E}+05$ & $1.34 \mathrm{E}+06$ & $1.46 \mathrm{E}+04$ & $1.30 \mathrm{E}+06$ & $4.09 \mathrm{E}+0$ \\
\hline 56 & $5.20 \mathrm{E}+05$ & $3.55 \mathrm{E}+05$ & $2.17 \mathrm{E}+05$ & $1.63 \mathrm{E}+05$ & $1.33 \mathrm{E}+06$ & $1.48 \mathrm{E}+04$ & $1.30 \mathrm{E}+06$ & $4.07 \mathrm{E}+0$ \\
\hline 57 & $4.94 \mathrm{E}+05$ & $3.55 \mathrm{E}+05$ & $2.13 \mathrm{E}+05$ & $1.64 \mathrm{E}+05$ & $1.32 \mathrm{E}+06$ & $1.49 \mathrm{E}+04$ & $1.29 \mathrm{E}+06$ & $4.06 \mathrm{E}+0$ \\
\hline 58 & $4.84 \mathrm{E}+05$ & $3.56 \mathrm{E}+05$ & $2.08 \mathrm{E}+05$ & $1.64 \mathrm{E}+05$ & $1.31 \mathrm{E}+06$ & $1.50 \mathrm{E}+04$ & $1.29 \mathrm{E}+06$ & $4.04 \mathrm{E}+0$ \\
\hline 59 & $4.80 \mathrm{E}+05$ & $3.56 \mathrm{E}+05$ & $2.02 \mathrm{E}+05$ & $1.64 \mathrm{E}+05$ & $1.30 \mathrm{E}+06$ & $1.51 \mathrm{E}+04$ & $1.28 \mathrm{E}+06$ & $4.02 \mathrm{E} \div 0$ \\
\hline 60 & $4.77 \mathrm{E}+05$ & $3.56 \mathrm{E}+05$ & $1.96 \mathrm{E}+05$ & $1.64 \mathrm{E}+05$ & $1.29 \mathrm{E}+06$ & $1.53 \mathrm{E}+04$ & $1.28 \mathrm{E}+06$ & $4.01 \mathrm{E}+0$ \\
\hline 61 & $4.74 \mathrm{E}+05$ & $3.57 \mathrm{E}+05$ & $1.90 \mathrm{E}+05$ & $1.64 \mathrm{E}+05$ & $1.27 \mathrm{E}+06$ & $1.54 \mathrm{E}+04$ & $1.27 \mathrm{E}+06$ & $3.99 \mathrm{E}+0$ \\
\hline 62 & $4.72 E+05$ & $3.57 \mathrm{E}+05$ & $1.86 \mathrm{E}+05$ & $1.64 \mathrm{E}+05$ & $1.26 \mathrm{E}+06$ & $1.55 \mathrm{E}+04$ & $1.27 \mathrm{E}+06$ & $3.97 \mathrm{E}+05$ \\
\hline 63 & $4.69 \mathrm{E}+05$ & $3.57 \mathrm{E}+05$ & $1.82 \mathrm{E}+05$ & $1.65 \mathrm{E}+05$ & $1.25 \mathrm{E}+06$ & $1.57 \mathrm{E}+04$ & $1.26 \mathrm{E}+06$ & $3.96 \mathrm{E}+0$ \\
\hline 64 & $4.67 \mathrm{E}+05$ & $3.58 \mathrm{E}+05$ & $1.78 \mathrm{E}+05$ & $1.65 \mathrm{E}+05$ & $1.24 \mathrm{E}+06$ & $1.58 \mathrm{E}+04$ & $1.26 \mathrm{E}+06$ & $3.94 \mathrm{E}+05$ \\
\hline 65 & $4.65 \mathrm{E}+05$ & $3.58 \mathrm{E}+05$ & $1.76 \mathrm{E}+05$ & $1.65 \mathrm{E}+05$ & $1.23 \mathrm{E}+06$ & $1.59 \mathrm{E}+04$ & $1.25 \mathrm{E}+06$ & $3.92 \mathrm{E}+0$ \\
\hline 66 & $4.63 \mathrm{E}+05$ & $3.58 \mathrm{E}+05$ & $1.73 \mathrm{E}+05$ & $1.65 \mathrm{E}+05$ & $1.23 \mathrm{E}+06$ & $1.62 \mathrm{E}+04$ & $1.19 \mathrm{E}+06$ & $6.38 \mathrm{E}+05$ \\
\hline 67 & $4.61 \mathrm{E}+05$ & $3.59 \mathrm{E}+05$ & $1.71 \mathrm{E}+05$ & $1.65 \mathrm{E}+05$ & $1.22 \mathrm{E}+06$ & $1.66 \mathrm{E}+04$ & $1.19 \mathrm{E}+06$ & $6.38 \mathrm{E}+0$ \\
\hline 68 & $4.59 \mathrm{E}+05$ & $3.59 \mathrm{E}+05$ & $1.70 \mathrm{E}+05$ & $1.65 \mathrm{E}+05$ & $1.22 \mathrm{E}+06$ & $1.69 \mathrm{E}+04$ & $1.18 \mathrm{E}+06$ & $6.37 \mathrm{E}+0$. \\
\hline 69 & $4.57 \mathrm{E}+05$ & $3.59 \mathrm{E}+05$ & $1.68 \mathrm{E}+05$ & $1.66 \mathrm{E}+05$ & $1.21 \mathrm{E}+06$ & $1.72 \mathrm{E}+04$ & $1.18 \mathrm{E}+06$ & $6.36 \mathrm{E}+05$ \\
\hline 168 & $2.11 \mathrm{E}+05$ & $3.60 \mathrm{E}+05$ & $2.08 \mathrm{E}+05$ & $1.80 \mathrm{E}+05$ & $6.91 \mathrm{E}+05$ & $4.14 \mathrm{E}+04$ & $7.60 \mathrm{E}+05$ & $4.98 \mathrm{E}+05$ \\
\hline 336 & $1.11 \mathrm{E}+05$ & $3.07 \mathrm{E}+05$ & $1.10 \mathrm{E}+05$ & $1.96 \mathrm{E}+05$ & $2.93 \mathrm{E}+05$ & $7.36 \mathrm{E}+04$ & $4.48 \mathrm{E}+05$ & $3.74 \mathrm{E}+05$ \\
\hline 504 & $8.21 \mathrm{E}+04$ & $2.58 \mathrm{E}+05$ & $7.59 \mathrm{E}+04$ & $2.06 \mathrm{E}+05$ & $1.45 \mathrm{E}+05$ & $9.12 \mathrm{E}+04$ & $2.02 \mathrm{E}+05$ & $2.52 \mathrm{E}+05$ \\
\hline 720 & $6.00 \mathrm{E}+04$ & $2.05 \mathrm{E}+05$ & $5.10 \mathrm{E}+04$ & $2.14 \mathrm{E}+05$ & $7.30 \mathrm{E}+04$ & $1.01 \mathrm{E}+05$ & $1.00 \mathrm{E}+05$ & $1.76 \mathrm{E}+05$ \\
\hline 2160 & $1.13 E+04$ & $4.27 \mathrm{E}+04$ & $5.76 \mathrm{E}+03$ & $2.31 \mathrm{E}+05$ & $6.48 \mathrm{E}+03$ & $5.98 \mathrm{E}+04$ & $2.26 \mathrm{E}+04$ & $6.12 \overline{\mathrm{E}+04}$ \\
\hline 4320 & $1.09 E+03$ & $4.03 E+03$ & $2.90 \mathrm{E}+04$ & $2.18 \mathrm{E}+05$ & $1.14 \mathrm{E}+03$ & $1.44 \mathrm{E}+04$ & $2.82 \mathrm{E}+03$ & $9.77 \mathrm{E}+03$ \\
\hline 4800 & $6.47 \mathrm{E}+02$ & $2.39 \mathrm{E}+03$ & $2.89 \mathrm{E}+04$ & $2.17 \mathrm{E}+05$ & $8.29 \mathrm{E}+02$ & $1.04 \mathrm{E}+04$ & $9.72 \mathrm{E}+02$ & $3.25 \mathrm{E}+03$ \\
\hline 8760 & $8.89 \mathrm{E}+00$ & $3.22 \mathrm{E}+01$ & $2.81 \mathrm{E}+04$ & $2.12 \mathrm{E}+05$ & $7.02 \mathrm{E}+01$ & $7.11 \mathrm{E}+02$ & $3.07 \mathrm{E}+02$ & $9.67 \mathrm{E}+02$ \\
\hline$\overline{\mathrm{BT}}$ & $\begin{array}{l}2.4 \mathrm{E} 6 \\
\mathrm{pCi} / \mathrm{L}\end{array}$ & $\begin{array}{l}8.88 \mathrm{E}+4 \\
\mathrm{~Bq} / \mathrm{kg}\end{array}$ & $8 \mathrm{~Bq} / \mathrm{g}$ & & & & & \\
\hline $\mathbf{O}$ & $\begin{array}{l}5.9 \mathrm{E} 6 \\
\mathrm{pCi} / \mathrm{L}\end{array}$ & $\begin{array}{l}2.18 \mathrm{E}+5 \\
\mathrm{~Bq} / \mathrm{kg}\end{array}$ & $218.3 \mathrm{~Bq} / \mathrm{g}$ & & & & & \\
\hline & & & & & & & & \\
\hline & \multicolumn{2}{|c|}{ VEGETABLES } & \multicolumn{2}{|c|}{ WHEAT SEED } & \multicolumn{2}{|l|}{ MEĀT } & \multicolumn{2}{|l|}{ MILK } \\
\hline \multirow[t]{2}{*}{ HOUR } & HTO & $\overline{\mathrm{OBT}}$ & HTO & OBT & HTO & $\overline{\text { OBT }}$ & HTO & $\overline{\mathrm{OBT}}$ \\
\hline & $(\mathrm{Bq} / \mathrm{g})$ & $(\mathrm{Bq} / \mathrm{g})$ & $(\mathrm{Bq} / \mathrm{g})$ & $(\mathrm{Bq} / \mathrm{g})$ & $(\mathrm{Bq} / \mathrm{g})$ & $(\mathrm{Bq} / \mathrm{g})$ & $(\mathrm{Bq} / \mathrm{g})$ & $(\mathrm{Bq} / \mathrm{g})$ \\
\hline ak HTO & & & & & & & & \\
\hline
\end{tabular}




\begin{tabular}{|c|c|c|c|c|c|c|c|c|}
\hline $1 / 3 / 6 / 6$ & $3.66 \mathrm{E}+07$ & $1.97 \mathrm{E}+05$ & $1.97 \bar{E}+07$ & $9.58 \mathrm{E}+04$ & $1.76 \mathrm{E}+06$ & $1.76 \mathrm{E}+03$ & $1.70 \mathrm{E}+06$ & $5.30 \mathrm{E}+05$ \\
\hline SUM & $3.68 \mathrm{E}+07$ & & $1.98 \mathrm{E}+07$ & & $1.76 \mathrm{E}+06$ & & $2.23 \mathrm{E}+06$ & \\
\hline $\mathrm{Ci} / \mathrm{g}$ & $9.95 E-04$ & & $5.35 E-04$ & & $4.75 \mathrm{E}-05$ & & $6.03 \mathrm{E}-05$ & \\
\hline & & & & & & & & \\
\hline DIL HTO & $5.9 \mathrm{E}-09$ & & & & & & & \\
\hline \multicolumn{9}{|l|}{$(\mathrm{Ci} / \mathrm{g})$} \\
\hline & & & & & & & & \\
\hline 168 & $2.11 \mathrm{E}+05$ & $3.60 \mathrm{E}+05$ & $2.08 \mathrm{E}+05$ & $1.80 \mathrm{E}+05$ & $6.91 \mathrm{E}+05$ & $4.14 \mathrm{E}+04$ & $7.60 \mathrm{E}+05$ & $4.98 \mathrm{E}+05$ \\
\hline SUM & $5.71 \mathrm{E}+05$ & & $3.88 \mathrm{E}+05$ & & $7.32 \mathrm{E}+05$ & & $1.26 \mathrm{E}+06$ & \\
\hline $\mathrm{Ci} / \mathrm{g}$ & $1.54 \mathrm{E}-05$ & & $1.05 E-05$ & & $1.98 \mathrm{E}-05$ & & $3.40 \mathrm{E}-05$ & \\
\hline & & & & & & & & \\
\hline DIL OBT & $2.4 \mathrm{E}-09$ & & & & & & & \\
\hline \multicolumn{9}{|l|}{$\mathrm{Ci} / \mathrm{g}$} \\
\hline & & & & & & & & \\
\hline 8760 & $8.89 \mathrm{E}+00$ & $3.22 \mathrm{E}+01$ & $2.81 \mathrm{E}+04$ & $2.12 \mathrm{E}+05$ & $7.02 \mathrm{E}+01$ & $7.11 \mathrm{E}+02$ & $3.07 \mathrm{E}+02$ & $9.67 \mathrm{E}+02$ \\
\hline SUM & $4.11 \mathrm{E}+01$ & & $2.40 \mathrm{E}+05$ & & $7.81 \mathrm{E}+02$ & & $1.27 \mathrm{E}+03$ & \\
\hline $\mathrm{Ci} / \mathrm{g}$ & $1.11 \mathrm{E}-09$ & & $6.48 \mathrm{E}-06$ & & $2.11 \mathrm{E}-08$ & & $3.44 \mathrm{E}-08$ & \\
\hline & & & & & & & & \\
\hline DIL OBT & $2.4 \mathrm{E}-09$ & & & & & & & \\
\hline $\mathrm{Ci} / \mathrm{g}$ & & & & & & & & \\
\hline & & & & & & & & \\
\hline
\end{tabular}




\begin{tabular}{|c|c|c|c|c|c|c|c|c|}
\hline & \multicolumn{7}{|c|}{ Tritium Facilities, Beyond Design Basis Fire, Average Met Conditions } & \\
\hline & \multicolumn{4}{|c|}{ Concentration Values for $10 \mathrm{~km}$ from release } & & & & \\
\hline \multicolumn{9}{|c|}{ Only Wheat Seed is examined since this is the edible portion of the Wheat. The "WHEAT" category is } \\
\hline \multicolumn{9}{|c|}{ ctually the unedible plant body and is not considered in the dose estimate. } \\
\hline & & & & & & & & \\
\hline & \multicolumn{2}{|c|}{ VEGETABLES } & \multicolumn{2}{|c|}{ WHEAT SEED } & \multicolumn{2}{|l|}{ MEAT } & \multicolumn{2}{|l|}{ MILK } \\
\hline \multirow[t]{3}{*}{ HOUR } & HTO & OBT & HTO & OBT & HTO & OBT & HTO & TH \\
\hline & $(\mathrm{Bq} / \mathrm{g})$ & $(\mathrm{Bq} / \mathrm{g})$ & $(\mathrm{Bq} / \mathrm{g})$ & $(\mathrm{Bq} / \mathrm{g})$ & $(\mathrm{Bq} / \mathrm{g})$ & $(\mathrm{Bq} / \mathrm{g})$ & $(\mathrm{Bq} / \mathrm{g})$ & $(\mathrm{Bq} / \mathrm{g})$ \\
\hline & & & & & & & & \\
\hline 1 & $1.96 \mathrm{E}+06$ & $8.43 E+03$ & $5.65 \mathrm{E}+05$ & $1.87 \mathrm{E}+03$ & $4.27 \mathrm{E}+04$ & $8.71 \mathrm{E}+00$ & $5.18 \mathrm{E}+03$ & $2.24 \mathrm{E}+03$ \\
\hline 2 & $1.26 \mathrm{E}+06$ & $1.39 \mathrm{E}+04$ & $9.83 \mathrm{E}+05$ & $3.79 \mathrm{E}+03$ & $7.48 \mathrm{E}+04$ & $2.42 \mathrm{E}+01$ & $1.27 \mathrm{E}+04$ & $5.50 \mathrm{E}+0$ \\
\hline 3 & $5.96 \mathrm{E}+05$ & $1.60 \mathrm{E}+04$ & $1.12 \bar{E}+06$ & $5.20 \mathrm{E}+03$ & $9.11 \mathrm{E}+04$ & $4.32 \mathrm{E}+01$ & $2.02 \mathrm{E}+04$ & $8.82 \mathrm{E}+0$ \\
\hline 4 & $3.53 \mathrm{E}+05$ & $1.70 \mathrm{E}+04$ & $1.10 \mathrm{E}+06$ & $6.22 \mathrm{E}+03$ & $9.98 \mathrm{E}+04$ & $6.41 \mathrm{E}+01$ & $2.72 \mathrm{E}+04$ & $1.19 \mathrm{E}+04$ \\
\hline 5 & $2.54 \mathrm{E}+05$ & $1.76 \mathrm{E}+04$ & $1.00 \mathrm{E}+06$ & $6.96 \mathrm{E}+03$ & $1.05 \mathrm{E}+05$ & $8.62 \mathrm{E}+01$ & $3.34 \mathrm{E}+04$ & $1.46 \mathrm{E}+0$ \\
\hline 6 & $2.08 \mathrm{E}+05$ & $1.80 \mathrm{E}+04$ & $8.74 \bar{E}+05$ & $7.50 \mathrm{E}+03$ & $1.04 \mathrm{E}+05$ & $9.73 \mathrm{E}+01$ & $1.01 \mathrm{E}+05$ & $3.14 \mathrm{E}+04$ \\
\hline 7 & $1.84 \mathrm{E}+05$ & $1.84 \mathrm{E}+04$ & $7.40 \mathrm{E}+05$ & $7.90 \mathrm{E}+03$ & $1.03 \mathrm{E}+05$ & $1.08 \mathrm{E}+02$ & $1.00 \mathrm{E}+05$ & $3.13 \mathrm{E}+04$ \\
\hline 8 & $1.68 E+05$ & $1.87 \mathrm{E}+04$ & $6.16 \mathrm{E}+05$ & $8.21 \mathrm{E}+03$ & $1.02 \mathrm{E}+05$ & $1.19 \mathrm{E}+02$ & $1.00 \mathrm{E}+05$ & $3.11 E+04$ \\
\hline 9 & $1.58 \mathrm{E}+05$ & $1.89 \mathrm{E}+04$ & $5.08 \bar{E}+05$ & $8.44 \mathrm{E}+03$ & $1.02 \mathrm{E}+05$ & $1.30 \mathrm{E}+02$ & $9.96 \mathrm{E}+04$ & $3.10 \mathrm{E}+04$ \\
\hline 10 & $1.49 \mathrm{E}+05$ & $1.92 \mathrm{E}+04$ & $4.17 \mathrm{E}+05$ & $8.64 E+03$ & $1.01 \mathrm{E}+05$ & $1.41 \mathrm{E}+02$ & $9.92 \mathrm{E}+04$ & $3.09 \mathrm{E}+04$ \\
\hline 11 & $1.43 \mathrm{E}+05$ & $1.94 \mathrm{E}+04$ & $3.43 \mathrm{E}+05$ & $8.79 \mathrm{E}+03$ & $1.00 \mathrm{E}+05$ & $1.52 \mathrm{E}+02$ & $9.88 \mathrm{E}+04$ & $3.08 \mathrm{E}+0$ \\
\hline 12 & $1.37 \mathrm{E}+05$ & $1.96 \mathrm{E}+04$ & $2.83 \mathrm{E}+05$ & $8.93 \mathrm{E}+03$ & $9.92 \mathrm{E}+04$ & $1.62 \mathrm{E}+02$ & $9.84 \mathrm{E}+04$ & $3.07 \mathrm{E}+\mathrm{C}$ \\
\hline 13 & $1.32 \mathrm{E}+05$ & $1.98 \mathrm{E}+04$ & $2.36 \overline{\mathrm{E}}+05$ & $9.04 \mathrm{E}+03$ & $9.84 \mathrm{E}+04$ & $1.73 \mathrm{E}+02$ & $9.81 \mathrm{E}+04$ & $3.06 \mathrm{E}+04$ \\
\hline 14 & $1.28 \mathrm{E}+05$ & $2.00 \mathrm{E}+04$ & $1.98 \bar{E}+05$ & $9.15 \mathrm{E}+03$ & $9.76 \mathrm{E}+04$ & $1.83 \mathrm{E}+02$ & $9.77 \mathrm{E}+04$ & $3.04 \mathrm{E}+04$ \\
\hline 15 & $1.23 \mathrm{E}+05$ & $2.02 E+04$ & $1.69 \bar{E}+05$ & $9.24 \mathrm{E}+03$ & $9.69 \mathrm{E}+04$ & $1.93 \mathrm{E}+02$ & $9.73 E+04$ & $3.03 \mathrm{E}+0$ \\
\hline 16 & $1.19 \mathrm{E}+05$ & $2.04 \mathrm{E}+04$ & $1.46 \mathrm{E}+05$ & $9.33 \mathrm{E}+03$ & $9.61 \mathrm{E}+04$ & $2.03 \mathrm{E}+02$ & $9.69 \mathrm{E}+04$ & $3.02 \mathrm{E}+04$ \\
\hline 17 & $1.15 \mathrm{E}+05$ & $2.06 \mathrm{E}+04$ & $1.28 \mathrm{E}+05$ & $9.41 \mathrm{E}+03$ & $9.53 \mathrm{E}+04$ & $2.14 \mathrm{E}+02$ & $9.65 \mathrm{E}+04$ & $3.01 \mathrm{E}+0$ \\
\hline 18 & $1.12 \mathrm{E}+05$ & $2.07 \mathrm{E}+04$ & $1.14 \mathrm{E}+05$ & $9.49 \mathrm{E}+03$ & $9.58 \mathrm{E}+04$ & $2.37 \mathrm{E}+02$ & $9.29 \mathrm{E}+04$ & $4.49 \mathrm{E}+0$ \\
\hline 19 & $1.08 \mathrm{E}+05$ & $2.09 \mathrm{E}+04$ & $1.03 \mathrm{E}+05$ & $9.56 \mathrm{E}+03$ & $9.62 \mathrm{E}+04$ & $2.60 \mathrm{E}+02$ & $9.31 \mathrm{E}+04$ & $4.50 \mathrm{E}+0$ \\
\hline 20 & $1.05 \mathrm{E}+05$ & $2.11 \mathrm{E}+04$ & $9.38 \mathrm{E}+04$ & $9.63 \mathrm{E}+03$ & $9.66 \mathrm{E}+04$ & $2.84 \mathrm{E}+02$ & $9.33 E+04$ & $4.51 \mathrm{E}+0$ \\
\hline 21 & $1.02 \mathrm{E}+05$ & $2.12 \mathrm{E}+04$ & $8.65 \mathrm{E}+04$ & $9.70 \mathrm{E}+03$ & $9.70 \mathrm{E}+04$ & $3.07 \mathrm{E}+02$ & $9.34 \mathrm{E}+04$ & $4.52 \mathrm{E}+0$ \\
\hline 22 & $9.92 \mathrm{E}+04$ & $2.14 \mathrm{E}+04$ & $8.06 \mathrm{E}+04$ & $9.76 \mathrm{E}+03$ & $9.73 \mathrm{E}+04$ & $3.31 \mathrm{E}+02$ & $9.36 \mathrm{E}+04$ & $4.53 \mathrm{E}+0$ \\
\hline 23 & $9.64 \mathrm{E}+04$ & $2.15 \mathrm{E}+04$ & $7.57 \mathrm{E}+04$ & $9.82 \mathrm{E}+03$ & $9.76 \mathrm{E}+04$ & $3.55 \mathrm{E}+02$ & $9.38 \mathrm{E}+04$ & $4.54 \mathrm{E}+0$ \\
\hline 24 & $9.36 \mathrm{E}+04$ & $2.16 \mathrm{E}+04$ & $7.15 \mathrm{E}+04$ & $9.88 \mathrm{E}+03$ & $9.78 \mathrm{E}+04$ & $3.79 \mathrm{E}+02$ & $9.39 \mathrm{E}+04$ & $4.55 \mathrm{E}+0$ \\
\hline 25 & $9.10 \mathrm{E}+04$ & $2.18 \mathrm{E}+04$ & $6.79 E+04$ & $9.94 \mathrm{E}+03$ & $9.80 \mathrm{E}+04$ & $4.02 \mathrm{E}+02$ & $9.40 \mathrm{E}+04$ & $4.56 \mathrm{E}+0$ \\
\hline 26 & $8.85 \mathrm{E}+04$ & $2.19 \mathrm{E}+04$ & $6.47 \mathrm{E}+04$ & $9.99 \mathrm{E}+03$ & $9.82 \mathrm{E}+04$ & $4.26 \mathrm{E}+02$ & $9.42 \mathrm{E}+04$ & $4.57 \mathrm{E}+0$ \\
\hline 27 & $8.60 \mathrm{E}+04$ & $2.20 \mathrm{E}+04$ & $6.19 \mathrm{E}+04$ & $1.01 \mathrm{E}+04$ & $9.83 \mathrm{E}+04$ & $4.50 \mathrm{E}+02$ & $9.43 \mathrm{E}+04$ & $4.57 \mathrm{E}+04$ \\
\hline 28 & $8.36 \mathrm{E}+04$ & $2.21 \mathrm{E}+04$ & $5.94 \mathrm{E}+04$ & $1.01 \mathrm{E}+04$ & $9.84 \mathrm{E}+04$ & $4.74 \mathrm{E}+02$ & $9.44 \mathrm{E}+04$ & $4.58 \mathrm{E}+0$ \\
\hline 29 & $8.13 \mathrm{E}+04$ & $2.23 \mathrm{E}+04$ & $5.70 \mathrm{E}+04$ & $1.02 \mathrm{E}+04$ & $9.85 \mathrm{E}+04$ & $4.99 \mathrm{E}+02$ & $9.45 \mathrm{E}+04$ & $4.59 \mathrm{E}+0$ \\
\hline 30 & $7.91 \mathrm{E}+04$ & $2.24 \mathrm{E}+04$ & $5.49 \mathrm{E}+04$ & $1.02 \mathrm{E}+04$ & $9.77 \mathrm{E}+04$ & $5.09 \mathrm{E}+02$ & $9.47 \mathrm{E}+04$ & $2.96 \mathrm{E}+0$ \\
\hline 31 & $7.67 \mathrm{E}+04$ & $2.25 \mathrm{E}+04$ & $5.29 \mathrm{E}+04$ & $1.02 \mathrm{E}+04$ & $9.69 \mathrm{E}+04$ & $5.19 \mathrm{E}+02$ & $9.43 \mathrm{E}+04$ & $2.95 \mathrm{E}+0$ \\
\hline 32 & $7.46 \mathrm{E}+04$ & $2.26 \mathrm{E}+04$ & $5.09 \mathrm{E}+04$ & $1.03 \mathrm{E}+04$ & $9.61 \mathrm{E}+04$ & $5.29 \mathrm{E}+02$ & $9.39 \mathrm{E}+04$ & $2.94 \mathrm{E}+0$ \\
\hline 33. & $7.25 \mathrm{E}+04$ & $2.27 \mathrm{E}+04$ & $4.91 \mathrm{E}+04$ & $1.03 E+04$ & $9.54 \mathrm{E}+04$ & $5.39 \mathrm{E}+02$ & $9.36 \mathrm{E}+04$ & $2.92 \mathrm{E}+0$ \\
\hline 34 & $7.04 \mathrm{E}+04$ & $2.28 \mathrm{E}+04$ & $4.73 \mathrm{E}+04$ & $1.04 \mathrm{E}+04$ & $9.46 \mathrm{E}+04$ & $5.48 \mathrm{E}+02$ & $9.32 \mathrm{E}+04$ & $2.91 E+0$ \\
\hline 35 & $6.84 \mathrm{E}+04$ & $2.29 \mathrm{E}+04$ & $4.56 \mathrm{E}+04$ & $1.04 \mathrm{E}+04$ & $9.38 \mathrm{E}+04$ & $5.58 \mathrm{E}+02$ & $9.28 \mathrm{E}+04$ & $2.90 \mathrm{E}+0$ \\
\hline 36 & $6.66 \mathrm{E}+04$ & $2.29 \mathrm{E}+04$ & $4.40 \mathrm{E}+04$ & $1.05 \mathrm{E}+04$ & $9.30 \mathrm{E}+04$ & $5.68 \mathrm{E}+02$ & $9.24 \mathrm{E}+04$ & $2.89 \mathrm{E}+0$ \\
\hline 37 & $6.50 \mathrm{E}+04$ & $2.30 \mathrm{E}+04$ & $4.24 \mathrm{E}+04$ & $1.05 \mathrm{E}+04$ & $9.23 \mathrm{E}+04$ & $5.77 \mathrm{E}+02$ & $9.21 \mathrm{E}+04$ & $2.88 \mathrm{E}+0$ \\
\hline 38 & $6.34 \mathrm{E}+04$ & $2.31 \mathrm{E}+04$ & $4.09 \mathrm{E}+04$ & $1.05 \mathrm{E}+04$ & $9.15 \mathrm{E}+04$ & $5.87 \mathrm{E}+02$ & $9.17 \mathrm{E}+04$ & $2.87 \mathrm{E}+\mathrm{C}$ \\
\hline
\end{tabular}




\begin{tabular}{|c|c|c|c|c|c|c|c|c|}
\hline 39 & $6.19 \mathrm{E}+04$ & $2.32 \mathrm{E}+04$ & $3.95 \mathrm{E}+04$ & $1.06 \mathrm{E}+04$ & $9.08 \mathrm{E}+04$ & $5.96 \mathrm{E}+02$ & $9.13 E+04$ & $2.85 \mathrm{E}+0$ \\
\hline 40 & $6.04 \mathrm{E}+04$ & $2.33 \mathrm{E}+04$ & $3.82 \mathrm{E}+04$ & $1.06 \mathrm{E}+04$ & $9.00 \mathrm{E}+04$ & $6.05 \mathrm{E}+02$ & $9.09 \mathrm{E}+04$ & $2.84 \mathrm{E}+$ \\
\hline 41 & $5.91 \mathrm{E}+04$ & $2.33 \mathrm{E}+04$ & $3.69 \mathrm{E}+04$ & $1.06 \mathrm{E}+04$ & $8.93 E+04$ & $6.15 \mathrm{E}+02$ & $9.06 \overline{\mathrm{E}+04}$ & $2.83 \mathrm{E}+0$ \\
\hline 42 & $5.78 \mathrm{E}+04$ & $2.34 \mathrm{E}+04$ & $3.57 \mathrm{E}+04$ & $1.07 \mathrm{E}+04$ & $8.92 \mathrm{E}+04$ & $6.38 \mathrm{E}+02$ & $8.64 \mathrm{E}+04$ & $4.47 \mathrm{E}+\mathrm{C}$ \\
\hline 43 & $5.65 \mathrm{E}+04$ & $2.35 \mathrm{E}+04$ & $3.46 \mathrm{E}+04$ & $1.07 \mathrm{E}+04$ & $8.91 \mathrm{E}+04$ & $6.62 \mathrm{E}+02$ & $8.64 \mathrm{E}+04$ & $4.47 \mathrm{E}+0$ \\
\hline 44 & $5.54 \mathrm{E}+04$ & $2.35 \mathrm{E}+04$ & $3.35 \mathrm{E}+04$ & $1.07 \mathrm{E}+04$ & $8.89 \mathrm{E}+04$ & $6.86 \mathrm{E}+02$ & $8.63 \mathrm{E}+04$ & $4.47 \mathrm{E}+$ \\
\hline 45 & $5.43 \mathrm{E}+04$ & $2.36 \mathrm{E}+04$ & $3.24 \mathrm{E}+04$ & $1.08 \mathrm{E}+04$ & $8.88 \mathrm{E}+04$ & $7.09 \mathrm{E}+02$ & $8.62 \mathrm{E}+04$ & $4.47 \mathrm{E}+\mathrm{C}$ \\
\hline 46 & $5.33 \mathrm{E}+04$ & $2.37 \mathrm{E}+04$ & $3.15 E+04$ & $1.08 \mathrm{E}+04$ & $8.86 \mathrm{E}+04$ & $7.33 \mathrm{E}+02$ & $8.62 \mathrm{E}+04$ & $4.47 \mathrm{E}+0$ \\
\hline 47 & $21 \mathrm{E}+04$ & $2.37 \mathrm{E}+04$ & $3.06 \mathrm{E}+04$ & $1.08 \mathrm{E}+04$ & $8.85 \mathrm{E}+04$ & $7.57 \mathrm{E}+02$ & $8.61 \mathrm{E}+04$ & $4.47 \mathrm{E}+$ \\
\hline 48 & $5.11 \mathrm{E}+04$ & $2.38 \mathrm{E}+04$ & $2.97 \mathrm{E}+04$ & $1.08 \mathrm{E}+04$ & $8.83 \mathrm{E}+04$ & $7.80 \mathrm{E}+02$ & $8.60 \mathrm{E}+04$ & $4.47 \mathrm{E}+0$ \\
\hline 49 & $5.03 \mathrm{E}+04$ & $2.38 \mathrm{E}+04$ & $2.88 \mathrm{E}+04$ & $1.09 \mathrm{E}+04$ & $8.81 E+04$ & $8.04 \mathrm{E}+02$ & $8.60 \mathrm{E}+04$ & $4.46 \mathrm{E}+04$ \\
\hline 50 & $4.95 \mathrm{E}+04$ & $2.39 \mathrm{E}+04$ & $2.80 \mathrm{E}+04$ & $1.09 \mathrm{E}+04$ & $8.79 \mathrm{E}+04$ & $8.27 \mathrm{E}+02$ & $8.59 \mathrm{E}+04$ & $4.46 \mathrm{E}+0$ \\
\hline 51 & $4.88 \mathrm{E}+04$ & $2.39 \mathrm{E}+04$ & $2.73 \mathrm{E}+04$ & $1.09 \mathrm{E}+04$ & $8.78 \mathrm{E}+04$ & $8.51 \mathrm{E}+02$ & $8.58 \mathrm{E}+04$ & $4.46 \mathrm{E}+0$ \\
\hline 52 & $4.81 \mathrm{E}+04$ & $2.40 \mathrm{E}+04$ & $2.66 \mathrm{E}+04$ & $1.09 \mathrm{E}+04$ & $8.75 E+04$ & $8.74 \mathrm{E}+02$ & $8.57 \mathrm{E}+04$ & $\overline{4.46 \mathrm{E}+02}$ \\
\hline 53 & $4.74 \mathrm{E}+04$ & $2.40 \mathrm{E}+04$ & $2.59 \mathrm{E}+04$ & $1.10 \mathrm{E}+04$ & $8.73 E+04$ & $8.98 \mathrm{E}+02$ & $8.56 \mathrm{E}+04$ & $4.46 \mathrm{E}+0$ \\
\hline 54 & $4.68 \mathrm{E}+04$ & $2.41 \mathrm{E}+04$ & $2.53 E+04$ & $1.10 \mathrm{E}+04$ & $8.66 \mathrm{E}+04$ & $9.07 \mathrm{E}+02$ & $8.40 \mathrm{E}+04$ & $2.63 \mathrm{E}+0$ \\
\hline 55 & $4.62 \mathrm{E}+04$ & $2.41 \mathrm{E}+04$ & $2.48 \mathrm{E}+04$ & $1.10 \mathrm{E}+04$ & $8.59 \mathrm{E}+04$ & $9.15 \mathrm{E}+02$ & $8.36 \mathrm{E}+04$ & $2.62 \mathrm{E}+0$ \\
\hline 56 & $4.57 \mathrm{E} \div 04$ & $2.42 \mathrm{E}+04$ & $2.42 \mathrm{E}+04$ & $1.10 \mathrm{E}+04$ & $8.52 \mathrm{E}+04$ & $9.24 \mathrm{E}+02$ & $8.33 \mathrm{E}+04$ & $2.61 \mathrm{E}+0$ \\
\hline 57 & $4.48 \mathrm{E}+04$ & $2.42 \mathrm{E}+04$ & $2.38 \mathrm{E}+04$ & $1.10 \mathrm{E}+04$ & $8.45 E+04$ & $9.32 \mathrm{E}+02$ & $8.29 \mathrm{E}+04$ & $2.60 \mathrm{E}+0$ \\
\hline 58 & $4.42 \mathrm{E}+04$ & $2.43 \mathrm{E}+04$ & $2.33 \mathrm{E}+04$ & $1.11 \mathrm{E}+04$ & $8.38 \mathrm{E}+04$ & $9.41 \mathrm{E}+02$ & $8.26 \mathrm{E}+04$ & $2.59 \mathrm{E}+0$ \\
\hline 59 & $4.37 \mathrm{E}+04$ & $2.43 \mathrm{E}+04$ & $2.28 \mathrm{E}+04$ & $1.11 \mathrm{E}+04$ & $8.31 \mathrm{E}+04$ & $9.49 \mathrm{E}+02$ & $8.22 \mathrm{E}+04$ & $2.58 \mathrm{E}+0$ \\
\hline 60 & $4.32 \mathrm{E}+04$ & $2.44 \mathrm{E}+04$ & $2.23 \mathrm{E}+04$ & $1.11 \mathrm{E}+04$ & $8.24 \mathrm{E}+04$ & $9.57 \mathrm{E}+02$ & $8.19 \mathrm{E}+04$ & $2.57 \mathrm{E}+0$ \\
\hline 61 & $4.28 \mathrm{E}+04$ & $2.44 \mathrm{E}+04$ & $2.19 \mathrm{E}+04$ & $1.11 \mathrm{E}+04$ & $8.17 \mathrm{E}+04$ & $9.65 \mathrm{E}+02$ & $8.16 \mathrm{E}+04$ & $2.56 \mathrm{E}+0$ \\
\hline 62 & $4.18 \mathrm{E}+04$ & $2.44 \mathrm{E}+04$ & $2.15 \mathrm{E}+04$ & $1.11 \mathrm{E}+04$ & $8.10 \mathrm{E}+04$ & $9.73 \mathrm{E}+02$ & $8.12 \mathrm{E}+04$ & $2.55 \mathrm{E}+0$ \\
\hline 63 & $4.13 E+04$ & $2.45 \mathrm{E}+04$ & $2.10 \mathrm{E}+04$ & $1.12 \mathrm{E}+04$ & $8.04 \mathrm{E}+04$ & $9.81 \mathrm{E}+02$ & $8.09 \mathrm{E}+04$ & $2.54 \mathrm{E}+0$ \\
\hline 64 & $4.08 \mathrm{E}+04$ & $2.45 \mathrm{E}+04$ & $2.06 \mathrm{E}+04$ & $1.12 \mathrm{E}+04$ & $7.97 \mathrm{E}+04$ & $9.89 \mathrm{E}+02$ & $8.06 \mathrm{E}+04$ & $2.53 \mathrm{E}+04$ \\
\hline 65 & $4.05 \mathrm{E}+04$ & $2.46 \mathrm{E}+04$ & $2.02 \mathrm{E}+04$ & $1.12 \mathrm{E}+04$ & $7.90 \mathrm{E}+04$ & $9.97 \mathrm{E}+02$ & $8.02 \mathrm{E}+04$ & $2.52 \mathrm{E}+04$ \\
\hline 66 & $4.02 \mathrm{E}+04$ & $2.46 \mathrm{E}+04$ & $1.98 \mathrm{E}+04$ & $1.12 \mathrm{E}+04$ & $7.88 \mathrm{E}+04$ & $1.02 \mathrm{E}+03$ & $7.64 \mathrm{E}+04$ & $4.22 \mathrm{E}+04$ \\
\hline 67 & $3.99 \mathrm{E}+04$ & $2.46 \mathrm{E}+04$ & $1.94 \mathrm{E}+04$ & $1.12 \mathrm{E}+04$ & $7.86 \mathrm{E}+04$ & $1.04 \mathrm{E}+03$ & $7.63 \mathrm{E}+04$ & $4.22 \mathrm{E}+04$ \\
\hline 68 & $3.96 \mathrm{E}+04$ & $2.47 \mathrm{E}+04$ & $1.91 \mathrm{E}+04$ & $1.13 E+04$ & $7.84 \mathrm{E}+04$ & $1.07 \mathrm{E}+03$ & $7.62 \mathrm{E}+04$ & $4.22 \mathrm{E}+04$ \\
\hline 69 & $3.93 \mathrm{E}+04$ & $2.47 \mathrm{E}+04$ & $1.88 \mathrm{E}+04$ & $1.13 \mathrm{E}+04$ & $7.81 \mathrm{E}+04$ & $1.09 \mathrm{E}+03$ & $7.61 \mathrm{E}+04$ & $4.21 \mathrm{E}+04$ \\
\hline 168 & $1.45 \mathrm{E}+04$ & $2.51 \mathrm{E}+04$ & $1.46 \mathrm{E}+04$ & $1.25 \mathrm{E}+04$ & $4.63 E+04$ & $2.75 \mathrm{E}+03$ & $5.08 \mathrm{E}+04$ & $3.41 \mathrm{E}+04$ \\
\hline 336 & $7.48 \mathrm{E}+03$ & $2.14 \mathrm{E}+04$ & $7.58 \mathrm{E}+03$ & $1.36 \mathrm{E}+04$ & $1.99 \mathrm{E}+04$ & $5.00 \mathrm{E}+03$ & $3.02 E+04$ & $2.58 \mathrm{E}+04$ \\
\hline 504 & $5.54 \mathrm{E}+03$ & $1.80 \mathrm{E}+04$ & $5.22 \mathrm{E}+03$ & $1.43 \mathrm{E}+04$ & $9.89 \mathrm{E}+03$ & $6.24 \mathrm{E}+03$ & $1.37 \mathrm{E}+04$ & $1.75 \mathrm{E}+04$ \\
\hline 720 & $4.04 \mathrm{E}+03$ & $1.43 \mathrm{E}+04$ & $3.51 \mathrm{E}+03$ & $1.49 \mathrm{E}+04$ & $5.01 \mathrm{E}+03$ & $6.92 \mathrm{E}+03$ & $6.87 \mathrm{E}+03$ & $1.22 \mathrm{E}+0$ \\
\hline 2160 & $7.59 \mathrm{E}+02$ & $2.94 \mathrm{E}+03$ & $3.95 \mathrm{E}+02$ & $1.61 \mathrm{E}+04$ & $4.42 \mathrm{E}+02$ & $4.12 \mathrm{E}+03$ & $1.55 \mathrm{E}+03$ & $4.24 \mathrm{E}+03$ \\
\hline 4320 & $7.30 \mathrm{E}+01$ & $2.74 \mathrm{E}+02$ & $1.99 \mathrm{E}+03$ & $1.51 \mathrm{E}+04$ & $7.65 \mathrm{E}+01$ & $9.94 \mathrm{E}+02$ & $1.91 \mathrm{E}+02$ & $6.71 \mathrm{E}+02$ \\
\hline 4800 & $4.34 \mathrm{E}+01$ & $1.62 \mathrm{E}+02$ & $1.98 \mathrm{E}+03$ & $1.51 \mathrm{E}+04$ & $5.58 \mathrm{E}+01$ & $7.17 \mathrm{E}+02$ & $6.55 \mathrm{E}+01$ & $2.22 \mathrm{E}+02$ \\
\hline 8760 & $5.97 \mathrm{E}-01$ & $2.17 \mathrm{E}+00$ & $1.93 \mathrm{E}+03$ & $1.47 \mathrm{E}+04$ & $4.70 \mathrm{E}+00$ & $4.87 \mathrm{E}+01$ & $2.06 \mathrm{E}+01$ & $6.60 \mathrm{E}+0$ \\
\hline BT & $\begin{array}{l}2.4 \mathrm{E} 6 \\
\mathrm{pCi} / \mathrm{L} \\
\end{array}$ & $\begin{array}{l}8 \mathrm{E}+4 \\
\mathrm{~kg}\end{array}$ & $88.8 \mathrm{~Bq} / \mathrm{g}$ & & & & & \\
\hline To & $\begin{array}{l}5.9 \mathrm{E} 6 \\
\mathrm{pCi} / \mathrm{L}\end{array}$ & \begin{tabular}{|l|}
$2.18 \mathrm{E}+5$ \\
$\mathrm{~Bq} / \mathrm{kg}$ \\
\end{tabular} & $218.3 \mathrm{~Bq} / \mathrm{g}$ & & & & & \\
\hline & \multicolumn{2}{|c|}{ VEGETABLES } & \multicolumn{2}{|c|}{ WHEAT SEED } & \multicolumn{2}{|l|}{ MEAT } & \multicolumn{2}{|l|}{ MILK } \\
\hline \multirow[t]{2}{*}{ HOUR } & $\mathrm{O}$ & OBT & HTO & OBT & HTO & OBT & HTO & $\mathrm{BBT}$ \\
\hline & $(\mathrm{Bq} / \mathrm{g})$ & $(\mathrm{Bq} / \mathrm{g})$ & $(\mathrm{Bq} / \mathrm{g})$ & $(\mathrm{Bq} / \mathrm{g})$ & $(\mathrm{Bq} / \mathrm{g})$ & $(\mathrm{Bq} / \mathrm{g})$ & $(\mathrm{Bq} / \mathrm{g})$ & $(\mathrm{Bq} / \mathrm{g})$ \\
\hline K HIO & & & & & & & & \\
\hline
\end{tabular}


a Tritium Oxide Release from SRS Tritium Facilities (U)

\begin{tabular}{|c|c|c|c|c|c|c|c|c|}
\hline $1 / 3 / 6 / 6$ & $1.96 \mathrm{E}+06$ & $8.43 E+03$ & $1.12 \mathrm{E}+06$ & $5.20 \mathrm{E}+03$ & $1.04 \mathrm{E}+05$ & $9.73 \mathrm{E}+01$ & $1.01 \mathrm{E}+05$ & $3.14 \mathrm{E}+04$ \\
\hline SUM & $1.97 \mathrm{E}+06$ & & $1.13 \mathrm{E}+06$ & & $1.04 \mathrm{E}+05$ & & $1.32 \mathrm{E}+05$ & \\
\hline $\mathrm{Ci} / \mathrm{g}$ & $5.31 \mathrm{E}-05$ & & $3.05 \mathrm{E}-05$ & & $2.81 \mathrm{E}-06$ & & $3.57 \mathrm{E}-06$ & \\
\hline & & & & & & & & \\
\hline DIL HTO & $5.9 \mathrm{E}-09$ & & & & & & & \\
\hline \multicolumn{9}{|l|}{$(\mathrm{Ci} / \mathrm{g})$} \\
\hline & & & & & & & & \\
\hline 168 & $1.45 \mathrm{E}+04$ & $2.51 E+04$ & $1.46 \mathrm{E}+04$ & $1.25 \mathrm{E}+04$ & $4.63 \mathrm{E}+04$ & $2.75 \mathrm{E}+03$ & $5.08 \mathrm{E}+04$ & $3.41 \mathrm{E}+04$ \\
\hline SUM & $3.96 \mathrm{E}+04$ & & $2.71 \mathrm{E}+04$ & & $4.91 \mathrm{E}+04$ & & $8.49 \mathrm{E}+04$ & \\
\hline $\mathrm{Ci} / \mathrm{g}$ & $1.07 \mathrm{E}-06$ & & 7.33E-07 & & $1.33 \mathrm{E}-06$ & & $2.30 \mathrm{E}-06$ & \\
\hline & & & & & & & & \\
\hline DIL OBT & $2.4 \mathrm{E}-09$ & & & & & & & \\
\hline \multicolumn{9}{|l|}{$\mathrm{Ci} / \mathrm{g}$} \\
\hline & & & & & & & & \\
\hline 8760 & $5.97 \mathrm{E}-01$ & $2.17 \mathrm{E}+00$ & $1.93 \mathrm{E}+03$ & $1.47 \mathrm{E}+04$ & $4.70 \mathrm{E}+00$ & $4.87 \mathrm{E}+01$ & $2.06 \mathrm{E}+01$ & $6.60 \mathrm{E}+01$ \\
\hline SUM & $2.77 \mathrm{E}+00$ & & $1.66 \mathrm{E}+04$ & & $5.34 \mathrm{E}+01$ & & $8.67 \mathrm{E}+01$ & \\
\hline $\mathrm{Ci} / \mathrm{g}$ & $7.49 \mathrm{E}-11$ & & 4.49E-07 & & $1.44 \mathrm{E}-09$ & & $2.34 \mathrm{E}-09$ & \\
\hline & & & & & & & & \\
\hline DIL OBT & $2.4 \mathrm{E}-09$ & & & & & & & \\
\hline $\mathrm{Ci} / \mathrm{g}$ & & & & & & & & \\
\hline
\end{tabular}




\begin{tabular}{|c|c|c|c|c|c|c|c|c|}
\hline & \multicolumn{7}{|c|}{ Tritium Facilities, Beyond Design Basis Fire, Average Met Conditions } & \\
\hline & \multicolumn{4}{|c|}{ Concentration Values for $11.54 \mathrm{~km}$ from release } & & & & \\
\hline \multicolumn{9}{|c|}{ Only Wheat Seed is examined since this is the edible portion of the Wheat. The "WHEAT" category is } \\
\hline \multicolumn{9}{|c|}{ actually the unedible plant body and is not considered in the dose estimate. } \\
\hline & & & & & & & & \\
\hline & \multicolumn{2}{|c|}{ VEGETABLES } & \multicolumn{2}{|c|}{ WHEAT SEED } & \multicolumn{2}{|l|}{ MEAT } & \multicolumn{2}{|l|}{ MILK } \\
\hline \multirow[t]{3}{*}{ HOUR } & HTO & $\overline{\mathrm{OBT}}$ & HTO & OBT & HTO & OBT & HTO & $\mathrm{T}$ \\
\hline & $(\mathrm{Bq} / \mathrm{g})$ & $(\mathrm{Bq} / \mathrm{g})$ & $(\mathrm{Bq} / \mathrm{g})$ & $(\mathrm{Bq} / \mathrm{g})$ & $(\mathrm{Bq} / \mathrm{g})$ & $(\mathrm{Bq} / \mathrm{g})$ & (B & T) \\
\hline & & & & & & & & \\
\hline 1 & $1.12 \mathrm{E}+06$ & $3.11 \mathrm{E}+03$ & $2.08 \mathrm{E}+05$ & $6.88 \mathrm{E}+02$ & $1.61 \mathrm{E}+04$ & $3.25 \overline{\mathrm{E}}+00$ & $1.95 \mathrm{E}+03$ & $8.36 \mathrm{E}+02$ \\
\hline 2 & $1.92 \mathrm{E}+06$ & $1.14 \mathrm{E}+04$ & $7.77 \mathrm{E}+05$ & $2.77 \mathrm{E}+03$ & $5.91 E+04$ & $1.54 \mathrm{E}+01$ & $8.10 \mathrm{E}+03$ & +03 \\
\hline 3 & $8.99 \bar{E}+05$ & $1.46 \mathrm{E}+04$ & $1.03 E+06$ & $4.36 \mathrm{E}+03$ & $8.11 \mathrm{E}+04$ & $3.23 \mathrm{E}+01$ & $1.51 \mathrm{E}+04$ & $6.59 \mathrm{E}+03$ \\
\hline 4 & $5.14 \mathrm{E}+05$ & $1.62 \mathrm{E}+04$ & $1.09 \mathrm{E}+06$ & $5.54 \mathrm{E}+03$ & $9.33 \mathrm{E}+04$ & $5.18 \mathrm{E}+01$ & $2.20 \mathrm{E}+04$ & $9.60 \mathrm{E}+03$ \\
\hline 5 & $3.57 \mathrm{E}+05$ & $1.70 \mathrm{E}+04$ & $1.03 \mathrm{E}+06$ & $6.41 \mathrm{E}+03$ & $1.00 \mathrm{E}+05$ & $7.29 \mathrm{E}+01$ & $2.83 \mathrm{E}+04$ & $1.24 \mathrm{E}+04$ \\
\hline$\overline{6}$ & $2.84 \mathrm{E}+05$ & $1.76 \mathrm{E}+04$ & $9.29 \mathrm{E}+05$ & $7.07 \mathrm{E}+03$ & $9.97 \mathrm{E}+04$ & $8.35 \mathrm{E}+01$ & $9.66 \mathrm{E}+04$ & $3.01 \mathrm{E}+0$ \\
\hline 7 & $2.46 \mathrm{E}+05$ & $1.81 \mathrm{E}+04$ & $8.07 \mathrm{E}+05$ & $7.56 \mathrm{E}+03$ & $9.90 \mathrm{E}+04$ & $9.41 \mathrm{E}+01$ & $9.63 \mathrm{E}+04$ & $3.00 \mathrm{E}+04$ \\
\hline 8 & $2.23 \mathrm{E}+05$ & $1.85 \mathrm{E}+04$ & $6.87 \mathrm{E}+05$ & $7.94 \mathrm{E}+03$ & $9.82 \mathrm{E}+04$ & $1.05 \mathrm{E}+02$ & $9.59 E+04$ & $2.99 \mathrm{E}+04$ \\
\hline 9 & $2.07 \mathrm{E}+05$ & $1.88 \mathrm{E}+04$ & $5.77 \mathrm{E}+05$ & $8.24 \mathrm{E}+03$ & $9.75 \mathrm{E}+04$ & $1.15 \mathrm{E}+02$ & $9.56 \mathrm{E}+04$ & $2.98 \mathrm{E}+04$ \\
\hline 10 & $1.96 \mathrm{E}+05$ & $1.92 \mathrm{E}+04$ & $4.83 \mathrm{E}+05$ & $8.49 \mathrm{E}+03$ & $9.68 \mathrm{E}+04$ & $1.25 \mathrm{E}+02$ & $9.52 \mathrm{E}+04$ & $2.97 \mathrm{E}+04$ \\
\hline 11 & $1.86 \mathrm{E}+05$ & $1.95 \mathrm{E}+04$ & $4.04 \mathrm{E}+05$ & $8.69 \mathrm{E}+03$ & $9.60 \mathrm{E}+04$ & $1.36 \mathrm{E}+02$ & $9.49 \mathrm{E}+04$ & $2.96 \mathrm{E}+04$ \\
\hline 12 & $1.78 \mathrm{E}+05$ & $1.98 \mathrm{E}+04$ & $3.39 \mathrm{E}+05$ & $8.87 \mathrm{E}+03$ & $9.53 \mathrm{E}+04$ & $1.46 \mathrm{E}+02$ & $.45 \mathrm{E}+04$ & $2.94 \mathrm{E}+04$ \\
\hline 13 & $1.72 \mathrm{E}+05$ & $2.00 \mathrm{E}+04$ & $2.87 \mathrm{E}+05$ & $9.03 E+03$ & $9.46 \mathrm{E}+04$ & $1.56 \mathrm{E}+02$ & $9.42 E+04$ & $2.93 \mathrm{E}+04$ \\
\hline 14 & $1.65 \mathrm{E}+05$ & $2.03 \mathrm{E}+04$ & $2.46 \mathrm{E}+05$ & $9.17 \mathrm{E}+03$ & $9.39 \mathrm{E}+04$ & $1.66 \mathrm{E}+02$ & $9.38 \mathrm{E}+04$ & $2.92 \mathrm{E}+04$ \\
\hline 15 & $1.60 \mathrm{E}+05$ & $2.06 \mathrm{E}+04$ & $2.13 \mathrm{E}+05$ & $9.29 \mathrm{E}+03$ & $9.31 \mathrm{E}+04$ & $1.76 \mathrm{E}+02$ & $9.34 \mathrm{E}+04$ & $2.91 \mathrm{E}+04$ \\
\hline 16 & $1.54 \mathrm{E}+05$ & $2.08 \mathrm{E}+04$ & $1.86 \mathrm{E}+05$ & $9.41 \mathrm{E}+03$ & $9.24 \mathrm{E}+04$ & $1.86 \mathrm{E}+02$ & $9.31 \mathrm{E}+04$ & $2.90 \mathrm{E}+0$ \\
\hline 17 & $1.49 \mathrm{E}+05$ & $2.10 \mathrm{E}+04$ & $1.66 \mathrm{E}+05$ & $9.52 \mathrm{E}+03$ & $9.17 \mathrm{E}+04$ & $1.95 \mathrm{E}+02$ & $9.27 \mathrm{E}+04$ & $2.89 \mathrm{E}+04$ \\
\hline 18 & $1.44 \mathrm{E}+05$ & $2.13 \mathrm{E}+04$ & $1.49 \mathrm{E}+05$ & $9.63 E+03$ & $9.26 \mathrm{E}+04$ & $2.19 E+02$ & $8.97 \mathrm{E}+04$ & $4.43 \mathrm{E}+04$ \\
\hline 19 & $1.40 \mathrm{E}+05$ & $2.15 \mathrm{E}+04$ & $1.36 \mathrm{E}+05$ & $9.73 \mathrm{E}+03$ & $9.34 \mathrm{E}+04$ & $2.42 \mathrm{E}+02$ & $9.01 \mathrm{E}+04$ & $4.45 \mathrm{E}+04$ \\
\hline 20 & $1.35 \mathrm{E}+05$ & $2.17 \mathrm{E}+04$ & $1.25 \mathrm{E}+05$ & $9.82 \mathrm{E}+03$ & $9.42 \mathrm{E}+04$ & $2.66 \mathrm{E}+02$ & $9.05 \mathrm{E}+04$ & $4.47 \mathrm{E}+04$ \\
\hline 21 & $1.31 \mathrm{E}+05$ & $2.19 \mathrm{E}+04$ & $1.16 \mathrm{E}+05$ & $9.91 \mathrm{E}+03$ & $9.48 \mathrm{E}+04$ & $2.89 \mathrm{E}+02$ & $9.09 \mathrm{E}+04$ & $4.49 \mathrm{E}+04$ \\
\hline 22 & $1.27 \mathrm{E}+05$ & $2.21 \mathrm{E}+04$ & $1.08 \mathrm{E}+05$ & $1.00 \mathrm{E}+04$ & $9.55 \mathrm{E}+04$ & $3.13 \mathrm{E}+02$ & $9.12 \mathrm{E}+04$ & $4.50 \mathrm{E}+04$ \\
\hline 23 & $1.24 \mathrm{E}+05$ & $2.23 \mathrm{E}+04$ & $1.02 \mathrm{E}+05$ & $1.0 \mathrm{i} \mathrm{E}+04$ & $9.61 \mathrm{E}+04$ & $3.38 \mathrm{E}+02$ & $9.15 \mathrm{E}+04$ & $4.52 \mathrm{E}+04$ \\
\hline 24 & $1.20 \mathrm{E}+05$ & $2.25 \mathrm{E}+04$ & $9.67 \mathrm{E}+04$ & $1.02 \mathrm{E}+04$ & $9.66 \mathrm{E}+04$ & $3.62 \mathrm{E}+02$ & $9.18 \mathrm{E}+04$ & $4.54 \mathrm{E}+04$ \\
\hline 25 & $1.16 \mathrm{E}+05$ & $2.26 \mathrm{E}+04$ & $9.20 \mathrm{E}+04$ & $1.02 \mathrm{E}+04$ & $9.71 E+04$ & $3.86 \mathrm{E}+02$ & $9.21 \mathrm{E}+04$ & $4.55 \mathrm{E}+04$ \\
\hline 26 & $1.13 \mathrm{E}+05$ & $2.28 \mathrm{E}+04$ & $8.78 \mathrm{E}+04$ & $1.03 \mathrm{E}+04$ & $9.76 \mathrm{E}+04$ & $4.11 \mathrm{E}+02$ & $9.24 \mathrm{E}+04$ & $4.57 \mathrm{E}+04$ \\
\hline 27 & $1.09 \mathrm{E}+05$ & $2.30 \mathrm{E}+04$ & $8.40 \mathrm{E}+04$ & $1.04 \mathrm{E}+04$ & $9.80 \mathrm{E}+04$ & $4.35 \mathrm{E}+02$ & $9.27 \mathrm{E}+04$ & $4.58 \mathrm{E}+04$ \\
\hline 28 & $1.06 \mathrm{E}+05$ & $2.31 \mathrm{E}+04$ & $8.05 E+04$ & $1.05 \mathrm{E}+04$ & $9.84 \mathrm{E}+04$ & $4.60 \mathrm{E}+02$ & $9.29 \mathrm{E}+04$ & $4.59 \mathrm{E}+04$ \\
\hline 29 & $1.03 \mathrm{E}+05$ & $2.33 \mathrm{E}+04$ & $7.73 E+04$ & $1.05 \mathrm{E}+04$ & $9.87 \mathrm{E}+04$ & $4.85 \mathrm{E}+02$ & $9.31 \mathrm{E}+04$ & $4.61 \mathrm{E}+04$ \\
\hline 30 & $9.99 \mathrm{E}+04$ & $2.34 \mathrm{E}+04$ & $7.43 \mathrm{E}+04$ & $1.06 \mathrm{E}+04$ & $9.79 \mathrm{E}+04$ & $4.95 \mathrm{E}+02$ & $9.49 \mathrm{E}+04$ & $2.96 \mathrm{E}+04$ \\
\hline 31 & $9.67 \mathrm{E}+04$ & $2.36 \mathrm{E}+04$ & $7.15 \mathrm{E}+04$ & $1.07 \mathrm{E}+04$ & $9.71 \mathrm{E}+04$ & $5.05 \mathrm{E}+02$ & $9.45 \mathrm{E}+04$ & $2.95 \mathrm{E}+\overline{04}$ \\
\hline 32 & $9.38 \mathrm{E}+04$ & $2.37 \mathrm{E}+04$ & $6.88 \mathrm{E}+04$ & $1.07 \mathrm{E}+04$ & $9.63 \mathrm{E}+04$ & $5.15 \mathrm{E}+02$ & $9.41 \mathrm{E}+04$ & $2.94 \mathrm{E}+04$ \\
\hline 33 & $9.11 \mathrm{E}+04$ & $2.38 \mathrm{E}+04$ & $6.63 E+04$ & $1.08 \mathrm{E}+04$ & $9.56 \mathrm{E}+04$ & $5.25 \mathrm{E}+02$ & $9.38 \mathrm{E}+04$ & $2.9 \overline{\mathrm{E}+04}$ \\
\hline 34 & $8.25 \mathrm{E}+04$ & $2.40 \mathrm{E}+04$ & $6.38 \mathrm{E}+04$ & $1.08 \mathrm{E}+04$ & $9.48 \mathrm{E}+04$ & $5.35 \mathrm{E}+02$ & $9.34 \mathrm{E}+04$ & $2.92 \mathrm{E}+04$ \\
\hline 35 & $7.85 \mathrm{E}+04$ & $2.41 \mathrm{E}+04$ & $6.08 \mathrm{E}+04$ & $1.09 \mathrm{E}+04$ & $9.40 \mathrm{E}+04$ & $5.45 \mathrm{E}+02$ & $9.30 \mathrm{E}+04$ & $2.91 \mathrm{E}+04$ \\
\hline 36 & $7.58 \mathrm{E}+04$ & $2.42 \mathrm{E}+04$ & $5.77 \mathrm{E}+04$ & $1.09 \mathrm{E}+04$ & $9.33 \mathrm{E}+04$ & $5.54 \mathrm{E}+02$ & $9.26 \mathrm{E}+04$ & $2.89 \overline{\mathrm{E}+04}$ \\
\hline 37 & $7.35 \mathrm{E}+04$ & $2.43 E+04$ & $5.47 \mathrm{E}+04$ & $1.10 \mathrm{E}+04$ & $9.25 \mathrm{E}+04$ & $5.64 \mathrm{E}+02$ & $9.23 \mathrm{E}+04$ & $2.88 \mathrm{E}+04$ \\
\hline 38 & $7.14 \mathrm{E}+04$ & $2.44 \mathrm{E}+04$ & $5.18 \mathrm{E}+04$ & $1.10 \mathrm{E}+04$ & $9.17 \mathrm{E}+04$ & $5.73 \mathrm{E}+02$ & $9.19 \mathrm{E}+04$ & $2.87 \mathrm{E}+04$ \\
\hline
\end{tabular}


Foodstuff Concentrations and Relocation Considerations Following a Tritium Oxide Release from SRS Tritium Facilities (U)

\begin{tabular}{|c|c|c|c|c|c|c|c|c|}
\hline 39 & $6.95 \mathrm{E}+04$ & $2.44 \mathrm{E}+04$ & $4.92 \mathrm{E}+04$ & $1.11 \mathrm{E}+04$ & $9.10 \mathrm{E}+04$ & $5.83 \mathrm{E}+02$ & $9.15 \mathrm{E}+04$ & $2.86 \mathrm{E}+$ \\
\hline 40 & $6.77 \mathrm{E}+04$ & $2.45 E+04$ & $4.68 \mathrm{E}+04$ & $1.11 \mathrm{E}+04$ & $9.02 E+04$ & $5.92 \mathrm{E}+02$ & $9.12 E+04$ & $2.85 \mathrm{E}+$ \\
\hline 41 & $6.59 \mathrm{E}+04$ & $2.46 \mathrm{E}+04$ & $4.47 \mathrm{E}+04$ & $1.11 \mathrm{E}+04$ & $8.95 E+04$ & $6.01 \mathrm{E}+02$ & $9.08 \mathrm{E}+04$ & $84 \mathrm{E}+$ \\
\hline 42 & $6.43 \mathrm{E}+04$ & $2.47 \mathrm{E}+04$ & $4.27 \mathrm{E}+04$ & $1.12 \mathrm{E}+04$ & $8.95 E+04$ & $6.26 \mathrm{E}+02$ & $8.67 \mathrm{E}+04$ & $4.58 \mathrm{E}+$ \\
\hline 43 & $6.28 \mathrm{E}+04$ & $2.48 \mathrm{E}+04$ & $4.09 \mathrm{E}+04$ & $1.12 \mathrm{E}+04$ & $8.94 \mathrm{E}+04$ & $6.50 \mathrm{E}+02$ & $8.67 \mathrm{E}+04$ & $1.58 \mathrm{E}+$ \\
\hline 44 & $6.13 E+04$ & $2.49 \mathrm{E}+04$ & $3.93 \mathrm{E}+04$ & $1.12 \mathrm{E}+04$ & $8.94 E+04$ & $6.75 \mathrm{E}+02$ & $8.67 \mathrm{E}+04$ & $.58 \mathrm{E}+$ \\
\hline 45 & $5.99 \mathrm{E}+04$ & $2.49 \mathrm{E}+04$ & $3.78 \mathrm{E}+04$ & $1.13 \mathrm{E}+04$ & $8.93 \mathrm{E}+04$ & $6.99 \mathrm{E}+02$ & $8.66 \mathrm{E}+04$ & $4.58 \mathrm{E}-$ \\
\hline 46 & $5.87 \mathrm{E}+04$ & $2.50 \mathrm{E}+04$ & $3.64 \mathrm{E}+04$ & $1.13 \mathrm{E}+04$ & $8.92 \mathrm{E}+04$ & $7.24 \mathrm{E}+02$ & $8.66 \mathrm{E}+04$ & $4.58 \mathrm{E}$ \\
\hline 47 & $5.31 \mathrm{E}+04$ & $2.51 \mathrm{E}+04$ & $3.51 \mathrm{E}+04$ & $1.13 \mathrm{E}+04$ & $8.91 \mathrm{E}+04$ & $7.49 \mathrm{E}+02$ & $8.66 \mathrm{E}+04$ & $4.58 \mathrm{E}-$ \\
\hline 48 & $4.94 \mathrm{E}+04$ & $2.51 \mathrm{E}+04$ & $3.34 \mathrm{E}+04$ & $1.14 \mathrm{E}+04$ & $8.89 \mathrm{E}+04$ & $7.73 \mathrm{E}+02$ & $8.65 E+04$ & $4.58 \mathrm{E}+$ \\
\hline 49 & $4.79 \mathrm{E}+04$ & $2.52 \mathrm{E}+04$ & $3.15 \mathrm{E}+04$ & $1.14 \mathrm{E}+04$ & $8.87 \mathrm{E}+04$ & $7.98 \mathrm{E}+02$ & $8.64 \mathrm{E}+04$ & $4.58 \mathrm{E}-$ \\
\hline 50 & $4.71 \mathrm{E}+04$ & $2.52 \mathrm{E}+04$ & $2.96 \mathrm{E}+04$ & $1.14 \mathrm{E}+04$ & $8.85 E+04$ & $8.22 \mathrm{E}+02$ & $8.64 \mathrm{E}+04$ & $4.58 \mathrm{E}$ \\
\hline 51 & $4.64 \mathrm{E}+04$ & $2.53 E+04$ & $2.79 \mathrm{E}+04$ & $1.14 \mathrm{E}+04$ & $8.83 E+04$ & $8.47 \mathrm{E}+02$ & $8.63 \mathrm{E}+04$ & $4.58 \mathrm{E}-$ \\
\hline 52 & $4.59 \mathrm{E}+04$ & $2.53 E+04$ & $2.64 \mathrm{E}+04$ & $1.15 \mathrm{E}+04$ & $8.81 E+04$ & $8.71 \mathrm{E}+02$ & $8.62 \mathrm{E}+04$ & $4.58 \mathrm{E}$ \\
\hline 53 & $4.53 \mathrm{E}+04$ & $2.54 \mathrm{E}+04$ & $2.51 \mathrm{E}+04$ & $1.15 \mathrm{E}+04$ & $8.78 E+04$ & $8.95 \mathrm{E}+02$ & $8.61 \mathrm{E}+04$ & $4.57 \mathrm{E}+$ \\
\hline 54 & $4.48 \mathrm{E}+04$ & $2.54 \mathrm{E}+04$ & $2.40 \mathrm{E}+04$ & $1.15 \mathrm{E}+04$ & $8.71 E+04$ & $9.04 \mathrm{E}+02$ & $8.44 \mathrm{E}+04$ & $2.65 \mathrm{E}-$ \\
\hline 55 & $4.43 \mathrm{E}+04$ & $2.54 \mathrm{E}+04$ & $2.31 \mathrm{E}+04$ & $1.15 \mathrm{E}+04$ & $8.64 \mathrm{E}+04$ & $9.13 \mathrm{E}+02$ & $8.41 \mathrm{E}+04$ & $2.64 \mathrm{E}+$ \\
\hline 56 & $4.39 \mathrm{E}+04$ & $2.55 \mathrm{E}+04$ & $2.23 \mathrm{E}+04$ & $1.15 \mathrm{E}+04$ & $8.57 \mathrm{E}+04$ & $9.21 \mathrm{E}+02$ & $8.37 \mathrm{E}+04$ & $2.63 \mathrm{E} t$ \\
\hline 57 & $4.32 \mathrm{E}+04$ & $2.55 \mathrm{E}+04$ & $2.17 \mathrm{E}+04$ & $1.16 \mathrm{E}+04$ & $8.50 \mathrm{E}+04$ & $9.30 \mathrm{E}+02$ & $8.34 \mathrm{E}+04$ & $2.61 \mathrm{E}+$ \\
\hline 58 & $4.27 \mathrm{E}+04$ & $2.56 \mathrm{E}+04$ & $2.11 \mathrm{E}+04$ & $1.16 \mathrm{E}+04$ & $8.43 E+04$ & $9.38 \mathrm{E}+02$ & $8.30 \mathrm{E}+04$ & $2.60 \mathrm{E}+$ \\
\hline 59 & $4.23 \mathrm{E}+04$ & $2.56 \mathrm{E}+04$ & $2.05 E+04$ & $1.16 \mathrm{E}+04$ & $8.36 \mathrm{E}+04$ & $9.46 \mathrm{E}+02$ & $8.27 \mathrm{E}+04$ & $2.59 \mathrm{E}+0$ \\
\hline 60 & $4.19 \mathrm{E}+04$ & $2.56 \mathrm{E}+04$ & $2.00 \mathrm{E}+04$ & $1.16 \mathrm{E}+04$ & $8.29 \mathrm{E}+04$ & $9.55 \mathrm{E}+02$ & $8.24 \mathrm{E}+04$ & $2.58 \mathrm{E}+\mathrm{C}$ \\
\hline 61 & $4.15 \mathrm{E}+04$ & $2.57 \mathrm{E}+04$ & $1.96 \mathrm{E}+04$ & $1.16 \mathrm{E}+04$ & $8.22 \mathrm{E}+04$ & $9.63 \mathrm{E}+02$ & $8.20 \mathrm{E}+04$ & $2.57 \mathrm{E}+0$ \\
\hline 62 & $4.08 \mathrm{E}+04$ & $2.57 \mathrm{E}+04$ & $1.92 \mathrm{E}+04$ & $1.17 \mathrm{E}+04$ & $8.15 \mathrm{E}+04$ & $9.71 \mathrm{E}+02$ & $8.17 \mathrm{E}+04$ & $2.56 \mathrm{E}+04$ \\
\hline 63 & $4.03 \mathrm{E}+04$ & $2.58 \mathrm{E}+04$ & $1.89 \mathrm{E}+04$ & $1.17 \mathrm{E}+04$ & $8.08 E+04$ & $9.79 \mathrm{E}+02$ & $8.13 E+04$ & $2.55 \mathrm{E}+04$ \\
\hline 64 & $4.00 \mathrm{E}+04$ & $2.58 \mathrm{E}+04$ & $1.85 \mathrm{E}+04$ & $1.17 \mathrm{E}+04$ & $8.01 \mathrm{E}+04$ & $9.87 \mathrm{E}+02$ & $8.10 \mathrm{E}+04$ & $2.54 \mathrm{E}+0$ \\
\hline 65 & $3.97 \mathrm{E}+04$ & $2.58 \mathrm{E}+04$ & $1.82 \mathrm{E}+04$ & $1.17 \mathrm{E}+04$ & $7.95 \mathrm{E}+04$ & $9.95 \mathrm{E}+02$ & $8.07 \mathrm{E}+04$ & $2.53 \mathrm{E}+0$ \\
\hline 66 & $3.94 \mathrm{E}+04$ & $2.59 \mathrm{E}+04$ & $1.78 \mathrm{E}+04$ & $1.17 \mathrm{E}+04$ & $7.92 \mathrm{E}+04$ & $1.02 \mathrm{E}+03$ & $7.68 \mathrm{E}+04$ & $4.33 \mathrm{E}+0$ \\
\hline 67 & $3.91 \mathrm{E}+04$ & $2.59 \mathrm{E}+04$ & $1.76 \mathrm{E}+04$ & $1.17 \mathrm{E}+04$ & $7.90 \mathrm{E}+04$ & $1.04 \mathrm{E}+03$ & $7.67 \mathrm{E}+04$ & $4.33 \mathrm{E}+0$ \\
\hline 68 & $3.89 \mathrm{E}+04$ & $2.59 \mathrm{E}+04$ & $1.73 \mathrm{E}+04$ & $1.18 \mathrm{E}+04$ & $7.88 \mathrm{E}+04$ & $1.07 \mathrm{E}+03$ & $7.66 \mathrm{E}+04$ & $4.32 \mathrm{E}+0$ \\
\hline 69 & $3.86 \mathrm{E}+04$ & $2.60 \mathrm{E}+04$ & $1.71 \mathrm{E}+04$ & $1.18 \mathrm{E}+04$ & $7.85 \mathrm{E}+04$ & $1.09 \mathrm{E}+03$ & $7.65 \mathrm{E}+04$ & $4.32 \mathrm{E}+04$ \\
\hline 168 & $1.53 \mathrm{E}+04$ & $2.63 \mathrm{E}+04$ & $1.55 \mathrm{E}+04$ & $1.30 \mathrm{E}+04$ & $4.68 \mathrm{E}+04$ & $2.81 \mathrm{E}+03$ & $5.12 \mathrm{E}+04$ & $3.51 \mathrm{E}+04$ \\
\hline 336 & $7.81 \mathrm{E}+03$ & $2.24 \mathrm{E}+04$ & $7.98 \mathrm{E}+03$ & $1.42 \mathrm{E}+04$ & $2.04 \mathrm{E}+04$ & $5.15 \mathrm{E}+03$ & $3.08 \mathrm{E}+04$ & $2.68 \mathrm{E}+0$ \\
\hline 504 & $5.76 \mathrm{E}+03$ & $1.88 \mathrm{E}+04$ & $5.49 \mathrm{E}+03$ & $1.49 \mathrm{E}+04$ & $1.03 \mathrm{E}+04$ & $6.46 \mathrm{E}+03$ & $1.42 \mathrm{E}+04$ & $1.82 \mathrm{E}+04$ \\
\hline 720 & $4.20 \mathrm{E}+03$ & $1.49 \mathrm{E}+04$ & $3.68 \mathrm{E}+03$ & $1.55 \mathrm{E}+04$ & $5.22 \mathrm{E}+03$ & $7.19 \mathrm{E}+03$ & $7.15 \mathrm{E}+03$ & $1.28 \mathrm{E}+04$ \\
\hline 2160 & $7.88 \mathrm{E}+02$ & $3.07 \mathrm{E}+03$ & $4.15 \mathrm{E}+02$ & $1.67 \mathrm{E}+04$ & $4.59 \mathrm{E}+02$ & $4.29 \mathrm{E}+03$ & $1.62 \mathrm{E}+03$ & $4.42 \mathrm{E}+0$ \\
\hline 4320 & $7.58 \mathrm{E}+01$ & $2.86 \mathrm{E}+02$ & $2.09 \mathrm{E}+03$ & $1.58 \mathrm{E}+04$ & $7.89 \mathrm{E}+01$ & $1.03 \mathrm{E}+03$ & $1.98 \mathrm{E}+02$ & $6.98 \mathrm{E}+02$ \\
\hline 4800 & $4.51 \mathrm{E}+01$ & $1.69 \mathrm{E}+02$ & $2.08 \mathrm{E}+03$ & $1.57 \mathrm{E}+04$ & $5.76 \mathrm{E}+01$ & $7.46 \mathrm{E}+02$ & $6.76 \mathrm{E}+01$ & $2.31 \mathrm{E}+02$ \\
\hline 8760 & $6.19 \mathrm{E}-01$ & $2.26 \mathrm{E}+00$ & $2.03 \mathrm{E}+03$ & $1.53 \mathrm{E}+04$ & $4.83 \mathrm{E}+00$ & $5.06 \mathrm{E}+01$ & $2.13 \mathrm{E}+01$ & $6.85 \mathrm{E}+01$ \\
\hline $3 T$ & $\begin{array}{l}2.4 \mathrm{E} 6 \\
\mathrm{pCi} / \mathrm{L}\end{array}$ & $\begin{array}{l}8.88 \mathrm{E}+4 \\
\mathrm{~Bq} / \mathrm{kg}\end{array}$ & $8 \mathrm{~Bq} / \mathrm{g}$ & & & & & \\
\hline ГO & $\begin{array}{l}5.9 \mathrm{E} 6 \\
\mathrm{pCi} / \mathrm{L} \\
\end{array}$ & $\begin{array}{l}2.18 \mathrm{E}+5 \\
\mathrm{~Bq} / \mathrm{kg} \\
\end{array}$ & $218.3 \mathrm{~Bq} / \mathrm{g}$ & & & & & \\
\hline & \multicolumn{2}{|c|}{ VEGETABLES } & \multicolumn{2}{|c|}{ WHEAT SEED } & \multicolumn{2}{|l|}{ MEAT } & \multicolumn{2}{|l|}{ MILK } \\
\hline UUR & HTO & OBT & 0 & OBT & HTO & BT & TO & OBT \\
\hline & $(\mathrm{Bq} / \mathrm{g})$ & $(\mathrm{Bq} / \mathrm{g})$ & $(\mathrm{Bq} / \mathrm{g})$ & $(\mathrm{Bq} / \mathrm{g})$ & $(\mathrm{Bq} / \mathrm{g})$ & $(\mathrm{Bq} / \mathrm{g})$ & $(\mathrm{Bq} / \mathrm{g})$ & $(\mathrm{Bq} / \mathrm{g})$ \\
\hline ak HTO & & & & & & & & \\
\hline
\end{tabular}


Foodstuff Concentrations and Relocation Considerations Following

a Tritium Oxide Release from SRS Tritium Facilities (U)

\begin{tabular}{|c|c|c|c|c|c|c|c|c|}
\hline $2 / 4 / 5 / 6$ & $1.92 \mathrm{E}+06$ & $1.14 \mathrm{E}+04$ & $1.09 \mathrm{E}+06$ & $5.54 \mathrm{E}+03$ & $1.00 \mathrm{E}+05$ & $7.29 \mathrm{E}+01$ & $9.66 \mathrm{E}+04$ & $3.01 \mathrm{E}+04$ \\
\hline SUM & $1.93 \mathrm{E}+06$ & & $1.09 \mathrm{E}+06$ & & $1.00 \mathrm{E}+05$ & & $1.27 \mathrm{E}+05$ & \\
\hline $\mathrm{Ci} / \mathrm{g}$ & $5.22 \mathrm{E}-05$ & & $2.95 \mathrm{E}-05$ & & $2.72 \mathrm{E}-06$ & & $3.42 \mathrm{E}-06$ & \\
\hline & & & & & & & & \\
\hline DIL HTO & $5.9 \mathrm{E}-09$ & & & & & & & \\
\hline \multicolumn{9}{|l|}{$(\mathrm{Ci} / \mathrm{g})$} \\
\hline & & & & & & & & \\
\hline 168 & $1.53 \mathrm{E}+04$ & $2.63 \mathrm{E}+04$ & $1.55 \mathrm{E}+04$ & $1.30 \mathrm{E}+04$ & $4.68 \mathrm{E}+04$ & $2.81 \mathrm{E}+03$ & $5.12 \mathrm{E}+04$ & $3.51 \mathrm{E}+04$ \\
\hline SUM & $4.16 \mathrm{E}+04$ & & $2.85 \mathrm{E}+04$ & & $4.96 \mathrm{E}+04$ & & $8.63 \mathrm{E}+04$ & \\
\hline $\mathrm{Ci} / \mathrm{g}$ & $1.12 \mathrm{E}-06$ & & $7.69 \mathrm{E}-07$ & & 1.34E-06 & & 2.33E-06 & \\
\hline & & & & & & & & \\
\hline DIL OBT & $2.4 \mathrm{E}-09$ & & & & & & & \\
\hline \multicolumn{9}{|l|}{$\overline{\mathrm{Ci} / \mathrm{g}}$} \\
\hline & & & & . & & & & \\
\hline 8760 & $6.19 \mathrm{E}-01$ & $2.26 \mathrm{E}+00$ & $2.03 \mathrm{E}+03$ & $1.53 \mathrm{E}+04$ & $4.83 \mathrm{E}+00$ & $5.06 \mathrm{E}+01$ & $2.13 E+01$ & $6.85 \mathrm{E}+01$ \\
\hline SUM & $2.88 \mathrm{E}+00$ & & $1.73 \mathrm{E}+04$ & & $5.54 \mathrm{E}+01$ & & $8.97 \mathrm{E}+01$ & \\
\hline $\mathrm{Ci} / \mathrm{g}$ & $7.78 \mathrm{E}-11$ & & 4.69E-07 & & $1.50 \mathrm{E}-09$ & & $2.43 \mathrm{E}-09$ & \\
\hline & & & & & & & & \\
\hline DIL OBT & $2.4 \mathrm{E}-09$ & & & & & & & \\
\hline $\mathrm{Ci} / \mathrm{g}$ & & & & & & & & \\
\hline
\end{tabular}




\begin{tabular}{|c|c|c|c|c|c|c|c|c|}
\hline & \multicolumn{7}{|c|}{ Tritium Facilities, Beyond Design Basis Fire, Average Met Conditions } & \\
\hline & \multicolumn{4}{|c|}{ Concentration Values for $50 \mathrm{~km}$ from release } & & & & \\
\hline \multicolumn{9}{|c|}{ Only Wheat Seed is examined since this is the edible portion of the Wheat. The "WHEAT" category is } \\
\hline \multicolumn{7}{|c|}{ actually the unedible plant body and is not considered in the dose estimate. } & & \\
\hline & & & & & & & & \\
\hline & \multicolumn{2}{|c|}{ VEGETABLES } & \multicolumn{2}{|c|}{ WHEAT SEED } & \multicolumn{2}{|l|}{ MEAT } & \multicolumn{2}{|l|}{ MILK } \\
\hline \multirow[t]{3}{*}{ HOUR } & HTO & OBT & HTO & OBT & HTO & OBT & HTO & OBT \\
\hline & (B & $/ \mathrm{g})$ & $(\mathrm{Bq} / \mathrm{g})$ & $(\mathrm{Bq} / \mathrm{g})$ & ) & $(\mathrm{Bq} / \mathrm{g})$ & $(\mathrm{Bc}$ & $(\mathrm{Bq} / \mathrm{g})$ \\
\hline & & & & & & & & \\
\hline 1 & $6.85 \mathrm{E}+04$ & $0.00 \mathrm{E}+00$ & $0.00 \mathrm{E}+00$ & $0.00 \overline{\mathrm{E}}+00$ & $5.77 \mathrm{E}+01$ & $6.20 \mathrm{E}-03$ & $6.99 \mathrm{E}+00$ & $2.18 \mathrm{E}+00$ \\
\hline 2 & $9.98 \mathrm{E}+04$ & $1.17 \mathrm{E}+02$ & $7.36 \mathrm{E}+03$ & $2.43 \mathrm{E}+01$ & $6.55 \mathrm{E}+02$ & $1.34 \mathrm{E}-01$ & $7.67 \mathrm{E}+01$ & $3.16 \mathrm{E}+01$ \\
\hline 3 & $1.45 \mathrm{E}+05$ & $2.88 \mathrm{E}+02$ & $1.88 \mathrm{E}+04$ & $6.92 \mathrm{E}+01$ & $1.61 \mathrm{E}+03$ & $4.57 \mathrm{E}-01$ & $2.25 \mathrm{E}+02$ & $9.52 \mathrm{E}+01$ \\
\hline 4 & $1.32 \mathrm{E}+05$ & $5.95 \mathrm{E}+02$ & $3.89 \mathrm{E}+04$ & $1.54 \mathrm{E}+02$ & $3.25 \mathrm{E}+03$ & $1.12 \mathrm{E}+00$ & $4.91 \mathrm{E}+02$ & $2.11 \mathrm{E}+02$ \\
\hline 5 & $5.58 \mathrm{E}+05$ & $3.47 \mathrm{E}+03$ & $2.33 \mathrm{E}+05$ & $8.33 \mathrm{E}+02$ & $1.79 \mathrm{E}+04$ & $4.80 \mathrm{E}+00$ & $1.89 \mathrm{E}+03$ & $8.20 \mathrm{E}+02$ \\
\hline 6 & $2.58 \mathrm{E}+05$ & $4.62 \mathrm{E}+03$ & $3.25 \mathrm{E}+05$ & $1.36 \mathrm{E}+03$ & $1.79 \mathrm{E}+04$ & $6.72 \mathrm{E}+00$ & $1.73 \mathrm{E}+04$ & 5.4 \\
\hline 7 & $1.29 \mathrm{E}+05$ & $5.06 \mathrm{E}+03$ & $3.44 \mathrm{E}+05$ & $1.74 \mathrm{E}+03$ & $1.78 \mathrm{E}+04$ & $8.63 \mathrm{E}+00$ & $1.73 \mathrm{E}+04$ & $E+03$ \\
\hline 8 & $8.67 \mathrm{E}+04$ & $5.27 \mathrm{E}+03$ & $3.25 \mathrm{E}+05$ & $2.01 \mathrm{E}+03$ & $1.77 \mathrm{E}+04$ & $1.05 \mathrm{E}+01$ & $1.72 \mathrm{E}+04$ & $5.36 \mathrm{E}+03$ \\
\hline 9 & $7.06 \mathrm{E}+04$ & $5.42 \mathrm{E}+03$ & $2.89 \mathrm{E}+05$ & $2.20 \mathrm{E}+03$ & $1.75 \mathrm{E}+04$ & $1.24 \mathrm{E}+01$ & $1.72 \mathrm{E}+04$ & 5.3 \\
\hline 10 & $6.33 \mathrm{E}+04$ & $5.53 \mathrm{E}+03$ & $2.48 \mathrm{E}+05$ & $2.35 \mathrm{E}+03$ & $1.74 \mathrm{E}+04$ & $1.43 \mathrm{E}+01$ & $1.71 \mathrm{E}+04$ & $5.33 \mathrm{E}+03$ \\
\hline 11 & $5.91 \mathrm{E}+04$ & $5.63 E+03$ & $2.09 \mathrm{E}+05$ & $2.46 \mathrm{E}+03$ & $1.73 \mathrm{E}+04$ & $1.61 \mathrm{E}+01$ & $1.71 \mathrm{E}+04$ & $5.31 \mathrm{E}+03$ \\
\hline 12 & $5.62 \mathrm{E}+04$ & $5.72 \mathrm{E}+03$ & $1.74 \mathrm{E}+05$ & $2.54 \mathrm{E}+03$ & $1.72 \mathrm{E}+04$ & $1.80 \mathrm{E}+01$ & $1.70 \mathrm{E}+04$ & +05 \\
\hline 13 & $5.40 \mathrm{E}+04$ & $5.81 E+03$ & $1.44 \mathrm{E}+05$ & $2.61 \mathrm{E}+03$ & $1.71 \mathrm{E}+04$ & $1.98 \mathrm{E}+01$ & $1.69 \mathrm{E}+\overline{04}$ & $5.28 \mathrm{E}+03$ \\
\hline 14 & $5.20 \mathrm{E}+04$ & $5.90 \bar{E}+03$ & $1.19 \mathrm{E}+05$ & $2.67 \mathrm{E}+03$ & $1.69 \mathrm{E}+04$ & $2.16 \mathrm{E}+01$ & $1.69 \mathrm{E}+\overline{04}$ & $5.26 \mathrm{E}+03$ \\
\hline 15 & $5.03 E+04$ & $5.98 \mathrm{E}+03$ & $9.91 \mathrm{E}+04$ & $2.72 \mathrm{E}+03$ & $1.68 \mathrm{E}+04$ & $2.34 \mathrm{E}+01$ & $1.68 \mathrm{E}+04$ & $5.24 \mathrm{E}+03$ \\
\hline 16 & $4.88 \mathrm{E}+04$ & $6.06 \mathrm{E}+03$ & $8.33 \mathrm{E}+04$ & $2.76 \mathrm{E}+03$ & $1.67 \mathrm{E}+04$ & $2.52 \mathrm{E}+01$ & $1.68 \mathrm{E}+04$ & $5.22 \mathrm{E}+0$ \\
\hline 17 & $4.74 \mathrm{E}+04$ & $6.13 \mathrm{E}+03$ & $7.08 \mathrm{E}+04$ & $2.80 \overline{\mathrm{E}}+03$ & $1.66 \mathrm{E}+04$ & $2.69 \mathrm{E} \div 01$ & $1.67 \mathrm{E}+04$ & $5.20 \mathrm{E}+03$ \\
\hline 18 & $4.60 \mathrm{E}+04$ & $6.21 \mathrm{E}+03$ & $6.10 \mathrm{E}+04$ & $2.84 \mathrm{E}+03$ & $1.70 \mathrm{E}+04$ & $3.27 \mathrm{E}+01$ & $1.64 \mathrm{E}+04$ & $9.89 \mathrm{E}+03$ \\
\hline 19 & $4.48 \mathrm{E}+04$ & $6.28 \mathrm{E}+03$ & $5.33 \mathrm{E}+04$ & $2.87 \bar{E}+03$ & $1.73 \mathrm{E}+04$ & $3.85 \mathrm{E}+01$ & $1.66 \mathrm{E}+04$ & $9.97 \mathrm{E}+03$ \\
\hline 20 & $4.36 \mathrm{E}+04$ & $6.34 \mathrm{E}+03$ & $4.73 \mathrm{E}+04$ & $2.90 \overline{\mathrm{E}}+03$ & $1.77 \mathrm{E}+04$ & $4.44 \mathrm{E}+01$ & $1.68 \mathrm{E}+04$ & $1.01 \mathrm{E}+04$ \\
\hline 21 & $4.24 \mathrm{E}+04$ & $6.41 \mathrm{E}+03$ & $4.26 \mathrm{E}+04$ & $2.93 \mathrm{E}+03$ & $1.80 \mathrm{E}+04$ & $5.03 \mathrm{E}+01$ & $1.70 \mathrm{E}+04$ & $1.01 \mathrm{E}+04$ \\
\hline 22 & $4.13 \mathrm{E}+04$ & $6.48 \mathrm{E}+03$ & $3.88 \mathrm{E}+04$ & $2.96 \mathrm{E}+03$ & $1.83 \mathrm{E}+04$ & $5.63 \mathrm{E}+01$ & $1.71 \mathrm{E}+04$ & 1.02 \\
\hline 23 & $4.03 E+04$ & $6.54 \mathrm{E}+03$ & $3.58 E+04$ & $2.99 \bar{E}+03$ & $1.86 \mathrm{E}+04$ & $6.24 \mathrm{E}+01$ & $1.73 \mathrm{E}+04$ & $1.03 \mathrm{E}+04$ \\
\hline 24 & $3.92 \mathrm{E}+04$ & $6.60 \mathrm{E}+03$ & $3.34 \mathrm{E}+04$ & $3.01 \mathrm{E}+03$ & $1.89 \mathrm{E}+04$ & $6.86 \mathrm{E}+01$ & $1.74 \mathrm{E}+04$ & $1.03 E+04$ \\
\hline 25 & $3.82 \mathrm{E}+04$ & $6.66 \mathrm{E}+03$ & $3.15 \mathrm{E}+04$ & $3.04 \mathrm{E}+03$ & $1.92 \mathrm{E}+04$ & $7.48 \mathrm{E}+01$ & $1.76 \mathrm{E}+04$ & $1.04 \mathrm{E}+04$ \\
\hline 26 & $3.72 \mathrm{E}+04$ & $6.71 \mathrm{E}+03$ & $2.98 \mathrm{E}+04$ & $3.06 \mathrm{E}+03$ & $1.94 \mathrm{E}+04$ & $8.10 \mathrm{E}+01$ & $1.77 \mathrm{E}+04$ & $1.05 \mathrm{E}+04$ \\
\hline 27 & $3.62 \mathrm{E}+04$ & $6.77 \mathrm{E}+03$ & $2.84 \mathrm{E}+04$ & $3.09 \mathrm{E}+03$ & $1.97 \mathrm{E}+04$ & $8.73 E+01$ & $1.78 \mathrm{E}+04$ & $1.05 \mathrm{E}+04$ \\
\hline 28 & $3.53 \mathrm{E}+04$ & $6.82 \mathrm{E}+03$ & $2.71 \mathrm{E}+04$ & $3.11 \mathrm{E}+03$ & $1.99 \mathrm{E}+04$ & $9.36 \mathrm{E}+01$ & $1.80 \mathrm{E}+04$ & $1.06 \mathrm{E}+04$ \\
\hline 29 & $3.44 \mathrm{E}+04$ & $6.87 \mathrm{E}+03$ & $2.61 E+04$ & $3.14 \mathrm{E}+03$ & $2.01 \mathrm{E}+04$ & $1.00 \mathrm{E}+02$ & $1.81 \mathrm{E}+04$ & $1.07 \mathrm{E}+04$ \\
\hline 30 & $3.35 \mathrm{E}+04$ & $6.92 \mathrm{E}+03$ & $2.51 \mathrm{E}+04$ & $3.16 \mathrm{E}+03$ & $2.00 \mathrm{E}+04$ & $1.02 \mathrm{E}+02$ & $1.94 \mathrm{E}+\overline{04}$ & $6.05 \mathrm{E}+03$ \\
\hline 31 & $3.25 \mathrm{E}+04$ & $6.97 \mathrm{E}+03$ & $2.42 \mathrm{E}+04$ & $3.18 \mathrm{E}+03$ & $1.98 \mathrm{E}+04$ & $1.04 \mathrm{E}+02$ & $1.93 \mathrm{E}+\overline{04}$ & $6.02 E+03$ \\
\hline 32 & $3.16 \mathrm{E}+04$ & $7.02 \mathrm{E}+03$ & $2.33 \mathrm{E}+04$ & $3.20 \mathrm{E}+03$ & $1.97 \mathrm{E}+04$ & $1.06 \mathrm{E}+02$ & $1.92 \mathrm{E}+04$ & $6.00 \mathrm{E}+03$ \\
\hline 33 & $3.08 \mathrm{E}+04$ & $7.06 \mathrm{E}+03$ & $2.25 \mathrm{E}+04$ & $3.22 \mathrm{E}+03$ & $1.95 \mathrm{E}+04$ & $1.08 \mathrm{E}+02$ & $1.91 \mathrm{E}+04$ & $5.98 \mathrm{E}+03$ \\
\hline 34 & $2.98 \mathrm{E}+04$ & $7.10 \mathrm{E}+03$ & $2.18 \mathrm{E}+04$ & $3.24 \mathrm{E}+03$ & $1.94 \mathrm{E}+04$ & $1.10 \mathrm{E}+02$ & $1.91 \mathrm{E}+04$ & $5.95 \mathrm{E}+03$ \\
\hline 35 & $2.89 \mathrm{E}+04$ & $7.15 \mathrm{E}+03$ & $2.10 \mathrm{E}+04$ & $3.26 \mathrm{E}+03$ & $1.92 \mathrm{E}+04$ & $1.12 \mathrm{E}+02$ & $1.90 \mathrm{E}+04$ & $5.93 \mathrm{E}+03$ \\
\hline 36 & $2.81 \mathrm{E}+04$ & $7.19 \mathrm{E}+03$ & $2.03 \mathrm{E}+04$ & $3.27 \mathrm{E}+03$ & $1.91 \mathrm{E}+04$ & $1.14 \mathrm{E}+02$ & $1.89 \mathrm{E}+04$ & $5.91 \mathrm{E}+03$ \\
\hline 37 & $2.73 \mathrm{E}+04$ & $7.22 \mathrm{E}+03$ & $1.96 \mathrm{E}+04$ & $3.29 \mathrm{E}+03$ & $1.89 \mathrm{E}+04$ & $1.16 \mathrm{E}+02$ & $1.88 \mathrm{E}+04$ & $5.88 \mathrm{E}+03$ \\
\hline 38 & $2.66 \mathrm{E}+04$ & $7.26 \mathrm{E}+03$ & $1.89 \mathrm{E}+04$ & $3.31 \mathrm{E}+03$ & $1.88 \mathrm{E}+04$ & $1.18 \mathrm{E}+02$ & $1.88 \mathrm{E}+04$ & $5.86 \mathrm{E}+03$ \\
\hline
\end{tabular}


Foodstuff Concentrations and Relocation Considerations Following

\begin{tabular}{|c|c|c|c|c|c|c|c|c|}
\hline 39 & $2.59 \mathrm{E}+04$ & $7.30 \mathrm{E}+03$ & $1.83 \mathrm{E}+04$ & $3.32 \mathrm{E}+03$ & $1.86 \mathrm{E}+04$ & $1.20 \mathrm{E}+02$ & $1.87 \mathrm{E}+04$ & $5.84 \mathrm{E}+0$ \\
\hline 40 & $2.52 \mathrm{E}+04$ & $7.33 \mathrm{E}+03$ & $1.77 \mathrm{E}+04$ & $3.34 \mathrm{E}+03$ & $1.85 \mathrm{E}+04$ & $1.22 \mathrm{E}+02$ & $1.86 \mathrm{E}+04$ & $5.82 \mathrm{E}+0$ \\
\hline 41 & $2.46 \mathrm{E}+04$ & $7.37 \mathrm{E}+03$ & $1.71 \mathrm{E}+04$ & $3.36 \mathrm{E}+03$ & $1.83 \mathrm{E}+04$ & $1.24 \mathrm{E}+02$ & $1.85 \mathrm{E}+04$ & $5.79 \mathrm{E}+$ \\
\hline 42 & $2.40 \mathrm{E}+04$ & $7.40 \mathrm{E}+03$ & $1.65 \mathrm{E}+04$ & $3.37 \mathrm{E}+03$ & $1.84 \mathrm{E}+04$ & $1 . \overline{30 \mathrm{E}+02}$ & $1.79 \mathrm{E}+04$ & $1.12 \mathrm{E}+\mathrm{C}$ \\
\hline 43 & $2.34 \mathrm{E}+04$ & $7.43 \mathrm{E}+03$ & $1.60 \mathrm{E}+04$ & $3.38 \mathrm{E}+03$ & $1.85 \mathrm{E}+04$ & $1.37 \mathrm{E}+02$ & $1.79 \mathrm{E}+04$ & $1.12 \mathrm{E}-\mathrm{T}$ \\
\hline 44 & $2.29 \mathrm{E}+04$ & $7.46 \mathrm{E}+03$ & $1.55 \mathrm{E}+04$ & $3.40 \mathrm{E}+03$ & $1.86 \mathrm{E}+04$ & $1.43 \mathrm{E}+02$ & $1.80 \mathrm{E}+04$ & $1.12 \mathrm{E}+$ \\
\hline 45 & $2.24 \mathrm{E}+04$ & $7.49 \mathrm{E}+03$ & $1.50 \mathrm{E}+04$ & $3.41 \mathrm{E}+03$ & $1.87 \mathrm{E}+04$ & $1.50 \mathrm{E}+02$ & $1.80 \mathrm{E}+04$ & $1.13 \mathrm{E}+0$ \\
\hline 46 & $2.19 \mathrm{E}+04$ & $7.52 \mathrm{E}+03$ & $1.45 \mathrm{E}+04$ & $3.42 \mathrm{E}+03$ & $1.88 \mathrm{E}+04$ & $1.57 E+02$ & $1.81 \mathrm{E}+04$ & $1.13 \mathrm{E}+$ \\
\hline 47 & $2.13 \mathrm{E}+04$ & $7.54 \mathrm{E}+03$ & $1.41 \mathrm{E}+04$ & $3.44 \mathrm{E}+03$ & $1.89 \mathrm{E}+04$ & $1.63 \mathrm{E}+02$ & $1.81 \mathrm{E}+04$ & $1.13 \mathrm{E}+\mathrm{C}$ \\
\hline 48 & $2.08 \mathrm{E}+04$ & $7.57 \mathrm{E}+03$ & $1.36 \mathrm{E}+04$ & $3.45 \mathrm{E}+03$ & $1.90 \mathrm{E}+04$ & $1.70 \mathrm{E}+02$ & $1.81 \mathrm{E}+04$ & $1.13 \mathrm{E}+\mathrm{c}$ \\
\hline 49 & $2.03 E+04$ & $7.60 \mathrm{E}+03$ & $1.32 \mathrm{E}+04$ & $3.46 \mathrm{E}+03$ & $1.90 \mathrm{E}+04$ & $1.76 \mathrm{E}+02$ & $1.82 \mathrm{E}+04$ & $1.14 \mathrm{E}+0$ \\
\hline 50 & $1.99 \mathrm{E}+04$ & $7.62 \mathrm{E}+03$ & $1.28 \mathrm{E}+04$ & $3.47 \mathrm{E}+03$ & $1.91 \mathrm{E}+04$ & $1.83 \mathrm{E}+02$ & $1.82 \mathrm{E}+04$ & $1 . \overline{14 \mathrm{E}+0}$ \\
\hline 51 & $1.96 \mathrm{E}+04$ & $7.65 \mathrm{E}+03$ & $1.24 \mathrm{E}+04$ & $3.48 \mathrm{E}+03$ & $1.92 \mathrm{E}+04$ & $1.90 \mathrm{E}+02$ & $1.83 \mathrm{E}+04$ & $1.14 \mathrm{E}+0$ \\
\hline 52 & $1.92 \mathrm{E}+04$ & $7.67 \mathrm{E}+03$ & $1.21 \mathrm{E}+04$ & $3.49 \mathrm{E}+03$ & $1.92 \mathrm{E}+04$ & $1.96 \mathrm{E}+02$ & $1.83 \mathrm{E}+04$ & $1.14 \mathrm{E}+04$ \\
\hline 53 & $1.89 \mathrm{E}+04$ & $7.69 \mathrm{E}+03$ & $1.17 \mathrm{E}+04$ & $3.50 \mathrm{E}+03$ & $1.93 \mathrm{E}+04$ & $2.03 \mathrm{E}+02$ & $1.83 \mathrm{E}+04$ & $1.14 \mathrm{E}+0$ \\
\hline 54 & $1.86 \mathrm{E}+04$ & $7.71 \mathrm{E}+03$ & $1.14 \mathrm{E}+04$ & $3.51 \mathrm{E}+03$ & $1.91 \mathrm{E}+04$ & $2.05 \mathrm{E}+02$ & $1.85 \mathrm{E}+04$ & $5.81 \mathrm{E}+0$ \\
\hline 55 & $1.83 \bar{E}+04$ & $7.74 \mathrm{E}+03$ & $1.11 \mathrm{E}+04$ & $3.52 \mathrm{E}+03$ & $1.90 \mathrm{E}+04$ & $2.07 \mathrm{E}+02$ & $1.84 \mathrm{E}+04$ & $5.78 \mathrm{E}+0$ \\
\hline 56 & $1.81 \mathrm{E}+04$ & $7.76 \mathrm{E}+03$ & $1.08 \mathrm{E}+04$ & $3.53 \mathrm{E}+03$ & $1.88 \mathrm{E}+04$ & $2.09 E+02$ & $1.84 \mathrm{E}+04$ & $5.76 \mathrm{E}+0$ \\
\hline 57 & $1.78 \mathrm{E}+04$ & $7.78 \mathrm{E}+03$ & $1.06 \mathrm{E}+04$ & $3.54 \mathrm{E}+03$ & $1.86 \mathrm{E}+04$ & $2.11 \mathrm{E}+02$ & $1.83 \mathrm{E}+04$ & $5.74 \mathrm{E}+0$ \\
\hline 58 & $1.7 \overline{\mathrm{E}}+04$ & $7.80 \mathrm{E}+03$ & $1.03 E+04$ & $3.55 \mathrm{E}+03$ & $1.85 \mathrm{E}+04$ & $2.12 \mathrm{E}+02$ & $1.82 \mathrm{E}+04$ & $5.71 \mathrm{E}+0$ \\
\hline 59 & $1.72 \mathrm{E}+04$ & $7.82 \mathrm{E}+03$ & $1.01 \mathrm{E}+04$ & $3.56 \mathrm{E}+03$ & $1.83 \mathrm{E}+04$ & $2.14 \mathrm{E}+02$ & $1.81 \mathrm{E}+04$ & $5.69 \mathrm{E}+0$ \\
\hline 60 & $1.70 \mathrm{E}+04$ & $7.84 \mathrm{E}+03$ & $9.87 \mathrm{E}+03$ & $3.57 \mathrm{E}+03$ & $1.82 \mathrm{E}+04$ & $2.16 \mathrm{E}+02$ & $1.81 \mathrm{E}+04$ & $5.67 \mathrm{E}+0$ \\
\hline 61 & $1.68 \mathrm{E}+04$ & $7.85 \mathrm{E}+03$ & $9.66 \mathrm{E}+03$ & $3.58 \mathrm{E}+03$ & $1.80 \mathrm{E}+04$ & $2.18 \mathrm{E}+02$ & $1.80 \mathrm{E}+04$ & $\overline{5.65 \mathrm{E}+0}$ \\
\hline 62 & $1.65 \mathrm{E}+04$ & $7.87 \mathrm{E}+03$ & $9.46 \mathrm{E}+03$ & $3.59 \mathrm{E}+03$ & $1.79 E+04$ & $2.20 \mathrm{E}+02$ & $1.79 \mathrm{E}+04$ & $5.62 \mathrm{E}+0$ \\
\hline 63 & $1.63 E+04$ & $7.89 \mathrm{E}+03$ & $9.27 \mathrm{E}+03$ & $3.60 \mathrm{E}+03$ & $1.78 \mathrm{E}+04$ & $2.21 \mathrm{E}+02$ & $1.79 \mathrm{E}+04$ & $5.60 \mathrm{E}+0$ \\
\hline 64 & $1.61 \mathrm{E}+04$ & $7.91 \mathrm{E}+03$ & $9.08 \mathrm{E}+03$ & $3.61 \mathrm{E}+03$ & $1.76 \mathrm{E}+04$ & $2.23 \mathrm{E}+02$ & $1.78 \mathrm{E}+04$ & $5.58 \mathrm{E}+0$ \\
\hline 65 & $1.60 \mathrm{E}+04$ & $7.93 \mathrm{E}+03$ & $8.91 \mathrm{E}+03$ & $3.61 \mathrm{E}+03$ & $1.75 \mathrm{E}+04$ & $2.25 \mathrm{E}+02$ & $1.77 \mathrm{E}+04$ & $5.56 \mathrm{E}+0$ \\
\hline 66 & $1.58 \mathrm{E}+04$ & $7.94 \mathrm{E}+03$ & $8.74 \mathrm{E}+03$ & $3.62 \mathrm{E}+03$ & $1.75 \mathrm{E}+04$ & $2.31 \mathrm{E}+02$ & $1.70 \mathrm{E}+04$ & $1.12 \mathrm{E}+0$ \\
\hline 67 & $1.57 \mathrm{E}+04$ & $7.96 \mathrm{E}+03$ & $8.59 \mathrm{E}+03$ & $3.63 \mathrm{E}+03$ & $1.75 \mathrm{E}+04$ & $2.38 \mathrm{E}+02$ & $1.70 \mathrm{E}+04$ & $1.13 \mathrm{E}+0$ \\
\hline 68 & $1.55 \mathrm{E}+04$ & $7.98 \mathrm{E}+03$ & $8.45 \mathrm{E}+03$ & $3.64 \mathrm{E}+03$ & $1.75 \mathrm{E}+04$ & $2.45 \mathrm{E}+02$ & $1.70 \mathrm{E}+04$ & $1.13 \mathrm{E}+0$ \\
\hline 69 & $1.54 \mathrm{E}+04$ & $7.99 \mathrm{E}+03$ & $8.32 \mathrm{E}+03$ & $3.65 \mathrm{E}+03$ & $1.76 \mathrm{E}+04$ & $2.51 \mathrm{E}+02$ & $1.70 \mathrm{E}+04$ & $1.13 \mathrm{E}+0$ \\
\hline 168 & $4.75 \mathrm{E}+03$ & $8.32 \mathrm{E}+03$ & $4.92 \mathrm{E}+03$ & $4.15 \mathrm{E}+03$ & $1.22 \mathrm{E}+04$ & $7.57 \mathrm{E}+02$ & $1.31 \mathrm{E}+04$ & $1.02 \mathrm{E}+0$ \\
\hline 336 & $2.34 \mathrm{E}+03$ & $7.08 \mathrm{E}+03$ & $2.46 \mathrm{E}+03$ & $4.51 \mathrm{E}+03$ & $5.80 \mathrm{E}+03$ & $1.50 \mathrm{E}+03$ & $8.36 \mathrm{E}+03$ & $8.05 \mathrm{E}+0$ \\
\hline 504 & $1.72 \mathrm{E}+03$ & $5.93 \mathrm{E}+0 \overline{3}$ & $1.68 \mathrm{E}+03$ & $4.73 \mathrm{E}+03$ & $3.06 \mathrm{E}+03$ & $1.92 \mathrm{E}+03$ & $4.12 \mathrm{E}+03$ & $5.65 \mathrm{E}+0$ \\
\hline 720 & $1.25 \mathrm{E}+03$ & $4.69 \mathrm{E}+03$ & $1.13 \mathrm{E}+03$ & $4.92 \mathrm{E}+03$ & $1.60 \mathrm{E}+03$ & $2.17 \mathrm{E}+03$ & $2.17 \mathrm{E}+03$ & $4.00 \mathrm{E}+03$ \\
\hline 2160 & $2.33 \mathrm{E}+02$ & $9.47 \mathrm{E}+02$ & $1.26 \mathrm{E}+02$ & $5.30 \mathrm{E}+03$ & $1.40 \mathrm{E}+02$ & $1.32 \mathrm{E}+03$ & $5.00 \mathrm{E}+02$ & $1.38 \mathrm{E}+03$ \\
\hline 4320 & $2.24 \mathrm{E}+01$ & $8.67 \mathrm{E}+01$ & $6.36 \mathrm{E}+02$ & $5.00 \mathrm{E}+03$ & $2.36 \mathrm{E}+01$ & $3.18 \mathrm{E}+02$ & $5.98 \mathrm{E}+01$ & $2.15 \mathrm{E}+0$ \\
\hline 4800 & $1.33 \mathrm{E}+01$ & $5.11 \mathrm{E}+01$ & $6.34 \mathrm{E}+02$ & $4.98 \mathrm{E}+03$ & $1.72 \mathrm{E}+01$ & $2.29 \mathrm{E}+02$ & $2.01 \mathrm{E}+01$ & $7.03 \mathrm{E}+0$ \\
\hline 8760 & $1.83 \mathrm{E}-01$ & $6.73 \mathrm{E}-01$ & $6.18 \mathrm{E}+02$ & $4.85 \mathrm{E}+03$ & $1.43 \mathrm{E}+00$ & $1.54 \mathrm{E}+01$ & $6.32 \mathrm{E}+00$ & $2.08 \mathrm{E}+0$ \\
\hline D) & $\begin{array}{l}\mathrm{4E6} \\
\mathrm{i} / \mathrm{L} \\
\end{array}$ & $\begin{array}{l}8.88 \mathrm{E}+4 \\
\mathrm{~Bq} / \mathrm{kg} \\
\end{array}$ & $8 \mathrm{~Bq} / \mathrm{g}$ & & & & & \\
\hline ro & $\begin{array}{l}5.9 \overline{\mathrm{E} 6} \\
\mathrm{pCi} / \mathrm{L}\end{array}$ & $\begin{array}{l}2.18 \mathrm{E}+5 \\
\mathrm{~Bq} / \mathrm{kg} \\
\end{array}$ & $218.3 \mathrm{~Bq} / \mathrm{g}$ & & & & & \\
\hline & \multirow{2}{*}{\multicolumn{2}{|c|}{ VEGETABLES }} & & & \multirow{2}{*}{\multicolumn{2}{|c|}{ MEAT }} & & \\
\hline & & & \multicolumn{2}{|c|}{ WHEAT SEED } & & & \multicolumn{2}{|l|}{ MILK } \\
\hline \multirow[t]{2}{*}{ HOUR } & ro & OBT & HTO & OBT & HTO & BT & HTO & OBT \\
\hline & $(\mathrm{Bq} / \mathrm{g})$ & $(\mathrm{Bq} / \mathrm{g})$ & $(\mathrm{Bq} / \mathrm{g})$ & $(\mathrm{Bq} / \mathrm{g})$ & $(\mathrm{Bq} / \mathrm{g})$ & $(\mathrm{Bq} / \mathrm{g})$ & $(\mathrm{Bq} / \mathrm{g})$ & $(\mathrm{Bq} / \mathrm{g})$ \\
\hline eak $\mathrm{HTO}$ & & & & & & & & \\
\hline
\end{tabular}


a Tritium Oxide Release from SRS Tritium Facilities (U)

\begin{tabular}{|c|c|c|c|c|c|c|c|c|}
\hline $5 / 7 / 29 / 30$ & $5.58 \mathrm{E}+05$ & $3.47 \mathrm{E}+03$ & $3.44 \mathrm{E}+05$ & $1.74 \mathrm{E}+03$ & $2.01 E+04$ & $1.00 \mathrm{E}+02$ & $1.94 \mathrm{E}+04$ & $6.05 \mathrm{E}+03$ \\
\hline$\overline{\text { SUM }}$ & $5.61 \mathrm{E}+05$ & & $3.46 \mathrm{E}+05$ & & $2.02 E+04$ & & $2.54 \mathrm{E}+04$ & \\
\hline $\mathrm{Ci} / \mathrm{g}$ & 1.52E-05 & & $9.34 \mathrm{E}-06$ & & $5.46 \mathrm{E}-07$ & & $6.86 \mathrm{E}-07$ & \\
\hline & & & & & & & & \\
\hline DIL HTO & $5.9 \mathrm{E}-09$ & & & & & & & \\
\hline \multicolumn{9}{|l|}{$(\mathrm{Ci} / \mathrm{g})$} \\
\hline & & & & & & & & \\
\hline 168 & $4.75 \mathrm{E}+03$ & $8.32 \mathrm{E}+03$ & $4.92 \mathrm{E}+03$ & $4.15 \mathrm{E}+03$ & $1.22 \mathrm{E}+04$ & $7.57 \mathrm{E}+02$ & $1.31 \mathrm{E}+04$ & $1.02 \mathrm{E}+04$ \\
\hline SUM & $1.31 \mathrm{E}+04$ & & $9.07 \mathrm{E}+03$ & & $1.30 \mathrm{E}+04$ & & $2.32 \mathrm{E}+04$ & \\
\hline $\mathrm{Ci} / \mathrm{g}$ & $3.53 \mathrm{E}-07$ & & 2.45E-07 & & 3.50E-07 & & $6.27 \mathrm{E}-07$ & \\
\hline & & & & & & & & \\
\hline DIL OBT & $2.4 \mathrm{E}-09$ & & & & & & & \\
\hline \multicolumn{9}{|l|}{$\mathrm{Ci} / \mathrm{g}$} \\
\hline & & & & & & & & \\
\hline 8760 & $1.83 \mathrm{E}-01$ & $6.73 \mathrm{E}-01$ & $6.18 \mathrm{E}+02$ & $4.85 \mathrm{E}+03$ & $1.43 \mathrm{E}+00$ & $1.54 \mathrm{E}+01$ & $6.32 \mathrm{E}+00$ & $2.08 \mathrm{E}+01$ \\
\hline SUM & $8.56 \mathrm{E}-01$ & & $5.47 \mathrm{E}+03$ & & $1.68 \mathrm{E}+01$ & & $2.71 \mathrm{E}+01$ & \\
\hline $\mathrm{Ci} / \mathrm{g}$ & $2.31 \mathrm{E}-11$ & & $1.48 \mathrm{E}-07$ & & $4.55 \mathrm{E}-10$ & & $7.33 \mathrm{E}-10$ & \\
\hline & & & & & & & & \\
\hline DIL OBT & $2.4 \mathrm{E}-09$ & & & & & & & \\
\hline $\mathrm{Ci} / \mathrm{g}$ & & & & & & & & \\
\hline
\end{tabular}




\begin{tabular}{|c|c|c|c|c|c|c|c|c|}
\hline & \multicolumn{7}{|c|}{ Tritium Facilities, Beyond Design Basis Fire, Average Met Conditions } & \\
\hline & \multicolumn{4}{|c|}{ Concentration Values for $100 \mathrm{~km}$ from release } & & & & \\
\hline \multicolumn{9}{|c|}{ Only Wheat Seed is examined since this is the edible portion of the Wheat. The "WHEAT" category is } \\
\hline \multicolumn{9}{|c|}{ ctually the unedible plant body and is not considered in the dose estimate. } \\
\hline & & & & & & & & \\
\hline & \multicolumn{2}{|c|}{ VEGETABLES } & \multicolumn{2}{|c|}{ WHEAT SEED } & \multicolumn{2}{|l|}{ MEAT } & \multicolumn{2}{|l|}{ MILK } \\
\hline \multirow[t]{3}{*}{ HOUR } & HTO & OBT & HTO & OBT & HTO & OBT & HTO & OBT \\
\hline & $(\mathrm{Bq} / \mathrm{g})$ & $(\mathrm{Bq} / \mathrm{g})$ & $(\mathrm{Bq} / \mathrm{g})$ & $(\mathrm{Bq} / \mathrm{g})$ & $(\mathrm{Bq} / \mathrm{g})$ & $(\mathrm{Bq} / \mathrm{g})$ & $(\mathrm{Bq} / \mathrm{g})$ & $(\mathrm{Bq} / \mathrm{g})$ \\
\hline & & & & & & & & \\
\hline 1 & $1.11 \mathrm{E}+04$ & $0.00 \mathrm{E}+00$ & $0.00 \mathrm{E}+00$ & $0.00 \mathrm{E}+00$ & $9.34 \mathrm{E}+00$ & $1.01 \mathrm{E}-03$ & $1.13 \mathrm{E}+00$ & $3.53 \mathrm{E}-0$ \\
\hline 2 & $1.90 \mathrm{E}+04$ & $1.90 \mathrm{E}+01$ & $1.19 \mathrm{E}+03$ & $3.94 \mathrm{E}+00$ & $1.09 \mathrm{E}+02$ & $2.20 \mathrm{E}-02$ & $1.27 \mathrm{E}+01$ & $5.20 \mathrm{E}+0$ \\
\hline 3 & $3.06 \mathrm{E}+04$ & $5.16 \mathrm{E}+01$ & $3.35 \mathrm{E}+03$ & $1.22 \mathrm{E}+01$ & $2.91 \mathrm{E}+02$ & $7.99 \mathrm{E}-02$ & $3.96 \mathrm{E}+01$ & $1.67 E+c$ \\
\hline 4 & $3.08 \mathrm{E}+04$ & $1.04 \mathrm{E}+02$ & $6.77 \mathrm{E}+03$ & $2.68 \mathrm{E}+01$ & $5.75 E+02$ & $1.97 \mathrm{E}-01$ & $8.66 \bar{E}+01$ & $3.71 \mathrm{E}+0$ \\
\hline 5 & $4 . \overline{1 E}+04$ & $1.57 \mathrm{E}+02$ & $1.01 \mathrm{E}+04$ & $4.44 \mathrm{E}+01$ & $8.94 \mathrm{E}+02$ & $3.80 \mathrm{E}-01$ & $1.52 \mathrm{E}+02$ & $6.53 \mathrm{E}+0$ \\
\hline 6 & $4.39 \mathrm{E}+04$ & $2.37 \mathrm{E}+02$ & $1.49 \mathrm{E}+04$ & $7.00 \mathrm{E}+01$ & $9.12 \mathrm{E}+02$ & $4.78 \mathrm{E}-01$ & $8.84 \mathrm{E}+02$ & $2.75 \mathrm{E}+02$ \\
\hline 7 & $5.97 \mathrm{E}+04$ & $3.12 \mathrm{E}+02$ & $1.90 \mathrm{E}+04$ & $9.80 \mathrm{E}+01$ & $9.44 \mathrm{E}+02$ & $5.79 \mathrm{E}-01$ & $8.99 \mathrm{E}+02$ & $2.80 \mathrm{E}+02$ \\
\hline 8 & $6.21 \mathrm{E}+04$ & $4.19 E+02$ & $2.46 \mathrm{E}+04$ & $1.35 \mathrm{E}+02$ & $9.75 \mathrm{E}+02$ & $6.84 \mathrm{E}-01$ & $9.15 \mathrm{E}+02$ & $2.85 \bar{E}+0$ \\
\hline 9 & $1.46 \mathrm{E}+05$ & $1.06 \mathrm{E}+03$ & $6.70 \mathrm{E}+04$ & $2.98 \mathrm{E}+02$ & $1.26 \mathrm{E}+03$ & $8.19 \mathrm{E}-01$ & $9.92 \mathrm{E}+02$ & $3.09 \mathrm{E}+0$ \\
\hline 10 & $2.16 \mathrm{E}+05$ & $2.13 \mathrm{E}+03$ & $1.41 \mathrm{E}+05$ & $6.09 E+02$ & $1.69 \mathrm{E}+03$ & $1.00 \mathrm{E}+00$ & $1.12 \mathrm{E}+03$ & $3.49 \overline{\mathrm{E}}+02$ \\
\hline 11 & $9.51 \mathrm{E}+04$ & $2.50 \mathrm{E}+03$ & $1.67 \mathrm{E}+05$ & $8.31 \mathrm{E}+02$ & $1.70 \mathrm{E}+03$ & $1.18 \mathrm{E}+00$ & $1.21 \mathrm{E}+03$ & $3.76 \mathrm{E}+0$ \\
\hline 12 & $5.70 \mathrm{E}+04$ & $2.66 \mathrm{E}+03$ & $1.67 \mathrm{E}+05$ & $9.90 \mathrm{E}+02$ & $1.70 \mathrm{E}+03$ & $1.36 \mathrm{E}+00$ & $1.27 \mathrm{E}+03$ & $3.96 \mathrm{E}+0$ \\
\hline 13 & $4.39 \mathrm{E}+04$ & $2.75 \mathrm{E}+03$ & $1.53 \mathrm{E}+05$ & $1.11 \mathrm{E}+03$ & $1.71 \mathrm{E}+03$ & $1.55 \mathrm{E}+00$ & $1.32 \mathrm{E}+03$ & $4.11 \mathrm{E}+02$ \\
\hline 14 & $3.87 \mathrm{E}+04$ & $2.82 \mathrm{E}+03$ & $1.34 \mathrm{E}+05$ & $1.19 \mathrm{E}+03$ & $1.71 \mathrm{E}+03$ & $1 . \overline{73 \mathrm{E}+00}$ & $1.36 \mathrm{E}+03$ & $4.23 \mathrm{E}+0$ \\
\hline 15 & $3.62 \mathrm{E}+04$ & $2.88 \mathrm{E}+03$ & $1.15 \mathrm{E}+05$ & $1.26 \mathrm{E}+03$ & $1.71 \mathrm{E}+03$ & $1.91 \mathrm{E}+00$ & $1.39 \mathrm{E}+03$ & $4.32 \overline{\mathrm{E}+0}$ \\
\hline 16 & $3.46 \mathrm{E}+04$ & $2.94 \mathrm{E}+03$ & $9.70 \mathrm{E}+04$ & $1.31 \mathrm{E}+03$ & $1.71 \mathrm{E}+03$ & $2.10 \mathrm{E}+00$ & $1.41 \mathrm{E}+03$ & $4.40 \mathrm{E}+0$ \\
\hline 17 & $3.35 \mathrm{E}+04$ & $3.00 \mathrm{E}+03$ & $8.14 \mathrm{E}+04$ & $1.35 \mathrm{E}+03$ & $1.71 \mathrm{E}+03$ & $2.28 \mathrm{E}+00$ & $1.43 \mathrm{E}+03$ & $4.46 \mathrm{E}+02$ \\
\hline 18 & $3.26 \mathrm{E}+04$ & $3.05 E+03$ & $6.83 \mathrm{E}+04$ & $1.39 \mathrm{E}+03$ & $2.08 \mathrm{E}+03$ & $4.43 \mathrm{E}+00$ & $2.01 \mathrm{E}+03$ & $2.96 \mathrm{E}+0$ \\
\hline 19 & $3.18 \mathrm{E}+04$ & $3.10 \mathrm{E}+03$ & $5.77 \mathrm{E}+04$ & $1.42 \mathrm{E}+03$ & $2.42 \mathrm{E}+03$ & $6.64 \mathrm{E}+00$ & $2.18 \mathrm{E}+03$ & $3.04 \mathrm{E}+0$ \\
\hline 20 & $3.11 \mathrm{E}+04$ & $3.15 \mathrm{E}+03$ & $4.92 E+04$ & $1.45 \mathrm{E}+03$ & $2.75 E+03$ & $8.92 \mathrm{E}+00$ & $2.34 \mathrm{E}+03$ & $3.11 \mathrm{E}+0$ \\
\hline 21 & $3.04 \mathrm{E}+04$ & $3.20 \mathrm{E}+03$ & $4.24 \mathrm{E}+04$ & $1.47 \mathrm{E}+03$ & $3.07 \mathrm{E}+03$ & $1.13 \mathrm{E}+01$ & $2.50 \mathrm{E}+03$ & $3.17 \mathrm{E}+0$ \\
\hline 22 & $2.98 \mathrm{E}+04$ & $3.25 \mathrm{E}+03$ & $3.71 \mathrm{E}+04$ & $1.49 \mathrm{E}+03$ & $3.38 \mathrm{E}+03$ & $1.37 \mathrm{E}+01$ & $2.66 \mathrm{E}+03$ & $3.24 \mathrm{E}+\mathrm{C}$ \\
\hline 23 & $2.92 \mathrm{E}+04$ & $3.30 \mathrm{E}+03$ & $3.30 \mathrm{E}+04$ & $1.52 \mathrm{E}+03$ & $3.67 \mathrm{E}+03$ & $1.61 \mathrm{E}+01$ & $2.81 \mathrm{E}+03$ & $3.30 \mathrm{E}+0$ \\
\hline 24 & $2.87 \mathrm{E}+04$ & $3.34 \mathrm{E}+03$ & $2.98 \mathrm{E}+04$ & $1.54 \mathrm{E}+03$ & $3.96 \mathrm{E}+03$ & $1.86 \mathrm{E}+01$ & $2.95 \mathrm{E}+03$ & $3.37 \mathrm{E}+0$ \\
\hline 25 & $2.81 \mathrm{E}+04$ & $3.39 \mathrm{E}+03$ & $2.72 \mathrm{E}+04$ & $1.56 \mathrm{E}+03$ & $4.24 \mathrm{E}+03$ & $2.12 \mathrm{E}+01$ & $3.10 \mathrm{E}+03$ & $3.43 \mathrm{E}+0$ \\
\hline 26 & $2.76 \mathrm{E}+04$ & $3.43 \mathrm{E}+03$ & $2.52 \mathrm{E}+04$ & $1.58 \mathrm{E}+03$ & $4.51 \mathrm{E}+03$ & $2.38 \mathrm{E}+01$ & $3.24 \mathrm{E}+03$ & $3.49 \mathrm{E}+0$ \\
\hline 27 & $2.70 \mathrm{E}+04$ & $3.47 \mathrm{E}+03$ & $2.36 \mathrm{E}+04$ & $1.60 \mathrm{E}+03$ & $4.77 \mathrm{E}+03$ & $2.65 \mathrm{E}+01$ & $3.38 \mathrm{E}+03$ & $3.55 \overline{\mathrm{E}+0}$ \\
\hline 28 & $2.65 \mathrm{E}+04$ & $3.52 \mathrm{E}+03$ & $2.24 \mathrm{E}+04$ & $1.62 \mathrm{E}+03$ & $5.03 \mathrm{E}+03$ & $2.92 \mathrm{E}+01$ & $3.52 \mathrm{E}+03$ & $3.61 \mathrm{E}+0$ \\
\hline 29 & $2.60 \mathrm{E}+04$ & $3.56 \mathrm{E}+03$ & $2.13 \mathrm{E}+04$ & $1.63 \mathrm{E}+03$ & $5.27 \mathrm{E}+03$ & $3.20 \mathrm{E}+01$ & $3.65 \mathrm{E}+03$ & $3.66 \mathrm{E}+0$ \\
\hline 30 & $2.54 \mathrm{E}+04$ & $3.60 \mathrm{E}+03$ & $2.04 \mathrm{E}+04$ & $1.65 \mathrm{E}+03$ & $5.24 \mathrm{E}+03$ & $3 . \overline{25 \mathrm{E}+01}$ & $5.08 \mathrm{E}+03$ & $1.59 \mathrm{E}+03$ \\
\hline 31 & $2.49 \mathrm{E}+04$ & $3.63 \mathrm{E}+03$ & $1.97 \mathrm{E}+04$ & $1.67 \mathrm{E}+03$ & $5.21 \mathrm{E}+03$ & $3.31 \mathrm{E}+01$ & $5.06 \mathrm{E}+03$ & $1.58 \mathrm{E}+0$ \\
\hline 32 & $2.43 \mathrm{E}+04$ & $3.67 \mathrm{E}+03$ & $1.90 \mathrm{E}+04$ & $1.69 \mathrm{E}+03$ & $5.17 \mathrm{E}+03$ & $3.36 \mathrm{E}+01$ & $5.05 \mathrm{E}+03$ & $1.58 \mathrm{E}+0$ \\
\hline 33 & $2.38 \mathrm{E}+04$ & $3.71 \mathrm{E}+03$ & $1.84 \mathrm{E}+04$ & $1.70 \mathrm{E}+03$ & $5.14 \mathrm{E}+03$ & $3.41 \mathrm{E}+01$ & $5.03 E+03$ & $1.57 \mathrm{E}+03$ \\
\hline 34 & $2.32 \mathrm{E}+04$ & $3.74 \mathrm{E}+03$ & $1.79 \mathrm{E}+04$ & $1.72 \mathrm{E}+03$ & $5.11 \mathrm{E}+03$ & $3.46 \mathrm{E}+01$ & $5.01 \mathrm{E}+03$ & $1.57 \mathrm{E}+03$ \\
\hline 35 & $2.26 \mathrm{E}+04$ & $3.78 \mathrm{E}+03$ & $1.74 \mathrm{E}+04$ & $1.73 \mathrm{E}+03$ & $5.07 \mathrm{E}+03$ & $3.52 \mathrm{E}+01$ & $5.00 \mathrm{E}+03$ & $1.56 \mathrm{E}+03$ \\
\hline 36 & $2.21 \mathrm{E}+04$ & $3.81 \mathrm{E}+03$ & $1.69 \mathrm{E}+04$ & $1.75 \mathrm{E}+03$ & $5.04 \mathrm{E}+03$ & $3.57 \mathrm{E}+01$ & $4.98 \mathrm{E}+03$ & $1.56 \mathrm{E}+03$ \\
\hline 37 & $2.16 \mathrm{E}+04$ & $3.85 \mathrm{E}+03$ & $1.64 \mathrm{E}+04$ & $1.76 \mathrm{E}+03$ & $5.01 \mathrm{E}+03$ & $3.62 \mathrm{E}+01$ & $4.97 \mathrm{E}+03$ & $1.55 \mathrm{E}+03$ \\
\hline 38 & $2.10 \mathrm{E}+04$ & $3.88 \mathrm{E}+03$ & $1.60 \mathrm{E}+04$ & $1.78 \mathrm{E}+03$ & $4.97 \overline{\mathrm{E}+03}$ & $3.67 \mathrm{E}+01$ & $4.95 \mathrm{E}+03$ & $1.55 \mathrm{E}+0$ \\
\hline
\end{tabular}




\begin{tabular}{|c|c|c|c|c|c|c|c|c|}
\hline 39 & $2.05 \mathrm{E}+04$ & $3.91 \bar{E}+03$ & $1.55 \mathrm{E}+04$ & $1.79 \mathrm{E}+03$ & $4.94 \mathrm{E}+03$ & $3.72 \mathrm{E}+01$ & $4.93 \mathrm{E}+03$ & $1.54 \overline{\mathrm{E}+03}$ \\
\hline 40 & $2.00 \mathrm{E}+04$ & $3.94 \mathrm{E}+03$ & $1.51 \mathrm{E}+04$ & $1.81 \mathrm{E}+03$ & $4.90 \mathrm{E}+03$ & $3.77 \mathrm{E}+01$ & $4.92 \mathrm{E}+03$ & $1.54 \overline{\mathrm{E}+0}$ \\
\hline 41 & $1.95 \mathrm{E}+04$ & $3.97 \mathrm{E}+03$ & $1.47 \mathrm{E}+04$ & $1.82 \mathrm{E}+03$ & $4.87 \mathrm{E}+03$ & $3.82 \mathrm{E}+01$ & $4.90 \mathrm{E}+03$ & $1.53 \mathrm{E}+0$ \\
\hline 42 & $1.90 \mathrm{E}+04$ & $4.00 \mathrm{E}+03$ & $1.43 \mathrm{E}+04$ & $1.83 \mathrm{E}+03$ & $5.04 \mathrm{E}+03$ & $4.12 \mathrm{E}+01$ & $4.89 \mathrm{E}+03$ & $4.56 \mathrm{E}+\mathrm{C}$ \\
\hline 43 & $1.86 \mathrm{E}+04$ & $4.02 \mathrm{E}+03$ & $1.39 \mathrm{E}+04$ & $1.84 \mathrm{E}+03$ & $5.21 \mathrm{E}+03$ & $4.43 \mathrm{E}+01$ & $4.97 \mathrm{E}+03$ & $4.59 \mathrm{E}-$ \\
\hline 44 & $1.81 \mathrm{E}+04$ & $4.05 \mathrm{E}+03$ & $1.35 \mathrm{E}+04$ & $1.86 \mathrm{E}+03$ & $5.36 \mathrm{E}+03$ & $4.73 \mathrm{E}+01$ & $5.04 \mathrm{E}+03$ & $4.63 \mathrm{E}+0$ \\
\hline 45 & $1.77 \mathrm{E}+04$ & $4.08 \mathrm{E}+03$ & $1.31 \mathrm{E}+04$ & $1.87 \mathrm{E}+03$ & $5.52 \mathrm{E}+03$ & $5.04 \mathrm{E}+01$ & $5.12 \mathrm{E}+03$ & $4.66 \mathrm{E}+0$ \\
\hline 46 & $1.73 \mathrm{E}+04$ & $4.10 \mathrm{E}+03$ & $1.27 \mathrm{E}+04$ & $1.88 \mathrm{E}+03$ & $5.66 \mathrm{E}+03$ & $5.35 \mathrm{E}+0 \mathrm{i}$ & $5.19 E+03$ & $4.69 \mathrm{E}-$ \\
\hline 47 & $1.69 \mathrm{E}+04$ & $4.12 \mathrm{E}+03$ & $1.24 \mathrm{E}+04$ & $1.89 \mathrm{E}+03$ & $5.80 \mathrm{E}+03$ & $5.67 \mathrm{E}+01$ & $5.26 \mathrm{E}+03$ & $4.72 \mathrm{E}+0$ \\
\hline 48 & $1.65 \mathrm{E}+04$ & $4.15 \mathrm{E}+03$ & $1.20 \mathrm{E}+04$ & $1.90 \mathrm{E}+03$ & $5.94 \mathrm{E}+03$ & $5.99 \mathrm{E}+01$ & $5.33 \mathrm{E}+03$ & $4.75 \mathrm{E}+0$ \\
\hline 49 & $1.61 \mathrm{E}+04$ & $4.17 \mathrm{E}+03$ & $1.17 \mathrm{E}+04$ & $1.91 \mathrm{E}+03$ & $6.07 \mathrm{E}+03$ & $6.30 \mathrm{E}+01$ & $5.40 \mathrm{E}+03$ & $4.77 \mathrm{E}+\mathrm{C}$ \\
\hline 50 & $1.58 \mathrm{E}+04$ & $4.19 \mathrm{E}+03$ & $1.14 \mathrm{E}+04$ & $1.92 \mathrm{E}+03$ & $6.19 \mathrm{E}+03$ & $6.63 \mathrm{E}+01$ & $5.4 \overline{7} \mathrm{E}+03$ & $4.80 \overline{\mathrm{E}+c}$ \\
\hline 51 & $1.55 E+04$ & $4.21 \mathrm{E}+03$ & $1.11 \mathrm{E}+04$ & $1.93 \mathrm{E}+03$ & $6.31 \mathrm{E}+03$ & $6.95 \mathrm{E}+01$ & $5.53 \mathrm{E}+03$ & $4.83 \mathrm{E}+0$ \\
\hline 52 & $1.51 \mathrm{E}+04$ & $4.24 \mathrm{E}+03$ & $1.08 \mathrm{E}+04$ & $1.94 \mathrm{E}+03$ & $6.42 \mathrm{E}+03$ & $7.27 \mathrm{E}+01$ & $5.60 \mathrm{E}+03$ & $4.85 \mathrm{E}+0$ \\
\hline 53 & $1.49 \mathrm{E}+04$ & $4.26 \mathrm{E}+03$ & $1.05 \mathrm{E}+04$ & $1.95 \mathrm{E}+03$ & $6.53 \mathrm{E}+03$ & $7.60 \mathrm{E}+01$ & $5.66 \mathrm{E}+03$ & $4.88 \mathrm{E}+$ \\
\hline 54 & $1.46 \mathrm{E}+04$ & $4.28 \mathrm{E}+03$ & $1.02 \mathrm{E}+04$ & $1.96 \mathrm{E}+03$ & $6.48 \mathrm{E}+03$ & $7.67 \mathrm{E}+01$ & $6.28 \mathrm{E}+03$ & $1.97 \mathrm{E}+0$ \\
\hline 55 & $1.43 \mathrm{E}+04$ & $4.29 E+03$ & $9.93 \mathrm{E}+03$ & $1.97 \mathrm{E}+03$ & $6.43 \mathrm{E}+03$ & $7.73 \mathrm{E}+01$ & $6.26 \mathrm{E}+03$ & $1.96 \mathrm{E}+0$ \\
\hline 56 & $1.41 \mathrm{E}+04$ & $4.31 \mathrm{E}+03$ & $9.68 \mathrm{E}+03$ & $1.98 \mathrm{E}+03$ & $6.39 \mathrm{E}+03$ & $7.79 \mathrm{E}+01$ & $6.24 \mathrm{E}+03$ & $1.96 \mathrm{E}+$ \\
\hline 57 & $1.38 \mathrm{E}+04$ & $4.33 \mathrm{E}+03$ & $9.45 \mathrm{E}+03$ & $1.99 \mathrm{E}+03$ & $6.34 \mathrm{E}+03$ & $7.85 \mathrm{E}+01$ & $6.21 \mathrm{E}+03$ & $1.95 \mathrm{E}+\mathrm{C}$ \\
\hline 58 & $1 . \overline{36 \mathrm{E}+04}$ & $4.35 \mathrm{E}+03$ & $9.22 \mathrm{E}+03$ & $1.99 \mathrm{E}+03$ & $6.29 \mathrm{E}+03$ & $7.92 \mathrm{E}+01$ & $6.19 \mathrm{E}+03$ & $1.94 \mathrm{E}+0$ \\
\hline 59 & $1.34 \mathrm{E}+04$ & $4.37 \mathrm{E}+03$ & $9.01 \mathrm{E}+03$ & $2.00 \mathrm{E}+03$ & $6.24 \mathrm{E}+03$ & $7.98 \mathrm{E}+01$ & $6.17 \mathrm{E}+03$ & $1.94 \mathrm{E}+0$ \\
\hline 60 & $1.32 \mathrm{E}+04$ & $4.38 \mathrm{E}+03$ & $8.80 \mathrm{E}+03$ & $2.01 \mathrm{E}+03$ & $6.19 \mathrm{E}+03$ & $8.04 \mathrm{E}+01$ & $6.14 \mathrm{E}+03$ & $1.93 \mathrm{E}+0$ \\
\hline 61 & $1.30 \mathrm{E}+04$ & $4.40 \mathrm{E}+03$ & $8.61 \mathrm{E}+03$ & $2.02 \mathrm{E}+03$ & $6.14 \mathrm{E}+03$ & $8.10 \mathrm{E}+01$ & $6.12 \mathrm{E}+03$ & $1.92 \mathrm{E}+0$ \\
\hline 62 & $1.28 \mathrm{E}+04$ & $4.42 \mathrm{E}+03$ & $8.42 \mathrm{E}+03$ & $2.03 \mathrm{E}+03$ & $6.10 \mathrm{E}+03$ & $8.16 \mathrm{E}+01$ & $6 . \overline{10 E}+03$ & $1.91 \mathrm{E}+0$ \\
\hline 63 & $1.26 \mathrm{E}+04$ & $4.43 \mathrm{E}+03$ & $8.25 \mathrm{E}+03$ & $2.03 \mathrm{E}+03$ & $6.05 \mathrm{E}+03$ & $8.22 \mathrm{E}+01$ & $6.07 \mathrm{E}+03$ & $1.91 \mathrm{E}+0$ \\
\hline 64 & $1.24 \mathrm{E}+04$ & $4.45 \mathrm{E}+03$ & $8.08 \mathrm{E}+03$ & $2.04 \mathrm{E}+03$ & $6.00 \bar{E}+03$ & $8.28 \mathrm{E}+01$ & $6.05 E+03$ & $1.90 \mathrm{E}+0$ \\
\hline 65 & $1.22 \mathrm{E}+04$ & $4.46 \mathrm{E}+03$ & $7.92 \mathrm{E}+03$ & $2.05 \mathrm{E}+03$ & $5.96 \mathrm{E}+03$ & $8.34 \mathrm{E}+01$ & $6.03 \bar{E}+03$ & $1.89 \mathrm{E}+03$ \\
\hline 66 & $1.21 \mathrm{E}+04$ & $4.48 \mathrm{E}+03$ & $7.76 \mathrm{E}+03$ & $2.06 \mathrm{E}+03$ & $6.04 \mathrm{E}+03$ & $8.67 \mathrm{E}+01$ & $5.85 \mathrm{E}+03$ & $5.18 \mathrm{E}+03$ \\
\hline 67 & $1.19 \mathrm{E}+04$ & $4.49 \mathrm{E}+03$ & $7.62 \mathrm{E}+03$ & $2.06 \mathrm{E}+03$ & $6.12 \mathrm{E}+03$ & $9.01 \mathrm{E}+01$ & $5.89 \mathrm{E}+03$ & $5.20 \mathrm{E}+0$ \\
\hline 68 & $1.18 \mathrm{E}+04$ & $4.51 \mathrm{E}+03$ & $7.49 \bar{E}+03$ & $2.07 \mathrm{E}+03$ & $6.20 \mathrm{E}+03$ & $9.34 \mathrm{E}+0 \mathrm{I}$ & $5.93 \mathrm{E}+03$ & $5.21 \mathrm{E}+0$ \\
\hline 69 & $1.17 \mathrm{E}+04$ & $4.52 \mathrm{E}+03$ & $7.36 \mathrm{E}+03$ & $2.08 \mathrm{E}+03$ & $6.27 \mathrm{E}+03$ & $9.68 \mathrm{E}+01$ & $5.97 \mathrm{E}+03$ & $5.23 \mathrm{E}+03$ \\
\hline 168 & $2.76 \mathrm{E}+03$ & $4.90 \mathrm{E}+03$ & $2.99 \mathrm{E}+03$ & $2.47 \mathrm{E}+03$ & $5.85 \mathrm{E}+03$ & $3.73 \mathrm{E}+02$ & $6.06 \mathrm{E}+03$ & $5.48 \mathrm{E}+03$ \\
\hline 336 & $1.33 \mathrm{E}+03$ & $4.16 \mathrm{E}+03$ & $1.45 \mathrm{E}+03$ & $2.69 \mathrm{E}+03$ & $3.08 \mathrm{E}+03$ & $8.05 \mathrm{E}+02$ & $4.22 \mathrm{E}+03$ & $4.52 \mathrm{E}+0$ \\
\hline 504 & $9.69 \mathrm{E}+02$ & $3.48 \mathrm{E}+03$ & $9.90 \mathrm{E}+02$ & $2.82 \mathrm{E}+03$ & $1.71 \mathrm{E}+03$ & $1.06 \mathrm{E}+03$ & $2.24 \mathrm{E}+03$ & $3.26 \mathrm{E}+03$ \\
\hline 720 & $7.03 \mathrm{E}+02$ & $2.75 \mathrm{E}+03$ & $6.61 \mathrm{E}+02$ & $2.93 \mathrm{E}+03$ & $9.23 \mathrm{E}+02$ & $1.22 \mathrm{E}+03$ & $1.23 \mathrm{E}+03$ & $2.33 \overline{\mathrm{E}+03}$ \\
\hline 2160 & $1.31 \mathrm{E}+02$ & $5.48 \mathrm{E}+02$ & $7.40 \mathrm{E}+01$ & $3.15 \mathrm{E}+03$ & $8.07 \mathrm{E}+01$ & $7.54 \mathrm{E}+02$ & $2.90 \mathrm{E}+02$ & $8.04 \mathrm{E}+02$ \\
\hline 4320 & $1.26 \mathrm{E}+01$ & $4.96 \mathrm{E}+01$ & $3.73 \mathrm{E}+02$ & $2.97 \mathrm{E}+03$ & $1.34 \mathrm{E}+01$ & $1.82 \mathrm{E}+02$ & $3.42 \mathrm{E}+01$ & $1.23 \mathrm{E}+02$ \\
\hline 4800 & $7.49 \mathrm{E}+00$ & $2.92 \mathrm{E}+01$ & $3.72 \mathrm{E}+02$ & $2.96 \mathrm{E}+03$ & $9.73 \mathrm{E}+00$ & $1.31 \mathrm{E}+02$ & $1.14 \mathrm{E}+01$ & $4.02 \bar{E}+01$ \\
\hline 8760 & $1.03 \mathrm{E}-01$ & $3.81 \mathrm{E}-01$ & $3.62 \mathrm{E}+02$ & $2.89 \mathrm{E}+03$ & $8.07 \mathrm{E}-01$ & $8.81 \mathrm{E}+00$ & $3.58 \mathrm{E}+00$ & $1.19 \mathrm{E}+01$ \\
\hline $3 \mathbf{T}$ & $\begin{array}{l}\text { E6 } \\
\text { /L }\end{array}$ & $\begin{array}{l}8 \mathrm{E}+4 \\
\mathrm{~kg}\end{array}$ & $8 \mathrm{~Bq} / \mathrm{g}$ & & & & & \\
\hline 0 & $\begin{array}{l}5.9 \mathrm{E} 6 \\
\mathrm{pCi} / \mathrm{L}\end{array}$ & $\begin{array}{l}2.18 \mathrm{E}+5 \\
\mathrm{~Bq} / \mathrm{kg}\end{array}$ & $8.3 \mathrm{~Bq} / \mathrm{g}$ & & & & & \\
\hline & \multirow{2}{*}{\multicolumn{2}{|c|}{ VEGETABLES }} & & & & & & \\
\hline & & & \multicolumn{2}{|c|}{ WHEAT SEED } & \multicolumn{2}{|l|}{$\overline{\text { MEAT }}$} & \multicolumn{2}{|l|}{ MILK } \\
\hline \multirow[t]{2}{*}{ HOUR } & 0 & OBT & ro & $\mathrm{OBT}$ & HTO & OBT & HTO & $\overline{\mathrm{OBT}}$ \\
\hline & $(\mathrm{Bq} / \mathrm{g})$ & $(\mathrm{Bq} / \mathrm{g})$ & $(\mathrm{Bq} / \mathrm{g})$ & $(\mathrm{Bq} / \mathrm{g})$ & $(\mathrm{Bq} / \mathrm{g})$ & $(\mathrm{Bq} / \mathrm{g})$ & $(\mathrm{Bq} / \mathrm{g})$ & $(\mathrm{Bq} / \mathrm{g})$ \\
\hline ak HTO & & & & & & & & \\
\hline
\end{tabular}


Foodstuff Concentrations and Relocation Considerations Following

a Tritium Oxide Release from SRS Tritium Facilities (U)

\begin{tabular}{|c|c|c|c|c|c|c|c|c|}
\hline $\begin{array}{l}10 / 11 / 53 / 5 \\
4\end{array}$ & $2.16 \mathrm{E}+05$ & $2.13 E+03$ & $1.67 \mathrm{E}+05$ & $8.31 \mathrm{E}+02$ & $6.53 \mathrm{E}+03$ & $7.60 \mathrm{E}+01$ & $6.28 \mathrm{E}+03$ & $1.97 \mathrm{E}+03$ \\
\hline SUM & $2.19 \mathrm{E}+05$ & & $1.68 \mathrm{E}+05$ & & $6.61 \mathrm{E}+03$ & & $8.26 \mathrm{E}+03$ & \\
\hline $\mathrm{Ci} / \mathrm{g}$ & $5.91 \mathrm{E}-06$ & & $4.54 \mathrm{E}-06$ & & 1.79E-07 & & $2.23 \mathrm{E}-07$ & \\
\hline & & & & & & & & \\
\hline DIL HTO & $5.9 \mathrm{E}-09$ & & & & & & & \\
\hline \multicolumn{9}{|l|}{$(\mathrm{Ci} / \mathrm{g})$} \\
\hline & & & & & & & & \\
\hline 168 & $2.76 \mathrm{E}+03$ & $4.90 \mathrm{E}+03$ & $2.99 \mathrm{E}+03$ & $2.47 \mathrm{E}+03$ & $5.85 \mathrm{E}+03$ & $3.73 E+02$ & $6.06 E+03$ & $5.48 \mathrm{E}+03$ \\
\hline$\overline{\text { SUM }}$ & $7.67 \mathrm{E}+03$ & & $5.46 \mathrm{E}+03$ & & $6.22 \mathrm{E}+03$ & & $1.15 \mathrm{E}+04$ & \\
\hline $\mathrm{Ci} / \mathrm{g}$ & 2.07E-07 & & 1.48E-07 & & 1.68E-07 & & 3.12E-07 & \\
\hline & & & & & & & & \\
\hline DIL OBT & $2.4 \mathrm{E}-09$ & & & & & & & \\
\hline \multicolumn{9}{|l|}{$\mathrm{Ci} / \mathrm{g}$} \\
\hline & & & & & & & & \\
\hline 8760 & $1.03 \mathrm{E}-01$ & $3.81 \mathrm{E}-01$ & $3.62 \mathrm{E}+02$ & $2.89 \mathrm{E}+03$ & $8.07 \mathrm{E}-01$ & $8.81 \mathrm{E}+00$ & $3.58 \mathrm{E}+00$ & $1.19 \mathrm{E}+01$ \\
\hline SUM & $4.84 \mathrm{E}-01$ & & $3.25 \mathrm{E}+03$ & & $9.62 \mathrm{E}+00$ & & $1.54 \mathrm{E}+01$ & \\
\hline $\mathrm{Ci} / \mathrm{g}$ & $1.31 \mathrm{E}-11$ & & 8.78E-08 & & $2.60 \mathrm{E}-10$ & & $4.18 \mathrm{E}-10$ & \\
\hline DIL OBT & 215001 & & & & & & & \\
\hline $\mathrm{Ci} / \mathrm{g}$ & $2.4 \mathrm{~L}-\mathrm{V}$ & & & & & & & \\
\hline & & & & & & & & \\
\hline
\end{tabular}




\begin{tabular}{|c|c|c|c|c|c|c|c|c|}
\hline & \multicolumn{6}{|c|}{ Tritium Facilities, Design Basis Fire, Adverse Met Conditions } & & \\
\hline & \multicolumn{4}{|c|}{ Concentration Values for $150 \mathrm{~m}$ from release } & & & & \\
\hline \multicolumn{9}{|c|}{ Only Wheat Seed is examined since this is the edible portion of the Wheat. The "WHEAT" category is } \\
\hline \multicolumn{7}{|c|}{ ctually the unedible plant body and is not considered in the dose estimate. } & & \\
\hline & & & & & & & & \\
\hline & \multicolumn{2}{|c|}{ VEGETABLES } & \multicolumn{2}{|c|}{ WHEAT SEED } & \multicolumn{2}{|l|}{ MEAT } & \multicolumn{2}{|l|}{ MILK } \\
\hline \multirow[t]{3}{*}{ HOUR } & HTO & OBT & HTO & OBT & HTO & OBT & HTO & OBT \\
\hline & $(\mathrm{Bq} / \mathrm{g})$ & $(\mathrm{Bq} / \mathrm{g})$ & $(\mathrm{Bq} / \mathrm{g})$ & $(\mathrm{Bq} / \mathrm{g})$ & $(\mathrm{Bq} / \mathrm{g})$ & $\mathrm{q} / \mathrm{g})$ & $(\mathrm{Bq} / \mathrm{g})$ & $(\mathrm{Ba} / \mathrm{g})$ \\
\hline & & & & & & & & \\
\hline 1 & $6.64 \mathrm{E}+05$ & $3.20 \mathrm{E}+03$ & $1.20 \mathrm{E}+05$ & $3.97 \mathrm{E}+02$ & $1.24 \mathrm{E}+04$ & $2.48 \mathrm{E}+00$ & $1.50 \mathrm{E}+03$ & $6.40 \mathrm{E}+02$ \\
\hline 2 & $2.62 \mathrm{E}+05$ & $4.43 \mathrm{E}+03$ & $1.80 \mathrm{E}+05$ & $7.12 \mathrm{E}+02$ & $1.85 \mathrm{E}+04$ & $6.29 \mathrm{E}+00$ & $3.33 \mathrm{E}+03$ & $1.44 \mathrm{E}+03$ \\
\hline 3 & $1.08 \mathrm{E}+05$ & $4.91 \mathrm{E}+03$ & $2.04 \mathrm{E}+05$ & $9.62 \mathrm{E}+02$ & $2.22 \mathrm{E}+04$ & $1.09 \mathrm{E}+01$ & $5.14 \mathrm{E}+03$ & 2.2 \\
\hline 4 & $4.83 \mathrm{E}+04$ & $5.11 \mathrm{E}+03$ & $2.04 \mathrm{E}+05$ & $1.16 \mathrm{E}+03$ & $2.42 \mathrm{E}+04$ & $1.59 \mathrm{E}+01$ & $6.81 \mathrm{E}+03$ & $2.96 \mathrm{E}+03$ \\
\hline 5 & $2.53 \mathrm{E}+04$ & $5.19 \mathrm{E}+03$ & $1.93 \mathrm{E}+05$ & $1.32 \mathrm{E}+03$ & $2.54 \mathrm{E}+04$ & $2.13 \mathrm{E}+01$ & $8.30 \mathrm{E}+03$ & $3.62 \mathrm{E}+0 \mathrm{3}$ \\
\hline 6 & $1.64 \mathrm{E}+04$ & $5.23 \mathrm{E}+03$ & $1.75 \mathrm{E}+05$ & $1.45 \mathrm{E}+03$ & $2.52 \mathrm{E}+04$ & $2.40 \mathrm{E}+01$ & $2.45 \mathrm{E}+04$ & $7.62 \mathrm{E}+0$ \\
\hline 7 & $1.29 \mathrm{E}+04$ & $5.25 \mathrm{E}+03$ & $1.55 \mathrm{E}+05$ & $1.55 \mathrm{E}+03$ & $2.50 \mathrm{E}+04$ & $2.66 \mathrm{E}+01$ & $2.43 \mathrm{E}+04$ & $7.58 \mathrm{E}+03$ \\
\hline 8 & $1.15 \mathrm{E}+04$ & $5.27 \mathrm{E}+03$ & $1.34 \mathrm{E}+05$ & $1.63 \mathrm{E}+03$ & $2.48 \mathrm{E}+04$ & $2.93 \mathrm{E}+01$ & $2.42 \mathrm{E}+04$ & $7.55 \mathrm{E}+03$ \\
\hline 9 & $1.08 \mathrm{E}+04$ & $5.29 \mathrm{E}+03$ & $1.15 \mathrm{E}+05$ & $1.70 \mathrm{E}+03$ & $2.46 \mathrm{E}+04$ & $3.19 \mathrm{E}+01$ & $2.41 \mathrm{E}+04$ & $7.52 \bar{E}+0$ \\
\hline 10 & $1.05 \mathrm{E}+04$ & $5.30 \mathrm{E}+03$ & $9.69 \mathrm{E}+04$ & $1.75 \mathrm{E}+03$ & $2.44 \mathrm{E}+04$ & $3.45 \mathrm{E}+01$ & $2.40 \mathrm{E}+04$ & $7.49 \mathrm{E}+03$ \\
\hline 11 & $1.03 \mathrm{E}+04$ & $5.31 \mathrm{E}+03$ & $8.14 \mathrm{E}+04$ & $1.79 \mathrm{E}+03$ & $2.42 \mathrm{E}+04$ & $3.71 \mathrm{E}+01$ & $2.39 \mathrm{E}+04$ & $7.46 \mathrm{E}+03$ \\
\hline 12 & $1.01 \mathrm{E}+04$ & $5.32 \mathrm{E}+03$ & $6.80 \mathrm{E}+04$ & $1.83 \mathrm{E}+03$ & $2.40 \mathrm{E}+04$ & $3.97 \mathrm{E}+01$ & $2.38 \mathrm{E}+04$ & $7.43 \mathrm{E}+0$ \\
\hline 13 & $9.91 \mathrm{E}+03$ & $5.34 \mathrm{E}+03$ & $5.66 \mathrm{E}+04$ & $1.85 \mathrm{E}+03$ & $2.38 \mathrm{E}+04$ & $4.22 \mathrm{E}+0 \mathrm{I}$ & $2.37 \mathrm{E}+04$ & $7.40 \mathrm{E}+0$ \\
\hline 14 & $9.76 \mathrm{E}+03$ & $5.35 \mathrm{E}+03$ & $4.70 \mathrm{E}+04$ & $1.88 \mathrm{E}+03$ & $2.36 \mathrm{E}+04$ & $4.47 \mathrm{E}+01$ & $2.36 \mathrm{E}+04$ & $7.37 \mathrm{E}+03$ \\
\hline 15 & $9.62 \mathrm{E}+03$ & $5.36 \mathrm{E}+03$ & $3.89 \mathrm{E}+04$ & $1.90 \mathrm{E}+03$ & $2.34 \mathrm{E}+04$ & $4.72 \mathrm{E}+01$ & $2.36 \mathrm{E}+04$ & $7.34 \mathrm{E}+0$ \\
\hline 16 & $9.48 \mathrm{E}+03$ & $5.37 \mathrm{E}+03$ & $3.23 \mathrm{E}+04$ & $1.91 \mathrm{E}+03$ & $2.32 \mathrm{E}+04$ & $4.96 \mathrm{E}+01$ & $2.35 \mathrm{E}+04$ & $7.31 \mathrm{E}+03$ \\
\hline 17 & $9.34 \mathrm{E}+03$ & $5.38 \mathrm{E}+03$ & $2.68 \mathrm{E}+04$ & $1.92 \mathrm{E}+03$ & $2.30 \mathrm{E}+04$ & $5.21 \mathrm{E}+01$ & $2.34 \mathrm{E}+04$ & $7.28 \mathrm{E}+03$ \\
\hline 18 & $9.20 \mathrm{E}+03$ & $5.39 \mathrm{E}+03$ & $2.23 \mathrm{E}+04$ & $1.94 \mathrm{E}+03$ & $2.29 \mathrm{E}+04$ & $5.74 \mathrm{E}+01$ & $2.22 \mathrm{E}+04$ & $1.04 \mathrm{E}+04$ \\
\hline 19 & $9.07 \mathrm{E}+03$ & $5.40 \mathrm{E}+03$ & $1.86 \mathrm{E}+04$ & $1.94 \mathrm{E}+03$ & $2.28 \mathrm{E}+04$ & $6.27 \mathrm{E}+01$ & $2.22 \mathrm{E}+04$ & $1.04 \mathrm{E}+0$ \\
\hline 20 & $8.94 \mathrm{E}+03$ & $5.41 \mathrm{E}+03$ & $1.57 \mathrm{E}+04$ & $1.95 \mathrm{E}+03$ & $2.27 \mathrm{E}+04$ & $6.80 \mathrm{E}+01$ & $2.21 \mathrm{E}+04$ & $1.04 \mathrm{E}+0$ \\
\hline 21 & $8.82 \mathrm{E}+03$ & $5.42 \mathrm{E}+03$ & $1.32 \mathrm{E}+04$ & $1.96 \mathrm{E}+03$ & $2.26 \mathrm{E}+04$ & $7.33 \mathrm{E}+01$ & $2.21 \mathrm{E}+04$ & $1.04 \mathrm{E}+04$ \\
\hline 22 & $8.70 \mathrm{E}+03$ & $5.43 \mathrm{E}+03$ & $1.12 \mathrm{E}+04$ & $1.97 \mathrm{E}+03$ & $2.25 \mathrm{E}+04$ & $7.86 \mathrm{E}+01$ & $2.20 \mathrm{E}+04$ & $1.04 \mathrm{E}+0$ \\
\hline 23 & $8.58 \mathrm{E}+03$ & $5.44 \mathrm{E}+03$ & $9.65 \mathrm{E}+03$ & $1.97 \mathrm{E}+03$ & $2.24 \mathrm{E}+04$ & $8.38 \mathrm{E}+01$ & $2.20 \mathrm{E}+04$ & $1.04 \mathrm{E}+0$ \\
\hline 24 & $8.47 \mathrm{E}+03$ & $5.44 \mathrm{E}+03$ & $8.36 \mathrm{E}+03$ & $1.98 \mathrm{E}+03$ & $2.23 \mathrm{E}+04$ & $8.91 E+01$ & $2.19 \mathrm{E}+04$ & $1.04 \mathrm{E}+04$ \\
\hline 25 & $8.36 \mathrm{E}+03$ & $5.45 \mathrm{E}+03$ & $7.31 \mathrm{E}+03$ & $1.98 \mathrm{E}+03$ & $2.23 \mathrm{E}+04$ & $9.43 \mathrm{E}+01$ & $2.19 \mathrm{E}+04$ & $1.04 \mathrm{E}+04$ \\
\hline 26 & $8.25 \mathrm{E}+03$ & $5.46 \mathrm{E}+03$ & $6.47 \mathrm{E}+03$ & $1.99 \mathrm{E}+03$ & $2.22 \mathrm{E}+04$ & $9.95 \mathrm{E}+01$ & $2.18 \mathrm{E}+04$ & $1.03 \mathrm{E}+0$ \\
\hline 27 & $8.14 \mathrm{E}+03$ & $5.47 \mathrm{E}+03$ & $5.78 \mathrm{E}+03$ & $1.99 \mathrm{E}+03$ & $2.21 \mathrm{E}+\overline{04}$ & $1.05 \mathrm{E}+02$ & $2.18 \mathrm{E}+04$ & $1.03 \mathrm{E}+04$ \\
\hline 28 & $8.04 E+03$ & $5.48 \mathrm{E}+03$ & $5.23 \mathrm{E}+03$ & $1.99 \mathrm{E}+03$ & $2.20 \mathrm{E}+04$ & $1.10 \mathrm{E}+02$ & $2.18 \mathrm{E}+04$ & $1.03 E+04$ \\
\hline 29 & $7.94 \mathrm{E}+03$ & $5.48 \mathrm{E}+03$ & $4.78 \mathrm{E}+03$ & $2.00 \mathrm{E}+03$ & $2.19 \mathrm{E}+04$ & $1.15 \mathrm{E}+02$ & $2.17 \mathrm{E}+04$ & $1.03 \mathrm{E}+0$ \\
\hline 30 & $7.85 \mathrm{E}+03$ & $5.49 \mathrm{E}+03$ & $4.42 \mathrm{E}+03$ & $2.00 \mathrm{E}+03$ & $2.17 \mathrm{E}+04$ & $1.17 \mathrm{E}+02$ & $2.10 \mathrm{E}+04$ & $6.56 \mathrm{E}+03$ \\
\hline 31 & $7.75 \mathrm{E}+03$ & $5.50 \mathrm{E}+03$ & $4.12 \mathrm{E}+03$ & $2.00 \mathrm{E}+03$ & $2.15 \mathrm{E}+04$ & $1.20 \mathrm{E}+02$ & $2.09 \mathrm{E}+04$ & $6.54 \mathrm{E}+0$ \\
\hline 32 & $7.66 \mathrm{E}+03$ & $5.51 \mathrm{E}+03$ & $3.87 \mathrm{E}+03$ & $2.01 \mathrm{E}+03$ & $2.13 \mathrm{E}+04$ & $1.22 \mathrm{E}+02$ & $2.08 \mathrm{E}+04$ & $6.51 \mathrm{E}+0$ \\
\hline 33 & $7.57 \mathrm{E}+03$ & $5.51 \mathrm{E}+03$ & $3.67 \mathrm{E}+03$ & $2.01 \mathrm{E}+03$ & $2.11 \mathrm{E}+04$ & $1.24 \mathrm{E}+02$ & $2.07 \mathrm{E}+04$ & $6.48 \mathrm{E}+03$ \\
\hline 34 & $7.49 \mathrm{E}+03$ & $5.52 \mathrm{E}+03$ & $3.51 \mathrm{E}+03$ & $2.01 \mathrm{E}+03$ & $2.10 \mathrm{E}+04$ & $1.26 \mathrm{E}+02$ & $2.07 \mathrm{E}+04$ & $6.45 \mathrm{E}+03$ \\
\hline 35 & $7.40 \mathrm{E}+03$ & $5.53 \mathrm{E}+03$ & $3.37 \mathrm{E}+03$ & $2.02 \mathrm{E}+03$ & $2.08 \mathrm{E}+04$ & $1.28 \mathrm{E}+02$ & $2.06 \mathrm{E}+04$ & $6.43 \mathrm{E}+03$ \\
\hline 36 & $7.32 \mathrm{E}+03$ & $5.53 \mathrm{E}+03$ & $3.25 \mathrm{E}+03$ & $2.02 E+03$ & $2.06 \mathrm{E}+04$ & $1.30 \mathrm{E}+02$ & $2.05 \mathrm{E}+04$ & $6.40 \mathrm{E}+03$ \\
\hline 37 & $7.24 \mathrm{E}+03$ & $5.54 \mathrm{E}+03$ & $3.15 \mathrm{E}+03$ & $2.02 \mathrm{E}+03$ & $2.04 \mathrm{E}+04$ & $1.33 \mathrm{E}+02$ & $2.04 \mathrm{E}+04$ & $6.38 \mathrm{E}+03$ \\
\hline 38 & $7.17 \mathrm{E}+03$ & $5.55 \mathrm{E}+03$ & $3.07 \mathrm{E}+03$ & $2.02 \mathrm{E}+03$ & $2.03 \mathrm{E}+04$ & $1.35 \mathrm{E}+02$ & $2.03 \mathrm{E}+04$ & $6.35 \mathrm{E}+03$ \\
\hline
\end{tabular}


Foodstuff Concentrations and Relocation Considerations Following

\begin{tabular}{|c|c|c|c|c|c|c|c|c|}
\hline 39 & $7.09 \mathrm{E}+03$ & $5.55 \mathrm{E}+03$ & $3.00 \mathrm{E}+03$ & $2.03 \mathrm{E}+03$ & $2.01 \mathrm{E}+04$ & $1.37 \mathrm{E}+02$ & $2.02 \mathrm{E}+04$ & $6.32 \mathrm{E}+\mathrm{c}$ \\
\hline 40 & $7.02 \mathrm{E}+03$ & $5.56 \mathrm{E}+03$ & $2.94 \mathrm{E}+03$ & $2.03 \mathrm{E}+03$ & $1.99 \mathrm{E}+04$ & $1.39 \mathrm{E}+02$ & $2.02 \mathrm{E}+04$ & $6.30 \mathrm{E}^{+}$ \\
\hline 41 & $6.95 \mathrm{E}+03$ & $5.56 \mathrm{E}+03$ & $2.88 \mathrm{E}+03$ & $2.03 \mathrm{E}+03$ & $1.98 \mathrm{E}+04$ & $1.41 \mathrm{E}+02$ & $2.01 E+04$ & $6.27 \mathrm{E}-$ \\
\hline 42 & $6.88 \mathrm{E}+03$ & $5.57 \mathrm{E}+03$ & $2.83 \mathrm{E}+03$ & $2.03 \mathrm{E}+03$ & $1.97 \mathrm{E}+04$ & $1.46 \mathrm{E}+02$ & $1.91 \mathrm{E}+04$ & $9.53 \mathrm{E}+$ \\
\hline 43 & $6.81 \mathrm{E}+03$ & $5.57 \mathrm{E}+03$ & $2.79 \mathrm{E}+03$ & $2.04 \mathrm{E}+03$ & $1.96 \mathrm{E}+04$ & $1.51 \mathrm{E}+02$ & $1.90 \mathrm{E}+04$ & $9.52 \mathrm{E}+0$ \\
\hline 44 & $6.75 \mathrm{E}+03$ & $5.58 \mathrm{E}+03$ & $2.75 \mathrm{E}+03$ & $2.04 \mathrm{E}+03$ & $1.95 \mathrm{E}+04$ & $1.56 \mathrm{E}+02$ & $1.90 \mathrm{E}+04$ & $9.50 \mathrm{E}+$ \\
\hline 45 & $6.69 \mathrm{E}+03$ & $5.58 \mathrm{E}+03$ & $2.71 \mathrm{E}+03$ & $2.04 \mathrm{E}+03$ & $1.94 \mathrm{E}+04$ & $1.61 \mathrm{E}+02$ & $1.89 \mathrm{E}+04$ & $9.49 \mathrm{E}+\mathrm{C}$ \\
\hline 46 & $6.63 \mathrm{E}+03$ & $5.59 \mathrm{E}+03$ & $2.68 \mathrm{E}+03$ & $2.05 \mathrm{E}+03$ & $1.93 \mathrm{E}+04$ & $1.65 \mathrm{E}+02$ & $1.89 \mathrm{E}+04$ & $9.47 \mathrm{E}+0$ \\
\hline 47 & $6.57 \mathrm{E}+03$ & $5.59 \mathrm{E}+03$ & $2.65 \mathrm{E}+03$ & $2.05 \mathrm{E}+03$ & $1.92 \mathrm{E}+04$ & $1.70 \mathrm{E}+02$ & $1.88 \mathrm{E}+04$ & $9.46 \mathrm{E}+$ \\
\hline 48 & $6.51 \mathrm{E}+03$ & $5.60 \mathrm{E}+03$ & $2.62 \mathrm{E}+03$ & $2.05 \mathrm{E}+03$ & $1.91 \mathrm{E}+04$ & $1.75 \mathrm{E}+02$ & $1.88 E+04$ & $9 . \overline{44 \mathrm{E}+}$ \\
\hline 49 & $6.46 \mathrm{E}+03$ & $5.60 \mathrm{E}+03$ & $2.59 \mathrm{E}+03$ & $2.05 \mathrm{E}+03$ & $1.90 \mathrm{E}+04$ & $1.80 \mathrm{E}+02$ & $1.87 \mathrm{E}+04$ & $9.43 \mathrm{E}+\mathrm{c}$ \\
\hline 50 & $6.40 \mathrm{E}+03$ & $5.61 \mathrm{E}+03$ & $2.57 \mathrm{E}+03$ & $2.05 \mathrm{E}+03$ & $1.89 \mathrm{E}+04$ & $1.85 \mathrm{E}+02$ & $1.87 \mathrm{E}+04$ & $9.41 \mathrm{E}+$ \\
\hline 51 & $6.35 \mathrm{E}+03$ & $5.61 \mathrm{E}+03$ & $2.54 \mathrm{E}+03$ & $2.06 \mathrm{E}+03$ & $1.88 \mathrm{E}+04$ & $1.90 \mathrm{E}+02$ & $1.87 \mathrm{E}+04$ & $9.40 \mathrm{E}+\mathrm{C}$ \\
\hline 52 & $6.30 \mathrm{E}+03$ & $5.62 \mathrm{E}+03$ & $2.52 \mathrm{E}+03$ & $2.06 \mathrm{E}+03$ & $1.87 \mathrm{E}+04$ & $1.94 \mathrm{E}+02$ & $1.86 \mathrm{E}+04$ & $9.38 \mathrm{E}+\mathrm{C}$ \\
\hline 53 & $6.25 \mathrm{E}+03$ & $5.62 \mathrm{E}+03$ & $2.50 \mathrm{E}+03$ & $2.06 \mathrm{E}+03$ & $1.86 \mathrm{E}+04$ & $1.99 \mathrm{E}+02$ & $1.86 \mathrm{E}+04$ & $9.37 \mathrm{E}+$ \\
\hline 54 & $6.21 \mathrm{E}+03$ & $5.62 \mathrm{E}+03$ & $2.48 \mathrm{E}+03$ & $2.06 \mathrm{E}+03$ & $1.85 \mathrm{E}+04$ & $2.01 \mathrm{E}+02$ & $1.79 \mathrm{E}+04$ & $5.62 \mathrm{E}+\mathrm{c}$ \\
\hline 55 & $6.16 \mathrm{E}+03$ & $5.63 \mathrm{E}+03$ & $2.46 \mathrm{E}+03$ & $2.07 \mathrm{E}+03$ & $1.83 \mathrm{E}+04$ & $2.03 \mathrm{E}+02$ & $1.78 \mathrm{E}+04$ & $5.59 \mathrm{E}+\mathrm{C}$ \\
\hline 56 & $6.12 \mathrm{E}+03$ & $5.63 \mathrm{E}+03$ & $2.44 \mathrm{E}+03$ & $2.07 \mathrm{E}+03$ & $1.82 \mathrm{E}+04$ & $2.05 \mathrm{E}+02$ & $1.78 \mathrm{E}+04$ & $5.57 \mathrm{E}+\mathrm{c}$ \\
\hline 57 & $6.07 \mathrm{E}+03$ & $5.64 \mathrm{E}+03$ & $2.42 \mathrm{E}+03$ & $2.07 \mathrm{E}+03$ & $1.80 \mathrm{E}+04$ & $2.07 \mathrm{E}+02$ & $1.77 \mathrm{E}+04$ & $5.55 \mathrm{E}+\mathrm{C}$ \\
\hline 58 & $6.03 E+03$ & $5.64 \mathrm{E}+03$ & $2.40 \mathrm{E}+03$ & $2.07 \mathrm{E}+03$ & $1.79 \mathrm{E}+04$ & $2.08 \mathrm{E}+02$ & $1.76 \mathrm{E}+04$ & $5.53 \mathrm{E}+0$ \\
\hline 59 & $5.99 \mathrm{E}+03$ & $5.64 \mathrm{E}+03$ & $2.38 \mathrm{E}+03$ & $2.08 \mathrm{E}+03$ & $1.77 \mathrm{E}+04$ & $2.10 \mathrm{E}+02$ & $1.75 \mathrm{E}+04$ & $5.50 \mathrm{E}+\mathrm{C}$ \\
\hline 60 & $5.95 \mathrm{E}+03$ & $5.65 \mathrm{E}+03$ & $2.36 \mathrm{E}+03$ & $2.08 \mathrm{E}+03$ & $1.76 \mathrm{E}+04$ & $2.12 \mathrm{E}+02$ & $1.75 \mathrm{E}+04$ & $5.48 \mathrm{E}+0$ \\
\hline 61 & $5.91 \mathrm{E}+03$ & $5 . \overline{65 \mathrm{E}}+03$ & $2.34 \mathrm{E}+03$ & $2.08 \mathrm{E}+03$ & $1.74 \mathrm{E}+04$ & $2.14 \mathrm{E}+02$ & $1.74 \mathrm{E}+04$ & $5.46 \overline{\mathrm{E}}+0$ \\
\hline 62 & $5.88 \mathrm{E}+03$ & $5.65 \mathrm{E}+03$ & $2.33 \mathrm{E}+03$ & $2.08 \mathrm{E}+03$ & $1.73 \mathrm{E}+04$ & $2.15 \mathrm{E}+02$ & $1.73 \mathrm{E}+04$ & $5.44 \mathrm{E}+\mathrm{C}$ \\
\hline 63 & $5.84 \mathrm{E}+03$ & $5.66 \mathrm{E}+03$ & $2.31 \mathrm{E}+03$ & $2.08 \mathrm{E}+03$ & $1.71 \mathrm{E}+04$ & $2.17 \mathrm{E}+02$ & $1.73 \mathrm{E}+04$ & $5.41 \mathrm{E}+0$ \\
\hline 64 & $5.81 \mathrm{E}+03$ & $5.66 \mathrm{E}+03$ & $2.29 \mathrm{E}+03$ & $2.09 \mathrm{E}+03$ & $1.70 \mathrm{E}+04$ & $2.19 \mathrm{E}+02$ & $1.72 \mathrm{E}+04$ & $5.39 \mathrm{E}+0$ \\
\hline 65 & $5.78 \mathrm{E}+03$ & $5.66 \mathrm{E}+03$ & $2.28 \mathrm{E}+03$ & $2.09 \mathrm{E}+03$ & $1.69 \mathrm{E}+04$ & $2.20 \mathrm{E}+02$ & $1.71 \mathrm{E}+04$ & $5.37 \mathrm{E}+0$ \\
\hline 66 & $5.74 \mathrm{E}+03$ & $5.67 \mathrm{E}+03$ & $2.26 \mathrm{E}+03$ & $2.09 \mathrm{E}+03$ & $1.68 \mathrm{E}+04$ & $2.25 \mathrm{E}+02$ & $1.63 \mathrm{E}+04$ & $8.67 \mathrm{E}+0$ \\
\hline 67 & $5.71 \mathrm{E}+03$ & $5.67 \mathrm{E}+03$ & $2.25 \mathrm{E}+03$ & $2.09 \mathrm{E}+03$ & $1.67 \mathrm{E}+04$ & $2.29 \mathrm{E}+02$ & $1.62 \mathrm{E}+04$ & $8.65 \mathrm{E}+0$ \\
\hline 68 & $5.68 \mathrm{E}+03$ & $5.67 \mathrm{E}+03$ & $2.23 \mathrm{E}+03$ & $2.10 \mathrm{E}+03$ & $1.66 \mathrm{E}+04$ & $2.34 \mathrm{E}+02$ & $1.62 \mathrm{E}+04$ & $8.64 \mathrm{E}+0$ \\
\hline 69 & $5.66 \mathrm{E}+03$ & $5.68 \mathrm{E}+03$ & $2.22 \mathrm{E}+03$ & $2.10 \mathrm{E}+03$ & $1.65 \mathrm{E}+04$ & $2.38 \mathrm{E}+02$ & $1.61 \mathrm{E}+04$ & $8.63 \mathrm{E}+0$ \\
\hline 168 & $2.38 \mathrm{E}+03$ & $5.68 \mathrm{E}+03$ & $1.82 \mathrm{E}+03$ & $2.26 \mathrm{E}+03$ & $8.95 \mathrm{E}+03$ & $5.17 \mathrm{E}+02$ & $9.37 \mathrm{E}+03$ & $6.37 \mathrm{E}+0$ \\
\hline 336 & $1.13 \mathrm{E}+03$ & $4.75 \mathrm{E}+03$ & $9.23 \mathrm{E}+02$ & $2.40 \mathrm{E}+03$ & $3.46 \mathrm{E}+03$ & $9.33 \mathrm{E}+02$ & $5.56 \mathrm{E}+03$ & $4.79 \mathrm{E}+0$ \\
\hline 504 & $8.30 \mathrm{E}+02$ & $3.92 \mathrm{E}+03$ & $6.32 \mathrm{E}+02$ & $2.48 \mathrm{E}+03$ & $1.61 \mathrm{E}+03$ & $1.15 \mathrm{E}+03$ & $2.31 \mathrm{E}+03$ & $3.13 \mathrm{E}+0$ \\
\hline 720 & $6.05 \mathrm{E}+02$ & $3.06 \mathrm{E}+03$ & $4.24 \mathrm{E}+02$ & $2.55 \mathrm{E}+03$ & $7.95 \mathrm{E}+02$ & $1.27 \mathrm{E}+03$ & $1.10 \mathrm{E}+03$ & $2.16 \mathrm{E}+0$ \\
\hline 2160 & $1.14 \mathrm{E}+02$ & $5.74 \mathrm{E}+02$ & $4.77 \mathrm{E}+01$ & $2.69 \mathrm{E}+03$ & $7.06 \mathrm{E}+01$ & $7.38 \mathrm{E}+02$ & $2.49 \mathrm{E}+02$ & $7.40 \mathrm{E}+0$ \\
\hline 4320 & $1.09 \mathrm{E}+01$ & $4.85 \mathrm{E}+01$ & $2.40 \mathrm{E}+02$ & $2.56 \mathrm{E}+03$ & $1.14 \mathrm{E}+01$ & $1.75 \mathrm{E}+02$ & $2.96 \mathrm{E}+01$ & $1.15 \mathrm{E}+0$ \\
\hline 4800 & $6.50 \mathrm{E}+00$ & $2.82 \mathrm{E}+01$ & $2.39 \mathrm{E} \div 02$ & $2.56 \mathrm{E}+03$ & $8.25 \mathrm{E}+00$ & $1.26 \mathrm{E}+02$ & $9.72 \mathrm{E}+00$ & $3.74 \mathrm{E}+0$ \\
\hline 8760 & $8.92 \mathrm{E}-02$ & $3.45 \mathrm{E}-01$ & $2.33 \mathrm{E}+02$ & $2.49 \mathrm{E}+03$ & $6.60 \mathrm{E}-01$ & $8.26 \mathrm{E}+00$ & $3.00 \mathrm{E}+00$ & $1.09 \mathrm{E}+\mathrm{c}$ \\
\hline DIL OBT & $\begin{array}{l}2.4 \mathrm{E} 6 \\
\mathrm{pCi} / \mathrm{L}\end{array}$ & $\begin{array}{l}8 \mathrm{E}+4 \\
\mathrm{~kg}\end{array}$ & $8 \mathrm{~Bq} / \mathrm{g}$ & & & & & \\
\hline TO & $\begin{array}{l}5.9 \mathrm{E} 6 \\
\mathrm{pCi} / \mathrm{L}\end{array}$ & $\begin{array}{l}2.18 \mathrm{E}+5 \\
\mathrm{~Bq} / \mathrm{kg}\end{array}$ & $218.3 \mathrm{~Bq} / \mathrm{g}$ & & & & & \\
\hline & \multicolumn{2}{|c|}{ VEGETABLES } & \multicolumn{2}{|c|}{ WHEAT SEED } & \multicolumn{2}{|l|}{ MEAT } & \multicolumn{2}{|l|}{ MILK } \\
\hline \multirow[t]{2}{*}{ HOUR } & HTO & OBT & HTO & OBT & HTO & OBT & HTO & OBT \\
\hline & $(\mathrm{Bq} / \mathrm{g})$ & $(\mathrm{Bq} / \mathrm{g})$ & $(\mathrm{Bq} / \mathrm{g})$ & $(\mathrm{Bq} / \mathrm{g})$ & $(\mathrm{Bq} / \mathrm{g})$ & $(\mathrm{Bq} / \mathrm{g})$ & $(\mathrm{Bq} / \mathrm{g})$ & $(\mathrm{Bq} / \mathrm{g})$ \\
\hline ak HTO & & & & & & & & \\
\hline
\end{tabular}


Foodstuff Concentrations and Relocation Considerations Following

a Tritium Oxide Release from SRS Tritium Facilities (U)

\begin{tabular}{|c|c|c|c|c|c|c|c|c|}
\hline $1 / 4 / 6 / 6$ & $6.64 \mathrm{E}+05$ & $3.20 \mathrm{E}+03$ & $2.04 \mathrm{E}+05$ & $1.16 \mathrm{E}+03$ & $2.52 \mathrm{E}+04$ & $2.40 \mathrm{E}+01$ & $2.45 \mathrm{E}+04$ & $7.62 \mathrm{E}+03$ \\
\hline SUM & $6.67 \mathrm{E}+05$ & & $2.06 \mathrm{E}+05$ & & $2.52 \mathrm{E}+04$ & & $3.21 \mathrm{E}+04$ & \\
\hline $\mathrm{Ci} / \mathrm{g}$ & $1.80 \mathrm{E}-05$ & & $5.56 \mathrm{E}-06$ & & 6.82E-07 & & $8.67 \mathrm{E}-07$ & \\
\hline & & & & & & & & \\
\hline DIL HTO & $5.9 \mathrm{E}-09$ & & & & & & & \\
\hline \multicolumn{9}{|l|}{$(\mathrm{Ci} / \mathrm{g})$} \\
\hline & & & & & & & & \\
\hline 168 & $2.38 \mathrm{E}+03$ & $5.68 \mathrm{E}+03$ & $1.82 \mathrm{E}+03$ & $2.26 \mathrm{E}+03$ & $8.95 \mathrm{E}+03$ & $5.17 \mathrm{E}+02$ & $9.37 \mathrm{E}+03$ & $6.37 \mathrm{E}+03$ \\
\hline SUM & $8.06 \mathrm{E}+03$ & & $4.08 \mathrm{E}+03$ & & $9.46 \mathrm{E}+03$ & & $1.57 \mathrm{E}+04$ & \\
\hline $\mathrm{Ci} / \mathrm{g}$ & $2.18 E-07$ & & $1.10 \mathrm{E}-07$ & & $2.56 \mathrm{E}-07$ & & $4.25 \mathrm{E}-07$ & \\
\hline & & & & & & & & \\
\hline DIL OBT & $2.4 \mathrm{E}-09$ & & & & & & & \\
\hline \multicolumn{9}{|l|}{$\mathrm{Ci} / \mathrm{g}$} \\
\hline & & & & & & & & \\
\hline 8760 & $8.92 \mathrm{E}-02$ & $3.45 \mathrm{E}-01$ & $2.33 \mathrm{E}+02$ & $2.49 \mathrm{E}+03$ & $6.60 \mathrm{E}-01$ & $8.26 \mathrm{E}+00$ & $3.00 \mathrm{E}+00$ & $1.09 \mathrm{E}+01$ \\
\hline SUM & $4.34 \mathrm{E}-01$ & & $2.72 \mathrm{E}+03$ & & $8.92 \mathrm{E}+00$ & & $1.39 \mathrm{E}+01$ & \\
\hline $\mathrm{Ci} / \mathrm{g}$ & $1.17 \mathrm{E}-11$ & & $7.36 \mathrm{E}-08$ & & $2.41 \mathrm{E}-10$ & & $3.76 \mathrm{E}-10$ & \\
\hline & & & & & & & & \\
\hline DIL OBT & $2.4 \mathrm{E}-09$ & & & & & & & \\
\hline $\mathrm{Ci} / \mathrm{g}$ & & & & & & & & \\
\hline
\end{tabular}




\begin{tabular}{|c|c|c|c|c|c|c|c|c|}
\hline & \multicolumn{6}{|c|}{ Tritium Facilities, Design Basis Fire, Adverse Met Conditions } & & \\
\hline & \multicolumn{4}{|c|}{ Concentration Values for $1 \mathrm{~km}$ from release } & & & & \\
\hline \multicolumn{9}{|c|}{ Only Wheat Seed is examined since this is the edible portion of the Wheat. The "WHEAT" category is } \\
\hline \multicolumn{9}{|c|}{ actually the unedible plant body and is not considered in the dose estimate. } \\
\hline & & & & & & & & \\
\hline & \multicolumn{2}{|c|}{ VEGETABLES } & \multicolumn{2}{|c|}{ WHEAT SEED } & \multicolumn{2}{|l|}{ MEAT } & \multicolumn{2}{|l|}{ MILK } \\
\hline \multirow[t]{2}{*}{ HOUR } & HTO & OBT & HTO & OBT & HTO & OBT & HTO & OBT \\
\hline & $(\mathrm{Bq} / \mathrm{g})$ & $(\mathrm{Bq} / \mathrm{g})$ & $(\mathrm{Bq} / \mathrm{g})$ & $(\mathrm{Bq} / \mathrm{g})$ & $(\mathrm{Bq} / \mathrm{g})$ & $a / \mathbf{g})$ & 3) & $8 \mathrm{~g} / \mathbf{g})$ \\
\hline & & & & & & & & \\
\hline 1 & $2.08 \mathrm{E}+07$ & $9.62 \mathrm{E}+04$ & $3.61 \mathrm{E}+06$ & $1.19 \mathrm{E}+04$ & $3.72 \mathrm{E}+05$ & $7.44 \mathrm{E}+01$ & $4.51 \mathrm{E}+04$ & $1.93 \mathrm{E}+04$ \\
\hline 2 & $8.70 \mathrm{E}+06$ & $1.35 \mathrm{E}+05$ & $5.47 \mathrm{E}+06$ & $2.16 \mathrm{E}+04$ & $5.64 \mathrm{E}+05$ & $1.90 \mathrm{E}+02$ & $1.01 \mathrm{E}+05$ & $4.35 \mathrm{E}+04$ \\
\hline 3 & $3.86 \mathrm{E}+06$ & $1.51 \mathrm{E}+05$ & $6.2 \overline{\mathrm{E}+06}$ & $2.94 \mathrm{E}+04$ & $6.78 \mathrm{E}+05$ & $3.31 \mathrm{E}+02$ & $1.56 \mathrm{E}+05$ & $z+04$ \\
\hline 4 & $1.91 \mathrm{E}+06$ & $1.58 \mathrm{E}+05$ & $6.31 \mathrm{E}+06$ & $3.56 \mathrm{E}+04$ & $7.47 \mathrm{E}+05$ & $4.87 \mathrm{E}+02$ & $2.08 \mathrm{E}+05$ & $9.05 \mathrm{E}+04$ \\
\hline 5 & $1.10 \mathrm{E}+06$ & $1.61 \mathrm{E}+05$ & $5.99 \mathrm{E}+06$ & $4.07 \mathrm{E}+04$ & $7.87 \mathrm{E}+05$ & $6.52 \mathrm{E}+02$ & $2.54 \mathrm{E}+05$ & $1.11 \mathrm{E}+05$ \\
\hline 6 & $7.61 \mathrm{E}+05$ & $1.63 \mathrm{E}+05$ & $5.47 \mathrm{E}+06$ & $4.47 \mathrm{E}+04$ & $7.81 E+05$ & $7.35 \mathrm{E}+02$ & $7.57 \mathrm{E}+05$ & $2.36 \mathrm{E}+0$ \\
\hline 7 & $6.09 \mathrm{E}+05$ & $1.64 \mathrm{E}+05$ & $4.86 \mathrm{E}+06$ & $4.80 \mathrm{E}+04$ & $7.74 \mathrm{E}+05$ & $8.18 \mathrm{E}+02$ & $7.54 \mathrm{E}+05$ & $2.35 \mathrm{E}+0$ \\
\hline 8 & $5.35 \mathrm{E}+05$ & $1.65 \mathrm{E}+05$ & $4.24 \mathrm{E}+06$ & $5.06 \mathrm{E}+04$ & $7.68 \mathrm{E}+05$ & $9.00 \mathrm{E}+02$ & $7.51 \mathrm{E}+05$ & $2.34 \mathrm{E}+05$ \\
\hline 9 & $4.93 \mathrm{E}+05$ & $1.66 \mathrm{E}+05$ & $3.65 \mathrm{E}+06$ & $5.28 \mathrm{E}+04$ & $7.62 \mathrm{E}+05$ & $9.81 \mathrm{E}+02$ & $7.48 \mathrm{E}+05$ & $2.33 \mathrm{E}+0$ \\
\hline 10 & $4.67 \mathrm{E}+05$ & $1.66 \mathrm{E}+05$ & $3.11 \mathrm{E}+06$ & $5.45 \mathrm{E}+04$ & $7.55 \mathrm{E}+05$ & $1.06 \mathrm{E}+03$ & $7.44 \mathrm{E}+05$ & $2.32 \mathrm{E}+0$ \\
\hline 11 & $4.49 \mathrm{E}+05$ & $1.67 \mathrm{E}+05$ & $2.63 E+06$ & $5.59 \mathrm{E}+04$ & $7.49 \mathrm{E}+05$ & $1.14 \mathrm{E}+03$ & $7.41 \mathrm{E}+05$ & $2.31 \mathrm{E}+05$ \\
\hline 12 & $4.35 \mathrm{E}+05$ & $1.68 \mathrm{E} \div 05$ & $2.22 \mathrm{E}+06$ & $5.71 \mathrm{E}+04$ & $7.43 \mathrm{E}+05$ & $1.22 \mathrm{E}+03$ & $7.38 \mathrm{E}+05$ & $2.30 \mathrm{l}$ \\
\hline 13 & $4.24 \mathrm{E}+05$ & $1.68 \mathrm{E}+05$ & $1.86 \mathrm{E}+06$ & $5.81 \mathrm{E}+04$ & $7.37 \mathrm{E}+05$ & $1.30 \mathrm{E}+03$ & $7.35 \mathrm{E}+05$ & $2.29 \mathrm{E}+05$ \\
\hline$\overline{14}$ & $4.13 E+05$ & $1.69 \mathrm{E}+05$ & $1.56 \mathrm{E}+06$ & $5.89 \mathrm{E}+04$ & $7.31 \mathrm{E}+05$ & $1.38 \mathrm{E}+03$ & $7.32 \mathrm{E}+05$ & $2.28 \mathrm{E}+05$ \\
\hline 15 & $4.03 \mathrm{E}+05$ & $1.69 \mathrm{E}+05$ & $1.31 \mathrm{E}+06$ & $5.95 \mathrm{E}+04$ & $7.25 \mathrm{E}+05$ & $1.45 \mathrm{E}+03$ & $7.29 \mathrm{E}+05$ & $2.27 \mathrm{E}+05$ \\
\hline 16 & $3.94 \mathrm{E}+05$ & $1.70 \mathrm{E}+05$ & $1.10 \mathrm{E}+06$ & $6.01 \mathrm{E}+04$ & $7.19 \mathrm{E}+05$ & $1.53 \mathrm{E}+03$ & $7.26 \mathrm{E}+05$ & $2.26 \mathrm{E}+05$ \\
\hline 17 & $3.86 \mathrm{E}+05$ & $1.70 \mathrm{E}+05$ & $9.21 \mathrm{E}+05$ & $6.06 \mathrm{E}+04$ & $7.13 \mathrm{E}+05$ & $1.61 \mathrm{E}+03$ & $7.23 \mathrm{E}+05$ & $2.25 \mathrm{E}+05$ \\
\hline 18 & $3.78 \mathrm{E}+05$ & $1.71 \mathrm{E}+05$ & $7.78 \mathrm{E}+05$ & $6.10 \mathrm{E}+04$ & $7.11 \mathrm{E}+05$ & $1.77 \mathrm{E}+03$ & $6.89 \mathrm{E}+05$ & $3.26 \mathrm{E}+05$ \\
\hline 19 & $3.70 \mathrm{E}+05$ & $1.71 \mathrm{E}+0 \mathrm{~S}$ & $6.60 \mathrm{E}+05$ & $6.14 \mathrm{E}+04$ & $7.09 \mathrm{E}+05$ & $1.94 \mathrm{E}+03$ & $6.88 \mathrm{E}+05$ & $3.26 \mathrm{E}+05$ \\
\hline 20 & $3.63 \mathrm{E}+05$ & $1.72 \mathrm{E}+05$ & $5.64 \mathrm{E}+05$ & $6.17 \mathrm{E}+04$ & $7.07 \mathrm{E}+05$ & $2.11 \mathrm{E}+03$ & $6.87 \mathrm{E}+05$ & $3.26 \mathrm{E}+05$ \\
\hline 21 & $3.56 \mathrm{E}+05$ & $1.72 \mathrm{E}+05$ & $4.85 \mathrm{E}+05$ & $6.20 \mathrm{E}+04$ & $7.05 \mathrm{E}+05$ & $2.27 \mathrm{E}+03$ & $6.86 \mathrm{E}+05$ & $3.26 \mathrm{E}+05$ \\
\hline 22 & $3.49 \mathrm{E}+05$ & $1.73 \mathrm{E}+05$ & $4.20 \mathrm{E}+05$ & $6.22 \mathrm{E}+04$ & $7.03 \mathrm{E}+05$ & $2.44 \mathrm{E}+03$ & $6.85 \mathrm{E}+05$ & $3.26 \mathrm{E}+05$ \\
\hline 23 & $3.43 \mathrm{E}+05$ & $1.73 \mathrm{E}+05$ & $3.68 \mathrm{E}+05$ & $6.25 \mathrm{E}+04$ & $7.01 \mathrm{E}+05$ & $2.61 \mathrm{E}+03$ & $6.84 \mathrm{E}+05$ & $3.25 \mathrm{E}+05$ \\
\hline 24 & $3.34 \mathrm{E}+05$ & $1.74 \mathrm{E}+05$ & $3.25 \mathrm{E}+05$ & $6.27 \mathrm{E}+04$ & $6.99 \mathrm{E}+05$ & $2.77 \mathrm{E}+03$ & $6.83 \mathrm{E}+05$ & $3.25 \mathrm{E}+05$ \\
\hline 25 & $3.27 \mathrm{E}+05$ & $1.74 \mathrm{E}+05$ & $2.89 \mathrm{E}+05$ & $6.29 \mathrm{E}+04$ & $6.97 \mathrm{E}+05$ & $2.94 \mathrm{E}+03$ & $6.82 \mathrm{E}+05$ & $3.25 \mathrm{E}+05$ \\
\hline 26 & $3.21 \mathrm{E}+05$ & $1.74 \mathrm{E}+05$ & $2.61 \mathrm{E}+05$ & $6.31 \mathrm{E}+04$ & $6.94 \mathrm{E}+05$ & $3.10 \mathrm{E}+03$ & $6.81 \mathrm{E}+05$ & $3.25 \mathrm{E}+05$ \\
\hline 27 & $3.16 \mathrm{E}+05$ & $1.75 \mathrm{E}+05$ & $2.37 \mathrm{E}+05$ & $6.32 \mathrm{E}+04$ & $6.92 \mathrm{E}+05$ & $3.27 \mathrm{E}+03$ & $6.80 \mathrm{E}+05$ & $3.24 \mathrm{E}+05$ \\
\hline 28 & $3.10 \mathrm{E}+05$ & $1.75 \mathrm{E}+05$ & $2.17 \mathrm{E}+05$ & $6.34 \mathrm{E}+04$ & $6.90 \mathrm{E}+05$ & $3.43 \mathrm{E}+03$ & $6.79 \mathrm{E}+05$ & $3.24 \mathrm{E}+05$ \\
\hline 29 & $3.06 \mathrm{E}+05$ & $1.75 \mathrm{E}+05$ & $2.01 \mathrm{E}+05$ & $6.35 \mathrm{E}+04$ & $6.87 \mathrm{E}+05$ & $3.60 \mathrm{E}+03$ & $6.78 \mathrm{E}+05$ & $3.24 \mathrm{E}+05$ \\
\hline 30 & $3.01 \mathrm{E}+05$ & $1.76 \mathrm{E}+05$ & $1.87 \mathrm{E}+05$ & $6.37 \mathrm{E}+04$ & $6.81 \mathrm{E}+05$ & $3.67 \mathrm{E}+03$ & $6.60 \mathrm{E}+05$ & $2.06 \mathrm{E}+05$ \\
\hline 31 & $2.96 \mathrm{E}+05$ & $1.76 \mathrm{E}+05$ & $1.76 \mathrm{E}+05$ & $6.38 \mathrm{E}+04$ & $6.76 \mathrm{E}+05$ & $3.74 \mathrm{E}+03$ & $6.58 \mathrm{E}+05$ & $2.06 \mathrm{E}+05$ \\
\hline 32 & $2.92 \mathrm{E}+05$ & $1.76 \mathrm{E}+05$ & $1.67 \mathrm{E}+05$ & $6.40 \mathrm{E}+04$ & $6.70 \mathrm{E}+05$ & $3.81 \mathrm{E}+03$ & $6.55 \mathrm{E}+05$ & $2.05 \mathrm{E}+05$ \\
\hline 33 & $2.87 \mathrm{E}+05$ & $1.77 \mathrm{E}+05$ & $1.59 \mathrm{E}+05$ & $6.41 \mathrm{E}+04$ & $6.65 \mathrm{E}+05$ & $3.88 \mathrm{E}+03$ & $6.52 \mathrm{E}+05$ & $2.04 \mathrm{E}+05$ \\
\hline 34 & $2.83 \mathrm{E}+05$ & $1.77 \mathrm{E}+05$ & $1.52 \mathrm{E}+05$ & $6.42 \mathrm{E}+04$ & $6.59 \mathrm{E}+05$ & $3.94 \mathrm{E}+03$ & $6.50 \mathrm{E}+05$ & $2.03 \mathrm{E}+05$ \\
\hline 35 & $2.79 \mathrm{E}+05$ & $1.77 \mathrm{E}+05$ & $1.46 \mathrm{E}+05$ & $6.44 \mathrm{E}+04$ & $6.54 \mathrm{E}+05$ & $4.01 \mathrm{E}+03$ & $6.47 \mathrm{E}+05$ & $2.02 \mathrm{E}+05$ \\
\hline$\overline{36}$ & $2.75 \mathrm{E}+05$ & $1.78 \mathrm{E}+05$ & $1.41 \mathrm{E}+05$ & $6.45 \mathrm{E}+04$ & $6.48 \mathrm{E}+05$ & $4.08 \mathrm{E}+03$ & $6.44 \mathrm{E}+05$ & $2.01 \overline{\mathrm{E}+05}$ \\
\hline 37 & $2.67 \mathrm{E}+05$ & $1.78 \mathrm{E}+05$ & $1.37 \mathrm{E}+05$ & $6.46 \mathrm{E}+04$ & $6.43 \mathrm{E}+05$ & $4.14 E+03$ & $6.42 \mathrm{E}+05$ & $2.01 \mathrm{E}+05$ \\
\hline 38 & $2.61 \mathrm{E}+05$ & $1.78 \mathrm{E}+05$ & $1.33 \mathrm{E}+05$ & $6.47 \mathrm{E}+04$ & $6.37 \mathrm{E}+05$ & $4.21 \mathrm{E}+03$ & $6.39 \mathrm{E}+05$ & $2.00 \mathrm{E}+05$ \\
\hline
\end{tabular}




\begin{tabular}{|c|c|c|c|c|c|c|c|c|}
\hline$\overline{39}$ & $2.5 \overline{\mathrm{E}+05}$ & $1.78 \mathrm{E}+05$ & $1.29 \mathrm{E}+05$ & $6.49 \mathrm{E}+04$ & $6.32 \mathrm{E}+05$ & $4.28 \mathrm{E}+03$ & $6.36 \mathrm{E}+05$ & $1.99 \mathrm{E}+0$ \\
\hline$\overline{40}$ & $2.53 \mathrm{E}+05$ & $1.79 \mathrm{E}+05$ & $1.26 \mathrm{E}+05$ & $6.50 \mathrm{E}+04$ & $6.27 \mathrm{E}+05$ & $4.34 \mathrm{E}+03$ & $6.34 \mathrm{E}+05$ & $1.98 \mathrm{E}+$ \\
\hline 41 & $2.50 \mathrm{E}+05$ & $1.79 \mathrm{E}+05$ & $1.22 \mathrm{E}+05$ & $6.51 \mathrm{E}+04$ & $6.22 \mathrm{E}+05$ & $4 . \overline{40 \mathrm{E}+03}$ & $6.31 \mathrm{E}+05$ & $1.97 \mathrm{E}+$ \\
\hline 42 & $2.47 \mathrm{E}+05$ & $1.79 \mathrm{E}+05$ & $1.19 \mathrm{E}+05$ & $6.52 \mathrm{E}+04$ & $6.19 \mathrm{E}+05$ & $4.56 \mathrm{E}+03$ & $6.00 \mathrm{E}+05$ & $3.03 E+$ \\
\hline 43 & $2.43 \mathrm{E}+05$ & $1.79 \mathrm{E}+05$ & $1.17 \mathrm{E}+05$ & $6.53 \mathrm{E}+04$ & $6.16 \mathrm{E}+05$ & $4.72 \mathrm{E}+03$ & $5 . \overline{99 \mathrm{E}+05}$ & $3.02 \mathrm{E}+0$ \\
\hline 44 & $2.40 \mathrm{E}+05$ & $1.79 \mathrm{E}+05$ & $1.14 \overline{\mathrm{E}}+05$ & $6.54 \mathrm{E}+04$ & $6.14 \mathrm{E}+05$ & $\overline{4.88 \mathrm{E}+03}$ & $5.97 \mathrm{E}+05$ & $3.02 \bar{E}+$ \\
\hline 45 & $2.38 \mathrm{E}+05$ & $1.80 \mathrm{E}+05$ & $1.12 \mathrm{E}+05$ & $6.55 \mathrm{E}+04$ & $6.11 \mathrm{E}+05$ & $5.03 \mathrm{E}+03$ & $5.96 \mathrm{E}+05$ & $3.01 \mathrm{E}+0$ \\
\hline 46 & $2 . \overline{35 \mathrm{E}+05}$ & $1.80 \mathrm{E}+05$ & $1.10 \mathrm{E}+05$ & $6.56 \mathrm{E}+04$ & $6.08 \mathrm{E}+05$ & $5.19 \mathrm{E}+03$ & $5.95 \mathrm{E}+05$ & $3.01 \mathrm{E}+\mathrm{c}$ \\
\hline 47 & $2.32 \mathrm{E}+05$ & $1.80 \mathrm{E}+05$ & $1.08 \mathrm{E}+05$ & $6.57 \mathrm{E}+04$ & $6.06 \mathrm{E}+05$ & $5.35 \overline{\mathrm{E}}+03$ & $5.94 \mathrm{E}+05$ & $3.01 \mathrm{E}+0$ \\
\hline 48 & $2.29 \mathrm{E}+05$ & $1.80 \mathrm{E}+05$ & $1.06 \mathrm{E}+05$ & $6.58 \mathrm{E}+04$ & $6.03 \mathrm{E}+05$ & $5.5 \overline{0 \mathrm{E}}+03$ & $5.92 \mathrm{E}+05$ & $3.00 \mathrm{E}+0$ \\
\hline 49 & $2.27 \mathrm{E}+05$ & $1.80 \mathrm{E}+05$ & $1.04 \mathrm{E}+05$ & $6.59 \mathrm{E}+04$ & $6.00 \mathrm{E}+05$ & $5.66 \mathrm{E}+03$ & $5.91 \mathrm{E}+05$ & $3.00 \mathrm{E}+0$ \\
\hline 50 & $2.25 \mathrm{E}+05$ & $1.81 \mathrm{E}+05$ & $1.03 \mathrm{E}+05$ & $6.60 \mathrm{E}+04$ & $5.98 \mathrm{E}+05$ & $5.81 \mathrm{E}+03$ & $5.90 \mathrm{E}+05$ & $2 . \overline{99 \mathrm{E}+0}$ \\
\hline 51 & $2.22 \mathrm{E}+05$ & $1.81 \mathrm{E}+05$ & $1.01 \mathrm{E}+05$ & $6.61 \mathrm{E}+04$ & $5.95 \mathrm{E}+05$ & $5.97 \mathrm{E}+03$ & $5.88 E+05$ & $2.99 \mathrm{E}+0$ \\
\hline 52 & $2.20 \mathrm{E}+05$ & $1.81 \mathrm{E}+05$ & $9.96 \mathrm{E}+04$ & $6.62 \mathrm{E}+04$ & $5.92 \mathrm{E}+05$ & $6.12 \mathrm{E}+03$ & $5.87 \mathrm{E}+05$ & $2.99 \mathrm{E}+0$ \\
\hline 53 & $2.18 \mathrm{E}+05$ & $1.81 \mathrm{E}+05$ & $9.83 \mathrm{E}+04$ & $6.63 E+04$ & $5.90 \mathrm{E}+05$ & $6.27 \mathrm{E}+03$ & $5.86 \mathrm{E}+05$ & $2.98 \mathrm{E}+0$ \\
\hline 54 & $2.16 \mathrm{E}+05$ & $1.81 \mathrm{E}+05$ & $9.70 \mathrm{E}+04$ & $6.64 \mathrm{E}+04$ & $5.85 \bar{E}+05$ & $6.33 \mathrm{E}+03$ & $5.67 \mathrm{E}+05$ & $1.78 \mathrm{E}+0$ \\
\hline 55 & $2.14 \mathrm{E}+05$ & $1.81 \mathrm{E}+05$ & $9.57 \mathrm{E}+04$ & $6.65 \mathrm{E}+04$ & $5.80 \mathrm{E}+05$ & $6.39 \mathrm{E}+03$ & $5.65 \mathrm{E}+05$ & $1.77 \mathrm{E}+0$ \\
\hline 56 & $2.12 \mathrm{E}+05$ & $1.82 \mathrm{E}+05$ & $9.45 \mathrm{E}+04$ & $6.65 \mathrm{E}+04$ & $5.75 \mathrm{E}+05$ & $6.45 \mathrm{E}+03$ & $5.62 \mathrm{E}+05$ & $1.76 \mathrm{E}+0$ \\
\hline 57 & $2.10 \mathrm{E}+05$ & $1.82 \mathrm{E}+05$ & $9.33 \mathrm{E}+04$ & $6.66 \mathrm{E}+04$ & $5.70 \mathrm{E}+05$ & $6.50 \mathrm{E}+03$ & $5.60 \mathrm{E}+05$ & $1.76 \mathrm{E}+0$ \\
\hline 58 & $2.08 \mathrm{E}+05$ & $1.82 \mathrm{E}+05$ & $9.22 \mathrm{E}+04$ & $6.67 \mathrm{E}+04$ & $5.66 \mathrm{E}+05$ & $6.56 \mathrm{E}+03$ & $5.58 \mathrm{E}+05$ & $1.75 \mathrm{E}+0$ \\
\hline 59 & $2.07 \mathrm{E}+05$ & $1.82 \mathrm{E}+05$ & $9.11 \mathrm{E}+04$ & $6.68 \mathrm{E}+04$ & $5.61 \mathrm{E}+05$ & $6.62 \mathrm{E}+03$ & $5.55 \mathrm{E}+05$ & $1.74 \mathrm{E}+0$ \\
\hline 60 & $2.05 \mathrm{E}+05$ & $1.82 \mathrm{E}+05$ & $9.00 \mathrm{E}+04$ & $6.69 \mathrm{E}+04$ & $5.56 \mathrm{E}+05$ & $6.67 \mathrm{E}+03$ & $5.53 \mathrm{E}+05$ & $1.73 \mathrm{E}+0$ \\
\hline 61 & $2.03 \mathrm{E}+05$ & $1.82 \mathrm{E}+05$ & $8.90 \mathrm{E}+04$ & $6.70 \mathrm{E}+04$ & $5.52 \mathrm{E}+05$ & $6.73 \mathrm{E}+03$ & $5.51 \mathrm{E}+05$ & $1.73 \mathrm{E}+0$ \\
\hline 62 & $2.02 \mathrm{E}+05$ & $1.83 \mathrm{E}+05$ & $8.79 \mathrm{E}+04$ & $6.70 \mathrm{E}+04$ & $5.47 \mathrm{E}+05$ & $6.78 \mathrm{E}+03$ & $5.48 \mathrm{E}+05$ & $1.72 \mathrm{E}+0$ \\
\hline 63 & $2.00 \mathrm{E}+05$ & $1.83 \mathrm{E}+05$ & $8.70 \mathrm{E}+04$ & $6.71 \mathrm{E}+04$ & $5.42 \mathrm{E}+05$ & $6.83 \mathrm{E}+03$ & $5.46 \mathrm{E}+05$ & $1.71 \mathrm{E}+0$ \\
\hline 64 & $1.99 \mathrm{E}+05$ & $1.83 E+05$ & $8.60 \mathrm{E}+04$ & $6.72 \mathrm{E}+04$ & $5.38 \mathrm{E}+05$ & $6.89 \mathrm{E}+03$ & $5 . \overline{44 \mathrm{E}+05}$ & $1.7 \overline{1 E+0}$ \\
\hline 65 & $1.97 \mathrm{E}+05$ & $1.83 \mathrm{E}+05$ & $8.51 \mathrm{E}+04$ & $6.73 \mathrm{E}+04$ & $5.33 \mathrm{E}+05$ & $6.94 \mathrm{E}+03$ & $5.42 \mathrm{E}+05$ & $1.70 \mathrm{E}+0$ \\
\hline 66 & $1.91 \mathrm{E}+05$ & $1.83 \mathrm{E}+05$ & $8.42 \overline{\mathrm{E}}+04$ & $6.74 \mathrm{E}+04$ & $5.31 \mathrm{E}+05$ & $7.09 \mathrm{E}+03$ & $5.15 \mathrm{E}+05$ & $2.77 \mathrm{E}+0$ \\
\hline 67 & $1.87 \mathrm{E}+05$ & $1.83 E+05$ & $8.28 \mathrm{E}+04$ & $6.74 \mathrm{E}+04$ & $5.29 \mathrm{E}+05$ & $7.23 \mathrm{E}+03$ & $5.13 E+05$ & $2.77 \mathrm{E}+0$ \\
\hline 68 & $1.86 \mathrm{E}+05$ & $1.83 \mathrm{E}+05$ & $8.13 \mathrm{E}+04$ & $6.75 \mathrm{E}+04$ & $5.26 \mathrm{E}+05$ & $7.38 \bar{E}+03$ & $5.12 \mathrm{E}+05$ & $2.76 \mathrm{E}+0$ \\
\hline 69 & $1.84 \mathrm{E}+05$ & $1.83 E+05$ & $7.97 \mathrm{E}+04$ & $6.76 \mathrm{E}+04$ & $5.24 \mathrm{E}+05$ & $7.53 \mathrm{E}+03$ & $5.11 \mathrm{E}+05$ & $2.7 \overline{6 \mathrm{E}+0}$ \\
\hline 168 & $7.72 \mathrm{E}+04$ & $1.84 \mathrm{E}+05$ & $5.93 \mathrm{E}+04$ & $7.30 \mathrm{E}+04$ & $2.85 \mathrm{E}+05$ & $1.65 E+04$ & $2.98 \mathrm{E}+05$ & $2.05 \mathrm{E}+0$ \\
\hline 336 & $3.65 \mathrm{E}+04$ & $1.53 \mathrm{E}+05$ & $3.00 \mathrm{E}+04$ & $7.74 \mathrm{E}+04$ & $1.11 \mathrm{E}+05$ & $3.00 \mathrm{E}+04$ & $1.78 \mathrm{E}+05$ & $1.54 \mathrm{E}+0$ \\
\hline 504 & $2.68 \mathrm{E}+04$ & $1.27 \mathrm{E}+05$ & $2.05 E+04$ & $8.01 \mathrm{E}+04$ & $5.20 \mathrm{E}+04$ & $3.71 \mathrm{E}+04$ & $7.43 \mathrm{E}+04$ & $1.01 \overline{\mathrm{E}}+0$ \\
\hline 720 & $1.95 \mathrm{E}+04$ & $9.89 \mathrm{E} \div 04$ & $1.37 \overline{\mathrm{E}+04}$ & $8.24 \mathrm{E}+04$ & $2.57 \mathrm{E}+04$ & $4.09 \mathrm{E}+04$ & $3.56 \mathrm{E}+04$ & $6 . \overline{98 \mathrm{E}+0}$ \\
\hline 2160 & $3.66 \mathrm{E}+03$ & $1.85 \mathrm{E}+04$ & $1.54 \mathrm{E}+03$ & $8.67 \mathrm{E}+04$ & $2.28 \mathrm{E}+03$ & $2.38 \mathrm{E}+04$ & $8.06 \mathrm{E}+03$ & $2 . \overline{40 \mathrm{E}+0}$ \\
\hline$\overline{4320}$ & $3.52 \mathrm{E}+02$ & $1.57 \mathrm{E}+03$ & $7.77 \overline{\mathrm{E}}+03$ & $8.28 \mathrm{E}+04$ & $3.68 \mathrm{E}+02$ & $5.66 \mathrm{E}+03$ & $9.56 \mathrm{E}+02$ & $3 . \overline{72 \mathrm{E}+03}$ \\
\hline 4800 & $2.09 \mathrm{E}+02$ & $9.10 \mathrm{E}+02$ & $7.74 \mathrm{E}+03$ & $8.25 \mathrm{E}+04$ & $2.66 \mathrm{E}+02$ & $4.07 \mathrm{E}+03$ & $3.13 \mathrm{E}+02$ & 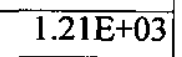 \\
\hline 8760 & $2.88 \mathrm{E}+00$ & $1.11 \mathrm{E}+01$ & $7.55 \mathrm{E}+03$ & $8.04 \mathrm{E}+04$ & $2.12 \mathrm{E}+01$ & $2.67 \mathrm{E}+02$ & $9.66 \mathrm{E}+01$ & $3.53 \mathrm{E}+0$ \\
\hline BT & $\begin{array}{l}2.4 \mathrm{E} 6 \\
\mathrm{pCi} / \mathrm{L}\end{array}$ & $\begin{array}{l}8.88 \mathrm{E}+4 \\
\mathrm{~Bq} / \mathrm{kg}\end{array}$ & $8 \mathrm{~Bq} / \mathrm{g}$ & & & & & \\
\hline To & $\begin{array}{l}5.9 \mathrm{E} 6 \\
\mathrm{pCi} / \mathrm{L}\end{array}$ & $\begin{array}{l}2.18 \mathrm{E}+5 \\
\mathrm{~Bq} / \mathrm{kg}\end{array}$ & $8.3 \mathrm{~Bq} / \mathrm{g}$ & & & & & \\
\hline & \multirow{2}{*}{\multicolumn{2}{|c|}{ VEGETABLES }} & & & & & & \\
\hline & & & \multicolumn{2}{|c|}{ WHEAT SEED } & \multicolumn{2}{|l|}{ MEAT } & \multicolumn{2}{|l|}{ MILK } \\
\hline \multirow[t]{2}{*}{ HOUR } & TO & $\mathrm{OBT}$ & HTO & OBT & HTO & OBT & HTO & $\overline{\mathrm{OBT}}$ \\
\hline & $(\mathrm{Bq} / \mathrm{g})$ & $(\mathrm{Bq} / \mathrm{g})$ & $(\mathrm{Bq} / \mathrm{g})$ & $(\mathrm{Bq} / \mathrm{g})$ & $(\overline{B q} / \mathrm{g})$ & $(\mathrm{Bq} / \mathrm{g})$ & $(\mathrm{Bq} / \mathrm{g})$ & $(\mathrm{Bq} / \mathrm{g})$ \\
\hline & & & & & & & & \\
\hline
\end{tabular}


Foodstuff Concentrations and Relocation Considerations Following

a Tritium Oxide Release from SRS Tritium Facilities (U)

\begin{tabular}{|c|c|c|c|c|c|c|c|c|}
\hline $1 / 4 / 6 / 6$ & $2.08 \mathrm{E}+07$ & $9.62 \mathrm{E}+04$ & $6.31 \mathrm{E}+06$ & $3.56 \mathrm{E}+04$ & $7.81 \mathrm{E}+05$ & $7.35 \mathrm{E}+02$ & $7.57 \mathrm{E}+05$ & $2.36 \mathrm{E}+05$ \\
\hline SUM & $2.09 \mathrm{E}+07$ & & $6.34 \mathrm{E}+06$ & & $7.82 \mathrm{E}+05$ & & $9.93 \overline{\mathrm{E}+05}$ & \\
\hline $\mathrm{Ci} / \mathrm{g}$ & $5.65 \mathrm{E}-04$ & & $1.71 \mathrm{E}-04$ & & $2.11 \mathrm{E}-05$ & & $2.68 \mathrm{E}-05$ & \\
\hline & & & & & & & & \\
\hline DIL HTO & $5.9 \mathrm{E}-09$ & & & & & & & \\
\hline \multicolumn{9}{|l|}{$(\mathrm{Ci} / \mathrm{g})$} \\
\hline & & & & & & & & \\
\hline 168 & $7.72 \mathrm{E}+04$ & $1.84 \mathrm{E}+05$ & $5.93 \mathrm{E}+04$ & $7.30 \mathrm{E}+04$ & $2.85 \mathrm{E}+05$ & $1.65 \mathrm{E}+04$ & $2.98 \mathrm{E}+05$ & $2.05 \mathrm{E}+05$ \\
\hline SUM & $2.61 \mathrm{E}+05$ & & $1.32 \mathrm{E}+05$ & & $3.01 \mathrm{E}+05$ & & $5.03 \mathrm{E}+05$ & \\
\hline $\mathrm{Ci} / \mathrm{g}$ & $7.05 \mathrm{E}-06$ & & $3.57 \mathrm{E}-06$ & & $8.14 \mathrm{E}-06$ & & $1 . \overline{36 \mathrm{E}-05}$ & \\
\hline & & & & & & & & \\
\hline DIL OBT & $2.4 \mathrm{E}-09$ & & & & & & & \\
\hline \multicolumn{9}{|l|}{$\mathrm{Ci} / \mathrm{g}$} \\
\hline & & & & & & & & \\
\hline 8760 & $2.88 \mathrm{E}+00$ & $1.11 \mathrm{E}+01$ & $7.55 \mathrm{E}+03$ & $8.04 \mathrm{E}+04$ & $2.12 \mathrm{E}+01$ & $2.67 \mathrm{E}+02$ & $9.66 \mathrm{E}+01$ & $3.53 \mathrm{E}+02$ \\
\hline SUM & $1.40 \mathrm{E}+01$ & & $8.80 \mathrm{E}+04$ & & $2.88 \mathrm{E}+02$ & & $4.49 \mathrm{E}+02$ & \\
\hline $\mathrm{Ci} / \mathrm{g}$ & $3.78 \mathrm{E}-10$ & & $2.38 \mathrm{E}-06$ & & $7.78 \mathrm{E}-09$ & & $1.21 \mathrm{E}-08$ & \\
\hline & & & & & & & & \\
\hline DIL OBT & $2.4 \mathrm{E}-09$ & & & & & & & \\
\hline $\mathrm{Ci} / \mathrm{g}$ & & & & & & & & \\
\hline
\end{tabular}




\begin{tabular}{|c|c|c|c|c|c|c|c|c|}
\hline & \multicolumn{6}{|c|}{ Tritium Facilities, Design Basis Fire, Adverse Met Conditions } & & \\
\hline & \multicolumn{4}{|c|}{ Concentration Values for $10 \mathrm{~km}$ from release } & & & & \\
\hline \multicolumn{9}{|c|}{ Only Wheat Seed is examined since this is the edible portion of the Wheat. The "WHEAT" category is } \\
\hline \multicolumn{9}{|c|}{ actually the unedible plant body and is not considered in the dose estimate. } \\
\hline & & & & & & & & \\
\hline & \multicolumn{2}{|c|}{ VEGETABLES } & \multicolumn{2}{|c|}{ WHEAT SEED } & \multicolumn{2}{|l|}{ MEAT } & \multicolumn{2}{|l|}{ MILK } \\
\hline \multirow[t]{3}{*}{ HOUR } & HTO & OBT & HTO & OBT & HTO & OBT & HTO & $\overline{\mathrm{OBT}}$ \\
\hline & $(\mathrm{Bq} / \mathrm{g})$ & $(\mathrm{Bq} / \mathrm{g})$ & $(\mathrm{Bq} / \mathrm{g})$ & $(\mathrm{Ba} / \mathrm{g})$ & $(\mathrm{Bq} / \mathrm{g})$ & ) & $(\mathrm{Bq} / \mathrm{g})$ & $\mathrm{Bo} / \mathrm{g})$ \\
\hline & & & & & & & & \\
\hline 1 & $1.79 \mathrm{E}+06$ & $6.83 \mathrm{E}+03$ & $2.56 \mathrm{E}+05$ & $8.46 \mathrm{E}+02$ & $2.67 \mathrm{E}+04$ & $5.32 \mathrm{E}+00$ & $3.24 \mathrm{E}+03$ & $1.38 \mathrm{E}+03$ \\
\hline 2 & $1.06 \mathrm{E}+06$ & $1.08 \mathrm{E}+04$ & $4.33 \mathrm{E}+05$ & $1.68 \mathrm{E}+03$ & $4.48 \mathrm{E}+04$ & $1.45 \mathrm{E}+01$ & $7.71 \mathrm{E}+03$ & $3.31 \mathrm{E}+03$ \\
\hline 3 & $5.76 \mathrm{E}+05$ & $1.27 \mathrm{E}+04$ & $5.21 \mathrm{E}+05$ & $2.39 \mathrm{E}+03$ & $5.66 \mathrm{E}+04$ & $2.62 \mathrm{E}+01$ & $1.24 \mathrm{E}+04$ & $5.37 \mathrm{E}+03$ \\
\hline 4 & $3.48 \mathrm{E}+05$ & $1.38 \mathrm{E}+04$ & $5.50 \mathrm{E}+05$ & $2.99 \mathrm{E}+03$ & $6.44 \mathrm{E}+04$ & $3.96 \mathrm{E}+01$ & $1.70 \mathrm{E}+04$ & $7.37 \mathrm{E}+03$ \\
\hline 5 & $2.36 \mathrm{E}+05$ & $1.44 \mathrm{E}+04$ & $5.41 \mathrm{E}+05$ & $3.49 \mathrm{E}+03$ & $6.96 \mathrm{E}+04$ & $5.41 \mathrm{E}+01$ & $2.12 \mathrm{E}+04$ & $9.22 \mathrm{E}+03$ \\
\hline 6 & $1.79 \mathrm{E}+05$ & $1.48 \mathrm{E}+04$ & $5.10 \mathrm{E}+05$ & $3.91 \mathrm{E}+03$ & $6.9 \overline{\mathrm{IE}}+04$ & $6.15 \mathrm{E}+01$ & $6.70 \mathrm{E}+04$ & $2.09 \mathrm{E}+04$ \\
\hline 7 & $1.47 \mathrm{E}+05$ & $1.51 \mathrm{E}+04$ & $4.66 \mathrm{E}+05$ & $4.25 \mathrm{E}+03$ & $6.86 \mathrm{E}+04$ & $6.89 \mathrm{E}+01$ & $6.67 \mathrm{E}+04$ & $2.08 \mathrm{E}+04$ \\
\hline 8 & $1.28 \mathrm{E}+05$ & $1.54 \mathrm{E}+04$ & $4.18 E+05$ & $4.55 \mathrm{E}+03$ & $6.80 \mathrm{E}+04$ & $7.61 \mathrm{E}+01$ & $6.64 \mathrm{E}+04$ & $2.07 \mathrm{E}+04$ \\
\hline 9 & $1.16 \mathrm{E}+05$ & $1.56 \mathrm{E}+04$ & $3.70 \mathrm{E}+05$ & $4.79 \mathrm{E}+03$ & $6.75 \mathrm{E}+04$ & $8.33 \mathrm{E}+01$ & $6.62 \mathrm{E}+04$ & $2.06 \mathrm{E}+04$ \\
\hline 10 & $1.08 \mathrm{E}+05$ & $1.58 \mathrm{E}+04$ & $3.25 \mathrm{E}+05$ & $5.00 \mathrm{E}+03$ & $6.70 \mathrm{E}+04$ & $9.05 \mathrm{E}+01$ & $6.59 \mathrm{E}+04$ & $2.05 E+04$ \\
\hline 11 & $1.02 \mathrm{E}+05$ & $1.60 \mathrm{E}+04$ & $2.83 \mathrm{E}+05$ & $5.18 \mathrm{E}+03$ & $6.65 \mathrm{E}+04$ & $9.76 \mathrm{E}+01$ & $6.57 \mathrm{E}+04$ & $2.05 \mathrm{E}+04$ \\
\hline 12 & $9.66 \mathrm{E}+04$ & $1.62 \mathrm{E}+04$ & $2.46 \mathrm{E}+05$ & $5.33 \mathrm{E}+03$ & $6.60 \mathrm{E}+04$ & $1.05 \mathrm{E}+02$ & $6.54 \mathrm{E}+04$ & $2.04 \mathrm{E}+04$ \\
\hline 13 & $9.26 \mathrm{E}+04$ & $1.63 \mathrm{E}+04$ & $2.14 \mathrm{E}+05$ & $5.46 \mathrm{E}+03$ & $6.54 \mathrm{E}+04$ & $1.12 \mathrm{E}+02$ & $6.52 \mathrm{E}+04$ & $2.03 \mathrm{E}+04$ \\
\hline 14 & $8.92 \mathrm{E}+04$ & $1.65 \mathrm{E}+04$ & $1.86 \mathrm{E}+05$ & $5.57 \mathrm{E}+03$ & $6.49 E+04$ & $1.19 \mathrm{E}+02$ & $6.49 \mathrm{E}+04$ & $2.02 \mathrm{E}+04$ \\
\hline 15 & $8.61 \mathrm{E}+04$ & $1.66 \mathrm{E}+04$ & $1.62 \mathrm{E}+05$ & $5.67 \mathrm{E}+03$ & $6.44 \mathrm{E}+04$ & $1.25 \mathrm{E}+02$ & $6.47 \mathrm{E}+04$ & $2.02 E+04$ \\
\hline 16 & $8.33 \mathrm{E}+04$ & $1.67 \mathrm{E}+04$ & $1.41 \mathrm{E}+05$ & $5.76 \mathrm{E}+03$ & $6.39 \mathrm{E}+04$ & $1.32 \mathrm{E}+02$ & $6.44 \mathrm{E}+04$ & $E+0$ \\
\hline 17 & $8.07 \mathrm{E}+04$ & $1.69 \mathrm{E}+04$ & $1.24 \mathrm{E}+05$ & $5.84 \mathrm{E}+03$ & $6.34 \mathrm{E}+04$ & $1.39 \mathrm{E}+02$ & $6.42 \mathrm{E}+04$ & $2.00 \mathrm{E}+04$ \\
\hline 18 & $7.84 \mathrm{E}+04$ & $1.70 \mathrm{E}+04$ & $1.10 \mathrm{E}+05$ & $5.91 E+03$ & $6.38 \mathrm{E}+04$ & $1.55 \mathrm{E}+02$ & $6.18 \mathrm{E}+04$ & $3.03 \mathrm{E}+04$ \\
\hline 19 & $7.62 \mathrm{E}+04$ & $1.71 E+04$ & $9.74 \mathrm{E}+04$ & $5.98 \mathrm{E}+03$ & $6.41 \mathrm{E}+04$ & $1.71 \mathrm{E}+02$ & $6.19 \mathrm{E}+04$ & $3.04 \mathrm{E}+04$ \\
\hline 20 & $7.41 \mathrm{E}+04$ & $1.72 \mathrm{E}+04$ & $8.72 \mathrm{E}+04$ & $6.04 \mathrm{E}+03$ & $6.43 \mathrm{E}+04$ & $1.87 \mathrm{E}+02$ & $6.21 \mathrm{E}+04$ & $3.05 \mathrm{E}+04$ \\
\hline 21 & $7.21 \mathrm{E}+04$ & $1.74 \mathrm{E}+04$ & $7.87 \mathrm{E}+04$ & $6.09 \mathrm{E}+03$ & $6.46 \mathrm{E}+04$ & $2.03 \mathrm{E}+02$ & $6.22 \mathrm{E}+04$ & $3.06 \mathrm{E}+04$ \\
\hline 22 & $7.03 \mathrm{E}+04$ & $1.75 \mathrm{E}+04$ & $7.15 \mathrm{E}+04$ & $6.15 \mathrm{E}+03$ & $6.49 \mathrm{E}+04$ & $2.19 \mathrm{E}+02$ & $6.23 \mathrm{E}+04$ & $3.06 \mathrm{E}+04$ \\
\hline 23 & $6.86 \mathrm{E}+04$ & $1.76 \mathrm{E}+04$ & $6.55 \mathrm{E}+04$ & $6.20 \mathrm{E}+03$ & $6.51 \mathrm{E}+04$ & $2.35 \mathrm{E}+02$ & $6.25 \mathrm{E}+04$ & $3.07 \mathrm{E}+04$ \\
\hline 24 & $6.68 \mathrm{E}+04$ & $1.77 \mathrm{E}+04$ & $6.05 \mathrm{E}+04$ & $6.24 \mathrm{E}+03$ & $6.53 \mathrm{E}+04$ & $2.52 \mathrm{E}+02$ & $6.26 \mathrm{E}+04$ & $3.08 \mathrm{E}+04$ \\
\hline 25 & $6.52 \mathrm{E}+04$ & $1.78 \mathrm{E}+04$ & $5.62 \mathrm{E}+04$ & $6.29 \mathrm{E}+03$ & $6.55 \mathrm{E}+04$ & $2.68 \mathrm{E}+02$ & $6.27 \mathrm{E}+04$ & $3.08 \mathrm{E}+04$ \\
\hline 26 & $6.37 \mathrm{E}+04$ & $1.79 \mathrm{E}+04$ & $5.25 \mathrm{E}+04$ & $6.33 \mathrm{E}+03$ & $6.56 \mathrm{E}+04$ & $2.84 \mathrm{E}+02$ & $6.28 \mathrm{E}+04$ & $3.09 \mathrm{E}+04$ \\
\hline 27 & $6.22 \mathrm{E}+04$ & $1.80 \mathrm{E}+04$ & $4.94 \mathrm{E}+04$ & $6.37 \mathrm{E}+03$ & $6.58 \mathrm{E}+04$ & $3.01 \mathrm{E}+02$ & $6.29 \mathrm{E}+04$ & $3.10 \mathrm{E}+04$ \\
\hline 28 & $6.09 \mathrm{E}+04$ & $1.81 \mathrm{E}+04$ & $4.68 \mathrm{E}+04$ & $6.41 \mathrm{E}+03$ & $6.59 \mathrm{E}+04$ & $3.17 \mathrm{E}+02$ & $6.30 \mathrm{E}+04$ & $3.10 \mathrm{E}+04$ \\
\hline 29 & $5.97 \mathrm{E}+04$ & $1.82 \mathrm{E}+04$ & $4.45 \mathrm{E}+04$ & $6.45 \mathrm{E}+03$ & $6.61 \mathrm{E}+04$ & $3.34 \mathrm{E}+02$ & $6.31 \mathrm{E}+04$ & $3.11 \mathrm{E}+04$ \\
\hline 30 & $5.85 \mathrm{E}+04$ & $1.82 \mathrm{E}+04$ & $4.25 \mathrm{E}+04$ & $6.48 \mathrm{E}+03$ & $6.55 \mathrm{E}+04$ & $3.40 \mathrm{E}+02$ & $6.35 \mathrm{E}+04$ & $1.98 \mathrm{E}+04$ \\
\hline 31 & $5.73 E+04$ & $1.83 \mathrm{E}+04$ & $4.08 \mathrm{E}+04$ & $6.52 \mathrm{E}+03$ & $6.50 \mathrm{E}+04$ & $3.47 \mathrm{E}+02$ & $6.33 \mathrm{E}+04$ & $1.98 \mathrm{E}+04$ \\
\hline 32 & $5.62 \mathrm{E}+04$ & $1.84 \mathrm{E}+04$ & $3.93 \mathrm{E}+04$ & $6.55 \mathrm{E}+03$ & $6.45 \mathrm{E}+04$ & $3.54 \mathrm{E}+02$ & $6.30 \mathrm{E}+04$ & $1.97 \mathrm{E}+04$ \\
\hline 33 & $5.52 \mathrm{E}+04$ & $1.85 \mathrm{E}+04$ & $3.79 \mathrm{E}+04$ & $6.59 \mathrm{E}+03$ & $6.40 \mathrm{E}+04$ & $3.61 \mathrm{E}+02$ & $6.28 \mathrm{E}+04$ & $1.96 \mathrm{E}+04$ \\
\hline 34 & $5.41 \mathrm{E}+04$ & $1.86 \mathrm{E}+04$ & $3.68 \mathrm{E}+04$ & $6.62 \mathrm{E}+03$ & $6.34 \mathrm{E}+04$ & $3.67 \mathrm{E}+02$ & $6.25 \mathrm{E}+04$ & $1.95 \mathrm{E}+04$ \\
\hline 35 & $5.31 \mathrm{E}+04$ & $1.86 \mathrm{E}+04$ & $3.57 \mathrm{E}+04$ & $6.65 \mathrm{E}+03$ & $6.29 \mathrm{E}+04$ & $3.74 \mathrm{E}+02$ & $6.23 \mathrm{E}+04$ & $1.95 \mathrm{E}+04$ \\
\hline 36 & $5.21 \mathrm{E}+04$ & $1.87 \mathrm{E}+04$ & $3.48 \mathrm{E}+04$ & $6.68 E+03$ & $6.24 \mathrm{E}+04$ & $3.80 \mathrm{E}+02$ & $6.20 \mathrm{E}+04$ & $1.94 \mathrm{E}+04$ \\
\hline 37 & $5.11 \mathrm{E}+04$ & $1.88 \mathrm{E}+04$ & $3.39 \mathrm{E}+04$ & $6.71 \mathrm{E}+03$ & $6.19 \mathrm{E}+04$ & $3.86 \mathrm{E}+02$ & $6.18 \mathrm{E}+04$ & $1.93 \bar{E}+04$ \\
\hline 38 & $5.01 \mathrm{E}+04$ & $1.89 \mathrm{E}+04$ & $3.31 \mathrm{E}+04$ & $6.74 \mathrm{E}+03$ & $6.14 \mathrm{E}+04$ & $3.93 \mathrm{E}+02$ & $6.15 \mathrm{E}+04$ & $1.92 \mathrm{E}+04$ \\
\hline
\end{tabular}




\begin{tabular}{|c|c|c|c|c|c|c|c|c|}
\hline 39 & $4.91 \mathrm{E}+04$ & $1.89 \mathrm{E}+04$ & $3.24 \mathrm{E}+04$ & $6.77 \mathrm{E}+03$ & $6.09 \mathrm{E}+04$ & $3.99 \mathrm{E}+02$ & $6.13 \mathrm{E}+04$ & $1.91 \mathrm{E}+04$ \\
\hline 40 & $4.82 \mathrm{E}+04$ & $1.90 \mathrm{E}+04$ & $3.17 \mathrm{E}+04$ & $6.80 \mathrm{E}+03$ & $6.04 \mathrm{E}+04$ & $4.05 \mathrm{E}+02$ & $6.10 \mathrm{E}+04$ & $1.91 \mathrm{E}+0$ \\
\hline 41 & $4.73 E+04$ & $1.91 \mathrm{E}+04$ & $3.11 \mathrm{E}+04$ & $6.83 \mathrm{E}+03$ & $5.99 \mathrm{E}+04$ & $4.11 \mathrm{E}+02$ & $6.08 \mathrm{E}+04$ & $1.90 \mathrm{E}+$ \\
\hline 42 & $4.65 \mathrm{E}+04$ & $1.91 \mathrm{E}+04$ & $3.05 \mathrm{E}+04$ & $6.86 \mathrm{E}+03$ & $5.99 \mathrm{E}+04$ & $4.28 \mathrm{E}+02$ & $5.81 \mathrm{E}+04$ & $3.06 \mathrm{E}+0$ \\
\hline 43 & $4.56 \mathrm{E}+04$ & $1.92 \mathrm{E}+04$ & $2.99 \mathrm{E}+04$ & $6.88 \mathrm{E}+03$ & $6.00 \mathrm{E}+04$ & $4.44 \mathrm{E}+02$ & $5.81 \mathrm{E}+04$ & $3.06 \mathrm{E}+0$ \\
\hline 44 & $4.48 \mathrm{E}+04$ & $1.92 \mathrm{E}+04$ & $2.94 \mathrm{E}+04$ & $6.91 \mathrm{E}+03$ & $6.00 \mathrm{E}+04$ & $4.61 \mathrm{E}+02$ & $5.81 \mathrm{E}+04$ & $3.06 \mathrm{E}+0$ \\
\hline$\overline{45}$ & $4.41 \mathrm{E}+04$ & $1.93 \mathrm{E}+04$ & $2.89 E+04$ & $6.94 \mathrm{E}+03$ & $6.00 \mathrm{E}+04$ & $4.77 \mathrm{E}+02$ & $5.81 \mathrm{E}+04$ & $3.06 \mathrm{E}+$ \\
\hline$\overline{46}$ & $4.33 \mathrm{E}+04$ & $1.94 \mathrm{E}+04$ & $2.84 \mathrm{E}+04$ & $6.97 \mathrm{E}+03$ & $5.99 \mathrm{E}+04$ & $4.94 \mathrm{E}+02$ & $5.81 \mathrm{E}+04$ & $3.06 \mathrm{E}+\mathrm{C}$ \\
\hline 47 & $4.26 \mathrm{E}+04$ & $1.94 \mathrm{E}+04$ & $2.80 \mathrm{E}+04$ & $6.99 \mathrm{E}+03$ & $5.99 \mathrm{E}+04$ & $5.10 \mathrm{E}+02$ & $5.81 \mathrm{E}+04$ & $3.07 \mathrm{E}+0$ \\
\hline 48 & $4.19 \mathrm{E}+04$ & $1.95 \mathrm{E}+04$ & $2.76 \mathrm{E}+04$ & $7.02 \mathrm{E}+03$ & $5.99 \mathrm{E}+04$ & $5.26 \mathrm{E}+02$ & $5.81 E+04$ & $3.07 \mathrm{E}+04$ \\
\hline 49 & $4.12 \mathrm{E}+04$ & $1.95 \mathrm{E}+04$ & $2.72 \mathrm{E}+04$ & $7.04 \mathrm{E}+03$ & $5.98 \mathrm{E}+04$ & $5.43 \mathrm{E}+02$ & $5.81 \mathrm{E}+04$ & $3.07 \mathrm{E}+0$ \\
\hline 50 & $4.06 \mathrm{E}+04$ & $1.96 \mathrm{E}+04$ & $2.68 \mathrm{E}+04$ & $7.07 \mathrm{E}+03$ & $5.98 \mathrm{E}+04$ & $5.59 \mathrm{E}+02$ & $5.81 \mathrm{E}+04$ & $3.07 E+0$ \\
\hline 51 & $4.00 \mathrm{E}+04$ & $1.96 \mathrm{E}+04$ & $2.64 \mathrm{E}+04$ & $7.09 \mathrm{E}+03$ & $5.97 E+04$ & $5.76 \mathrm{E}+02$ & $5.81 E+04$ & $3.07 \mathrm{E}+04$ \\
\hline 52 & $3.94 E+04$ & $1.97 \mathrm{E}+04$ & $2.60 \mathrm{E}+04$ & $7.12 \mathrm{E}+03$ & $5.97 \mathrm{E}+04$ & $5.92 \mathrm{E}+02$ & $5.80 \mathrm{E}+04$ & $3.07 \bar{E}+04$ \\
\hline 53 & $3.89 \mathrm{E}+04$ & $1.97 \mathrm{E}+04$ & $2.56 \mathrm{E}+04$ & $7.14 \mathrm{E}+03$ & $5.96 \mathrm{E}+04$ & $6.08 \mathrm{E}+02$ & $5.80 \mathrm{E}+04$ & $3.07 \mathrm{E}+\mathrm{C}$ \\
\hline 54 & $3.83 \mathrm{E}+04$ & $1.98 \mathrm{E}+04$ & $2.52 \mathrm{E}+04$ & $7.16 \mathrm{E}+03$ & $5.91 \mathrm{E}+04$ & $6.14 \mathrm{E}+02$ & $5.73 \mathrm{E}+04$ & $1.80 \mathrm{E}+0$ \\
\hline 55 & $3.78 \mathrm{E}+04$ & $1.98 \mathrm{E}+04$ & $2.48 \mathrm{E}+04$ & $7.19 \mathrm{E}+03$ & $5.86 \mathrm{E}+04$ & $6.20 \mathrm{E}+02$ & $5.71 \mathrm{E}+04$ & $1.79 \mathrm{E}+04$ \\
\hline 56 & $3.73 \mathrm{E}+04$ & $1.98 \mathrm{E}+04$ & $2.44 \mathrm{E}+04$ & $7.21 \mathrm{E}+03$ & $5.82 \mathrm{E}+04$ & $6.26 \mathrm{E}+02$ & $5.68 \mathrm{E}+04$ & $1.78 \mathrm{E}+0$ \\
\hline 57 & $3.68 \mathrm{E}+04$ & $1.99 \mathrm{E}+04$ & $2.41 \mathrm{E}+04$ & $7.23 \mathrm{E}+03$ & $5.77 \mathrm{E}+04$ & $6.32 \mathrm{E}+02$ & $5.66 \mathrm{E}+04$ & $1.77 \mathrm{E}+0$ \\
\hline 58 & $3.63 \mathrm{E}+04$ & $1.99 \mathrm{E}+04$ & $2.37 \mathrm{E}+04$ & $7.25 \mathrm{E}+03$ & $5.72 \mathrm{E}+04$ & $6.37 \mathrm{E}+02$ & $5.64 \mathrm{E}+04$ & $1.77 \mathrm{E}+04$ \\
\hline 59 & $3.59 \mathrm{E}+04$ & $2.00 \mathrm{E}+04$ & $2.33 \mathrm{E}+04$ & $7.27 \mathrm{E}+03$ & $5.67 \mathrm{E}+04$ & $6.43 \mathrm{E}+02$ & $5.61 \mathrm{E}+04$ & $1.76 \mathrm{E}+0$ \\
\hline 60 & $3.54 \mathrm{E}+04$ & $2.00 \mathrm{E}+04$ & $2.29 \mathrm{E}+04$ & $7.30 \mathrm{E}+03$ & $5.63 \mathrm{E}+04$ & $6.49 \mathrm{E}+02$ & $5.59 \mathrm{E}+04$ & $1.75 \mathrm{E}+04$ \\
\hline 61 & $3.50 \mathrm{E}+04$ & $2.00 \mathrm{E}+04$ & $2.25 \mathrm{E}+04$ & $7.32 \mathrm{E}+03$ & $5.58 \mathrm{E}+04$ & $6.54 \mathrm{E}+02$ & $5.57 \mathrm{E}+04$ & $1.75 \mathrm{E}+04$ \\
\hline 62 & $3.45 \mathrm{E}+04$ & $2.01 \mathrm{E}+04$ & $2.22 \mathrm{E}+04$ & $7.34 \mathrm{E}+03$ & $5.53 \mathrm{E}+04$ & $6.60 \mathrm{E}+02$ & $5.55 \mathrm{E}+04$ & $1.74 \mathrm{E}+04$ \\
\hline 63 & $3.41 \mathrm{E}+04$ & $2.01 \mathrm{E}+04$ & $2.18 \mathrm{E}+04$ & $7.36 \mathrm{E}+03$ & $5.49 \mathrm{E}+04$ & $6.65 \mathrm{E}+02$ & $5.52 \mathrm{E}+04$ & $1.73 \mathrm{E}+0$ \\
\hline 64 & $3.37 \mathrm{E}+04$ & $2.02 \mathrm{E}+04$ & $2.14 \mathrm{E}+04$ & $7.38 \mathrm{E}+03$ & $5.44 \mathrm{E}+04$ & $6.71 E+02$ & $5.50 \mathrm{E}+04$ & $1.73 \mathrm{E}+04$ \\
\hline 65 & $3.33 \mathrm{E}+04$ & $2.02 \mathrm{E}+04$ & $2.11 \mathrm{E}+04$ & $7.40 \mathrm{E}+03$ & $5.40 \mathrm{E}+04$ & $6.76 \mathrm{E}+02$ & $5.48 \mathrm{E}+04$ & $1.72 \mathrm{E}+04$ \\
\hline 66 & $3.28 \mathrm{E}+04$ & $2.02 \mathrm{E}+04$ & $2.07 \mathrm{E}+04$ & $7.42 \mathrm{E}+03$ & $5.39 \mathrm{E}+04$ & $6.92 \mathrm{E}+02$ & $5.22 \mathrm{E}+04$ & $2.94 \mathrm{E}+04$ \\
\hline 67 & $3.23 \mathrm{E}+04$ & $2.03 \mathrm{E}+04$ & $2.04 \mathrm{E}+04$ & $7.44 \mathrm{E}+03$ & $5.38 \mathrm{E}+04$ & $7.08 \mathrm{E}+02$ & $5.22 \mathrm{E}+04$ & $2.94 \mathrm{E}+04$ \\
\hline 68 & $3.19 \mathrm{E}+04$ & $2.03 \mathrm{E}+04$ & $2.01 \mathrm{E}+04$ & $7.45 \mathrm{E}+03$ & $5.37 \mathrm{E}+04$ & $7.24 \mathrm{E}+02$ & $5.21 \mathrm{E}+04$ & $2.94 \mathrm{E}+04$ \\
\hline 69 & $3.15 \mathrm{E}+04$ & $2.03 \mathrm{E}+04$ & $1.97 \mathrm{E}+04$ & $7.47 \mathrm{E}+03$ & $5.36 \mathrm{E}+04$ & $7.40 \mathrm{E}+02$ & $5.21 \mathrm{E}+04$ & $2.94 \mathrm{E}+04$ \\
\hline 168 & $9.04 \mathrm{E}+03$ & $2.13 E+04$ & $8.09 \mathrm{E}+03$ & $8.57 \mathrm{E}+03$ & $3.19 \mathrm{E}+04$ & $1.76 \mathrm{E}+03$ & $3.32 \mathrm{E}+04$ & $2.36 \mathrm{E}+04$ \\
\hline 336 & $4.13 E+03$ & $1.78 \mathrm{E}+04$ & $3.55 \mathrm{E}+03$ & $9.11 \mathrm{E}+03$ & $1.27 \mathrm{E}+04$ & $3.37 \mathrm{E}+03$ & $2.01 \mathrm{E}+04$ & $1.80 \mathrm{E}+04$ \\
\hline 504 & $3.01 \mathrm{E}+03$ & $1.47 \mathrm{E}+04$ & $2.41 \mathrm{E}+03$ & $9.42 \mathrm{E}+03$ & $6.01 \mathrm{E}+03$ & $4.24 \mathrm{E}+03$ & $8.54 \mathrm{E}+03$ & $1.19 \mathrm{E}+04$ \\
\hline 720 & $2.19 \mathrm{E}+03$ & $1.14 \mathrm{E}+04$ & $1.61 \mathrm{E}+03$ & $9.69 \mathrm{E}+03$ & $3.00 \mathrm{E}+03$ & $4.71 \mathrm{E}+03$ & $4.14 \mathrm{E}+03$ & $8.24 \mathrm{E}+03$ \\
\hline 2160 & $4.09 \mathrm{E}+02$ & $2.13 \mathrm{E}+03$ & $1.80 \mathrm{E}+02$ & $1.02 \mathrm{E}+04$ & $2.64 \mathrm{E}+02$ & $2.78 \mathrm{E}+03$ & $9.42 \mathrm{E}+02$ & $2.82 \mathrm{E}+03$ \\
\hline 4320 & $3.94 \mathrm{E}+01$ & $1.78 \mathrm{E}+02$ & $9.09 \mathrm{E}+02$ & $9.73 \mathrm{E}+03$ & $4.18 \mathrm{E}+01$ & $6.59 \mathrm{E}+02$ & $1.10 \mathrm{E}+02$ & $4.33 \mathrm{E}+02$ \\
\hline 4800 & $2.34 \mathrm{E}+01$ & $1.03 \mathrm{E}+02$ & $9.06 \mathrm{E}+02$ & $9.70 \mathrm{E}+03$ & $3.02 \mathrm{E}+01$ & $4.73 \mathrm{E}+02$ & $3.56 \mathrm{E}+01$ & $1.40 \mathrm{E}+02$ \\
\hline 8760 & $3.21 \mathrm{E}-01$ & $1.25 \mathrm{E}+00$ & $8.83 E+02$ & $9.46 \mathrm{E}+03$ & $2.39 \mathrm{E}+00$ & $3.09 \mathrm{E}+01$ & $1.09 \mathrm{E}+01$ & $4.07 \mathrm{E}+01$ \\
\hline$\overline{\mathbf{B T}}$ & & $\begin{array}{l}8 \mathrm{8E}+4 \\
\mathrm{~kg}\end{array}$ & $8 \mathrm{~Bq} / \mathbf{g}$ & & & & & \\
\hline $\mathbf{O}$ & $\begin{array}{l}5.9 \mathrm{E} 6 \\
\mathrm{pCi} / \mathrm{L}\end{array}$ & $\begin{array}{l}2.18 \mathrm{E}+5 \\
\mathrm{~Bq} / \mathrm{kg}\end{array}$ & $8.3 \mathrm{~Bq} / \mathrm{g}$ & & & & & \\
\hline & \multirow{2}{*}{\multicolumn{2}{|c|}{ VEGETABLES }} & & & & & & \\
\hline & & & \multicolumn{2}{|c|}{ WHEAT SEED } & \multicolumn{2}{|l|}{ MEAT } & \multicolumn{2}{|l|}{ MILK } \\
\hline \multirow[t]{2}{*}{ HOUR } & TO & OBT & TO & OBT & HTO & $\overline{B T}$ & HTO & OBT \\
\hline & $(\mathrm{Bq} / \mathrm{g})$ & $(\mathrm{Bq} / \mathrm{g})$ & $(\mathrm{Bq} / \mathrm{g})$ & $(\mathrm{Bq} / \mathrm{g})$ & $(\overline{\mathrm{Bq}} / \mathrm{g})$ & $(\mathrm{Bq} / \mathrm{g})$ & $(\mathrm{Bq} / \mathrm{g})$ & $(\mathrm{Bq} / \mathrm{g})$ \\
\hline ak HTO & & & & & & & & \\
\hline
\end{tabular}


a Tritium Oxide Release from SRS Tritium Facilities (U)

\begin{tabular}{|c|c|c|c|c|c|c|c|c|}
\hline $1 / 4 / 5 / 6$ & $1.79 \mathrm{E}+06$ & $6.83 \mathrm{E}+03$ & $5.50 \mathrm{E}+05$ & $2.99 \mathrm{E}+03$ & $6.96 \mathrm{E}+04$ & $5.41 \mathrm{E}+01$ & $6.70 \mathrm{E}+04$ & $2.09 \mathrm{E}+04$ \\
\hline$\overline{\text { SUM }}$ & $1.79 \mathrm{E}+06$ & & $5.53 \mathrm{E}+05$ & & $6.96 \mathrm{E}+04$ & & $8.78 \mathrm{E}+04$ & \\
\hline $\mathrm{Ci} / \mathrm{g}$ & $4.84 \mathrm{E}-05$ & & $1.50 \mathrm{E}-05$ & & $1.88 \mathrm{E}-06$ & & $2.37 \mathrm{E}-06$ & \\
\hline DU UTO & $50=0$ & & & & & & & \\
\hline \multirow{2}{*}{\multicolumn{9}{|c|}{$(\mathrm{Ci} / \mathrm{g})$}} \\
\hline & & & & & & & & \\
\hline 168 & $9.04 E+03$ & $2.13 E+04$ & $8.09 E+03$ & $8.57 E+03$ & $3.19 \mathrm{E}+04$ & $1.76 \mathrm{E}+03$ & $3.32 \mathrm{E}+04$ & $2.36 E+04$ \\
\hline$\overline{\text { SUM }}$ & $3.03 E+04$ & & $1.67 \mathrm{E}+04$ & & $3.37 \mathrm{E}+04$ & & $5.69 \mathrm{E}+04$ & \\
\hline $\mathrm{Ci} / \mathrm{g}$ & 8.19E-07 & & $4.50 \mathrm{E}-07$ & & 9.10E-07 & & $1.54 \mathrm{E}-06$ & \\
\hline & & & & & & & & \\
\hline DIL OBT & $2.4 \mathrm{E}-09$ & & & & & & & \\
\hline \multicolumn{9}{|l|}{$\mathrm{Ci} / \mathrm{g}$} \\
\hline & & & & & & & & \\
\hline 8760 & $3.21 \mathrm{E}-01$ & $1.25 \mathrm{E}+00$ & $8.83 \mathrm{E}+02$ & $9.46 \mathrm{E}+03$ & $2.39 \mathrm{E}+00$ & $3.09 \mathrm{E}+01$ & $1.09 \mathrm{E}+0 \mathrm{I}$ & $4.07 \mathrm{E}+01$ \\
\hline$\overline{\text { SUM }}$ & $1.57 \mathrm{E}+00$ & & $1.03 E+04$ & & $3.33 \mathrm{E}+01$ & & $5.17 \mathrm{E}+01$ & \\
\hline $\mathrm{Ci} / \mathrm{g}$ & $4.25 \mathrm{E}-11$ & & $2.79 \mathrm{E}-07$ & & $8.99 \mathrm{E}-10$ & & $1.40 \mathrm{E}-09$ & \\
\hline & & & & & & & & \\
\hline DIL OBT & $2.4 \mathrm{E}-09$ & & & & & & & \\
\hline $\mathrm{Ci} / \mathrm{g}$ & & & & & & & & \\
\hline
\end{tabular}




\begin{tabular}{|c|c|c|c|c|c|c|c|c|}
\hline & \multicolumn{6}{|c|}{ Tritium Facilities, Design Basis Fire, Adverse Met Conditions } & & \\
\hline & \multicolumn{6}{|c|}{ Concentration Values for $11.54 \mathrm{~km}$ from release } & & \\
\hline \multicolumn{9}{|c|}{ Only Wheat Seed is examined since this is the edible portion of the Wheat. The "WHEAT" category is } \\
\hline \multicolumn{9}{|c|}{ actually the unedible plant body and is not considered in the dose estimate. } \\
\hline & & & & & & & & \\
\hline & \multicolumn{2}{|c|}{ VEGETABLES } & \multicolumn{2}{|c|}{ WHEAT SEED } & \multicolumn{2}{|l|}{ MEAT } & \multicolumn{2}{|l|}{ MILK } \\
\hline \multirow[t]{3}{*}{ HOUR } & HTO & OBT & HTO & OBT & HTO & OBT & HTO & $\overline{\mathrm{OBT}}$ \\
\hline & $(\mathrm{Bq} / \mathrm{g})$ & $(\mathrm{Bq} / \mathrm{g})$ & $(\mathrm{Bq} / \mathrm{g})$ & $(\mathrm{Bq} / \mathrm{g})$ & $(\mathrm{Bq} / \mathrm{g})$ & $(\mathrm{Ba} / \mathrm{g})$ & $(\mathrm{Bq} / \mathrm{g})$ & $(\mathrm{Bq} / \mathrm{g})$ \\
\hline & & & & & & & & \\
\hline 1 & $4.20 \mathrm{E}+05$ & $2.73 \mathrm{E}+02$ & $1.02 \mathrm{E}+04$ & $3.38 \mathrm{E}+01$ & $1.43 \mathrm{E}+03$ & $2.5 \overline{1 \mathrm{E}}-01$ & $1.73 \mathrm{E}+02$ & $6.86 \mathrm{E}+01$ \\
\hline 2 & $1.84 \mathrm{E}+06$ & $7.30 \mathrm{E}+03$ & $2.74 \mathrm{E}+05$ & $9.14 \mathrm{E}+02$ & $2.87 \mathrm{E}+04$ & $5.96 \mathrm{E}+00$ & $3.24 \mathrm{E}+03$ & $1.38 \mathrm{E}+03$ \\
\hline 3 & $9.60 \mathrm{E}+05$ & $1.07 \mathrm{E}+04$ & $4.30 \mathrm{E}+05$ & $1.69 \mathrm{E}+03$ & $4.49 \mathrm{E}+04$ & $1.52 \mathrm{E}+01$ & $7.27 \mathrm{E}+03$ & $3.12 \mathrm{E}+03$ \\
\hline 4 & $5.56 \mathrm{E}+05$ & $1.25 \mathrm{E}+04$ & $5.08 \mathrm{E}+05$ & $2.37 \mathrm{E}+03$ & $5.59 \mathrm{E}+04$ & $2.67 \mathrm{E}+01$ & $1.15 \mathrm{E}+04$ & $4.98 \mathrm{E}+03$ \\
\hline 5 & $3.61 \mathrm{E}+05$ & $1.35 \mathrm{E}+04$ & $5.33 \mathrm{E}+05$ & $2.94 \mathrm{E}+03$ & $6.33 \mathrm{E}+04$ & $3.99 \mathrm{E}+01$ & $1.57 \mathrm{E}+04$ & $6.81 \mathrm{E}+03$ \\
\hline 6 & $2.63 \mathrm{E}+05$ & $1.41 \mathrm{E}+04$ & $5.24 \mathrm{E}+05$ & $3.43 \mathrm{E}+03$ & $6.29 \mathrm{E}+04$ & $4.66 \mathrm{E}+01$ & $6.09 \mathrm{E}+04$ & $1.90 \mathrm{E}+0$ \\
\hline$\overline{7}$ & $2.10 \mathrm{E}+05$ & $1.46 \mathrm{E}+04$ & $4.95 \mathrm{E}+05$ & $3.84 \mathrm{E}+03$ & $6.24 \mathrm{E}+04$ & $5.33 \mathrm{E}+01$ & $6.07 \mathrm{E}+04$ & $1.89 \mathrm{E}+04$ \\
\hline 8 & $1.80 \mathrm{E}+05$ & $1.50 \mathrm{E}+04$ & $4.56 \mathrm{E}+05$ & $4.19 \mathrm{E}+03$ & $6.20 \mathrm{E}+04$ & $5.99 \mathrm{E}+01$ & $6.05 \mathrm{E}+04$ & $1.89 \mathrm{E}+04$ \\
\hline 9 & $1.60 \mathrm{E}+05$ & $1.53 \mathrm{E}+04$ & $4.12 \mathrm{E}+05$ & $4.48 \mathrm{E}+03$ & $6.15 \mathrm{E}+04$ & $6.65 \mathrm{E}+01$ & $6.03 \mathrm{E}+04$ & $1.88 \mathrm{E}+04$ \\
\hline 10 & $1.47 \mathrm{E}+05$ & $1.56 \mathrm{E}+04$ & $3.68 \mathrm{E}+05$ & $4.73 \mathrm{E}+03$ & $6.11 \mathrm{E}+04$ & $7.30 \mathrm{E}+01$ & $6.01 E+04$ & $1.87 \mathrm{E}+04$ \\
\hline 11 & $1.38 \mathrm{E}+05$ & $1.58 \mathrm{E}+04$ & $3.26 \mathrm{E}+05$ & $4.95 \mathrm{E}+03$ & $6.07 \mathrm{E}+04$ & $7.95 \mathrm{E}+01$ & $5.99 \mathrm{E}+04$ & $1.87 \mathrm{E}+04$ \\
\hline 12 & $1.31 \mathrm{E}+05$ & $1.61 \mathrm{E}+04$ & $2.88 \mathrm{E}+05$ & $5.14 \mathrm{E}+03$ & $6.02 \mathrm{E}+04$ & $8.59 \mathrm{E}+01$ & $5.96 \mathrm{E}+04$ & $1.86 \mathrm{E}+04$ \\
\hline 13 & $1.25 \mathrm{E}+05$ & $1.63 \mathrm{E}+04$ & $2.53 \mathrm{E}+05$ & $5.30 \mathrm{E}+03$ & $5.98 \mathrm{E}+04$ & $9.23 \mathrm{E}+01$ & $5.94 \mathrm{E}+04$ & $1.85 \mathrm{E}+04$ \\
\hline 14 & $1.20 \mathrm{E}+05$ & $1.65 \mathrm{E}+04$ & $2.23 \mathrm{E}+05$ & $5.45 \mathrm{E}+03$ & $5.93 \mathrm{E}+04$ & $9.86 \mathrm{E}+01$ & $5.92 \mathrm{E}+04$ & $1.85 \mathrm{E}+04$ \\
\hline 15 & $1.16 \mathrm{E}+05$ & $1.67 \mathrm{E}+04$ & $1.97 \mathrm{E}+05$ & $5.58 \mathrm{E}+03$ & $5.89 \mathrm{E}+04$ & $1.05 E+02$ & $5.90 \mathrm{E}+04$ & $1.84 E+04$ \\
\hline 16 & $1.12 \mathrm{E}+05$ & $1.69 \mathrm{E}+04$ & $1.74 \mathrm{E}+05$ & $5.69 \mathrm{E}+03$ & $5.84 \mathrm{E}+04$ & $1.11 \mathrm{E}+02$ & $5.88 \mathrm{E}+04$ & $1.83 \mathrm{E}+0$ \\
\hline 17 & $1.08 \mathrm{E}+05$ & $1.71 \mathrm{E}+04$ & $1.55 \mathrm{E}+05$ & $5.79 \mathrm{E}+03$ & $5.80 \mathrm{E}+04$ & $1.17 \mathrm{E}+02$ & $5.86 \mathrm{E}+04$ & $1.83 \mathrm{E}+04$ \\
\hline 18 & $1.05 \mathrm{E}+05$ & $1.73 \mathrm{E}+04$ & $1.38 \mathrm{E}+05$ & $5.89 \mathrm{E}+03$ & $5.87 \mathrm{E}+04$ & $1.33 \mathrm{E}+02$ & $5.68 \mathrm{E}+04$ & $2.89 \mathrm{E}+04$ \\
\hline 19 & $1.02 \mathrm{E}+05$ & $1.74 \mathrm{E}+04$ & $1.24 \mathrm{E}+05$ & $5.98 \mathrm{E}+03$ & $5.93 \mathrm{E}+04$ & $1.48 \mathrm{E}+02$ & $5.72 \mathrm{E}+04$ & $2.91 \mathrm{E}+04$ \\
\hline 20 & $9.91 \mathrm{E}+04$ & $1.76 \mathrm{E}+04$ & $1.13 \mathrm{E}+05$ & $6.06 \mathrm{E}+03$ & $5.99 \mathrm{E}+04$ & $1.64 \mathrm{E}+02$ & $5.75 \mathrm{E}+04$ & $2.92 \mathrm{E}+04$ \\
\hline 21 & $9.64 \mathrm{E}+04$ & $1.77 \mathrm{E}+04$ & $1.03 \mathrm{E}+05$ & $6.13 E+03$ & $6.05 \mathrm{E}+04$ & $1.80 \mathrm{E}+02$ & $5.77 \mathrm{E}+04$ & $2.94 \mathrm{E}+04$ \\
\hline 22 & $9.39 E+04$ & $1.79 \mathrm{E}+04$ & $9.41 \mathrm{E}+04$ & $6.21 \mathrm{E}+03$ & $6.10 \mathrm{E}+04$ & $1.96 \mathrm{E}+02$ & $5.80 \mathrm{E}+04$ & $2.95 \mathrm{E}+0$ \\
\hline 23 & $9.15 \mathrm{E}+04$ & $1.81 \mathrm{E}+04$ & $8.70 \mathrm{E}+04$ & $6.27 \mathrm{E}+03$ & $6.15 \mathrm{E}+04$ & $2.12 \mathrm{E}+02$ & $5.83 \mathrm{E}+04$ & $2.96 \mathrm{E}+0$ \\
\hline 24 & $8.91 \mathrm{E}+04$ & $1.82 \mathrm{E}+04$ & $8.09 \mathrm{E}+04$ & $6.34 \mathrm{E}+03$ & $6.20 \mathrm{E}+04$ & $2.28 \mathrm{E}+02$ & $5.85 \mathrm{E}+04$ & $2.98 \mathrm{E}+04$ \\
\hline 25 & $8.69 \mathrm{E}+04$ & $1.83 \mathrm{E}+04$ & $7.57 \mathrm{E}+04$ & $6.40 \mathrm{E}+03$ & $6.25 \mathrm{E}+04$ & $2.45 \mathrm{E}+02$ & $5.88 \mathrm{E}+04$ & $2.99 \mathrm{E}+04$ \\
\hline 26 & $8.48 \mathrm{E}+04$ & $1.85 \mathrm{E}+04$ & $7.12 \mathrm{E}+04$ & $6.46 \mathrm{E}+03$ & $6.29 \mathrm{E}+04$ & $2.61 \mathrm{E}+02$ & $5.90 \mathrm{E}+04$ & $3.00 \overline{\mathrm{E}+0}$ \\
\hline 27 & $8.29 E+04$ & $1.86 \mathrm{E}+04$ & $6.74 \mathrm{E}+04$ & $6.51 \mathrm{E}+03$ & $6.33 E+04$ & $2.78 \mathrm{E}+02$ & $5.93 \mathrm{E}+04$ & $3.01 \mathrm{E}+04$ \\
\hline$\overline{28}$ & $8.10 \mathrm{E}+04$ & $1.87 \mathrm{E}+04$ & $6.41 \mathrm{E}+04$ & $6.57 \mathrm{E}+03$ & $6.38 \mathrm{E}+04$ & $2.94 \mathrm{E}+02$ & $5.95 \mathrm{E}+04$ & $3.03 \mathrm{E}+04$ \\
\hline 29 & $7.93 \mathrm{E}+04$ & $1.89 \mathrm{E}+04$ & $6.12 \mathrm{E}+04$ & $6.62 \mathrm{E}+03$ & $6.41 \mathrm{E}+04$ & $3.11 \mathrm{E}+02$ & $5.97 \mathrm{E}+04$ & $3.04 \mathrm{E}+04$ \\
\hline 30 & $7.77 \mathrm{E}+04$ & $1.90 \mathrm{E}+04$ & $5.87 \mathrm{E}+04$ & $6.67 \mathrm{E}+03$ & $6.36 \mathrm{E}+04$ & $3.18 \mathrm{E}+02$ & $6.17 \mathrm{E}+04$ & $1.93 \mathrm{E}+04$ \\
\hline 31 & $7.61 \mathrm{E}+04$ & $1.91 \mathrm{E}+04$ & $5.65 \mathrm{E}+04$ & $6.72 \mathrm{E}+03$ & $6.31 \mathrm{E}+04$ & $3.24 \mathrm{E}+02$ & $6.14 \mathrm{E}+04$ & $1.92 \mathrm{E}+04$ \\
\hline 32 & $7.46 \mathrm{E}+04$ & $1.92 \mathrm{E}+04$ & $5.45 \mathrm{E}+04$ & $6.77 \mathrm{E}+03$ & $6.26 \mathrm{E}+04$ & $3.31 \mathrm{E}+02$ & $6.12 \mathrm{E}+04$ & $1.91 \mathrm{E}+04$ \\
\hline$\overline{33}$ & $7.31 \mathrm{E}+04$ & $1.93 \mathrm{E}+04$ & $5.28 \mathrm{E}+04$ & $6.81 \mathrm{E}+03$ & $6.21 \mathrm{E}+04$ & $3.37 \mathrm{E}+02$ & $6.09 \mathrm{E}+04$ & $1.90 \mathrm{E}+04$ \\
\hline 34 & $7.16 \mathrm{E}+04$ & $1.94 \mathrm{E}+04$ & $5.12 E+04$ & $6.86 \mathrm{E}+03$ & $6.16 \mathrm{E}+04$ & $3.44 \mathrm{E}+02$ & $6.07 \mathrm{E}+04$ & $1.90 \mathrm{E}+04$ \\
\hline 35 & $7.02 \mathrm{E}+04$ & $1.96 \mathrm{E}+04$ & $4.98 \mathrm{E}+04$ & $6.90 \mathrm{E}+03$ & $6.11 \mathrm{E}+04$ & $3.50 \mathrm{E}+02$ & $6.05 \mathrm{E}+04$ & $1.89 \mathrm{E}+04$ \\
\hline 36 & $6.88 \mathrm{E}+04$ & $1.97 \mathrm{E}+04$ & $4.86 \mathrm{E}+04$ & $6.95 \mathrm{E}+03$ & $6.07 \mathrm{E}+04$ & $3.56 \mathrm{E}+02$ & $6.02 \bar{E}+04$ & $1.88 \mathrm{E}+04$ \\
\hline 37 & $6.74 \mathrm{E}+04$ & $1.98 \mathrm{E}+04$ & $4.74 \mathrm{E}+04$ & $6.99 \mathrm{E}+03$ & $6.02 \mathrm{E}+04$ & $3.62 \mathrm{E}+02$ & $6.00 \mathrm{E}+04$ & $1.87 \mathrm{E}+04$ \\
\hline 38 & $6.60 \mathrm{E}+04$ & $1.99 \mathrm{E}+04$ & $4.63 \mathrm{E}+04$ & $7.03 \mathrm{E}+03$ & $5.97 \mathrm{E}+04$ & $3.69 \mathrm{E}+02$ & $5.98 \bar{E}+04$ & $1.87 \mathrm{E}+04$ \\
\hline
\end{tabular}




\begin{tabular}{|c|c|c|c|c|c|c|c|c|}
\hline 39 & $6.47 \mathrm{E}+04$ & $2.00 \mathrm{E}+04$ & $4.53 \mathrm{E}+04$ & $7.08 \mathrm{E}+03$ & $5.92 \mathrm{E}+04$ & $3.75 \mathrm{E}+02$ & $5.95 \mathrm{E}+04$ & $1.86 \mathrm{E}+04$ \\
\hline 40 & $6.34 \mathrm{E}+04$ & $2.00 \mathrm{E}+04$ & $4.44 \mathrm{E}+04$ & $7.12 \mathrm{E}+03$ & $5.87 \mathrm{E}+04$ & $3.81 \mathrm{E}+02$ & $5.93 \mathrm{E}+04$ & $1.85 \mathrm{E}+0$ \\
\hline 41 & $6.22 \mathrm{E}+04$ & $2.01 \mathrm{E}+04$ & $4.35 \mathrm{E}+04$ & $7.16 \mathrm{E}+03$ & $5.83 \mathrm{E}+04$ & $3.87 \mathrm{E}+02$ & $5.90 \mathrm{E}+04$ & $1.85 \mathrm{E}+04$ \\
\hline 42 & $6.10 \mathrm{E}+04$ & $2.02 \mathrm{E}+04$ & $4.27 \mathrm{E}+04$ & $7.20 \mathrm{E}+03$ & $5.85 \mathrm{E}+04$ & $4.04 \mathrm{E}+02$ & $5.67 \mathrm{E}+04$ & $3.10 \mathrm{E}+04$ \\
\hline 43 & $.98 \mathrm{E}+04$ & $2.03 \mathrm{E}+04$ & $4.19 \mathrm{E}+04$ & $7.23 \mathrm{E}+03$ & $5.87 \mathrm{E}+04$ & $4.21 \mathrm{E}+02$ & $5.68 \mathrm{E}+04$ & $3.10 \overline{\mathrm{E}+0}$ \\
\hline 44 & $5.86 \mathrm{E}+04$ & $2.04 \mathrm{E}+04$ & $4.12 \mathrm{E}+04$ & $7.27 E+03$ & $5.89 \mathrm{E}+04$ & $4.38 \mathrm{E}+02$ & $5.69 \mathrm{E}+04$ & $3.11 \mathrm{E}+\mathrm{c}$ \\
\hline 45 & $5.75 \mathrm{E}+04$ & $2.05 \mathrm{E}+04$ & $4.05 \mathrm{E}+04$ & $7.31 \mathrm{E}+03$ & $5.90 \mathrm{E}+04$ & $4.55 \mathrm{E}+02$ & $5.70 \mathrm{E}+04$ & $3.11 \mathrm{E}+0$ \\
\hline 46 & $5.64 \mathrm{E}+04$ & $2.06 \mathrm{E}+04$ & $3.99 \mathrm{E}+04$ & $7.35 \mathrm{E}+03$ & $5.92 \mathrm{E}+04$ & $4.72 \mathrm{E}+02$ & $5.70 \mathrm{E}+04$ & $3.12 \mathrm{E}+$ \\
\hline 47 & $5.54 \mathrm{E}+04$ & $2.06 \mathrm{E}+04$ & $3.92 \mathrm{E}+04$ & $7.38 \mathrm{E}+03$ & $5.93 \mathrm{E}+04$ & $4.89 \mathrm{E}+02$ & $5.71 \mathrm{E}+04$ & $3.12 \mathrm{E}+\mathrm{C}$ \\
\hline 48 & $5.44 \mathrm{E}+04$ & $2.07 \mathrm{E}+04$ & $3.86 \mathrm{E}+04$ & $7.42 \mathrm{E}+03$ & $5.94 \bar{E}+04$ & $5.06 \mathrm{E}+02$ & $5.72 \mathrm{E}+04$ & $3.12 \bar{E}+04$ \\
\hline 49 & $5.34 \mathrm{E}+04$ & $2.08 \mathrm{E}+04$ & $3.81 \mathrm{E}+04$ & $7.45 \mathrm{E}+03$ & $5.95 \mathrm{E}+04$ & $5.23 \mathrm{E}+02$ & $5.73 \mathrm{E}+04$ & $3.13 E+04$ \\
\hline 50 & $5.25 \mathrm{E}+04$ & $2.09 \mathrm{E}+04$ & $3.75 \mathrm{E}+04$ & $7.49 \mathrm{E}+03$ & $5.96 \mathrm{E}+04$ & $5.40 \mathrm{E}+02$ & $5.73 E+04$ & $3.13 \mathrm{E}+0$ \\
\hline 51 & $5.17 \mathrm{E}+04$ & $2.09 \mathrm{E}+04$ & $3.70 \mathrm{E}+04$ & $7.52 \mathrm{E}+03$ & $5.97 \mathrm{E}+04$ & $5.58 \mathrm{E}+02$ & $5.74 \mathrm{E}+04$ & $3.14 \mathrm{E}+0$ \\
\hline 52 & $5.09 \mathrm{E}+04$ & $2.10 \mathrm{E}+04$ & $3.64 \mathrm{E}+04$ & $7.56 \mathrm{E}+03$ & $5.98 \mathrm{E}+04$ & $5.75 \mathrm{E}+02$ & $5.74 \mathrm{E}+04$ & $3.14 \mathrm{E}+04$ \\
\hline 53 & $5.01 \mathrm{E}+04$ & $2.11 \mathrm{E}+04$ & $3.58 \mathrm{E}+04$ & $7.59 \mathrm{E}+03$ & $5.99 \mathrm{E}+04$ & $5.92 \mathrm{E}+02$ & $5.75 \mathrm{E}+04$ & $3.14 \mathrm{E}+0$ \\
\hline 54 & $4.94 \mathrm{E}+04$ & $2.11 \mathrm{E}+04$ & $3.53 \mathrm{E}+04$ & $7.62 \mathrm{E}+03$ & $5.94 \mathrm{E}+04$ & $5.98 \mathrm{E}+02$ & $5.75 \mathrm{E}+04$ & $1.80 \mathrm{E}+\mathrm{c}$ \\
\hline 55 & $4.86 \mathrm{E}+04$ & $2.12 \mathrm{E}+04$ & $3.47 \mathrm{E}+04$ & $7.66 \mathrm{E}+03$ & $5.89 E+04$ & $6.04 \mathrm{E}+02$ & $5.73 \mathrm{E}+04$ & $1.80 \mathrm{E}+0$ \\
\hline 56 & $4.79 \mathrm{E}+04$ & $2.13 \mathrm{E}+04$ & $3.41 \mathrm{E}+04$ & $7.69 \mathrm{E}+03$ & $5.84 \mathrm{E}+04$ & $6.10 \mathrm{E}+02$ & $5.71 \mathrm{E}+04$ & $1.79 \mathrm{E}+0$ \\
\hline 57 & $4.72 \mathrm{E}+04$ & $2.13 \mathrm{E}+04$ & $3.35 \mathrm{E}+04$ & $7.72 E+03$ & $5.79 \overline{\mathrm{E}}+04$ & $6.16 \mathrm{E}+02$ & $5.69 \mathrm{E}+04$ & $1.78 \mathrm{E}+0$ \\
\hline 58 & $4.65 \mathrm{E}+04$ & $2.14 \mathrm{E}+04$ & $3.30 \mathrm{E}+04$ & $7.75 \mathrm{E}+03$ & $5.75 \mathrm{E}+04$ & $6.21 \mathrm{E}+02$ & $5.66 \mathrm{E}+04$ & $1.78 \mathrm{E}+04$ \\
\hline 59 & $4.59 \mathrm{E}+04$ & $2.14 \mathrm{E}+04$ & $3.24 \mathrm{E}+04$ & $7.78 \mathrm{E}+03$ & $5.70 \mathrm{E}+04$ & $6.27 \mathrm{E}+02$ & $5.64 \mathrm{E}+04$ & $1.77 \mathrm{E}+0$ \\
\hline 60 & $4.52 \mathrm{E}+04$ & $2.15 \mathrm{E}+04$ & $3.18 \mathrm{E}+04$ & $7.81 \mathrm{E}+03$ & $5.65 \mathrm{E}+04$ & $6.33 \mathrm{E}+02$ & $5.62 \mathrm{E}+04$ & $1.76 \mathrm{E}+0$ \\
\hline 61 & $4.46 \mathrm{E}+04$ & $2.15 \mathrm{E}+04$ & $3.13 \mathrm{E}+04$ & $7.84 \mathrm{E}+03$ & $5.61 \mathrm{E}+04$ & $6.38 \mathrm{E}+02$ & $5.59 \mathrm{E}+04$ & $1.75 \mathrm{E}+02$ \\
\hline 62 & $4.39 \mathrm{E}+04$ & $2.16 \mathrm{E}+04$ & $3.07 \mathrm{E}+04$ & $7.87 \mathrm{E}+03$ & $5.56 \mathrm{E}+04$ & $6.44 \mathrm{E}+02$ & $5.57 \mathrm{E}+04$ & $1.75 \mathrm{E}+0$ \\
\hline 63 & $4.33 E+04$ & $2.17 \mathrm{E}+04$ & $3.02 \mathrm{E}+04$ & $7.89 \mathrm{E}+03$ & $5.52 \mathrm{E}+04$ & $6.49 \mathrm{E}+02$ & $5.55 \mathrm{E}+04$ & $1.74 \mathrm{E}+0$ \\
\hline 64 & $4.27 \mathrm{E}+04$ & $2.17 \mathrm{E}+04$ & $2.97 \mathrm{E}+04$ & $7.92 E+03$ & $5.47 \mathrm{E}+04$ & $6.55 \mathrm{E}+02$ & $5.53 \mathrm{E}+04$ & $1.73 \mathrm{E}+0$ \\
\hline $6 \overline{5}$ & $4.22 \mathrm{E}+04$ & $2.18 \mathrm{E}+04$ & $2.91 \mathrm{E}+04$ & $7.95 \mathrm{E}+03$ & $5.43 \mathrm{E}+04$ & $6.60 \mathrm{E}+02$ & $5.50 \mathrm{E}+04$ & $1.73 \mathrm{E}+04$ \\
\hline 66 & $4.15 \mathrm{E}+04$ & $2.18 \mathrm{E}+04$ & $2.86 \mathrm{E}+04$ & $7.97 \mathrm{E}+03$ & $5.43 \mathrm{E}+04$ & $6.77 \mathrm{E}+02$ & $5.26 \mathrm{E}+04$ & $3.07 \mathrm{E}+04$ \\
\hline 67 & $4.09 \mathrm{E}+04$ & $2.19 \mathrm{E}+04$ & $2.81 \mathrm{E}+04$ & $8.00 \mathrm{E}+03$ & $5.43 \mathrm{E}+04$ & $6.94 \mathrm{E}+02$ & $5.26 \mathrm{E}+04$ & $3.07 \mathrm{E}+02$ \\
\hline 68 & $4.03 E+04$ & $2.19 \mathrm{E}+04$ & $2.76 \mathrm{E}+04$ & $8.03 \mathrm{E}+03$ & $5.43 \mathrm{E}+04$ & $7.11 \mathrm{E}+02$ & $5.26 \mathrm{E}+04$ & $3.07 \mathrm{E}+04$ \\
\hline 69 & $3.98 E+04$ & $2.19 \mathrm{E}+04$ & $2.71 \mathrm{E}+04$ & $8.05 \mathrm{E}+03$ & $5.43 \mathrm{E}+04$ & $7.28 \mathrm{E}+02$ & $5.26 \mathrm{E}+04$ & $3.07 \mathrm{E}+04$ \\
\hline 168 & $9.98 \mathrm{E}+03$ & $2.33 \mathrm{E}+04$ & $9.44 \mathrm{E}+03$ & $9.43 \mathrm{E}+03$ & $3.37 \mathrm{E}+04$ & $1.84 \mathrm{E}+03$ & $3.51 \mathrm{E}+04$ & $2.56 \mathrm{E}+04$ \\
\hline 336 & $4.45 \mathrm{E}+03$ & $1.95 \mathrm{E}+04$ & $3.92 \mathrm{E}+03$ & $1.00 \mathrm{E}+04$ & $1.36 \mathrm{E}+04$ & $3.62 \mathrm{E}+03$ & $2.14 \mathrm{E}+04$ & $1.97 \mathrm{E}+04$ \\
\hline 504 & $3.23 \mathrm{E}+03$ & $1.61 \mathrm{E}+04$ & $2.65 \mathrm{E}+03$ & $1.04 \mathrm{E}+04$ & $6.53 \mathrm{E}+03$ & $4.59 \mathrm{E}+03$ & $9.23 \mathrm{E}+03$ & $1.31 \mathrm{E}+04$ \\
\hline 720 & $2.35 \mathrm{E}+03$ & $1.25 \mathrm{E}+04$ & $1.77 \mathrm{E}+03$ & $1.07 \mathrm{E}+04$ & $3.29 \mathrm{E}+03$ & $5.13 \mathrm{E}+03$ & $4.51 \mathrm{E}+03$ & $9.09 \mathrm{E}+03$ \\
\hline 2160 & $4.38 \mathrm{E}+02$ & $2.32 \mathrm{E}+03$ & $1.98 \mathrm{E}+02$ & $1.12 \mathrm{E}+04$ & $2.88 \mathrm{E}+02$ & $3.04 \mathrm{E}+03$ & $1.03 \mathrm{E}+03$ & $3.10 \mathrm{E}+03$ \\
\hline 4320 & $4.21 \mathrm{E}+01$ & $1.93 \mathrm{E}+02$ & $9.97 \mathrm{E}+02$ & $1.07 \mathrm{E}+04$ & $4.50 \mathrm{E}+01$ & $7.21 \mathrm{E}+02$ & $1.19 \mathrm{E}+02$ & $4.74 \mathrm{E}+02$ \\
\hline 4800 & $2.50 \mathrm{E}+01$ & $1.12 \mathrm{E}+02$ & $9.94 \mathrm{E}+02$ & $1.07 \mathrm{E}+04$ & $3.24 \mathrm{E}+01$ & $5.18 \mathrm{E}+02$ & $3.83 \mathrm{E}+01$ & $1.52 \mathrm{E}+02$ \\
\hline 8760 & $3.43 \mathrm{E}-01$ & $1.34 \mathrm{E}+00$ & $9.69 \mathrm{E}+02$ & $1.04 \mathrm{E}+04$ & $2.55 \mathrm{E}+00$ & $3.37 \mathrm{E}+01$ & $1.17 \mathrm{E}+01$ & $4.43 \mathrm{E}+01$ \\
\hline L OBT & $\begin{array}{l}\mathrm{E} 6 \\
/ \mathrm{L}\end{array}$ & $\begin{array}{l}8 \mathrm{E}+4 \\
/ \mathrm{kg}\end{array}$ & $8 \mathrm{~Bq} / \mathrm{g}$ & & & & & \\
\hline TO & $\begin{array}{l}5.9 \mathrm{E} 6 \\
\mathrm{pCi} / \mathrm{L}\end{array}$ & $\begin{array}{l}2.18 \mathrm{E}+5 \\
\mathrm{~Bq} / \mathrm{kg}\end{array}$ & $218.3 \mathrm{~Bq} / \mathrm{g}$ & & & & & \\
\hline & \multirow{2}{*}{\multicolumn{2}{|c|}{ VEGETABLES }} & & & \multirow{2}{*}{\multicolumn{2}{|c|}{ MEAT }} & & \\
\hline & & & \multicolumn{2}{|c|}{ WHEAT SEED } & & & \multicolumn{2}{|l|}{ MILK } \\
\hline \multirow[t]{2}{*}{ HOUR } & TO & OBT & HTO & OBT & HTO & $\mathrm{BT}$ & HTO & $\overline{\mathrm{BT}}$ \\
\hline & $(\mathrm{Bq} / \mathrm{g})$ & $(\mathrm{Bq} / \mathrm{g})$ & $(\mathrm{Bq} / \mathrm{g})$ & $(\mathrm{Bq} / \mathrm{g})$ & $(\mathrm{Bq} / \mathrm{g})$ & $(\mathrm{Bq} / \mathrm{g})$ & $(\mathrm{Bq} / \mathrm{g})$ & $(\mathrm{Bq} / \mathrm{g})$ \\
\hline ak HTO & & & & & & & & \\
\hline
\end{tabular}


Foodstuff Concentrations and Relocation Considerations Following

a Tritium Oxide Release from SRS Tritium Facilities (U)

\begin{tabular}{|c|c|c|c|c|c|c|c|c|}
\hline $2 / 5 / 5 / 6$ & $1.84 \mathrm{E}+06$ & $7.30 \mathrm{E}+03$ & $5.33 \mathrm{E}+05$ & $2.94 \mathrm{E}+03$ & $6.33 \mathrm{E}+04$ & $3.99 \mathrm{E}+01$ & $6.09 \mathrm{E}+04$ & $1.90 \mathrm{E}+04$ \\
\hline SUM & $1.84 \mathrm{E}+06$ & & $5.36 \mathrm{E}+05$ & & $6.33 \mathrm{E}+04$ & & $7.99 \mathrm{E}+04$ & \\
\hline $\mathrm{Ci} / \mathrm{g}$ & $4.98 \mathrm{E}-05$ & & $1.45 \mathrm{E}-05$ & & $1.71 \mathrm{E}-06$ & & $2.16 \mathrm{E}-06$ & \\
\hline & & & & & & & & \\
\hline DIL HTO & 5.9E-09 & & & & & & & \\
\hline \multicolumn{9}{|l|}{$(\mathrm{Ci} / \mathrm{g})$} \\
\hline & & & & & & & & \\
\hline 168 & $9.98 \mathrm{E}+03$ & $2.33 E+04$ & $9.44 \mathrm{E}+03$ & $9.43 \mathrm{E}+03$ & $3.37 \mathrm{E}+04$ & $1.84 \mathrm{E}+03$ & $3.51 \mathrm{E}+04$ & $2.56 \mathrm{E}+04$ \\
\hline SUM & $3.33 \mathrm{E}+04$ & & $1.89 \mathrm{E}+04$ & & $3.56 \mathrm{E}+04$ & & $6.07 \mathrm{E}+04$ & \\
\hline $\mathrm{Ci} / \mathrm{g}$ & $9.00 \mathrm{E}-07$ & & 5.10E-07 & & $9.62 \mathrm{E}-07$ & & $1.64 \mathrm{E}-06$ & \\
\hline & & & & & & & & \\
\hline DIL OBT & $2.4 \mathrm{E}-09$ & & & & & & & \\
\hline \multicolumn{9}{|l|}{$\mathrm{Ci} / \mathrm{g}$} \\
\hline & & & & & & & & \\
\hline 8760 & $3.43 \mathrm{E}-01$ & $1.34 \mathrm{E}+00$ & $9.69 \mathrm{E}+02$ & $1.04 \mathrm{E}+04$ & $2.55 \mathrm{E}+00$ & $3.37 \mathrm{E}+01$ & $1.17 \mathrm{E}+01$ & $4.43 \mathrm{E}+01$ \\
\hline SUM & $1.69 \mathrm{E}+00$ & & $1.14 \mathrm{E}+04$ & & $3.62 \mathrm{E}+01$ & & $5.60 \mathrm{E}+01$ & \\
\hline $\mathrm{Ci} / \mathrm{g}$ & $4.55 \mathrm{E}-11$ & & 3.08E-07 & & $9.79 \mathrm{E}-10$ & & $1.51 \mathrm{E}-09$ & \\
\hline DIL OBT & $24 E_{-} 00$ & & & & & & & \\
\hline $\mathrm{Ci} / \mathrm{g}$ & $2.4 \mathrm{E}-\mathrm{GS}$ & & & & & & & \\
\hline & & & & & & & & \\
\hline
\end{tabular}




\begin{tabular}{|c|c|c|c|c|c|c|c|c|}
\hline & \multicolumn{6}{|c|}{ Tritium Facilities, Design Basis Fire, Adverse Met Conditions } & & \\
\hline & \multicolumn{4}{|c|}{ Concentration Values for $50 \mathrm{~km}$ from release } & & & & \\
\hline \multicolumn{9}{|c|}{ Only Wheat Seed is examined since this is the edible portion of the Wheat. The "WHEAT" category is } \\
\hline \multicolumn{9}{|c|}{ actually the unedible plant body and is not considered in the dose estimate. } \\
\hline & & & & & & & & \\
\hline \multirow{3}{*}{ HOUR } & \multicolumn{2}{|c|}{ VEGETABLES } & \multicolumn{2}{|c|}{ WHEAT SEED } & \multicolumn{2}{|l|}{$\overline{M E A T}$} & \multicolumn{2}{|l|}{ MILK } \\
\hline & HTO & OBT & HTO & OBT & HTO & OBT & HTO & $\overline{\mathrm{BT}}$ \\
\hline & $(\mathrm{Bq} / \mathrm{g})$ & $(\mathrm{Bq} / \mathrm{g})$ & $(\mathrm{Bq} / \mathrm{g})$ & $(\mathrm{Bq} / \mathrm{g})$ & $(\mathrm{Bq} / \mathrm{g})$ & $(\mathrm{Bq} / \mathrm{g})$ & $(\mathrm{Bq} / \mathrm{g})$ & $\mathrm{qq} / \mathrm{g})$ \\
\hline & & & & & & & & \\
\hline 1 & $1.50 \mathrm{E}+04$ & $0.00 \mathrm{E}+00$ & $0.00 \mathrm{E}+00$ & $0.00 \mathrm{E}+00$ & $1.53 \mathrm{E}+01$ & $1.65 \mathrm{E}-03$ & $1.86 \mathrm{E}+00$ & $5.79 \mathrm{E}-$ \\
\hline 2 & $3.55 \mathrm{E}+04$ & $2.78 \mathrm{E}+01$ & $9.99 \mathrm{E}+02$ & $3.30 \mathrm{E}+00$ & $1.38 \mathrm{E}+02$ & $2.64 \mathrm{E}-02$ & $1.65 \mathrm{E}+01$ & $6.46 \mathrm{E}+00$ \\
\hline 3 & $6.97 \mathrm{E}+04$ & $9.36 \mathrm{E}+01$ & $3.48 \mathrm{E}+03$ & $1.25 \mathrm{E}+01$ & $4.31 \mathrm{E}+02$ & $1.08 \mathrm{E}-01$ & $5.66 \mathrm{E}+01$ & $2.31 \mathrm{E}+01$ \\
\hline 4 & $6.83 \mathrm{E}+04$ & $2.23 \mathrm{E}+02$ & $8.40 \mathrm{E}+03$ & $3.21 \mathrm{E}+01$ & $9.54 \mathrm{E}+02$ & $2.99 \mathrm{E}-01$ & $1.36 \mathrm{E}+02$ & $5.69 \mathrm{E}+01$ \\
\hline 5 & $4.53 \mathrm{E}+05$ & $2.17 \mathrm{E}+03$ & $8.16 \mathrm{E}+04$ & $2.82 \mathrm{E}+02$ & $8.57 \mathrm{E}+03$ & $2.02 \mathrm{E}+00$ & $8.16 \mathrm{E}+02$ & $3.48 \mathrm{E}+02$ \\
\hline 6 & $2.24 \mathrm{E}+05$ & $3.06 \mathrm{E}+03$ & $1.23 \mathrm{E}+05$ & $4.98 \mathrm{E}+02$ & $8.56 \mathrm{E}+03$ & $2.94 \mathrm{E}+00$ & $8.30 \mathrm{E}+03$ & $2.58 \mathrm{E}+03$ \\
\hline 7 & $1.17 \mathrm{E}+05$ & $3.48 \mathrm{E}+03$ & $1.41 \mathrm{E}+05$ & $6.77 \bar{E}+02$ & $8.51 \mathrm{E}+03$ & $3.85 \mathrm{E}+00$ & $8.27 \mathrm{E}+03$ & $2.58 \mathrm{E}+03$ \\
\hline 8 & $7.20 \mathrm{E}+04$ & $3.69 \mathrm{E}+03$ & $1.45 \mathrm{E}+05$ & $8.25 \mathrm{E}+02$ & $8.46 \mathrm{E}+03$ & $4.76 \mathrm{E}+00$ & $8.25 \mathrm{E}+03$ & $2.57 \mathrm{E}+03$ \\
\hline 9 & $5.18 \mathrm{E}+04$ & $3.82 \mathrm{E}+03$ & $1.40 \mathrm{E}+05$ & $9.48 \mathrm{E}+02$ & $8.41 \mathrm{E}+03$ & $5.66 \mathrm{E}+00$ & $8.23 \mathrm{E}+03$ & $2.56 \mathrm{E}+03$ \\
\hline 10 & $4.22 \mathrm{E}+04$ & $3.91 \mathrm{E}+03$ & $1.30 \mathrm{E}+05$ & $1.05 \mathrm{E}+03$ & $8.36 \mathrm{E}+03$ & $6.56 \mathrm{E}+00$ & $8.20 \mathrm{E}+03$ & $2.55 \mathrm{E}+03$ \\
\hline 11 & $3.71 \mathrm{E}+04$ & $3.98 \mathrm{E}+03$ & $1.18 \mathrm{E}+05$ & $1.14 \mathrm{E}+03$ & $8.30 \mathrm{E}+03$ & $7.44 \mathrm{E}+00$ & $8.18 \mathrm{E}+03$ & $2.55 \mathrm{E}+03$ \\
\hline 12 & $3.41 \mathrm{E}+04$ & $4.05 \mathrm{E}+03$ & $1.05 \mathrm{E}+05$ & $1.21 \mathrm{E}+03$ & $8.25 \mathrm{E}+03$ & $8.33 \mathrm{E}+00$ & $8.15 E+03$ & $2.54 \mathrm{E}+03$ \\
\hline 13 & $3.21 \mathrm{E}+04$ & $4.11 \mathrm{E}+03$ & $9.28 \mathrm{E}+04$ & $1.27 \mathrm{E}+03$ & $8.19 \mathrm{E}+03$ & $9.20 \mathrm{E}+00$ & $8.12 \mathrm{E}+03$ & $2.53 \mathrm{E}+03$ \\
\hline 14 & $3.07 \mathrm{E}+04$ & $4.16 \mathrm{E}+03$ & $8.14 \mathrm{E}+04$ & $1.32 \mathrm{E}+03$ & $8.14 \mathrm{E}+03$ & $1.01 \mathrm{E}+01$ & $8.10 \mathrm{E}+03$ & $2.52 \mathrm{E}+03$ \\
\hline 15 & $2.95 \mathrm{E}+04$ & $4.21 \mathrm{E}+03$ & $7.11 \mathrm{E}+04$ & $1 . \overline{37 \mathrm{E}+03}$ & $8.08 \mathrm{E}+03$ & $1.09 \mathrm{E}+01$ & $8.07 \mathrm{E}+03$ & $2.51 \mathrm{E}+03$ \\
\hline 16 & $2.86 \mathrm{E}+04$ & $4.26 \mathrm{E}+03$ & $6.20 \mathrm{E}+04$ & $1.40 \mathrm{E}+03$ & $8.03 E+03$ & +01 & $8.05 E+03$ & $2.51 \mathrm{E}+03$ \\
\hline 17 & $2.77 \mathrm{E}+04$ & $4.31 \mathrm{E}+03$ & $5.42 \mathrm{E}+04$ & $1.44 \mathrm{E}+03$ & $7.97 \mathrm{E}+03$ & $1.26 \mathrm{E}+01$ & $8.02 \mathrm{E}+03$ & $2.50 \mathrm{E}+03$ \\
\hline 18 & $2.70 \mathrm{E}+04$ & $4.35 \mathrm{E}+0 \overline{3}$ & $4.75 \mathrm{E}+04$ & $1.4 \overline{\mathrm{E}}+03$ & $8.21 \mathrm{E}+03$ & $1.59 \mathrm{E}+01$ & $7.96 \mathrm{E}+03$ & $5.32 \mathrm{E}+03$ \\
\hline 19 & $2.63 \mathrm{E}+04$ & $4.40 \mathrm{E}+03$ & $4.18 \mathrm{E}+04$ & $1.50 \mathrm{E}+03$ & $8.44 \mathrm{E}+03$ & $1.91 \mathrm{E}+01$ & $8.07 \mathrm{E}+03$ & $5.37 \mathrm{E}+03$ \\
\hline 20 & $2.56 \mathrm{E}+04$ & $4.44 \mathrm{E}+03$ & $3.70 \mathrm{E}+04$ & $1.52 \mathrm{E}+03$ & $8.65 E+03$ & $2.24 \mathrm{E}+01$ & $8.17 \mathrm{E}+03$ & $5.42 \mathrm{E}+03$ \\
\hline 21 & $2.51 \mathrm{E}+04$ & $4.48 \mathrm{E}+03$ & $3.29 \mathrm{E}+04$ & $1.54 \mathrm{E}+03$ & $8.86 \mathrm{E}+03$ & $2.58 \mathrm{E}+01$ & $8.28 E+03$ & $5.46 \mathrm{E}+03$ \\
\hline 22 & $2.45 \mathrm{E}+04$ & $4.52 \mathrm{E}+03$ & $2.95 \mathrm{E}+04$ & $1.56 \mathrm{E}+03$ & $9.06 \mathrm{E}+03$ & $2.92 \mathrm{E}+01$ & $8.38 \mathrm{E}+03$ & $5.51 \mathrm{E}+03$ \\
\hline 23 & $2.40 \mathrm{E}+04$ & $4.56 \mathrm{E}+03$ & $2.67 \mathrm{E}+04$ & $1.58 \mathrm{E}+03$ & $9.25 \mathrm{E}+03$ & $3.26 \mathrm{E}+01$ & $8.48 \mathrm{E}+03$ & $5.55 \mathrm{E}+03$ \\
\hline 24 & $2.35 \mathrm{E}+04$ & $4.60 \mathrm{E}+03$ & $2.43 \mathrm{E}+04$ & $1.60 \mathrm{E}+03$ & $9.44 \mathrm{E}+03$ & $3.60 \mathrm{E}+01$ & $8.57 \mathrm{E}+03$ & $5.59 \mathrm{E}+03$ \\
\hline 25 & $2.31 \mathrm{E}+04$ & $4.64 \mathrm{E}+03$ & $2.23 \mathrm{E}+04$ & $1.62 \mathrm{E}+03$ & $9.62 \mathrm{E}+03$ & $3.95 \bar{E}+01$ & $8.67 \mathrm{E}+03$ & $5.63 \mathrm{E}+03$ \\
\hline 26 & $2.26 \mathrm{E}+04$ & $4.68 \mathrm{E}+03$ & $2.07 \mathrm{E}+04$ & $1.63 \mathrm{E}+03$ & $9.79 \mathrm{E}+03$ & $4.31 \mathrm{E}+01$ & $8.76 \mathrm{E}+03$ & $5.67 \mathrm{E}+03$ \\
\hline 27 & $2.22 \mathrm{E}+04$ & $4.71 \mathrm{E}+03$ & $1.93 \mathrm{E}+04$ & $1.65 \mathrm{E}+03$ & $9.97 \mathrm{E}+03$ & $4.66 \mathrm{E}+01$ & $8.85 E+03$ & $5.71 \mathrm{E}+03$ \\
\hline 28 & $2.19 \mathrm{E}+04$ & $4.75 \mathrm{E}+03$ & $1.82 \mathrm{E}+04$ & $1.66 \mathrm{E}+03$ & $1.01 \mathrm{E}+04$ & $5.02 \mathrm{E}+01$ & $8.94 \mathrm{E}+03$ & $5.75 \mathrm{E}+03$ \\
\hline 29 & $2.15 E+04$ & $4.78 \mathrm{E}+03$ & $1.72 \mathrm{E}+04$ & $1.68 \mathrm{E}+03$ & $1.03 \mathrm{E}+04$ & $5.39 \mathrm{E}+01$ & $9.02 \mathrm{E}+03$ & $5.79 \mathrm{E}+03$ \\
\hline 30 & $2.13 \mathrm{E}+04$ & $4.81 \mathrm{E}+03$ & $1.64 \mathrm{E}+04$ & $1.69 \mathrm{E}+03$ & $1.02 \mathrm{E}+04$ & $5.49 \mathrm{E}+01$ & $9.91 \mathrm{E}+03$ & $3.10 \mathrm{E}+03$ \\
\hline 31 & $2.10 \mathrm{E}+04$ & $4.85 \mathrm{E}+03$ & $1.57 \mathrm{E}+04$ & $1.70 \mathrm{E}+03$ & $1.01 \mathrm{E}+04$ & $5.60 \mathrm{E}+01$ & $9.87 \mathrm{E}+03$ & $3.08 \mathrm{E}+03$ \\
\hline 32 & $2.07 \mathrm{E}+04$ & $4.88 \mathrm{E}+03$ & $1.51 \mathrm{E}+04$ & $1.72 \mathrm{E}+03$ & $1.01 \mathrm{E}+04$ & $5.70 \mathrm{E}+01$ & $9.83 \mathrm{E}+03$ & $3.07 \mathrm{E}+03$ \\
\hline 33 & $2.05 \mathrm{E}+04$ & $4.91 \mathrm{E}+03$ & $1.46 \mathrm{E}+04$ & $1.73 \mathrm{E}+03$ & $9.99 \mathrm{E}+03$ & $5.80 \mathrm{E}+01$ & $9.79 \mathrm{E}+03$ & $3.06 \mathrm{E}+03$ \\
\hline 34 & $2.02 E+04$ & $4.94 \mathrm{E}+03$ & $1.42 \mathrm{E}+04$ & $1.74 \mathrm{E}+03$ & $9.91 \mathrm{E}+03$ & $5.91 \mathrm{E}+01$ & $9.76 \mathrm{E}+03$ & $3.05 \mathrm{E}+03$ \\
\hline 35 & $2.00 \mathrm{E}+04$ & $4.97 \mathrm{E}+03$ & $1.39 \mathrm{E}+04$ & $1.76 \mathrm{E}+03$ & $9.84 \mathrm{E}+03$ & $6.01 \mathrm{E}+01$ & $9.72 \mathrm{E}+03$ & $3.04 \mathrm{E}+03$ \\
\hline 36 & $1.98 \mathrm{E}+04$ & $5.00 \mathrm{E}+03$ & $1.36 \mathrm{E}+04$ & $1.77 \mathrm{E}+03$ & $9.76 \mathrm{E}+03$ & $6.11 \mathrm{E}+01$ & $9.68 \mathrm{E}+03$ & $3.03 E+03$ \\
\hline 37 & $1.95 \mathrm{E}+04$ & $5.03 \mathrm{E}+03$ & $1.33 \mathrm{E}+04$ & $1.78 E+03$ & $9.69 \mathrm{E}+03$ & $6.21 \mathrm{E}+01$ & $9.65 \mathrm{E}+03$ & $3.02 \mathrm{E}+03$ \\
\hline 38 & $1.93 \mathrm{E}+04$ & $5.06 \mathrm{E}+03$ & $1.31 \mathrm{E}+04$ & $1.79 \mathrm{E}+03$ & $9.62 \mathrm{E}+03$ & $6.31 \mathrm{E}+01$ & $9.61 \mathrm{E}+03$ & $3.00 \mathrm{E}+03$ \\
\hline
\end{tabular}




\begin{tabular}{|c|c|c|c|c|c|c|c|c|}
\hline 39 & $1.91 \mathrm{E}+04$ & $5.09 \mathrm{E}+03$ & $1.29 \mathrm{E}+04$ & $1.81 \mathrm{E}+03$ & $9.54 \mathrm{E}+03$ & $6.41 \mathrm{E}+01$ & $9.57 \mathrm{E}+03$ & $2.99 \mathrm{E}+0$ \\
\hline 40 & $1.88 \mathrm{E}+04$ & $5.12 \mathrm{E}+03$ & $1.28 \mathrm{E}+04$ & $1.82 \mathrm{E}+03$ & $9.47 \mathrm{E}+03$ & $6.50 \mathrm{E}+01$ & $9.54 \mathrm{E}+03$ & $2.98 \mathrm{E}+0$ \\
\hline 41 & $1.86 \mathrm{E}+04$ & $5.15 \mathrm{E}+03$ & $1.26 \mathrm{E}+04$ & $1.83 \mathrm{E}+03$ & $9.40 \mathrm{E}+03$ & $6.60 \mathrm{E}+01$ & $9.50 \mathrm{E}+03$ & $2.97 \mathrm{E}+03$ \\
\hline 42 & $1.84 \mathrm{E}+04$ & $5.18 \mathrm{E}+03$ & $1.25 \mathrm{E}+04$ & $1.84 \mathrm{E}+03$ & $9.53 \mathrm{E}+03$ & $6.98 \mathrm{E}+01$ & $9.24 \mathrm{E}+03$ & $6.29 \mathrm{E}+0$ \\
\hline 43 & $1.81 \mathrm{E}+04$ & $5.20 \mathrm{E}+03$ & $1.24 \mathrm{E}+04$ & $1.85 \mathrm{E}+03$ & $9.66 \mathrm{E}+03$ & $7.36 \mathrm{E}+01$ & $9.30 \mathrm{E}+03$ & $6.32 \mathrm{E}+0$ \\
\hline 44 & $1.79 \mathrm{E}+04$ & $5.23 \mathrm{E}+03$ & $1.23 \mathrm{E}+04$ & $1.86 \mathrm{E}+03$ & $9.78 \mathrm{E}+03$ & $7.74 \mathrm{E}+01$ & $9.36 \mathrm{E}+03$ & $6.35 \mathrm{E}+0$ \\
\hline 45 & $1.77 \mathrm{E}+04$ & $5.26 \mathrm{E}+03$ & $1.22 \mathrm{E}+04$ & $1.88 \mathrm{E}+03$ & $9.90 \mathrm{E}+03$ & $8.13 \mathrm{E}+01$ & $9.42 \mathrm{E}+03$ & $6.37 \mathrm{E}+0$ \\
\hline 46 & $1.75 \mathrm{E}+04$ & $5.28 \mathrm{E}+03$ & $1.21 \mathrm{E}+04$ & $1.89 \mathrm{E}+03$ & $1.00 \mathrm{E}+04$ & $8.52 \mathrm{E}+01$ & $9.48 \mathrm{E}+03$ & $6.40 \mathrm{E}+0$ \\
\hline 47 & $1.72 \mathrm{E}+04$ & $5.31 \mathrm{E}+03$ & $1.20 \mathrm{E}+04$ & $1.90 \mathrm{E}+03$ & $1.01 \mathrm{E}+04$ & $8.91 \mathrm{E}+01$ & $9.53 \mathrm{E}+03$ & $6.42 \mathrm{E}+0$ \\
\hline 48 & $1.70 \mathrm{E}+04$ & $5.33 \mathrm{E}+03$ & $1.20 \mathrm{E}+04$ & $1.91 \mathrm{E}+03$ & $1.02 \mathrm{E}+04$ & $9.30 \mathrm{E}+01$ & $9.59 \mathrm{E}+03$ & $6.45 \mathrm{E}+0$ \\
\hline 49 & $1.68 \mathrm{E}+04$ & $5.36 \mathrm{E}+03$ & $1.19 \mathrm{E}+04$ & $1.92 \mathrm{E}+03$ & $1.03 \mathrm{E}+04$ & $9.69 \mathrm{E}+01$ & $9.64 \mathrm{E}+03$ & $6.47 \mathrm{E}+0$ \\
\hline 50 & $1.66 \mathrm{E}+04$ & $5.38 \mathrm{E}+03$ & $1.19 \mathrm{E}+04$ & $1.93 \mathrm{E}+03$ & $1.04 \mathrm{E}+04$ & $1.01 \mathrm{E}+02$ & $9.70 \mathrm{E}+03$ & $6.50 \mathrm{E}+0$ \\
\hline 51 & $1.64 \mathrm{E}+04$ & $5.41 \mathrm{E}+03$ & $1.18 \mathrm{E}+04$ & $1.94 \mathrm{E}+03$ & $1.05 \mathrm{E}+04$ & $1.05 \mathrm{E}+02$ & $9.75 \mathrm{E}+03$ & $6.52 \mathrm{E}+0$ \\
\hline 52 & $1.62 \mathrm{E}+04$ & $5.43 \mathrm{E}+03$ & $1.17 \mathrm{E}+04$ & $1.96 \mathrm{E}+03$ & $1.06 \mathrm{E}+04$ & $1.09 \mathrm{E}+02$ & $9.80 \mathrm{E}+03$ & $6.54 \mathrm{E}+0$ \\
\hline 53 & $1.61 \mathrm{E}+04$ & $5.45 \mathrm{E}+03$ & $1.16 \mathrm{E}+04$ & $1.97 \mathrm{E}+03$ & $1.07 \mathrm{E}+04$ & $1.13 \mathrm{E}+02$ & $9.85 \mathrm{E}+03$ & $6.56 \mathrm{E}+0$ \\
\hline 54 & $1.59 \mathrm{E}+04$ & $5.48 \mathrm{E}+03$ & $1.15 \mathrm{E}+04$ & $1.98 \mathrm{E}+03$ & $1.07 \mathrm{E}+04$ & $1.14 \mathrm{E}+02$ & $1.03 \mathrm{E}+04$ & $3.24 \mathrm{E}+0$ \\
\hline 55 & $1.57 \mathrm{E}+04$ & $5.50 \mathrm{E}+03$ & $1.14 \mathrm{E}+04$ & $1.99 \mathrm{E}+03$ & $1.06 \mathrm{E}+04$ & $1.15 \mathrm{E}+02$ & $1.03 \mathrm{E}+04$ & $3.22 \mathrm{E}+0$ \\
\hline 56 & $1.55 \mathrm{E}+04$ & $5.52 \mathrm{E}+03$ & $1.13 \mathrm{E}+04$ & $2.00 \mathrm{E}+03$ & $1.05 \mathrm{E}+04$ & $1.16 \mathrm{E}+02$ & $1.02 \mathrm{E}+04$ & $3.21 \mathrm{E}+0$ \\
\hline 57 & $1.53 \mathrm{E}+04$ & $5.54 \mathrm{E}+03$ & $1.12 \mathrm{E}+04$ & $2.01 \mathrm{E}+03$ & $1.04 \mathrm{E}+04$ & $1.17 \mathrm{E}+02$ & $1.02 \mathrm{E}+04$ & $3.20 \mathrm{E}+0$ \\
\hline 58 & $1.52 \mathrm{E}+04$ & $5.56 \mathrm{E}+03$ & $1.11 \mathrm{E}+04$ & $2.02 \mathrm{E}+03$ & $1.03 \mathrm{E}+04$ & $1.18 \mathrm{E}+02$ & $1.02 \mathrm{E}+04$ & $3.19 \mathrm{E}+0$ \\
\hline 59 & $1.50 \mathrm{E}+04$ & $5.58 \mathrm{E}+03$ & $1.10 \mathrm{E}+04$ & $2.03 \mathrm{E}+03$ & $1.02 \mathrm{E}+04$ & $1.19 \mathrm{E}+02$ & $1.01 \mathrm{E}+04$ & $3.17 \mathrm{E}+0$ \\
\hline 60 & $1.48 \mathrm{E}+04$ & $5.60 \mathrm{E}+03$ & $1.09 \mathrm{E}+04$ & $2.04 \mathrm{E}+03$ & $1.02 \mathrm{E}+04$ & $1.20 \mathrm{E}+02$ & $1.01 \mathrm{E}+04$ & $3.16 \mathrm{E}+0$ \\
\hline 61 & $1.46 \mathrm{E}+04$ & $5.62 \mathrm{E}+03$ & $1.07 \mathrm{E}+04$ & $2.05 \mathrm{E}+03$ & $1.01 \mathrm{E}+04$ & $1.21 \mathrm{E}+02$ & $1.00 \mathrm{E}+04$ & $3.15 \mathrm{E}+0$ \\
\hline 62 & $1.45 \mathrm{E}+04$ & $5.64 \mathrm{E}+03$ & $1.06 \mathrm{E}+04$ & $2.06 \mathrm{E}+03$ & $9.99 \mathrm{E}+03$ & $1.22 \mathrm{E}+02$ & $1.00 \mathrm{E}+04$ & $3.14 \mathrm{E}+0$ \\
\hline 63 & $1.43 \mathrm{E}+04$ & $5.66 \mathrm{E}+03$ & $1.05 \mathrm{E}+04$ & $2.07 \mathrm{E}+03$ & $9.92 \mathrm{E}+03$ & $1.23 \mathrm{E}+02$ & $9.96 \mathrm{E}+03$ & $3.13 \mathrm{E}+0$ \\
\hline 64 & $1.41 \mathrm{E}+04$ & $5.68 \mathrm{E}+03$ & $1.04 \mathrm{E}+04$ & $2.08 \mathrm{E}+03$ & $9.84 \mathrm{E}+03$ & $1.24 \mathrm{E}+02$ & $9.92 \mathrm{E}+03$ & $3.11 \mathrm{E}+03$ \\
\hline 65 & $1.40 \mathrm{E}+04$ & $5.70 \mathrm{E}+03$ & $1.02 \mathrm{E}+04$ & $2.09 \mathrm{E}+03$ & $9.76 \mathrm{E}+03$ & $1.25 \mathrm{E}+02$ & $9.88 \mathrm{E}+03$ & $3.10 \mathrm{E}+0$ \\
\hline 66 & $1.38 \mathrm{E}+04$ & $5.72 \mathrm{E}+03$ & $1.01 \mathrm{E}+04$ & $2.10 \mathrm{E}+03$ & $9.83 \mathrm{E}+03$ & $1.29 \mathrm{E}+02$ & $9.53 \mathrm{E}+03$ & $6.74 \mathrm{E}+0$ \\
\hline 67 & $1.36 \mathrm{E}+04$ & $5.74 \mathrm{E}+03$ & $9.95 \mathrm{E}+03$ & $2.11 \mathrm{E}+03$ & $9.90 \mathrm{E}+03$ & $1.33 \mathrm{E}+02$ & $9.56 \mathrm{E}+03$ & $6.75 \mathrm{E}+03$ \\
\hline 68 & $1.34 \mathrm{E}+04$ & $5.75 \mathrm{E}+03$ & $9.82 \mathrm{E}+03$ & $2.12 \mathrm{E}+03$ & $9.97 \mathrm{E}+03$ & $1.37 \mathrm{E}+02$ & $9.60 \mathrm{E}+03$ & $6.77 \mathrm{E}+03$ \\
\hline 69 & $1.33 \mathrm{E}+04$ & $5.77 \mathrm{E}+03$ & $9.68 \mathrm{E}+03$ & $2.13 \mathrm{E}+03$ & $1.00 \mathrm{E}+04$ & $1.41 \mathrm{E}+02$ & $9.63 E+03$ & $6.78 \mathrm{E}+0$ \\
\hline 168 & $2.74 \mathrm{E}+03$ & $6.42 \mathrm{E}+03$ & $2.96 \mathrm{E}+03$ & $2.64 \mathrm{E}+03$ & $7.75 \mathrm{E}+03$ & $4.22 \mathrm{E}+02$ & $7.96 \mathrm{E}+03$ & $6.58 \mathrm{E}+03$ \\
\hline 336 & $1.18 \mathrm{E}+03$ & $5.36 \mathrm{E}+03$ & $1.08 \mathrm{E}+03$ & $2.81 \mathrm{E}+03$ & $3.39 \mathrm{E}+03$ & $9.18 \mathrm{E}+02$ & $5.10 \mathrm{E}+03$ & $5.22 \mathrm{E}+0$ \\
\hline 504 & $8.52 \mathrm{E}+02$ & $4.41 \mathrm{E}+03$ & $7.27 \mathrm{E}+02$ & $2.91 \mathrm{E}+03$ & $1.71 \mathrm{E}+03$ & $1.20 E+03$ & $2.35 \mathrm{E}+03$ & $3.58 \mathrm{E}+03$ \\
\hline 720 & $6.16 \mathrm{E}+02$ & $3.43 \mathrm{E}+03$ & $4.83 \mathrm{E}+02$ & $2.99 \mathrm{E}+03$ & $8.92 \mathrm{E}+02$ & $1.36 \mathrm{E}+03$ & $1.21 \mathrm{E}+03$ & $2.51 \mathrm{E}+03$ \\
\hline 2160 & $1.15 \mathrm{E}+02$ & $6.29 \mathrm{E}+02$ & $5.38 \mathrm{E}+01$ & $3.14 \mathrm{E}+03$ & $7.81 \mathrm{E}+01$ & $8.22 \mathrm{E}+02$ & $2.83 E+02$ & $8.56 \mathrm{E}+02$ \\
\hline 4320 & $1.10 \mathrm{E}+01$ & $5.17 \mathrm{E}+01$ & $2.72 \mathrm{E}+02$ & $3.00 \mathrm{E}+03$ & $1.20 \mathrm{E}+01$ & $1.95 \mathrm{E}+02$ & $3.20 \mathrm{E}+01$ & $1.29 \mathrm{E}+02$ \\
\hline 4800 & $6.54 \mathrm{E}+00$ & $2.99 \mathrm{E}+01$ & $2.71 \mathrm{E}+02$ & $2.99 \mathrm{E}+03$ & $8.60 \mathrm{E}+00$ & $1.40 \mathrm{E}+02$ & $1.02 \mathrm{E}+01$ & $4.11 \mathrm{E}+01$ \\
\hline 8760 & $8.98 \mathrm{E}-02$ & $3.54 \mathrm{E}-01$ & $2.64 \mathrm{E}+02$ & $2.91 \mathrm{E}+03$ & 6.71E-01 & $9.08 \mathrm{E}+00$ & $3.10 \mathrm{E}+00$ & $1.19 \mathrm{E}+0$ \\
\hline BT & $\begin{array}{l}\mathrm{E} 6 \\
\mathrm{i} / \mathrm{L}\end{array}$ & $\begin{array}{l}8 \overline{\mathrm{E}+4} \\
\mathrm{~kg}\end{array}$ & $\mathbf{B ~ B q} / \mathbf{g}$ & & & & & \\
\hline TO & $\begin{array}{l}5.9 \mathrm{E} 6 \\
\mathrm{pCi} / \mathrm{L}\end{array}$ & $\begin{array}{l}2.18 \mathrm{E}+5 \\
\mathrm{~Bq} / \mathrm{kg}\end{array}$ & $218.3 \mathrm{~Bq} / \mathrm{g}$ & & & & & \\
\hline & \multicolumn{2}{|c|}{ VEGETABLES } & \multicolumn{2}{|c|}{ WHEAT SEED } & \multicolumn{2}{|l|}{ MEAT } & \multicolumn{2}{|l|}{ MILK } \\
\hline \multirow[t]{2}{*}{ HOUR } & $\mathrm{O}$ & $\mathrm{OBT}$ & HTO & OBT & HTO & OBT & HTO & $\mathrm{OBT}$ \\
\hline & $(\mathrm{Bq} / \mathrm{g})$ & $(\mathrm{Bq} / \mathrm{g})$ & $(\mathrm{Bq} / \mathrm{g})$ & $(\mathrm{Bq} / \mathrm{g})$ & $(\mathrm{Bq} / \mathrm{g})$ & $(\mathrm{Bq} / \mathrm{g})$ & $(\mathrm{Bq} / \mathrm{g})$ & $(\mathrm{Bq} / \mathrm{g})$ \\
\hline . & & & & & & & & \\
\hline
\end{tabular}


Foodstuff Concentrations and Relocation Considerations Following

a Tritium Oxide Release from SRS Tritium Facilities (U)

\begin{tabular}{|c|c|c|c|c|c|c|c|c|}
\hline $5 / 8 / 29 / 54$ & $4.53 \mathrm{E}+05$ & $2.17 \mathrm{E}+03$ & $1.45 \mathrm{E}+05$ & $8.25 \mathrm{E}+02$ & $1.03 \mathrm{E}+04$ & $5.39 \mathrm{E}+01$ & $1.03 \mathrm{E}+04$ & $3.24 \mathrm{E}+03$ \\
\hline SUM & $4.55 \mathrm{E}+05$ & & $1.46 \mathrm{E}+05$ & & $1.04 \mathrm{E}+04$ & & $1.36 \mathrm{E}+04$ & \\
\hline$\overline{\mathrm{Ci} / \mathrm{g}}$ & $1.23 \mathrm{E}-05$ & & $3.94 \mathrm{E}-06$ & & $2.80 \mathrm{E}-07$ & & $3.66 \mathrm{E}-07$ & \\
\hline DII & $50 \mathrm{c} 00$ & & & & & & & \\
\hline DIL HTO & $5.9 \mathrm{E}-09$ & & & & & & & \\
\hline \multicolumn{9}{|l|}{$(\mathrm{Ci} / \mathrm{g})$} \\
\hline & & & & & & & & \\
\hline 168 & $2.74 \mathrm{E}+03$ & $6.42 \mathrm{E}+03$ & $2.96 \mathrm{E}+03$ & $2.64 \mathrm{E}+03$ & $7.75 \mathrm{E}+03$ & $4.22 \mathrm{E}+02$ & $7.96 \mathrm{E}+03$ & $6.58 \mathrm{E}+03$ \\
\hline SUM & $9.16 \mathrm{E}+03$ & & $5.60 \mathrm{E}+03$ & & $8.17 \mathrm{E}+03$ & & $1.45 \mathrm{E}+04$ & \\
\hline $\mathrm{Ci} / \mathrm{g}$ & $2.47 \mathrm{E}-07$ & & $1.51 \mathrm{E}-07$ & & $2.21 \mathrm{E}-07$ & & $3.93 E-07$ & \\
\hline & & & & & & & & \\
\hline DIL OBT & $2.4 \mathrm{E}-09$ & & & & & & & \\
\hline \multicolumn{9}{|l|}{$\mathrm{Ci} / \mathrm{g}$} \\
\hline & & & & & & & & \\
\hline 8760 & $8.98 \mathrm{E}-02$ & $3.54 \mathrm{E}-01$ & $2.64 \mathrm{E}+02$ & $2.91 \mathrm{E}+03$ & $6.71 \mathrm{E}-01$ & $9.08 \mathrm{E}+00$ & $3.10 \mathrm{E}+00$ & $1.19 \mathrm{E}+01$ \\
\hline SUM & $4.44 \mathrm{E}-01$ & & $3.18 \mathrm{E}+03$ & & $9.75 \mathrm{E}+00$ & & $1.50 \mathrm{E}+01$ & \\
\hline $\mathrm{Ci} / \mathrm{g}$ & $1.20 \mathrm{E}-11$ & & $8.59 \mathrm{E}-08$ & & $2.63 \mathrm{E}-10$ & & $4.06 \mathrm{E}-10$ & \\
\hline & & & & & & & & \\
\hline DIL OBT & $2.4 \mathrm{E}-09$ & & & & & & & \\
\hline $\mathrm{Ci} / \mathrm{g}$ & & & & & & & & \\
\hline
\end{tabular}




\begin{tabular}{|c|c|c|c|c|c|c|c|c|}
\hline & \multicolumn{6}{|c|}{ Tritium Facilities, Design Basis Fire, Adverse Met Conditions } & & \\
\hline & \multicolumn{4}{|c|}{ Concentration Values for $100 \mathrm{~km}$ from release } & & & & \\
\hline \multicolumn{9}{|c|}{ Only Wheat Seed is examined since this is the edible portion of the Wheat. The "WHEAT" category is } \\
\hline \multicolumn{7}{|c|}{ ctually the unedible plant body and is not considered in the dose estimate. } & & \\
\hline & & & & & & & & \\
\hline & \multicolumn{2}{|c|}{ VEGETABLES } & \multicolumn{2}{|c|}{ WHEAT SEED } & \multicolumn{2}{|l|}{ MEAT } & \multicolumn{2}{|l|}{ MILK } \\
\hline \multirow[t]{3}{*}{ HOUR } & HTO & OBT & HTO & OBT & HTO & OBT & HTO & $\overline{B T}$ \\
\hline & $(\mathrm{Bq} / \mathrm{g})$ & $\mathrm{g} / \mathrm{g})$ & $(\mathrm{Bq} / \mathrm{g})$ & $(\mathrm{Bq} / \mathrm{g})$ & $(\mathrm{Bq} / \mathrm{g})$ & ) & $(\mathrm{Bq} / \mathrm{g})$ & $\mathrm{Bq} / \mathrm{g})$ \\
\hline & & & & & & & & \\
\hline 1 & $5.08 \mathrm{E}+02$ & $0.00 \mathrm{E}+00$ & $0.00 \mathrm{E}+00$ & $0.00 \mathrm{E}+00$ & $5.21 \mathrm{E}-01$ & $5.61 \mathrm{E}-05$ & $6.32 \mathrm{E}-02$ & $1.97 \mathrm{E}-02$ \\
\hline 2 & $1.54 \mathrm{E}+03$ & $9.44 \mathrm{E}-01$ & $3.39 \mathrm{E}+01$ & $1.12 \mathrm{E}-01$ & $5.02 \mathrm{E}+00$ & $9.33 \mathrm{E}-04$ & $5.97 \mathrm{E}-01$ & $2.31 \mathrm{E}-01$ \\
\hline 3 & $3.62 \mathrm{E}+03$ & $3.81 \mathrm{E}+00$ & $1.41 \mathrm{E}+02$ & $4.98 \mathrm{E}-01$ & $1.82 \mathrm{E}+01$ & $4.32 \mathrm{E}-03$ & $2.30 \mathrm{E}+00$ & $9.31 \mathrm{E}$ \\
\hline 4 & $4.75 \mathrm{E}+03$ & $1.05 \mathrm{E}+01$ & $3.94 \mathrm{E}+02$ & $1.47 \mathrm{E}+00$ & $4.61 \mathrm{E}+01$ & $1.34 \mathrm{E}-02$ & $6.15 E+00$ & $2.56 \mathrm{E}+00$ \\
\hline 5 & $9.79 \mathrm{E}+03$ & $1.93 \mathrm{E}+01$ & $7.36 \mathrm{E}+02$ & $2.98 \mathrm{E}+00$ & $8.90 \mathrm{E}+01$ & $3.08 \mathrm{E}-02$ & $1.28 \mathrm{E}+01$ & $5.38 \mathrm{E}+00$ \\
\hline 6 & $1.13 \mathrm{E}+04$ & $3.75 \mathrm{E}+01$ & $1.41 \mathrm{E}+03$ & $5.93 \mathrm{E}+00$ & $9.58 \mathrm{E}+01$ & $4.11 \mathrm{E}-02$ & $9.29 \mathrm{E}+01$ & $2.89 \mathrm{E}+01$ \\
\hline 7 & $1.79 \mathrm{E}+04$ & $5.84 \mathrm{E}+01$ & $2.21 \mathrm{E}+03$ & $9.92 \mathrm{E}+00$ & $1.09 \mathrm{E}+02$ & $5.28 \mathrm{E}-02$ & $9.91 \mathrm{E}+01$ & $3.09 \mathrm{E}+01$ \\
\hline 8 & $2.59 \mathrm{E}+04$ & $9.16 \mathrm{E}+01$ & $3.42 \mathrm{E}+03$ & $1.61 \mathrm{E}+01$ & $1.27 \overline{\mathrm{E}}+02$ & $6.64 \mathrm{E}-02$ & $1.07 \mathrm{E}+02$ & $3.33 \mathrm{E}+0$ \\
\hline 9 & $3.47 \mathrm{E}+04$ & $1.89 \mathrm{E}+02$ & $6.98 \mathrm{E}+03$ & $3.11 \mathrm{E}+01$ & $1.67 \mathrm{E}+02$ & $8.42 \mathrm{E}-02$ & $1.21 \mathrm{E}+02$ & $3.76 \mathrm{E}+01$ \\
\hline 10 & $1.88 \mathrm{E}+05$ & $9.92 \mathrm{E}+02$ & $3.70 \mathrm{E}+04$ & $1.37 \mathrm{E}+02$ & $5.85 \mathrm{E}+02$ & $1.47 \mathrm{E}-0 \mathrm{I}$ & $2.10 \mathrm{E}+02$ & $6.54 \mathrm{E}+01$ \\
\hline 11 & $8.84 E+04$ & $1.34 \mathrm{E}+03$ & $5.30 \mathrm{E}+04$ & $2.26 \mathrm{E}+02$ & $5.94 \mathrm{E}+02$ & $2.11 \mathrm{E}-01$ & $2.71 \mathrm{E}+02$ & $8.44 \mathrm{E}+01$ \\
\hline 12 & $4.73 \mathrm{E}+04$ & $1.50 \mathrm{E}+03$ & $5.98 \mathrm{E}+04$ & $2.99 \mathrm{E}+02$ & $6.00 \mathrm{E}+02$ & $2.75 \mathrm{E}-01$ & $3.15 \mathrm{E}+02$ & $\overline{9.8}$ \\
\hline 13 & $2.98 \mathrm{E}+04$ & $1.59 \mathrm{E}+03$ & $6.08 \mathrm{E}+04$ & $3.60 \mathrm{E}+02$ & $6.05 \mathrm{E}+02$ & $3.40 \mathrm{E}-01$ & $3.49 \mathrm{E}+02$ & $1.09 \mathrm{E}+02$ \\
\hline 14 & $2.20 \mathrm{E}+04$ & $1 . \overline{64 \mathrm{E}+03}$ & $5.84 \mathrm{E}+04$ & $4.11 \mathrm{E}+02$ & $6.08 \mathrm{E}+02$ & $4.05 \mathrm{E}-0 \mathrm{I}$ & $3.76 \mathrm{E}+02$ & $1.17 \mathrm{E}+02$ \\
\hline 15 & $1.82 \mathrm{E}+04$ & $1.68 \mathrm{E}+03$ & $5.41 \mathrm{E}+04$ & $4.53 \mathrm{E}+02$ & $6.10 \mathrm{E}+02$ & $4.71 \mathrm{E}-01$ & $3.97 \mathrm{E}+02$ & $1.24 \mathrm{E}+02$ \\
\hline 16 & $1.63 \mathrm{E}+04$ & $1 . \overline{71 E+03}$ & $4.90 \mathrm{E}+04$ & $4.89 \mathrm{E}+02$ & $6.12 \mathrm{E}+02$ & $5.36 \mathrm{E}-01$ & $4.15 \mathrm{E}+02$ & $1.29 \mathrm{E}+02$ \\
\hline 17 & $1.51 \mathrm{E}+04$ & $1.74 \mathrm{E}+03$ & $4.38 E+04$ & $5.19 \mathrm{E}+02$ & $6.13 \mathrm{E}+02$ & $6.02 \mathrm{E}-01$ & $4.30 \mathrm{E}+02$ & $1.34 \mathrm{E}+02$ \\
\hline 18 & $1.44 \mathrm{E}+04$ & $1.77 \mathrm{E}+03$ & $3.87 \mathrm{E}+04$ & $5.44 \mathrm{E}+02$ & $8.13 \mathrm{E}+02$ & $1.63 \mathrm{E}+00$ & $7.88 \mathrm{E}+02$ & $1 . \overline{39 \mathrm{E}+03}$ \\
\hline 19 & $1.38 \mathrm{E}+04$ & $1.79 \mathrm{E}+03$ & $3.40 \mathrm{E}+04$ & $5.66 \mathrm{E}+02$ & $9.87 \mathrm{E}+02$ & $2.70 \mathrm{E}+00$ & $8.72 \mathrm{E}+02$ & $1.43 \mathrm{E}+03$ \\
\hline 20 & $1.34 \mathrm{E}+04$ & $1.81 \mathrm{E}+03$ & $2.98 \mathrm{E}+04$ & $5.85 \mathrm{E}+02$ & $1.14 \mathrm{E}+03$ & $3.79 \mathrm{E}+00$ & $9.51 \mathrm{E}+02$ & $1.46 \mathrm{E}+03$ \\
\hline 21 & $1.30 \mathrm{E}+04$ & $1.84 \mathrm{E}+03$ & $2.61 \mathrm{E}+04$ & $6.02 \mathrm{E}+02$ & $1.29 \mathrm{E}+03$ & $4.92 \mathrm{E}+00$ & $1.03 \mathrm{E}+03$ & $1 . \overline{49 \mathrm{E}+03}$ \\
\hline 22 & $1.27 \mathrm{E}+04$ & $1.86 \mathrm{E}+03$ & $2.29 \mathrm{E}+04$ & $6.16 \mathrm{E}+02$ & $1.43 \mathrm{E}+03$ & $6.07 \mathrm{E}+00$ & $1.10 \mathrm{E}+03$ & $1.52 \mathrm{E}+03$ \\
\hline 23 & $1.25 \mathrm{E}+04$ & $1.88 \overline{\mathrm{E}}+03$ & $2.02 \mathrm{E}+04$ & $6.30 \mathrm{E}+02$ & $1.56 \mathrm{E}+03$ & $7.24 \mathrm{E}+00$ & $1.17 \mathrm{E}+03$ & $1 . \overline{55 \mathrm{E}+03}$ \\
\hline 24 & $1.22 \mathrm{E}+04$ & $1.90 \mathrm{E}+03$ & $1.79 E+04$ & $6.42 \mathrm{E}+02$ & $1.69 \mathrm{E}+03$ & $8.44 \mathrm{E}+00$ & $1.23 \mathrm{E}+03$ & $1.58 \mathrm{E}+03$ \\
\hline 25 & $1.20 \overline{\mathrm{E}}+04$ & $1.92 \mathrm{E}+03$ & $1.60 \mathrm{E}+04$ & $6.52 \mathrm{E}+02$ & $1.81 \mathrm{E}+03$ & $9.66 \mathrm{E}+00$ & $1.30 \mathrm{E}+03$ & $1.61 \mathrm{E}+03$ \\
\hline 26 & $1.18 \mathrm{E}+04$ & $1.94 \mathrm{E}+03$ & $1.44 \mathrm{E}+04$ & $6.63 \mathrm{E}+02$ & $1.93 \mathrm{E}+03$ & $1.09 \mathrm{E}+01$ & $1.36 \mathrm{E}+03$ & $1.63 \mathrm{E}+03$ \\
\hline 27 & $1.16 \mathrm{E}+04$ & $1.96 \mathrm{E}+03$ & $1.30 \mathrm{E}+04$ & $6.72 \mathrm{E}+02$ & $2.05 \mathrm{E}+03$ & $1.22 \mathrm{E}+01$ & $1.42 \mathrm{E}+03$ & $1.66 \mathrm{E}+\overline{03}$ \\
\hline 28 & $1.15 \mathrm{E}+04$ & $1.98 \mathrm{E}+03$ & $1.19 \mathrm{E}+04$ & $6.81 \mathrm{E}+02$ & $2.16 \mathrm{E}+03$ & $1.35 \mathrm{E}+01$ & $1.48 \mathrm{E}+03$ & $1.69 \mathrm{E}+03$ \\
\hline 29 & $1.14 \mathrm{E}+04$ & $2.00 \mathrm{E}+03$ & $1.10 \mathrm{E}+04$ & $6.89 \overline{\mathrm{E}+02}$ & $2.28 \mathrm{E}+03$ & $1.48 \mathrm{E}+01$ & $1.54 \mathrm{E}+03$ & $1.71 \mathrm{E}+03$ \\
\hline 30 & $1.13 \mathrm{E}+04$ & $2.01 \mathrm{E}+03$ & $1.02 \mathrm{E}+04$ & $6.97 \mathrm{E}+02$ & $2.26 \mathrm{E}+03$ & $1.50 \mathrm{E}+01$ & $2.19 \mathrm{E}+03$ & $6.85 \mathrm{E}+02$ \\
\hline 31 & $1.12 \mathrm{E}+04$ & $2.03 \mathrm{E}+03$ & $9.56 \mathrm{E}+03$ & $7.05 \mathrm{E}+02$ & $2.25 \mathrm{E}+03$ & $1.52 \mathrm{E}+01$ & $2.18 \mathrm{E}+03$ & $6 . \overline{83 \mathrm{E}+02}$ \\
\hline 32 & $1.11 \mathrm{E}+04$ & $2.05 \mathrm{E}+03$ & $9.05 \mathrm{E}+03$ & $7.13 \mathrm{E}+02$ & $2.23 \mathrm{E}+03$ & $1.55 \mathrm{E}+01$ & $2.18 \mathrm{E}+03$ & $6.81 \mathrm{E}+02$ \\
\hline 33 & $1.11 \mathrm{E}+04$ & $2.07 \mathrm{E}+03$ & $8.63 \mathrm{E}+03$ & $7.20 \mathrm{E}+02$ & $2.22 \mathrm{E}+03$ & $1.57 \mathrm{E}+01$ & $2.17 \mathrm{E}+03$ & $6.79 \mathrm{E}+02$ \\
\hline 34 & $1.10 \mathrm{E}+04$ & $2.09 \mathrm{E}+03$ & $8.30 \mathrm{E}+03$ & $7.27 \mathrm{E}+02$ & $2.20 \mathrm{E}+03$ & $1.59 \mathrm{E}+01$ & $2.16 \mathrm{E}+03$ & $6.77 \overline{\mathrm{E}}+02$ \\
\hline 35 & $1.10 \mathrm{E}+04$ & $2.10 \mathrm{E}+03$ & $8.03 E+03$ & $7.34 \mathrm{E}+02$ & $2.19 \mathrm{E}+03$ & $1.61 \mathrm{E}+01$ & $2.16 \overline{\mathrm{E}+03}$ & $6.75 \mathrm{E}+02$ \\
\hline 36 & $1.09 \mathrm{E}+04$ & $2.12 \mathrm{E}+03$ & $7.81 \mathrm{E}+03$ & $7.41 \mathrm{E}+02$ & $2.18 \mathrm{E}+03$ & $1.64 \mathrm{E}+01$ & $2.15 \overline{\mathrm{E}+03}$ & $6.73 E+02$ \\
\hline 37 & $1.09 \mathrm{E}+04$ & $2.14 \mathrm{E}+03$ & $7.64 \mathrm{E}+03$ & $7.48 \mathrm{E}+02$ & $2.16 \mathrm{E}+03$ & $1.66 \mathrm{E}+01$ & $2.14 \mathrm{E}+03$ & $6.70 \mathrm{E}+02$ \\
\hline 38 & $1.08 \mathrm{E}+04$ & $2.16 \mathrm{E}+03$ & $7.51 \mathrm{E}+03$ & $7.55 \mathrm{E}+02$ & $2.15 \mathrm{E}+03$ & $1.68 \mathrm{E}+01$ & $2.14 \mathrm{E}+03$ & $6.68 \overline{\mathrm{E}}+02$ \\
\hline
\end{tabular}




\begin{tabular}{|c|c|c|c|c|c|c|c|c|}
\hline 39 & $1.08 \mathrm{E}+04$ & $2.17 \mathrm{E}+03$ & $7.41 \mathrm{E}+03$ & $7.62 \mathrm{E}+02$ & $2.14 \mathrm{E}+03$ & $1.70 \mathrm{E}+0 \mathrm{I}$ & $2.13 \mathrm{E}+03$ & $6.66 \mathrm{E}+02$ \\
\hline 40 & $1.07 \mathrm{E}+04$ & $2.19 \mathrm{E}+03$ & $7.34 \mathrm{E}+03$ & $7.69 \mathrm{E}+02$ & $2.12 \mathrm{E}+03$ & $1.72 \mathrm{E}+01$ & $2.12 \widehat{E}+03$ & $6.64 \mathrm{E}+0$ \\
\hline 41 & $1.07 \mathrm{E}+04$ & $2.21 \mathrm{E}+03$ & $7.28 \mathrm{E}+03$ & $7.76 \mathrm{E}+02$ & $2.11 \mathrm{E}+03$ & $1.75 \mathrm{E}+01$ & $2.12 \mathrm{E}+03$ & $6.62 \bar{E}+02$ \\
\hline 42 & $1.06 \mathrm{E}+04$ & $2.22 \mathrm{E}+03$ & $7.25 \mathrm{E}+03$ & $7.83 \mathrm{E}+02$ & $2.21 \mathrm{E}+03$ & $1.89 \mathrm{E}+01$ & $2.15 \mathrm{E}+03$ & $2.15 \mathrm{E}+03$ \\
\hline$\overline{43}$ & $1.06 \mathrm{E}+04$ & $2.24 \mathrm{E}+03$ & $7.23 \mathrm{E}+03$ & $7.90 \mathrm{E}+02$ & $2.32 \mathrm{E}+03$ & $2.04 \mathrm{E}+01$ & $2.19 \mathrm{E}+03$ & $2.17 \mathrm{E}+0$ \\
\hline 44 & $1.05 \mathrm{E}+04$ & $2.26 \mathrm{E}+03$ & $7.22 \mathrm{E}+03$ & $7.97 \mathrm{E}+02$ & $2.42 \mathrm{E}+03$ & $2.18 \mathrm{E}+01$ & $2.24 \mathrm{E}+03$ & $2.19 \mathrm{E}+0$. \\
\hline 45 & $1.05 \mathrm{E}+04$ & $2.27 \mathrm{E}+03$ & $7.22 \mathrm{E}+03$ & $8.04 \mathrm{E}+02$ & $2.52 \mathrm{E}+03$ & $2.33 \mathrm{E}+01$ & $2.29 \mathrm{E}+03$ & $2.21 \mathrm{E}+03$ \\
\hline 46 & $1.04 \mathrm{E}+04$ & $2.29 \mathrm{E}+03$ & $7.23 \mathrm{E}+03$ & $8.11 \mathrm{E}+02$ & $2.61 \mathrm{E}+03$ & $2.49 \mathrm{E}+01$ & $2.34 \mathrm{E}+03$ & $2.23 \mathrm{E}+0$ \\
\hline 47 & $1.04 \mathrm{E}+04$ & $2.31 \mathrm{E}+03$ & $7.25 \mathrm{E}+03$ & $8.18 \mathrm{E}+02$ & $2.71 E+03$ & $2.64 \mathrm{E}+01$ & $2.39 \mathrm{E}+03$ & $2.25 \mathrm{E}+0$ \\
\hline 48 & $1.03 E+04$ & $2.32 \mathrm{E}+03$ & $7.28 \mathrm{E}+03$ & $8.25 \mathrm{E}+02$ & $2.80 \mathrm{E}+03$ & $2.79 \mathrm{E}+01$ & $2.43 \mathrm{E}+03$ & $2.27 \mathrm{E}+03$ \\
\hline 49 & $1.03 \mathrm{E}+04$ & $2.34 \mathrm{E}+03$ & $7.31 \mathrm{E}+03$ & $8.32 \mathrm{E}+02$ & $2.90 \mathrm{E}+03$ & $2.95 \mathrm{E}+01$ & $2.48 \mathrm{E}+03$ & $2.29 \mathrm{E}+0$ \\
\hline 50 & $1.02 \mathrm{E}+04$ & $2.36 \mathrm{E}+03$ & $7.34 \mathrm{E}+03$ & $8.39 \mathrm{E}+02$ & $2.99 \mathrm{E}+03$ & $3.11 \mathrm{E}+01$ & $2.53 \mathrm{E}+03$ & $2.31 \mathrm{E}+0$ \\
\hline 51 & $1.02 \mathrm{E}+04$ & $2.37 \mathrm{E}+03$ & $7.37 \mathrm{E}+03$ & $8.46 \mathrm{E}+02$ & $3.08 \mathrm{E}+03$ & $3.27 \mathrm{E}+01$ & $2.57 \mathrm{E}+03$ & $2.33 \mathrm{E}+03$ \\
\hline 52 & $1.01 \mathrm{E}+04$ & $2.39 \mathrm{E}+03$ & $7.39 \mathrm{E}+03$ & $8.54 \mathrm{E}+02$ & $3.17 \mathrm{E}+03$ & $3.43 \mathrm{E}+01$ & $2.62 \mathrm{E}+03$ & $2.35 \mathrm{E}+0$ \\
\hline 53 & $1.01 \mathrm{E}+04$ & $2.40 \mathrm{E}+03$ & $7.41 \mathrm{E}+03$ & $8.61 \mathrm{E}+02$ & $3.25 \mathrm{E}+03$ & $3.59 \mathrm{E}+01$ & $2.66 \mathrm{E}+03$ & $2.37 \overline{\mathrm{E}+0}$ \\
\hline 54 & $1.00 \mathrm{E}+04$ & $2.42 \mathrm{E}+03$ & $7.42 \mathrm{E}+03$ & $8.68 \mathrm{E}+02$ & $3.23 \mathrm{E}+03$ & $3.63 \mathrm{E}+01$ & $3.13 \mathrm{E}+03$ & $9.82 \bar{E}+02$ \\
\hline 55 & $9.94 \mathrm{E}+03$ & $2.43 \mathrm{E}+03$ & $7.42 \mathrm{E}+03$ & $8.75 \mathrm{E}+02$ & $3.21 \mathrm{E}+03$ & $3.66 \mathrm{E}+01$ & $3.12 \mathrm{E}+03$ & $9.79 \mathrm{E}+02$ \\
\hline 56 & $9.89 \mathrm{E}+03$ & $2.45 \mathrm{E}+03$ & $7.41 \mathrm{E}+03$ & $8.82 \mathrm{E}+02$ & $3.19 \mathrm{E}+03$ & $3.69 \mathrm{E}+01$ & $3.11 \mathrm{E}+03$ & $9.75 \mathrm{E}+02$ \\
\hline 57 & $9.83 \mathrm{E}+03$ & $2.46 \mathrm{E}+03$ & $7.40 \mathrm{E}+03$ & $8.89 \mathrm{E}+02$ & $3.16 \mathrm{E}+03$ & $3.72 \mathrm{E}+01$ & $3.10 \mathrm{E}+03$ & $9.7 \overline{2 E+02}$ \\
\hline 58 & $9.77 \mathrm{E}+03$ & $2.48 \mathrm{E}+03$ & $7.39 \mathrm{E}+03$ & $8.96 \mathrm{E}+02$ & $3.14 \mathrm{E}+03$ & $3.75 \mathrm{E}+01$ & $3.09 \mathrm{E}+03$ & $9.69 \mathrm{E}+02$ \\
\hline 59 & $9.71 \mathrm{E}+03$ & $2.49 \mathrm{E}+03$ & $7.37 \mathrm{E}+03$ & $9.04 \mathrm{E}+02$ & $3.12 \mathrm{E}+03$ & $3.78 \mathrm{E}+01$ & $3.08 \mathrm{E}+03$ & $9.65 \mathrm{E}+0$ \\
\hline 60 & $9.65 \mathrm{E}+03$ & $2.51 \mathrm{E}+03$ & $7.34 \mathrm{E}+03$ & $9.11 \mathrm{E}+02$ & $3.10 \mathrm{E}+03$ & $3.81 \mathrm{E}+01$ & $3.07 \mathrm{E}+03$ & $9.62 \mathrm{E}+0$ \\
\hline 61 & $9.58 \mathrm{E}+03$ & $2.52 \mathrm{E}+03$ & $7.31 \mathrm{E}+03$ & $9.18 \mathrm{E}+02$ & $3.07 \mathrm{E}+03$ & $3.84 \mathrm{E}+01$ & $3.06 \mathrm{E}+03$ & $9.59 \mathrm{E}+02$ \\
\hline 62 & $9.51 \mathrm{E}+03$ & $2.54 \mathrm{E}+03$ & $7.28 \mathrm{E}+03$ & $9.25 \mathrm{E}+02$ & $3.05 \mathrm{E}+03$ & $3.88 \mathrm{E}+01$ & $3.04 E+03$ & $9.55 \mathrm{E}+02$ \\
\hline 63 & $9.44 \mathrm{E}+03$ & $2.55 \mathrm{E}+03$ & $7.24 \mathrm{E}+03$ & $9.31 \mathrm{E}+02$ & $3.03 \mathrm{E}+03$ & $3.90 \mathrm{E}+01$ & $3.03 \mathrm{E}+03$ & $9.52 \mathrm{E}+02$ \\
\hline 64 & $9.37 \mathrm{E}+03$ & $2.57 \mathrm{E}+03$ & $7.20 \mathrm{E}+03$ & $9.38 \mathrm{E}+02$ & $3.01 E+03$ & $3.93 \mathrm{E}+01$ & $3.02 \mathrm{E}+03$ & $9.49 \mathrm{E}+02$ \\
\hline 65 & $9.29 \mathrm{E}+03$ & $2.58 \mathrm{E}+03$ & $7.16 \mathrm{E}+03$ & $9.45 \mathrm{E}+02$ & $2.99 \mathrm{E}+03$ & $3.96 \mathrm{E}+01$ & $3.01 \mathrm{E}+03$ & $9.45 \mathrm{E}+02$ \\
\hline 66 & $9.21 \mathrm{E}+03$ & $2.59 \mathrm{E}+03$ & $7.12 \mathrm{E}+03$ & $9.52 \mathrm{E}+02$ & $3.07 \mathrm{E}+03$ & $4.14 \mathrm{E}+01$ & $2.97 \mathrm{E}+03$ & $2.66 \mathrm{E}+03$ \\
\hline 67 & $9.13 \mathrm{E}+03$ & $2.61 \mathrm{E}+03$ & $7.07 \mathrm{E}+03$ & $9.59 \mathrm{E}+02$ & $3.15 \mathrm{E}+03$ & $4.31 \mathrm{E}+01$ & $3.01 \mathrm{E}+03$ & $2.68 \mathrm{E}+03$ \\
\hline$\overline{68}$ & $9.04 \mathrm{E}+03$ & $2.62 \mathrm{E}+03$ & $7.02 \mathrm{E}+03$ & $9.65 \mathrm{E}+02$ & $3.22 \mathrm{E}+03$ & $4.49 \mathrm{E}+01$ & $3.05 \mathrm{E}+03$ & $2.69 \mathrm{E}+03$ \\
\hline 69 & $8.96 \mathrm{E}+03$ & $2.63 \bar{E}+03$ & $6.96 \mathrm{E}+03$ & $9.72 \mathrm{E}+02$ & $3.30 \mathrm{E}+03$ & $4.66 \mathrm{E}+01$ & $3.09 \mathrm{E}+03$ & $2.71 \mathrm{E}+03$ \\
\hline 168 & $1.38 \mathrm{E}+03$ & $3.21 \mathrm{E}+03$ & $1.84 \mathrm{E}+03$ & $1.36 \mathrm{E}+03$ & $3.70 \mathrm{E}+03$ & $1.82 \mathrm{E}+02$ & $3.76 \mathrm{E}+03$ & $3.29 \mathrm{E}+03$ \\
\hline 336 & $5.73 \mathrm{E}+02$ & $2.68 \mathrm{E}+03$ & $5.55 \mathrm{E}+02$ & $1.45 \mathrm{E}+03$ & $1.68 \mathrm{E}+03$ & $4.40 \mathrm{E}+02$ & $2.48 \mathrm{E}+03$ & $2.65 \mathrm{E}+03$ \\
\hline 504 & $4.11 \mathrm{E}+02$ & $2.21 \mathrm{E}+03$ & $3.72 \mathrm{E}+02$ & $1.50 \mathrm{E}+03$ & $8.65 \mathrm{E}+02$ & $5.88 \mathrm{E}+02$ & $1.18 \mathrm{E}+03$ & $1.84 \mathrm{E}+03$ \\
\hline 720 & $2.97 \mathrm{E}+02$ & $1.71 \mathrm{E}+03$ & $2.46 \mathrm{E}+02$ & $1.54 \mathrm{E}+03$ & $4.57 \mathrm{E}+02$ & $6.76 \mathrm{E}+02$ & $6.13 \mathrm{E}+02$ & $1.29 \mathrm{E}+03$ \\
\hline 2160 & $5.49 \mathrm{E}+01$ & $3.11 \mathrm{E}+02$ & $2.73 \mathrm{E}+01$ & $1.62 \mathrm{E}+03$ & $3.96 \mathrm{E}+01$ & $4.15 \mathrm{E}+02$ & $1.45 \mathrm{E}+02$ & $4.39 \mathrm{E}+02$ \\
\hline 4320 & $5.27 \mathrm{E}+00$ & $2.53 \mathrm{E}+01$ & $1.38 \mathrm{E}+02$ & $1.55 \mathrm{E}+03$ & $5.99 \mathrm{E}+00$ & $9.87 E+01$ & $1.61 \mathrm{E}+01$ & $6.52 \mathrm{E}+01$ \\
\hline 4800 & $3.14 \mathrm{E}+00$ & $1.46 \mathrm{E}+01$ & $1.38 \mathrm{E}+02$ & $1.54 \mathrm{E}+03$ & $4.31 \mathrm{E}+00$ & $7.08 \mathrm{E}+01$ & $5.09 \mathrm{E}+00$ & $2.07 \mathrm{E}+01$ \\
\hline 8760 & $4.30 \mathrm{E}-02$ & $1.71 \mathrm{E}-01$ & $1.34 \mathrm{E}+02$ & $1.50 \mathrm{E}+03$ & $3.35 \mathrm{E}-01$ & $4.58 \mathrm{E}+00$ & $1.55 \overline{\mathrm{E}}+00$ & $6.01 \mathrm{E}+00$ \\
\hline $\mathbf{T}$ & $\begin{array}{l}\mathrm{E} 6 \\
\mathrm{~L}\end{array}$ & $\begin{array}{l}8.88 \mathrm{E}+4 \\
\mathrm{~Bq} / \mathrm{kg}\end{array}$ & $8 \mathrm{~Bq} / \mathrm{g}$ & & & & & \\
\hline $\bar{O}$ & $\begin{array}{l}5.9 \mathrm{E} 6 \\
\mathrm{pCi} / \mathrm{L}\end{array}$ & $\begin{array}{l}2.18 \mathrm{E}+5 \\
\mathrm{~Bq} / \mathrm{kg}\end{array}$ & $8.3 \mathrm{~Bq} / \mathrm{g}$ & & & & & \\
\hline & \multicolumn{2}{|c|}{ VEGETABLES } & \multicolumn{2}{|c|}{ WHEAT SEED } & \multicolumn{2}{|l|}{$\overline{\text { MEAT }}$} & \multicolumn{2}{|l|}{ MILK } \\
\hline HOUR & $\mathrm{O}$ & $\overline{\mathrm{OBT}}$ & HTO & $\mathrm{OBT}$ & HTO & OBT & HTO & $\overline{\mathrm{OBT}}$ \\
\hline & $(\mathrm{Bq} / \mathrm{g})$ & $(\mathrm{Bq} / \mathrm{g})$ & $(\mathrm{Bq} / \mathrm{g})$ & $(\mathrm{Bq} / \mathrm{g})$ & $(\mathrm{Bq} / \mathrm{g})$ & $(\mathrm{Bq} / \mathrm{g})$ & $(\mathrm{Bq} / \mathrm{g})$ & $(\overline{\mathrm{Bq} / \mathrm{g})}$ \\
\hline ak HTO & & & & & & & & \\
\hline
\end{tabular}




\begin{tabular}{|c|c|c|c|c|c|c|c|c|}
\hline $\begin{array}{l}10 / 13 / 53 / 5 \\
4\end{array}$ & $1.88 \mathrm{E}+05$ & $9.92 \mathrm{E}+02$ & $6.08 \mathrm{E}+04$ & $3.60 \mathrm{E}+02$ & $3.25 \mathrm{E}+03$ & $3.59 \mathrm{E}+01$ & $3.13 \mathrm{E}+0 \overline{3}$ & $9.82 \overline{\mathrm{E}}+02$ \\
\hline SUM & $1.89 \mathrm{E}+05$ & & $6.12 \mathrm{E}+04$ & & $3.29 \mathrm{E}+03$ & & $4.11 \mathrm{E}+03$ & \\
\hline $\mathrm{Ci} / \mathrm{g}$ & $5.10 \mathrm{E}-06$ & & $1.65 \mathrm{E}-06$ & & $8.89 \mathrm{E}-08$ & & $1.11 \mathrm{E}-07$ & \\
\hline & & & & & & & & \\
\hline DIL HTO & $5.9 \mathrm{E}-09$ & & & & & & & \\
\hline \multicolumn{9}{|l|}{$(\mathrm{Ci} / \mathrm{g})$} \\
\hline & & & & & & & & \\
\hline 168 & $1.38 \mathrm{E}+03$ & $3.21 \mathrm{E}+03$ & $1.84 \mathrm{E}+03$ & $1.36 \mathrm{E}+03$ & $3.70 \mathrm{E}+03$ & $1.82 \mathrm{E}+\overline{02}$ & $3.76 \mathrm{E}+03$ & $3.29 \bar{E}+03$ \\
\hline$\overline{\text { SUM }}$ & $4.59 \mathrm{E}+03$ & & $3.20 \mathrm{E}+03$ & & $3.88 \mathrm{E}+03$ & & $7.04 \mathrm{E}+03$ & \\
\hline $\mathrm{Ci} / \mathrm{g}$ & $1.24 \mathrm{E}-07$ & & $8.64 E-08$ & & 1.05E-07 & & $1.90 \mathrm{E}-07$ & \\
\hline & & & & & & & & \\
\hline DIL OBT & $2.4 \mathrm{E}-09$ & & & & & & & \\
\hline \multicolumn{9}{|l|}{$\mathrm{Ci} / \mathrm{g}$} \\
\hline & & & & & & & & \\
\hline 8760 & $4.30 \mathrm{E}-02$ & $1.71 \mathrm{E}-01$ & $1.34 \mathrm{E}+02$ & $1.50 \mathrm{E}+03$ & $3.35 \mathrm{E}-01$ & $4.58 \mathrm{E}+00$ & $1.55 \mathrm{E}+00$ & $6.01 \mathrm{E}+00$ \\
\hline SUM & $2.14 \mathrm{E}-01$ & & $1.64 \mathrm{E}+03$ & & $4.92 \mathrm{E}+00$ & & $7.55 \mathrm{E}+00$ & \\
\hline $\mathrm{Ci} / \mathrm{g}$ & $5.79 \mathrm{E}-12$ & & $4.42 \mathrm{E}-08$ & & $1.33 \mathrm{E}-10$ & & $2.04 \mathrm{E}-10$ & \\
\hline DIL OBT & $2.4 \mathrm{E}-09$ & & & & & & & \\
\hline $\mathrm{Ci} / \mathrm{g}$ & & & & & & & & \\
\hline
\end{tabular}




\begin{tabular}{|c|c|c|c|c|c|c|c|c|}
\hline & \multicolumn{6}{|c|}{ Tritium Facilities, Design Basis Fire, Average Met Conditions } & & \\
\hline & \multicolumn{4}{|c|}{ Concentration Values for $150 \mathrm{~m}$ from release } & & & & \\
\hline \multicolumn{9}{|c|}{ Only Wheat Seed is examined since this is the edible portion of the Wheat. The "WHEAT" category is } \\
\hline \multicolumn{9}{|c|}{ actually the unedible plant body and is not considered in the dose estimate. } \\
\hline & & & & & & & & \\
\hline & \multicolumn{2}{|c|}{ VEGETABLES } & \multicolumn{2}{|c|}{ WHEAT SEED } & \multicolumn{2}{|l|}{ MEAT } & \multicolumn{2}{|l|}{ MILK } \\
\hline \multirow[t]{3}{*}{ HOUR } & HTO & OBT & HTO & $\widehat{\mathrm{OBT}}$ & HTO & OBT & HTO & $\overline{\mathrm{BT}}$ \\
\hline & $(\mathrm{Bq} / \mathrm{g})$ & $(\mathrm{Bq} / \mathrm{g})$ & $(\mathrm{Bq} / \mathrm{g})$ & $(\mathrm{Bq} / \mathrm{g})$ & $(\mathrm{Bq} / \mathrm{g})$ & $(\mathrm{Bo} / \mathrm{g})$ & $(\mathrm{Bq} / \mathrm{g})$ & $30 / \mathrm{g})$ \\
\hline & & & & & & & & \\
\hline$\overline{1}$ & $4.26 \mathrm{E}+07$ & $2.16 \mathrm{E}+05$ & $1.32 \mathrm{E}+07$ & $4.46 \mathrm{E}+04$ & $1.06 \mathrm{E}+06$ & $2.17 \bar{E}+02$ & $1.28 \mathrm{E}+05$ & $5 . \overline{55 \mathrm{E}+}$ \\
\hline 2 & $1.53 \mathrm{E}+07$ & $2.90 \overline{\mathrm{E}}+05$ & $1.89 \mathrm{E}+07$ & $7.67 \mathrm{E}+04$ & $1.54 \mathrm{E}+06$ & $5.39 \overline{\mathrm{E}}+02$ & $2.80 \mathrm{E}+05$ & $1.22 \mathrm{E}+0$ \\
\hline 3 & $5 . \overline{86 \mathrm{E}+06}$ & $3.16 \mathrm{E}+05$ & $2.03 \mathrm{E}+07$ & $9.99 \mathrm{E}+04$ & $1.79 \overline{\mathrm{E}+06}$ & $9.15 \mathrm{E}+02$ & $4.25 \mathrm{E}+05$ & $1.86 \overline{\mathrm{E}+0}$ \\
\hline 4 & $2 . \overline{54 \mathrm{E}+06}$ & $3.26 \mathrm{E}+05$ & $1.93 \mathrm{E}+07$ & $1.17 \mathrm{E}+05$ & $1.91 \mathrm{E}+06$ & $1.32 \mathrm{E}+03$ & $5.55 \mathrm{E}+05$ & $2.44 \mathrm{E}+0$ \\
\hline 5 & $1 . \overline{39 \mathrm{E}+06}$ & $3.30 \mathrm{E}+05$ & $1.73 \mathrm{E}+07$ & $1.29 \mathrm{E}+05$ & $1.97 \overline{\mathrm{E}+06}$ & $1.74 E+03$ & $6.69 \mathrm{E}+05$ & $2.94 \mathrm{E}+0$ \\
\hline 6 & $9.85 \mathrm{E}+05$ & $3.32 \mathrm{E}+05$ & $1.48 \mathrm{E}+07$ & $1.38 \mathrm{E}+05$ & $1.96 \bar{E}+06$ & $1.94 \mathrm{E}+03$ & $1.90 \mathrm{E}+06$ & $5.91 \overline{\mathrm{E}+0}$ \\
\hline 7 & $8.38 \mathrm{E}+05$ & $3.33 \mathrm{E}+05$ & $1.24 \mathrm{E}+07$ & $1.44 \mathrm{E}+05$ & $1.94 \mathrm{E}+06$ & $2.15 \mathrm{E}+03$ & $1.89 \mathrm{E}+06$ & $5.89 \bar{E}+0$ \\
\hline 8 & $7.79 \mathrm{E}+05$ & $3.34 \mathrm{E}+05$ & $1.01 \mathrm{E}+07$ & $1.49 \mathrm{E}+05$ & $1 . \overline{93 E+06}$ & $2.36 \mathrm{E}+03$ & $1.88 \mathrm{E}+06$ & $5.8 \overline{6 E+05}$ \\
\hline$\overline{9}$ & $7.50 \mathrm{E}+05$ & $3.35 \mathrm{E}+05$ & $8.19 E+06$ & $1.52 \mathrm{E}+05$ & $1.91 \mathrm{E}+06$ & $2.56 \mathrm{E}+03$ & $1.87 \mathrm{E}+06$ & $5.84 \mathrm{E}+0$. \\
\hline 10 & $7.31 \mathrm{E}+05$ & $3.36 \mathrm{E}+05$ & $6.55 \mathrm{E}+06$ & $1.54 \mathrm{E}+05$ & $1.89 \mathrm{E}+06$ & $2 . \overline{76 \mathrm{E}+03}$ & $1.87 \mathrm{E}+06$ & $5.81 \mathrm{E}+0$ \\
\hline 11 & $7.15 \mathrm{E}+05$ & $3.37 \mathrm{E}+05$ & $5.20 \mathrm{E}+06$ & $1.56 \overline{\mathrm{E}}+05$ & $1.88 \mathrm{E}+06$ & $\overline{2.96 \mathrm{E}+03}$ & $1.86 \mathrm{E}+06$ & $5.7 \overline{9 \mathrm{E}+0}$ \\
\hline 12 & $7 . \overline{02 \mathrm{E}+05}$ & $3.38 \mathrm{E}+05$ & $4.10 \mathrm{E}+06$ & $1.58 \mathrm{E}+05$ & $1.86 \bar{E}+06$ & $3.16 \mathrm{E}+03$ & $1.85 \mathrm{E}+06$ & $5.77 \overline{\mathrm{E}+0 .}$ \\
\hline 13 & $6.89 \mathrm{E}+05$ & $3.39 \mathrm{E}+05$ & $3.23 \mathrm{E}+06$ & $1.59 \mathrm{E}+05$ & $1.85 \mathrm{E}+06$ & $3.36 \mathrm{E}+03$ & $1.84 \mathrm{E}+06$ & $5.74 \mathrm{E}+0$ \\
\hline 14 & $6.76 \mathrm{E}+05$ & $3.39 \mathrm{E}+05$ & $2.54 \mathrm{E}+06$ & $1.60 \mathrm{E}+05$ & $1.83 E+06$ & $3.55 \widehat{\mathrm{E}+03}$ & $1.84 \mathrm{E}+06$ & $5.72 E+05$ \\
\hline 15 & $6.64 \mathrm{E}+05$ & $3 . \overline{40 \mathrm{E}+05}$ & $2.00 \mathrm{E}+06$ & $1.60 \mathrm{E}+05$ & $1.82 \mathrm{E}+06$ & $3 . \overline{74 \mathrm{E}+03}$ & $1.83 \mathrm{E}+06$ & $5.69 \mathrm{E}+05$ \\
\hline 16 & $6.52 \mathrm{E}+05$ & $3.41 \mathrm{E}+05$ & $1.59 \mathrm{E}+06$ & $1.61 \mathrm{E}+05$ & $1.80 \mathrm{E}+06$ & $3.94 \mathrm{E}+03$ & $1.82 \mathrm{E}+06$ & $5.67 \mathrm{E}+0$ \\
\hline 17 & $6.40 \mathrm{E}+05$ & $3.42 \bar{E}+05$ & $1.26 \mathrm{E}+06$ & $1.62 \mathrm{E}+05$ & $1.79 \mathrm{E}+06$ & $4.13 \mathrm{E}+03$ & $1.81 \mathrm{E}+06$ & $5.65 \mathrm{E}+05$ \\
\hline 18 & $6.29 \mathrm{E}+05$ & $3.42 \mathrm{E}+05$ & $1.02 \mathrm{E}+06$ & $1.62 \mathrm{E}+05$ & $1.78 \mathrm{E}+06$ & $4.53 \mathrm{E}+03$ & $1.72 \bar{E}+06$ & $8.05 \mathrm{E}+05$ \\
\hline 19 & $6.18 \mathrm{E}+05$ & $3.43 \mathrm{E}+05$ & $8.26 \mathrm{E}+05$ & $1.62 \mathrm{E}+05$ & $1.77 \mathrm{E}+06$ & $4.94 \widetilde{E}+03$ & $1.72 \mathrm{E}+06$ & $8.04 \mathrm{E}+05$ \\
\hline 20 & $6.08 \mathrm{E}+05$ & $3.44 \mathrm{E}+05$ & $6.8 \overline{1 \mathrm{E}+05}$ & $1.63 \mathrm{E}+05$ & $1.76 \mathrm{E}+06$ & $5.35 \mathrm{E}+03$ & $1.72 \mathrm{E}+06$ & $8.03 \mathrm{E}+0$ \\
\hline 21 & $5.98 \mathrm{E}+05$ & $3.44 \mathrm{E}+05$ & $5.71 \mathrm{E}+05$ & $1.63 \mathrm{E}+05$ & $1.76 \mathrm{E}+06$ & $5.76 \mathrm{E}+03$ & $1.71 \mathrm{E}+06$ & $8.02 \mathrm{E}+05$ \\
\hline$\overline{22}$ & $5.88 \mathrm{E}+05$ & $3.45 \mathrm{E}+05$ & $4.87 \mathrm{E}+05$ & $1.6 \overline{\mathrm{E}+05}$ & $1.75 \mathrm{E}+06$ & $6.16 \mathrm{E}+03$ & $1.71 \mathrm{E}+06$ & $8.01 \mathrm{E}+05$ \\
\hline 23 & $5.79 \mathrm{E}+05$ & $3.45 \mathrm{E}+05$ & $4.22 \mathrm{E}+05$ & $1.63 \mathrm{E}+05$ & $1.74 \mathrm{E}+06$ & $6.57 \mathrm{E}+03$ & $1.7 \overline{1 E}+06$ & $8.01 \mathrm{E}+0$ \\
\hline 24 & $5.70 \mathrm{E}+05$ & $3.46 \mathrm{E}+05$ & $3.73 \mathrm{E}+05$ & $1.64 \mathrm{E}+05$ & $1.74 \mathrm{E}+06$ & $6.97 \mathrm{E}+03$ & $1.70 \mathrm{E}+06$ & $7.99 \mathrm{E}+05$ \\
\hline 25 & $5.61 E+05$ & $3.46 \mathrm{E}+05$ & $3.36 \mathrm{E}+05$ & $1.64 \mathrm{E}+05$ & $1.73 E+06$ & $7.37 \mathrm{E}+03$ & $1.70 \mathrm{E}+06$ & $7.98 \mathrm{E}+05$ \\
\hline 26 & $5.53 \mathrm{E}+05$ & $3.47 \mathrm{E}+05$ & $3.07 \mathrm{E}+05$ & $1.64 \mathrm{E}+05$ & $1.72 \mathrm{E}+06$ & $7.77 \mathrm{E}+03$ & $1.70 \mathrm{E}+06$ & $7.97 \mathrm{E}+05$ \\
\hline 27 & $5.45 \mathrm{E}+05$ & $3.47 \mathrm{E}+05$ & $2.85 \mathrm{E}+05$ & $1.64 \mathrm{E}+05$ & $1.71 \mathrm{E}+06$ & $8.17 \widetilde{\mathrm{E}+03}$ & $1.69 \mathrm{E}+06$ & $7.96 \bar{E}+05$ \\
\hline 28 & $5.37 \mathrm{E}+05$ & $3.48 \mathrm{E}+05$ & $2.68 \mathrm{E}+05$ & $1.65 \mathrm{E}+05$ & $1.71 E+06$ & $8.57 \mathrm{E}+03$ & $1.69 \mathrm{E}+06$ & $7.95 \overline{\mathrm{E}+05}$ \\
\hline 29 & $5.30 \mathrm{E}+05$ & $3.48 \mathrm{E}+05$ & $2.54 \mathrm{E}+05$ & $1.65 \mathrm{E}+05$ & $1.70 \mathrm{E}+06$ & $8.97 \widehat{E}+03$ & $1.69 \mathrm{E}+06$ & $7.94 \mathrm{E}+05$ \\
\hline 30 & $5.23 \mathrm{E}+05$ & $3.49 \mathrm{E}+05$ & $2.43 \mathrm{E}+05$ & $1.65 \mathrm{E}+05$ & $1.68 \mathrm{E}+06$ & $9.15 \widehat{\mathrm{E}}+03$ & $1.63 \mathrm{E}+06$ & $5.1 \overline{0 \mathrm{E}+05}$ \\
\hline 31 & $5.16 \mathrm{E}+05$ & $3.49 \mathrm{E}+05$ & $2.35 \mathrm{E}+05$ & $1.65 \mathrm{E}+05$ & $1.67 \mathrm{E}+06$ & $9.32 \mathrm{E}+03$ & $1.63 \mathrm{E}+06$ & $5.08 \mathrm{E}+05$ \\
\hline 32 & $5.10 \mathrm{E}+05$ & $3.50 \mathrm{E}+05$ & $2.27 \bar{E}+05$ & $1.65 \mathrm{E}+05$ & $1.66 \mathrm{E}+06$ & $9.49 \mathrm{E}+03$ & $1.62 \overline{E+06}$ & $5.06 \mathrm{E}+05$ \\
\hline 33 & $5.03 \mathrm{E}+05$ & $3.50 \mathrm{E}+05$ & $2.21 \mathrm{E}+05$ & $1.66 \mathrm{E}+05$ & $1.64 \mathrm{E}+06$ & $9.66 \mathrm{E}+03$ & $1.61 \mathrm{E}+06$ & $5.04 \overline{\mathrm{E}+05}$ \\
\hline 34 & $4.97 \mathrm{E}+05$ & $3.51 \mathrm{E}+05$ & $2.16 \mathrm{E}+05$ & $1.66 \mathrm{E}+05$ & $1.63 \mathrm{E}+06$ & $9.83 \mathrm{E}+03$ & $1.61 \mathrm{E}+06$ & $5.02 \mathrm{E}+05$ \\
\hline 35 & $4.92 \mathrm{E}+05$ & $3.51 \mathrm{E}+05$ & $2.12 \mathrm{E}+05$ & $1.66 \mathrm{E}+05$ & $1.61 \mathrm{E}+06$ & $9.99 \mathrm{E}+03$ & $1.60 \mathrm{E}+06$ & $4.99 \mathrm{E}+05$ \\
\hline 36 & $4.86 \mathrm{E}+05$ & $3.51 \mathrm{E}+05$ & $2.08 \mathrm{E}+05$ & $1.66 \mathrm{E}+05$ & $1.60 \mathrm{E}+06$ & $1.02 \mathrm{E}+04$ & $1.59 \mathrm{E}+06$ & $4.97 \overline{\mathrm{E}+05}$ \\
\hline 37 & $4.81 \mathrm{E}+05$ & $3.52 \mathrm{E}+05$ & $2.05 \mathrm{E}+05$ & $1.66 \mathrm{E}+05$ & $1.59 \mathrm{E}+06$ & $1.03 \mathrm{E}+04$ & $1.59 \overline{\mathrm{E}+06}$ & $4.95 \mathrm{E}+05$ \\
\hline$\overline{38}$ & $4 . \overline{75 E}+05$ & $3.52 \mathrm{E}+05$ & $2.02 \mathrm{E}+05$ & $1.67 \mathrm{E}+05$ & $1.57 \mathrm{E}+06$ & $1.05 \mathrm{E}+04$ & $1.58 \mathrm{E}+06$ & $4.93 \mathrm{E}+05$ \\
\hline
\end{tabular}




\begin{tabular}{|c|c|c|c|c|c|c|c|c|}
\hline 39 & $4.71 \mathrm{E}+05$ & $3.52 \mathrm{E}+05$ & $1.99 \mathrm{E}+05$ & $1.67 \mathrm{E}+05$ & $1.56 \mathrm{E}+06$ & $1.07 \mathrm{E}+04$ & $1.57 \mathrm{E}+06$ & $4 . \overline{91 \mathrm{E}+0}$ \\
\hline 40 & $4.66 \mathrm{E}+05$ & $3.53 \mathrm{E}+05$ & $1.96 \mathrm{E}+05$ & $1.67 \bar{E}+05$ & $1.55 \mathrm{E}+06$ & $1.08 \mathrm{E}+04$ & $1.57 \bar{E}+06$ & $4 . \overline{89 \mathrm{E}+0}$ \\
\hline 41 & $4.61 \mathrm{E}+05$ & $3.53 \mathrm{E}+05$ & $1.94 \mathrm{E}+05$ & $1.67 \mathrm{E}+05$ & $1.54 \mathrm{E}+06$ & $1.10 \mathrm{E}+04$ & $1.56 \mathrm{E}+06$ & $4.87 \mathrm{E}+$ \\
\hline 42 & $4.57 \mathrm{E}+05$ & $3 . \overline{54 \mathrm{E}+05}$ & $1.91 \mathrm{E}+05$ & $1.67 \mathrm{E}+05$ & $1.53 \mathrm{E}+06$ & $1.13 \mathrm{E}+04$ & $1.48 \mathrm{E}+06$ & $7.36 \mathrm{E}+\mathrm{c}$ \\
\hline 43 & $4.53 \mathrm{E}+05$ & $3.54 \mathrm{E}+05$ & $1.89 \mathrm{E}+05$ & $1.68 \mathrm{E}+05$ & $1.52 \mathrm{E}+06$ & $1.17 \mathrm{E}+04$ & $1.48 \mathrm{E}+06$ & $7.35 \mathrm{E}+0$ \\
\hline$\overline{44}$ & $4.4 \overline{9 E+05}$ & $3.54 \mathrm{E}+05$ & $1.87 \mathrm{E}+05$ & $1.68 \mathrm{E}+05$ & $1.51 \mathrm{E}+06$ & $1.21 \mathrm{E}+04$ & $1.47 \mathrm{E}+06$ & $7.34 \mathrm{E}+$ \\
\hline 45 & $4.45 \mathrm{E}+05$ & $3.54 \mathrm{E}+05$ & $1.85 \mathrm{E}+05$ & $1.68 \mathrm{E}+05$ & $1.51 \mathrm{E}+06$ & $1.25 \mathrm{E}+04$ & $1.47 \mathrm{E}+06$ & $7 . \overline{33 \mathrm{E}+\mathrm{c}}$ \\
\hline 46 & $4.41 \mathrm{E}+05$ & $3.55 \mathrm{E}+05$ & $1.83 \mathrm{E}+05$ & $1.68 \mathrm{E}+05$ & $1.50 \mathrm{E}+06$ & $1.29 \mathrm{E}+04$ & $1.47 \mathrm{E}+06$ & $\overline{7.32 \mathrm{E}+0}$ \\
\hline 47 & $4.38 \mathrm{E}+05$ & $3.55 \mathrm{E}+05$ & $1.81 \mathrm{E}+05$ & $1.68 \mathrm{E}+05$ & $1.49 \mathrm{E}+06$ & $1.32 \mathrm{E}+04$ & $1.46 \mathrm{E}+06$ & $7.31 \mathrm{E}+$ \\
\hline 48 & $4.34 \mathrm{E}+05$ & $3.55 \mathrm{E}+05$ & $1.80 \mathrm{E}+05$ & $1.68 \mathrm{E}+05$ & $1.49 \mathrm{E}+06$ & $1.36 \mathrm{E}+04$ & $1.46 \mathrm{E}+06$ & $7.29 \mathrm{E}+\mathrm{c}$ \\
\hline 49 & $4.31 \mathrm{E}+05$ & $3.56 \mathrm{E}+05$ & $1.78 \mathrm{E}+05$ & $1.69 \mathrm{E}+05$ & $1.48 \mathrm{E}+06$ & $1.40 \mathrm{E}+04$ & $1.46 \mathrm{E}+06$ & $7.28 \overline{\mathrm{E}+0}$ \\
\hline 50 & $4.28 \mathrm{E}+05$ & $3.56 \mathrm{E}+05$ & $1.76 \mathrm{E}+05$ & $1.69 \mathrm{E}+05$ & $1.47 \mathrm{E}+06$ & $1.44 \mathrm{E}+04$ & $1.45 \mathrm{E}+06$ & $7.27 \overline{\mathrm{E}+0}$ \\
\hline 51 & $4.25 \mathrm{E}+05$ & $3.56 \mathrm{E}+05$ & $1.75 \mathrm{E}+05$ & $1.69 \mathrm{E}+05$ & $1.47 \mathrm{E}+06$ & $1.47 \mathrm{E}+04$ & $1.45 E+06$ & $7.26 \mathrm{E}+0$ \\
\hline 52 & $4.22 \mathrm{E}+05$ & $3.56 \mathrm{E}+05$ & $1.73 E+05$ & $1.69 \mathrm{E}+05$ & $1 . \overline{46 \mathrm{E}+06}$ & $1.51 \mathrm{E}+04$ & $1.45 \mathrm{E}+06$ & $7.2 \overline{5 \mathrm{E}+0}$ \\
\hline 53 & $4.19 \mathrm{E}+05$ & $3.57 \mathrm{E}+05$ & $1.72 \mathrm{E}+05$ & $1.69 \mathrm{E}+05$ & $1.45 \mathrm{E}+06$ & $1.55 \mathrm{E}+04$ & $1.44 \mathrm{E}+06$ & $7.24 \mathrm{E}+0$ \\
\hline 54 & $4.16 \mathrm{E}+05$ & $3.57 \mathrm{E}+05$ & $1.70 \mathrm{E}+05$ & $1.69 \mathrm{E}+05$ & $1.44 \mathrm{E}+06$ & $1.56 \mathrm{E}+04$ & $1.39 \mathrm{E}+06$ & $4.37 \mathrm{E}+0$ \\
\hline 55 & $4.14 \mathrm{E}+05$ & $3.57 \mathrm{E}+05$ & $1.69 \overline{\mathrm{E}+05}$ & $1 . \overline{70 \mathrm{E}+05}$ & $1.43 \mathrm{E}+06$ & $1.58 \mathrm{E}+04$ & $1.39 \mathrm{E}+06$ & $4.35 \mathrm{E}+0$ \\
\hline 56 & $4.11 \mathrm{E}+05$ & $3.58 \mathrm{E}+05$ & $1.68 \mathrm{E}+05$ & $1.70 \mathrm{E}+05$ & $1.42 \mathrm{E}+06$ & $1.59 \mathrm{E}+04$ & $1.38 \mathrm{E}+06$ & $4 . \overline{34 \mathrm{E}+0}$ \\
\hline 57 & $4.09 \mathrm{E}+05$ & $3.58 \mathrm{E}+05$ & $1.67 \mathrm{E}+05$ & $1.70 \mathrm{E}+05$ & $1.40 \mathrm{E}+06$ & $1.60 \mathrm{E}+04$ & $1.38 \mathrm{E}+06$ & $4.32 \mathrm{E}+0$ \\
\hline 58 & $4.06 \mathrm{E}+05$ & $3.58 \mathrm{E}+05$ & $1.65 \mathrm{E}+05$ & $1.70 \mathrm{E}+05$ & $1.39 \mathrm{E}+06$ & $1.62 \mathrm{E}+04$ & $1.37 \mathrm{E}+06$ & $4.30 \mathrm{E}+0$ \\
\hline 59 & $4.04 \mathrm{E}+05$ & $3.58 \mathrm{E}+05$ & $1.64 \mathrm{E}+05$ & $1.70 \mathrm{E}+05$ & $1.38 \mathrm{E}+06$ & $1.63 \mathrm{E}+04$ & $1.37 \mathrm{E}+06$ & $4.28 \mathrm{E}+0$ \\
\hline 60 & $4.02 \mathrm{E}+05$ & $3.58 \mathrm{E}+05$ & $1.63 \mathrm{E}+05$ & $1.70 \mathrm{E}+05$ & $1.37 \mathrm{E}+06$ & $1.64 \mathrm{E}+04$ & $1.36 \mathrm{E}+06$ & $4 . \overline{27 \mathrm{E}+0}$ \\
\hline 61 & $4.00 \mathrm{E}+05$ & $3.59 \mathrm{E}+05$ & $1.62 \mathrm{E}+05$ & $1.71 E+05$ & $1.36 \mathrm{E}+06$ & $1.66 \mathrm{E}+04$ & $1.35 \mathrm{E}+06$ & $4 . \overline{25 \mathrm{E}+0}$ \\
\hline 62 & $3.98 \mathrm{E}+05$ & $3.59 \mathrm{E}+05$ & $1.61 \mathrm{E}+05$ & $1.71 \mathrm{E}+05$ & $1.35 \mathrm{E}+06$ & $1.67 \mathrm{E}+04$ & $1.35 \mathrm{E}+06$ & $4 . \overline{23 \mathrm{E}+0}$ \\
\hline 63 & $3.96 \mathrm{E}+05$ & $3.59 \mathrm{E}+05$ & $1.60 \mathrm{E}+05$ & $1.71 \mathrm{E}+05$ & $1.33 \mathrm{E}+06$ & $1.68 \mathrm{E}+04$ & $1.34 \mathrm{E}+06$ & $4.21 \mathrm{E}+0$ \\
\hline 64 & $3.94 \mathrm{E}+05$ & $3.59 \mathrm{E} \div 05$ & $1.59 \mathrm{E}+05$ & $1.71 \mathrm{E}+05$ & $1.32 \mathrm{E}+06$ & $1.70 \mathrm{E}+04$ & $1.34 \mathrm{E}+06$ & $4.20 \mathrm{E}+0$ \\
\hline 65 & $3.92 \mathrm{E}+05$ & $3.60 \mathrm{E}+05$ & $1.58 \mathrm{E}+05$ & $1.71 \mathrm{E}+05$ & $1.31 \mathrm{E}+06$ & $1.71 E+04$ & $1.33 \mathrm{E}+06$ & $4.18 \mathrm{E}+0$ \\
\hline 66 & $3.90 \mathrm{E}+05$ & $3.60 \mathrm{E}+05$ & $1.57 \mathrm{E}+05$ & $1.71 E+05$ & $1.31 \mathrm{E}+06$ & $1.75 \mathrm{E}+04$ & $1.27 \mathrm{E}+06$ & $6.71 \mathrm{E}+0$ \\
\hline 67 & $3.89 \mathrm{E}+05$ & $3.60 \mathrm{E}+05$ & $1.56 \mathrm{E}+05$ & $1.72 \mathrm{E}+05$ & $1.30 \overline{\mathrm{E}}+06$ & $1.78 \mathrm{E}+04$ & $1.26 \mathrm{E}+06$ & $6.70 \mathrm{E}+0$ \\
\hline 68 & $3.87 E+05$ & $3.60 \mathrm{E}+05$ & $1.55 \mathrm{E}+05$ & $1.72 \mathrm{E}+05$ & $1.29 \mathrm{E}+06$ & $1.82 \mathrm{E}+04$ & $1.26 \mathrm{E}+06$ & $6.69 \mathrm{E}+0$ \\
\hline 69 & $3.85 \mathrm{E}+05$ & $3.60 \mathrm{E}+05$ & $1.54 \mathrm{E}+05$ & $1.72 \mathrm{E}+05$ & $1.2 \overline{\mathrm{E}}+06$ & $1.85 \mathrm{E}+04$ & $1.26 \mathrm{E}+06$ & $6.68 \mathrm{E}+0$ \\
\hline 168 & $1.79 \mathrm{E}+05$ & $3.55 \mathrm{E}+05$ & $1.68 \mathrm{E}+05$ & $1.86 \mathrm{E}+05$ & $6.63 \mathrm{E}+05$ & $4.46 \mathrm{E}+04$ & $7.34 \mathrm{E}+05$ & $4.95 \bar{E}+0$. \\
\hline 336 & $9.55 \mathrm{E}+04$ & $3.01 \overline{\mathrm{E}+05}$ & $9.00 \mathrm{E}+04$ & $1.99 \mathrm{E}+05$ & $2.75 \mathrm{E}+05$ & $7.61 \mathrm{E}+04$ & $4.25 \mathrm{E}+05$ & $3.69 \mathrm{E}+0$ \\
\hline 504 & $7.10 \mathrm{E}+04$ & $2.51 \mathrm{E}+05$ & $6.24 \mathrm{E}+04$ & $2.07 \mathrm{E}+05$ & $1.35 \mathrm{E}+05$ & $9.31 \mathrm{E}+04$ & $1.89 \mathrm{E}+05$ & $2 . \overline{48} \mathrm{E}+0$ \\
\hline 720 & $5.20 \mathrm{E}+04$ & $1.99 \mathrm{E}+05$ & $4.21 \mathrm{E}+04$ & $2.14 \mathrm{E}+05$ & $6.85 \mathrm{E}+04$ & $1.02 \mathrm{E}+05$ & $9.39 \mathrm{E}+04$ & $1.73 \mathrm{E}+0$ \\
\hline 2160 & $9.81 \mathrm{E}+03$ & $4.00 \mathrm{E}+04$ & $4.76 \mathrm{E}+03$ & $2.28 \mathrm{E}+05$ & $6.19 \mathrm{E}+03$ & $5.96 \mathrm{E}+04$ & $2.15 \mathrm{E}+04$ & $6.01 \overline{\mathrm{E}+04}$ \\
\hline 4320 & $9.4 \bar{E}+02$ & $3.66 \mathrm{E}+03$ & $2.39 \mathrm{E}+04$ & $2.16 \mathrm{E}+05$ & $1.06 \mathrm{E}+03$ & $1.43 \mathrm{E}+04$ & $2.67 \mathrm{E}+03$ & $9.57 \overline{\mathrm{E}+0}$ \\
\hline 4800 & $5.61 \mathrm{E}+02$ & $2.16 \mathrm{E}+03$ & $2.38 \mathrm{E}+04$ & $2.15 \mathrm{E}+05$ & $7.75 \mathrm{E}+02$ & $1.03 \mathrm{E}+04$ & $9.10 \mathrm{E}+02$ & $3.17 \bar{E}+03$ \\
\hline 8760 & $7.71 \mathrm{E}+00$ & $2.84 \mathrm{E}+01$ & $2.32 \overline{E+04}$ & $2.10 \mathrm{E}+05$ & $6.47 \mathrm{E}+01$ & $6.95 \mathrm{E}+02$ & $2.86 \mathrm{E}+02$ & $9.38 \mathrm{E}+02$ \\
\hline DIL & $\begin{array}{l}2.4 \mathrm{E} 6 \\
\mathrm{pCi} / \mathrm{L}\end{array}$ & $\begin{array}{l}8 \mathrm{E}+4 \\
\mathrm{~kg}\end{array}$ & $8 \mathrm{~Bq} / \mathrm{g}$ & & & & & \\
\hline 5 & $\begin{array}{l}5.9 \mathrm{EE} 6 \\
\mathrm{pCi} / \mathrm{L}\end{array}$ & $\begin{array}{l}2.18 \mathrm{E}+5 \\
\mathrm{~Bq} / \mathrm{kg}\end{array}$ & $218.3 \mathrm{~Bq} / \mathrm{g}$ & & & & & \\
\hline & \multicolumn{2}{|c|}{ VEGETABLES } & \multicolumn{2}{|c|}{ WHEAT SEED } & \multicolumn{2}{|l|}{ MEAT } & \multicolumn{2}{|l|}{ MILK } \\
\hline \multirow[t]{2}{*}{ HOUR } & $\mathrm{O}$ & $\overline{\mathrm{OBT}}$ & ro & OBT & HTO & OBT & HTO & DBT \\
\hline & $(\mathrm{Bq} / \mathrm{g})$ & $(\mathrm{Bq} / \mathrm{g})$ & $(\mathrm{Bq} / \mathrm{g})$ & $(\mathrm{Bq} / \mathrm{g})$ & $(\mathrm{Bq} / \mathrm{g})$ & $(\mathrm{Bq} / \mathrm{g})$ & $(\mathrm{Bq} / \mathrm{g})$ & $(\mathrm{Bq} / \mathrm{g})$ \\
\hline $\mathrm{k} \mathrm{HTO}$ & & & & & & & & \\
\hline
\end{tabular}


Foodstuff Concentrations and Relocation Considerations Following

WSRC-TR-99-00118

a Tritium Oxide Release from SRS Tritium Facilities (U)

\begin{tabular}{|c|c|c|c|c|c|c|c|c|}
\hline $1 / 3 / 5 / 6$ & $4.26 \mathrm{E}+07$ & $2.16 \mathrm{E}+05$ & $2.03 \mathrm{E}+07$ & $9.99 \mathrm{E}+04$ & $1.97 \mathrm{E}+06$ & $1.74 \mathrm{E}+03$ & $1.90 \mathrm{E}+06$ & $5.91 \mathrm{E}+05$ \\
\hline SUM & $4.28 \mathrm{E}+07$ & & $2.04 \mathrm{E}+07$ & & $1.98 \mathrm{E}+06$ & & $2.49 \mathrm{E}+06$ & \\
\hline $\mathrm{Ci} / \mathrm{g}$ & $1.16 \mathrm{E}-03$ & & $5.50 \mathrm{E}-04$ & & $5.34 \mathrm{E}-05$ & & $6.72 \mathrm{E}-05$ & \\
\hline & & & & & & & & \\
\hline DIL HTO & $5.9 \mathrm{E}-09$ & & & & & & & \\
\hline \multicolumn{9}{|l|}{$(\mathrm{Ci} / \mathrm{g})$} \\
\hline & & & & & & & & \\
\hline 168 & $1.79 \mathrm{E}+05$ & $3.55 \mathrm{E}+05$ & $1.68 \mathrm{E}+05$ & $1.86 \mathrm{E}+05$ & $6.63 \mathrm{E}+05$ & $4.46 \mathrm{E}+04$ & $7.34 \mathrm{E}+05$ & $4.95 \overline{\mathrm{E}+05}$ \\
\hline SUM & $5.34 \mathrm{E}+05$ & & $3.54 \mathrm{E}+05$ & & $7.07 \mathrm{E}+05$ & & $1.23 \mathrm{E}+06$ & \\
\hline $\mathrm{Ci} / \mathrm{g}$ & $1.44 \mathrm{E}-05$ & & 9.57E-06 & & $1.91 \mathrm{E}-05$ & & $3.32 \mathrm{E}-05$ & \\
\hline & & & & & & & & \\
\hline$\overline{\mathrm{DIL}} \overline{\mathrm{OBT}}$ & $2.4 \mathrm{E}-09$ & & & & & & & \\
\hline \multicolumn{9}{|l|}{$\mathrm{Ci} / \mathrm{g}$} \\
\hline & & & & & & & & \\
\hline 8760 & $7.71 \mathrm{E}+00$ & $2.84 \mathrm{E}+01$ & $2.32 \mathrm{E}+04$ & $2.10 \mathrm{E}+05$ & $6.47 \mathrm{E}+01$ & $6.95 \mathrm{E}+02$ & $2.86 \mathrm{E}+02$ & $9.38 \mathrm{E}+02$ \\
\hline SUM & $3.61 \mathrm{E}+01$ & & $2.33 \mathrm{E}+05$ & & $7.60 \mathrm{E}+02$ & & $1.22 \mathrm{E}+03$ & \\
\hline $\mathrm{Ci} / \mathrm{g}$ & $9.76 \mathrm{E}-10$ & & $6.30 \mathrm{E}-06$ & & $2.05 \mathrm{E}-08$ & & $3.31 \mathrm{E}-08$ & \\
\hline DIL OBT & $25^{2} 0$ & & & & & & & \\
\hline $\begin{array}{l}\mathrm{Ci} / \mathrm{g} \\
\end{array}$ & $2.4 \mathrm{E}-09$ & & & & & & & \\
\hline & & & & & & & & \\
\hline
\end{tabular}




\begin{tabular}{|c|c|c|c|c|c|c|c|c|}
\hline & \multicolumn{6}{|c|}{ Tritium Facilities, Design Basis Fire, Average Met Conditions } & & \\
\hline & \multicolumn{4}{|c|}{ Concentration Values for $1 \mathrm{~km}$ from release } & & & & \\
\hline \multicolumn{9}{|c|}{ Only Wheat Seed is examined since this is the edible portion of the Wheat. The "WHEAT" category is } \\
\hline \multicolumn{9}{|c|}{ actually the unedible plant body and is not considered in the dose estimate. } \\
\hline & & & & & & & & \\
\hline & \multicolumn{2}{|c|}{ VEGETABLES } & \multicolumn{2}{|c|}{ WHEAT SEED } & \multicolumn{2}{|l|}{ MEAT } & \multicolumn{2}{|l|}{ MILK } \\
\hline \multirow[t]{2}{*}{ HOUR } & HTO & OBT & HTO & OBT & HTO & OBT & HTO & $\mathrm{OB}$ \\
\hline & $(\mathrm{Bq} / \mathrm{g})$ & $(\mathrm{Bq} / \mathrm{g})$ & $(\mathrm{Bq} / \mathrm{g})$ & $(\mathrm{Bq} / \mathrm{g})$ & $(\mathrm{Bq} / \mathrm{g})$ & $(\mathrm{Bq} / \mathrm{g})$ & $(\mathrm{Bq} / \mathrm{g})$ & $\mathrm{Bg} / \mathrm{g})$ \\
\hline & & & & & & & & \\
\hline 1 & $9.48 \mathrm{E}+06$ & $4.33 \mathrm{E}+04$ & $2.66 \mathrm{E}+06$ & $8.96 \mathrm{E}+03$ & $2.13 \mathrm{E}+05$ & $4.37 \mathrm{E}+01$ & $2.58 \mathrm{E}+04$ & $1.12 \mathrm{E}+\mathrm{C}$ \\
\hline 2 & $3.90 \mathrm{E}+06$ & $5.99 \mathrm{E}+04$ & $3.89 \mathrm{E}+06$ & $1.57 \mathrm{E}+04$ & $3.18 \mathrm{E}+05$ & $1.10 \mathrm{E}+02$ & $5.72 \mathrm{E}+04$ & $2.50 \mathrm{E}+04$ \\
\hline 3 & $1.78 \mathrm{E}+06$ & $6.66 \mathrm{E}+04$ & $4.26 \mathrm{E}+06$ & $2.08 \mathrm{E}+04$ & $3.76 \mathrm{E}+05$ & $1.89 \mathrm{E}+02$ & $8.79 \mathrm{E}+04$ & $3.85 \mathrm{E}+04$ \\
\hline 4 & $9.51 \mathrm{E}+05$ & $6.97 \mathrm{E}+04$ & $4.13 \mathrm{E}+06$ & $2.45 \mathrm{E}+04$ & $4.07 \mathrm{E}+05$ & $2.75 \mathrm{E}+02$ & $1.16 \mathrm{E}+05$ & $5.08 \mathrm{E}+04$ \\
\hline 5 & $6.11 \mathrm{E}+05$ & $7.12 \mathrm{E}+04$ & $3.75 \mathrm{E}+06$ & $2.73 \mathrm{E}+04$ & $4.24 \mathrm{E}+05$ & $3.64 \mathrm{E}+02$ & $1.40 \mathrm{E}+05$ & $6.17 \mathrm{E}+04$ \\
\hline 6 & $4.62 \mathrm{E}+05$ & $7.22 \mathrm{E}+04$ & $3.27 \mathrm{E}+06$ & $2.94 \mathrm{E}+04$ & $4.21 \mathrm{E}+05$ & $4.09 \mathrm{E}+02$ & $4.08 \mathrm{E}+05$ & $1.27 \mathrm{E}+05$ \\
\hline 7 & $3.91 \mathrm{E}+05$ & $7.29 \mathrm{E}+04$ & $2.78 \mathrm{E}+06$ & $3.10 \mathrm{E}+04$ & $4.18 \mathrm{E}+05$ & $4.54 \mathrm{E}+02$ & $4.06 \mathrm{E}+05$ & $1.27 \mathrm{E}+05$ \\
\hline 8 & $3.51 \mathrm{E}+05$ & $7.35 \mathrm{E}+04$ & $2.32 \mathrm{E}+06$ & $3.21 \mathrm{E}+04$ & $4.14 \mathrm{E}+05$ & $4.98 \mathrm{E}+02$ & $4.05 \mathrm{E}+05$ & $1.26 \mathrm{E}+05$ \\
\hline 9 & $3.27 \mathrm{E}+05$ & $7.40 \mathrm{E}+04$ & $1.91 \mathrm{E}+06$ & $3.30 \mathrm{E}+04$ & $4.11 \mathrm{E}+05$ & $5.42 \mathrm{E}+02$ & $4.03 \mathrm{E}+05$ & $1.26 \mathrm{E}+05$ \\
\hline 10 & $3.09 \mathrm{E}+05$ & $7.45 \mathrm{E}+04$ & $1.56 \mathrm{E}+06$ & $3.37 \mathrm{E}+04$ & $4.07 \mathrm{E}+05$ & $5.85 \mathrm{E}+02$ & $E+05$ & $1.25 \mathrm{E}+05$ \\
\hline 11 & $2.95 \mathrm{E}+05$ & $7.49 \mathrm{E}+04$ & $1.26 \mathrm{E}+06$ & $3.43 \mathrm{E}+04$ & $4.04 \mathrm{E}+05$ & $6.28 \mathrm{E}+02$ & $4.00 \mathrm{E}+05$ & $1.25 \mathrm{E}+05$ \\
\hline 12 & $2.83 \mathrm{E}+05$ & $7.54 \mathrm{E}+04$ & $1.03 \mathrm{E}+06$ & $3.47 \mathrm{E}+04$ & $4.01 \mathrm{E}+05$ & $6.71 \mathrm{E}+02$ & $3.98 \mathrm{E}+05$ & $1.24 \mathrm{E}+05$ \\
\hline 13 & $2.73 \mathrm{E}+05$ & $7.57 \mathrm{E}+04$ & $8.33 \mathrm{E}+05$ & $3.51 \mathrm{E}+04$ & $3.97 \mathrm{E}+05$ & $7.13 \mathrm{E}+02$ & $3.97 \mathrm{E}+05$ & $1.24 \mathrm{E}+05$ \\
\hline 14 & $2.64 \mathrm{E}+05$ & $7.61 \mathrm{E}+04$ & $6.79 \mathrm{E}+05$ & $3.54 \mathrm{E}+04$ & $3.94 \mathrm{E}+05$ & $7.55 \mathrm{E}+02$ & $3.95 \mathrm{E}+05$ & $1.23 \mathrm{E}+05$ \\
\hline 15 & $2.55 \mathrm{E}+05$ & $7.65 \mathrm{E}+04$ & $5.57 \mathrm{E}+05$ & $3.57 \mathrm{E}+04$ & $3.91 \mathrm{E}+05$ & $7.97 \mathrm{E}+02$ & $3.93 \mathrm{E}+05$ & $1.23 \mathrm{E}+05$ \\
\hline 16 & $2.48 \mathrm{E}+05$ & $7.68 \mathrm{E}+04$ & $4.61 E+05$ & $3.59 \mathrm{E}+04$ & $3.88 \mathrm{E}+05$ & $8.38 \mathrm{E}+02$ & $3.92 \mathrm{E}+05$ & $1.22 \mathrm{E}+05$ \\
\hline 17 & $2.39 \mathrm{E}+05$ & $7.72 \mathrm{E}+04$ & $3.85 \mathrm{E}+05$ & $3.61 \mathrm{E}+04$ & $3.84 \mathrm{E}+05$ & $8.79 \mathrm{E}+02$ & $3.90 \mathrm{E}+05$ & $1.22 \mathrm{E}+05$ \\
\hline 18 & $2.31 \mathrm{E}+05$ & $7.75 \mathrm{E}+04$ & $3.25 \mathrm{E}+05$ & $3.63 \mathrm{E}+04$ & $3.84 \mathrm{E}+05$ & $9.69 \mathrm{E}+02$ & $3.72 \mathrm{E}+05$ & $1.77 \mathrm{E}+05$ \\
\hline 19 & $2.24 \mathrm{E}+05$ & $7.78 \mathrm{E}+04$ & $2.79 E+05$ & $3.64 \mathrm{E}+04$ & $3.84 \mathrm{E}+05$ & $1.06 \mathrm{E}+03$ & $3.72 \mathrm{E}+05$ & $1.77 \mathrm{E}+05$ \\
\hline 20 & $2.18 \mathrm{E}+05$ & $7.81 \mathrm{E}+04$ & $2.42 \mathrm{E}+05$ & $3.66 \mathrm{E}+04$ & $3.83 \mathrm{E}+05$ & $1.15 \mathrm{E}+03$ & $3.72 \mathrm{E}+05$ & $1.77 \mathrm{E}+05$ \\
\hline 21 & $2.13 \mathrm{E}+05$ & $7.83 \mathrm{E}+04$ & $2.13 \mathrm{E}+05$ & $3.67 \mathrm{E}+04$ & $3.83 \mathrm{E}+05$ & $1.24 \mathrm{E}+03$ & $3.72 \mathrm{E}+05$ & $1.77 \mathrm{E}+05$ \\
\hline 22 & $2.07 \mathrm{E}+05$ & $7.86 \mathrm{E}+04$ & $1.90 \mathrm{E}+05$ & $3.68 \mathrm{E}+04$ & $3.82 \mathrm{E}+05$ & $1.33 \mathrm{E}+03$ & $3.71 \mathrm{E}+05$ & $1.77 \mathrm{E}+05$ \\
\hline 23 & $2.02 E+05$ & $7.89 \mathrm{E}+04$ & $1.72 \mathrm{E}+05$ & $3.70 \mathrm{E}+04$ & $3.81 \mathrm{E}+05$ & $1.42 \mathrm{E}+03$ & $3.71 \mathrm{E}+05$ & $1.77 \mathrm{E}+05$ \\
\hline 24 & $1.97 \mathrm{E}+05$ & $7.91 \mathrm{E}+04$ & $1.57 \mathrm{E}+05$ & $3.71 \mathrm{E}+04$ & $3.81 \mathrm{E}+05$ & $1.51 \mathrm{E}+03$ & $3.71 \mathrm{E}+05$ & $1.77 \mathrm{E}+05$ \\
\hline 25 & $1.92 \mathrm{E}+05$ & $7.94 \mathrm{E}+04$ & $1.45 \mathrm{E}+05$ & $3.72 \mathrm{E}+04$ & $3.80 \mathrm{E}+05$ & $1.60 \mathrm{E}+03$ & $3.71 \mathrm{E}+05$ & $1.77 \mathrm{E}+05$ \\
\hline 26 & $1.87 \mathrm{E}+05$ & $7.96 \mathrm{E}+04$ & $1.35 \mathrm{E}+05$ & $3.73 E+04$ & $3.79 \mathrm{E}+05$ & $1.69 \mathrm{E}+03$ & $3.70 \mathrm{E}+05$ & $1.77 \mathrm{E}+05$ \\
\hline 27 & $1.83 \mathrm{E}+05$ & $7.98 \mathrm{E}+04$ & $1.27 \mathrm{E}+05$ & $3.74 \mathrm{E}+04$ & $3.78 \mathrm{E}+05$ & $1.79 \mathrm{E}+03$ & $3.70 \mathrm{E}+05$ & $1.77 \mathrm{E}+05$ \\
\hline 28 & $1.79 \mathrm{E}+05$ & $8.00 \mathrm{E}+04$ & $1.21 \mathrm{E}+05$ & $3.75 \mathrm{E}+04$ & $3.77 \mathrm{E}+05$ & $1.88 \mathrm{E}+03$ & $3.69 \mathrm{E}+05$ & $1.77 \mathrm{E}+05$ \\
\hline 29 & $1.75 \mathrm{E}+05$ & $8.02 E+04$ & $1.15 \mathrm{E}+05$ & $3.76 \mathrm{E}+04$ & $3.76 \mathrm{E}+05$ & $1.97 \mathrm{E}+03$ & $3.69 \mathrm{E}+05$ & $1.77 \mathrm{E}+05$ \\
\hline 30 & $1.71 \mathrm{E}+05$ & $8.04 \mathrm{E}+04$ & $1.10 \mathrm{E}+05$ & $3.77 \mathrm{E}+04$ & $3.73 \mathrm{E}+05$ & $2.00 \mathrm{E}+03$ & $3.62 \mathrm{E}+05$ & $1.13 \mathrm{E}+05$ \\
\hline 31 & $1.67 \mathrm{E}+05$ & $8.06 \mathrm{E}+04$ & $1.05 \mathrm{E}+05$ & $3.78 \mathrm{E}+04$ & $3.70 \mathrm{E}+05$ & $2.04 \mathrm{E}+03$ & $3.60 \mathrm{E}+05$ & $1.13 \mathrm{E}+05$ \\
\hline 32 & $1.64 E+05$ & $8.08 \mathrm{E}+04$ & $1.01 \mathrm{E}+05$ & $3.79 \mathrm{E}+04$ & $3.67 \mathrm{E}+05$ & $2.08 \mathrm{E}+03$ & $3.59 \mathrm{E}+05$ & $1.12 \bar{E}+05$ \\
\hline 33 & $1.60 \mathrm{E}+05$ & $8.10 \mathrm{E}+04$ & $9.77 \mathrm{E}+04$ & $3.80 \mathrm{E}+04$ & $3.64 \mathrm{E}+05$ & $2.12 \mathrm{E}+03$ & $3.57 \mathrm{E}+05$ & $1.12 \mathrm{E}+05$ \\
\hline 34 & $1.52 \mathrm{E}+05$ & $8.12 \mathrm{E}+04$ & $9.44 \mathrm{E}+04$ & $3.81 \mathrm{E}+04$ & $3.61 \mathrm{E}+05$ & $2.16 \mathrm{E}+03$ & $3.56 \mathrm{E}+05$ & $1.11 \mathrm{E}+05$ \\
\hline 35 & $1.48 \mathrm{E}+05$ & $8.13 \mathrm{E}+04$ & $9.09 \mathrm{E}+04$ & $3.81 \mathrm{E}+04$ & $3.58 \mathrm{E}+05$ & $2.19 \bar{E}+03$ & $3.54 \mathrm{E}+05$ & $1.11 \mathrm{E}+05$ \\
\hline 36 & $1.44 \mathrm{E}+05$ & $8.15 \mathrm{E}+04$ & $8.75 \mathrm{E}+04$ & $3.82 \mathrm{E}+04$ & $3.55 \mathrm{E}+05$ & $2.23 \mathrm{E}+03$ & $3.53 \mathrm{E}+05$ & $1.10 \mathrm{E}+05$ \\
\hline 37 & $1.41 \mathrm{E}+05$ & $8.16 \mathrm{E}+04$ & $8.42 \mathrm{E}+04$ & $3.83 E+04$ & $3.52 \mathrm{E}+05$ & $2.27 \mathrm{E}+03$ & $3.51 \mathrm{E}+05$ & $1.10 \mathrm{E}+05$ \\
\hline 38 & $1.39 \mathrm{E}+05$ & $8.18 E+04$ & $8.10 \bar{E}+04$ & $3.84 \mathrm{E}+04$ & $3.49 \mathrm{E}+05$ & $2.30 \mathrm{E}+03$ & $3.50 \mathrm{E}+05$ & $1.09 \mathrm{E}+05$ \\
\hline
\end{tabular}




\begin{tabular}{|c|c|c|c|c|c|c|c|c|}
\hline$\overline{39}$ & $1.36 \overline{\mathrm{E}}+05$ & $8.19 \mathrm{E}+04$ & $7.82 \mathrm{E}+04$ & $3.84 \mathrm{E}+04$ & $3.46 \mathrm{E}+05$ & $2.34 \mathrm{E}+03$ & $3.48 \bar{E}+05$ & $1.09 \mathrm{E}+$ \\
\hline 40 & $1.34 \mathrm{E}+05$ & $8.20 \mathrm{E}+04$ & $7.55 \mathrm{E}+04$ & $3.85 \mathrm{E}+04$ & $3.43 \mathrm{E}+05$ & $2.37 \mathrm{E}+03$ & $3.47 \mathrm{E}+05$ & $1.08 \mathrm{E}+\mathrm{C}$ \\
\hline 41 & $1.31 \mathrm{E}+05$ & $8.21 \mathrm{E}+04$ & $7.31 \mathrm{E}+04$ & $3.86 \mathrm{E}+04$ & $3.40 \mathrm{E}+05$ & $2.41 \mathrm{E}+03$ & $3.46 \mathrm{E}+05$ & $1.08 \mathrm{E}+0$ \\
\hline 42 & $1.29 \mathrm{E}+05$ & $8.23 \mathrm{E}+04$ & $7.08 \overline{\mathrm{E}+04}$ & $3.86 \mathrm{E}+04$ & $3.39 \mathrm{E}+05$ & $2.49 \mathrm{E}+03$ & $3.29 \mathrm{E}+05$ & $1.66 \mathrm{E}+$ \\
\hline $4 \overline{3}$ & $1.27 \mathrm{E}+05$ & $8.24 \mathrm{E}+04$ & $6.8 \overline{7} \bar{E}+04$ & $3.87 \overline{E+04}$ & $3.38 \mathrm{E}+05$ & $2.58 \mathrm{E}+03$ & $3.28 \mathrm{E}+05$ & $1.66 \mathrm{E}+0$ \\
\hline$\overline{44}$ & $1.25 \mathrm{E}+05$ & $8.25 \mathrm{E}+04$ & $6.68 \mathrm{E}+04$ & $3.88 \mathrm{E}+04$ & $3.37 \mathrm{E}+05$ & $2.67 \mathrm{E}+03$ & $3.27 \mathrm{E}+05$ & $1.66 \mathrm{E}+0$ \\
\hline$\overline{45}$ & $1.23 \mathrm{E}+05$ & $8.26 \mathrm{E}+04$ & $6.50 \mathrm{E}+04$ & $3.88 \mathrm{E}+04$ & $3.35 \mathrm{E}+05$ & $2.75 \mathrm{E}+03$ & $3.27 \mathrm{E}+05$ & $1.66 \mathrm{E}+\mathrm{c}$ \\
\hline 46 & $1.21 \mathrm{E}+05$ & $8.27 \mathrm{E}+04$ & $6.34 \mathrm{E}+04$ & $3.89 \mathrm{E}+04$ & $3.34 \mathrm{E}+05$ & $2.84 \mathrm{E}+03$ & $3.26 \mathrm{E}+05$ & $1.66 \mathrm{E}+0$ \\
\hline 47 & $1.20 \mathrm{E}+05$ & $8.28 \mathrm{E}+04$ & $6.18 \mathrm{E}+04$ & $3.89 \mathrm{E}+04$ & $3.33 \mathrm{E}+05$ & $2.93 \mathrm{E}+03$ & $3.26 \mathrm{E}+05$ & $1.65 \mathrm{E}+0$ \\
\hline 48 & $1.16 \mathrm{E}+05$ & $8.29 \mathrm{E}+04$ & $6.04 \mathrm{E}+04$ & $3.90 \mathrm{E}+04$ & $3.31 \mathrm{E}+05$ & $3.01 \mathrm{E}+03$ & $3.25 \mathrm{E}+05$ & $1.6 \overline{5 \mathrm{E}+0}$ \\
\hline 49 & $1.14 \mathrm{E}+05$ & $8.30 \mathrm{E}+04$ & $5.88 \mathrm{E}+04$ & $3 . \overline{91 E+04}$ & $3.30 \mathrm{E}+05$ & $3.10 \mathrm{E}+03$ & $3.24 \mathrm{E}+05$ & $1.65 \mathrm{E}+\mathrm{C}$ \\
\hline 50 & $1.12 \mathrm{E}+05$ & $8.31 \mathrm{E}+04$ & $5.72 \mathrm{E}+04$ & $3.91 \mathrm{E}+04$ & $3.29 \mathrm{E}+05$ & $3.18 \mathrm{E}+03$ & $3.24 \mathrm{E}+05$ & $1.65 \mathrm{E}+0$ \\
\hline 51 & $1.11 \mathrm{E}+05$ & $8.32 \mathrm{E}+04$ & $5.56 \mathrm{E}+04$ & $3.92 \mathrm{E}+04$ & $3.27 \mathrm{E}+05$ & $3.27 \mathrm{E}+03$ & $3.23 \mathrm{E}+05$ & $1.65 \mathrm{E}+\mathrm{C}$ \\
\hline 52 & $1.10 \mathrm{E}+05$ & $8.33 \mathrm{E}+04$ & $5.42 \mathrm{E}+04$ & $3.92 \mathrm{E}+04$ & $3.26 \mathrm{E}+05$ & $3.35 \mathrm{E}+03$ & $3.22 \mathrm{E}+05$ & $1.64 \mathrm{E}+0$ \\
\hline 53 & $1.08 \mathrm{E}+05$ & $8.33 \mathrm{E}+04$ & $5.29 \mathrm{E}+04$ & $3.93 \mathrm{E}+04$ & $3.25 \mathrm{E}+05$ & $3 . \overline{44 \mathrm{E}+03}$ & $3.22 \mathrm{E}+05$ & $1.64 \overline{\mathrm{E}+0}$ \\
\hline 54 & $1.07 \mathrm{E}+05$ & $8.34 \mathrm{E}+04$ & $5.17 \mathrm{E}+04$ & $3.93 \mathrm{E}+04$ & $3.22 \mathrm{E}+05$ & $3 . \overline{47 \mathrm{E}+03}$ & $3.12 \mathrm{E}+05$ & $9.78 \bar{E}+c$ \\
\hline 55 & $1.06 \mathrm{E}+05$ & $8.35 \mathrm{E}+04$ & $5.06 \mathrm{E}+04$ & $3.93 \mathrm{E}+04$ & $3.19 \mathrm{E}+05$ & $3.50 \mathrm{E}+03$ & $3.11 \mathrm{E}+05$ & $9.74 \mathrm{E}+0$ \\
\hline 56 & $1.05 \mathrm{E}+05$ & $8.36 \mathrm{E}+04$ & $4.96 \mathrm{E}+04$ & $3.94 \mathrm{E}+04$ & $3.17 \mathrm{E}+05$ & $3.53 \mathrm{E}+03$ & $3.09 \mathrm{E}+05$ & $9.70 \mathrm{E}+0$ \\
\hline 57 & $1.05 \mathrm{E}+05$ & $8.36 \mathrm{E}+04$ & $4.87 \mathrm{E}+04$ & $3.94 \mathrm{E}+04$ & $3.14 \mathrm{E}+05$ & $3.57 \mathrm{E}+03$ & $3.08 \mathrm{E}+05$ & $9.66 \mathrm{E}+0$ \\
\hline 58 & $1.04 \mathrm{E}+05$ & $8.37 \mathrm{E}+04$ & $4.79 \mathrm{E}+04$ & $3.95 \mathrm{E}+04$ & $3.11 \mathrm{E}+05$ & $3.60 \mathrm{E}+03$ & $3.07 \mathrm{E}+05$ & $9.62 \mathrm{E}+0$ \\
\hline 59 & $9.81 \mathrm{E}+04$ & $8.38 \mathrm{E}+04$ & $4.71 \mathrm{E}+04$ & $3.95 \mathrm{E}+04$ & $3.09 \mathrm{E}+05$ & $3.63 \mathrm{E}+03$ & $3.06 \mathrm{E}+05$ & $9.58 \mathrm{E}+\mathrm{c}$ \\
\hline 60 & $9.59 \mathrm{E}+04$ & $8.38 \mathrm{E}+04$ & $4.60 \mathrm{E}+04$ & $3.96 \mathrm{E}+04$ & $3.06 \mathrm{E}+05$ & $3.66 \mathrm{E}+03$ & $3.04 \mathrm{E}+05$ & $9.54 \mathrm{E}+0$ \\
\hline 61 & $9.48 \mathrm{E}+04$ & $8.39 \mathrm{E}+04$ & $4.46 \mathrm{E}+04$ & $3.96 \mathrm{E}+04$ & $3.04 \mathrm{E}+05$ & $3.69 \mathrm{E}+03$ & $3.03 \mathrm{E}+05$ & $9.50 \mathrm{E}+0$ \\
\hline 62 & $9.41 \mathrm{E}+04$ & $8.40 \mathrm{E}+04$ & $4.33 \mathrm{E}+04$ & $3.97 \mathrm{E}+04$ & $3.01 \mathrm{E}+05$ & $3.72 \mathrm{E}+03$ & $3.02 \mathrm{E}+05$ & $9.46 \mathrm{E}+($ \\
\hline 63 & $9.36 \mathrm{E}+04$ & $8.40 \mathrm{E}+04$ & $4.21 \mathrm{E}+04$ & $3.97 \mathrm{E}+04$ & $2.98 \mathrm{E}+05$ & $3.75 E+03$ & $3.00 \mathrm{E}+05$ & $9.43 \mathrm{E}+\mathrm{c}$ \\
\hline 64 & $9.31 \mathrm{E}+04$ & $8.41 \mathrm{E}+04$ & $4.11 \mathrm{E}+04$ & $3.97 \mathrm{E}+04$ & $2.96 \mathrm{E}+05$ & $3.78 \mathrm{E}+03$ & $2.99 \mathrm{E}+05$ & $\overline{9.39 \mathrm{E}+0}$ \\
\hline 65 & $9.27 \mathrm{E}+04$ & $8.41 \mathrm{E}+04$ & $\overline{4.01 \mathrm{E}+04}$ & $3.98 \mathrm{E}+04$ & $2.93 \mathrm{E}+05$ & $3.80 \mathrm{E}+03$ & $2.98 \mathrm{E}+05$ & $9.35 \mathrm{E}+0$ \\
\hline 66 & $9.22 \mathrm{E}+04$ & $8.42 \mathrm{E}+04$ & $3.93 \mathrm{E}+04$ & $3.98 \mathrm{E}+04$ & $2.92 \mathrm{E}+05$ & $3.89 \mathrm{E}+03$ & $2.83 \mathrm{E}+05$ & $1.53 \mathrm{E}+0$ \\
\hline 67 & $9.18 \mathrm{E}+04$ & $8.42 \mathrm{E}+04$ & $3.87 \mathrm{E}+04$ & $3.98 \mathrm{E}+04$ & $2.91 \mathrm{E}+05$ & $3.97 \mathrm{E}+03$ & $2.83 \mathrm{E}+05$ & $1.53 \mathrm{E}+0$ \\
\hline 68 & $9.14 \mathrm{E}+04$ & $8.43 \mathrm{E}+04$ & $3.81 \mathrm{E}+04$ & $3.99 \mathrm{E}+04$ & $2.90 \mathrm{E}+05$ & $4.05 \mathrm{E}+03$ & $2.82 \mathrm{E}+05$ & $1.53 \mathrm{E}+0$ \\
\hline 69 & $9.10 \mathrm{E}+04$ & $8.43 \mathrm{E}+04$ & $3.76 \mathrm{E}+04$ & $3.99 \mathrm{E}+04$ & $2.88 \mathrm{E}+05$ & $4.13 \mathrm{E}+03$ & $2.81 \mathrm{E}+05$ & $1.52 \mathrm{E}+0$ \\
\hline 168 & $4.20 \mathrm{E}+04$ & $8.31 \mathrm{E}+04$ & $3.98 \mathrm{E}+04$ & $4.33 \mathrm{E}+04$ & $1.50 \mathrm{E}+05$ & $1.02 \mathrm{E}+04$ & $1.66 \mathrm{E}+05$ & $1.14 \mathrm{E}+0$ \\
\hline 336 & $2.22 \mathrm{E}+04$ & $7.04 \mathrm{E}+04$ & $2.12 \mathrm{E}+04$ & $4.63 \mathrm{E}+04$ & $6.32 \mathrm{E}+04$ & $1.75 \mathrm{E}+04$ & $9.68 \mathrm{E}+04$ & $8.56 \mathrm{E}+0$ \\
\hline 504 & $1.65 \mathrm{E}+04$ & $5.88 \mathrm{E}+04$ & $1.47 \mathrm{E}+04$ & $4.82 \mathrm{E}+04$ & $3.13 \mathrm{E}+04$ & $2.15 \mathrm{E}+04$ & $4.35 \mathrm{E}+04$ & $5.78 \mathrm{E}+0$ \\
\hline 720 & $1.21 \mathrm{E}+04$ & $4.65 \mathrm{E}+04$ & $9.87 \mathrm{E}+03$ & $4.99 \mathrm{E}+04$ & $1.59 \mathrm{E}+04$ & $2.37 \mathrm{E}+04$ & $2.18 \mathrm{E}+04$ & $4.04 \mathrm{E}+0$ \\
\hline 2160 & $2.28 \mathrm{E}+03$ & $9.34 \mathrm{E}+03$ & $1.12 \mathrm{E}+03$ & $5.31 \bar{E}+04$ & $1.44 \mathrm{E}+03$ & $1.39 \mathrm{E}+04$ & $5.00 \mathrm{E}+03$ & $1.40 \mathrm{E}+0$ \\
\hline $4 \overline{320}$ & $2.19 \mathrm{E}+02$ & $8.52 \mathrm{E}+02$ & $5.60 \mathrm{E}+03$ & $5.04 \mathrm{E}+04$ & $2.46 \mathrm{E}+02$ & $3.33 \mathrm{E}+03$ & $6.19 \mathrm{E}+02$ & $2.23 \mathrm{E}+0$ \\
\hline 4800 & $1.30 \mathrm{E}+02$ & $5.02 \mathrm{E}+02$ & $5.59 \mathrm{E}+03$ & $5.02 \mathrm{E}+04$ & $1.79 \mathrm{E}+02$ & $2.40 \mathrm{E}+03$ & $2.10 \mathrm{E}+02$ & $7.35 \mathrm{E}+0$ \\
\hline 8760 & $1.79 \mathrm{E}+00$ & $6.59 \mathrm{E}+00$ & $5.44 \mathrm{E}+03$ & $4.89 \mathrm{E}+04$ & $1 . \overline{49 E+01}$ & $1.61 \mathrm{E}+02$ & $6.60 \mathrm{E}+01$ & $2.1 \overline{8 \mathrm{E}+0}$ \\
\hline BT & $\begin{array}{l}\mathrm{E} 6 \\
/ \mathrm{L}\end{array}$ & $\begin{array}{l}8 \overline{\mathrm{E}+4} \\
\mathrm{~kg}\end{array}$ & $8 \mathrm{~Bq} / \mathrm{g}$ & & & & & \\
\hline DIL HTO & $\begin{array}{l}5.9 \mathrm{E} 6 \\
\mathrm{pCi} / \mathrm{L}\end{array}$ & $\begin{array}{l}2.18 \mathrm{E}+5 \\
\mathrm{~Bq} / \mathrm{kg}\end{array}$ & $218.3 \mathrm{~Bq} / \mathrm{g}$ & & & & & \\
\hline & \multicolumn{2}{|c|}{ VEGETABLES } & \multicolumn{2}{|c|}{ WHEAT SEED } & \multicolumn{2}{|l|}{ MEAT } & \multicolumn{2}{|l|}{ MILK } \\
\hline \multirow[t]{2}{*}{ HOUR } & $\mathrm{O}$ & $\overline{\mathrm{OBT}}$ & $\mathrm{CO}$ & OBT & $\overline{\text { HTO }}$ & $\mathrm{BT}$ & HTO & $\mathrm{DBT}$ \\
\hline & $(\mathrm{Bq} / \mathrm{g})$ & $(\mathrm{Bq} / \mathrm{g})$ & $(\mathrm{Bq} / \mathrm{g})$ & $(\mathrm{Bq} / \mathrm{g})$ & $(\mathrm{Bq} / \mathrm{g})$ & $(\mathrm{Bq} / \mathrm{g})$ & $(\mathrm{Bq} / \mathrm{g})$ & $(\overline{\mathrm{Bq}} / \mathrm{g})$ \\
\hline & & & & & & & & \\
\hline
\end{tabular}


Foodstuff Concentrations and Relocation Considerations Following

a Tritium Oxide Release from SRS Tritium Facilities (U)

\begin{tabular}{|c|c|c|c|c|c|c|c|c|}
\hline $1 / 3 / 5 / 6$ & $9.48 \mathrm{E}+06$ & $4.33 \mathrm{E}+04$ & $4.26 \mathrm{E}+06$ & $2.08 \overline{\mathrm{E}}+04$ & $4.24 \mathrm{E}+05$ & $3.64 \mathrm{E}+02$ & $4.08 \mathrm{E}+05$ & $1.27 \mathrm{E}+05$ \\
\hline SUM & $9.53 \mathrm{E}+06$ & & $4.28 \mathrm{E}+06$ & & $4.25 \mathrm{E}+05$ & & $5.35 \mathrm{E}+05$ & \\
\hline $\mathrm{Ci} / \mathrm{g}$ & $2.57 \mathrm{E}-04$ & & $1.16 \mathrm{E}-04$ & & $1.15 \mathrm{E}-05$ & & $1.45 \mathrm{E}-05$ & \\
\hline & & & & & & & & \\
\hline DIL HTO & $5.9 \mathrm{E}-09$ & & & & & & & \\
\hline \multicolumn{9}{|l|}{$(\mathrm{Ci} / \mathrm{g})$} \\
\hline & & & & & & & & \\
\hline 168 & $4.20 \mathrm{E}+04$ & $8.31 E+04$ & $3.98 \mathrm{E}+04$ & $4.33 \mathrm{E}+04$ & $1.50 \mathrm{E}+05$ & $1.02 E+04$ & $1.66 \mathrm{E}+05$ & $1.14 \mathrm{E}+05$ \\
\hline SUM & $1.25 \mathrm{E}+05$ & & $8.31 \mathrm{E}+04$ & & $1.60 \mathrm{E}+05$ & & $2.80 \mathrm{E}+05$ & \\
\hline $\mathrm{Ci} / \mathrm{g}$ & $3.38 \mathrm{E}-06$ & & $2.24 \mathrm{E}-06$ & & $4.33 \mathrm{E}-06$ & & $7.57 \mathrm{E}-06$ & \\
\hline & & & & & & & & \\
\hline DIL OBT & $2.4 \mathrm{E}-09$ & & & & & & & \\
\hline \multicolumn{9}{|l|}{$\mathrm{Ci} / \mathrm{g}$} \\
\hline & & & & & & & & \\
\hline 8760 & $1.79 \mathrm{E}+00$ & $6.59 \mathrm{E}+00$ & $5.44 \mathrm{E}+03$ & $4.89 \mathrm{E}+04$ & $1.49 \mathrm{E}+01$ & $1.61 \mathrm{E}+02$ & $6.60 \mathrm{E}+01$ & $2.18 \mathrm{E}+02$ \\
\hline SUM & $8.38 \mathrm{E}+00$ & & $5.44 \mathrm{E}+04$ & & $1.76 \mathrm{E}+02$ & & $2.84 \mathrm{E}+02$ & \\
\hline $\mathrm{Ci} / \mathrm{g}$ & $2.27 \mathrm{E}-10$ & & $1.47 \mathrm{E}-06$ & & $4.77 \mathrm{E}-09$ & & $7.67 \mathrm{E}-09$ & \\
\hline & & & & & & & & \\
\hline \begin{tabular}{|l}
$\mathrm{DIL}$ OB I \\
$\mathrm{Ci} / \mathrm{g}$
\end{tabular} & $2.4 \mathrm{E}-09$ & & & & & & & \\
\hline $\mathrm{Ci} / \mathrm{g}$ & & & & & & & & \\
\hline
\end{tabular}




\begin{tabular}{|c|c|c|c|c|c|c|c|c|}
\hline & \multicolumn{6}{|c|}{ Tritium Facilities, Design Basis Fire, Average Met Conditions } & & \\
\hline & \multicolumn{4}{|c|}{ Concentration Values for $10 \mathrm{~km}$ from release } & & & & \\
\hline \multicolumn{9}{|c|}{ nly Wheat Seed is examined since this is the edible portion of the Wheat. The "WHEAT" category is } \\
\hline \multicolumn{9}{|c|}{ ctually the unedible plant body and is not considered in the dose estimate. } \\
\hline & & & & & & & & \\
\hline & \multicolumn{2}{|c|}{ VEGETABLES } & \multicolumn{2}{|c|}{ WHEAT SEED } & \multicolumn{2}{|l|}{ MEAT } & \multicolumn{2}{|l|}{ MILK } \\
\hline \multirow[t]{2}{*}{ HOUR } & HTO & OBT & HTO & OBT & HTO & OBT & HTO & OBT \\
\hline & $(\mathrm{Bq} / \mathrm{g})$ & $(\mathrm{Bq} / \mathrm{g})$ & $(\mathrm{Bq} / \mathrm{g})$ & $(\mathrm{Bq} / \mathrm{g})$ & $(\mathrm{Bq} / \mathrm{g})$ & $(\mathrm{Bq} / \mathrm{g})$ & $(\mathrm{Bq} / \mathrm{g})$ & $\overline{B q} / \mathrm{g})$ \\
\hline & & & & & & & & \\
\hline 1 & $4.87 \mathrm{E}+05$ & $1.85 \mathrm{E}+03$ & $1.14 \mathrm{E}+05$ & $3.83 \mathrm{E}+02$ & $9.17 \mathrm{E}+03$ & $1.88 \mathrm{E}+00$ & $1.11 \mathrm{E}+03$ & $4.81 \mathrm{E}+$ \\
\hline 2 & $3.40 \mathrm{E}+05$ & $3.17 \mathrm{E}+03$ & $2.03 \mathrm{E}+05$ & $7.97 \mathrm{E}+02$ & $1.66 \mathrm{E}+04$ & $5.33 \mathrm{E}+00$ & $2.78 \mathrm{E}+03$ & $1.21 \mathrm{E}+03$ \\
\hline 3 & $1.72 \mathrm{E}+05$ & $3.76 \mathrm{E}+03$ & $2.40 \mathrm{E}+05$ & $1.12 \mathrm{E}+03$ & $2.09 E+04$ & $9.7 \overline{1 E+00}$ & $4.53 E+03$ & $1.98 \mathrm{E}+03$ \\
\hline 4 & $1.00 \mathrm{E}+05$ & $4.06 \mathrm{E}+03$ & $2.44 \mathrm{E}+05$ & $1.37 \mathrm{E}+03$ & $2.35 \mathrm{E}+04$ & $1.46 \mathrm{E}+01$ & $6.18 \mathrm{E}+03$ & $2.71 \mathrm{E}+03$ \\
\hline 5 & $6.80 \mathrm{E}+04$ & $4.23 \mathrm{E}+03$ & $2.29 \mathrm{E}+05$ & $1.56 \mathrm{E}+03$ & $2.50 \mathrm{E}+04$ & $1.99 \mathrm{E}+01$ & $7.68 \mathrm{E}+03$ & $3.37 \mathrm{E}+03$ \\
\hline 6 & $5.23 \mathrm{E}+04$ & $4.34 \mathrm{E}+03$ & $2.06 \mathrm{E}+05$ & $1.71 \mathrm{E}+03$ & $2.48 \mathrm{E}+04$ & $2.26 \mathrm{E}+01$ & $2.40 \mathrm{E}+04$ & $7.48 \mathrm{E}+03$ \\
\hline 7 & $4.39 \mathrm{E}+04$ & $4.43 \mathrm{E}+03$ & $1.80 \mathrm{E}+05$ & $1.82 \mathrm{E}+03$ & $2.46 \mathrm{E}+04$ & $2.52 \mathrm{E}+01$ & $2.39 \mathrm{E}+04$ & $7.45 \mathrm{E}+03$ \\
\hline 8 & $3.90 \mathrm{E}+04$ & $4.50 \mathrm{E}+03$ & $1.54 \mathrm{E}+05$ & $1.91 \mathrm{E}+03$ & $2.44 \mathrm{E}+04$ & $2.78 \mathrm{E}+01$ & $2.38 \mathrm{E}+04$ & $7.43 \mathrm{E}+03$ \\
\hline 9 & $3.57 \mathrm{E}+04$ & $4.56 \mathrm{E}+03$ & $1.30 \mathrm{E}+05$ & $1.99 \mathrm{E}+03$ & $2.42 \mathrm{E}+04$ & $3.04 \mathrm{E}+01$ & $2.37 \mathrm{E}+04$ & $7.40 \mathrm{E}+03$ \\
\hline 10 & $3.34 \mathrm{E}+04$ & $4.62 \mathrm{E}+03$ & $1.10 \mathrm{E}+05$ & $2.04 \mathrm{E}+03$ & $2.40 \mathrm{E}+04$ & $3.29 \mathrm{E}+01$ & $2.37 \mathrm{E}+04$ & $7.37 \mathrm{E}+03$ \\
\hline 11 & $3.16 \mathrm{E}+04$ & $4.67 \mathrm{E}+03$ & $9.17 \mathrm{E}+04$ & $2.09 \mathrm{E}+03$ & $2.38 \mathrm{E}+04$ & $3.55 \mathrm{E}+01$ & $2.36 \mathrm{E}+04$ & $7.34 \mathrm{E}+03$ \\
\hline 12 & $3.01 E+04$ & $4.72 \mathrm{E}+03$ & $7.68 \mathrm{E}+04$ & $2.13 E+03$ & $2.36 \mathrm{E}+04$ & $3.80 \mathrm{E}+01$ & $2.35 \mathrm{E}+04$ & $7.31 \mathrm{E}+03$ \\
\hline 13 & $2.89 \mathrm{E}+04$ & $4.76 \mathrm{E}+03$ & $6.45 \mathrm{E}+04$ & $2.17 \mathrm{E}+03$ & $2.35 \mathrm{E}+04$ & $4.05 \mathrm{E}+01$ & $2.34 \mathrm{E}+04$ & $7.28 \mathrm{E}+03$ \\
\hline 14 & $2.77 \mathrm{E}+04$ & $4.81 \mathrm{E}+03$ & $5.45 \mathrm{E}+04$ & $2.20 \mathrm{E}+03$ & $2.33 \mathrm{E}+04$ & $4.30 \mathrm{E}+01$ & $2.33 \mathrm{E}+04$ & $7.25 \mathrm{E}+03$ \\
\hline 15 & $2.67 \mathrm{E}+04$ & $4.85 E+03$ & $4.64 \mathrm{E}+04$ & $2.22 \mathrm{E}+03$ & $2.31 \mathrm{E}+04$ & $4.54 \mathrm{E}+01$ & $2.32 \mathrm{E}+04$ & $7.23 \mathrm{E}+03$ \\
\hline 16 & $2.58 \mathrm{E}+04$ & $4.89 \mathrm{E}+03$ & $3.99 \mathrm{E}+04$ & $2.25 \mathrm{E}+03$ & $2.29 \mathrm{E}+04$ & $4.7 \overline{\mathrm{E}+01}$ & $2.31 \mathrm{E}+04$ & $7.20 \mathrm{E}+03$ \\
\hline 17 & $2.49 \mathrm{E}+04$ & $4.93 \mathrm{E}+03$ & $3.47 \mathrm{E}+04$ & $2.27 \mathrm{E}+03$ & $2.27 \mathrm{E}+04$ & $5.03 \mathrm{E}+01$ & $2.30 \mathrm{E}+04$ & $7.17 \mathrm{E}+03$ \\
\hline 18 & $2.41 \mathrm{E}+04$ & $4.97 \mathrm{E}+03$ & $3.05 \mathrm{E}+04$ & $2.29 \mathrm{E}+03$ & $2.28 \mathrm{E}+04$ & $5.59 \mathrm{E}+01$ & $2.21 \mathrm{E}+04$ & $1.08 \mathrm{E}+04$ \\
\hline 19 & $2.33 \mathrm{E}+04$ & $5.00 \mathrm{E}+03$ & $2.72 \mathrm{E}+04$ & $2.31 \mathrm{E}+03$ & $2.29 \mathrm{E}+04$ & $6.15 \mathrm{E}+01$ & $2.22 \mathrm{E}+04$ & $1.08 \mathrm{E}+04$ \\
\hline 20 & $2.26 \mathrm{E}+04$ & $5.04 \mathrm{E}+03$ & $2.44 \mathrm{E}+04$ & $2.32 \mathrm{E}+03$ & $2.30 \mathrm{E}+04$ & $6.72 \mathrm{E}+01$ & $2.22 \mathrm{E}+04$ & $1.08 \mathrm{E}+04$ \\
\hline 21 & $2.20 \mathrm{E}+04$ & $5.07 \mathrm{E}+03$ & $2.22 \mathrm{E}+04$ & $2.34 \mathrm{E}+03$ & $2.31 \mathrm{E}+04$ & $7.29 \mathrm{E}+01$ & $2.22 \mathrm{E}+04$ & $1.09 \mathrm{E}+04$ \\
\hline 22 & $2.13 \mathrm{E}+04$ & $5.10 \mathrm{E}+03$ & $2.04 \mathrm{E}+04$ & $2.36 \mathrm{E}+03$ & $2.31 \mathrm{E}+04$ & $7.86 \mathrm{E}+01$ & $2.23 \mathrm{E}+04$ & $1.09 \mathrm{E}+04$ \\
\hline 23 & $2.07 E+04$ & $5.13 \mathrm{E}+03$ & $1.89 \mathrm{E}+04$ & $2.37 \mathrm{E}+03$ & $2.32 \mathrm{E}+04$ & $8.43 \mathrm{E}+01$ & $2.23 \mathrm{E}+04$ & $1.09 \mathrm{E}+04$ \\
\hline 24 & $2.01 \mathrm{E}+04$ & $5.16 \mathrm{E}+03$ & $1.77 \mathrm{E}+04$ & $2.39 \mathrm{E}+03$ & $2.32 \mathrm{E}+04$ & $9.00 \mathrm{E}+01$ & $2.23 E+04$ & $1.09 \mathrm{E}+04$ \\
\hline 25 & $1.96 \mathrm{E}+04$ & $5.19 \mathrm{E}+03$ & $1.66 \mathrm{E}+04$ & $2.40 \mathrm{E}+03$ & $2.33 \mathrm{E}+04$ & $9.58 \mathrm{E}+01$ & $2.24 \mathrm{E}+04$ & $1.09 \mathrm{E}+04$ \\
\hline 26 & $1.90 \mathrm{E}+04$ & $5.22 \mathrm{E}+03$ & $1.57 \mathrm{E}+04$ & $2.41 \mathrm{E}+03$ & $2.33 \mathrm{E}+04$ & $1.02 \mathrm{E}+02$ & $2.24 \mathrm{E}+04$ & $1.10 \mathrm{E}+04$ \\
\hline 27 & $1.85 \mathrm{E}+04$ & $5.24 \mathrm{E}+03$ & $1.49 \mathrm{E}+04$ & $2.43 \mathrm{E}+03$ & $2.34 \mathrm{E}+04$ & $1.07 \mathrm{E}+02$ & $2.24 \mathrm{E}+04$ & $1.10 \mathrm{E}+04$ \\
\hline 28 & $1.80 \mathrm{E}+04$ & $5.27 \mathrm{E}+03$ & $1.42 \mathrm{E}+04$ & $2.44 \mathrm{E}+03$ & $2.34 \mathrm{E}+04$ & $1.13 \mathrm{E}+02$ & $2.24 \mathrm{E}+04$ & $1.10 \mathrm{E}+04$ \\
\hline 29 & $1.75 \mathrm{E}+04$ & $5.29 \mathrm{E}+03$ & $1.36 \mathrm{E}+04$ & $2.45 \mathrm{E}+03$ & $2.34 \mathrm{E}+04$ & $1.19 \mathrm{E}+02$ & $2.25 \mathrm{E}+04$ & $1.10 \mathrm{E}+04$ \\
\hline 30 & $1.71 \mathrm{E}+04$ & $5.32 \mathrm{E}+03$ & $1.31 \mathrm{E}+04$ & $2.46 \mathrm{E}+03$ & $2.32 \mathrm{E}+04$ & $1.21 \mathrm{E}+02$ & $2.25 \mathrm{E}+04$ & $7.02 \mathrm{E}+03$ \\
\hline 31 & $1.66 \mathrm{E}+04$ & $5.34 \mathrm{E}+03$ & $1.26 \mathrm{E}+04$ & $2.47 \mathrm{E}+03$ & $2.30 \mathrm{E}+04$ & $1.24 \mathrm{E}+02$ & $2.24 \mathrm{E}+04$ & $6.99 \mathrm{E}+03$ \\
\hline 32 & $1.61 \mathrm{E}+04$ & $5.36 \mathrm{E}+03$ & $1.21 \mathrm{E}+04$ & $2.48 \mathrm{E}+03$ & $2.28 \mathrm{E}+04$ & $1.26 \mathrm{E}+02$ & $2.23 \mathrm{E}+04$ & $6.97 \mathrm{E}+03$ \\
\hline 33 & $1.57 \mathrm{E}+04$ & $5.38 \mathrm{E}+03$ & $1.16 \mathrm{E}+04$ & $2.49 \mathrm{E}+03$ & $2.26 \mathrm{E}+04$ & $1.28 \mathrm{E}+02$ & $2.22 \mathrm{E}+04$ & $6.94 \mathrm{E}+03$ \\
\hline 34 & $1.52 \mathrm{E}+04$ & $5.40 \mathrm{E}+03$ & $1.12 \mathrm{E}+04$ & $2.50 \mathrm{E}+03$ & $2.24 \mathrm{E}+04$ & $1.31 \mathrm{E}+02$ & $2.21 \mathrm{E}+04$ & $6.91 E+03$ \\
\hline 35 & $1.48 \mathrm{E}+04$ & $5.42 \mathrm{E}+03$ & $1.08 \mathrm{E}+04$ & $2.51 \mathrm{E}+03$ & $2.23 \mathrm{E}+04$ & $1.33 \mathrm{E}+02$ & $2.20 \mathrm{E}+04$ & $6.88 \mathrm{E}+03$ \\
\hline 36 & $1.44 \mathrm{E}+04$ & $5.44 \mathrm{E}+03$ & $1.05 \mathrm{E}+04$ & $2.52 \mathrm{E}+03$ & $2.21 \mathrm{E}+04$ & $1.35 \mathrm{E}+02$ & $2.19 E+04$ & $6.85 \mathrm{E}+03$ \\
\hline 37 & $1.41 \mathrm{E}+04$ & $5.46 \mathrm{E}+03$ & $1.01 \mathrm{E}+04$ & $2.53 \mathrm{E}+03$ & $2.19 \mathrm{E}+04$ & $1.38 \mathrm{E}+02$ & $2.19 E+04$ & $6.83 \mathrm{E}+03$ \\
\hline 38 & $1.37 \mathrm{E}+04$ & $5.48 \mathrm{E}+03$ & $9.75 \mathrm{E}+03$ & $2.54 \mathrm{E}+03$ & $2.17 \mathrm{E}+04$ & $1.40 \mathrm{E}+02$ & $2.18 \mathrm{E}+04$ & $6.80 \mathrm{E}+03$ \\
\hline
\end{tabular}




\begin{tabular}{|c|c|c|c|c|c|c|c|c|}
\hline 39 & $1.34 \mathrm{E}+\overline{04}$ & $5.49 \mathrm{E}+03$ & $9.43 \mathrm{E}+03$ & $2.55 \overline{\mathrm{E}}+03$ & $2.15 \mathrm{E}+04$ & $1.42 \mathrm{E}+02$ & $2.17 \mathrm{E}+04$ & $6.77 \mathrm{E}+0$ \\
\hline 40 & $1.31 \mathrm{E}+04$ & $5.51 \mathrm{E}+03$ & $9.12 \mathrm{E}+03$ & $2.56 \mathrm{E}+03$ & $2.14 \mathrm{E}+04$ & $1.44 \mathrm{E}+02$ & $2.16 \mathrm{E}+04$ & $6.75 \mathrm{E}+\mathrm{c}$ \\
\hline 41 & $1.28 \mathrm{E}+04$ & $5.52 \mathrm{E}+03$ & $8.83 \mathrm{E}+03$ & $2.57 \mathrm{E}+03$ & $2.12 \mathrm{E}+04$ & $1.46 \mathrm{E}+02$ & $2.15 \mathrm{E}+04$ & $6.72 \mathrm{E}+\mathrm{C}$ \\
\hline 42 & $1.25 \mathrm{E}+04$ & $5.54 \mathrm{E}+03$ & $8.55 \mathrm{E}+03$ & $2.57 \mathrm{E}+03$ & $2.12 \mathrm{E}+04$ & $1.52 \mathrm{E}+02$ & $2.05 \mathrm{E}+04$ & $1.07 \mathrm{E}+0$ \\
\hline 43 & $1.23 \mathrm{E}+04$ & $5.55 \mathrm{E}+03$ & $8.29 \mathrm{E}+03$ & $2.58 \mathrm{E}+03$ & $2.11 \mathrm{E}+04$ & $1.58 \mathrm{E}+02$ & $2.05 \mathrm{E}+04$ & $1.07 \mathrm{E}+$ \\
\hline 44 & $1.20 \mathrm{E}+04$ & $5.57 \mathrm{E}+03$ & $8.03 \mathrm{E}+03$ & $2.59 \mathrm{E}+03$ & $2.1 \mathrm{IE}+04$ & $1.64 \mathrm{E}+02$ & $2.05 \mathrm{E}+04$ & $1.07 \mathrm{E}+$ \\
\hline 45 & $1.18 \mathrm{E}+04$ & $5.58 \mathrm{E}+03$ & $7.80 \mathrm{E}+03$ & $2.59 \bar{E}+03$ & $2.11 \mathrm{E}+04$ & $1.69 \mathrm{E}+02$ & $2.05 \mathrm{E}+04$ & $1.07 \bar{E}+c$ \\
\hline 46 & $1.15 \mathrm{E}+04$ & $5.59 \mathrm{E}+03$ & $7.57 \mathrm{E}+03$ & $2.60 \overline{\mathrm{E}+03}$ & $2.10 \mathrm{E}+04$ & $1.75 \mathrm{E}+02$ & $2.05 \mathrm{E}+04$ & $1.07 \mathrm{E}+$ \\
\hline 47 & $1.13 \mathrm{E}+04$ & $5.61 \mathrm{E}+03$ & $7.35 \mathrm{E}+03$ & $2.61 \mathrm{E}+03$ & $2.10 \mathrm{E}+04$ & $1.81 \mathrm{E}+02$ & $2.04 \mathrm{E}+04$ & $1.07 \mathrm{E}+$ \\
\hline 48 & $1.10 \mathrm{E}+04$ & $5.62 \mathrm{E}+03$ & $7.15 \mathrm{E}+03$ & $2.62 \mathrm{E}+03$ & $2.10 \mathrm{E}+04$ & $1.86 \mathrm{E}+02$ & $2.04 \mathrm{E}+04$ & $1.07 \mathrm{E}+\mathrm{C}$ \\
\hline 49 & $1.08 \mathrm{E}+\overline{04}$ & $5.63 \mathrm{E}+03$ & $6.94 \mathrm{E}+03$ & $2.62 \mathrm{E}+03$ & $2.09 \mathrm{E}+04$ & $1.92 \mathrm{E}+02$ & $2.04 \mathrm{E}+04$ & $1.07 \mathrm{E}+0$ \\
\hline 50 & $1.06 \mathrm{E}+\overline{04}$ & $5.64 \mathrm{E}+03$ & $6.75 \mathrm{E}+03$ & $2.63 \bar{E}+03$ & $2.09 \mathrm{E}+04$ & $1.98 \mathrm{E}+02$ & $2.04 \mathrm{E}+04$ & $1.07 \mathrm{E}+\mathrm{C}$ \\
\hline 51 & $1.04 \mathrm{E}+\overline{04}$ & $5.65 \mathrm{E}+03$ & $6.56 \mathrm{E}+03$ & $2.63 \bar{E}+03$ & $2.08 E+04$ & $2.03 \mathrm{E}+02$ & $2.04 E+04$ & $1.07 \mathrm{E}+0$ \\
\hline 52 & $1.02 \mathrm{E}+\overline{04}$ & $5.66 \mathrm{E}+03$ & $6.38 \mathrm{E}+03$ & $2.64 \bar{E}+03$ & $2.08 \mathrm{E}+04$ & $2.09 \mathrm{E}+02$ & $2.03 E+04$ & $1.07 \mathrm{E}+0$ \\
\hline 53 & $1.01 \mathrm{E}+\overline{04}$ & $5.67 \mathrm{E}+03$ & $6.22 \mathrm{E}+03$ & $2.65 \mathrm{E}+03$ & $2.07 \mathrm{E}+04$ & $2.15 \mathrm{E}+02$ & $2.03 E+04$ & $1.07 \mathrm{E}$ \\
\hline 54 & $9.94 \mathrm{E}+03$ & $5.69 \mathrm{E}+03$ & $6.06 \mathrm{E}+03$ & $2.65 \mathrm{E}+03$ & $2.05 \mathrm{E}+04$ & $2.17 \mathrm{E}+02$ & $1.99 \mathrm{E}+04$ & $6.24 \mathrm{E}+\mathrm{C}$ \\
\hline 55 & $9.81 \mathrm{E}+03$ & $5.69 \mathrm{E}+03$ & $5.91 \mathrm{E}+03$ & $2.66 \bar{E}+03$ & $2.04 \mathrm{E}+04$ & $2.19 \mathrm{E}+02$ & $1.98 \mathrm{E}+04$ & $6.22 \mathrm{E}+\mathrm{C}$ \\
\hline 56 & $9.68 \mathrm{E}+03$ & $5.70 \mathrm{E}+03$ & $5.77 \mathrm{E}+03$ & $2.66 \bar{E}+03$ & $2.02 \mathrm{E}+04$ & $2.21 \mathrm{E}+02$ & $1.97 \mathrm{E}+04$ & $6.19 \mathrm{E}+0$ \\
\hline 57 & $9.55 \mathrm{E}+03$ & $5.71 \mathrm{E}+03$ & $5.65 \mathrm{E}+03$ & $2.67 \mathrm{E}+03$ & $2.00 \mathrm{E}+04$ & $2.23 \mathrm{E}+02$ & $1.97 \mathrm{E}+04$ & $6.17 \mathrm{E}+0$ \\
\hline 58 & $9.43 E+03$ & $5.72 E+03$ & $5.53 \mathrm{E}+03$ & $2.67 \bar{E}+03$ & $1.99 \mathrm{E}+04$ & $2.25 \mathrm{E}+02$ & $1.96 \mathrm{E}+04$ & $6.14 \mathrm{E}+0$ \\
\hline 59 & $9.26 \mathrm{E}+03$ & $5.73 \mathrm{E}+03$ & $5.41 \mathrm{E}+03$ & $2.68 \bar{E}+03$ & $1.97 \mathrm{E}+04$ & $2.27 \mathrm{E}+02$ & $1.95 \mathrm{E}+04$ & $6.12 \mathrm{E}+0$ \\
\hline 60 & $9.12 \mathrm{E}+03$ & $5.74 \mathrm{E}+03$ & $5.30 \mathrm{E}+03$ & $2.68 \bar{E}+03$ & $1.95 \mathrm{E}+04$ & $2.29 \mathrm{E}+02$ & $1.94 \mathrm{E}+04$ & $6.09 \mathrm{E}+0$ \\
\hline 61 & $9.01 \mathrm{E}+03$ & $5.75 \mathrm{E}+03$ & $5.19 \mathrm{E}+03$ & $2.69 \mathrm{E}+03$ & $1.94 \mathrm{E}+04$ & $2.31 \mathrm{E}+02$ & $1.93 \mathrm{E}+04$ & $6.07 \mathrm{E}+0$ \\
\hline 62 & $8.84 \mathrm{E}+03$ & $5.76 \mathrm{E}+03$ & $5.08 \mathrm{E}+03$ & $2.69 \overline{\mathrm{E}+03}$ & $1.92 \mathrm{E}+04$ & $2.32 \mathrm{E}+02$ & $1.93 \mathrm{E}+04$ & $6.04 \mathrm{E}+0$ \\
\hline 63 & $8.65 \mathrm{E}+03$ & $5.76 \mathrm{E}+03$ & $4.98 \mathrm{E}+03$ & $2.70 \bar{E}+03$ & $1.91 \mathrm{E}+04$ & $2.34 \mathrm{E}+02$ & $1.92 \mathrm{E}+04$ & $6.02 \mathrm{E}+0$ \\
\hline 64 & $8.52 \mathrm{E}+03$ & $5.77 \mathrm{E}+03$ & $4.87 \mathrm{E}+03$ & $2.70 \bar{E}+03$ & $1.89 \mathrm{E}+04$ & $2.36 \mathrm{E}+02$ & $1.91 \mathrm{E}+04$ & $5.99 \mathrm{E}+0$ \\
\hline 65 & $8.43 E+03$ & $5.78 \mathrm{E}+03$ & $4.76 \mathrm{E}+03$ & $2.71 \mathrm{E}+03$ & $1.87 \mathrm{E}+04$ & $2.38 \mathrm{E}+02$ & $1.90 \mathrm{E}+04$ & $5.97 \mathrm{E}+0$ \\
\hline 66 & $8.34 \mathrm{E}+03$ & $5.79 \mathrm{E}+03$ & $4.65 \mathrm{E}+03$ & $2.71 \mathrm{E}+03$ & $1.87 \bar{E}+04$ & $2.43 \mathrm{E}+02$ & $1.81 \mathrm{E}+04$ & $1.01 \mathrm{E}+0$ \\
\hline 67 & $8.26 \mathrm{E}+03$ & $5.79 \mathrm{E}+03$ & $4.55 \mathrm{E}+03$ & $2.71 \mathrm{E}+03$ & $1.86 \mathrm{E}+04$ & $2.49 \mathrm{E}+02$ & $1.81 E+04$ & $1.01 \mathrm{E}+0$ \\
\hline 68 & $8.19 \mathrm{E}+03$ & $5.80 \mathrm{E}+03$ & $4.46 \mathrm{E}+03$ & $2.72 \mathrm{E}+03$ & $1.86 \mathrm{E}+04$ & $2.54 \mathrm{E}+02$ & $1.81 \mathrm{E}+04$ & $\overline{1.01 \mathrm{E}+0}$ \\
\hline 69 & $8.12 E+03$ & $5.81 \mathrm{E}+03$ & $4.38 \mathrm{E}+03$ & $2.72 \mathrm{E}+03$ & $1.85 \mathrm{E}+04$ & $2.60 \mathrm{E}+02$ & $1.80 \mathrm{E}+04$ & $1.01 \mathrm{E}+0$ \\
\hline 168 & $2.89 \mathrm{E}+03$ & $5.82 \mathrm{E}+03$ & $2.80 \mathrm{E}+03$ & $3.01 \mathrm{E}+03$ & $1.00 \mathrm{E}+04$ & $6.73 \mathrm{E}+02$ & $1.10 \mathrm{E}+04$ & $7.81 \mathrm{E}+0$ \\
\hline 336 & $1.50 \mathrm{E}+03$ & $4.92 \mathrm{E}+03$ & $1.47 \mathrm{E}+03$ & $3.22 \mathrm{E}+03$ & $4.28 \mathrm{E}+03$ & $1.19 \mathrm{E}+03$ & $6.51 \mathrm{E}+03$ & $5.90 \mathrm{E}+0$ \\
\hline 504 & $1.12 \mathrm{E}+03$ & $4.11 \mathrm{E}+03$ & $1.01 \mathrm{E}+03$ & $3.35 \mathrm{E}+03$ & $2.14 \mathrm{E}+03$ & $1.47 \mathrm{E}+03$ & $2.96 \mathrm{E}+03$ & $4.01 \mathrm{E}+0$ \\
\hline 720 & $8.14 \mathrm{E}+02$ & $3.24 \mathrm{E}+03$ & $6.80 \mathrm{E}+02$ & $3.47 \mathrm{E}+03$ & $1.10 \mathrm{E}+03$ & $1.63 \mathrm{E}+03$ & $1.49 \mathrm{E}+03$ & $2.81 \mathrm{E}+0$ \\
\hline 2160 & $1.53 \mathrm{E}+02$ & $6.45 \mathrm{E}+02$ & $7.68 \mathrm{E}+01$ & $3.69 \mathrm{E}+03$ & $9.83 \mathrm{E}+01$ & $9.57 \mathrm{E}+02$ & $3.44 \mathrm{E}+02$ & $9.73 \mathrm{E}+0$ \\
\hline 4320 & $1.47 \mathrm{E}+01$ & $5.83 \mathrm{E}+01$ & $3.86 \mathrm{E}+02$ & $3.50 \mathrm{E}+03$ & $1.66 \mathrm{E}+01$ & $2.30 \mathrm{E}+02$ & $4.21 \mathrm{E}+01$ & $1.53 \mathrm{E}+02$ \\
\hline 4800 & $8.76 \mathrm{E}+00$ & $3.43 \mathrm{E}+01$ & $3.85 \bar{E}+02$ & $3.49 \mathrm{E}+03$ & $1.21 \mathrm{E}+01$ & $1.65 \mathrm{E}+02$ & $1.42 \mathrm{E}+01$ & $5.04 \overline{\mathrm{E}+01}$ \\
\hline 8760 & $1.20 \mathrm{E}-01$ & $4.46 \mathrm{E}-01$ & $3.75 \mathrm{E}+02$ & $3.40 \mathrm{E}+03$ & $1.00 \mathrm{E}+00$ & $1.11 \mathrm{E}+01$ & $4.44 \mathrm{E}+00$ & $1.49 \mathrm{E}+0$ \\
\hline BT & $\begin{array}{l}\mathrm{E} 6 \\
/ \mathrm{L}\end{array}$ & $\begin{array}{l}8.88 \mathrm{E}+4 \\
\mathrm{~Bq} / \mathrm{kg}\end{array}$ & $8 \mathrm{~Bq} / \mathrm{g}$ & & & & & \\
\hline 50 & $\begin{array}{l}5.9 \mathrm{E} 6 \\
\mathrm{pCi} / \mathrm{L}\end{array}$ & $\begin{array}{l}2.18 \mathrm{E}+5 \\
\mathrm{~Bq} / \mathrm{kg}\end{array}$ & $8.3 \mathrm{~Bq} / \mathrm{g}$ & & & & & \\
\hline & \multirow{2}{*}{\multicolumn{2}{|c|}{ VEGETABLES }} & & & & & & \\
\hline & & & \multicolumn{2}{|c|}{ WHEAT SEED } & \multicolumn{2}{|l|}{ MEAT } & \multicolumn{2}{|l|}{ MILK } \\
\hline \multirow[t]{2}{*}{ HOUR } & $\overline{T O}$ & OBT & HTO & OBT & HTO & $\overline{\mathrm{BT}}$ & HTO & $\overline{\mathrm{OBT}}$ \\
\hline & $(\mathrm{Bq} / \mathrm{g})$ & $(\mathrm{Bq} / \mathrm{g})$ & $(\mathrm{Bq} / \mathrm{g})$ & $(\mathrm{Bq} / \mathrm{g})$ & $(\mathrm{Bq} / \mathrm{g})$ & $(\mathrm{Bq} / \mathrm{g})$ & $(\mathrm{Bq} / \mathrm{g})$ & $(\overline{\mathrm{Bq}} / \mathrm{g})$ \\
\hline $\mathrm{ak} \mathrm{HTO}$ & & & & & & & & \\
\hline
\end{tabular}


a Tritium Oxide Release from SRS Tritium Facilities (U)

\begin{tabular}{|c|c|c|c|c|c|c|c|c|}
\hline $1 / 4 / 6 / 6$ & $4.87 \mathrm{E}+05$ & $1.85 \mathrm{E}+03$ & $2.44 \bar{E}+05$ & $1.37 \bar{E}+03$ & $2.48 \mathrm{E}+04$ & $2.26 \mathrm{E}+01$ & $2.40 \mathrm{E}+04$ & $7.48 \mathrm{E}+03$ \\
\hline SUM & $4.88 \mathrm{E}+05$ & & $2.45 \mathrm{E}+05$ & & $2.48 \mathrm{E}+04$ & & $3.15 \mathrm{E}+04$ & \\
\hline $\mathrm{Ci} / \mathrm{g}$ & $1.32 \mathrm{E}-05$ & & $6.62 \mathrm{E}-06$ & & $6.70 \mathrm{E}-07$ & & $8.51 E-07$ & \\
\hline & & & & & & & & \\
\hline DIL HTO & $5.9 \mathrm{E}-09$ & & & & & & & \\
\hline \multicolumn{9}{|l|}{$(\mathrm{Ci} / \mathrm{g})$} \\
\hline & & & & & & & & \\
\hline 168 & $2.89 \mathrm{E}+03$ & $5.82 \mathrm{E}+03$ & $2.80 \mathrm{E}+03$ & $3.01 \mathrm{E}+03$ & $1.00 \mathrm{E}+04$ & $6.73 \mathrm{E}+02$ & $1.10 \mathrm{E}+04$ & $7.81 \mathrm{E}+03$ \\
\hline SUM & $8.72 E+03$ & & $5.81 \mathrm{E}+03$ & & $1.07 \mathrm{E}+04$ & & $1.89 \mathrm{E}+04$ & \\
\hline $\mathrm{Ci} / \mathrm{g}$ & 2.36E-07 & & $1.57 \bar{E}-07$ & & 2.89E-07 & & 5.10E-07 & \\
\hline & & & & & & & & \\
\hline DIL OBT & $2.4 \mathrm{E}-09$ & & & & & & & \\
\hline \multicolumn{9}{|l|}{$\mathrm{Ci} / \mathrm{g}$} \\
\hline & & & & & & & & \\
\hline 8760 & $1.20 \mathrm{E}-01$ & $4.46 \overline{\mathrm{E}}-01$ & $3.75 \mathrm{E}+02$ & $3.40 \mathrm{E}+03$ & $1.00 \mathrm{E}+00$ & $1.11 \mathrm{E}+01$ & $4.44 \mathrm{E}+00$ & $1.49 \mathrm{E}+01$ \\
\hline SUM & $5.67 \mathrm{E}-01$ & & $3.78 \mathrm{E}+03$ & & $1.21 \mathrm{E}+01$ & & $1.93 \mathrm{E}+01$ & \\
\hline Cí/g & $1.53 \mathrm{E}-11$ & & $1.02 \mathrm{E}-07$ & & $3.26 \mathrm{E}-10$ & & $5.23 \mathrm{E}-10$ & \\
\hline DIL OBT & 78 & & & & & & & \\
\hline \begin{tabular}{|l} 
DIL UBI \\
$\mathrm{Ci} / \mathrm{g}$
\end{tabular} & $2.4 \mathrm{E}-09$ & & & & & & & \\
\hline $\mathrm{Ci} / \mathrm{g}$ & & & & & & & & \\
\hline
\end{tabular}




\begin{tabular}{|c|c|c|c|c|c|c|c|c|}
\hline & \multicolumn{6}{|c|}{ Tritium Facilities, Design Basis Fire, Average Met Conditions } & & \\
\hline & \multicolumn{6}{|c|}{ Concentration Values for $11.54 \mathrm{~km}$ from release } & & \\
\hline \multicolumn{9}{|c|}{ Only Wheat Seed is examined since this is the edible portion of the Wheat. The "WHEAT" category is } \\
\hline \multicolumn{9}{|c|}{ ctually the unedible plant body and is not considered in the dose estimate. } \\
\hline & & & & & & & & \\
\hline & \multicolumn{2}{|c|}{ VEGETABLES } & \multicolumn{2}{|c|}{ WHEAT SEED } & \multicolumn{2}{|l|}{ MEAT } & \multicolumn{2}{|l|}{ MILK } \\
\hline \multirow[t]{3}{*}{ HOUR } & HTO & $\overline{\mathrm{OBT}}$ & HTO & $\overline{\mathrm{OBT}}$ & HTO & OB' & HTO & $\overline{\mathrm{OBT}}$ \\
\hline & $(\mathrm{Bq} / \mathrm{g})$ & /g) & $(\mathrm{Bq} / \mathrm{g})$ & $(\mathrm{Bq} / \mathrm{g})$ & $(\mathrm{Bq} / \mathrm{g})$ & r) & $\mathrm{q} / \mathrm{g})$ & ) \\
\hline & & & & & & & & \\
\hline 1 & $2.63 \mathrm{E}+05$ & $6.83 \mathrm{E}+02$ & $4.20 \mathrm{E}+04$ & $1.41 \mathrm{E}+02$ & $3.44 \mathrm{E}+03$ & $6.98 \mathrm{E}-01$ & $4.17 \mathrm{E}+02$ & 1.19ETVL \\
\hline 2 & $4.89 \mathrm{E}+05$ & $2.53 \mathrm{E}+03$ & $1.58 \mathrm{E}+05$ & $5.74 \bar{E}+02$ & $1.29 \mathrm{E}+04$ & $3.35 \mathrm{E}+00$ & $1 . \overline{76 \mathrm{E}+03}$ & $7.62 \mathrm{E}+02$ \\
\hline 3 & $2.50 \mathrm{E}+05$ & $3.39 E+03$ & $2.16 \mathrm{E}+05$ & $9.26 \overline{\mathrm{E}}+02$ & $1.83 \mathrm{E}+04$ & $7.18 \mathrm{E}+00$ & $3.36 \mathrm{E}+03$ & $1.46 \mathrm{E}+03$ \\
\hline 4 & $1.45 \mathrm{E}+05$ & $3.82 E+03$ & $2.36 \mathrm{E}+05$ & $1.21 \bar{E}+03$ & $2.17 \bar{E}+04$ & $1.17 \mathrm{E}+01$ & $4.96 \mathrm{E}+03$ & $2.17 \mathrm{E}+03$ \\
\hline 5 & $9.70 \mathrm{E}+04$ & $4.07 \mathrm{E}+03$ & $2.32 \mathrm{E}+05$ & $1.42 \mathrm{E}+03$ & $2.38 \mathrm{E}+04$ & $1.67 \mathrm{E}+01$ & $6.47 \mathrm{E}+03$ & $2.84 \mathrm{E}+03$ \\
\hline 6 & $7.32 \mathrm{E}+04$ & $4.23 E+03$ & $2.16 \mathrm{E}+05$ & $1.60 \mathrm{E}+03$ & $2.36 \mathrm{E}+04$ & $1.93 \mathrm{E}+01$ & $2.29 \mathrm{E}+04$ & $7.12 \mathrm{E}+03$ \\
\hline 7 & $6.04 \mathrm{E}+04$ & $4.36 \mathrm{E}+03$ & $1.93 \mathrm{E}+05$ & $1.73 \mathrm{E}+03$ & $2.34 \mathrm{E}+04$ & $2.18 \mathrm{E}+01$ & $2 . \overline{28 E}+04$ & $7.10 \mathrm{E}+03$ \\
\hline 8 & $5.29 \mathrm{E}+\overline{04}$ & $4.46 \mathrm{E}+03$ & $1.69 \mathrm{E}+05$ & $1.84 \mathrm{E}+03$ & $2.32 \mathrm{E}+04$ & $2.42 \mathrm{E}+01$ & $2.27 \mathrm{E}+04$ & $7.07 \mathrm{E}+03$ \\
\hline 9 & $4.81 \mathrm{E}+04$ & $4.54 \mathrm{E}+03$ & $1.46 \mathrm{E}+05$ & $1.93 \overline{\mathrm{E}}+03$ & $2.31 \mathrm{E}+04$ & $2.67 \mathrm{E}+01$ & $2.26 \mathrm{E}+04$ & $7.04 \mathrm{E}+03$ \\
\hline 10 & $4.46 \mathrm{E}+04$ & $4.62 \mathrm{E}+03$ & $1.25 \mathrm{E}+05$ & $2.00 \mathrm{E}+03$ & $2.29 E+04$ & $2.92 \mathrm{E}+01$ & $2.25 \mathrm{E}+04$ & $7.02 E+03$ \\
\hline 11 & $4.20 \mathrm{E}+\overline{04}$ & $4.69 \mathrm{E}+03$ & $1.06 \mathrm{E}+05$ & $2.06 \bar{E}+03$ & $2.27 \bar{E}+04$ & $3.16 \mathrm{E}+01$ & $2.24 \mathrm{E}+04$ & $6.99 \mathrm{E}+03$ \\
\hline 12 & $3.99 \mathrm{E}+04$ & $4.76 \mathrm{E}+03$ & $9.03 \mathrm{E}+04$ & $2.11 \bar{E}+03$ & $2.25 \mathrm{E}+04$ & $3.40 \mathrm{E}+01$ & $2.24 \mathrm{E}+04$ & $6.97 \mathrm{E}+03$ \\
\hline 13 & $3.81 \mathrm{E}+04$ & $4.82 \mathrm{E}+03$ & $7.71 \mathrm{E}+04$ & $2.16 \bar{E}+03$ & $2.24 \mathrm{E}+04$ & $3.64 \mathrm{E}+01$ & $2.23 \mathrm{E}+04$ & $6.94 \mathrm{E}+03$ \\
\hline 14 & $3.66 \mathrm{E}+04$ & $4.88 \mathrm{E}+03$ & $6.63 \mathrm{E}+04$ & $2.20 \bar{E}+03$ & $2.22 \mathrm{E}+04$ & $3.87 \mathrm{E}+01$ & $2.22 \mathrm{E}+04$ & $6.91 \mathrm{E}+03$ \\
\hline 15 & $3.52 \mathrm{E}+04$ & $4.94 \mathrm{E}+03$ & $5.73 \mathrm{E}+04$ & $2.23 \mathrm{E}+03$ & $2.20 \mathrm{E}+04$ & $4.11 \mathrm{E}+01$ & $2.21 \mathrm{E}+04$ & $6.89 \mathrm{E}+03$ \\
\hline 16 & $3.39 \mathrm{E}+04$ & $5.00 \mathrm{E}+03$ & $5.00 \mathrm{E}+04$ & $2.27 \overline{\mathrm{E}+03}$ & $2.18 \mathrm{E}+04$ & $E+01$ & $2.20 \mathrm{E}+04$ & $6.86 \mathrm{E}+03$ \\
\hline 17 & $3.27 \mathrm{E}+04$ & $5.05 \mathrm{E}+03$ & $4.41 \mathrm{E}+04$ & $2.29 \mathrm{E}+03$ & $2.17 \mathrm{E}+04$ & $4.57 \mathrm{E}+01$ & $2.19 \mathrm{E}+04$ & $6.83 \mathrm{E}+03$ \\
\hline 18 & $3.16 \mathrm{E}+04$ & $5.10 \mathrm{E}+03$ & $3.93 \mathrm{E}+04$ & $2.32 \mathrm{E}+03$ & $2.19 \mathrm{E}+04$ & $5.13 \mathrm{E}+01$ & $2.12 \mathrm{E}+04$ & $1.06 \mathrm{E}+04$ \\
\hline 19 & $3.06 \mathrm{E}+04$ & $5.15 \mathrm{E}+03$ & $3.54 \mathrm{E}+04$ & $2.35 \mathrm{E}+03$ & $2.21 \mathrm{E}+04$ & $5.69 \mathrm{E}+01$ & $2.13 E+04$ & $1.06 \mathrm{E}+04$ \\
\hline 20 & $2.96 \mathrm{E}+04$ & $5.19 \mathrm{E}+03$ & $3.21 \mathrm{E}+04$ & $2.37 \mathrm{E}+03$ & $2.22 \mathrm{E}+04$ & $\bar{E}+01$ & $2.14 \mathrm{E}+04$ & $1.07 \mathrm{E}+04$ \\
\hline 21 & $2.87 \mathrm{E}+04$ & $5.24 \mathrm{E}+03$ & $2.95 \mathrm{E}+04$ & $2.39 \mathrm{E}+03$ & $2.24 \mathrm{E}+04$ & $6.84 \mathrm{E}+01$ & $2.15 \mathrm{E}+04$ & $1.07 \mathrm{E}+04$ \\
\hline 22 & $2.78 \mathrm{E}+04$ & $5.28 \mathrm{E}+03$ & $2.73 \mathrm{E}+04$ & $2.42 \mathrm{E}+03$ & $2.25 \mathrm{E}+04$ & $7.41 \mathrm{E}+01$ & $2.15 \mathrm{E}+04$ & $1.08 \mathrm{E}+04$ \\
\hline 23 & $2.70 \mathrm{E}+04$ & $5.32 \mathrm{E}+03$ & $2.54 \mathrm{E}+04$ & $2.44 \mathrm{E}+03$ & $2.27 \mathrm{E}+04$ & $7.99 \mathrm{E}+01$ & $2.16 \mathrm{E}+04$ & $1.08 \mathrm{E}+04$ \\
\hline 24 & $2.62 \mathrm{E}+04$ & $5.36 \mathrm{E}+03$ & $2.39 \mathrm{E}+04$ & $2.46 \mathrm{E}+03$ & $2.28 \mathrm{E}+04$ & $8.57 \mathrm{E}+01$ & $2.17 \mathrm{E}+04$ & $1.08 \mathrm{E}+04$ \\
\hline 25 & $2.54 \mathrm{E}+04$ & $5.40 \mathrm{E}+03$ & $2.25 \mathrm{E}+04$ & $2.47 \mathrm{E}+03$ & $2.29 \mathrm{E}+04$ & $9.16 \mathrm{E}+01$ & $2.18 \mathrm{E}+04$ & $1.09 \mathrm{E}+04$ \\
\hline 26 & $2.47 \mathrm{E}+04$ & $5.44 \mathrm{E}+03$ & $2.14 \mathrm{E}+04$ & $2.49 \mathrm{E}+03$ & $2.30 \mathrm{E}+04$ & $9.75 \mathrm{E}+01$ & $2.18 \mathrm{E}+04$ & $1.09 \mathrm{E}+04$ \\
\hline 27 & $2.39 \mathrm{E}+04$ & $5.48 \mathrm{E}+03$ & $2.03 \mathrm{E}+04$ & $2.51 \mathrm{E}+03$ & $2.31 \mathrm{E}+04$ & $1.03 \mathrm{E}+02$ & $2.19 \mathrm{E}+04$ & $1.09 \mathrm{E}+04$ \\
\hline 28 & $2.32 \mathrm{E}+04$ & $5.51 \mathrm{E}+03$ & $1.94 \mathrm{E}+04$ & $2.53 \bar{E}+03$ & $2.32 \mathrm{E}+04$ & $1.09 \mathrm{E}+02$ & $2.19 \mathrm{E}+04$ & $1.10 \mathrm{E}+04$ \\
\hline 29 & $2.26 \mathrm{E}+04$ & $5.54 \mathrm{E}+03$ & $1.86 \mathrm{E}+04$ & $2.54 \mathrm{E}+03$ & $2.33 \mathrm{E}+04$ & $1.15 \mathrm{E}+02$ & $2.20 \mathrm{E}+04$ & $1.10 \mathrm{E}+04$ \\
\hline 30 & $2.19 \mathrm{E}+04$ & $5.58 \mathrm{E}+03$ & $1.78 \mathrm{E}+04$ & $2.56 \mathrm{E}+03$ & $2.31 \mathrm{E}+04$ & $1.18 \mathrm{E}+02$ & $2.24 \mathrm{E}+04$ & $6.99 \mathrm{E}+03$ \\
\hline 31 & $2.13 \mathrm{E}+04$ & $5.61 \mathrm{E}+03$ & $1.71 \mathrm{E}+04$ & $2.57 \mathrm{E}+03$ & $2.29 \mathrm{E}+04$ & $1.20 \mathrm{E}+02$ & $2.23 \mathrm{E}+04$ & $6.96 \mathrm{E}+03$ \\
\hline 32 & $2.06 \mathrm{E}+04$ & $5.64 \mathrm{E}+03$ & $1.65 \mathrm{E}+04$ & $2.59 \mathrm{E}+03$ & $2.27 \mathrm{E}+04$ & $1.22 \mathrm{E}+02$ & $2.22 \mathrm{E}+04$ & $6.94 \mathrm{E}+03$ \\
\hline 33 & $2.01 \mathrm{E}+04$ & $5.67 \mathrm{E}+03$ & $1.59 \mathrm{E}+04$ & $2.60 \mathrm{E}+03$ & $2.25 \mathrm{E}+04$ & $1.25 \mathrm{E}+02$ & $2.21 \mathrm{E}+04$ & $6.91 \mathrm{E}+03$ \\
\hline 34 & $1.94 \mathrm{E}+04$ & $5.69 \mathrm{E}+03$ & $1.53 \mathrm{E}+04$ & $2.62 \mathrm{E}+03$ & $2.24 \mathrm{E}+04$ & $1.27 \mathrm{E}+02$ & $2.20 \mathrm{E}+04$ & $6.88 \mathrm{E}+03$ \\
\hline 35 & $1.77 \mathrm{E}+04$ & $5.72 \mathrm{E}+03$ & $1.47 \mathrm{E}+04$ & $2.63 \mathrm{E}+03$ & $2.22 \mathrm{E}+04$ & $1.29 \mathrm{E}+02$ & $2.19 \mathrm{E}+04$ & $6.85 \mathrm{E}+03$ \\
\hline 36 & $1.68 \mathrm{E}+04$ & $5.74 \mathrm{E}+03$ & $1.41 \mathrm{E}+04$ & $2.64 \mathrm{E}+03$ & $2.20 \mathrm{E}+04$ & $1 . \overline{32} \mathrm{E}+02$ & $2.19 \mathrm{E}+04$ & $6.83 \mathrm{E}+03$ \\
\hline 37 & $1.62 \mathrm{E}+04$ & $5.76 \mathrm{E}+03$ & $1.34 \mathrm{E}+04$ & $2.65 \mathrm{E}+03$ & $2.18 \mathrm{E}+04$ & $1 . \overline{34 E+02}$ & $2.18 \mathrm{E}+04$ & $6.80 \mathrm{E}+03$ \\
\hline 38 & $1.57 \mathrm{E}+04$ & $5.79 \mathrm{E}+03$ & $1.27 \mathrm{E}+04$ & $2.67 \mathrm{E}+03$ & $2.16 \mathrm{E}+04$ & $1.36 \mathrm{E}+02$ & $2.17 \mathrm{E}+04$ & $6.77 \mathrm{E}+03$ \\
\hline
\end{tabular}




\begin{tabular}{|c|c|c|c|c|c|c|c|c|}
\hline 39 & $1.52 \mathrm{E}+04$ & $5.80 \mathrm{E}+03$ & $1.21 \mathrm{E}+04$ & $2.68 \mathrm{E}+03$ & $2.15 \mathrm{E}+04$ & $1.38 \mathrm{E}+02$ & $2.16 \mathrm{E}+04$ & $6.75 \mathrm{E}+0$ \\
\hline 40 & $1.48 \mathrm{E}+04$ & $5.82 \mathrm{E}+03$ & $1.15 \mathrm{E}+04$ & $2.69 \mathrm{E}+03$ & $2.13 E+04$ & $1.41 \mathrm{E}+02$ & $2.15 E+04$ & $6.72 \mathrm{E}+0$ \\
\hline 41 & $1.45 \mathrm{E}+04$ & $5.84 \mathrm{E}+03$ & $1.10 \mathrm{E}+04$ & $2.70 \mathrm{E}+03$ & $2.11 \mathrm{E}+04$ & $1.43 \mathrm{E}+02$ & $2.14 \mathrm{E}+04$ & $6.69 \mathrm{E}+\mathrm{C}$ \\
\hline$\overline{42}$ & $1.41 \mathrm{E}+04$ & $5.86 \mathrm{E}+03$ & $1.05 \mathrm{E}+04$ & $2.70 \mathrm{E}+03$ & $2.11 \mathrm{E}+04$ & $1.49 \mathrm{E}+02$ & $2.05 \mathrm{E}+04$ & $1.09 \mathrm{E}+$ \\
\hline 43 & $1.37 \mathrm{E}+04$ & $5.88 \mathrm{E}+03$ & $1.01 \mathrm{E}+04$ & $2.71 \mathrm{E}+03$ & $2.11 \mathrm{E}+04$ & $1.55 \mathrm{E}+02$ & $2.04 \mathrm{E}+04$ & $1.09 \mathrm{E}$ \\
\hline 44 & $1.34 \bar{E}+04$ & $5.89 \mathrm{E}+03$ & $9.64 \mathrm{E}+03$ & $2.72 \mathrm{E}+03$ & $2.11 \mathrm{E}+04$ & $1.60 \mathrm{E}+02$ & $2.04 \mathrm{E}+04$ & $1.09 \mathrm{E}+$ \\
\hline 45 & $1.31 \mathrm{E}+04$ & $5.91 \mathrm{E}+03$ & $9.27 \mathrm{E}+03$ & $2.73 E+03$ & $2.11 \mathrm{E}+04$ & $1.66 \mathrm{E}+02$ & $2.04 \mathrm{E}+04$ & $1.09 \mathrm{E}+0$ \\
\hline 46 & $1.28 \mathrm{E}+04$ & $5.92 \mathrm{E}+03$ & $8.92 \mathrm{E}+03$ & $2.74 \mathrm{E}+03$ & $2 . \overline{10 E+04}$ & $1 . \overline{72} \overline{\mathrm{E}+02}$ & $2.04 \mathrm{E}+04$ & $1.09 \mathrm{E}+\mathrm{c}$ \\
\hline 47 & $1.25 \mathrm{E}+04$ & $5.94 \mathrm{E}+03$ & $8.60 \mathrm{E}+03$ & $2 . \overline{75 E}+03$ & $2.10 E+04$ & $1 . \overline{78 \bar{E}+02}$ & $2.04 \mathrm{E}+04$ & $1.09 \mathrm{E}+\mathrm{c}$ \\
\hline 48 & $1.13 \mathrm{E}+04$ & $5.95 \mathrm{E}+03$ & $8.30 \mathrm{E}+03$ & $2.75 \mathrm{E}+03$ & $2.10 \mathrm{E}+04$ & $1.84 \mathrm{E}+02$ & $2.04 \mathrm{E}+04$ & $1.09 \mathrm{E}+\mathrm{C}$ \\
\hline 49 & $1.04 \mathrm{E}+04$ & $5.96 \mathrm{E}+03$ & $7.92 \mathrm{E}+03$ & $2.76 \mathrm{E}+03$ & $2.10 \mathrm{E}+04$ & $1.90 \mathrm{E}+02$ & $2.04 \mathrm{E}+04$ & $1 . \overline{09 \mathrm{E}+0}$ \\
\hline 50 & $1.00 \mathrm{E}+04$ & $5.97 \mathrm{E}+03$ & $7.49 \mathrm{E}+03$ & $2.77 \mathrm{E}+03$ & $2.09 \mathrm{E}+04$ & $1.96 \mathrm{E}+02$ & $2.04 \mathrm{E}+04$ & $1.09 \mathrm{E}+0$ \\
\hline 51 & $9.77 \mathrm{E}+03$ & $5.98 \mathrm{E}+03$ & $7.05 \mathrm{E}+03$ & $2.77 \mathrm{E}+03$ & $2.09 \mathrm{E}+04$ & $2.02 \mathrm{E}+02$ & $2.04 \mathrm{E}+04$ & $1.09 \mathrm{E}+0$ \\
\hline 52 & $9.61 \mathrm{E}+03$ & $5.99 \mathrm{E}+03$ & $6.65 \mathrm{E}+03$ & $2.78 \mathrm{E}+03$ & $2.08 \mathrm{E}+04$ & $2.08 \mathrm{E}+02$ & $2.0 \overline{\mathrm{E}}+04$ & $1.09 \mathrm{E}+0$ \\
\hline 53 & $9.48 \mathrm{E}+03$ & $6.00 \mathrm{E}+03$ & $6.28 \mathrm{E}+03$ & $2.78 \mathrm{E}+03$ & $2.07 \mathrm{E}+04$ & $2.13 \overline{\mathrm{E}+02}$ & $2.03 \mathrm{E}+04$ & $1.09 \mathrm{E}+$ \\
\hline 54 & $9.36 \mathrm{E}+03$ & $6.01 \mathrm{E}+03$ & $5.96 \mathrm{E}+03$ & $2.79 \mathrm{E}+03$ & $2.06 \mathrm{E}+04$ & $2.15 \overline{\mathrm{E}+02}$ & $1.99 \mathrm{E}+04$ & $6.25 \mathrm{E}+03$ \\
\hline 55 & $9.25 \mathrm{E}+03$ & $6.02 \mathrm{E}+03$ & $5.68 \mathrm{E}+03$ & $2.79 \mathrm{E}+03$ & $2.04 \mathrm{E}+04$ & $2.18 \overline{E+02}$ & $1.99 \mathrm{E}+04$ & $6.22 \mathrm{E}+0$ \\
\hline 56 & $9.15 \mathrm{E}+03$ & $6.03 \mathrm{E}+03$ & $5.44 \mathrm{E}+03$ & $2.80 \mathrm{E}+03$ & $2.02 \mathrm{E}+04$ & $2.20 \overline{\mathrm{E}}+02$ & $1.98 \mathrm{E}+04$ & $6.20 \mathrm{E}+0$ \\
\hline 57 & $9.05 \mathrm{E}+03$ & $6.03 E+03$ & $5.24 \mathrm{E}+03$ & $2.80 \mathrm{E}+03$ & $2.01 E+04$ & $2.22 \mathrm{E}+02$ & $1.97 \mathrm{E}+04$ & $6.17 \mathrm{E}+0$ \\
\hline 58 & $8.95 \mathrm{E}+03$ & $6.04 E+03$ & $5.06 \mathrm{E}+03$ & $2.81 \mathrm{E}+03$ & $1.99 \mathrm{E}+04$ & $2.24 \mathrm{E}+02$ & $1.96 \mathrm{E}+04$ & $6.15 \mathrm{E}+03$ \\
\hline 59 & $8.81 \mathrm{E}+03$ & $6.05 \mathrm{E}+03$ & $4.91 \mathrm{E}+03$ & $2.81 \mathrm{E}+03$ & $1.97 \mathrm{E}+04$ & $2.25 \mathrm{E}+02$ & $1.95 \mathrm{E}+04$ & $6.12 \mathrm{E}+03$ \\
\hline 60 & $8.70 \mathrm{E}+03$ & $6.06 \mathrm{E}+03$ & $4.77 \mathrm{E}+03$ & $2.82 \mathrm{E}+03$ & $1.96 \mathrm{E}+04$ & $2.27 \mathrm{E}+02$ & $1.95 E+04$ & $6.10 \mathrm{E}+0$ \\
\hline 61 & $8.61 \mathrm{E}+03$ & $6.06 \mathrm{E}+03$ & $4.65 \mathrm{E}+03$ & $2.82 \mathrm{E}+03$ & $1.94 \mathrm{E}+04$ & $2.29 \mathrm{E}+02$ & $1.94 \mathrm{E}+04$ & $6.07 \mathrm{E}+03$ \\
\hline 62 & $8.48 E+03$ & $6.07 \mathrm{E}+03$ & $4.54 \mathrm{E}+03$ & $2.82 \mathrm{E}+03$ & $1.92 \mathrm{E}+04$ & $2.31 \bar{E}+02$ & $1.93 \mathrm{E}+04$ & $6.05 \mathrm{E}+03$ \\
\hline 63 & $8.33 \mathrm{E}+03$ & $6.08 \mathrm{E}+03$ & $4.43 \mathrm{E}+03$ & $2.83 \mathrm{E}+03$ & $1.91 \mathrm{E}+04$ & $2.33 \mathrm{E}+02$ & $1.92 \mathrm{E}+04$ & $6.02 \mathrm{E}+0$ \\
\hline 64 & $8.23 \mathrm{E}+03$ & $6.08 \mathrm{E}+03$ & $4.33 \mathrm{E}+03$ & $2.83 \mathrm{E}+03$ & $1.89 E+04$ & $2.35 \mathrm{E}+02$ & $1.91 \mathrm{E}+04$ & $6 . \overline{00 E+0}$ \\
\hline 65 & $8.16 \mathrm{E}+03$ & $6.09 \mathrm{E}+03$ & $4.24 \mathrm{E}+03$ & $2.84 \mathrm{E}+03$ & $1.88 \mathrm{E}+04$ & $2.37 \mathrm{E}+02$ & $1.91 \mathrm{E}+04$ & $5.98 \mathrm{E}+03$ \\
\hline 66 & $8.09 \mathrm{E}+03$ & $6.10 \mathrm{E}+03$ & $4.15 \mathrm{E}+03$ & $2.84 \mathrm{E}+03$ & $1.87 \mathrm{E}+04$ & $2.43 \mathrm{E}+02$ & $1.81 \mathrm{E}+04$ & $1.03 E+04$ \\
\hline 67 & $8.03 \mathrm{E}+03$ & $6.10 \mathrm{E}+03$ & $4.07 \bar{E}+03$ & $2.84 \mathrm{E}+03$ & $1.87 \mathrm{E}+04$ & $2.48 \mathrm{E}+02$ & $1.81 \mathrm{E}+04$ & $1.03 \mathrm{E}+0$ \\
\hline 68 & $7.97 \mathrm{E}+03$ & $6.11 \mathrm{E}+03$ & $4.00 \mathrm{E}+03$ & $2.85 \mathrm{E}+03$ & $1.86 \mathrm{E}+04$ & $2.54 \mathrm{E}+02$ & $1.81 \mathrm{E}+04$ & $1.03 \mathrm{E}+0$ \\
\hline 69 & $7.91 \mathrm{E}+03$ & $6.12 \mathrm{E}+03$ & $3.93 \mathrm{E}+03$ & $2.85 \mathrm{E}+03$ & $1.85 \mathrm{E}+04$ & $2.60 \mathrm{E}+02$ & $1.8 \mathrm{1E}+04$ & $1.03 \mathrm{E}+04$ \\
\hline 168 & $3.07 \mathrm{E}+03$ & $6.11 \mathrm{E}+03$ & $2.98 \mathrm{E}+03$ & $3.14 \mathrm{E}+03$ & $1.01 \mathrm{E}+04$ & $6.87 \mathrm{E}+02$ & $1.11 \mathrm{E}+04$ & $8.05 \mathrm{E}+03$ \\
\hline 336 & $1.58 \mathrm{E}+03$ & $5.16 \mathrm{E}+03$ & $1.55 \mathrm{E}+03$ & $3.36 \mathrm{E}+03$ & $4.40 \mathrm{E}+03$ & $1.23 \mathrm{E}+03$ & $6.63 \mathrm{E}+03$ & $6.13 \mathrm{E}+0$ \\
\hline 504 & $1.17 \mathrm{E}+03$ & $4.31 \mathrm{E}+03$ & $1.07 \mathrm{E}+03$ & $3.50 \mathrm{E}+03$ & $2.23 \mathrm{E}+03$ & $1.53 \mathrm{E}+03$ & $3.06 \mathrm{E}+03$ & $4.19 \mathrm{E}+03$ \\
\hline 720 & $8.50 \mathrm{E}+02$ & $3.40 \mathrm{E}+03$ & $7.18 \mathrm{E}+02$ & $3.62 \mathrm{E}+03$ & $1.15 \mathrm{E}+03$ & $1.69 \mathrm{E}+03$ & $1.56 \mathrm{E}+03$ & $2.94 \mathrm{E}+03$ \\
\hline 2160 & $1.60 \mathrm{E}+02$ & $6.75 \mathrm{E}+02$ & $8.09 \mathrm{E}+01$ & $3.86 \mathrm{E}+03$ & $1.03 \mathrm{E}+02$ & $9.99 \mathrm{E}+02$ & $3.60 \mathrm{E}+02$ & $1.02 E+03$ \\
\hline 4320 & $1.54 \mathrm{E}+01$ & $6.09 \mathrm{E}+01$ & $4.07 \mathrm{E}+02$ & $3.66 \mathrm{E}+03$ & $1.72 \mathrm{E}+01$ & $2.40 \mathrm{E}+02$ & $4.37 \mathrm{E}+01$ & $1.60 \mathrm{E}+02$ \\
\hline 4800 & $9.13 \mathrm{E}+00$ & $3.58 \mathrm{E}+01$ & $4.06 \mathrm{E}+02$ & $3.65 \mathrm{E}+03$ & $1.25 \mathrm{E}+01$ & $1.72 \mathrm{E}+02$ & $1.47 \mathrm{E}+01$ & $5.25 \mathrm{E}+01$ \\
\hline 8760 & $1.26 \mathrm{E}-01$ & $4.66 \mathrm{E}-01$ & $3.95 \mathrm{E}+02$ & $3.55 \mathrm{E}+03$ & $1.03 \mathrm{E}+00$ & $1.15 \mathrm{E}+01$ & $4.60 \mathrm{E}+00$ & $1.55 \mathrm{E}+01$ \\
\hline$\overline{\mathbf{B T}}$ & $\begin{array}{l}2.4 \mathrm{E} 6 \\
\mathrm{pCi} / \mathrm{L}\end{array}$ & $\begin{array}{l}8.88 \mathrm{E}+4 \\
\mathrm{~Bq} / \mathrm{kg}\end{array}$ & $8 \mathrm{~Bq} / \mathrm{g}$ & & & & & \\
\hline 0 & $\begin{array}{l}5.9 \mathrm{E} 6 \\
\mathrm{pCi} / \mathrm{L}\end{array}$ & $\begin{array}{l}2.18 \mathrm{E}+5 \\
\mathrm{~Bq} / \mathrm{kg}\end{array}$ & $8.3 \mathrm{~Bq} / \mathrm{g}$ & & & & & \\
\hline & & & & & & & & \\
\hline & \multicolumn{2}{|c|}{ VEGETABLES } & \multicolumn{2}{|c|}{ WHEAT SEED } & \multicolumn{2}{|l|}{ MEAT } & \multicolumn{2}{|l|}{ MILK } \\
\hline \multirow[t]{2}{*}{ HOUR } & $\mathrm{HTO}$ & $\overline{\mathrm{OBT}}$ & TO & $\mathrm{OBT}$ & HTO & $\mathrm{OBT}$ & HTO & $\overline{\mathrm{OBT}}$ \\
\hline & $(\mathrm{Bq} / \mathrm{g})$ & $(\overline{\mathrm{Bq}} / \mathrm{g})$ & $(\mathrm{Bq} / \mathrm{g})$ & $(\mathrm{Bq} / \mathrm{g})$ & $(\mathrm{Bq} / \mathrm{g})$ & $(\mathrm{Bq} / \mathrm{g})$ & $(\mathrm{Bq} / \mathrm{g})$ & $(\mathrm{Bq} / \mathrm{g})$ \\
\hline $\mathrm{ak}$ HTO & & & & & & & & \\
\hline
\end{tabular}


a Tritium Oxide Release from SRS Tritium Facilities (U)

\begin{tabular}{|c|c|c|c|c|c|c|c|c|}
\hline $2 / 4 / 6 / 6$ & $4 . \overline{89} \mathrm{E}+05$ & $2.53 \mathrm{E}+03$ & $2.36 \mathrm{E}+05$ & $1.21 \mathrm{E}+03$ & $2.36 \mathrm{E}+04$ & $1.93 \mathrm{E}+01$ & $2.29 \mathrm{E}+04$ & $7.12 \mathrm{E}+03$ \\
\hline SUM & $4 . \overline{92}+05$ & & $2.37 \mathrm{E}+05$ & & $2.36 \mathrm{E}+04$ & & $3.00 \mathrm{E}+04$ & \\
\hline$\overline{\mathrm{Ci} / \mathrm{g}}$ & $1.33 \mathrm{E}-05$ & & $6.41 \mathrm{E}-06$ & & $6.38 \mathrm{E}-07$ & & $8.11 E-07$ & \\
\hline & & & & & & & & \\
\hline DIL HTO & $5.9 \mathrm{E}-09$ & & & & & & & \\
\hline \multicolumn{9}{|l|}{$(\mathrm{Ci} / \mathrm{g})$} \\
\hline & & & & & & & & \\
\hline 168 & $3.07 \mathrm{E}+03$ & $6.11 \mathrm{E}+03$ & $2.98 \mathrm{E}+03$ & $3.14 \mathrm{E}+03$ & $1.01 \mathrm{E}+04$ & $6.87 \mathrm{E}+02$ & $1.11 \mathrm{E}+04$ & $8.05 \mathrm{E}+03$ \\
\hline SUM & $9.17 \mathrm{E}+03$ & & $6.12 \mathrm{E}+03$ & & $1.08 \mathrm{E}+04$ & & $1.92 \mathrm{E}+04$ & \\
\hline $\mathrm{Ci} / \mathrm{g}$ & $2.48 E-07$ & & $1.65 \mathrm{E}-07$ & & $2.92 \mathrm{E}-07$ & & $5.18 \mathrm{E}-07$ & \\
\hline & & & & & & & & \\
\hline DIL OBT & $2.4 \mathrm{E}-09$ & & & & & & & \\
\hline \multicolumn{9}{|l|}{$\mathrm{Ci} / \mathrm{g}$} \\
\hline & & & & & & & & \\
\hline 8760 & $1.26 \mathrm{E}-01$ & $4.66 \mathrm{E}-01$ & $3.95 \mathrm{E}+02$ & $3.55 \mathrm{E}+03$ & $1.03 \mathrm{E}+00$ & $1.15 \mathrm{E}+01$ & $4.60 \mathrm{E}+00$ & $1.55 \mathrm{E}+01$ \\
\hline SUM & $5.91 \mathrm{E}-01$ & & $3.95 \mathrm{E}+03$ & & $1.26 \mathrm{E}+01$ & & $2.01 \mathrm{E}+01$ & \\
\hline $\mathrm{Ci} / \mathrm{g}$ & $1.60 \mathrm{E}-11$ & & 1.07E-07 & & $3.40 \mathrm{E}-10$ & & $5.43 \mathrm{E}-10$ & \\
\hline & & & & & & & & \\
\hline DIL OBT & $2.4 \mathrm{E}-09$ & & & & & & & \\
\hline $\mathrm{Ci} / \mathrm{g}$ & & & & & & & & \\
\hline
\end{tabular}




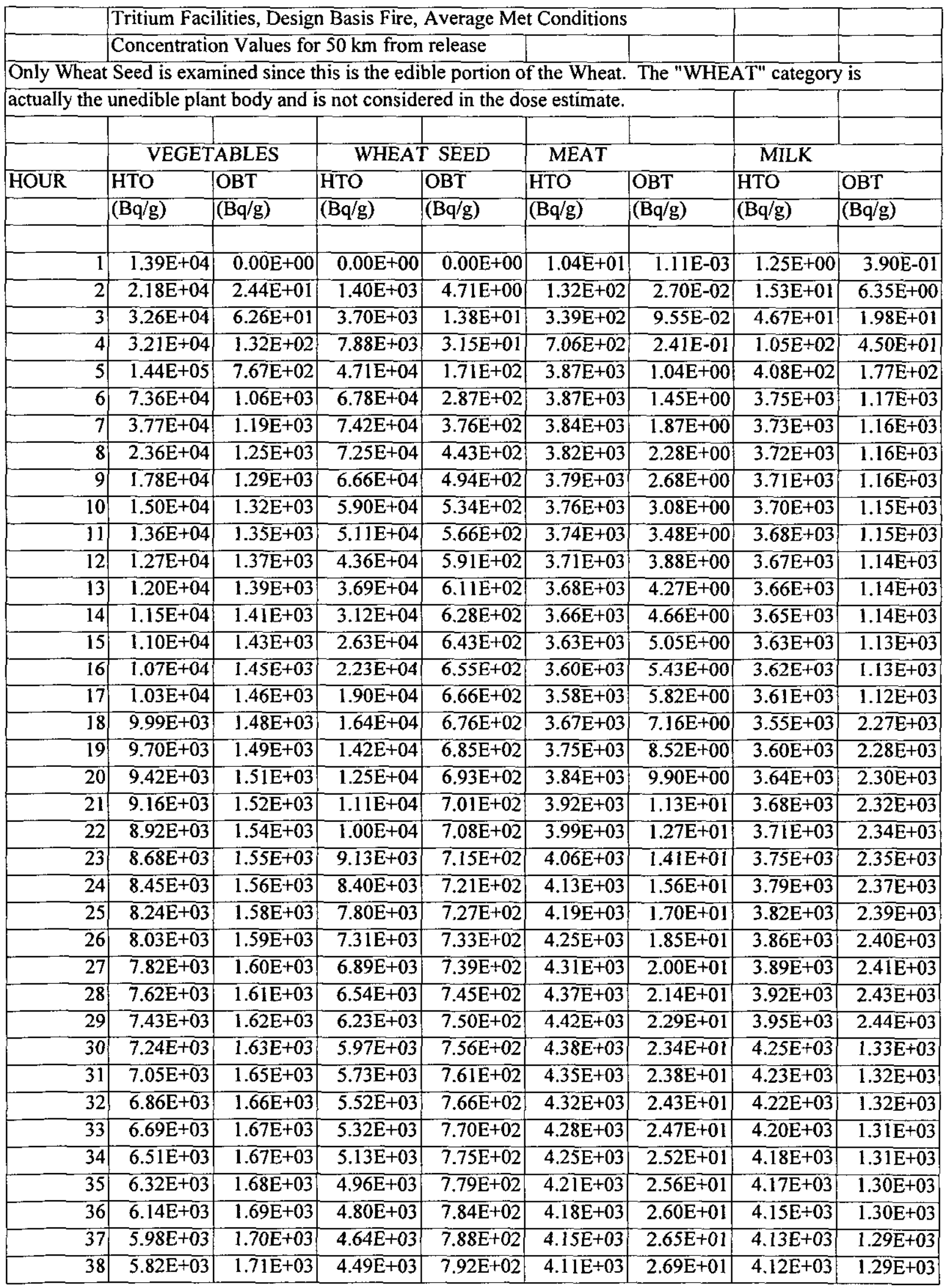




\begin{tabular}{|c|c|c|c|c|c|c|c|c|}
\hline 39 & $5.68 \mathrm{E}+03$ & $1.72 \mathrm{E}+03$ & $4.34 \mathrm{E}+03$ & $7.96 \mathrm{E}+02$ & $4.08 \mathrm{E}+03$ & $2.73 \mathrm{E}+01$ & $4.10 \mathrm{E}+03$ & $1.28 \mathrm{E}+03$ \\
\hline 40 & $5.54 \mathrm{E}+03$ & $1.73 \mathrm{E}+03$ & $4.21 \mathrm{E}+03$ & $8.00 \mathrm{E}+02$ & $4.05 \mathrm{E}+03$ & $2.77 \mathrm{E}+01$ & $4.09 \mathrm{E}+03$ & $1.28 \mathrm{E}+03$ \\
\hline 41 & $5.40 \mathrm{E}+03$ & $1.73 \mathrm{E}+03$ & $4.07 E+03$ & $8.03 E+02$ & $4.02 E+03$ & $2.81 \mathrm{E}+01$ & $4.07 \mathrm{E}+03$ & $1.27 \bar{E}+c$ \\
\hline 42 & $5.27 \mathrm{E}+03$ & $1.74 \mathrm{E}+03$ & $3.95 \mathrm{E}+03$ & $8.07 \mathrm{E}+02$ & $4.05 \mathrm{E}+03$ & $2.97 \mathrm{E}+01$ & $3.92 \mathrm{E}+03$ & $2.58 \mathrm{E}+03$ \\
\hline 43 & $5.15 \mathrm{E}+03$ & $1.75 \mathrm{E}+03$ & $3.82 \mathrm{E}+03$ & $8.10 \mathrm{E}+02$ & $4.07 \mathrm{E}+03$ & $3.12 \mathrm{E}+01$ & $3.94 \mathrm{E}+03$ & $2.58 \mathrm{E}+0$ \\
\hline 44 & $5.03 \mathrm{E}+03$ & $1.75 \mathrm{E}+03$ & $3.71 \overline{\mathrm{E}+03}$ & $8.14 \mathrm{E}+02$ & $4.10 \mathrm{E}+03$ & $3.27 \mathrm{E}+01$ & $3.95 \mathrm{E}+03$ & $2.59 \mathrm{E}+0$ \\
\hline 45 & $4.91 \mathrm{E}+03$ & $1.76 \mathrm{E}+03$ & $3.60 \mathrm{E}+03$ & $8.17 \mathrm{E}+02$ & $4.13 \mathrm{E}+03$ & $3.43 \mathrm{E}+01$ & $3.96 \mathrm{E}+03$ & $2.59 \mathrm{E}+03$ \\
\hline 46 & $4.80 \mathrm{E}+03$ & $1.77 \mathrm{E}+03$ & $3.49 E+03$ & $8.20 \mathrm{E}+02$ & $4.15 E+03$ & $3.58 \mathrm{E}+01$ & $3.97 E+03$ & $2.60 \mathrm{E}+0$ \\
\hline 47 & $4.70 \mathrm{E}+03$ & $1.77 \mathrm{E}+03$ & $3.39 \mathrm{E}+03$ & $8.23 \mathrm{E}+02$ & $4.17 \mathrm{E}+03$ & $3.74 \mathrm{E}+01$ & $3.99 \mathrm{E}+03$ & $2.61 \mathrm{E}+0$ \\
\hline 48 & $4.58 \mathrm{E}+03$ & $1.78 \mathrm{E}+03$ & $3.29 \mathrm{E}+03$ & $8.26 \mathrm{E}+02$ & $4.19 \mathrm{E}+03$ & $3.89 \mathrm{E}+01$ & $4.00 \mathrm{E}+03$ & $2.61 \mathrm{E}+03$ \\
\hline 49 & $4.45 \mathrm{E}+03$ & $1.78 \mathrm{E}+03$ & $3.19 \mathrm{E}+03$ & $8.29 \mathrm{E}+02$ & $4.21 \mathrm{E}+03$ & $4.05 \mathrm{E}+01$ & $4.01 \mathrm{E}+03$ & $2.62 \mathrm{E}+03$ \\
\hline 50 & $4.35 \mathrm{E}+03$ & $1.79 \mathrm{E}+03$ & $3.10 \mathrm{E}+03$ & $8 . \overline{32 E+02}$ & $4.23 E+03$ & $4.20 \mathrm{E}+01$ & $4.02 \mathrm{E}+03$ & $2.62 \mathrm{E}+0$ \\
\hline 51 & $4.25 E+03$ & $1.79 \mathrm{E}+03$ & $3.01 E+03$ & $8.35 E+02$ & $4.25 \mathrm{E}+03$ & $4.36 \mathrm{E}+01$ & $4.03 E+03$ & $2.62 \mathrm{E}+03$ \\
\hline 52 & $4.17 \mathrm{E}+03$ & $1.80 \mathrm{E}+03$ & $2.92 \mathrm{E}+03$ & $8.37 \mathrm{E}+02$ & $4.26 \mathrm{E}+03$ & $4.52 \mathrm{E}+01$ & $4.04 \mathrm{E}+03$ & $2.63 \mathrm{E}+03$ \\
\hline 53 & $4.09 \mathrm{E}+03$ & $1.80 \mathrm{E}+03$ & $2.83 \mathrm{E}+03$ & $8.40 \mathrm{E}+02$ & $4.27 \mathrm{E}+03$ & $4.67 \mathrm{E}+01$ & $4.05 \mathrm{E}+03$ & $2.63 \mathrm{E}+0$ \\
\hline 54 & $4.02 \mathrm{E}+03$ & $1.81 \mathrm{E}+03$ & $2.75 \mathrm{E}+03$ & $8.43 \mathrm{E}+02$ & $4.24 \mathrm{E}+03$ & $4.72 \mathrm{E}+0 \mathrm{l}$ & $4.11 \mathrm{E}+03$ & $1.29 \mathrm{E}+03$ \\
\hline 55 & $3.96 \mathrm{E}+03$ & $1.81 \mathrm{E}+03$ & $2.68 \mathrm{E}+03$ & $8.45 \mathrm{E}+02$ & $4.20 \mathrm{E}+03$ & $4.76 \mathrm{E}+01$ & $4.09 \mathrm{E}+03$ & $1.28 \mathrm{E}+03$ \\
\hline 56 & $3.89 \mathrm{E}+03$ & $1.82 \mathrm{E}+03$ & $2.61 \mathrm{E}+03$ & $8.47 \mathrm{E}+02$ & $4.17 \mathrm{E}+03$ & $4.80 \mathrm{E}+01$ & $4.08 \mathrm{E}+03$ & $1.28 \mathrm{E}+0$ \\
\hline 57 & $3.83 \mathrm{E}+03$ & $1.82 \mathrm{E}+03$ & $2.54 \mathrm{E}+03$ & $8.50 \mathrm{E}+02$ & $4.14 \mathrm{E}+03$ & $4.84 \mathrm{E}+01$ & $4.06 \mathrm{E}+03$ & $1.27 \overline{\mathrm{E}+03}$ \\
\hline 58 & $3.78 \mathrm{E}+03$ & $1.83 \mathrm{E}+03$ & $2.48 \mathrm{E}+03$ & $8.52 \mathrm{E}+02$ & $4.10 \mathrm{E}+03$ & $4.88 \mathrm{E}+01$ & $4.04 \mathrm{E}+03$ & $1.27 \mathrm{E}+03$ \\
\hline 59 & $3.71 \mathrm{E}+03$ & $1.83 \mathrm{E}+03$ & $2.42 \mathrm{E}+03$ & $8.54 \mathrm{E}+02$ & $4.07 \mathrm{E}+03$ & $4.92 \mathrm{E}+01$ & $4.03 \mathrm{E}+03$ & $1.26 \mathrm{E}+03$ \\
\hline 60 & $3.65 \mathrm{E}+03$ & $1.83 \mathrm{E}+03$ & $2.36 \mathrm{E}+03$ & $8.57 \mathrm{E}+02$ & $4.04 \mathrm{E}+03$ & $4.96 \mathrm{E}+01$ & $4.01 \mathrm{E}+03$ & $1.26 \mathrm{E}+03$ \\
\hline 61 & $3.60 \mathrm{E}+03$ & $1.84 \mathrm{E}+03$ & $2.31 \mathrm{E}+03$ & $8.59 \mathrm{E}+02$ & $4.00 \mathrm{E}+03$ & $5.00 \mathrm{E}+01$ & $3.99 \mathrm{E}+03$ & $1.25 \mathrm{E}+03$ \\
\hline 62 & $3.54 \mathrm{E}+03$ & $1.84 \mathrm{E}+03$ & $2.26 \mathrm{E}+03$ & $8.61 \mathrm{E}+02$ & $3.97 \mathrm{E}+03$ & $5.04 \mathrm{E}+01$ & $3.98 \mathrm{E}+03$ & $1.25 \mathrm{E}+03$ \\
\hline 63 & $3.48 \mathrm{E}+03$ & $1.85 \mathrm{E}+03$ & $2.21 \mathrm{E}+03$ & $8.63 E+02$ & $3.94 \mathrm{E}+03$ & $5.08 \mathrm{E}+01$ & $3.96 \mathrm{E}+03$ & $1.24 \mathrm{E}+0$ \\
\hline 64 & $3.43 E+03$ & $1 . \overline{85} \mathrm{E}+03$ & $2.16 \mathrm{E}+03$ & $8.65 \mathrm{E}+02$ & $3.90 \mathrm{E}+03$ & $5.12 \mathrm{E}+01$ & $3.94 E+03$ & $1.24 \mathrm{E}+03$ \\
\hline 65 & $3.38 \mathrm{E}+03$ & $1.85 \mathrm{E}+03$ & $2.12 \mathrm{E}+03$ & $8.67 \mathrm{E}+02$ & $3.87 \mathrm{E}+03$ & $5.15 \mathrm{E}+01$ & $3.93 \mathrm{E}+03$ & $1 . \overline{23 E}+03$ \\
\hline 66 & $3.34 \mathrm{E}+03$ & $1 . \overline{86} \mathrm{E}+03$ & $2.07 \mathrm{E}+03$ & $8.69 \mathrm{E}+02$ & $3.88 \mathrm{E}+03$ & $5.31 \mathrm{E}+01$ & $3.76 \mathrm{E}+03$ & $2.60 \mathrm{E}+03$ \\
\hline 67 & $3.30 \mathrm{E}+03$ & $1.86 \mathrm{E}+03$ & $2.03 E+03$ & $8 . \overline{71 E}+02$ & $3.89 \mathrm{E}+03$ & $5.46 \mathrm{E}+01$ & $3.77 \mathrm{E}+03$ & $2.60 \mathrm{E}+03$ \\
\hline 68 & $3.26 \mathrm{E}+03$ & $1.86 \mathrm{E}+03$ & $1.99 \mathrm{E}+03$ & $8 . \overline{72} \mathrm{E}+02$ & $3.90 \mathrm{E}+03$ & $5.62 \mathrm{E}+01$ & $3.77 \mathrm{E}+03$ & $2.61 \mathrm{E}+0$ \\
\hline 69 & $3.23 E+03$ & $1 . \overline{87} \mathrm{E}+03$ & $1.95 E+03$ & $8 . \overline{74 E}+02$ & $3.90 \mathrm{E}+03$ & $5.78 \mathrm{E}+01$ & $3.77 \mathrm{E}+03$ & $2.61 \mathrm{E}+03$ \\
\hline 168 & $9.43 \mathrm{E}+02$ & $1.93 \mathrm{E}+03$ & $9.44 \mathrm{E}+02$ & $9 . \overline{96 \mathrm{E}+02}$ & $2.55 \mathrm{E}+03$ & $1.81 \mathrm{E}+02$ & $2.73 \mathrm{E}+03$ & $2.30 \mathrm{E}+03$ \\
\hline 336 & $4.69 \mathrm{E}+02$ & $1.62 \mathrm{E}+03$ & $4.76 \mathrm{E}+02$ & $1.07 \mathrm{E}+03$ & $1.22 \mathrm{E}+03$ & $3.51 \mathrm{E}+02$ & $1.75 \mathrm{E}+03$ & $1.83 \mathrm{E}+03$ \\
\hline 504 & $3.45 \mathrm{E}+02$ & $1.35 \mathrm{E}+03$ & $3.27 \mathrm{E}+02$ & $1.11 \mathrm{E}+03$ & $6.55 \mathrm{E}+02$ & $4.48 \mathrm{E}+02$ & $8.74 \mathrm{E}+02$ & $1.29 \overline{\mathrm{E}+03}$ \\
\hline 720 & $2.51 \mathrm{E}+02$ & $1.06 \mathrm{E}+03$ & $2.19 \mathrm{E}+02$ & $1.15 \mathrm{E}+03$ & $3.49 \mathrm{E}+02$ & $5.04 \mathrm{E}+02$ & $4.67 \mathrm{E}+02$ & $9.15 \mathrm{E}+02$ \\
\hline 2160 & $4.70 \mathrm{E}+01$ & $2.08 \mathrm{E}+02$ & $2.46 \mathrm{E}+01$ & $1.22 \mathrm{E}+03$ & $3.11 \mathrm{E}+01$ & $3.04 \mathrm{E}+02$ & $1.11 \mathrm{E}+02$ & $3.16 \mathrm{E}+02$ \\
\hline 4320 & $4.52 \mathrm{E}+00$ & $1.84 \mathrm{E}+01$ & $1.24 \mathrm{E}+02$ & $1.16 \mathrm{E}+03$ & $5.11 \mathrm{E}+00$ & $7.29 \mathrm{E}+01$ & $1.31 \mathrm{E}+01$ & $4.88 \mathrm{E}+01$ \\
\hline 4800 & $2.69 \mathrm{E}+00$ & $1.08 \mathrm{E}+01$ & $1.23 \mathrm{E}+02$ & $1.15 \mathrm{E}+03$ & $3.71 \mathrm{E}+00$ & $5.25 \mathrm{E}+01$ & $4.36 \mathrm{E}+00$ & $1 . \overline{59 \mathrm{E}+01}$ \\
\hline 8760 & $3.69 \mathrm{E}-02$ & $1.38 \mathrm{E}-01$ & $1.20 \mathrm{E}+02$ & $1.12 \mathrm{E}+03$ & $3.03 \mathrm{E}-01$ & $3.49 \mathrm{E}+00$ & $1.36 \mathrm{E}+00$ & $4.67 \mathrm{E}+0 \mathrm{C}$ \\
\hline BT & & $\begin{array}{l}8.88 \mathrm{E}+4 \\
\mathrm{~Bq} / \mathrm{kg}\end{array}$ & $8 \mathrm{~Bq} / \mathrm{g}$ & & & & & \\
\hline $\bar{O}$ & $\begin{array}{l}5.9 \mathrm{E} 6 \\
\mathrm{pCi} / \mathrm{L}\end{array}$ & $\begin{array}{l}2.18 \mathrm{E}+5 \\
\mathrm{~Bq} / \mathrm{kg}\end{array}$ & $218.3 \mathrm{~Bq} / \mathrm{g}$ & & & & & \\
\hline & \multirow{2}{*}{\multicolumn{2}{|c|}{ VEGETABLES }} & & & & & & \\
\hline & & & \multicolumn{2}{|c|}{ WHEAT SEED } & \multicolumn{2}{|l|}{ MEAT } & \multicolumn{2}{|l|}{ MILK } \\
\hline \multirow[t]{2}{*}{ HOUR } & $\mathrm{O}$ & $\mathrm{OBT}$ & ro & OBT & HTO & $\mathrm{OBT}$ & HTO & $\overline{\mathrm{OBT}}$ \\
\hline & $(\mathrm{Bq} / \mathrm{g})$ & $(\mathrm{Bq} / \mathrm{g})$ & $(\mathrm{Bq} / \mathrm{g})$ & $(\mathrm{Bq} / \mathrm{g})$ & $(\mathrm{Bq} / \mathrm{g})$ & $(\mathrm{Bq} / \mathrm{g})$ & $(\mathrm{Bq} / \mathrm{g})$ & $(\mathrm{Bq} / \mathrm{g})$ \\
\hline TO & & & & & & & & \\
\hline
\end{tabular}


Foodstuff Concentrations and Relocation Considerations Following

a Tritium Oxide Release from SRS Tritium Facilities (U)

\begin{tabular}{|c|c|c|c|c|c|c|c|c|}
\hline $5 / 11 / 29 / 30$ & $1.44 \mathrm{E}+05$ & $7.67 \mathrm{E}+02$ & $7.25 \overline{\mathrm{E}}+04$ & $4 . \overline{43 E+02}$ & $4.42 \mathrm{E}+03$ & $2.29 \mathrm{E}+01$ & $4.25 E+03$ & $1.33 \mathrm{E}+03$ \\
\hline$\overline{\mathrm{SUM}}$ & $1.45 \mathrm{E}+05$ & & $7.29 \overline{\mathrm{E}+04}$ & & $4.44 \mathrm{E}+03$ & & $5.58 \mathrm{E}+03$ & \\
\hline $\mathrm{Ci} / \mathrm{g}$ & $3.92 \mathrm{E}-06$ & & $1.97 \mathrm{E}-06$ & & $1.20 \mathrm{E}-07$ & & $1.51 \mathrm{E}-07$ & \\
\hline & & & & & & & & \\
\hline DIL HTO & $5.9 \mathrm{E}-09$ & & & & & & & \\
\hline \multicolumn{9}{|l|}{$(\mathrm{Ci} / \mathrm{g})$} \\
\hline & & & & & & & & \\
\hline 168 & $9.43 \mathrm{E}+02$ & $1.93 E+03$ & $9.44 \bar{E}+02$ & $9 . \overline{96 \mathrm{E}+02}$ & $2.55 \mathrm{E}+03$ & $1.81 \mathrm{E}+02$ & $2.73 E+03$ & $2.30 \overline{\mathrm{E}}+03$ \\
\hline SUM & $2.87 \mathrm{E}+03$ & & $1.94 \overline{\mathrm{E}+03}$ & & $2.73 \mathrm{E}+03$ & & $5.03 \mathrm{E}+03$ & \\
\hline $\mathrm{Ci} / \mathrm{g}$ & $7.75 \mathrm{E}-08$ & & $5.24 \mathrm{E}-08$ & & $7.38 \mathrm{E}-08$ & & $1.36 \mathrm{E}-07$ & \\
\hline & & & & & & & & \\
\hline DIL OBT & $2.4 \mathrm{E}-09$ & & & & & & & \\
\hline \multicolumn{9}{|l|}{$\mathrm{Ci} / \mathrm{g}$} \\
\hline & & & & & & & & \\
\hline 8760 & $3.69 \mathrm{E}-02$ & $1.38 \mathrm{E}-01$ & $1.20 \mathrm{E}+02$ & $1.12 \mathrm{E}+03$ & $3.03 \mathrm{E}-01$ & $3.49 \mathrm{E}+00$ & $1.36 \mathrm{E}+00$ & $4.67 \overline{\mathrm{E}+00}$ \\
\hline SUM & $1.75 \mathrm{E}-01$ & & $1.24 \mathrm{E}+03$ & & $3.79 \mathrm{E}+00$ & & $6.03 E+00$ & \\
\hline $\mathrm{Ci} / \mathrm{g}$ & $4.73 \mathrm{E}-12$ & & $3.36 \mathrm{E}-08$ & & $1.03 \mathrm{E}-10$ & & $1.63 \mathrm{E}-10$ & \\
\hline & & & & & & & & \\
\hline DIL OBT & $2.4 \mathrm{E}-09$ & & & & & & & \\
\hline $\mathrm{Ci} / \mathrm{g}$ & & & & & & & & \\
\hline
\end{tabular}




\begin{tabular}{|c|c|c|c|c|c|c|c|c|}
\hline & \multicolumn{6}{|c|}{ Tritium Facilities, Design Basis Fire, Average Met Conditions } & & \\
\hline & \multicolumn{4}{|c|}{ Concentration Values for $100 \mathrm{~km}$ from release } & & & & \\
\hline \multicolumn{9}{|c|}{ Only Wheat Seed is examined since this is the edible portion of the Wheat. The "WHEAT" category is } \\
\hline \multicolumn{7}{|c|}{ actually the unedible plant body and is not considered in the dose estimate. } & & \\
\hline & & & & & & & & \\
\hline & \multicolumn{2}{|c|}{ VEGETABLES } & \multicolumn{2}{|c|}{ WHEAT SEED } & \multicolumn{2}{|l|}{ MEAT } & \multicolumn{2}{|l|}{ MILK } \\
\hline \multirow[t]{3}{*}{ HOUR } & HTO & OBT & HTO & $\overline{\mathrm{OBT}}$ & HTO & OBT & HTO & $\overline{\mathrm{OBT}}$ \\
\hline & $(\overline{B q} / g)$ & $(\mathrm{Bq} / \mathrm{g})$ & $(\mathrm{Bq} / \mathrm{g})$ & $(\mathrm{Bq} / \mathrm{g})$ & $(\mathrm{Bq} / \mathrm{g})$ & $(\mathrm{Bq} / \mathrm{g})$ & q/g) & $3 q / g)$ \\
\hline & & & & & & & & \\
\hline 1 & $2.19 \mathrm{E}+03$ & $0.00 \mathrm{E}+00$ & $0.00 \mathrm{E}+00$ & $0.00 \mathrm{E}+00$ & $1.62 \mathrm{E}+00$ & $1.75 \mathrm{E}-04$ & $1.97 \mathrm{E}-01$ & $6.12 \mathrm{E}-02$ \\
\hline 2 & $3.98 \mathrm{E}+03$ & $3.83 \mathrm{E}+00$ & $2.19 \mathrm{E}+02$ & $7.39 \mathrm{E}-01$ & $2.11 \mathrm{E}+01$ & $4.28 \mathrm{E}-03$ & $2.45 \mathrm{E}+00$ & $1.01 \mathrm{E}+00$ \\
\hline 3 & $6.57 \mathrm{E}+03$ & $1.08 \mathrm{E}+01$ & $6.36 \mathrm{E}+02$ & $2.36 \mathrm{E}+00$ & $5.88 \mathrm{E}+01$ & $1.6 \overline{\mathrm{E}-02}$ & $7.91 \mathrm{E}+00$ & $3.35 \mathrm{E}+00$ \\
\hline 4 & $6.98 \mathrm{E}+03$ & $2.23 \mathrm{E}+01$ & $1.32 \mathrm{E}+03$ & $5.28 \mathrm{E}+00$ & $1.20 \overline{\mathrm{E}+02}$ & $4.07 \mathrm{E}-02$ & $1.78 \mathrm{E}+01$ & $7.63 \mathrm{E}+00$ \\
\hline 5 & $1.05 \mathrm{E}+04$ & $3.45 E+01$ & $2.03 E+03$ & $8.98 \mathrm{E}+00$ & $1.92 \mathrm{E}+02$ & $8.02 \mathrm{E}-02$ & $3.18 \mathrm{E}+01$ & $1.37 \mathrm{E}+01$ \\
\hline 6 & $1.02 \mathrm{E}+04$ & $5.28 \mathrm{E}+01$ & $3.04 \mathrm{E}+03$ & $1.44 \mathrm{E}+01$ & $1.95 \mathrm{E}+02$ & $1.01 \mathrm{E}-01$ & $1.89 \mathrm{E}+02$ & $5.89 \mathrm{E}+01$ \\
\hline 7 & $1.36 \mathrm{E}+04$ & $7.06 \mathrm{E}+01$ & $3.98 \mathrm{E}+03$ & $2.05 \mathrm{E}+01$ & $2.01 \mathrm{E}+02$ & $1.23 \mathrm{E}-01$ & $1.92 \mathrm{E}+02$ & $5.98 \mathrm{E}+01$ \\
\hline 8 & $1.45 \mathrm{E}+04$ & $9.53 \mathrm{E}+01$ & $5.22 \mathrm{E}+03$ & $2.86 \mathrm{E}+01$ & $2.07 \mathrm{E}+02$ & $1.45 \mathrm{E}-01$ & $1.95 \mathrm{E}+02$ & $6.06 \mathrm{E}+01$ \\
\hline 9 & $3.68 \mathrm{E}+04$ & $2.37 \mathrm{E}+02$ & $1.38 \mathrm{E}+04$ & $6.26 \mathrm{E}+01$ & $2.61 \mathrm{E}+02$ & $1.73 \mathrm{E}-01$ & $2.09 \mathrm{E}+02$ & $6.52 \mathrm{E}+01$ \\
\hline 10 & $5.65 \mathrm{E}+04$ & $4.78 \mathrm{E}+02$ & $2.91 \mathrm{E}+04$ & $1.28 \mathrm{E}+02$ & $3.39 \mathrm{E}+02$ & $2.09 \mathrm{E}-01$ & $2.33 \mathrm{E}+02$ & $7.26 \mathrm{E}+01$ \\
\hline 11 & $2.68 \mathrm{E}+04$ & $5.76 \mathrm{E}+02$ & $3.5 \overline{\mathrm{E}}+04$ & $1.77 \mathrm{E}+02$ & $3.41 \mathrm{E}+02$ & $2.46 \mathrm{E}-01$ & $2 . \overline{49 E+02}$ & $7.77 \mathrm{E}+01$ \\
\hline 12 & $1.56 \mathrm{E}+04$ & $6.22 \mathrm{E}+02$ & $3.63 \mathrm{E}+04$ & $2.15 \mathrm{E}+02$ & $3.42 \mathrm{E}+02$ & $2.82 \mathrm{E}-01$ & $2.61 \mathrm{E}+02$ & $8.14 \mathrm{E}+01$ \\
\hline 13 & $1.12 \mathrm{E}+04$ & $6.49 \mathrm{E}+02$ & $3.44 \mathrm{E}+04$ & $2.45 \mathrm{E}+02$ & $3.43 \mathrm{E}+02$ & $3.19 \mathrm{E}-01$ & $2.70 \mathrm{E}+02$ & $8.41 \mathrm{E}+01$ \\
\hline 14 & $9.23 \mathrm{E}+03$ & $6.67 \mathrm{E}+02$ & $3.12 \mathrm{E}+04$ & $2.68 \mathrm{E}+02$ & $3.43 \mathrm{E}+02$ & $3.56 \mathrm{E}-01$ & $2.77 \mathrm{E}+02$ & $8.63 \mathrm{E}+01$ \\
\hline 15 & $8.29 \mathrm{E}+03$ & $6.83 \mathrm{E}+02$ & $2.74 \mathrm{E}+04$ & $2.86 \mathrm{E}+02$ & $3.44 \mathrm{E}+02$ & $3.93 \mathrm{E}-01$ & $2.83 \mathrm{E}+02$ & $8.81 \mathrm{E}+01$ \\
\hline 16 & $7.75 \mathrm{E}+03$ & $6.96 \mathrm{E}+02$ & $2.38 \mathrm{E}+04$ & $3.00 \mathrm{E}+02$ & $3 . \overline{44 \mathrm{E}+02}$ & $4.29 \mathrm{E}-0 \mathrm{I}$ & $2.87 \mathrm{E}+02$ & $8.95 \mathrm{E}+01$ \\
\hline 17 & $7.39 \mathrm{E}+03$ & $7.09 \mathrm{E}+02$ & $2.04 \mathrm{E}+04$ & $3.12 \mathrm{E}+02$ & $3.43 \mathrm{E}+02$ & $4.66 \mathrm{E}-01$ & $2.91 \mathrm{E}+02$ & $9.07 \mathrm{E}+01$ \\
\hline 18 & $7.13 \mathrm{E}+03$ & $7.21 \mathrm{E}+02$ & $1.74 \mathrm{E}+04$ & $3.23 \mathrm{E}+02$ & $4.32 \mathrm{E}+02$ & $9.75 \mathrm{E}-01$ & $4.19 \mathrm{E}+02$ & $6.92 \mathrm{E}+02$ \\
\hline 19 & $6.91 \mathrm{E}+03$ & $7.32 \mathrm{E}+02$ & $1.49 \mathrm{E}+04$ & $3.31 \mathrm{E}+02$ & $5.15 \mathrm{E}+02$ & $1.50 \mathrm{E}+00$ & $4.59 \mathrm{E}+02$ & $7.09 \mathrm{E}+02$ \\
\hline 20 & $6.72 \mathrm{E}+03$ & $7.44 \mathrm{E}+02$ & $1.28 \mathrm{E}+04$ & $3.39 \mathrm{E}+02$ & $5.92 \mathrm{E}+02$ & $2.04 \mathrm{E}+00$ & $4.97 \mathrm{E}+02$ & $7.25 \mathrm{E}+02$ \\
\hline 21 & $6.56 \mathrm{E}+03$ & $7.54 \mathrm{E}+02$ & $1.11 \mathrm{E}+04$ & $3.46 \mathrm{E}+02$ & $6.66 \mathrm{E}+02$ & $2.59 \mathrm{E}+00$ & $5.34 \mathrm{E}+02$ & $7.41 \mathrm{E}+02$ \\
\hline 22 & $6.41 \mathrm{E}+03$ & $7.65 \mathrm{E}+02$ & $9.65 \mathrm{E}+03$ & $3.52 \mathrm{E}+02$ & $7.37 \mathrm{E}+02$ & $3.16 \mathrm{E}+00$ & $5.70 \mathrm{E}+02$ & $7.56 \mathrm{E}+02$ \\
\hline 23 & $6.27 E+03$ & $7.75 \mathrm{E}+02$ & $8.52 \mathrm{E}+03$ & $3.57 \mathrm{E}+02$ & $8.05 E+02$ & $3.7 \bar{E}+00$ & $6.05 E+02$ & $7.71 E+02$ \\
\hline 24 & $6.14 \mathrm{E}+03$ & $7.85 \mathrm{E}+02$ & $7.61 \mathrm{E}+03$ & $3.63 \mathrm{E}+02$ & $8.70 \mathrm{E}+02$ & $4.33 \mathrm{E}+00$ & $6.39 \mathrm{E}+02$ & $7.85 \mathrm{E}+02$ \\
\hline 25 & $6.01 \mathrm{E}+03$ & $7.95 \mathrm{E}+02$ & $6.87 \mathrm{E}+03$ & $3.68 \mathrm{E}+02$ & $9.34 \mathrm{E}+02$ & $4.94 \mathrm{E}+00$ & $6.72 \mathrm{E}+02$ & $7.99 \mathrm{E}+02$ \\
\hline 26 & $5.89 \mathrm{E}+03$ & $8.04 E+02$ & $6.29 \mathrm{E}+03$ & $3.73 E+02$ & $9.95 \mathrm{E}+02$ & $5.56 \mathrm{E}+00$ & $7.05 \mathrm{E}+02$ & $8.13 E+02$ \\
\hline 27 & $5.77 \mathrm{E}+03$ & $8.13 \mathrm{E}+02$ & $5.81 \mathrm{E}+03$ & $3.77 \mathrm{E}+02$ & $1.06 \mathrm{E}+03$ & $6.18 \mathrm{E}+00$ & $7.37 \mathrm{E}+02$ & $8.27 \mathrm{E}+02$ \\
\hline 28 & $5.66 \mathrm{E}+03$ & $8.22 \mathrm{E}+02$ & $5.43 \mathrm{E}+03$ & $3.82 \mathrm{E}+02$ & $1.11 \mathrm{E}+03$ & $6.82 \mathrm{E}+00$ & $7.68 \mathrm{E}+02$ & $8.40 \mathrm{E}+02$ \\
\hline 29 & $5.55 E+03$ & $8.31 \mathrm{E}+02$ & $5.11 \mathrm{E}+03$ & $3.86 \mathrm{E}+02$ & $1.17 \mathrm{E}+03$ & $7.47 \mathrm{E}+00$ & $7.98 \mathrm{E}+02$ & $8.53 \mathrm{E}+02$ \\
\hline 30 & $5.44 \mathrm{E}+03$ & $8.40 \mathrm{E}+02$ & $4.85 \mathrm{E}+03$ & $3.90 \mathrm{E}+02$ & $1.16 \mathrm{E}+03$ & $7.59 \mathrm{E}+00$ & $1.13 \mathrm{E}+03$ & $3.52 \mathrm{E}+02$ \\
\hline 31 & $5.33 \mathrm{E}+03$ & $8.48 \mathrm{E}+02$ & $4.64 \mathrm{E}+03$ & $3.94 \mathrm{E}+02$ & $1.15 \mathrm{E}+03$ & $7.71 \mathrm{E}+00$ & $1.12 \mathrm{E}+03$ & $3.51 \mathrm{E}+02$ \\
\hline 32 & $5.22 \mathrm{E}+03$ & $8.56 \mathrm{E}+02$ & $4.45 \mathrm{E}+03$ & $3.98 \mathrm{E}+02$ & $1.15 \mathrm{E}+03$ & $7.83 \mathrm{E}+00$ & $1.12 \mathrm{E}+03$ & $3.49 \mathrm{E}+02$ \\
\hline 33 & $5.11 \mathrm{E}+03$ & $8.64 \mathrm{E}+02$ & $4.29 \mathrm{E}+03$ & $4.02 \mathrm{E}+02$ & $1.14 \mathrm{E}+03$ & $7.94 \mathrm{E}+00$ & $1.11 \mathrm{E}+03$ & $3.48 \mathrm{E}+02$ \\
\hline 34 & $5.00 \mathrm{E}+03$ & $8.72 \mathrm{E}+02$ & $4.15 \mathrm{E}+03$ & $4.06 \mathrm{E}+02$ & $1.13 \mathrm{E}+03$ & $8.06 \mathrm{E}+00$ & $1.11 \mathrm{E}+03$ & $3.47 \mathrm{E}+02$ \\
\hline 35 & $4.89 \mathrm{E}+03$ & $8.80 \mathrm{E}+02$ & $4.02 \mathrm{E}+03$ & $4.09 \mathrm{E}+02$ & $1.12 \mathrm{E}+03$ & $8.18 \mathrm{E}+00$ & $1.11 \mathrm{E}+03$ & $3.46 \mathrm{E}+02$ \\
\hline 36 & $4.78 \mathrm{E}+03$ & $8.87 \mathrm{E}+02$ & $3.90 \mathrm{E}+03$ & $4.13 \mathrm{E}+02$ & $1.11 \mathrm{E}+03$ & $8.29 \mathrm{E}+00$ & $1.10 \mathrm{E}+03$ & $3.45 \mathrm{E}+02$ \\
\hline 37 & $4.68 \mathrm{E}+03$ & $8.94 \mathrm{E}+02$ & $3.79 \mathrm{E}+03$ & $4.16 \mathrm{E}+02$ & $1.11 \mathrm{E}+03$ & $8.40 \mathrm{E}+00$ & $1.10 \mathrm{E}+03$ & $3.44 \mathrm{E}+02$ \\
\hline 38 & $4.57 \mathrm{E}+03$ & $9.01 \mathrm{E}+02$ & $3.69 \mathrm{E}+03$ & $4.20 \mathrm{E}+02$ & $1.10 \mathrm{E}+03$ & $8.51 \mathrm{E}+00$ & $1.09 \mathrm{E}+03$ & $3.42 \mathrm{E}+02$ \\
\hline
\end{tabular}




\begin{tabular}{|c|c|c|c|c|c|c|c|c|}
\hline 39 & $4.47 \overline{\mathrm{E}+03}$ & $9.08 \mathrm{E}+02$ & $3.59 \mathrm{E}+03$ & $4.23 \mathrm{E}+02$ & $1.09 \mathrm{E}+03$ & $8.63 \mathrm{E}+00$ & $1.09 \mathrm{E}+03$ & $\overline{3.41 \mathrm{E}}+0$ \\
\hline 40 & $4.37 \mathrm{E}+03$ & $9.15 \mathrm{E}+02$ & $3.50 \mathrm{E}+03$ & $4.26 \mathrm{E}+02$ & $1.08 \mathrm{E}+03$ & $8.74 \mathrm{E}+00$ & $1.09 \mathrm{E}+03$ & $3.40 \mathrm{E}+02$ \\
\hline 41 & $4.27 \mathrm{E}+03$ & $9.21 \mathrm{E}+02$ & $3.41 \mathrm{E}+03$ & $4.29 \mathrm{E}+02$ & $1.08 \mathrm{E}+03$ & $8.85 \mathrm{E}+00$ & $1.08 \mathrm{E}+03$ & $3.39 \mathrm{E}+02$ \\
\hline 42 & $4.18 E+03$ & $9.27 \mathrm{E}+02$ & $3.32 \mathrm{E}+03$ & $4.32 \mathrm{E}+02$ & $1.12 \mathrm{E}+03$ & $9.55 \mathrm{E}+00$ & $1.08 \mathrm{E}+03$ & $1.06 \overline{\mathrm{E}+03}$ \\
\hline 43 & $4.08 \mathrm{E}+03$ & $9.34 \mathrm{E}+02$ & $3.23 \mathrm{E}+03$ & $4.35 \mathrm{E}+02$ & $1.15 \mathrm{E}+03$ & $1.03 \mathrm{E}+01$ & $1.10 \mathrm{E}+03$ & $\overline{1.07 \overline{\mathrm{E}+0}}$ \\
\hline 44 & $3.99 \mathrm{E}+03$ & $9.39 \mathrm{E}+02$ & $3.15 \mathrm{E}+03$ & $4.38 \mathrm{E}+02$ & $1.19 \mathrm{E}+03$ & $1.10 \mathrm{E}+01$ & $1.12 \mathrm{E}+03$ & $\overline{1.07 \mathrm{E}+0}$ \\
\hline 45 & $3.90 \mathrm{E}+03$ & $9.45 \mathrm{E}+02$ & $3.07 \mathrm{E}+03$ & $4.41 \mathrm{E}+02$ & $1 . \overline{23} \mathrm{E}+03$ & $1.17 \mathrm{E}+01$ & $1.14 \mathrm{E}+03$ & $1.08 \mathrm{E}+03$ \\
\hline 46 & $3.82 \mathrm{E}+03$ & $9.51 \mathrm{E}+02$ & $2.99 \mathrm{E}+03$ & $4.44 \mathrm{E}+02$ & $1 . \overline{26 \mathrm{E}+03}$ & $1.24 \mathrm{E}+01$ & $1.15 \mathrm{E}+03$ & $1.09 \mathrm{E}+0$ \\
\hline 47 & $3.74 \mathrm{E}+03$ & $9.56 \mathrm{E}+02$ & $2.92 \mathrm{E}+03$ & $4.46 \mathrm{E}+02$ & $1.30 \mathrm{E}+03$ & $1.32 \mathrm{E}+01$ & $1.17 \mathrm{E}+03$ & $1.10 E+0$ \\
\hline 48 & $3.65 \mathrm{E}+03$ & $9.61 \mathrm{E}+02$ & $2.84 \mathrm{E}+03$ & $4.49 \mathrm{E}+02$ & $1.33 \mathrm{E}+03$ & $1.39 \mathrm{E}+01$ & $1.19 \mathrm{E}+03$ & $1.10 \mathrm{E}+0$ \\
\hline 49 & $3.56 \mathrm{E}+03$ & $9.67 \mathrm{E}+02$ & $2.77 \mathrm{E}+03$ & $4.52 \mathrm{E}+02$ & $1.36 \overline{\mathrm{E}+03}$ & $1 . \overline{47 \mathrm{E}+01}$ & $1.20 \mathrm{E}+03$ & $1.11 \mathrm{E}+03$ \\
\hline 50 & $3.48 \mathrm{E}+03$ & $9.72 \bar{E}+02$ & $2.70 \mathrm{E}+03$ & $4.54 \mathrm{E}+02$ & $1.39 \mathrm{E}+03$ & $1.54 \mathrm{E}+01$ & $1.22 \mathrm{E}+03$ & $1.12 \mathrm{E}+03$ \\
\hline 51 & $3.40 \mathrm{E}+03$ & $9.76 \mathrm{E}+02$ & $2.63 \mathrm{E}+03$ & $4.57 \mathrm{E}+02$ & $1.42 \mathrm{E}+03$ & $1.62 \mathrm{E}+01$ & $1.24 \mathrm{E}+03$ & $1.12 \mathrm{E}+0$ \\
\hline 52 & $3.33 \mathrm{E}+03$ & $9.8 \overline{1 E+02}$ & $2.56 \mathrm{E}+03$ & $4.59 \mathrm{E}+02$ & $1.45 \mathrm{E}+03$ & $1.70 \mathrm{E}+01$ & $1 . \overline{25 \mathrm{E}+03}$ & $1.13 \mathrm{E}+03$ \\
\hline 53 & $3.26 \mathrm{E}+03$ & $9.8 \overline{5 E+02}$ & $2.49 \mathrm{E}+03$ & $4.61 \mathrm{E}+02$ & $1.47 \mathrm{E}+03$ & $1.77 \mathrm{E}+01$ & $1.27 \mathrm{E}+03$ & $1.13 \mathrm{E}+03$ \\
\hline 54 & $3.20 \mathrm{E}+03$ & $9.90 \mathrm{E}+02$ & $2.43 \mathrm{E}+03$ & $4.63 \mathrm{E}+02$ & $1.46 \mathrm{E}+03$ & $1.79 \mathrm{E}+01$ & $1.42 \mathrm{E}+03$ & $4.45 \mathrm{E}+02$ \\
\hline 55 & $3.14 \mathrm{E}+03$ & $9.94 \mathrm{E}+02$ & $2.37 \mathrm{E}+03$ & $4.66 \mathrm{E}+02$ & $1.45 \mathrm{E}+03$ & $1.80 \mathrm{E}+01$ & $1.41 E+03$ & $4.43 \mathrm{E}+02$ \\
\hline 56 & $3.09 \mathrm{E}+03$ & $9.98 \mathrm{E}+02$ & $2.31 \mathrm{E}+03$ & $4.68 \mathrm{E}+02$ & $1.44 \mathrm{E}+03$ & $1.81 \mathrm{E}+01$ & $1.41 \mathrm{E}+03$ & $4.41 \mathrm{E}+02$ \\
\hline 57 & $3.03 \mathrm{E}+03$ & $1.00 \mathrm{E}+03$ & $2.25 \mathrm{E}+0 \overline{3}$ & $4.70 \mathrm{E}+02$ & $1.43 \mathrm{E}+03$ & $1.83 \mathrm{E}+01$ & $1.40 \mathrm{E}+03$ & $4.40 \mathrm{E}+02$ \\
\hline 58 & $2.98 \mathrm{E}+03$ & $1.01 \mathrm{E}+03$ & $2.20 \mathrm{E}+03$ & $4.72 \mathrm{E}+02$ & $1, \overline{42} \mathrm{E}+03$ & $1.84 \mathrm{E}+01$ & $1.40 \mathrm{E}+03$ & $4 . \overline{38 \mathrm{E}+02}$ \\
\hline 59 & $2.93 \mathrm{E}+03$ & $1.01 \mathrm{E}+03$ & $2.15 \mathrm{E}+03$ & $4.74 E+02$ & $1.41 \mathrm{E}+03$ & $1.86 \mathrm{E}+01$ & $1.39 \mathrm{E}+03$ & $\overline{4.36 \mathrm{E}+02}$ \\
\hline 60 & $2.88 \mathrm{E}+03$ & $1.01 \mathrm{E}+03$ & $2.10 \mathrm{E}+03$ & $4.76 \mathrm{E}+02$ & $1.40 \mathrm{E}+03$ & $1.87 \mathrm{E}+01$ & $1.38 \mathrm{E}+03$ & $\overline{4.35 \mathrm{E}+02}$ \\
\hline 61 & $2.83 E+03$ & $1.02 \mathrm{E}+03$ & $2.05 \mathrm{E}+03$ & $4.78 \mathrm{E}+02$ & $1.38 \mathrm{E}+03$ & $1.88 \mathrm{E}+01$ & $1.38 \mathrm{E}+03$ & $4.33 \mathrm{E}+02$ \\
\hline 62 & $2.78 \mathrm{E}+03$ & $1.02 \mathrm{E}+03$ & $2.01 \mathrm{E}+03$ & $4.80 \mathrm{E}+02$ & $1.37 \mathrm{E}+03$ & $1.90 \mathrm{E}+01$ & $1.37 \mathrm{E}+03$ & $4.31 \mathrm{E}+02$ \\
\hline$\overline{63}$ & $2.73 \mathrm{E}+03$ & $1.03 \bar{E}+03$ & $1.97 \mathrm{E}+03$ & $4.81 \mathrm{E}+02$ & $1.36 \mathrm{E}+03$ & $1.91 \mathrm{E}+01$ & $1.37 \mathrm{E}+03$ & $4.30 \mathrm{E}+0$ \\
\hline 64 & $2.69 \mathrm{E}+03$ & $1.03 \mathrm{E}+03$ & $1.92 \mathrm{E}+03$ & $4.83 \mathrm{E}+02$ & $1.35 \mathrm{E}+03$ & $1.92 \mathrm{E}+01$ & $1.36 \mathrm{E}+03$ & $4.28 \mathrm{E}+0$ \\
\hline 65 & $2.65 \mathrm{E}+03$ & $1.03 \mathrm{E}+03$ & $1.88 \mathrm{E}+03$ & $4.85 \mathrm{E}+02$ & $1.34 \mathrm{E}+03$ & $1.94 \mathrm{E}+01$ & $1.36 \mathrm{E}+03$ & $4.26 \mathrm{E}+02$ \\
\hline 66 & $2.61 \mathrm{E}+03$ & $1.04 \bar{E}+03$ & $1.84 \mathrm{E}+03$ & $4.87 \mathrm{E}+02$ & $1.36 \mathrm{E}+03$ & $2.02 \mathrm{E}+01$ & $1.32 \mathrm{E}+03$ & $1.21 \mathrm{E}+03$ \\
\hline 67 & $2.57 \mathrm{E}+03$ & $1.04 \mathrm{E}+03$ & $1.81 \mathrm{E}+03$ & $4.88 \mathrm{E}+02$ & $1.38 \mathrm{E}+03$ & $2.09 \mathrm{E}+01$ & $1.33 \mathrm{E}+03$ & $1.21 \mathrm{E}+03$ \\
\hline 68 & $2.54 \mathrm{E}+03$ & $1.04 \mathrm{E}+03$ & $1.77 \mathrm{E}+03$ & $4.90 \overline{\mathrm{E}+02}$ & $1.40 \mathrm{E}+03$ & $2.17 \mathrm{E}+0 \mathrm{l}$ & $1.34 \mathrm{E}+03$ & $1.21 \mathrm{E}+03$ \\
\hline 69 & $2.50 \mathrm{E}+03$ & $1.04 E+03$ & $1.74 \mathrm{E}+03$ & $4.92 \mathrm{E}+02$ & $1.42 \mathrm{E}+03$ & $2.25 \mathrm{E}+01$ & $1.3 \overline{5 E+03}$ & $1.22 \mathrm{E}+03$ \\
\hline 168 & $5.43 \mathrm{E}+02$ & $1.13 \mathrm{E}+03$ & $5.69 \mathrm{E}+02$ & $5.89 \mathrm{E}+02$ & $1.24 \mathrm{E}+03$ & $9.00 \mathrm{E}+01$ & $1.30 \mathrm{E}+03$ & $1.25 \mathrm{E}+03$ \\
\hline$\overline{336}$ & $2.64 \mathrm{E}+02$ & $9.49 \mathrm{E}+02$ & $2.79 \mathrm{E}+02$ & $6.30 \overline{\mathrm{E}+02}$ & $6.51 \mathrm{E}+02$ & $1.89 \mathrm{E}+02$ & $8.93 \mathrm{E}+02$ & $1.03 \mathrm{E}+03$ \\
\hline 504 & $1.93 E+02$ & $7.89 \mathrm{E}+02$ & $1.91 \mathrm{E}+02$ & $6.55 \mathrm{E}+02$ & $3.65 \mathrm{E}+02$ & $2.47 \mathrm{E}+02$ & $4.76 \mathrm{E}+02$ & $7.42 \mathrm{E}+02$ \\
\hline 720 & $1.40 \mathrm{E}+02$ & $6.19 \mathrm{E}+02$ & $1.27 \mathrm{E}+02$ & $6.76 \mathrm{E}+02$ & $2.00 \mathrm{E}+02$ & $2.82 \mathrm{E}+02$ & $2.64 \mathrm{E}+02$ & $5.30 \mathrm{E}+02$ \\
\hline 2160 & $2.61 E+01$ & $1.20 \mathrm{E}+02$ & $1.43 \mathrm{E}+01$ & $7.17 \mathrm{E}+02$ & $1.78 \mathrm{E}+01$ & $1.73 \mathrm{E}+02$ & $6.37 \mathrm{E}+01$ & $1.83 \mathrm{E}+02$ \\
\hline 4320 & $2.51 \mathrm{E}+00$ & $1.05 \mathrm{E}+01$ & $7.18 \mathrm{E}+01$ & $6.82 \mathrm{E}+02$ & $2.88 \mathrm{E}+00$ & $4.16 \mathrm{E}+01$ & $7.45 \mathrm{E}+00$ & $2.79 \mathrm{E}+0$ \\
\hline 4800 & $1.49 \mathrm{E}+00$ & $6.13 \mathrm{E}+00$ & $7.16 \mathrm{E}+01$ & $6.80 \mathrm{E}+02$ & $2.09 \mathrm{E}+00$ & $2.99 \mathrm{E}+01$ & $2.45 \mathrm{E}+00$ & $9.02 \mathrm{E}+00$ \\
\hline 8760 & $2.05 \mathrm{E}-02$ & $7.75 \mathrm{E}-02$ & $6.98 \mathrm{E}+01$ & $6.63 \mathrm{E}+02$ & $1.70 \mathrm{E}-01$ & $1.98 \mathrm{E}+00$ & $7.62 \mathrm{E}-01$ & $2.65 E+00$ \\
\hline $\mathbf{T}$ & $\begin{array}{l}2.4 \mathrm{E} 6 \\
\mathrm{pCi} / \mathrm{L}\end{array}$ & $\begin{array}{l}8.88 \mathrm{E}+4 \\
\mathrm{~Bq} / \mathrm{kg}\end{array}$ & $88.8 \mathrm{~Bq} / \mathrm{g}$ & & & & & \\
\hline$\overline{\mathbf{T}}$ & $\begin{array}{l}5.9 \mathrm{E} 6 \\
\mathrm{pCi} / \mathrm{L}\end{array}$ & $\begin{array}{l}2.18 \mathrm{E}+5 \\
\mathrm{~Bq} / \mathrm{kg}\end{array}$ & $218.3 \mathrm{~Bq} / \mathrm{g}$ & & & & & \\
\hline & \multirow{2}{*}{\multicolumn{2}{|c|}{ VEGETABLES }} & \multirow{2}{*}{\multicolumn{2}{|c|}{ WHEAT SEED }} & \multirow{2}{*}{\multicolumn{2}{|c|}{$\overline{\text { MEAT }}$}} & & \\
\hline \multirow{3}{*}{ HOUR } & & & & & & & \multicolumn{2}{|l|}{ MILK } \\
\hline & & & & & & BT & HTO & $\mathrm{BT}$ \\
\hline & $3 q / g)$ & $(\mathrm{Bq} / \mathrm{g})$ & $(\mathrm{Bq} / \mathrm{g})$ & $(\mathrm{Bq} /$ & $(B q / g)$ & $3 q$ & $3 q / g)$ & $3 q / g)$ \\
\hline $\mathrm{ak} \mathrm{H}$ & & & & & & & & \\
\hline
\end{tabular}


Foodstuff Concentrations and Relocation Considerations Following

WSRC-TR-99-00118

a Tritium Oxide Release from SRS Tritium Facilities (U)

\begin{tabular}{|c|c|c|c|c|c|c|c|c|}
\hline $\begin{array}{l}10 / 12 / 53 / 5 \\
4\end{array}$ & $5.65 \mathrm{E}+04$ & $4.78 \mathrm{E}+02$ & $3.63 \mathrm{E}+04$ & $2.15 \mathrm{E}+02$ & $1.47 \mathrm{E}+03$ & $1.77 \mathrm{E}+01$ & $1.42 \mathrm{E}+03$ & $4.45 \mathrm{E}+02$ \\
\hline SUM & $5.70 \mathrm{E}+04$ & & $3.65 \mathrm{E}+04$ & & $1.49 \mathrm{E}+03$ & & $1.86 \mathrm{E}+03$ & \\
\hline $\mathrm{Ci} / \mathrm{g}$ & $1.54 \mathrm{E}-06$ & & $9.87 \mathrm{E}-07$ & & 4.03E-08 & & $5.03 \mathrm{E}-08$ & \\
\hline & & & & & & & & \\
\hline DIL HTO & $5.9 \mathrm{E}-09$ & & & & & & & \\
\hline \multicolumn{9}{|l|}{$(\mathrm{Ci} / \mathrm{g})$} \\
\hline & & & & & & & & \\
\hline 168 & $5.43 \mathrm{E}+02$ & $1.13 \mathrm{E}+03$ & $5.69 \mathrm{E}+02$ & $5.89 \mathrm{E}+02$ & $1.24 \mathrm{E}+03$ & $9.00 \mathrm{E}+01$ & $1.30 \mathrm{E}+03$ & $1.25 \bar{E}+03$ \\
\hline SUM & $1.67 \mathrm{E}+03$ & & $1.16 \mathrm{E}+03$ & & $1.33 \mathrm{E}+03$ & & $2.55 \mathrm{E}+03$ & \\
\hline $\mathrm{Ci} / \mathrm{g}$ & $4.51 \mathrm{E}-08$ & & $3.13 \mathrm{E}-08$ & & $3.60 \mathrm{E}-08$ & & $6.89 \mathrm{E}-08$ & \\
\hline DI ORT & 20500 & & & & & & & \\
\hline \multicolumn{9}{|l|}{$\mathrm{Ci} / \mathrm{g}$} \\
\hline & & & & & & & & \\
\hline 8760 & $2.05 \mathrm{E}-02$ & $7.75 \mathrm{E}-02$ & $6.98 \mathrm{E}+01$ & $6.63 \mathrm{E}+02$ & $1.70 \mathrm{E}-01$ & $1.98 \mathrm{E}+00$ & $7.62 \mathrm{E}-01$ & $2.65 \mathrm{E}+00$ \\
\hline SUM & $9.80 \mathrm{E}-02$ & & $7.32 \mathrm{E}+02$ & & $2.15 \mathrm{E}+00$ & & $3.41 \mathrm{E}+00$ & \\
\hline $\mathrm{Ci} / \mathrm{g}$ & $2.65 \mathrm{E}-12$ & & $1.98 \mathrm{E}-08$ & & $5.82 \mathrm{E}-11$ & & $9.22 \mathrm{E}-11$ & \\
\hline DIL OBT & $2.4 \mathrm{E}-09$ & & & & & & & \\
\hline $\mathrm{Ci} / \mathrm{g}$ & & & & & & & & \\
\hline
\end{tabular}

